\title{
Highly Enantioselective Cross-Electrophile Aryl-Alkenylation of Unactivated Alkenes
}

Zhi-Xiong Tian, Jin-Bao Qiao, Guang-Li Xu, Xiaobo Pang, Liangliang Qi, Wei-Yuan Ma, Zhen-Zhen Zhao, Jicheng Duan, Yun-Fei Du, Peifeng Su, Xue-Yuan Liu, Xing-Zhong Shu*

State Key Laboratory of Applied Organic Chemistry (SKLAOC), College of Chemistry and Chemical Engineering, Lanzhou University, 222 South Tianshui Road, Lanzhou, 730000, China.

\section{Table of Contents}

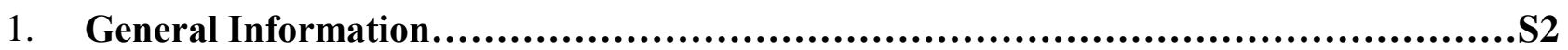

2. Optimization of Reaction Parameters.................................................

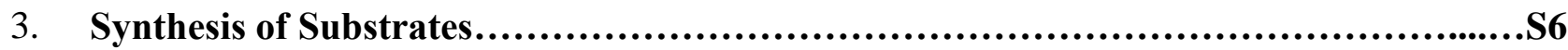

4. Ni-catalyzed Enantioselective Cross-electrophile Aryl-alkenylation of Alkene..............S22

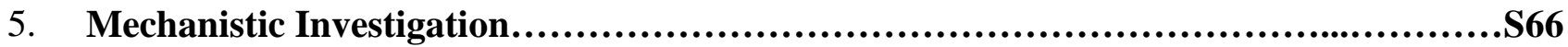

6. Crystallographic Data for Compound 3z.............................................S70

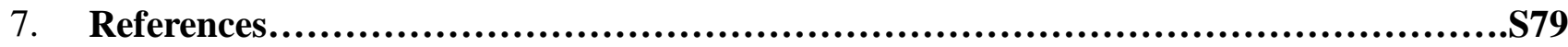

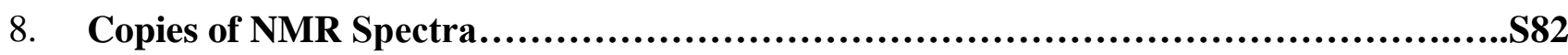




\section{General Information}

All reactions were carried out under an atmosphere of argon in sealed tube with magnetic stirring. Dry DMF, THF, $\mathrm{CH}_{2} \mathrm{Cl}_{2}$ were purified using a solvent-purification system that contained activated alumina and molecular sieves. Other solvents were dried and purified according to the procedure from "Purification of Laboratory Chemicals". 1

Nickel catalysts, reductants were purchased from Acros, Alfa Aesar, Aldrich, Ark Pharm, and Strem. Other chemicals were purchased from TCI, Adamas, and Energy chemicals, and were directly used without further purifications.

${ }^{1} \mathrm{H}$ and ${ }^{13} \mathrm{C}$ NMR spectra were collected on a Bruker AVANCE III 400MHz, JEOL JNM-ECS 400M and Agilent-NMR-inova $600 \mathrm{MHz}$ spectrometer at room temperature. ${ }^{1} \mathrm{H}$ NMR spectra were reported in parts per million (ppm) downfield of tetramethylsilane (TMS) and were referenced to the signal of TMS (0 ppm). ${ }^{13} \mathrm{C}$ NMR spectra were reported in ppm relative to residual $\mathrm{CHCl}_{3}(77.00 \mathrm{ppm})$. Coupling constants, $J$, are reported in hertz $(\mathrm{Hz}) .{ }^{19} \mathrm{~F}$ NMR spectra were also collected on Bruker AVANCE III $400 \mathrm{MHz}$ spectrometers and Agilent-NMR-inova $600 \mathrm{MHz}$ spectrometer at room temperature. Melting points were determined on a microscopic apparatus. IR spectra were collected using Bruker-TENSOR 27 spectrometer and Agilent Technologies Cary 630 FTIR, and only major peaks were reported in $\mathrm{cm}^{-1}$. HRMS was performed on Bruker Apex II FT-ICR mass instrument (ESI). GC analysis was performed on Thermo Scientific TRACE 1300. GC-MS data was collected on Thermo Scientific TRACE DSQ GC-MS. The enantiomeric excess (ee) of the products was determined by chiral HPLC (Thermo Scientific UltiMate 3000) using Daicel CHIRALCEL® columns and Daicel CHIRALPAK® columns (internal diameter $4.6 \mathrm{~mm}$, column length $250 \mathrm{~mm}$, particle size $5 \mu \mathrm{m})$. Optical rotations were measured on an AUTOPOL IV Automatic polarimeter (Rudolph Research Analytical). The X-RAY was measured on Agilent SUPERNOVA. Thin layer chromatography was carried out using XINNUO SGF254 TLC plates. Flash chromatography was performed using XINNUO silica gel (200-300 mesh). 


\section{Optimization of Reaction Parameters}

\section{General Procedure}

The procedure was conducted in an argon-filled glove box. To a reaction tube equipped with a magnetic stir bar was charged with catalyst (10 mol \%, $0.010 \mathrm{mmol}), \mathbf{L 1}(14 \mathrm{~mol} \%, 3.8 \mathrm{mg}, 0.014$ mmol), reductant (4 equiv, $0.4 \mathrm{mmol})$, and solvent $(0.5 \mathrm{~mL})$. The reaction mixture was stirred for 5 min. Substrates $\mathbf{1 a}(27.4 \mathrm{mg}, 0.1 \mathrm{mmol})$ and $\mathbf{2 a}(23.0 \mathrm{mg}, 0.1 \mathrm{mmol})$ were then added. The reaction tube was sealed with a rubber septum, and removed from the glove box. The reaction mixture was stirred at appreciate temperature for $24 \mathrm{~h}$. The reaction mixture was diluted with ethyl acetate (10 $\mathrm{mL}$ ), washed with water, brine, and dried over anhydrous $\mathrm{Na}_{2} \mathrm{SO}_{4}$. A $0.2 \mathrm{~mL}$ of solution was collected, diluted with ethyl acetate $(2 \mathrm{~mL})$, and analyzed by GC. The yield was determined versus the internal standard (dodecane). The rest solution was concentrated under the reduced pressure, and part of the residue was purified by thin layer chromatography on silica gel. The enantiomeric excess (ee) of the products was determined by chiral HPLC. 
Table S1. Effect of reductant, solvent and temperature ${ }^{a}$<smiles>C=C(C)COc1ccccc1I</smiles>

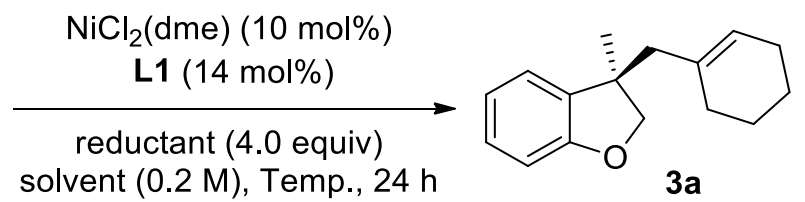

\begin{tabular}{|c|c|c|c|c|c|}
\hline entry & reductant & solvent & temperature & yield $(\%)$ & ee $(\%)$ \\
\hline 1 & $\mathrm{Mn}$ & DMF & rt. & 59 & 93 \\
\hline 2 & $\mathrm{Zn}$ & DMF & rt. & trace & - \\
\hline 3 & $\mathrm{Mg}$ & DMF & rt. & 0 & - \\
\hline 4 & Mn & $\mathrm{CH}_{3} \mathrm{CN}$ & rt. & 0 & - \\
\hline 5 & Mn & DMSO & rt. & 48 & 90 \\
\hline 6 & Mn & DMA & rt. & 52 & 91 \\
\hline 7 & $\mathrm{Mn}$ & Toluene & rt. & 0 & - \\
\hline 8 & Mn & THF & $\mathrm{rt}$. & trace & - \\
\hline 9 & Mn & Dioxane & rt. & 0 & - \\
\hline 10 & Mn & $\mathrm{DMF} / \mathrm{THF}(4 / 1)$ & $\mathrm{rt}$. & 60 & 94 \\
\hline 11 & $\mathrm{Mn}$ & $\mathrm{DMF} / \mathrm{THF}(3 / 2)$ & rt. & 63 & 94 \\
\hline 12 & $\mathrm{Mn}$ & DMF/THF(1/1) & rt. & 67 & 95 \\
\hline 13 & $\mathrm{Mn}$ & $\mathrm{DMF} / \mathrm{THF}(2 / 3)$ & rt. & 55 & 95 \\
\hline 14 & Mn & DMF/THF(1/4) & rt. & 31 & 94 \\
\hline 15 & Mn & $\mathrm{DMF} / \mathrm{THF}(1 / 1)$ & $0{ }^{\circ} \mathrm{C}$ & trace & - \\
\hline 16 & $\mathrm{Mn}$ & DMF/THF(1/1) & $10^{\circ} \mathrm{C}$ & 34 & 95 \\
\hline 17 & Mn & $\mathrm{DMF} / \mathrm{THF}(1 / 1)$ & $40{ }^{\circ} \mathrm{C}$ & 60 & 95 \\
\hline 18 & Mn & DMF/THF(1/1) & $60^{\circ} \mathrm{C}$ & 54 & 93 \\
\hline
\end{tabular}

${ }^{a} \mathbf{1 a}(0.1 \mathrm{mmol})$ and $\mathbf{2 a}(0.1 \mathrm{mmol})$ was used. The yields were determined by GC analysis with doecane as an internal standard. The ees were determined by chiral HPLC. 
Table S2. Effect of catalyst ${ }^{\mathrm{a}}$

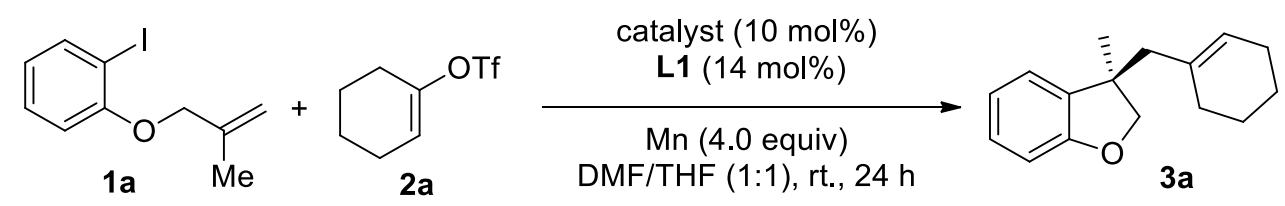

\begin{tabular}{cccc}
\hline entry & catalyst & yield $(\%)$ & ee $(\%)$ \\
\hline 1 & $\mathrm{NiF}_{2}$ & 0 & - \\
2 & $\mathrm{NiCl}_{2}$ & 30 & 91 \\
3 & $\mathrm{NiBr}_{2}$ & 69 & 97 \\
4 & $\mathrm{NiI}_{2}$ & $79(77)^{\mathrm{b}}$ & 98 \\
5 & $\mathrm{NiCl}_{2}(\mathrm{dme})$ & 67 & 95 \\
6 & $\mathrm{Ni}^{2}(\mathrm{cod})_{2}$ & 68 & 95 \\
7 & $\mathrm{NiCl}_{2}(\mathrm{dppp})$ & 45 & 94 \\
8 & $\mathrm{NiCl}_{2}(\mathrm{dppf})$ & 0 & - \\
9 & $\mathrm{CoCl}_{2}$ & 0 & - \\
10 & $\mathrm{CoBr}_{2}$ & 0 & - \\
11 & $\mathrm{PdCl}_{2}$ & 0 & - \\
12 & $\mathrm{CuCl}_{2}$ & 0 & - \\
\hline
\end{tabular}

${ }^{a} \mathbf{1 a}(0.1 \mathrm{mmol})$ and $\mathbf{2 a}(0.1 \mathrm{mmol})$ was used. The yields were determined by GC analysis with doecane as an internal standard. The ees were determined by chiral HPLC. ${ }^{b}$ Isolated yield. 


\section{Synthesis of Substrates}

\subsection{Synthesis of Aryl Iodide tethered Alkenes}

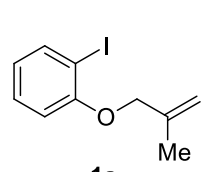

$1 \mathrm{a}$<smiles>C=C(C)COc1ccc(CC(C)(C)C)cc1I</smiles>

$1 \mathrm{~b}$

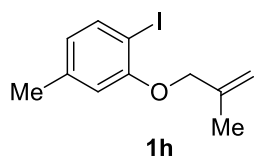

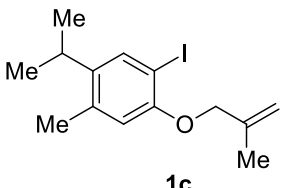

$1 c$

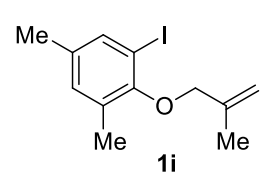

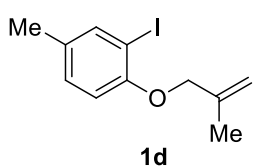
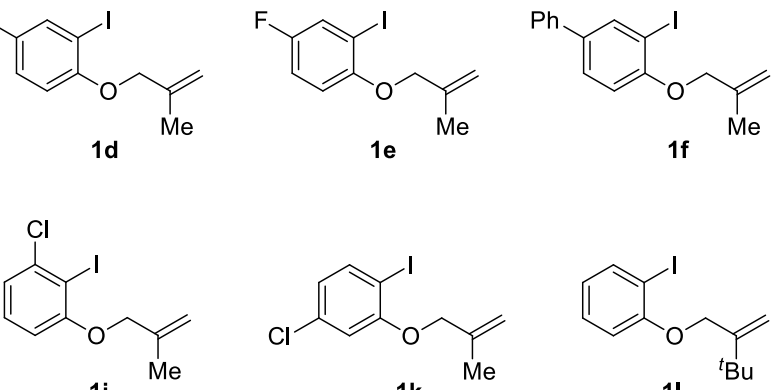

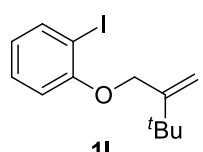

$1 \mathrm{k}$

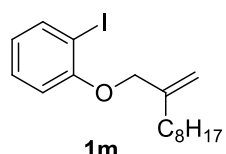<smiles>C=C(CCCCCCCC)COc1ccccc1I</smiles><smiles>C=CCCOCC(=C)COc1ccccc1I</smiles><smiles>C=C(C)CCOc1ccccc1I</smiles><smiles>C=C(C)CCCOc1ccccc1I</smiles><smiles>C=C(C)COc1ccc(C(=O)N2CCCCC2)cc1I</smiles><smiles>C=CCOc1ccccc1I</smiles>

$1 \mathrm{~s}$

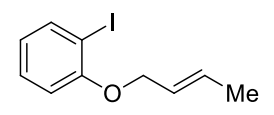

$1 t$

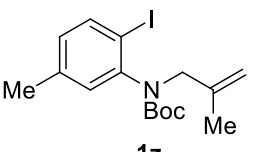

$1 z$

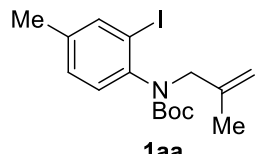<smiles>CC=C(C)COc1ccccc1I</smiles>

1v

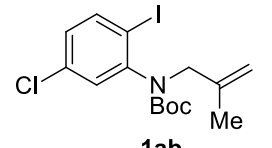

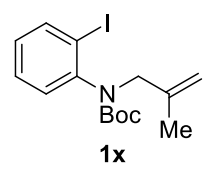

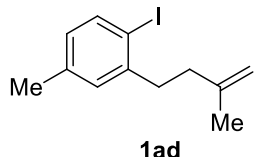

ad<smiles>C=C(C)CCc1cc2c(cc1I)OCO2</smiles>

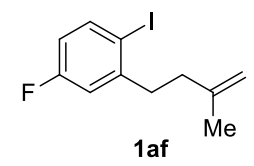

af $\mathrm{M}$

Known compounds $\mathbf{1 a},{ }^{2} \mathbf{1 b},{ }^{2} \mathbf{1 d},{ }^{3} \mathbf{1 e},{ }^{4} \mathbf{1 f},{ }^{5} \mathbf{1 h}^{5}, \mathbf{1 k}^{6}, \mathbf{1 y}^{2}$, were prepared according to the literature procedure in ref.2. Known compound $1 \mathbf{n}^{7}, \mathbf{1} \mathbf{p}^{2}, \mathbf{1 s}^{8}, \mathbf{1 t}^{9}, \mathbf{1} \mathbf{u}^{10}, \mathbf{1} \mathbf{v}^{11}, \mathbf{1} \mathbf{w}^{12}$, was prepared according to the literature procedure in ref.13. Known compound $\mathbf{1} \mathbf{x}^{14}$ was prepared according to the literature procedure in ref.14, Known compound $1 \mathbf{a c}^{15}$ was prepared according to the literature procedure in ref.15. The preparation of new compounds, and their characterization data are provided as follows. 


\section{General procedure A:}

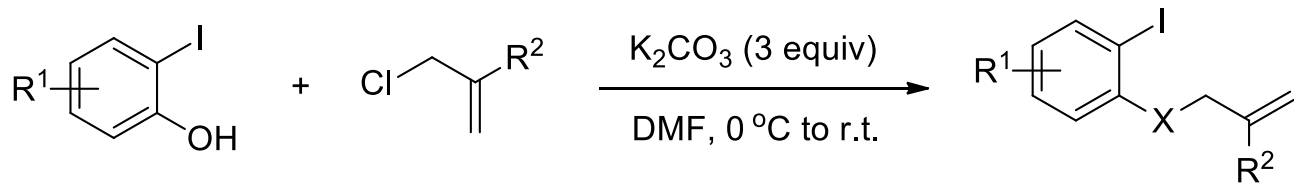

These compounds were synthesized according to the literature procedure. ${ }^{2}$ To a solution of phenol (10.0 mmol) in DMF (30.0 mL) was added $\mathrm{K}_{2} \mathrm{CO}_{3}(4.15 \mathrm{~g}, 30.0 \mathrm{mmol})$ at $0{ }^{\circ} \mathrm{C}$, followed by slowly addition of allyl halide $(10.0 \mathrm{mmol})$ after $20 \mathrm{~min}$. The reaction mixture was allowed to warm to room temperature and stirred overnight. The reaction was quenched with water $(40.0 \mathrm{~mL})$, and extracted with ethyl acetate $(3 \times 30.0 \mathrm{~mL})$. The combined organic layers were washed with saturated aqueous $\mathrm{Na}_{2} \mathrm{~S}_{2} \mathrm{O}_{3}$, brine, dried over anhydrous $\mathrm{Na}_{2} \mathrm{SO}_{4}$ and concentrated under reduced pressure. The residue was purified by flash chromatography on silica gel to give the product.

\section{General procedure B:}

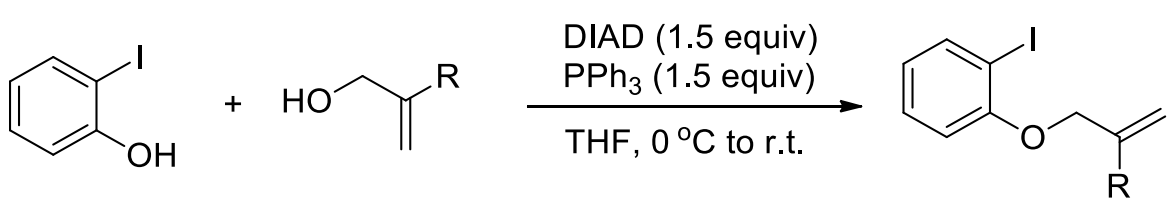

These compounds were synthesized according to the literature procedure. ${ }^{13}$ To a solution of alcohol $(10.0 \mathrm{mmol})$ and phenol $(10.0 \mathrm{mmol})$ in THF $(30.0 \mathrm{~mL})$ was added $\mathrm{PPh}_{3}(2.62 \mathrm{~g}, 10.0 \mathrm{mmol})$ at $0{ }^{\circ} \mathrm{C}$, followed by slowly addition of diisopropyl azodicarboxylate (DIAD, $2.02 \mathrm{~g}, 10.0 \mathrm{mmol}$ ) after 20 min under argon. The reaction mixture was allowed to warm to room temperature and stirred overnight. The reaction was quenched with water $(40.0 \mathrm{~mL})$, and extracted with ethyl acetate $(3 \times$ $30.0 \mathrm{~mL}$ ). The combined organic layers were washed with saturated aqueous $\mathrm{Na}_{2} \mathrm{~S}_{2} \mathrm{O}_{3}$, brine, dried over anhydrous $\mathrm{Na}_{2} \mathrm{SO}_{4}$ and concentrated under reduced pressure. The residue was purified by flash chromatography on silica gel to give the product.

\section{1-Iodo-5-isopropyl-4-methyl-2-((2-methylallyl)oxy)benzene (1c)}

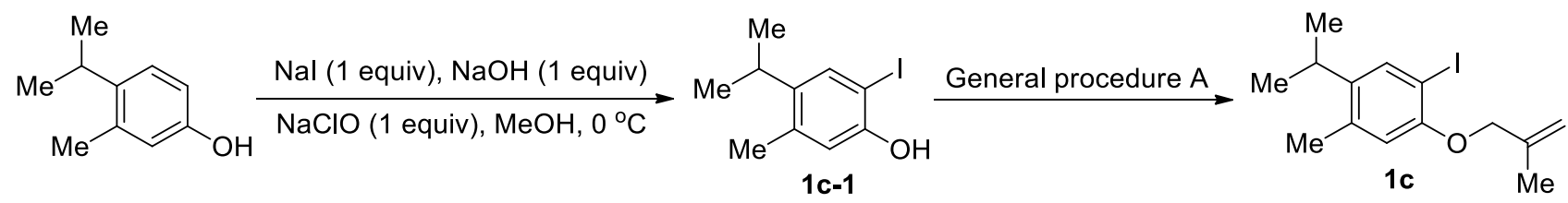

Step 1: Compound 1c-1 was synthesized according to the literature procedure. ${ }^{16}$ To a solution of phenol $(10.0 \mathrm{mmol})$ in $\mathrm{MeOH}(30.0 \mathrm{~mL})$ at $0{ }^{\circ} \mathrm{C}$ was added $\mathrm{NaI} \cdot 2 \mathrm{H}_{2} \mathrm{O}(1.86 \mathrm{~g}, 10.0 \mathrm{mmol})$ and 
$\mathrm{NaOH}(0.40 \mathrm{~g}, 10.0 \mathrm{mmol})$, followed by slowly addition of $\mathrm{NaClO}(15.0 \mathrm{ml}, 10.0 \mathrm{mmol}, 5 \%$ aqueous solution,) after $20 \mathrm{~min}$. The reaction mixture was stirred overnight at the same temperature. The $\mathrm{MeOH}$ was removed under reduced pressure, and water $(30.0 \mathrm{~mL})$ was added. The reaction mixture was neutralized with aqueous $\mathrm{HCl}(2.0 \mathrm{M})$ to $\mathrm{pH}<7$, and extracted with ethyl acetate $(3 \times$ $20.0 \mathrm{~mL}$ ). The combined organic layers were washed with water, saturated aqueous $\mathrm{Na}_{2} \mathrm{~S}_{2} \mathrm{O}_{3}$, brine, dried over anhydrous $\mathrm{Na}_{2} \mathrm{SO}_{4}$, and concentrated under reduced pressure. The residue was purified by flash chromatography on silica gel to give 2-iodo-4-isopropyl-5-methylphenol (1c-1).

$1.55 \mathrm{~g}, 56 \%$ yield, white solid, $\mathrm{mp}: 40-42{ }^{\circ} \mathrm{C}, \mathrm{R}_{\mathrm{f}}=0.3$ (silica gel, petroleum ether/ethyl acetate $=$ 10:1).

${ }^{1}$ H NMR (400 MHz, CDCl 3 ): $\delta 7.43(\mathrm{~s}, 1 \mathrm{H}), 6.78(\mathrm{~s}, 1 \mathrm{H}), 5.05(\mathrm{~s}, 1 \mathrm{H})$, 3.03-2.97 (m, $\left.1 \mathrm{H}\right), 2.25$ (s, $3 \mathrm{H}), 1.18(\mathrm{~d}, J=6.8 \mathrm{~Hz}, 6 \mathrm{H})$.

${ }^{13}$ C NMR (100 MHz, $\left.\mathbf{C D C l}_{3}\right): \delta$ 152.3, 141.6, 137.7, 134.3, 116.6, 82.4, 28.6, 23.3, 19.0.

IR (neat, $\mathbf{c m}^{-1}$ ): 2963, 1481, 1459, 1398, 1299, 1269, 1200, 880, 762, 725.

HRMS (ESI): $[\mathrm{M}+\mathrm{H}]^{+}$calcd for $\mathrm{C}_{10} \mathrm{H}_{14} \mathrm{IO} 277.0084$, found 277.0093 .

Step 2: Compound 1c was prepared from 2-iodo-4-isopropyl-5-methylphenol (1c-1, 1.38 g, 5.0 $\mathrm{mmol})$ and 3-chloro-2-methylprop-1-ene $(0.45 \mathrm{~g}, 5.0 \mathrm{mmol})$ according to the General procedure A. $1.39 \mathrm{~g}, 84 \%$ yield, colorless oil, $\mathrm{R}_{\mathrm{f}}=0.7$ (silica gel, petroleum ether).

${ }^{1}$ H NMR (400 MHz, CDCl $)$ ) $\delta 7.56$ (s, 1 H), 6.57 (s, $\left.1 \mathrm{H}\right), 5.19$ (s, $\left.1 \mathrm{H}\right), 5.00$ (s, $\left.1 \mathrm{H}\right), 4.43$ (s, 2 H), 3.03-2.96 (m, $1 \mathrm{H}), 2.27(\mathrm{~s}, 3 \mathrm{H}), 1.86(\mathrm{~s}, 3 \mathrm{H}), 1.18(\mathrm{~d}, J=6.8 \mathrm{~Hz}, 6 \mathrm{H})$.

${ }^{13}$ C NMR (100 MHz, $\left.\mathbf{C D C l}_{3}\right): \delta 154.8,141.4,140.5,136.4,135.7,114.4,112.6,83.3,72.6,28.6$, $23.3,19.5,19.4$.

IR (neat, $\mathbf{c m}^{-\mathbf{1}}$ ): 2963, 2872, 1654, 1591, 1490, 1252, 1053, 1030, 902, 716.

HRMS (ESI): $[\mathrm{M}+\mathrm{H}]^{+}$calcd for $\mathrm{C}_{14} \mathrm{H}_{20} \mathrm{IO} 331.0553$, found 331.0562 .

\section{2-Iodo-1-methyl-3-((2-methylallyl)oxy)benzene (1g)}

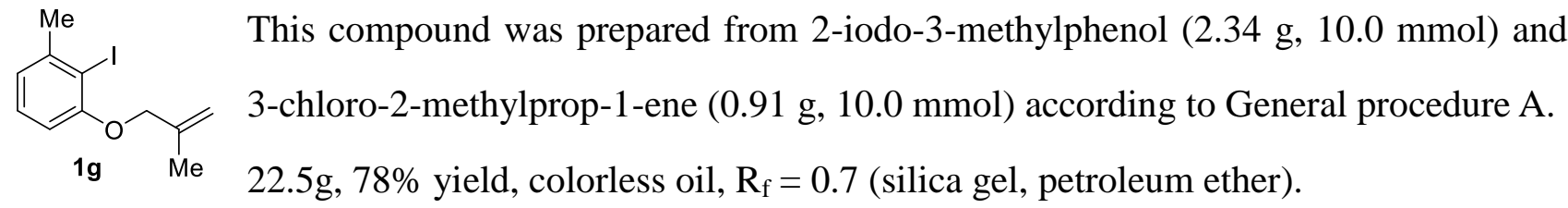

${ }^{1}$ H NMR (400 MHz, CDCl 3 ): $\delta 7.15(\mathrm{t}, J=8.0 \mathrm{~Hz}, 1 \mathrm{H}), 6.87(\mathrm{~d}, J=8.0 \mathrm{~Hz}, 1 \mathrm{H}), 6.60(\mathrm{~d}, J=8.0$ Hz, $1 \mathrm{H}), 5.22$ (s, $1 \mathrm{H}), 5.01$ (s, $1 \mathrm{H}), 4.47$ (s, $2 \mathrm{H}), 2.47$ (s, $3 \mathrm{H}), 1.88$ (s, $3 \mathrm{H})$.

${ }^{13}$ C NMR (100 MHz, $\left.\mathbf{C D C l}_{3}\right): \delta 157.2,143.5,140.4,128.5,122.4,112.8,109.2,93.6,72.7,28.8$, 19.5 . 
IR (neat, $\mathbf{c m}^{-\mathbf{1}}$ ): 3290, 2918, 1959, 1650, 1565, 1450, 1260, 1057, 902, 764.3

HRMS (ESI): $[\mathrm{M}+\mathrm{H}]{ }^{+}$calcd for $\mathrm{C}_{11} \mathrm{H}_{14} \mathrm{IO} 289.0084$, found 289.0091.

\section{1-Iodo-3,5-dimethyl-2-((2-methylallyl)oxy)benzene (1i)}

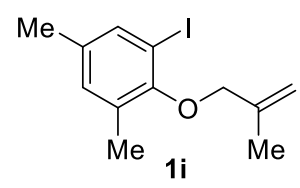

This compound was prepared from 2-iodo-4,6-dimethylphenol (2.48 g, 10.0 $\mathrm{mmol}$ ) and 3-chloro-2-methylprop-1-ene (0.91 g, $10.0 \mathrm{mmol})$ according to General procedure A.

$2.48 \mathrm{~g}, 82 \%$ yield, colorless oil, $\mathrm{R}_{\mathrm{f}}=0.7$ (silica gel, petroleum ether).

${ }^{1}$ H NMR (400 MHz, CDCl $): \delta 7.43$ (s, 1 H), 6.94 (s, 1 H), 5.19 (s, 1 H), 5.01 (s, 1 H), 4.23 (s, 2 H), 2.29 (s, $3 \mathrm{H}), 2.23(\mathrm{~s}, 3 \mathrm{H}), 1.93$ (s, $3 \mathrm{H})$.

${ }^{13}$ C NMR (100 MHz, $\left.\mathbf{C D C l}_{3}\right)$ : $\delta$ 154.6, 141.2, 137.3, 135.6, 132.2, 131.8, 113.0, 91.8, 76.04, 20.2, 19.9, 17.0.

IR (neat, $\mathbf{c m}^{-\mathbf{1}}$ ): 3077, 2973, 2858, 1653, 1469, 1272, 1123, 1041, 995, 853.

HRMS (ESI): $[\mathrm{M}+\mathrm{H}]^{+}$calcd for $\mathrm{C}_{12} \mathrm{H}_{16} \mathrm{IO} 303.0240$, found 303.0247.

\section{1-Chloro-2-iodo-3-((2-methylallyl)oxy)benzene (1j)}

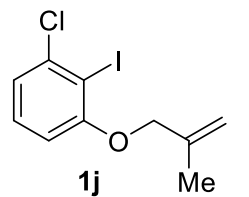

This compound was prepared from 3-chloro-2-iodophenol (2.54 g, $10.0 \mathrm{mmol})$ and 3-chloro-2-methylprop-1-ene (0.91 g, $10.0 \mathrm{mmol})$ according to General procedure A. $22.5 \mathrm{~g}, 78 \%$ yield, colorless oil, $\mathrm{R}_{\mathrm{f}}=0.7$ (silica gel, petroleum ether).

${ }^{1}$ H NMR (400 MHz, CDCl $): \delta 7.20(\mathrm{t}, J=8.0 \mathrm{~Hz}, 1 \mathrm{H}), 7.08(\mathrm{~d}, J=8.0 \mathrm{~Hz}, 1 \mathrm{H}), 6.65(\mathrm{~d}, J=8.0$ Hz, $1 \mathrm{H}), 5.20$ (s, $1 \mathrm{H}), 5.03$ (s, $1 \mathrm{H}), 4.48$ (s, $2 \mathrm{H}), 1.87$ (s, $3 \mathrm{H})$.

${ }^{13}$ C NMR (100 MHz, $\left.\mathbf{C D C l}_{3}\right): \delta 158.9,139.8,139.8,129.6,121.9,113.2,109.7,91.7,73.0,19.5$.

IR (neat, $\mathbf{c m}^{-1}$ ): 2975, 2920, 1572, 1440, 1259, 1060, 1014, 904, 766, 697

HRMS (ESI): $[\mathrm{M}+\mathrm{H}]{ }^{+}$calcd for $\mathrm{C}_{10} \mathrm{H}_{11} \mathrm{ClIO} 308.9538$, found 308.9542 .

\section{1-(3, 3-Dimethyl-2-methylenebutoxy)-2-iodobenzene (11)}

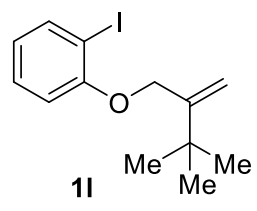

This compound was prepared from 2-iodophenol (2.20 g, $10.0 \mathrm{mmol})$ and 3,3-dimethyl-2-methylenebutan-1-ol (1.14 g, $10.0 \mathrm{mmol})$ according to General procedure B.

$1.96 \mathrm{~g}, 62 \%$ yield, colorless oil, $\mathrm{R}_{\mathrm{f}}=0.8$ (silica gel, petroleum ether).

${ }^{1}$ H NMR (400 MHz, $\left.\mathbf{C D C l}_{3}\right): \delta 7.77(\mathrm{dd}, J=1.6,8.0 \mathrm{~Hz}, 1 \mathrm{H}), 7.29-7.24(\mathrm{~m}, 1 \mathrm{H}), 6.79$ (dd, $J=1.2$, $8.4 \mathrm{~Hz}, 1 \mathrm{H}), 6.69$ (m, $1 \mathrm{H}), 5.33(\mathrm{~d}, J=1.2 \mathrm{~Hz}, 1 \mathrm{H}), 5.13$ (d, $J=0.8 \mathrm{~Hz}, 1 \mathrm{H}), 4.61(\mathrm{~s}, 2 \mathrm{H}), 1.18$ (s, $9 \mathrm{H})$. 
${ }^{13}$ C NMR (100 MHz, $\left.\mathbf{C D C l}_{3}\right): \delta 157.3,151.2,139.5,129.3,122.4,112.1,110.0,86.4,69.1,34.8$, 29.5 .

IR (neat, $\mathbf{c m}^{-1}$ ): 2961, 2868, 1638, 1582, 1472, 1438, 1274, 1019, 909, $747 \mathrm{~cm}^{-1}$.

HRMS (ESI): $[\mathrm{M}+\mathrm{H}]^{+}$calcd for $\mathrm{C}_{13} \mathrm{H}_{18} \mathrm{IO} 317.0397$, found 317.0400.

\section{1-Iodo-2-((2-methylenedecyl)oxy)benzene (1m)}

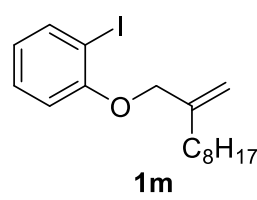

This compound was prepared from 2-iodophenol (2.20 g, $10.0 \mathrm{mmol})$ and 2-methylenedecan-1-ol (1.70 g, $10.0 \mathrm{mmol})$ according to the General procedure B. $2.42 \mathrm{~g}, 65 \%$ yield, colorless oil, $\mathrm{R}_{\mathrm{f}}=0.8$ (silica gel, petroleum ether).

${ }^{1}$ H NMR (400 MHz, $\left.\mathbf{C D C l}_{3}\right): \delta 7.77(\mathrm{dd}, J=1.6,7.6 \mathrm{~Hz}, 1 \mathrm{H}), 7.29-7.25(\mathrm{~m}, 1 \mathrm{H}), 6.79$ (dd, $J=0.8$, $8.4 \mathrm{~Hz}, 1 \mathrm{H}), 6.70(\mathrm{~m}, 1 \mathrm{H}), 5.22(\mathrm{~s}, 1 \mathrm{H}), 5.01(\mathrm{~d}, J=0.8 \mathrm{~Hz}, 1 \mathrm{H}), 4.50(\mathrm{~s}, 2 \mathrm{H}), 2.18(\mathrm{t}, J=8.0 \mathrm{~Hz}$, $2 \mathrm{H}), 1.54-1.47$ (m, $2 \mathrm{H}), 1.31-1.27(\mathrm{~m}, 10 \mathrm{H}), 0.88$ (t, $J=6.4 \mathrm{~Hz}, 3 \mathrm{H})$.

${ }^{13}$ C NMR (100 MHz, $\left.\mathbf{C D C l}_{3}\right): \delta$ 157.2, 144.3, 139.5, 129.3, 122.5, 112.3, 111.9, 86.6, 71.6, 33.2, $31.9,29.44,29.41,29.3,27.6,22.7,14.1$.

IR (neat, $\mathbf{c m}^{-\mathbf{1}}$ ): 2926, 2857, 1582, 1472, 1439, 1291, 1244, 1019, 746, 727.

HRMS (ESI): $[\mathrm{M}+\mathrm{H}]^{+}$calcd for $\mathrm{C}_{17} \mathrm{H}_{26} \mathrm{IO} 373.1023$, found 373.1024 .

\section{1-((2-((But-3-en-1-yloxy)methyl)allyl)oxy)-2-iodobenzene (10)}

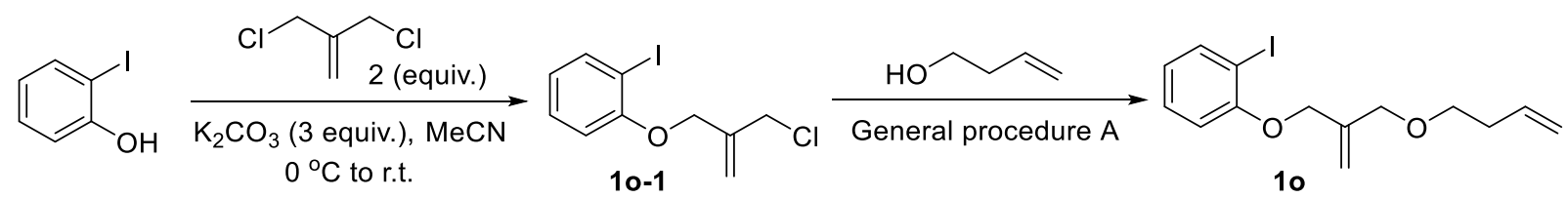

Compound 1o-1 was synthesized according to the literature procedure. ${ }^{17}$ To a solution of $\mathrm{K}_{2} \mathrm{CO}_{3}$ (4.15 g, $30.0 \mathrm{mmol}), 3$-chloro-2-(chloromethyl)prop-1-ene $(2.32 \mathrm{~mL}, 20.0 \mathrm{mmol})$ in acetonitrile $(25.0 \mathrm{~mL})$ was added 2-iodophenol $(2.20 \mathrm{~g}, 10.0 \mathrm{mmol})$ at $0{ }^{\circ} \mathrm{C}$. The reaction mixture was stirred at room temperature overnight, and filtered through a pad of celite. The filtrate was concentrated under reduced pressure. The residue was purified by flash chromatography on silica gel to afford $2.16 \mathrm{~g}$ of 10-1 (93\% purity, mixed with dichloromethyl ethylene).

Compound 1o was prepared from the above crude 1o-1 and but-3-en-1-ol (0.50 g, $7.0 \mathrm{mmol})$ according to the General procedure A.

$1.69 \mathrm{~g}, 49 \%$ yield for two steps, colorless oil, $\mathrm{R}_{\mathrm{f}}=0.3$ (silica gel, petroleum ether/ethyl acetate $=$ 50:1).

${ }^{1}$ H NMR (400 MHz, $\left.\mathbf{C D C l}_{3}\right): \delta 7.77$ (dd, $\left.J=8.0,1.6 \mathrm{~Hz}, 1 \mathrm{H}\right), 7.30-7.25(\mathrm{~m}, 1 \mathrm{H}), 6.82(\mathrm{dd}, J=8.4$, $1.2 \mathrm{~Hz}, 1 \mathrm{H}), 6.71(\mathrm{~m}, 1 \mathrm{H}), 5.87-5.77(\mathrm{~m}, 1 \mathrm{H}), 5.43(\mathrm{~d}, J=0.4 \mathrm{~Hz}, 1 \mathrm{H}), 5.29(\mathrm{~d}, J=0.8 \mathrm{~Hz}, 1 \mathrm{H})$, 
5.12-5.01 (m, 2 H), 4.59 (s, 2 H), 4.13 (s, 2 H), 3.52 (t, J = 6.8 Hz, 2 H), 2.38-2.33 (m, 2 H).

${ }^{13}$ C NMR (100 MHz, $\left.\mathbf{C D C l}_{3}\right): \delta 157.0,140.9,139.4,135.2,129.3,122.6,116.4,114.8,112.2,86.5$, $71.6,69.7,69.4,34.2$.

IR (neat, $\mathbf{c m}^{-\mathbf{1}}$ ): 3071, 2857, 1582, 1474, 1440, 1247, 1098, 1018, 917, 747.

HRMS (ESI): $[\mathrm{M}+\mathrm{H}]^{+}$calcd for $\mathrm{C}_{14} \mathrm{H}_{18} \mathrm{IO}_{2} 345.0346$, found 345.0352.

\section{1-Iodo-2-((4-methylpent-4-en-1-yl)oxy)benzene (1q)}

This compound was prepared from 2-iodophenol (2.20 g, $10.0 \mathrm{mmol})$ and 1q $\mathrm{Me}$ 4-methylpent-4-en-1-ol (1.00 g, $10.0 \mathrm{mmol})$ according to General procedure B. $2.05 \mathrm{~g}, 68 \%$ yield, colorless oil, $\mathrm{R}_{\mathrm{f}}=0.7$ (silica gel, petroleum ether).

${ }^{1}$ H NMR (400 MHz, $\left.\mathbf{C D C l}_{3}\right): \delta 7.76(\mathrm{dd}, J=1.6 \mathrm{~Hz}, J=7.6 \mathrm{~Hz}, 1 \mathrm{H}), 7.29-7.24$ (m, $\left.1 \mathrm{H}\right), 6.78$ (dd, $J=0.8 \mathrm{~Hz}, J=8.4 \mathrm{~Hz}, 1 \mathrm{H}), 6.68(\mathrm{dt}, J=1.2 \mathrm{~Hz}, J=7.6 \mathrm{~Hz}, 1 \mathrm{H}), 4.75(\mathrm{~s}, 2 \mathrm{H}), 4.00(\mathrm{t}, J=6.0 \mathrm{~Hz}$, $2 \mathrm{H}), 2.27(\mathrm{t}, J=7.2 \mathrm{~Hz}, 2 \mathrm{H}), 2.01-1.94(\mathrm{~m}, 2 \mathrm{H}), 1.77(\mathrm{~s}, 3 \mathrm{H})$.

${ }^{13}$ C NMR (100 MHz, $\left.\mathbf{C D C l}_{3}\right): \delta 157.5,144.8,139.3,129.3,122.3,112.0,110.5,86.7,68.4,34.0$, 27.0, 22.4.

IR (neat, $\mathbf{c m}^{-1}$ ): 3072, 2918, 2874, 1694, 1464, 1275, 1052, 1018, 889, 746

HRMS (ESI): $[\mathrm{M}+\mathrm{H}]{ }^{+}$calcd for $\mathrm{C}_{12} \mathrm{H}_{16} \mathrm{IO} 303.0240$, found 303.0248

\section{(3-Iodo-4-((2-methylallyl)oxy)phenyl)(piperidin-1-yl)methanone (1r)}<smiles>O=C(O)c1ccc(O)c(I)c1</smiles>

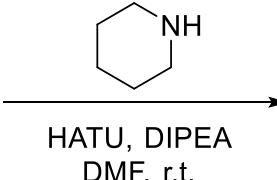

DMF, r.t.
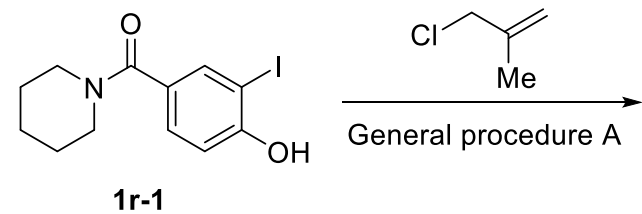

Step 1: Compound 1r-1 was synthesized according to the literature procedure. ${ }^{18}$ To a solution of 4-hydroxy-3-iodobenzoic acid $(2.64 \mathrm{~g}, 10.0 \mathrm{mmol})$ in $\mathrm{DMF}(25.0 \mathrm{~mL})$ at $0{ }^{\circ} \mathrm{C}$ was added 1-[Bis(dimethylamino)methylene]-1H-1,2,3-triazolo[4,5-b]pyridinium 3-oxid hexafluorophosphate (HATU, $5.70 \mathrm{~g}, 15.0 \mathrm{mmol}$ ) and $N, N$-Diisopropylethylamine (DIPEA, $2.50 \mathrm{~mL}, 15.0 \mathrm{mmol}$ ). The reaction mixture was stirred at room temperature for $1 \mathrm{~h}$, and piperidine $(1.0 \mathrm{~mL}, 12.0 \mathrm{mmol})$ was dropwise added. The reaction mixture was stirred at room temperature for $24 \mathrm{~h}$. The reaction was quenched with $\mathrm{H}_{2} \mathrm{O}$, and extracted with ethyl acetate $(3 \times 20.0 \mathrm{~mL})$. The combined organic layers were washed with saturated aqueous $\mathrm{NH}_{4} \mathrm{Cl}$, brine, dried over anhydrous $\mathrm{Na}_{2} \mathrm{SO}_{4}$, and concentrated under reduced pressure. The residue was purified by flash chromatography on silica gel to give 1r-1 as a white solid $\left(2.71 \mathrm{~g}, 82 \%\right.$ yield, mp: $\left.181-183{ }^{\circ} \mathrm{C}\right)$. 
${ }^{1}$ H NMR (400 MHz, CDCl $)$ : $\delta 7.72(\mathrm{~s}, 1 \mathrm{H}), 7.44(\mathrm{~s}, 1 \mathrm{H}), 7.20(\mathrm{~d}, J=6.8 \mathrm{~Hz}, 1 \mathrm{H}), 6.83(\mathrm{~d}, J=$ $8.0 \mathrm{~Hz}, 1 \mathrm{H}$ ), 3.67 (brs, $2 \mathrm{H}$ ), 3.41 (brs, $2 \mathrm{H}$ ), 1.68-1.60 (m, $6 \mathrm{H}$ ).

${ }^{13}$ C NMR (100 MHz, $\left.\mathbf{C D C l}_{3}\right): \delta 169.3,157.3,137.5,128.8,128.7,115.0,84.8,49.1,43.6,26.3$, 25.6, 24.4.

IR (neat, $\mathbf{c m}^{\mathbf{- 1}}$ ): 3728, 2937, 2621, 1699, 1507, 1277, 1114, 1025, 832, 761.

HRMS (ESI): $[\mathrm{M}+\mathrm{H}]^{+}$calcd for $\mathrm{C}_{12} \mathrm{H}_{15} \mathrm{INO}_{2} 332.0142$, found 332.0150.

Step 2: Compound 1r was prepared from 1r-1 (1.66 g, $5.0 \mathrm{mmol})$ and 3-chloro-2-methylprop-1-ene (0.5 mL, $5.0 \mathrm{mmol})$ according to the General procedure A.

$1.48 \mathrm{~g}, 77 \%$ yield, white solid, mp: $62-64{ }^{\circ} \mathrm{C}, \mathrm{R}_{\mathrm{f}}=0.3$ (silica gel, petroleum ether/ethyl acetate $=$ $4: 1)$.

${ }^{1}$ H NMR (400 MHz, $\left.\mathbf{C D C l}_{3}\right): \delta 7.84(\mathrm{~d}, J=2.0 \mathrm{~Hz}, 1 \mathrm{H}), 7.35(\mathrm{dd}, J=2.0,8.4 \mathrm{~Hz}, 1 \mathrm{H}), 6.78(\mathrm{~d}, J$ $=8.4 \mathrm{~Hz}, 1 \mathrm{H}), 5.19(\mathrm{~s}, 1 \mathrm{H}), 5.03(\mathrm{~s}, 1 \mathrm{H}), 4.51$ (s, $2 \mathrm{H}), 3.60$ (brs, $2 \mathrm{H}), 3.45$ (brs, $2 \mathrm{H}), 1.87$ (s, 3 H), 1.68-1.59 (m, $6 \mathrm{H})$.

${ }^{13}$ C NMR (100 MHz, $\left.\mathbf{C D C l}_{3}\right): \delta 168.3,157.7,139.5,138.1,130.3,128.4,113.0,111.3,86.0,72.4$, 48.8, 43.2, 26.2, 25.4, 24.4, 19.3.

IR (neat, $\mathbf{~ c m}^{-\mathbf{1}}$ ): 3474, 3459, 2910, 2823 1634, 1437, 1277, 1262, 776, 751.

HRMS (ESI): $[\mathrm{M}+\mathrm{H}]^{+}$calcd for $\mathrm{C}_{16} \mathrm{H}_{21} \mathrm{INO}_{2} 386.0611$, found 386.0616 .

\section{Tert-butyl (2-iodo-4-methylphenyl)(2-methylallyl)carbamate (1z)}

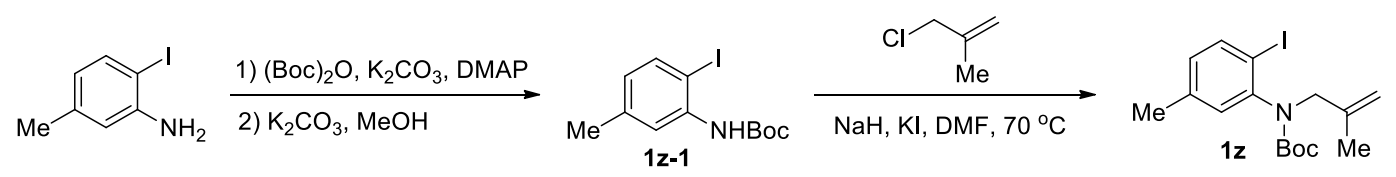

General Procedure:

Compound 1z-1 was synthesized according to the literature procedure. ${ }^{14}$ To a solution of 2-iodo-5-methylaniline $(2.33 \mathrm{~g}, 10.0 \mathrm{mmol})$ in THF $(30.0 \mathrm{~mL})$ was added $\mathrm{K}_{2} \mathrm{CO}_{3}(2.77 \mathrm{~g}, 20.0 \mathrm{mmol})$ and DMAP $(0.12 \mathrm{~g}, 1.0 \mathrm{mmol})$ at $0{ }^{\circ} \mathrm{C}$, followed by slowly addition of $(\mathrm{Boc}){ }_{2} \mathrm{O}(2.29 \mathrm{~g}, 10.5 \mathrm{mmol})$ after $10 \mathrm{~min}$. The reaction mixture was allowed to warm to room temperature and stirred for $3 \mathrm{~h}$. The reaction was quenched with saturated aqueous solution of $\mathrm{NaHCO}_{3}(30.0 \mathrm{~mL})$, and extracted with ethyl acetate $(3 \times 30.0 \mathrm{~mL})$. The combined organic layers were washed with saturated aqueous $\mathrm{Na}_{2} \mathrm{~S}_{2} \mathrm{O}_{3}$, brine, dried over anhydrous $\mathrm{Na}_{2} \mathrm{SO}_{4}$ and concentrated under reduced pressure to give the crude product. The residue was then engaged in the known procedure $\left(\mathrm{K}_{2} \mathrm{CO}_{3}(4.15 \mathrm{~g}, 30.0 \mathrm{mmol})\right.$, $\left.\mathrm{MeOH}(30.0 \mathrm{~mL}), 3 \mathrm{~h}, 70^{\circ} \mathrm{C}\right)$ to obtain the crude product $\mathbf{1 z - 1}$.

To a solution of the above crude compound 1z-1 in DMF (20.0 mL) was added KI (1.99 g, 12.0 $\mathrm{mmol})$ and $\mathrm{NaH}(0.36 \mathrm{~g}, 15.0 \mathrm{mmol})$ at $0{ }^{\circ} \mathrm{C}$, followed by slowly addition of 3-chloro-2-methylprop-1-ene $(0.91 \mathrm{~g}, 10.0 \mathrm{mmol})$ after $20 \mathrm{~min}$. The reaction mixture was allowed to 
warm to room temperature and stirred at $70{ }^{\circ} \mathrm{C}$ for $3 \mathrm{~h}$. The reaction was quenched with water (40.0 $\mathrm{mL})$, and extracted with ethyl acetate $(3 \times 30.0 \mathrm{~mL})$. The combined organic layers were washed with saturated aqueous $\mathrm{Na}_{2} \mathrm{~S}_{2} \mathrm{O}_{3}$, brine, dried over anhydrous $\mathrm{Na}_{2} \mathrm{SO}_{4}$, and concentrated under reduced pressure. The residue was purified by flash chromatography on silica gel to give the product $\mathbf{1 z}$.

$2.79 \mathrm{~g}, 72 \%$ yield, colorless oil, $\mathrm{R}_{\mathrm{f}}=0.7$ (silica gel, petroleum ether/ethyl acetate $=20: 1$ ).

${ }^{1} \mathrm{H}$ NMR (400 MHz, $\mathbf{C D C l}_{3}$, mixture of rotamers): $\delta 7.70$ (d, J=8.0 Hz, $1 \mathrm{H}$ ), [7.04 (s), 6.95 (s), 1 $\mathrm{H}], 6.79(\mathrm{~d}, J=7.2 \mathrm{~Hz}, 1 \mathrm{H}), 4.85(\mathrm{~s}, 1 \mathrm{H}), 4.77(\mathrm{~s}, 1 \mathrm{H}),[4.56(\mathrm{~d}, J=15.6 \mathrm{~Hz}), 4.48(\mathrm{~d}, J=16.0 \mathrm{~Hz})$, $1 \mathrm{H}], 3.48(\mathrm{~d}, J=15.6 \mathrm{~Hz}, 1 \mathrm{H}), 2.29$ (s, $3 \mathrm{H}), 1.82$ (s, $3 \mathrm{H})$, [1.52 (s), 1.37 (s), $9 \mathrm{H}]$.

${ }^{13} \mathbf{C}$ NMR (100 MHz, $\mathbf{C D C l}_{3}$, mixture of rotamers): $\delta 154.2,144.4,141.2,139.2,138.9,138.7$, $130.9,130.3,129.9,129.6,112.8,112.6,95.8,80.6,80.2,56.3,55.2,28.2,20.9,20.6$.

IR (neat, $\mathbf{c m}^{-1}$ ): 2974, 2925, 1706, 1593, 1367, 1299, 1170, 937, 861, 759.

HRMS (ESI): $[\mathrm{M}+\mathrm{H}]^{+}$calcd for $\mathrm{C}_{16} \mathrm{H}_{23} \mathrm{INO}_{2} 388.0768$, found 388.0774.

NOTE: Because of the amide bond rotation equilibrium, the rotamers of $\mathbf{1 z}$ were observed on the NMR. This phenomenon is seen with many tertiary amides. For related references, see: ref. 19-20.

\section{Tert-butyl (2-iodo-5-methylphenyl)(2-methylallyl)carbamate (1aa)}

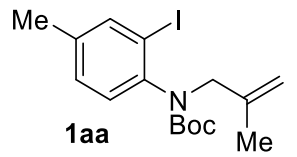

This compound was prepared from 2-iodo-4-methylaniline $(2.33 \mathrm{~g}, 10.0 \mathrm{mmol})$ according to the General Procedure for the synthesis of $\mathbf{1 z}$.

$2.94 \mathrm{~g}, 76 \%$ yield, colorless oil, $\mathrm{R}_{\mathrm{f}}=0.7$ (silica gel, petroleum ether/ethyl acetate $=20: 1$.

${ }^{1}$ H NMR (400 MHz, CDCl 3 , mixture of rotamers): $\delta 7.68(\mathrm{~s}, 1 \mathrm{H}), 7.26-6.99(\mathrm{~m}, 2 \mathrm{H}), 4.83(\mathrm{~s}, 1$ H), $4.74(\mathrm{~s}, 1 \mathrm{H}),[4.58(\mathrm{~d}, J=15.2 \mathrm{~Hz}), 4.49(\mathrm{~d}, J=15.2 \mathrm{~Hz}), 1 \mathrm{H}], 3.47(\mathrm{~d}, J=16.0 \mathrm{~Hz}, 1 \mathrm{H}), 2.29$ (s, $3 \mathrm{H}), 1.81(\mathrm{~s}, 3 \mathrm{H}),[1.52(\mathrm{~s}), 1.36(\mathrm{~s}), 9 \mathrm{H}]$.

${ }^{13} \mathbf{C}$ NMR (100 MHz, $\mathbf{C D C l}_{3}$, mixture of rotamers): $\delta 154.3,142.0,141.8,141.4,141.2,140.0$, 139.7, 139.0, 138.6, 129.6, 129.3, 129.1, 113.0, 112.8, 99.7, 80.5, 80.0, 56.2, 55.1, 28.2, 20.5, 20.4. IR (neat, $\mathbf{c m}^{-1}$ ): 3077, 2976, 2925, 1706, 1487, 1368, 1297, 1171, 866, 763.

HRMS (ESI): $[\mathrm{M}+\mathrm{H}]{ }^{+}$calcd for $\mathrm{C}_{16} \mathrm{H}_{23} \mathrm{INO}_{2}$ 388.0768, found 388.0774.

NOTE: Because of the amide bond rotation equilibrium, the rotamers of 1 aa were observed on the NMR. This phenomenon is seen with many tertiary amides. For related references, see: ref. 19-20.

\section{Tert-butyl (5-chloro-2-iodophenyl)(2-methylallyl)carbamate (1ab)}

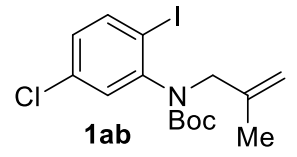

This compound was prepared from 5-chloro-2-iodoaniline $(2.53 \mathrm{~g}, 10.0 \mathrm{mmol})$ 
according to the General Procedure for the synthesis of $\mathbf{1 z}$.

$3.30 \mathrm{~g}, 81 \%$ yield, white solid, $\mathrm{mp}: 184-186{ }^{\circ} \mathrm{C}, \mathrm{R}_{\mathrm{f}}=0.6$ (silica gel, petroleum ether/ethyl acetate $=$ 20:1).

${ }^{1} \mathrm{H}$ NMR (400 MHz, $\mathbf{C D C l}_{3}$, mixture of rotamers): $\delta 7.77(\mathrm{~d}, J=8.4 \mathrm{~Hz}, 1 \mathrm{H}),[7.26(\mathrm{~s}), 7.20(\mathrm{~s}), 1$ $\mathrm{H}], 6.70(\mathrm{dd}, J=2.4 \mathrm{~Hz}, J=8.4 \mathrm{~Hz}, 1 \mathrm{H}), 4.88(\mathrm{~s}, 1 \mathrm{H}), 4.75(\mathrm{~s}, 1 \mathrm{H}), 4.58-4.54(\mathrm{~m}, 1 \mathrm{H}), 3.50-3.46$ (m, $1 \mathrm{H}), 1.82(\mathrm{~s}, 3 \mathrm{H}),[1.52(\mathrm{~s}), 1.37(\mathrm{~s}), 9 \mathrm{H}]$.

${ }^{13} \mathbf{C}$ NMR (100 MHz, $\mathbf{C D C l}_{3}$, mixture of rotamers): $\delta 153.7,145.7,140.8,140.0,134.2,129.8$, $128.8,113.5,97.6,80.7,56.1,55.0,28.2,20.48$.

IR (neat, $\mathbf{c m}^{-\mathbf{1}}$ ): 2976, 1708, 1572, 1463, 1366, 1289, 1165, 863, 729.

HRMS (ESI): $[\mathrm{M}+\mathrm{H}]^{+}$calcd for $\mathrm{C}_{15} \mathrm{H}_{20} \mathrm{ClINO}_{2}$ 408.0222, found 408.0219.

NOTE: Because of the amide bond rotation equilibrium, the rotamers of 1ab were observed on the NMR. This phenomenon is seen with many tertiary amides. For related references, see: ref. 19-20.

\section{1-Iodo-4-methyl-2-(3-methylbut-3-en-1-yl)benzene (1ad)}

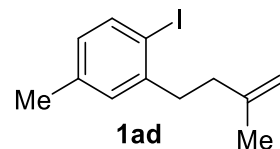

This compound was prepared from (2-iodo-5-methylphenyl)methanol (2.48 g, $10.0 \mathrm{mmol}$ ) according to the literature reference 15.

$1.86 \mathrm{~g}, 65 \%$ yield, colorless oil, $\mathrm{R}_{\mathrm{f}}=0.8$ (silica gel, petroleum ether).

${ }^{1}$ H NMR (400 MHz, $\left.\mathbf{C D C l}_{3}\right): \delta 7.66(\mathrm{~d}, J=8.0 \mathrm{~Hz}, 1 \mathrm{H}), 7.04(\mathrm{~d}, J=2.0 \mathrm{~Hz}, 1 \mathrm{H}), 6.70(\mathrm{dd}, J=2.0$ $\mathrm{Hz}, J=8.0 \mathrm{~Hz}, 1 \mathrm{H}), 4.76$ (s, $2 \mathrm{H}), 2.81-2.77$ (m, $2 \mathrm{H}), 2.31-2.23(\mathrm{~m}, 5 \mathrm{H}), 1.81(\mathrm{~s}, 3 \mathrm{H})$.

${ }^{13}$ C NMR (100 MHz, $\left.\mathbf{C D C l}_{3}\right): \delta$ 145.1, 144.4, 139.1, 138.2, 130.2, 128.7, 110.3, 96.3, 39.3, 38.4, 22.6, 20.9.

IR (neat, $\mathbf{c m}^{-\mathbf{1}}$ ): 3075, 2951, 2924, 2854, 1648, 1592, 1467, 1122, 1011, 886.

HRMS (APCI): $[\mathrm{M}+\mathrm{H}]^{+}$calcd for $\mathrm{C}_{12} \mathrm{H}_{15} \mathrm{I}$ 287.0291, found 287.0300.

\section{5-Iodo-6-(3-methylbut-3-en-1-yl)benzo[d][1,3]dioxole (1ae)}

This compound was prepared from 5-(bromomethyl)-6-iodobenzo[d][1,3]dioxole ${ }^{21}$ $(1.52 \mathrm{~g}, 10.0 \mathrm{mmol})$ according to the literature reference 15 .

$2.40 \mathrm{~g}, 76 \%$ yield, colorless oil, $\mathrm{R}_{\mathrm{f}}=0.4$ (silica gel, petroleum ether).

${ }^{1}$ H NMR (400 MHz, CDCl $): \delta 7.22(\mathrm{~s}, 1 \mathrm{H}), 6.73(\mathrm{~s}, 1 \mathrm{H}), 5.93(\mathrm{~s}, 2 \mathrm{H}), 4.75(\mathrm{~d}, J=9.6 \mathrm{~Hz}, 2 \mathrm{H})$, 2.78-2.74 (m, $2 \mathrm{H}), 2.24-2.20$ (m, $2 \mathrm{H}), 1.80$ (s, $3 \mathrm{H})$.

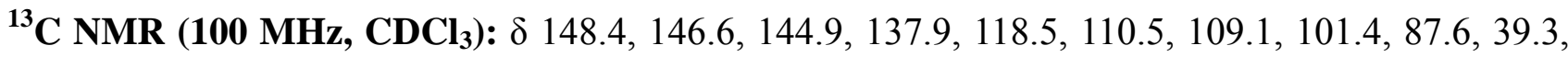
$38.4,22.6$. 
IR (neat, $\mathbf{c m}^{-\mathbf{1}}$ ): 3074, 2925, 1648, 1596, 1226, 1107, 1041, 935, 889, 826.

HRMS (ESI): $[\mathrm{M}+\mathrm{H}]^{+}$calcd for $\mathrm{C}_{12} \mathrm{H}_{14} \mathrm{IO}_{2}$ 317.0041, found 317.0033.

\section{4-Fluoro-1-iodo-2-(3-methylbut-3-en-1-yl)benzene (1af)}

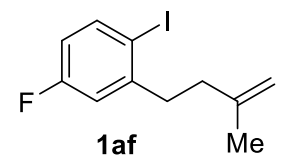

This compound was prepared from (5-fluoro-2-iodophenyl)methanol (2.52 g, 10.0 $\mathrm{mmol}$ ) according to the literature reference 15 .

$1.62 \mathrm{~g}, 56 \%$ yield, colorless oil, $\mathrm{R}_{\mathrm{f}}=0.4$ (silica gel, petroleum ether).

${ }^{1}$ H NMR (400 MHz, CDCl $): \delta$ 7.25-7.19 (m, $\left.1 \mathrm{H}\right), 6.96(\mathrm{~d}, J=8.0 \mathrm{~Hz}, 1 \mathrm{H}), 6.91-6.85(\mathrm{~m}, 1 \mathrm{H})$, 4.72-4.70 (m, 2 H), 2.77-2.73 (m, 2 H), 2.33-2.29 (m, 2 H), 1.76 (s, 3 H).

${ }^{13}$ C NMR (100 MHz, $\left.\mathbf{C D C l}_{3}\right): \delta 162.9\left(\mathrm{~d}, J_{C-F}=325 \mathrm{~Hz}\right), 144.8\left(\mathrm{~d}, J_{C-F}=8.0 \mathrm{~Hz}\right), 144.7,129.6(\mathrm{~d}$, $\left.J_{C-F}=9.0 \mathrm{~Hz}\right), 124.0,115.1\left(\mathrm{~d}, J_{C-F}=21.0 \mathrm{~Hz}\right), 112.6\left(\mathrm{~d}, J_{C-F}=21.0 \mathrm{~Hz}\right), 110.5,39.2,33.9,22.5$. 148.4, 146.6, 144.9, 137.9, 118.5, 110.5, 109.1, 101.4, 87.6, 39.3, 38.4, 22.6.

${ }^{19}$ F NMR (376 MHz, CDCl 3$)$ : $\delta-114.0$

IR (neat, $\mathbf{c m}^{-1}$ ): 2917, 2849, 1590, 1453, 1417, 1270, 1112, 887, 781, 688.

HRMS (ESI): $[\mathrm{M}+\mathrm{H}]^{+}$calcd for $\mathrm{C}_{11} \mathrm{H}_{13} \mathrm{FI} 291.0040$, found 291.0049 .

\subsection{Synthesis of Alkenyl Triflate Reagents 2a-2v}<smiles>[TeH2]OC1=CCCCC1</smiles><smiles>[TeH]C1=CCCC1</smiles>

$2 b$

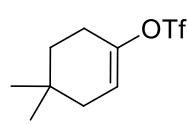

2c

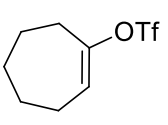

2d

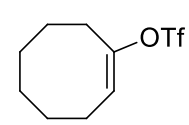

$2 e$

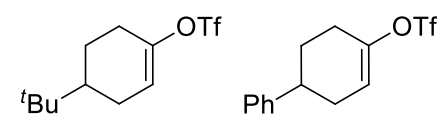

$2 f$ $2 \mathrm{~g}$

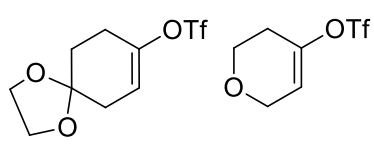

$2 h$

$2 \mathbf{i}$<smiles>[TeH2]OC1=CCSCC1</smiles><smiles>CCOC1=CCN(N)CC1</smiles><smiles>[Te+]C1=Cc2ccccc2C1</smiles><smiles>CCOC1=CCCc2ccccc21</smiles><smiles>C=C(CCCCCC(=C)OCC(=C)OC(C)=O)O[Ga]</smiles>

2j

$2 k$

2I

$2 \mathrm{~m}$

$2 n$

20

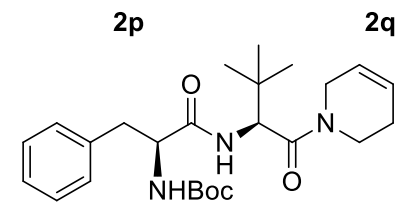

$2 u$<smiles>[O+]c1ccccc1</smiles>

$2 t$<smiles>Ic1ccccc1</smiles>

2v

$2 w$

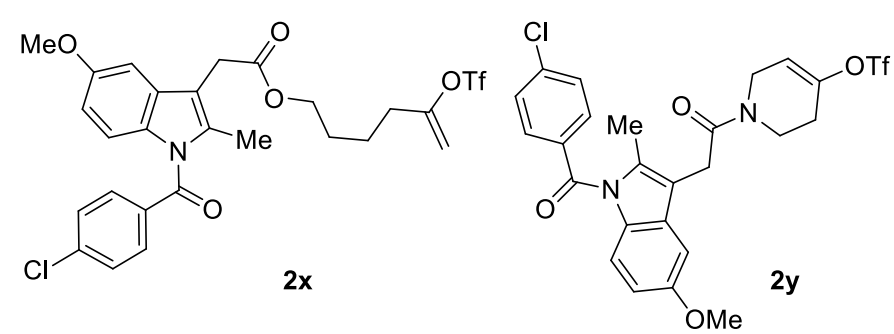

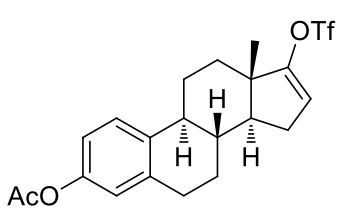

$2 z$

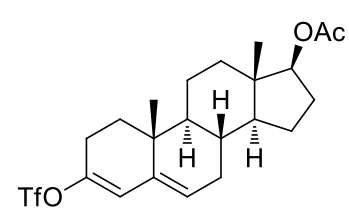

2aa

Alkenyl triflates $\mathbf{2 a},{ }^{22} \mathbf{2 b},{ }^{22} \mathbf{2} \mathbf{c}^{23,} \mathbf{2 d},{ }^{22} \mathbf{2} e^{22} \mathbf{2 f},{ }^{22} \mathbf{2} \mathbf{g},{ }^{24} \mathbf{2 h},{ }^{22} \mathbf{2 i},{ }^{22} \mathbf{2} \mathbf{j},{ }^{25} \mathbf{2} \mathbf{k},{ }^{26} \mathbf{2},{ }^{27} \mathbf{2} \mathbf{m},{ }^{28} \mathbf{2 n},{ }^{22} \mathbf{2 z},{ }^{29}$ $\mathbf{2} \mathbf{a a},{ }^{30}$ are known compounds, and were synthesized according the literature procedure. ${ }^{22}$ Known 
compound $2 \mathbf{r}$ was prepared according to the literature procedure. ${ }^{31}$ Known compound $2 \mathbf{s}$ was prepared according to the literature procedure. ${ }^{32}$ Known compound $\mathbf{2 t}$ was prepared according to the literature procedure. ${ }^{33}$ Known compound $\mathbf{2 u}$ was prepared according to the literature procedure. ${ }^{34}$ The preparation of new compounds, and their characterization data are provided as follows.

\section{General procedure C:}

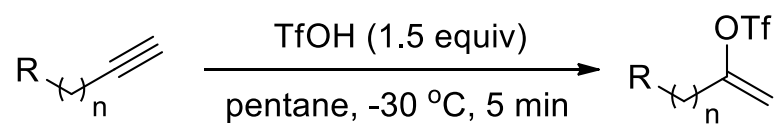

These compounds were synthesized according the literature procedure. ${ }^{22}$ To a solution of alkyne $(10.0 \mathrm{mmol})$ in pentane $(20.0 \mathrm{~mL})$ was dropwise added trifluoromethanesulfonic acid $(1.33 \mathrm{~mL}, 15.0$ mmol) at $-30{ }^{\circ} \mathrm{C}$. The reaction mixture was warmed to $0{ }^{\circ} \mathrm{C}$ after $1 \mathrm{~h}$, and quenched with saturated aqueous $\mathrm{NaHCO}_{3}$. The organic layer was separated after $5 \mathrm{~min}$, washed twice with saturated aqueous $\mathrm{NaHCO}_{3}$, dried over anhydrous $\mathrm{Na}_{2} \mathrm{SO}_{4}$, and concentrated under reduced pressure. The residue was purified by flash chromatography on silica gel to give alkenyl triflates.

\section{Hept-1-en-2-yl trifluoromethanesulfonate (2o)}

OTf This compound was prepared from hept-1-yne $(0.96 \mathrm{~g}, 10.0 \mathrm{mmol})$ according to the 20 General procedure C.

$1.79 \mathrm{~g}, 73 \%$ yield, colorless oil, $\mathrm{R}_{\mathrm{f}}=0.8$ (silica gel, petroleum ether).

${ }^{1}$ H NMR (600 MHz, CDCl $): \delta 5.08(\mathrm{~d}, J=3.6 \mathrm{~Hz}, 1 \mathrm{H}), 4.92(\mathrm{~d}, J=3.6 \mathrm{~Hz}, 1 \mathrm{H}), 2.33(\mathrm{t}, J=7.8$ Hz, 2 H), 1.56-1.54 (m, 2 H), 1.36-1.32 (m, 4 H), 0.92-0.90 (m, 3 H).

${ }^{13}$ C NMR (150 MHz, $\left.\mathbf{C D C l}_{3}\right): \delta 157.2,118.6\left(\mathrm{q}, J_{C-F}=318.0 \mathrm{~Hz}\right), 103.9,33.8,30.8,25.7,22.2$, 13.8 .

${ }^{19}$ F NMR (564 MHz, $\left.\mathrm{CDCl}_{3}\right): \delta-74.3$.

IR (neat, $\mathbf{c m}^{-\mathbf{1}}$ ): 2962, 2875, 1671, 1419, 1251, 1142, 1094, 947, 705, 613.

HRMS (ESI): $[\mathrm{M}+\mathrm{H}]^{+}$calcd for $\mathrm{C}_{8} \mathrm{H}_{14} \mathrm{~F}_{3} \mathrm{O}_{3} \mathrm{~S} 247.0610$, found 247.0610.

\section{Methyl 6-(((trifluoromethyl)sulfonyl)oxy)hept-6-enoate (2p)}

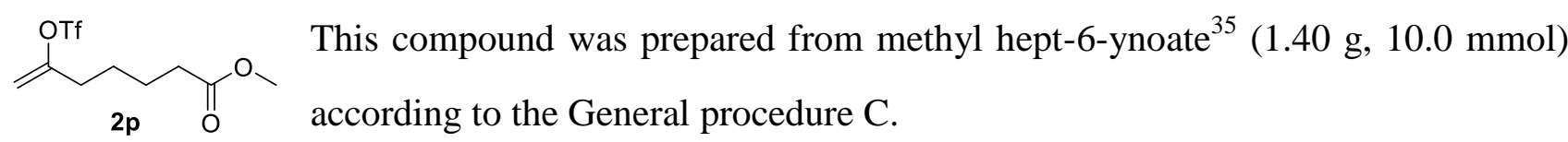

$2.37 \mathrm{~g}, 82 \%$ yield, colorless oil, $\mathrm{R}_{\mathrm{f}}=0.4$ (silica gel, petroleum ether/ethyl acetate $=10: 1$ ).

${ }^{1}$ H NMR (400 MHz, CDCl $): \delta 5.12(\mathrm{~d}, J=3.6 \mathrm{~Hz}, 1 \mathrm{H}), 4.97(\mathrm{~d}, J=3.6 \mathrm{~Hz}, 1 \mathrm{H}), 3.68(\mathrm{~s}, 3 \mathrm{H})$, 
2.39-2.34 (m, 4 H), 1.72-1.66 (m, 2 H), 1.63-1.57 (m, 2 H).

${ }^{13}$ C NMR (100 MHz, $\left.\mathbf{C D C l}_{3}\right): \delta 173.6,156.2,118.4\left(\mathrm{q}, J_{C-F}=318.0 \mathrm{~Hz}\right), 104.5,51.5,33.5,33.4$, 25.3, 23.8.

${ }^{19}$ F NMR (376 MHz, $\left.\mathrm{CDCl}_{3}\right): \delta-74.2$.

IR (neat, $\mathbf{c m}^{-1}$ ): 2956, 2874, 1740, 1417, 1211, 1073, 943, 830, 791, 638.

HRMS (ESI): $[\mathrm{M}+\mathrm{H}]^{+}$calcd for $\mathrm{C}_{9} \mathrm{H}_{14} \mathrm{~F}_{3} \mathrm{O}_{5} \mathrm{~S} 291.0509$, found 291.0510.

\section{6-(((Trifluoromethyl)sulfonyl)oxy)hept-6-en-1-yl acetate (2q)}

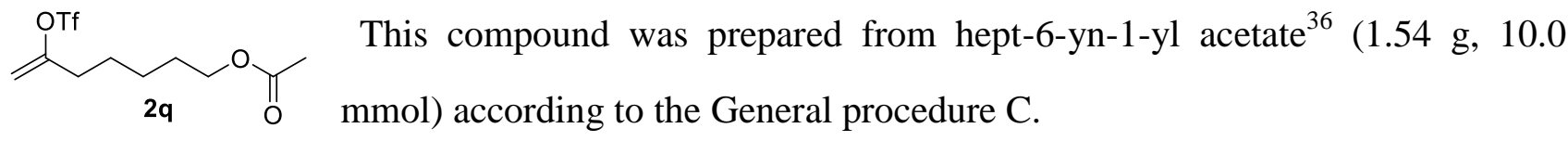

$2.37 \mathrm{~g}, 78 \%$ yield, colorless oil, $\mathrm{R}_{\mathrm{f}}=0.4$ (silica gel, petroleum ether/ethyl acetate $=10: 1$ ).

${ }^{1}$ H NMR (400 MHz, CDCl $): \delta 5.11(\mathrm{~d}, J=3.6 \mathrm{~Hz}, 1 \mathrm{H}), 4.95(\mathrm{~d}, J=3.6 \mathrm{~Hz}, 1 \mathrm{H}), 4.07$ (t, $J=6.4$ Hz, 2 H), 2.36 (t, J = 7.2 Hz, 2 H), 2.06 (s, 3 H), 1.70-1.55 (m, 4 H), 1.46-1.39 (m, 2 H).

${ }^{13}$ C NMR (100 MHz, $\left.\mathbf{C D C l}_{3}\right): \delta 171.2,156.5,118.4\left(\mathrm{q}, J_{C-F}=318.0 \mathrm{~Hz}\right), 104.3,64.1,33.7,28.1$, $25.5,25.0,20.9$.

${ }^{19}$ F NMR (376 MHz, $\left.\mathbf{C D C l}_{3}\right): \delta-74.2$.

IR (neat, $\mathbf{~ c m}^{-1}$ ): 2954, 2870, 1739, 1643, 1417, 1210, 1141, 900, 706, 637.

HRMS (ESI): $[\mathrm{M}+\mathrm{H}]^{+}$calcd for $\mathrm{C}_{10} \mathrm{H}_{16} \mathrm{~F}_{3} \mathrm{O}_{5} \mathrm{~S} 305.0665$, found 305.0665 .

1-((S)-2-((S)-2-((Tert-butoxycarbonyl)amino)-3-phenylpropanamido)-3,3-dimethylbutanoyl)-1, 2,3,6-tetrahydropyridin-4-yl trifluoromethanesulfonate (2w)
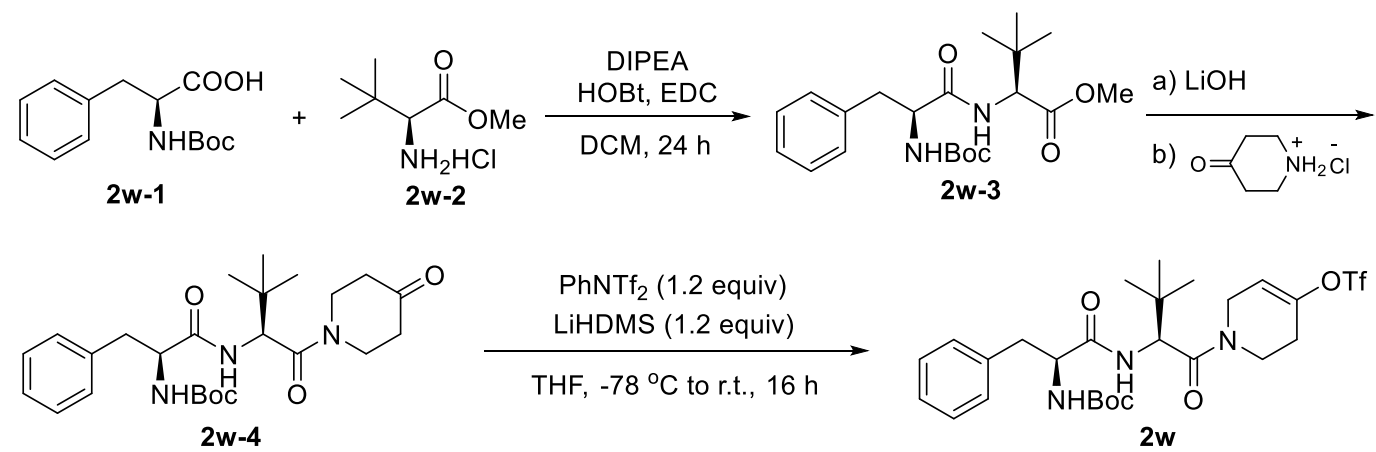

To a stirred solution of acid $\mathbf{2 w}-\mathbf{1}^{37}(2.65 \mathrm{~g}, 10.0 \mathrm{mmol})$ and ester $\mathbf{2} \mathbf{w}-\mathbf{2}^{38}(1.81 \mathrm{~g}, 10.0 \mathrm{mmol})$ in $\mathrm{CH}_{2} \mathrm{Cl}_{2}(20.0 \mathrm{~mL})$ at $0{ }^{\circ} \mathrm{C}$ was added DIPEA (1.65 mL, $\left.10.0 \mathrm{mmol}\right)$, hydroxybenzotriazole (HOBt, $1.49 \mathrm{~g}, 11.0 \mathrm{mmol}$ ) and 1-ethyl-3-(3-dimethylaminopropyl)carbodiimide (EDC, $1.92 \mathrm{~g}, 10.0 \mathrm{mmol}$ ). The reaction mixture was stirred at the same temperature for $10 \mathrm{~min}$, and then room temperature for $24 \mathrm{~h}$. The reaction was quenched with saturated aqueous $\mathrm{NaHCO}_{3}$, extracted with ethyl acetate $(3 \times$ 
$30.0 \mathrm{~mL}$ ). The combined organic layers were washed with brine, dried over anhydrous $\mathrm{Na}_{2} \mathrm{SO}_{4}$, and concentrated under reduced pressure. The residue was purified by flash chromatography on silica gel to give peptide $\mathbf{2 w - 3}$ as a white solid $\left(2.74 \mathrm{~g}, 70 \%\right.$ yield, mp: $\left.114-116{ }^{\circ} \mathrm{C}\right)$.

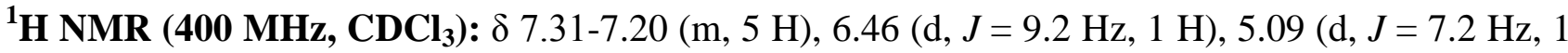
H), 4.39-4.33 (m, 2 H), 3.67 (s, 3 H), 3.06 (d, J = 6.8 Hz, 2 H), 1.42 (s, 9 H), 0.91 (s, 9 H).

${ }^{13}$ C NMR (100 MHz, $\left.\mathbf{C D C l}_{3}\right): \delta 171.3,170.9,155.4,136.6,129.2,128.6,126.8,80.1,60.0,56.0$, $51.7,37.8,34.7,28.2,26.4$.

IR (neat, $\mathbf{c m}^{-\mathbf{1}}$ ): $3317,2973,1743,1655,1537,1368,1168,1023,881,700$.

HRMS (ESI): $[\mathrm{M}+\mathrm{H}]{ }^{+}$calcd for $\mathrm{C}_{21} \mathrm{H}_{33} \mathrm{~N}_{2} \mathrm{O}_{5} 393.2384$, found 393.2388.

To a solution of peptide $\mathbf{2 w - 3}(5.0 \mathrm{mmol}, 1.96 \mathrm{~g})$ in $\mathrm{THF} / \mathrm{H}_{2} \mathrm{O}(10.0 \mathrm{~mL} / 10.0 \mathrm{~mL})$ was added $\mathrm{LiOH} \cdot \mathrm{H}_{2} \mathrm{O}(1.05 \mathrm{~g}, 25.0 \mathrm{mmol})$ at $0{ }^{\circ} \mathrm{C}$. The reaction mixture was stirred at the same temperature for $20 \mathrm{~min}$, then room temperature for $24 \mathrm{~h}$. The reaction was quenched with saturated aqueous $\mathrm{NaHCO}_{3}$, and extracted with ethyl acetate $(3 \times 30.0 \mathrm{~mL})$. The combined organic layers were washed with brine, dried over anhydrous $\mathrm{Na}_{2} \mathrm{SO}_{4}$, and concentrated under reduced pressure. The residue was used for the next step without purification.

To a stirred solution of the above residue in $\mathrm{THF} / \mathrm{CH}_{2} \mathrm{Cl}_{2}(10.0 \mathrm{~mL} / 10.0 \mathrm{~mL})$ at room temperature was added 1-[Bis(dimethylamino)methylene]-1H-1,2,3-triazolo[4,5-b]pyridinium 3-oxid hexafluorophosphate (HATU, $3.81 \mathrm{~g}, 10.0 \mathrm{mmol}$ ) and N,N-diisopropylethylamine (DIPEA, 2.06 $\mathrm{mL}, 12.5 \mathrm{mmol})$, followed by 4-oxopiperidinium chloride $(0.68 \mathrm{~g}, 5.0 \mathrm{mmol})$ after $10 \mathrm{~min}$. The reaction mixture was stirred at room temperature for $24 \mathrm{~h}$. The reaction was quenched with saturated aqueous $\mathrm{NaHCO}_{3}$, and extracted with ethyl acetate $(3 \times 20.0 \mathrm{~mL})$. The combined organic layers were washed with brine, dried over anhydrous $\mathrm{Na}_{2} \mathrm{SO}_{4}$, and concentrated under reduced pressure. The residue was used for the next step without purification.

To a stirred solution of above crude ketone in THF $(20.0 \mathrm{~mL})$ was dropwise added Lithium bis(trimethylsilyl)amide (LiHMDS, $0.80 \mathrm{~g}, 4.8 \mathrm{mmol})$ at $-78{ }^{\circ} \mathrm{C}$. A solution of $\mathrm{PhNTf}_{2}(1.72 \mathrm{~g}, 4.8$ $\mathrm{mmol})$ in THF $(10.0 \mathrm{~mL})$ was dropwise added after $1 \mathrm{~h}$. The reaction mixture was allowed to warm to room temperature and stirred for $16 \mathrm{~h}$. The reaction was quenched with $\mathrm{H}_{2} \mathrm{O}$ and extracted with ethyl acetate $(3 \times 20.0 \mathrm{~mL})$. The organic layers were washed with saturated aqueous $\mathrm{NH}_{4} \mathrm{Cl}$, brine, dried over anhydrous $\mathrm{Na}_{2} \mathrm{SO}_{4}$, and concentrated under reduced pressure. The residue was purified by flash chromatography on silica gel to give alkenyl triflate $\mathbf{2 w}$.

$1.89 \mathrm{~g}, 64 \%$ yield for 3 steps, white solid, $\mathrm{mp}: 70-72{ }^{\circ} \mathrm{C}, \mathrm{R}_{\mathrm{f}}=0.4$ (silica gel, petroleum ether/ethyl 
acetate $=4: 1)$.

${ }^{1} \mathbf{H}$ NMR (400 MHz, $\mathbf{C D C l}_{3}$, mixture of rotamors): $\delta$ 7.30-7.13 (m, $\left.5 \mathrm{H}\right), 7.03-7.01(\mathrm{~m}, 1 \mathrm{H})$, 5.81-5.79 (m, $1 \mathrm{H})$, 5.29-5.24 (m, $1 \mathrm{H})$, 4.91-4.80 (m, $1 \mathrm{H})$, 4.51-3.54 (m, $5 \mathrm{H}), 3.14-3.03$ (m, $2 \mathrm{H})$, 2.57-2.44 (m, 2 H), 1.40-1.37 (m, 9 H), 0.96-0.87 (m, 9 H).

${ }^{13} \mathrm{C}$ NMR (100 $\mathbf{M H z} \mathbf{C D C l}_{3}$, mixture of rotamors): $\delta 171.3,171.0,170.1,169.8,169.7,169.5$, 155.2 , 155.1, 147.5, 145.9, 136.6, 136.53, 136.47, 129.1, 129.0, 128.5, 128.3, 126.8, 126.6, 118.3(q, $\left.J_{C-F}=319.0 \mathrm{~Hz}\right), 115.5,115.4,114.7,79.8,55.6,54.7,54.11,54.05,43.7,43.0,42.8,40.2,40.1,38.6$, $38.5,38.4,37.9,35.7,35.6,35.5,35.3,28.5,28.10,28.05,27.7,26.3,26.23,26.19,26.1$.

${ }^{19}$ F NMR (376 MHz, $\left.\mathbf{C D C l}_{3}\right): \delta-73.76,-73.80$.

IR (neat, $\mathbf{c m}^{-1}$ ): 3423, 2976, 1701, 1638, 1422, 1368, 1215, 1142, 870, 747.

HRMS (ESI): $[\mathrm{M}+\mathrm{H}]^{+}$calcd for $\mathrm{C}_{26} \mathrm{H}_{37} \mathrm{~F}_{3} \mathrm{~N}_{3} \mathrm{O}_{7} \mathrm{~S} 592.2299$, found 592.2309.

NOTE: Because of the amide bond rotation equilibrium, the rotamers of $\mathbf{2 w}$ were observed on the NMR. This phenomenon is seen with many tertiary amides. For related references, see: ref. 19-20

\section{5-(((trifluoromethyl)sulfonyl)oxy)hex-5-en-1-yl}

2-(1-(4-chlorobenzoyl)-5-methoxy-2-methyl-1H-indol-3-yl)acetate (2x)

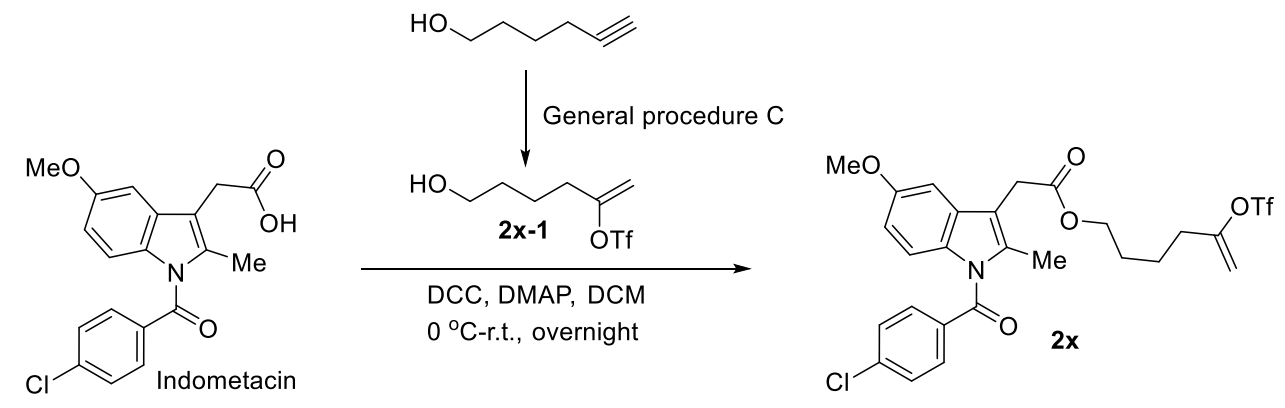

Step 1: Triflate 2x-1 (4.22 g, 85\% yield) was prepared as a colorless oil from hex-5-yn-1-ol (2.21 $\mathrm{mL}, 20.0 \mathrm{mmol}$ ) according to the General procedure $\mathrm{C}$.

${ }^{1}$ H NMR (400 MHz, CDCl 3$): \delta 5.11(\mathrm{~d}, J=3.6 \mathrm{~Hz}, 1 \mathrm{H}), 4.97(\mathrm{~d}, J=3.6 \mathrm{~Hz}, 1 \mathrm{H}), 3.66(\mathrm{q}, J=6.0$ Hz, $2 \mathrm{H}), 2.39$ (t, $J=6.8 \mathrm{~Hz}, 2 \mathrm{H}), 1.99$ (s, $1 \mathrm{H}), 1.69-1.58$ (m, $4 \mathrm{H})$.

${ }^{13}$ C NMR (100 MHz, CDCl 3 ): $\delta$ 156.6, $118.4\left(q, J_{C-F}=318.0 \mathrm{~Hz}\right), 104.3,62.1,33.5,31.4,22.3$.

${ }^{19}$ F NMR (376 MHz, $\left.\mathrm{CDCl}_{3}\right)$ : $\delta-74.2$.

IR (neat, $\mathbf{c m}^{-\mathbf{1}}$ ): 3347, 2947, 2878, 1672, 1418, 1213, 1148, 1066, 948, 706.

HRMS (ESI): $[\mathrm{M}+\mathrm{K}]^{+}$calcd for $\mathrm{C}_{7} \mathrm{H}_{11} \mathrm{~F}_{3} \mathrm{O}_{4} \mathrm{SK} 286.9962$, found 286.9967.

Step2: To a solution of Indomethacin (5.37 g, $15.0 \mathrm{mmol})$ in DCM (30.0 $\mathrm{mL})$ was added dicyclohexylcarbodiimide (DCC, $3.09 \mathrm{~g}, 15.0 \mathrm{mmol}$ ) and 4-dimethylaminopyridine (DMAP, $0.12 \mathrm{~g}$, 
$1.0 \mathrm{mmol})$ at $0{ }^{\circ} \mathrm{C}$. The reaction mixture was stirred for $30 \mathrm{~min}$, and a solution of triflate $\mathbf{2 x - 1}(2.48 \mathrm{~g}$, $10.0 \mathrm{mmol})$ in DCM $(10.0 \mathrm{~mL})$ was added. The reaction mixture was stirred at room temperature overnight. The solvent was removed under reduced pressure, and the residue was treated with water, extracted with ethyl acetate $(3 \times 30.0 \mathrm{~mL})$. The combined organic layers were washed with water, brine, dried over anhydrous $\mathrm{Na}_{2} \mathrm{SO}_{4}$, and concentrated under reduced pressure. The residue was purified by flash chromatography on silica gel to give the product $\mathbf{2 x}$.

$4.23 \mathrm{~g}, 72 \%$ yield, colorless oil, $\mathrm{R}_{\mathrm{f}}=0.4$ ( silica gel, petroleum ether/ethyl acetate $=4: 1$ ).

${ }^{1}$ H NMR (400 MHz, CDCl $): \delta 7.66(\mathrm{~d}, J=8.4 \mathrm{~Hz}, 2 \mathrm{H}), 7.47(\mathrm{~d}, J=8.8 \mathrm{~Hz}, 2 \mathrm{H}), 6.96(\mathrm{~d}, J=2.4$ $\mathrm{Hz}, 1 \mathrm{H}), 6.86(\mathrm{~d}, J=8.8 \mathrm{~Hz}, 1 \mathrm{H}), 6.67(\mathrm{dd}, J=2.4,9.2 \mathrm{~Hz}, 1 \mathrm{H}), 5.08(\mathrm{~d}, J=3.2 \mathrm{~Hz}, 1 \mathrm{H}), 4.86(\mathrm{~d}$, $J=3.6 \mathrm{~Hz}, 1 \mathrm{H}), 4.13(\mathrm{t}, J=6.4 \mathrm{~Hz}, 2 \mathrm{H}), 3.83(\mathrm{~s}, 3 \mathrm{H}), 3.67(\mathrm{~s}, 2 \mathrm{H}), 2.39(\mathrm{~s}, 3 \mathrm{H}), 2.33(\mathrm{t}, J=7.6$ $\mathrm{Hz}, 2 \mathrm{H}), 1.72-1.52$ (m, $4 \mathrm{H})$.

${ }^{13}$ C NMR (100 MHz, CDCl $): \delta 170.8,168.2,156.0,155.9,139.2,135.9,133.7,131.1,130.7,130.5$, $129.0,118.4\left(\mathrm{q}, J_{C-F}=318 \mathrm{~Hz}\right), 114.9,112.4,111.4,104.6,101.2,64.2,55.6,33.3,30.2,27.5,22.4$, 13.3.

\section{${ }^{19}$ F NMR (376 MHz, $\left.\mathrm{CDCl}_{3}\right): \delta-74.0$.}

IR (neat, $\mathbf{c m}^{-1}$ ): 3470, 2959, 1735, 1683, 1480, 1418, 1215, 1069, 926, 755.

HRMS (ESI): $[\mathrm{M}+\mathrm{H}]^{+}$calcd for $\mathrm{C}_{26} \mathrm{H}_{26} \mathrm{ClF}_{3} \mathrm{NO}_{7} \mathrm{~S} 588.1065$, found 588.1076.

\section{1-(2-(1-(4-chlorobenzoyl)-5-methoxy-2-methyl-1H-indol-3-yl)acetyl)-1,2,3,6-tetrahydropyridin-}

\section{4-yl trifluoromethanesulfonate $(2 y)$}
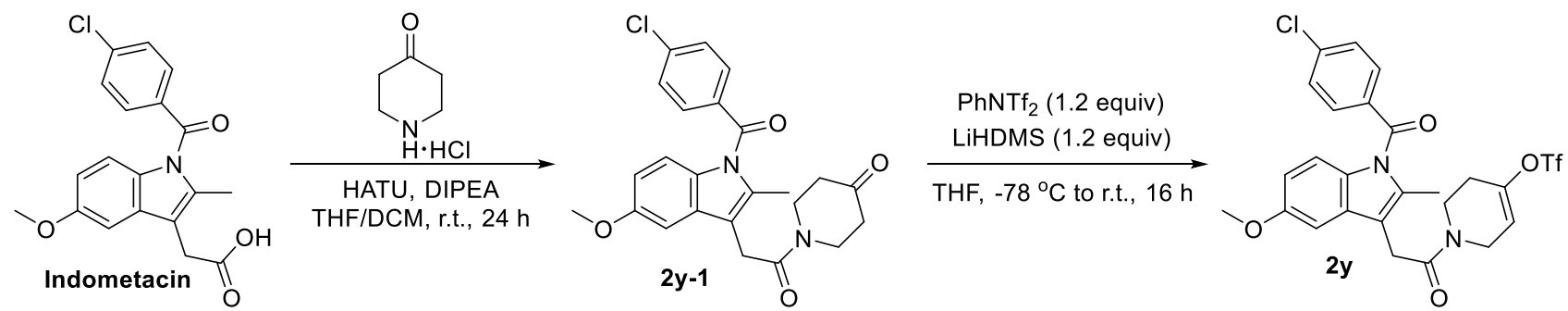

Step 1: To a solution of Indomethacin $(1.97 \mathrm{~g}, 5.5 \mathrm{mmol})$ in $\mathrm{THF} / \mathrm{CH}_{2} \mathrm{Cl}_{2}(10.0 \mathrm{~mL} / 10.0 \mathrm{~mL})$ was added 1-[Bis(dimethylamino)methylene]-1H-1,2,3-triazolo[4,5-b]pyridinium 3-oxid hexafluorophosphate (HATU, $4.18 \mathrm{~g}, 11.0 \mathrm{mmol}$ ) and N,N-diisopropylethylamine (DIPEA, $2.06 \mathrm{~mL}$, $12.0 \mathrm{mmol})$ at room temperature. 4-Oxopiperidinium chloride $(0.68 \mathrm{~g}, 5.0 \mathrm{mmol})$ was added after 10 min. The reaction mixture was stirred at room temperature for $24 \mathrm{~h}$. The reaction was quenched with saturated aqueous $\mathrm{NaHCO}_{3}$, and extracted with ethyl acetate $(3 \times 20.0 \mathrm{~mL})$. The combined organic layers were washed with brine, dried over anhydrous $\mathrm{Na}_{2} \mathrm{SO}_{4}$, and concentrated under reduced 
pressure. The residue was used for the next step without purification.

Step 2: To a solution of the above residue in THF $(30.0 \mathrm{~mL})$ was dropwise added Lithium bis(trimethylsilyl)amide (LiHMDS, $1.00 \mathrm{~g}, 6.0 \mathrm{mmol})$ at $-78{ }^{\circ} \mathrm{C}$. A solution of $\mathrm{PhNTf}_{2}(2.15 \mathrm{~g}, 6.0$ mmol) in THF $(10.0 \mathrm{~mL})$ was dropwise added after $1 \mathrm{~h}$. The reaction mixture was allowed to warm to room temperature, and stirred for $16 \mathrm{~h}$. The reaction was quenched with $\mathrm{H}_{2} \mathrm{O}$, and extracted with ethyl acetate $(3 \times 20.0 \mathrm{~mL})$. The organic layers were washed with saturated aqueous $\mathrm{NH}_{4} \mathrm{Cl}$, brine, dried over anhydrous $\mathrm{Na}_{2} \mathrm{SO}_{4}$, and concentrated under reduced pressure. The residue was purified by flash chromatography on silica gel to give alkenyl triflate $\mathbf{2 y}$.

$2.05 \mathrm{~g}, 72 \%$ yield for two steps, white solid, mp: $184-186{ }^{\circ} \mathrm{C}, \mathrm{R}_{\mathrm{f}}=0.4$ (silica gel, petroleum ether/ethyl acetate $=2: 1$ ), approximate $1.4: 1$ ratio of rotamers.

${ }^{1}$ H NMR (600 MHz, CDCl $): \delta 7.66(\mathrm{~d}, J=8.4 \mathrm{~Hz}, 2 \mathrm{H}), 7.47(\mathrm{~d}, J=8.4 \mathrm{~Hz}, 2 \mathrm{H}), 6.94(\mathrm{~m}, 1 \mathrm{H})$, 6.82-6.80 (m, $1 \mathrm{H}), 6.66-6.65(\mathrm{~m}, 1 \mathrm{H}),[5.81(\mathrm{~s}), 5.75(\mathrm{~s}), 1 \mathrm{H}),[4.25(\mathrm{~d}, J=3.0 \mathrm{~Hz}), 4.14(\mathrm{~d}, J=$ $1.8 \mathrm{~Hz}), 2 \mathrm{H}), 3.86-3.67$ (m, $7 \mathrm{H}), 2.47$ (s, $1 \mathrm{H}), 2.38$ (d, J=6.0 Hz, $3 \mathrm{H}), 2.32(\mathrm{~s}, 1 \mathrm{H})$.

${ }^{13}$ C NMR (150 MHz, CDCl 3 ): $\delta$ 169.0, 168.9, 168.2, 156.0, 147.7, 145.7, 139.3, 135.3, 131.1, 130.7, $130.3,129.1,118.3\left(\mathrm{q}, J_{C-F}=319.5 \mathrm{~Hz}\right), 116.0,114.9,114.4,112.4,111.6,111.4,101.2,55.6,43.2$, $42.3,40.4,38.6,30.6,30.5,28.4,27.7,13.3$.

${ }^{19}$ F NMR (376 MHz, $\left.\mathbf{C D C l}_{3}\right): \delta-73.6,-73.8$.

IR (neat, $\mathbf{c m}^{-1}$ ): 2928, 2842, 1679, 1418, 1316, 1213, 1142, 1053, 868, 776.

HRMS (ESI): $[\mathrm{M}+\mathrm{H}]^{+}$calcd for $\mathrm{C}_{25} \mathrm{H}_{23} \mathrm{ClF}_{3} \mathrm{~N}_{2} \mathrm{O}_{6} \mathrm{~S}$ 571.0912, found 571.0922.

NOTE: Because of the amide bond rotation equilibrium, two rotamers of $\mathbf{2 y}$ were observed on the NMR. This phenomenon is seen with many tertiary amides. For related references, see: ref. 37-38 


\section{Ni-catalyzed Enantioselective Cross-electrophile Aryl-alkenylation of Alkene}

\subsection{General Procedure}<smiles>[R]C(=C)CC([X])([R])C</smiles>

1 (1 equiv.)

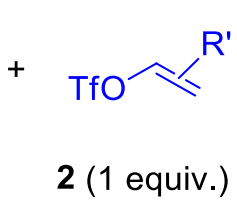

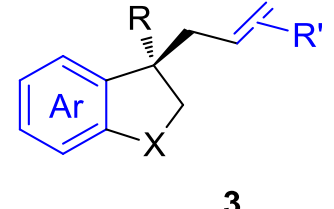

3

The procedure was conducted in an argon-filled glove box. To a reaction tube equipped with a magnetic stir bar was charged with $\mathrm{NiI}_{2}(6.3 \mathrm{mg}, 0.020 \mathrm{mmol}), \mathbf{L 1}(7.6 \mathrm{mg}, 0.028 \mathrm{mmol}), \mathrm{Mn}(44.0$ $\mathrm{mg}$, $0.8 \mathrm{mmol})$, and DMF/THF $(0.5 \mathrm{~mL} / 0.5 \mathrm{~mL})$. The reaction mixture was stirred for $5 \mathrm{~min}$. Substrates $1(0.2 \mathrm{mmol})$ and $2(0.2 \mathrm{mmol})$ were then added. The reaction tube was sealed with a rubber septum, and removed from the glove box. The reaction mixture was stirred at $25{ }^{\circ} \mathrm{C}$ for $24 \mathrm{~h}$. The reaction was quenched with water $(20.0 \mathrm{~mL})$, and extracted with ethyl acetate $(3 \times 15.0 \mathrm{~mL})$. The combined organic layers were washed with water, brine, dried over anhydrous $\mathrm{Na}_{2} \mathrm{SO}_{4}$, and concentrated under reduced pressure. The residue was purified by flash chromatography on silica gel to afford the desired product $\mathbf{3}$.

\subsection{Characterization Data of Products}

\section{(R)-3-(cyclohex-1-en-1-ylmethyl)-3-methyl-2,3-dihydrobenzofuran (3a)}

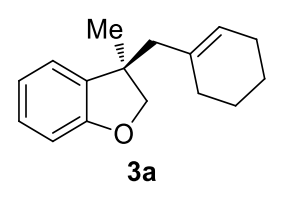

This compound was prepared according to the General procedure from the reaction of 1a (54.8 $\mathrm{mg}, 0.2 \mathrm{mmol})$ and $2 \mathbf{a}(46.0 \mathrm{mg}, 0.2 \mathrm{mmol})$.

$35.1 \mathrm{mg}, 77 \%$ yield, $98 \%$ ee, colorless oil.

Chiral HPLC: CHIRALPAK IA, $25{ }^{\circ} \mathrm{C},{ }^{i} \mathrm{PrOH}-$ hexanes $0.2 / 99.8,0.75 \mathrm{~mL} / \mathrm{min}, 280 \mathrm{~nm} . t_{\mathrm{R}}($ major$)=$ $9.1 \min , t_{\mathrm{R}}(\operatorname{minor})=11.1 \min$.

$[\boldsymbol{\alpha}]_{\mathbf{D}}{ }^{25}=-12\left(\mathrm{c}=1.0, \mathrm{CH}_{2} \mathrm{Cl}_{2}\right)$.

${ }^{1}$ H NMR (400 MHz, CDCl 3 ): $\delta$ 7.13-7.07 (m, $\left.2 \mathrm{H}\right), 6.86(\mathrm{dt}, J=0.8,7.6 \mathrm{~Hz}, 1 \mathrm{H}), 6.77(\mathrm{~d}, J=8.0$ Hz, $1 \mathrm{H}), 5.41$ (s, $1 \mathrm{H}), 4.47(\mathrm{~d}, J=8.4 \mathrm{~Hz}, 1 \mathrm{H}), 4.14$ (d, $J=8.4 \mathrm{~Hz}, 1 \mathrm{H}), 2.33-2.25$ (m, $2 \mathrm{H}), 1.99$ (s, $2 \mathrm{H}), 1.78-1.61$ (m, $2 \mathrm{H}), 1.54-1.44$ (m, $4 \mathrm{H}), 1.31$ (s, $3 \mathrm{H})$.

${ }^{13}$ C NMR (100 MHz, $\left.\mathbf{C D C l}_{3}\right): \delta$ 159.2, 135.8, 134.6, 127.9, 125.9, 122.9, 120.2, 109.5, 82.3, 49.0, $45.5,30.1,26.3,25.4,23.0,22.1$.

IR (neat, $\mathbf{c m}^{-1}$ ): 2926, 2838, 1597, 1482, 1459, 1230, 1016, 980, 831, 747.

HRMS (ESI): $[\mathrm{M}+\mathrm{H}]^{+}$calcd for $\mathrm{C}_{16} \mathrm{H}_{21} \mathrm{O} 229.1587$, found 229.1597. 

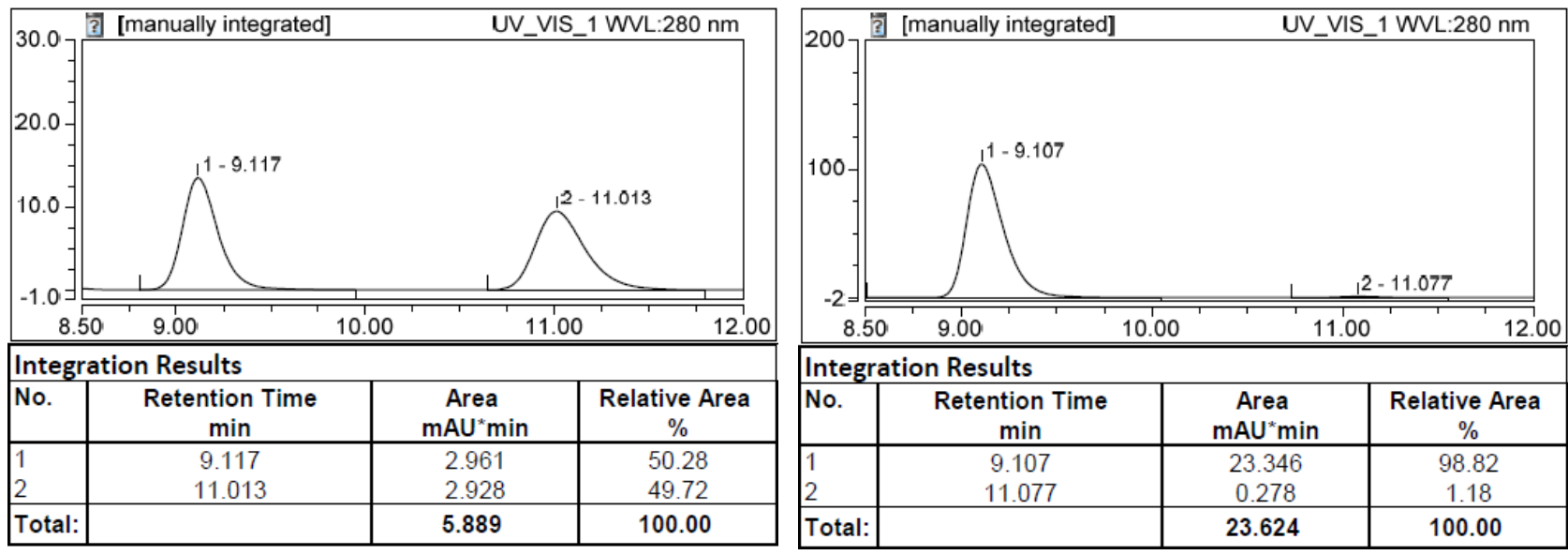

(R)-3-(cyclopent-1-en-1-ylmethyl)-3-methyl-2,3-dihydrobenzofuran (3b)

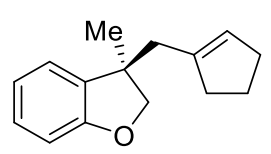

$3 \mathbf{b}$

The compound was prepared according to the General procedure from the reaction of $\mathbf{1 a}(54.8 \mathrm{mg}, 0.2 \mathrm{mmol})$ and $\mathbf{2 b}(43.2 \mathrm{mg}, 0.2 \mathrm{mmol})$.

$33.8 \mathrm{mg}, 79 \%$ yield, $98 \%$ ee, colorless oil.

Chiral HPLC: CHIRALPAK IA, $25{ }^{\circ} \mathrm{C},{ }^{i} \mathrm{PrOH}$-hexanes $0.2 / 99.8,0.75 \mathrm{~mL} / \mathrm{min}, 280 \mathrm{~nm}, t_{\mathrm{R}}($ major $)=$ $10.5 \min , t_{\mathrm{R}}($ minor $)=12.8 \mathrm{~min}$.

$[\boldsymbol{\alpha}]_{\mathbf{D}}{ }^{20}=+20\left(\mathrm{c}=1.0, \mathrm{CH}_{2} \mathrm{Cl}_{2}\right)$.

${ }^{1}$ H NMR (400 MHz, CDCl 3 ): $\delta$ 7.13-7.07 (m, $\left.2 \mathrm{H}\right), 6.86(\mathrm{~d}, J=7.2 \mathrm{~Hz}, 1 \mathrm{H}), 6.76(\mathrm{~d}, J=8.0 \mathrm{~Hz}, 1$ H), $5.37(\mathrm{t}, J=0.8 \mathrm{~Hz}, 1 \mathrm{H}), 4.45(\mathrm{~d}, J=8.4 \mathrm{~Hz}, 1 \mathrm{H}), 4.16(\mathrm{~d}, J=8.8 \mathrm{~Hz}, 1 \mathrm{H}), 2.51-2.40(\mathrm{~m}, 2 \mathrm{H})$, 2.28-2.24 (m, 2 H), 2.11-1.93 (m, 2 H), 1.82-1.74 (m, 2 H), 1.33 (s, $3 \mathrm{H})$.

${ }^{13} \mathbf{C}$ NMR (100 MHz, $\left.\mathbf{C D C l}_{3}\right): \delta$ 159.3, 140.8, 135.7, 128.5, 128.0, 122.8, 120.3, 109.5, 82.2, 45.3, $42.1,36.4,32.3,26.5,24.0$.

IR (neat, $\mathbf{c m}^{-1}$ ): 3051, 2957, 2849, 1599, 1482, 1232, 1018, 980, 833, 747.

HRMS (ESI): $[\mathrm{M}+\mathrm{H}]^{+}$calcd for $\mathrm{C}_{15} \mathrm{H}_{19} \mathrm{O} 215.1430$, found 215.1438 .
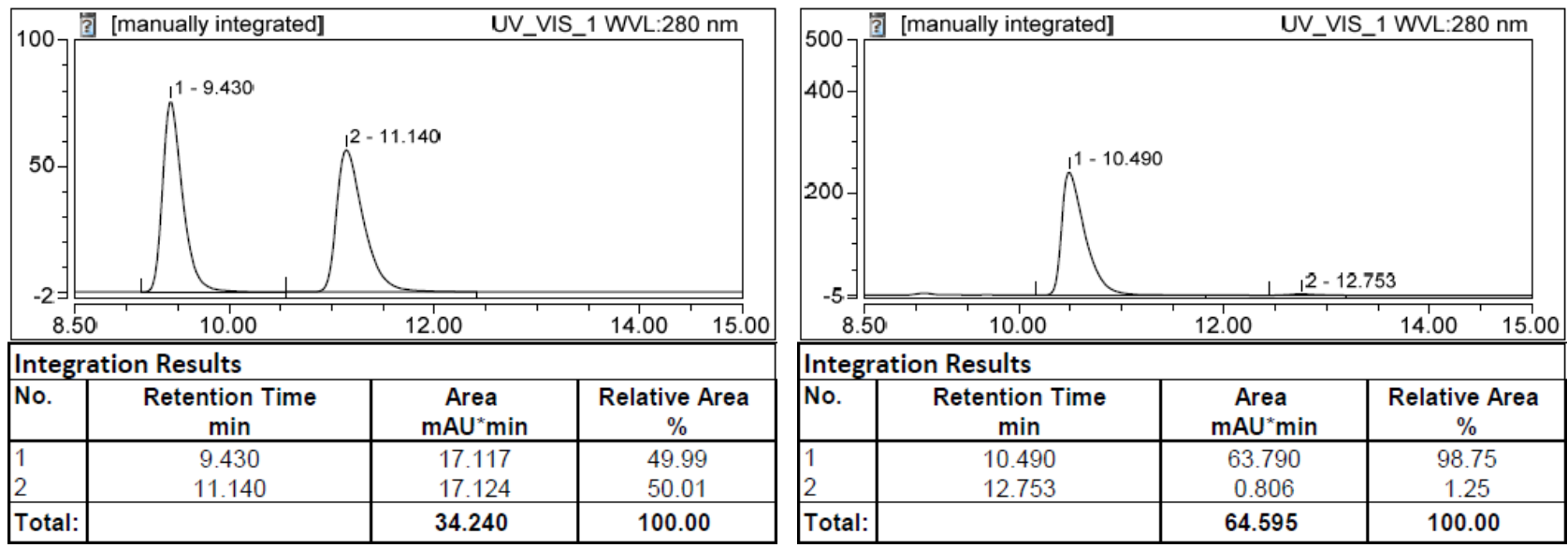


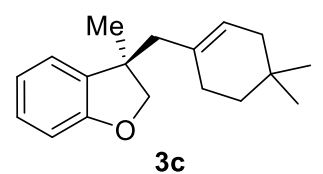

The compound was prepared according to the General Procedure from the reaction of 1a $(54.8 \mathrm{mg}, 0.2 \mathrm{mmol})$ with $2 \mathbf{c}(51.6 \mathrm{mg}, 0.2 \mathrm{mmol})$.

$36.9 \mathrm{mg}, 72 \%$ yield, $99 \%$ ee, colorless oil.

Chiral HPLC: CHIRALPAK IA, $25{ }^{\circ} \mathrm{C},{ }^{i} \mathrm{PrOH}$-hexanes $0.2 / 99.8,0.75 \mathrm{~mL} / \mathrm{min}, 280 \mathrm{~nm}, t_{\mathrm{R}}(\operatorname{minor})=$ $8.1 \mathrm{~min}, t_{\mathrm{R}}($ major $)=8.6 \min$.

$[\alpha]_{\mathbf{D}}{ }^{20}=-5\left(\mathrm{c}=1.0, \mathrm{CH}_{2} \mathrm{Cl}_{2}\right)$.

${ }^{1}$ H NMR (400 MHz, CDCl 3 ): $\delta$ 7.13-7.07 (m, $\left.2 \mathrm{H}\right), 6.86(\mathrm{dt}, J=0.8,7.2 \mathrm{~Hz}, 1 \mathrm{H}), 6.75(\mathrm{~d}, J=8.0$ Hz, $1 \mathrm{H}), 5.33(\mathrm{~s}, 1 \mathrm{H}), 4.48(\mathrm{~d}, J=8.8 \mathrm{~Hz}, 1 \mathrm{H}), 4.15(\mathrm{~d}, J=8.4 \mathrm{~Hz}, 1 \mathrm{H}), 2.34-2.27(\mathrm{~m}, 2 \mathrm{H})$, 1.77-1.59 (m, $4 \mathrm{H}), 1.32$ (s, $3 \mathrm{H}) .1 .26-1.23$ (m, $2 \mathrm{H}), 0.85$ (s, $3 \mathrm{H}), 0.83$ (s, $3 \mathrm{H})$.

${ }^{13} \mathbf{C}$ NMR (100 MHz, $\left.\mathbf{C D C l}_{3}\right): \delta 159.3,135.8,133.2,127.9,125.1,122.9,120.3,109.5,82.3,48.9$, $45.6,39.6,35.8,28.9,28.2,27.8,27.4,26.4$.

IR (neat, $\mathbf{c m}^{-1}$ ): 2956, 2920, 1613, 1482, 1459, 1232, 1018, 982, 833, 747.

HRMS (ESI): $[\mathrm{M}+\mathrm{H}]^{+}$calcd for $\mathrm{C}_{18} \mathrm{H}_{25} \mathrm{O}$ 257.1900, found 257.1896.
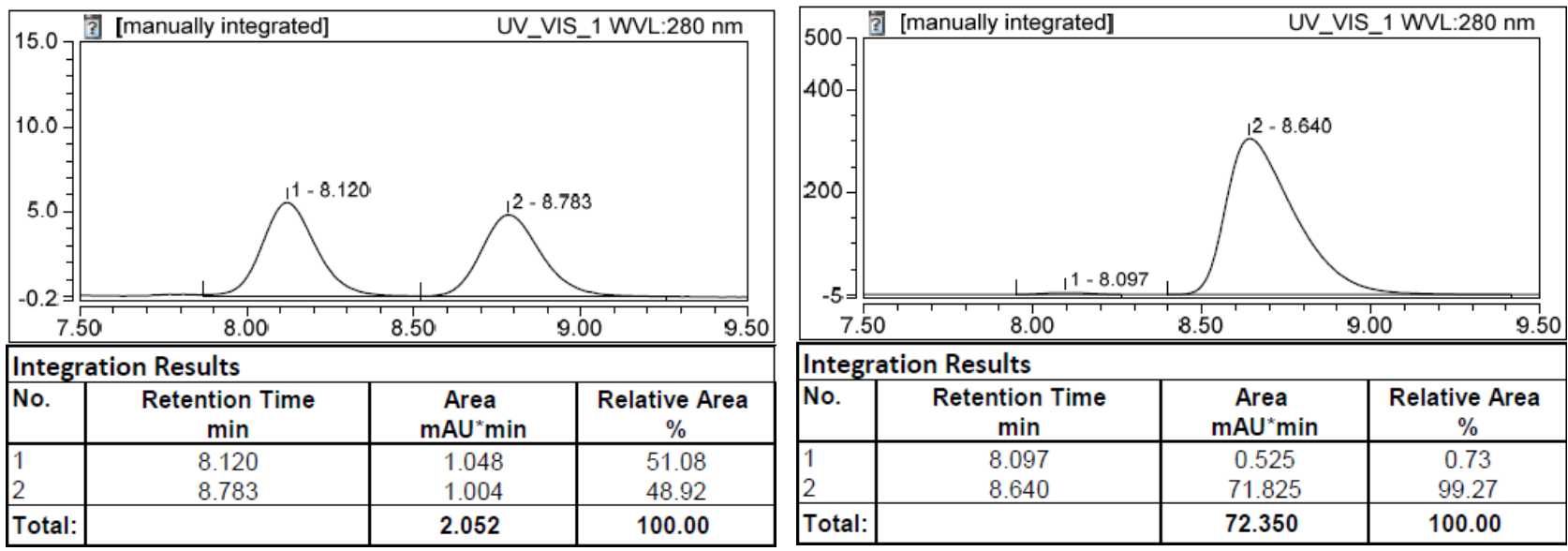

(R)-3-(cyclohept-1-en-1-ylmethyl)-3-methyl-2,3-dihydrobenzofuran (3d)

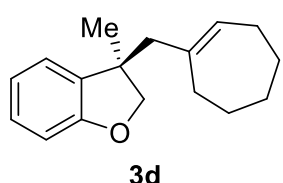

The compound was prepared according to the General Procedure from the reaction of $\mathbf{1 a}(54.8 \mathrm{mg}, 0.2 \mathrm{mmol})$ and $\mathbf{2 d}(48.8 \mathrm{mg}, 0.2 \mathrm{mmol})$.

$31.5 \mathrm{mg}, 65 \%$ yield, $97 \%$ ee, colorless oil.

Chiral HPLC: CHIRALPAK IA, $25{ }^{\circ} \mathrm{C},{ }^{i} \mathrm{PrOH}$-hexanes $0.2 / 99.8,0.75 \mathrm{~mL} / \mathrm{min}, 279 \mathrm{~nm}, t_{\mathrm{R}}($ major $)=$ $8.1 \min , t_{\mathrm{R}}(\operatorname{minor})=9.9 \min$.

$[\boldsymbol{\alpha}]_{\mathbf{D}}{ }^{19}=+2\left(\mathrm{c}=1.0, \mathrm{CH}_{2} \mathrm{Cl}_{2}\right)$.

${ }^{1}$ H NMR (400 MHz, $\left.\mathbf{C D C l}_{3}\right): \delta$ 7.12-7.07 (m, $\left.2 \mathrm{H}\right), 6.85(\mathrm{dt}, J=0.8,7.6 \mathrm{~Hz}, 1 \mathrm{H}), 6.76(\mathrm{~d}, J=8.0$ Hz, $1 \mathrm{H}), 5.53(\mathrm{t}, J=6.4 \mathrm{~Hz}, 1 \mathrm{H}), 4.51(\mathrm{~d}, J=8.4 \mathrm{~Hz}, 1 \mathrm{H}), 4.09(\mathrm{~d}, J=8.8 \mathrm{~Hz}, 1 \mathrm{H}), 2.35-2.27(\mathrm{~m}$, 2 H), 2.08-1.87 (m, 4 H), 1.69-1.65 (m, 2 H), 1.45-1.31 (m, 4 H), 1.33 (s, 3 H). 
${ }^{13}$ C NMR (100 MHz, $\left.\mathbf{C D C l}_{3}\right): \delta$ 159.4, 141.3, 135.6, 131.4, 127.9, 123.1, 120.2, 109.5, 81.8, 50.7, $45.9,34.4,32.5,28.6,27.0,26.5,26.1$.

IR (neat, $\mathbf{c m}^{-1}$ ): 2922, 2846, 1597, 1482, 1450, 1230, 1016, 978, 831, 747.

HRMS (ESI): $[\mathrm{M}+\mathrm{H}]{ }^{+}$calcd for $\mathrm{C}_{17} \mathrm{H}_{23} \mathrm{O} 243.1743$, found 243.1746 .
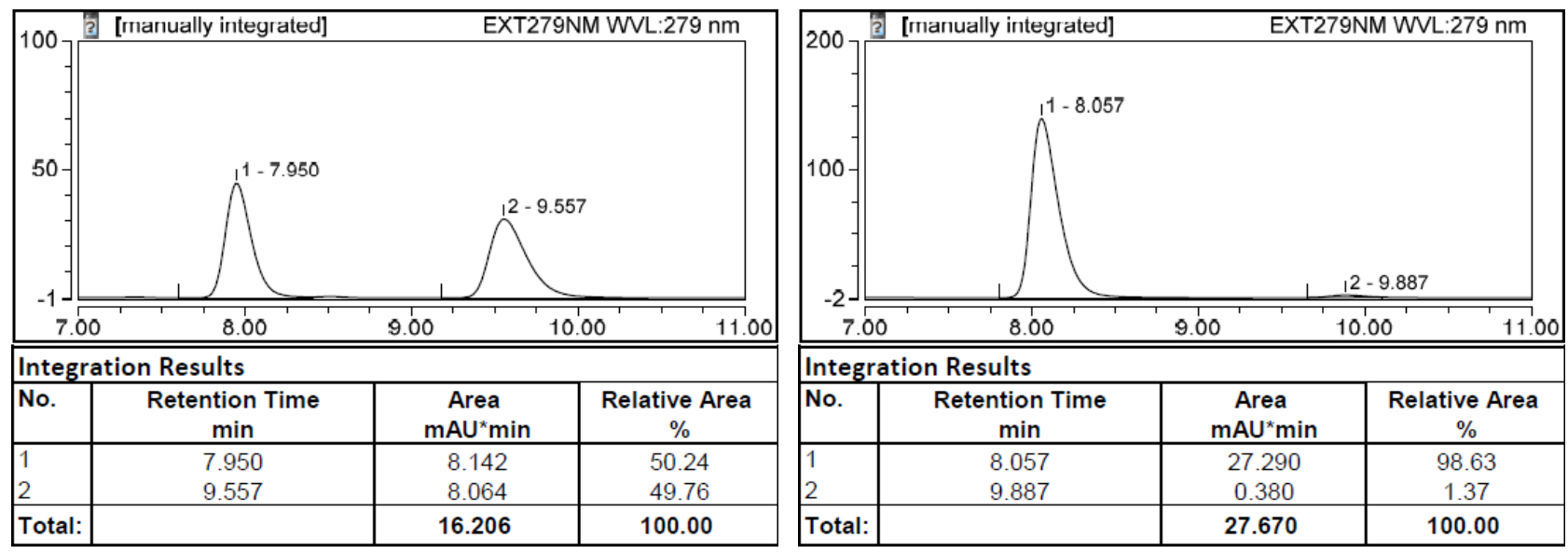

(R,E)-3-(cyclooct-1-en-1-ylmethyl)-3-methyl-2,3-dihydrobenzofuran (3e)

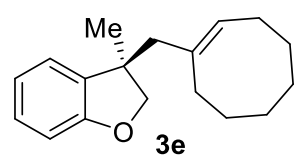

The compound was prepared according to the General Procedure from the reaction of $1 \mathbf{a}(54.8 \mathrm{mg}, 0.2 \mathrm{mmol})$ and $2 \mathbf{e}(51.6 \mathrm{mg}, 0.2 \mathrm{mmol})$.

$21.5 \mathrm{mg}, 42 \%$ yield, $97 \%$ ee, colorless oil.

Chiral HPLC: CHIRALPAK IA, $25{ }^{\circ} \mathrm{C},{ }^{i} \mathrm{PrOH}$-hexanes $0.2 / 99.8,0.75 \mathrm{~mL} / \mathrm{min}, 280 \mathrm{~nm}, t_{\mathrm{R}}($ major$)=$ $10.1 \min , t_{\mathrm{R}}(\operatorname{minor})=15.5 \min$.

$[\boldsymbol{\alpha}]_{\mathbf{D}}{ }^{19}=+2\left(\mathrm{c}=1.0, \mathrm{CH}_{2} \mathrm{Cl}_{2}\right)$.

${ }^{1}$ H NMR (400 MHz, CDCl $)$ ): $\delta$ 7.12-7.07 (m, $\left.2 \mathrm{H}\right), 6.87-6.83(\mathrm{t}, J=7.2 \mathrm{~Hz}, 1 \mathrm{H}), 6.77(\mathrm{~d}, J=8.0$ $\mathrm{Hz}, 1 \mathrm{H}), 5.35(\mathrm{t}, J=8.0 \mathrm{~Hz}, 1 \mathrm{H}), 4.50(\mathrm{~d}, J=8.4 \mathrm{~Hz}, 1 \mathrm{H}), 4.14(\mathrm{~d}, J=8.4 \mathrm{~Hz}, 1 \mathrm{H}), 2.36-2.25(\mathrm{~m}$, $2 \mathrm{H}), 2.10-1.90$ (m, $4 \mathrm{H}), 1.45-1.41$ (m, $8 \mathrm{H}), 1.33$ (s, $3 \mathrm{H})$.

${ }^{13} \mathbf{C}$ NMR (100 MHz, $\left.\mathbf{C D C l}_{3}\right): \delta$ 159.3, 137.1, 135.8, 129.0, 127.9, 123.0, 120.2, 109.5, 82.1, 46.8, 45.7, 29.9, 29.6, 28.3, 26.7, 26.6, 26.0, 26.0.

IR (neat, $\mathbf{c m}^{-1}$ ): 2924, 2853, 1482, 1459, 1277, 1262, 1018, 980, 713, 751.

HRMS (ESI): $[\mathrm{M}+\mathrm{H}]{ }^{+}$calcd for $\mathrm{C}_{18} \mathrm{H}_{25} \mathrm{O} 257.1900$, found 257.1902. 

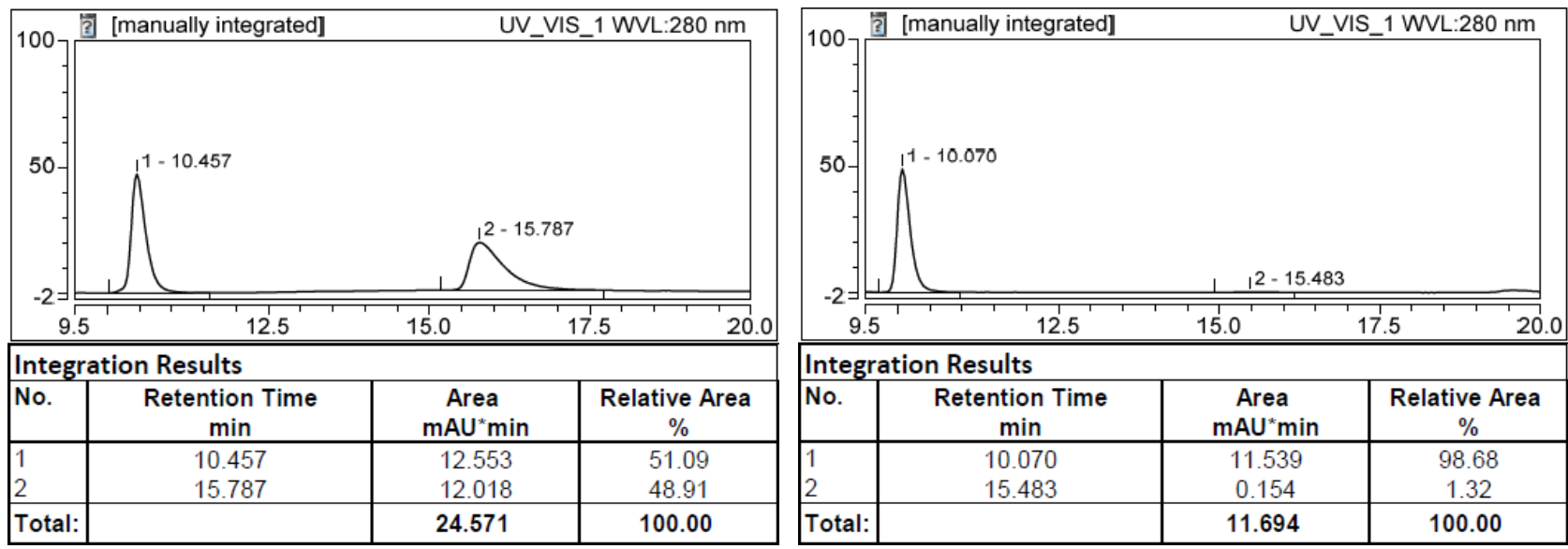

(3R)-3-((4-(tert-butyl)cyclohex-1-en-1-yl)methyl)-3-methyl-2,3-dihydrobenzofuran (3f)

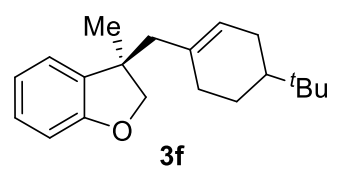

The compound was prepared according to the General Procedure from the reaction of $1 \mathbf{a}(54.8 \mathrm{mg}, 0.2 \mathrm{mmol})$ and $\mathbf{2 f}(57.2 \mathrm{mg}, 0.2 \mathrm{mmol})$.

$31.8 \mathrm{mg}, 56 \%$ yield, $98 \%$ ee, $\mathrm{dr}=1.2 / 1$, white solid, $\mathrm{mp} 34-36^{\circ} \mathrm{C}$.

Chiral HPLC: CHIRALCEL OD-H, $25{ }^{\circ} \mathrm{C},{ }^{i} \mathrm{PrOH}-h$ exanes $0.1 / 99.9,0.4 \mathrm{~mL} / \mathrm{min}, 280 \mathrm{~nm} . t_{\mathrm{R} 1}$ (major) $=24.2 \mathrm{~min}, t_{\mathrm{R} 1}(\operatorname{minor})=30.3 \mathrm{~min} ; t_{\mathrm{R} 2}($ major $)=25.0 \mathrm{~min}, t_{\mathrm{R} 2}($ minor $)=26.0 \mathrm{~min}$.

$[\boldsymbol{\alpha}]_{\mathbf{D}}{ }^{23}=+11\left(\mathrm{c}=1.0, \mathrm{CH}_{2} \mathrm{Cl}_{2}\right)$.

${ }^{1}$ H NMR (400 MHz, CDCl 3 ): $\delta$ 7.13-7.07 (m, $\left.2 \mathrm{H}\right), 6.88-6.84(\mathrm{~m}, 1 \mathrm{H}), 6.77(\mathrm{~d}, J=8.0 \mathrm{~Hz}, 1 \mathrm{H})$, [5.43 (t, $J=2.4 \mathrm{~Hz}), 5.39(\mathrm{~d}, J=3.2 \mathrm{~Hz}), 1 \mathrm{H}], 4.48-4.45(\mathrm{~m}, 1 \mathrm{H}), 4.16-4.12(\mathrm{~m}, 1 \mathrm{H}), 2.32-2.25(\mathrm{~m}$, $2 \mathrm{H}), 2.04-1.68$ (m, $5 \mathrm{H}), 1.33-1.30$ (m, $3 \mathrm{H}) .1 .20-1.07$ (m, $2 \mathrm{H}), 0.84$ (s, $9 \mathrm{H})$.

${ }^{13}$ C NMR (100 MHz, $\left.\mathbf{C D C l}_{3}\right): \delta$ 159.2, 136.0, 135.8, 134.5, 134.4, 127.93, 127.90, 126.3, 126.2, $122.9,120.3,120.2,109.5,82.3,82.2,48.6,48.3,45.6,45.5,43.83,43.76,32.1,31.7,31.6,27.18$, $27.15,27.1,26.4,26.2,24.5,24.3$.

IR (neat, $\mathbf{c m}^{-\mathbf{1}}$ ): 3008, 2965, 1654, 1547, 1480, 1460, 1277, 1262, 767, 751.

HRMS (ESI): $[\mathrm{M}+\mathrm{Na}]^{+}$calcd for $\mathrm{C}_{20} \mathrm{H}_{28} \mathrm{ONa} 302.2032$, found 302.2034.
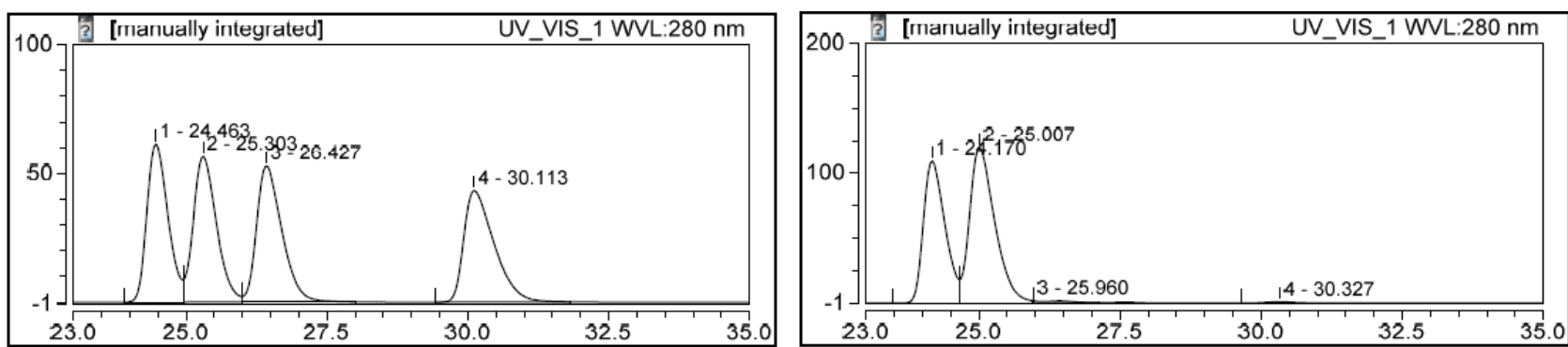

\begin{tabular}{|l|c|c|c|}
\hline \hline \multicolumn{3}{|l|}{ Integration Results } \\
\hline No. & $\begin{array}{c}\text { Retention Time } \\
\text { min }\end{array}$ & $\begin{array}{c}\text { Area } \\
\text { mAU*min }\end{array}$ & $\begin{array}{c}\text { Relative Area } \\
\%\end{array}$ \\
\hline 1 & 24.463 & 26.570 & 24.59 \\
2 & 25.303 & 27.070 & 25.06 \\
3 & 26.427 & 27.504 & 25.46 \\
4 & 30.113 & 26.898 & 24.90 \\
\hline Total: & & $\mathbf{1 0 8 . 0 4 3}$ & $\mathbf{1 0 0 . 0 0}$ \\
\hline
\end{tabular}

\begin{tabular}{|l|c|c|c|}
\hline \hline \multicolumn{3}{|l|}{ Integration Results } \\
\hline No. & $\begin{array}{c}\text { Retention Time } \\
\text { min }\end{array}$ & $\begin{array}{c}\text { Area } \\
\text { mAU*min }^{*}\end{array}$ & $\begin{array}{c}\text { Relative Area } \\
\%\end{array}$ \\
\hline 1 & 24.170 & 48.208 & 43.22 \\
2 & 25.007 & 61.938 & 55.53 \\
3 & 25.960 & 0.933 & 0.84 \\
4 & 30.327 & 0.464 & 0.42 \\
\hline Total: & & $\mathbf{1 1 1 . 5 4 4}$ & $\mathbf{1 0 0 . 0 0}$ \\
\hline
\end{tabular}




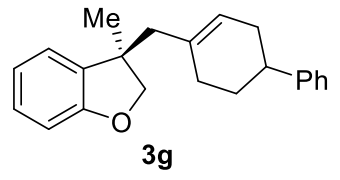

The compound was prepared according to the General Procedure from the reaction of $1 \mathbf{a}(54.8 \mathrm{mg}, 0.2 \mathrm{mmol})$ and $2 \mathrm{~g}(61.2 \mathrm{mg}, 0.2 \mathrm{mmol})$.

$34.7 \mathrm{mg}, 57 \%$ yield, $98 \%$ ee, $\mathrm{dr}=1.1: 1$, white solid, $\mathrm{mp}: 69-71^{\circ} \mathrm{C}$.

Chiral HPLC: CHIRALPAK IB, $25{ }^{\circ} \mathrm{C},{ }^{i} \mathrm{PrOH}-$ hexanes $0.2 / 99.8,1 \mathrm{~mL} / \mathrm{min}, 203 \mathrm{~nm} . t_{\mathrm{R} 1}(\operatorname{minor})=$ $17.0 \min , t_{\mathrm{R} 1}($ major $)=21.5 \mathrm{~min} ; t_{\mathrm{R} 2}($ minor $)=18.0 \mathrm{~min}, t_{\mathrm{R} 2}($ major $)=18.8 \mathrm{~min}$.

$[\alpha]_{\mathbf{D}}^{23}=+12\left(\mathrm{c}=1.0, \mathrm{CH}_{2} \mathrm{Cl}_{2}\right)$.

${ }^{1}$ H NMR (400 MHz, CDCl 3 ): $\delta$ 7.30-7.09 (m, 7 H), 6.89-6.85 (t, $\left.J=7.2 \mathrm{~Hz}, 1 \mathrm{H}\right), 6.80-6.76(\mathrm{~m}, 1$ H), [5.52 (d, $J=2.4 \mathrm{~Hz}), 5.48(\mathrm{~d}, J=1.2 \mathrm{~Hz}), 1 \mathrm{H}), 4.50(\mathrm{~d}, J=8.4 \mathrm{~Hz}, 1 \mathrm{H}), 4.17(\mathrm{t}, J=8.4 \mathrm{~Hz}, 1$ H), 2.71-2.67 (m, $1 \mathrm{H}), 2.37-1.63(\mathrm{~m}, 8 \mathrm{H}), 1.34(\mathrm{~d}, J=6.4 \mathrm{~Hz}, 3 \mathrm{H})$.

${ }^{13} \mathbf{C}$ NMR (100 MHz, $\left.\mathbf{C D C l}_{3}\right): \delta 159.2,147.0,146.9,135.7,135.6,134.6,134.5,128.29,128.26$, $128.01,127.96,126.82,126.78,125.92,125.90,125.6,125.5,122.9,120.3,109.5,82.2,48.7,48.5$, $45.6,45.4,39.7,39.5,33.8,33.4,30.8,30.3,30.1,30.0,26.4,26.2$.

IR (neat, $\mathbf{c m}^{-\mathbf{1}}$ ): 3407, 2917, 1655, 1482, 1459, 1277, 1262, 1016, 751, 699.

HRMS (ESI): $[\mathrm{M}+\mathrm{Na}]^{+}$calcd for $\mathrm{C}_{22} \mathrm{H}_{24} \mathrm{NaO} 327.1719$, found 327.1720.

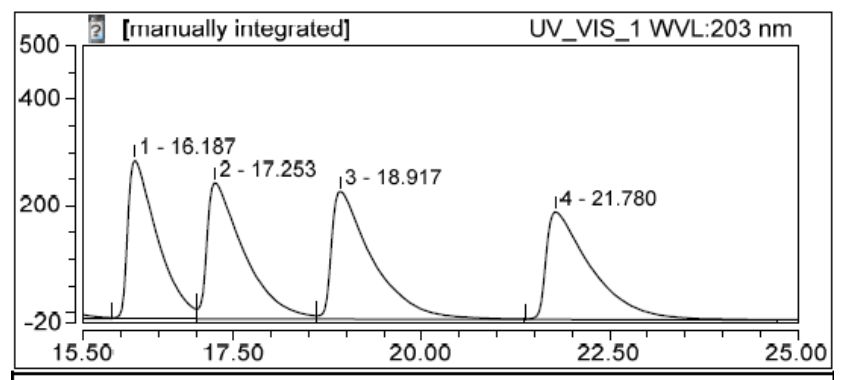

\begin{tabular}{|l|c|c|c|}
\hline 500 & [manually integrated] \\
\hline
\end{tabular}

\section{(R)-8-((3-methyl-2,3-dihydrobenzofuran-3-yl)methyl)-1,4-dioxaspiro[4.5]dec-7-ene (3h)}

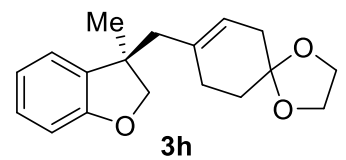

The compound was prepared according to the General Procedure from the reaction of $1 \mathbf{a}(54.8 \mathrm{mg}, 0.2 \mathrm{mmol})$ and $\mathbf{2 h}(57.6 \mathrm{mg}, 0.2 \mathrm{mmol})$ in DMF. $46.9 \mathrm{mg}, 82 \%$ yield, $98 \%$ ee, colorless oil.

Chiral HPLC: CHIRALCEL OJ-H, $25{ }^{\circ} \mathrm{C},{ }^{i}$ PrOH-hexanes 4/96, $0.8 \mathrm{~mL} / \mathrm{min}, 281 \mathrm{~nm}, t_{\mathrm{R}}($ major $)=$ $16.7 \mathrm{~min}, t_{\mathrm{R}}(\operatorname{minor})=18.7 \mathrm{~min}$.

$[\alpha]_{\mathbf{D}}{ }^{22}=-7\left(\mathrm{c}=1.0, \mathrm{CH}_{2} \mathrm{Cl}_{2}\right)$.

${ }^{1}$ H NMR (400 MHz, CDCl 3 ): $\delta$ 7.13-7.07 (m, $\left.2 \mathrm{H}\right), 6.86(\mathrm{t}, J=7.2 \mathrm{~Hz}, 1 \mathrm{H}), 6.77(\mathrm{~d}, J=8.0 \mathrm{~Hz}, 1$ 
H), $5.31(\mathrm{~s}, 1 \mathrm{H}), 4.45(\mathrm{~d}, J=8.8 \mathrm{~Hz}, 1 \mathrm{H}), 4.16(\mathrm{~d}, J=8.4 \mathrm{~Hz}, 1 \mathrm{H}), 3.97-3.93(\mathrm{~m}, 4 \mathrm{H}), 2.37-2.29$

(m, 2 H), 2.26 (s, $2 \mathrm{H}), 2.01-1.89$ (m, $2 \mathrm{H}), 1.67-1.63$ (m, $2 \mathrm{H}), 1.33$ (s, $3 \mathrm{H})$.

${ }^{13}$ C NMR (100 MHz, $\left.\mathbf{C D C l}_{3}\right): \delta$ 159.2, 135.5, 134.3, 128.0, 123.04, 122.95, 120.3, 109.6, 107.6, $82.4,64.3,47.7,45.5,35.8,31.2,29.1,25.9$.

IR (neat, $\mathbf{c m}^{-1}$ ): 2956, 2883, 1597, 1482, 1243, 1116, 1060, 1016, 833, 753.

HRMS (ESI): $[\mathrm{M}+\mathrm{H}]{ }^{+}$calcd for $\mathrm{C}_{18} \mathrm{H}_{23} \mathrm{O}_{3}$ 287.1642, found 287.1643.
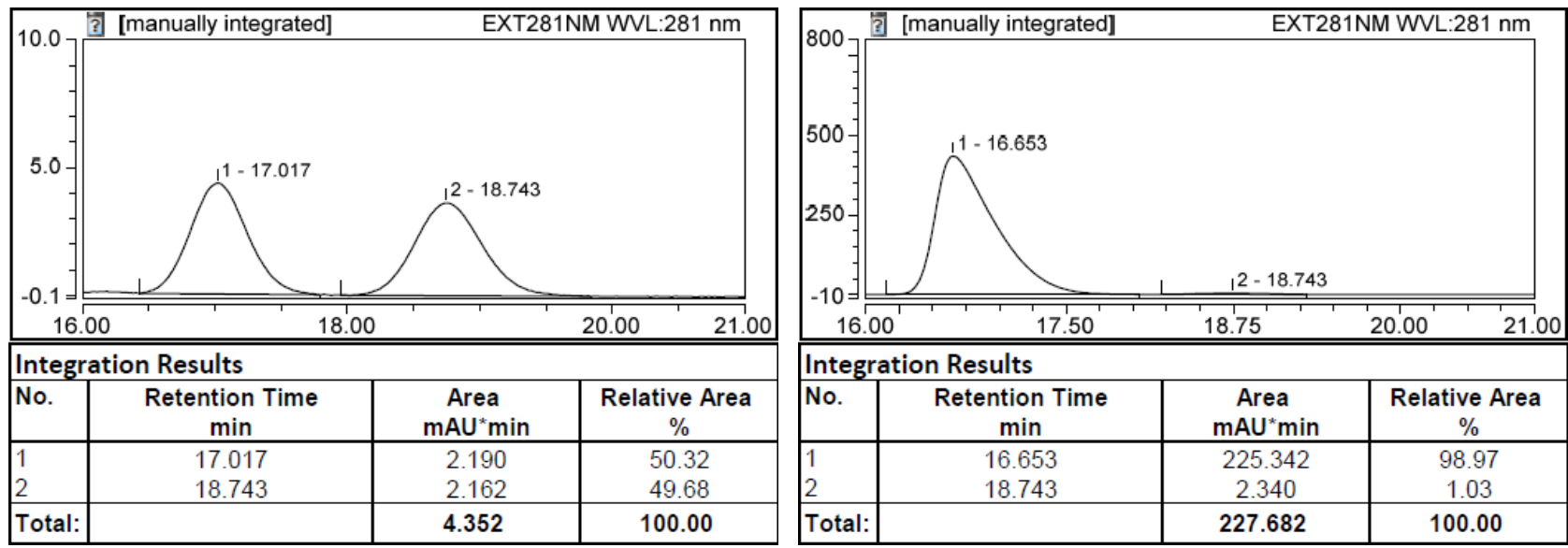

(R)-3-((3,6-dihydro-2H-pyran-4-yl)methyl)-3-methyl-2,3-dihydrobenzofuran (3i)<smiles>C[C@]1(CC2=CCOCC2)COc2ccccc21</smiles>

3i

The compound was prepared according to the General Procedure from the

reaction of 1a (54.8 $\mathrm{mg}, 0.2 \mathrm{mmol})$ and $\mathbf{2 i}(46.4 \mathrm{mg}, 0.2 \mathrm{mmol})$ in DMF.

$30.8 \mathrm{mg}, 67 \%$ yield, $98 \%$ ee, colorless oil.

Chiral HPLC: CHIRALPAK IA, $25{ }^{\circ} \mathrm{C}$, ${ }^{i} \mathrm{PrOH}$-hexanes 5/95, $1 \mathrm{~mL} / \mathrm{min}, 280 \mathrm{~nm}, t_{\mathrm{R}}$ (major) $=5.6$ $\min , t_{\mathrm{R}}($ minor $)=6.2 \mathrm{~min}$.

$[\boldsymbol{\alpha}]_{\mathbf{D}}{ }^{21}=+1\left(\mathrm{c}=1.0, \mathrm{CH}_{2} \mathrm{Cl}_{2}\right)$.

1H NMR (400 MHz, CDCl $)$ ): $\delta$ 7.14-7.08 (m, $2 \mathrm{H}), 6.88-6.84(\mathrm{dt}, J=0.8,7.2 \mathrm{~Hz}, 1 \mathrm{H}), 6.77(\mathrm{~d}, J=$ $8.0 \mathrm{~Hz}, 1 \mathrm{H}), 5.40(\mathrm{~s}, 1 \mathrm{H}), 4.46(\mathrm{~d}, J=8.8 \mathrm{~Hz}, 1 \mathrm{H}), 4.16(\mathrm{~d}, J=8.4 \mathrm{~Hz}, 1 \mathrm{H}), 4.10-4.06(\mathrm{~m}, 2 \mathrm{H})$, 3.69-3.59 (m, 2 H), 2.38-2.30 (m, 2 H), 1.86-1.74 (m, 2 H), 1.35 (s, 3 H).

${ }^{13} \mathbf{C}$ NMR (100 MHz, $\left.\mathbf{C D C l}_{3}\right): \delta$ 159.3, 135.2, 132.6, 128.1, 124.4, 122.9, 120.3, 109.6, 82.0, 65.4, $64.2,48.2,45.4,30.2,26.2$.

IR (neat, $\mathbf{c m}^{-\mathbf{1}}$ ): 2962, 2752, 1722, 1597, 1481, 1235, 1128, 978, 832, 752.

HRMS (ESI): $[\mathrm{M}+\mathrm{H}]{ }^{+}$calcd for $\mathrm{C}_{15} \mathrm{H}_{19} \mathrm{O}_{2} 231.1380$, found 231.1381 . 

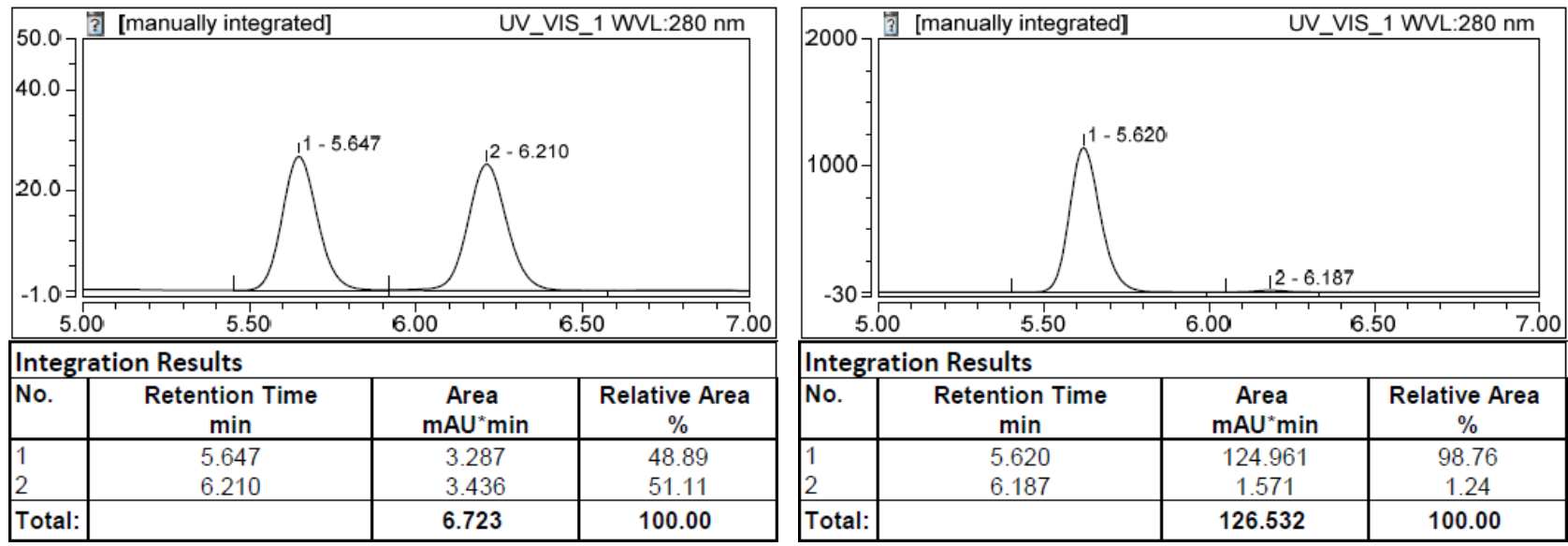

(R)-3-((3,6-dihydro-2H-thiopyran-4-yl)methyl)-3-methyl-2,3-dihydrobenzofuran (3j)

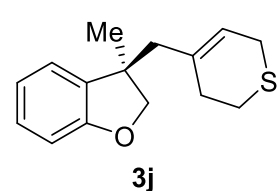

The compound was prepared according to the General Procedure from the reaction of $\mathbf{1 a}(54.8 \mathrm{mg}, 0.2 \mathrm{mmol})$ and $\mathbf{2 j}(49.6 \mathrm{mg}, 0.2 \mathrm{mmol})$ in DMF.

$32.0 \mathrm{mg}, 65 \%$ yield, $96 \%$ ee, colorless oil.

Chiral HPLC: CHIRALCEL OJ-H, $25{ }^{\circ} \mathrm{C}$, ${ }^{i}$ PrOH-hexanes 2/98, $1 \mathrm{~mL} / \mathrm{min}, 260 \mathrm{~nm}, t_{\mathrm{R}}($ major $)=$ $12.0 \mathrm{~min}, t_{\mathrm{R}}($ minor $)=13.2 \mathrm{~min}$.

$[\boldsymbol{\alpha}]_{\mathbf{D}}{ }^{21}=-17\left(\mathrm{c}=1.0, \mathrm{CH}_{2} \mathrm{Cl}_{2}\right)$.

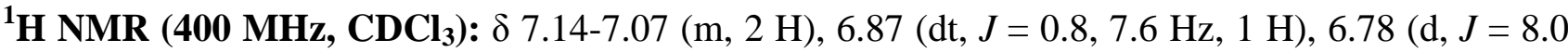
Hz, $1 \mathrm{H}), 5.57(\mathrm{~s}, 1 \mathrm{H}), 4.45(\mathrm{~d}, J=8.8 \mathrm{~Hz}, 1 \mathrm{H}), 4.14(\mathrm{~d}, J=8.8 \mathrm{~Hz}, 1 \mathrm{H}), 3.19-3.09$ (m, $2 \mathrm{H})$, 2.64-2.53 (m, 2 H), 2.36-2.27 (m, 2 H), 2.04-1.88 (m, 2 H), 1.34 (s, $3 \mathrm{H})$.

${ }^{13}$ C NMR (100 MHz, $\left.\mathbf{C D C l}_{3}\right): \delta 159.3,135.6,135.1,128.2,123.0,122.5,120.3,109.7,82.1,49.8$, $45.6,30.8,26.0,25.7,25.1$.

IR (neat, $\mathbf{c m}^{-1}$ ): 2961, 2883, 1663, 1596, 1480, 1230, 1017, 975, 831, 753.

HRMS (ESI): $[\mathrm{M}+\mathrm{H}]^{+}$calcd for $\mathrm{C}_{15} \mathrm{H}_{19} \mathrm{OS} 247.1151$, found 247.1153 .
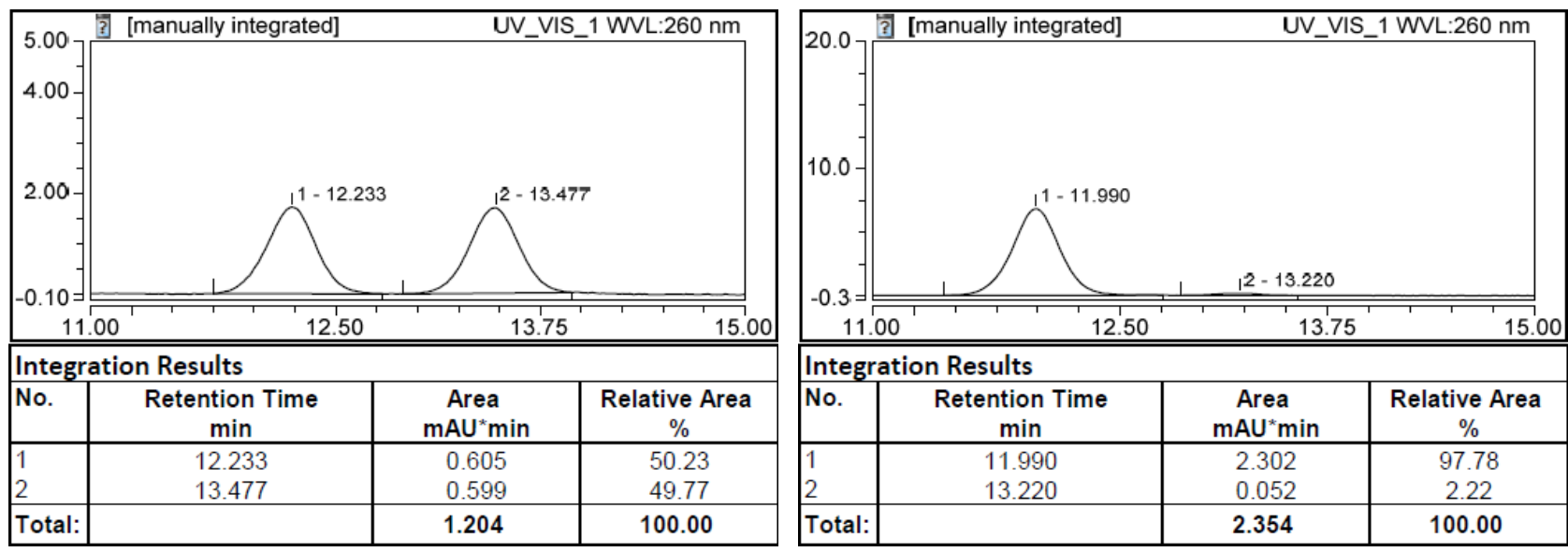

(R)-4-((3-methyl-2,3-dihydrobenzofuran-3-yl)methyl)-1-tosyl-1,2,3,6-tetrahydropyridine (3k) 


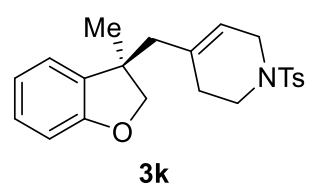

The compound was prepared according to the General Procedure from the reaction of 1a $(54.8 \mathrm{mg}, 0.2 \mathrm{mmol})$ and $2 \mathbf{k}(77.0 \mathrm{mg}, 0.2 \mathrm{mmol})$.

$59.7 \mathrm{mg}, 78 \%$ yield, $98 \%$ ee, white solid, mp: $87-89^{\circ} \mathrm{C}$.

Chiral HPLC: CHIRALPAK ID, $25{ }^{\circ} \mathrm{C},{ }^{i} \mathrm{PrOH}-h$ exanes 8/92, $1 \mathrm{~mL} / \mathrm{min}, 203 \mathrm{~nm}, t_{\mathrm{R}}(\operatorname{minor})=56.5$ $\min , t_{\mathrm{R}}($ major $)=57.8 \min$.

$[\alpha]_{\mathbf{D}}^{23}=-8\left(\mathrm{c}=1.0, \mathrm{CH}_{2} \mathrm{Cl}_{2}\right)$.

${ }^{1}$ H NMR (400 MHz, CDCl 3 ): $\delta 7.64(\mathrm{~d}, J=8.4 \mathrm{~Hz}, 2 \mathrm{H}), 7.31(\mathrm{~d}, J=8.0 \mathrm{~Hz}, 2 \mathrm{H}), 7.12-7.08$ (m, 1 H), $7.02(\mathrm{dd}, J=0.8,7.2 \mathrm{~Hz}, 1 \mathrm{H}), 6.84(\mathrm{dt}, J=0.8,7.6 \mathrm{~Hz}, 1 \mathrm{H}), 6.73(\mathrm{~d}, J=8.0 \mathrm{~Hz}, 1 \mathrm{H}), 5.29(\mathrm{~s}, 1$ H), $4.35(\mathrm{~d}, J=8.4 \mathrm{~Hz}, 1 \mathrm{H}), 4.08(\mathrm{~d}, J=8.8 \mathrm{~Hz}, 1 \mathrm{H}), 3.61-3.47(\mathrm{~m}, 2 \mathrm{H}), 3.12-2.94(\mathrm{~m}, 2 \mathrm{H}), 2.42$ (s, $3 \mathrm{H}), 2.32-2.24$ (m, 2 H), 1.94-1.78 (m, $2 \mathrm{H}), 1.28$ (s, $3 \mathrm{H})$.

${ }^{13}$ C NMR (100 MHz, CDCl $)$ ): $\delta$ 159.2, 143.5, 134.8, 133.5, 133.2, 129.6, 128.2, 127.6, 122.8, 121.0, 120.4, 109.6, 81.7, 47.9, 45.3, 44.7, 42.8, 29.9, 26.0, 21.4.

IR (neat, $\mathbf{c m}^{-1}$ ): 2963, 2922, 1597, 1482, 1344, 1165, 1094, 952, 754, 688.

HRMS (ESI): $[\mathrm{M}+\mathrm{H}]^{+}$calcd for $\mathrm{C}_{22} \mathrm{H}_{26} \mathrm{NO}_{3} \mathrm{~S} 384.1628$, found 384.1627.

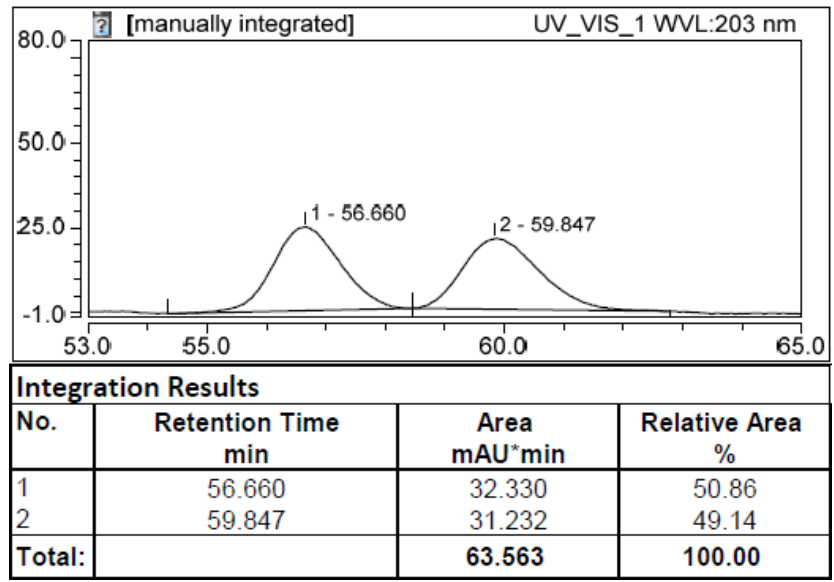

\begin{tabular}{|l|c|c|c|}
\hline 1500 & \multicolumn{3}{|c|}{ UV_VIS_1 WVL:203 nm } \\
\hline
\end{tabular}

\section{Tert-butyl (R)-4-((3-methyl-2,3-dihydrobenzofuran-3-yl)methyl)-3,6-dihydropyridine-1(2H)-} carboxylate (31)

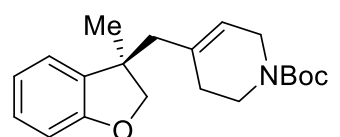

3I

The compound was prepared according to the General Procedure from the reaction of 1a $(54.8 \mathrm{mg}, 0.2 \mathrm{mmol})$ and $2 \mathbf{l}(66.2 \mathrm{mg}, 0.2 \mathrm{mmol})$. $52.0 \mathrm{mg}, 79 \%$ yield, $96 \%$ ee, colorless oil.

Chiral HPLC: CHIRALCEL OJ-H, $25{ }^{\circ} \mathrm{C},{ }^{i} \mathrm{PrOH}-$ hexanes 2/98, $1 \mathrm{~mL} / \mathrm{min}, 260 \mathrm{~nm}, t_{\mathrm{R}}$ (major) $=$ $12.4 \mathrm{~min}, t_{\mathrm{R}}($ minor $)=17.0 \mathrm{~min}$.

$[\boldsymbol{\alpha}]_{\mathrm{D}}^{23}=-6\left(\mathrm{c}=1.0, \mathrm{CH}_{2} \mathrm{Cl}_{2}\right)$.

${ }^{1}$ H NMR (400 MHz, CDCl $\left.)_{3}\right): \delta$ 7.14-7.06 (m, $\left.2 \mathrm{H}\right), 6.86(\mathrm{dt}, J=0.8,7.6 \mathrm{~Hz}, 1 \mathrm{H}), 6.77(\mathrm{~d}, J=8.0$ 
$\mathrm{Hz}, 1 \mathrm{H}), 5.34(\mathrm{~s}, 1 \mathrm{H}), 4.44$ (d, J = 8.4 Hz, $1 \mathrm{H}), 4.14$ (d, J = 8.8 Hz, $1 \mathrm{H}), 3.91-3.76$ (m, $2 \mathrm{H}), 3.41$

(s, $1 \mathrm{H}), 3.29-3.23$ (m, $1 \mathrm{H}), 2.38-2.31$ (m, $2 \mathrm{H}), 1.82-1.76$ (m, $2 \mathrm{H}), 1.45$ (s, $9 \mathrm{H}), 1.34$ (s, $3 \mathrm{H})$.

${ }^{13}$ C NMR (100 MHz, $\left.\mathbf{C D C l}_{3}\right): \delta 159.2,154.9,135.0,133.6,128.2,122.9,122.3,120.4,109.6,81.9$, $79.4,48.3,45.5,43.5,40.9,30.0,28.4,26.1$.

IR (neat, $\mathbf{c m}^{-1}$ ): 2976, 2932, 1698, 1481, 1420, 1366, 1172, 980, 845, 753.

HRMS (ESI): $[\mathrm{M}+\mathrm{H}]{ }^{+}$calcd for $\mathrm{C}_{20} \mathrm{H}_{28} \mathrm{NO}_{3} 330.2064$, found 330.2062.
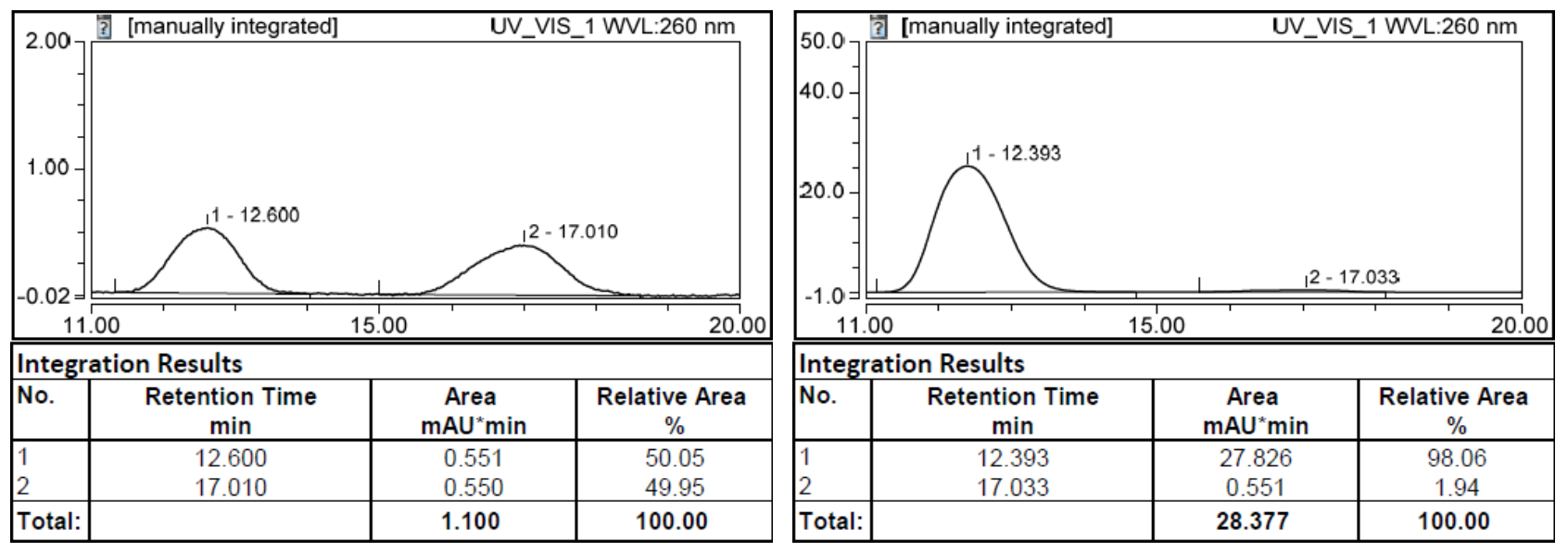

(R)-3-((1H-inden-2-yl)methyl)-3-methyl-2,3-dihydrobenzofuran (3m)

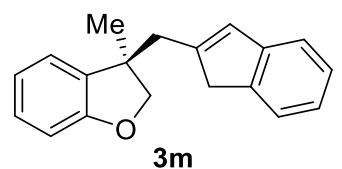

The compound was prepared according to the General Procedure from the reaction of $1 \mathbf{a}(54.8 \mathrm{mg}, 0.2 \mathrm{mmol})$ and $\mathbf{2 m}(52.8 \mathrm{mg}, 0.2 \mathrm{mmol})$ in DMF. $37.2 \mathrm{mg}, 71 \%$ yield, $96 \%$ ee, colorless oil.

Chiral HPLC: CHIRALCEL OD-H, $25{ }^{\circ} \mathrm{C},{ }^{i} \mathrm{PrOH}-$ hexanes 5/95, $1 \mathrm{~mL} / \mathrm{min}, 280 \mathrm{~nm}, t_{\mathrm{R}}($ major $)=$ $6.2 \min , t_{\mathrm{R}}(\operatorname{minor})=6.8 \min$.

$[\alpha]_{\mathbf{D}}^{22}=+66\left(\mathrm{c}=1.0, \mathrm{CH}_{2} \mathrm{Cl}_{2}\right)$.

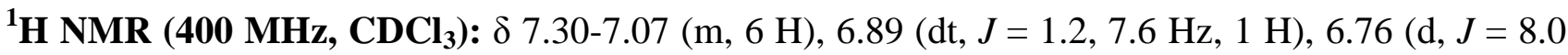
$\mathrm{Hz}, 1 \mathrm{H}), 6.52(\mathrm{~m}, 1 \mathrm{H}), 4.52(\mathrm{~d}, J=8.8 \mathrm{~Hz}, 1 \mathrm{H}), 4.19(\mathrm{~d}, J=8.4 \mathrm{~Hz}, 1 \mathrm{H}), 3.15-2.95(\mathrm{~m}, 2 \mathrm{H}), 2.81$ (s, $2 \mathrm{H}), 1.41(\mathrm{~s}, 3 \mathrm{H})$.

${ }^{13}$ C NMR (100 MHz, CDCl 3 ): $\delta$ 159.3, 145.8, 144.8, 143.4, 134.9, 130.4, 128.3, 126.2, 124.0, 123.3, $122.8,120.5,120.1,109.7,81.8,45.8,42.4,42.1,26.5$.

IR (neat, $\mathbf{c m}^{-1}$ ): 2962, 2885, 1610, 1481, 1392, 1265, 1101, 978, 831, 752.

HRMS (ESI): $[\mathrm{M}+\mathrm{H}]^{+}$calcd for $\mathrm{C}_{19} \mathrm{H}_{19} \mathrm{O} 263.1430$, found 263.1431 . 

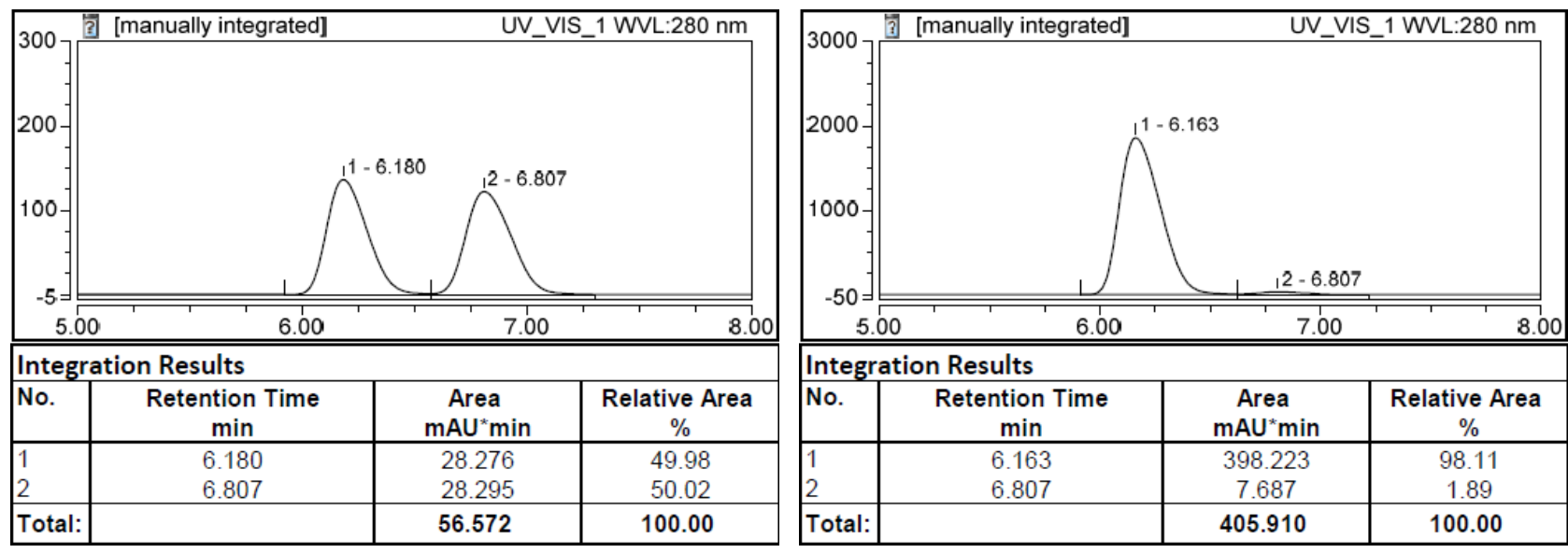

(R)-3-((3,4-dihydronaphthalen-1-yl)methyl)-3-methyl-2,3-dihydrobenzofuran (3n)

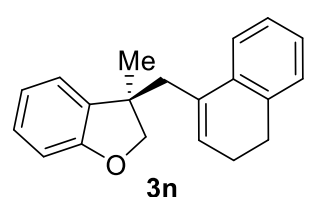

The compound was prepared according to the General Procedure from the reaction of $1 \mathbf{a}(49 \mathrm{mg}, 0.2 \mathrm{mmol})$ and $\mathbf{2 n}(55.6 \mathrm{mg}, 0.2 \mathrm{mmol})$ in DMF. $39.7 \mathrm{mg}, 72 \%$ yield, $97 \%$ ee, colorless oil.

Chiral HPLC: CHIRALCEL OJ-H, $25{ }^{\circ} \mathrm{C},{ }^{i} \mathrm{PrOH}-$ hexanes 5/95, $1 \mathrm{~mL} / \mathrm{min}, 260 \mathrm{~nm}, t_{\mathrm{R}}(\operatorname{minor})=$ $11.5 \min , t_{\mathrm{R}}($ major $)=15.3 \min$

$[\boldsymbol{\alpha}]_{\mathbf{D}}^{22}=-25\left(\mathrm{c}=1.0, \mathrm{CH}_{2} \mathrm{Cl}_{2}\right)$.

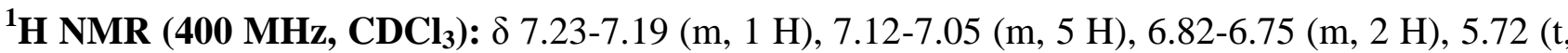
$J=4.8 \mathrm{~Hz}, 1 \mathrm{H}), 4.47(\mathrm{~d}, J=8.8 \mathrm{~Hz}, 1 \mathrm{H}), 4.00(\mathrm{~d}, J=8.8 \mathrm{~Hz}, 1 \mathrm{H}), 2.85-2.67(\mathrm{~m}, 4 \mathrm{H}), 2.20-2.15$ (m, $2 \mathrm{H}), 1.31(\mathrm{~s}, 3 \mathrm{H})$.

${ }^{13}$ C NMR (100 MHz, CDCl 3 ): $\delta$ 159.4, 136.5, 135.5, 135.4, 133.1, 129.4, 128.0, 127.5, 126.5, 126.1, $123.1,123.0,120.2,109.6,82.1,46.1,41.7,28.6,25.0,23.2$.

IR (neat, $\mathbf{c m}^{-1}$ ): 2963, 2881, 1655, 1597, 1480, 1234, 1016, 975, 833, 744.

HRMS (ESI): $[\mathrm{M}+\mathrm{K}]^{+}$calcd for $\mathrm{C}_{20} \mathrm{H}_{20} \mathrm{OK} 315.1146$, found 315.1145 .

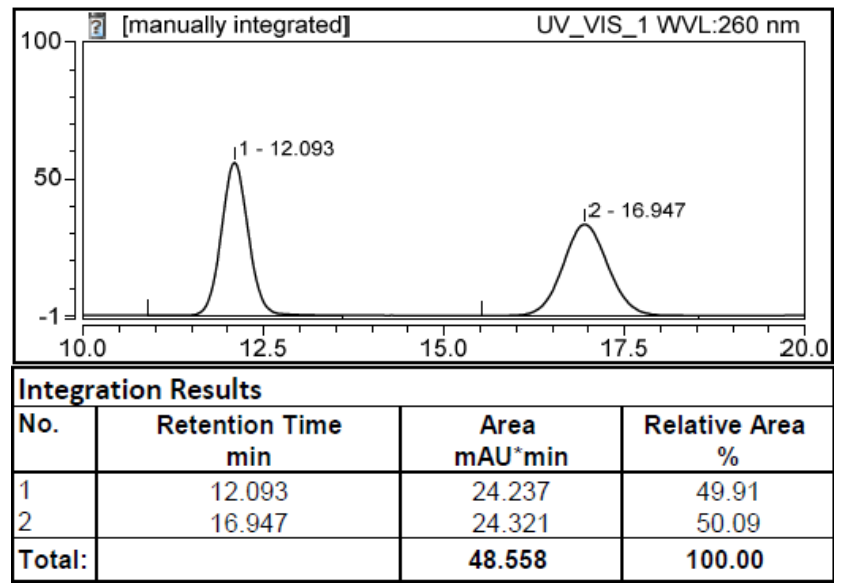

\begin{tabular}{|l|c|c|c|}
\hline 1000 & \multicolumn{3}{|c|}{ UV_VIS_1 WVL:260 nm } \\
\hline
\end{tabular}


<smiles>C=C(CCCCCCCCCCCCCC)CC1(C)COc2ccccc21</smiles>

The compound was prepared according to the General Procedure from the reaction of 1a (109.6 $\mathrm{mg}, 0.4 \mathrm{mmol})$ and $2 \mathbf{o}(49.2 \mathrm{mg}, 0.2 \mathrm{mmol})$ in THF. $34.6 \mathrm{mg}, 71 \%$ yield, $90 \%$ ee, colorless oil.

Chiral HPLC: CHIRALPAK IB, $25{ }^{\circ} \mathrm{C},{ }^{i} \mathrm{PrOH}$-hexanes $0.2 / 99.8,0.75 \mathrm{~mL} / \mathrm{min}, 280 \mathrm{~nm}, t_{\mathrm{R}}($ major $)=$ $7.3 \min , t_{\mathrm{R}}(\operatorname{minor})=11.6 \min$.

$[\boldsymbol{\alpha}]_{\mathbf{D}}{ }^{20}=+8\left(\mathrm{c}=1.0, \mathrm{CH}_{2} \mathrm{Cl}_{2}\right)$.

${ }^{1}$ H NMR (400 MHz, CDCl 3 ): $\delta$ 7.13-7.09 (m, $\left.2 \mathrm{H}\right), 6.88-6.84(\mathrm{~m}, 1 \mathrm{H}), 6.77(\mathrm{~d}, J=8.0 \mathrm{~Hz}, 1 \mathrm{H})$, $4.85(\mathrm{~d}, J=1.6 \mathrm{~Hz}, 1 \mathrm{H}), 4.70(\mathrm{~s}, 1 \mathrm{H}), 4.50(\mathrm{~d}, J=8.4 \mathrm{~Hz}, 1 \mathrm{H}), 4.16(\mathrm{~d}, J=8.8 \mathrm{~Hz}, 1 \mathrm{H}), 2.44-2.32$ (m, $2 \mathrm{H}), 1.84-1.68(\mathrm{~m}, 2 \mathrm{H}), 1.38-1.14(\mathrm{~m}, 9 \mathrm{H}), 0.86(\mathrm{t}, J=7.2 \mathrm{~Hz}, 3 \mathrm{H})$.

${ }^{13}$ C NMR (100 MHz, $\left.\mathbf{C D C l}_{3}\right): \delta$ 159.2, 146.6, 135.5, 128.0, 122.9, 120.3, 113.4, 109.6, 82.0, 46.3, $45.3,37.0,31.5,27.6,26.3,22.5,14.0$.

IR (neat, $\mathbf{c m}^{-1}$ ): 2958, 2876, 1638, 1599, 1482, 1232, 1018, 982, 896, 747.

HRMS (ESI): $[\mathrm{M}+\mathrm{H}]{ }^{+}$calcd for $\mathrm{C}_{17} \mathrm{H}_{25} \mathrm{O} 245.1900$, found 245.1902 .
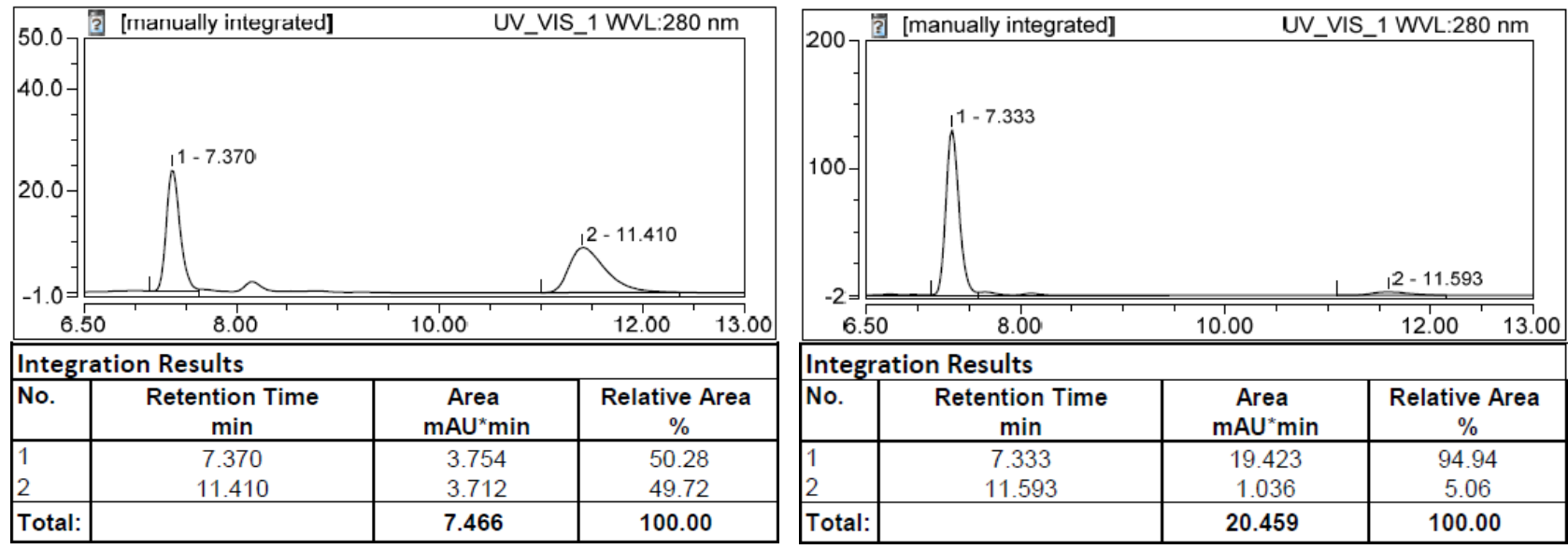

\section{Methyl (R)-6-((3-methyl-2,3-dihydrobenzofuran-3-yl)methyl)hept-6-enoate (3p)}

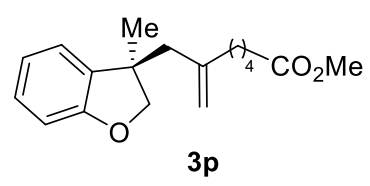

The compound was prepared according to the General Procedure from the reaction of $1 \mathbf{a}(164.4 \mathrm{mg}, 0.6 \mathrm{mmol})$ and $\mathbf{2 p}(58.0 \mathrm{mg}, 0.2 \mathrm{mmol})$. $32.8 \mathrm{mg}, 57 \%$ yield, $92 \%$ ee, colorless oil.

Chiral HPLC: CHIRALPAK ID, $25{ }^{\circ} \mathrm{C},{ }^{i} \mathrm{PrOH}$-hexanes 5/95, $1 \mathrm{~mL} / \mathrm{min}, 203 \mathrm{~nm}, t_{\mathrm{R}}$ (major) $=5.7$ $\min , t_{\mathrm{R}}(\operatorname{minor})=6.4 \min$.

$[\boldsymbol{\alpha}]_{\mathrm{D}}{ }^{20}=+4\left(\mathrm{c}=1.0, \mathrm{CH}_{2} \mathrm{Cl}_{2}\right)$.

${ }^{1}$ H NMR (400 MHz, CDCl $): \delta 7.14-7.09(\mathrm{~m}, 2 \mathrm{H}), 6.87(\mathrm{t}, J=7.2 \mathrm{~Hz}, 1 \mathrm{H}), 6.77(\mathrm{~d}, J=8.0 \mathrm{~Hz}, 1$ H), $4.86(\mathrm{~d}, J=0.8 \mathrm{~Hz}, 1 \mathrm{H}), 4.72(\mathrm{~s}, 1 \mathrm{H}), 4.50(\mathrm{~d}, J=8.8 \mathrm{~Hz}, 1 \mathrm{H}), 4.16(\mathrm{~d}, J=8.4 \mathrm{~Hz}, 1 \mathrm{H}), 3.66$ (s, $3 \mathrm{H}), 2.43-2.31(\mathrm{~m}, 2 \mathrm{H}), 2.27-2.23$ (m, $2 \mathrm{H}), 1.81-1.64(\mathrm{~m}, 2 \mathrm{H}), 1.56-1.42(\mathrm{~m}, 2 \mathrm{H}), 1.40-1.25$ 
$(\mathrm{m}, 5 \mathrm{H})$.

${ }^{13}$ C NMR (100 MHz, $\left.\mathbf{C D C l}_{3}\right): \delta 174.1,159.3,145.9,135.3,128.1,122.8,120.3,113.9,109.6,81.9$, $51.5,46.3,45.3,36.5,33.8,27.3,26.3,24.4$.

IR (neat, $\mathbf{c m}^{-1}$ ): 3006, 2959, 1739, 1482, 1459, 1276, 1262, 976, 776, 751.

HRMS (ESI): $[\mathrm{M}+\mathrm{H}]^{+}$calcd for $\mathrm{C}_{18} \mathrm{H}_{25} \mathrm{O}_{3} 289.1798$, found 289.1800 .
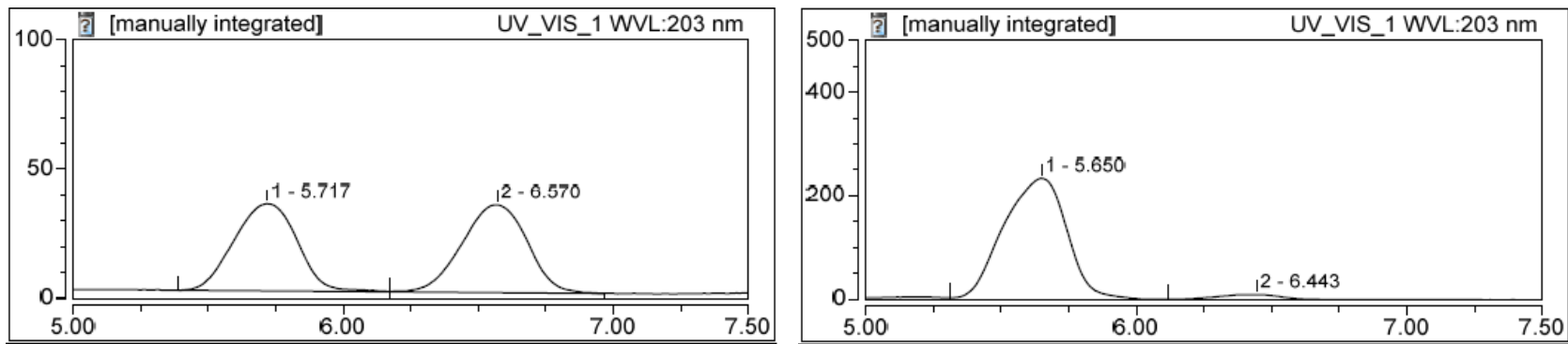

\begin{tabular}{|l|c|c|c|}
\hline \multicolumn{3}{|l|}{ Integration Results } \\
\hline No. & $\begin{array}{c}\text { Retention Time } \\
\text { min }\end{array}$ & $\begin{array}{c}\text { Area } \\
\text { mAU*min }\end{array}$ & $\begin{array}{c}\text { Relative Area } \\
\%\end{array}$ \\
\hline 1 & 5.717 & 9.172 & 49.02 \\
2 & 6.570 & 9.538 & 50.98 \\
\hline Total: & & 18.710 & 100.00 \\
\hline
\end{tabular}

\begin{tabular}{|l|c|c|c|}
\hline \multicolumn{3}{|l|}{ Integration Results } \\
\hline No. & $\begin{array}{c}\text { Retention Time } \\
\text { min }\end{array}$ & $\begin{array}{c}\text { Area } \\
\text { mAU*min }\end{array}$ & $\begin{array}{c}\text { Relative Area } \\
\%\end{array}$ \\
\hline 1 & 5.650 & 64.017 & 95.96 \\
2 & 6.443 & 2.696 & 4.04 \\
\hline Total: & & 66.714 & 100.00 \\
\hline
\end{tabular}

\section{(R)-6-((3-methyl-2,3-dihydrobenzofuran-3-yl)methyl)hept-6-en-1-yl acetate (3q)}<smiles>C=C(CC1(C)COc2ccccc21)NC(C)=O</smiles>

$3 q$
The compound was prepared according to the General Procedure from the reaction of $\mathbf{1 a}(164.4 \mathrm{mg}, 0.6 \mathrm{mmol})$ and $\mathbf{2 q}(60.8 \mathrm{mg}, 0.2 \mathrm{mmol})$.

$34.6 \mathrm{mg}, 54 \%$ yield, $93 \%$ ee, colorless oil.

Chiral HPLC: CHIRALPAK ID, $25{ }^{\circ} \mathrm{C}$, ${ }^{i} \mathrm{PrOH}$-hexanes 4/96, $1 \mathrm{~mL} / \mathrm{min}, 280 \mathrm{~nm}, t_{\mathrm{R}}$ (major) $=6.0$ $\min , t_{\mathrm{R}}(\operatorname{minor})=6.6 \mathrm{~min}$.

$[\boldsymbol{\alpha}]_{\mathbf{D}}{ }^{20}=+5\left(\mathrm{c}=1.0, \mathrm{CH}_{2} \mathrm{Cl}_{2}\right)$.

${ }^{1}$ H NMR (400 MHz, CDCl 3 ): $\delta$ 7.14-7.09 (m, $\left.2 \mathrm{H}\right), 6.86(\mathrm{dt}, J=0.8,7.2 \mathrm{~Hz}, 1 \mathrm{H}), 6.77(\mathrm{~d}, J=8.0$ $\mathrm{Hz}, 1 \mathrm{H}), 4.85(\mathrm{~d}, J=1.2 \mathrm{~Hz}, 1 \mathrm{H}), 4.72(\mathrm{~s}, 1 \mathrm{H}), 4.50(\mathrm{~d}, J=8.4 \mathrm{~Hz}, 1 \mathrm{H}), 4.16(\mathrm{~d}, J=8.8 \mathrm{~Hz}, 1 \mathrm{H})$, $4.02(\mathrm{t}, J=6.8 \mathrm{~Hz}, 2 \mathrm{H}), 2.43-2.31(\mathrm{~m}, 2 \mathrm{H}), 2.04(\mathrm{~s}, 3 \mathrm{H}), 1.84-1.64(\mathrm{~m}, 2 \mathrm{H}), 1.60-1.53(\mathrm{~m}, 2 \mathrm{H})$, 1.40-1.19 (m, $7 \mathrm{H})$.

${ }^{13}$ C NMR (100 MHz, $\left.\mathbf{C D C l}_{3}\right): \delta$ 171.2, 159.3, 146.1, 135.3, 128.1, 122.8, 120.3, 113.7, 109.6, 81.9, $64.5,46.3,45.3,36.8,28.4,27.4,26.3,25.5,21.0$.

IR (neat, $\mathbf{c m}^{-\mathbf{1}}$ ): 2939, 2866, 1739, 1597, 1482, 1366, 1239, 1046, 978, 751.

HRMS (ESI): $[\mathrm{M}+\mathrm{H}]^{+}$calcd for $\mathrm{C}_{19} \mathrm{H}_{27} \mathrm{O}_{3} 303.1955$, found 303.1953 . 

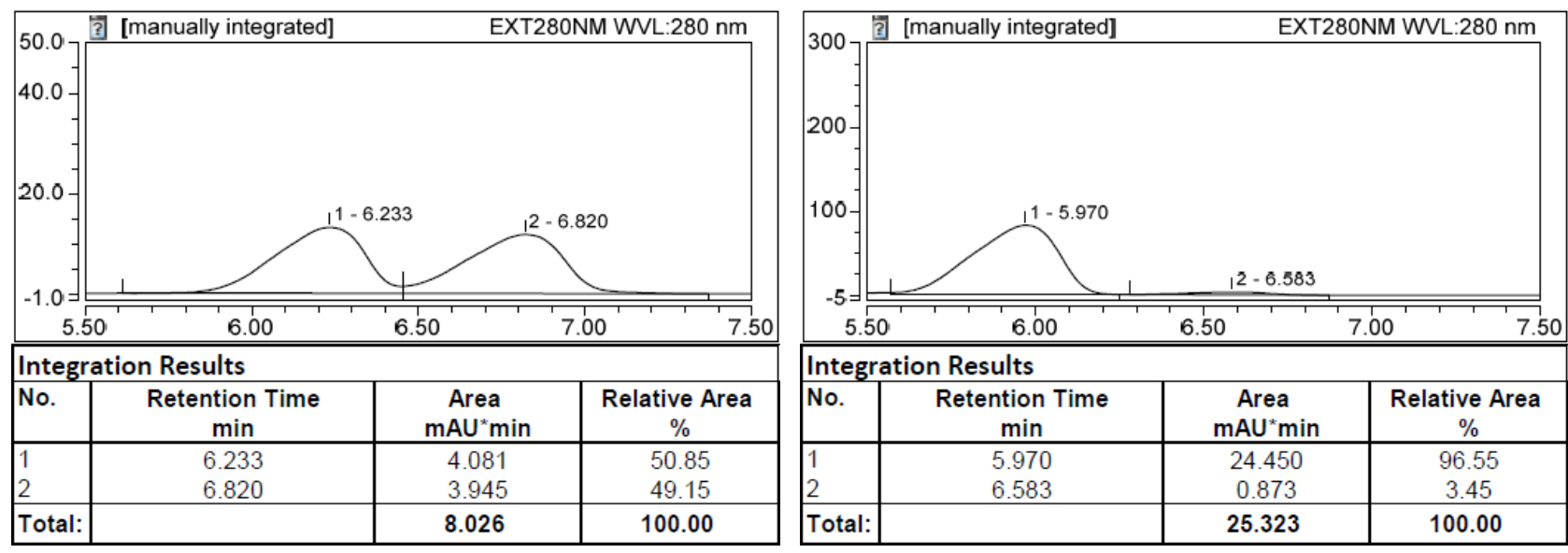

\section{(R)-5-(tert-butyl)-3-methyl-3-(2-propylpent-2-en-1-yl)-2,3-dihydrobenzofuran (3s)}

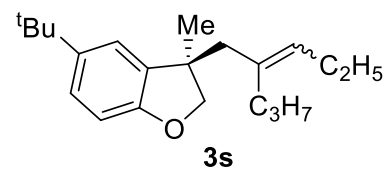

This compound was prepared according to the General Procedure from the reaction of $\mathbf{1 b}(66.0 \mathrm{mg}, 0.2 \mathrm{mmol})$ and $2 \mathrm{~s}(49.2 \mathrm{mg}, 0.2 \mathrm{mmol})$ in DMF. $18.0 \mathrm{mg}, 30 \%$ yield, $E / Z=2.5 / 1,91 \%$ ee, colorless oil. The $E$ - and Z-isomers were determined by 1 -D NOE experiments.

Chiral HPLC: CHIRALCEL OD-H, $25{ }^{\circ} \mathrm{C},{ }^{i} \mathrm{PrOH}-h$ exanes $0.4 / 99.6,0.3 \mathrm{~mL} / \mathrm{min}, 280 \mathrm{~nm}, t_{\mathrm{R} 1}$ (major) $=18.6 \mathrm{~min}, t_{\mathrm{R} 1}($ minor $)=21.9 \mathrm{~min} ; t_{\mathrm{R} 2}($ major $)=19.4 \mathrm{~min}, t_{\mathrm{R} 2}($ minor $)=20.9 \min$.

$[\alpha]_{\mathbf{D}}^{23}=+33\left(\mathrm{c}=1.0, \mathrm{CH}_{2} \mathrm{Cl}_{2}\right)$.

${ }^{1}$ H NMR (400 MHz, CDCl $): \delta 7.13(\mathrm{td}, J=2.0 \mathrm{~Hz}, J=8.4 \mathrm{~Hz}, 2 \mathrm{H}), 7.20(\mathrm{~d}, J=8.4 \mathrm{~Hz}, 1 \mathrm{H})$, $5.123(\mathrm{t}, J=6.8 \mathrm{~Hz}, 1 \mathrm{H}), 4.47(\mathrm{~d}, J=8.4 \mathrm{~Hz}, 1 \mathrm{H}), 4.12(\mathrm{~d}, J=8.4 \mathrm{~Hz}, 1 \mathrm{H}), 2.40-2.21(\mathrm{~m}, 2 \mathrm{H})$, 2.03-1.92 (m, $2 \mathrm{H}), 1.87-1.58(\mathrm{~m}, 2 \mathrm{H}), 1.32-1.30(\mathrm{~m}, 2 \mathrm{H}), 1.32(\mathrm{~s}, 3 \mathrm{H}), 1.30(\mathrm{~s}, 9 \mathrm{H}), 0.92(\mathrm{t}, J=$ $7.6 \mathrm{~Hz}, 3 \mathrm{H}), 0.78(\mathrm{t}, J=7.2 \mathrm{~Hz}, 3 \mathrm{H})$.

${ }^{13}$ C NMR (100 MHz, $\left.\mathbf{C D C l}_{3}\right): \delta 157.1,143.2,135.5,135.1,131.9,124.6,119.9,108.6,82.3,46.7$, $45.9,40.0,34.3,31.8,26.2,21.7,21.1,14.6,14.0$.

IR (neat, $\mathbf{c m}^{-1}$ ): 2960, 2931, 1594, 1490, 1363, 1261, 1186, 1057, 989, 816.

HRMS (ESI): $[\mathrm{M}+\mathrm{H}]{ }^{+}$calcd for $\mathrm{C}_{21} \mathrm{H}_{33} \mathrm{O}$ 301.2526, found 301.2533. 

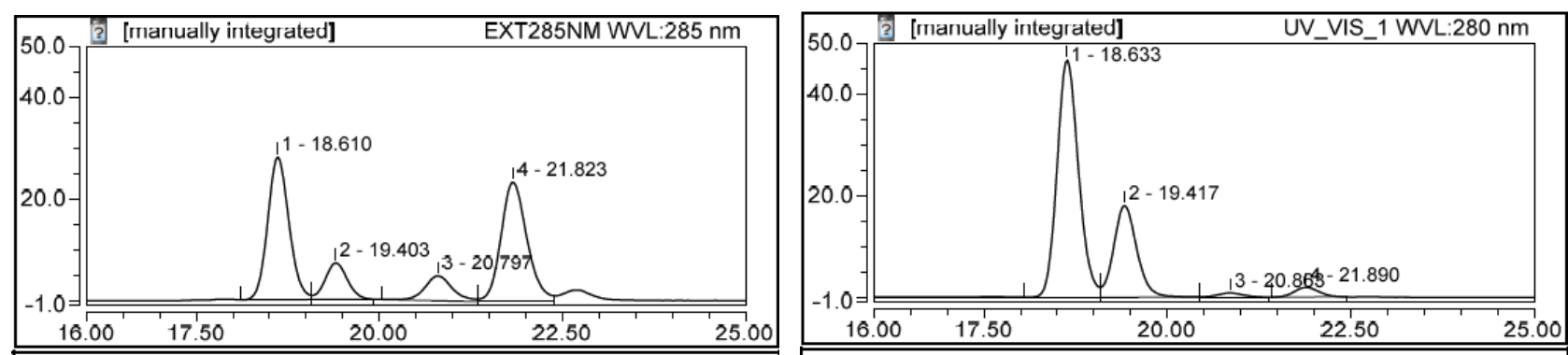

\begin{tabular}{|l|c|c|c|}
\hline \hline \multicolumn{3}{|l|}{ Integration Results } \\
\hline No. & $\begin{array}{c}\text { Retention Time } \\
\text { min }\end{array}$ & $\begin{array}{c}\text { Area } \\
\text { mAU*min }\end{array}$ & $\begin{array}{c}\text { Relative Area } \\
\%\end{array}$ \\
\hline 1 & 18.610 & 9.506 & 39.79 \\
2 & 19.403 & 2.530 & 10.59 \\
3 & 20.797 & 2.145 & 8.98 \\
4 & 21.823 & 9.708 & 40.64 \\
\hline Total: & & $\mathbf{2 3 . 8 8 9}$ & $\mathbf{1 0 0 . 0 0}$ \\
\hline
\end{tabular}

\begin{tabular}{|l|c|c|c|}
\hline \hline \multicolumn{3}{|l|}{ Integration Results } \\
\hline No. & $\begin{array}{c}\text { Retention Time } \\
\text { min }\end{array}$ & $\begin{array}{c}\text { Area } \\
\text { mAU*min }\end{array}$ & $\begin{array}{c}\text { Relative Area } \\
\%\end{array}$ \\
\hline 1 & 18.633 & 15.919 & 67.28 \\
2 & 19.417 & 6.668 & 28.18 \\
3 & 20.863 & 0.328 & 1.39 \\
4 & 21.890 & 0.745 & 3.15 \\
\hline Total: & & $\mathbf{2 3 . 6 5 9}$ & $\mathbf{1 0 0 . 0 0}$ \\
\hline
\end{tabular}

(R)-5-(tert-butyl)-3-(2,3-dimethylbut-2-en-1-yl)-3-methyl-2,3-dihydrobenzofuran (3t)<smiles>CC(C)=C(C)C[C@@]1(C)Oc2ccc(Br)cc21</smiles>

The compound was prepared according to the General Procedure from the reaction of $\mathbf{1 b}(66.0 \mathrm{mg}, 0.2 \mathrm{mmol})$ and $\mathbf{2 t}(43.6 \mathrm{mg}, 0.2 \mathrm{mmol})$ in DMF.

$23.4 \mathrm{mg}, 43 \%$ yield, $99.8 \%$ ee, colorless oil.

Chiral HPLC: CHIRALCEL OD-H, $25{ }^{\circ} \mathrm{C},{ }^{i} \mathrm{PrOH}-$ hexanes $0.4 / 99.6,0.3 \mathrm{~mL} / \mathrm{min}, 280 \mathrm{~nm}, t_{\mathrm{R}}(\operatorname{minor})$ $=21.6 \mathrm{~min}, t_{\mathrm{R}}($ major $)=22.7 \mathrm{~min}$.

$[\boldsymbol{\alpha}]_{\mathbf{D}}^{22}=+38\left(\mathrm{c}=1.0, \mathrm{CH}_{2} \mathrm{Cl}_{2}\right)$.

${ }^{1}$ H NMR (400 MHz, CDCl $): \delta 7.13(\mathrm{dd}, J=2.0 \mathrm{~Hz}, J=8.0 \mathrm{~Hz}, 1 \mathrm{H}), 7.20(\mathrm{~d}, J=2.0 \mathrm{~Hz}, 1 \mathrm{H})$, $7.08(\mathrm{~d}, J=8.4 \mathrm{~Hz}, 1 \mathrm{H}), 4.37(\mathrm{~d}, J=8.4 \mathrm{~Hz}, 1 \mathrm{H}), 4.09$ (d, $J=8.4 \mathrm{~Hz}, 1 \mathrm{H}), 2.61(\mathrm{~d}, J=13.6 \mathrm{~Hz}, 1$ H), $2.23(\mathrm{~d}, J=13.6 \mathrm{~Hz}, 1 \mathrm{H}), 1.63(\mathrm{~s}, 3 \mathrm{H}), 1.56(\mathrm{~s}, 3 \mathrm{H}), 1.41(\mathrm{~s}, 3 \mathrm{H}), 1.35(\mathrm{~s}, 3 \mathrm{H}), 1.28(\mathrm{~m}, 9 \mathrm{H})$.

${ }^{13}$ C NMR (100 MHz, $\left.\mathbf{C D C l}_{3}\right): \delta$ 157.2, 143.2, 135.0, 128.6, 124.6, 124.5, 120.2, 108.5, 83.4, 46.7, $44.5,34.3,31.8,25.4,20.9,20.84,20.82$.

IR (neat, $\mathbf{c m}^{-\mathbf{1}}$ ): 2961, 2917, 1738, 1648, 1490, 1462, 1262, 1057, 993, 815.

HRMS (ESI): $[\mathrm{M}+\mathrm{H}]^{+}$calcd for $\mathrm{C}_{19} \mathrm{H}_{29} \mathrm{O}$ 273.2213, found 273.2219.
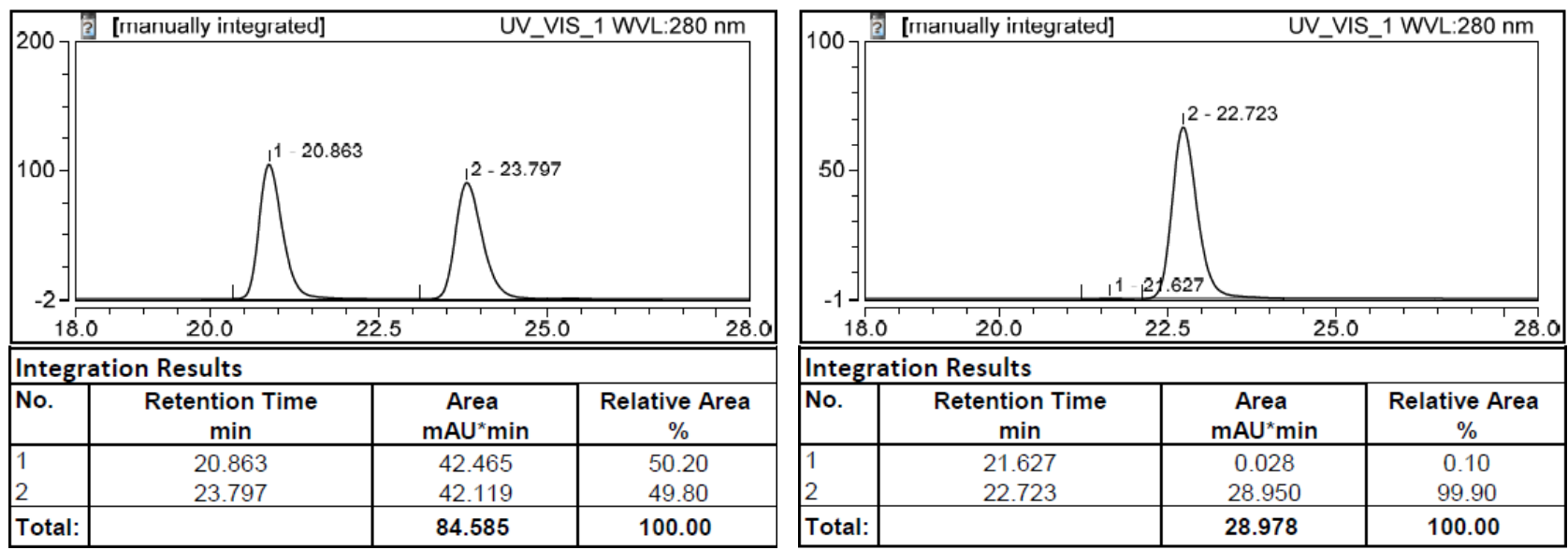


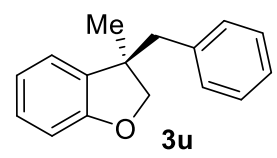

The compound was prepared according to the General Procedure from the reaction of $\mathbf{1 a}(54.8 \mathrm{mg}, 0.2 \mathrm{mmol})$ and $\mathbf{2 v}(40.8 \mathrm{mg}, 0.2 \mathrm{mmol})$.

$31.4 \mathrm{mg}, 70 \%$ yield, $98 \%$ ee, colorless oil.

Chiral HPLC: CHIRALPAK IA, $25{ }^{\circ} \mathrm{C},{ }^{i} \mathrm{PrOH}$-hexanes $0.2 / 99.8,0.75 \mathrm{~mL} / \mathrm{min}, 220 \mathrm{~nm}, t_{\mathrm{R}}($ major $)=$ $19.1 \mathrm{~min}, t_{\mathrm{R}}($ minor $)=22.7 \mathrm{~min}$.

$[\boldsymbol{\alpha}]_{\mathbf{D}}{ }^{21}=+1\left(\mathrm{c}=1.0, \mathrm{CH}_{2} \mathrm{Cl}_{2}\right)$.

${ }^{1}$ H NMR (400 MHz, $\left.\mathbf{C D C l}_{3}\right): \delta$ 7.25-7.20 (m, $\left.3 \mathrm{H}\right), 7.15-7.11(\mathrm{~m}, 1 \mathrm{H})$, 7.00-6.98 (m, $\left.2 \mathrm{H}\right)$, 6.95-6.92 (m, $1 \mathrm{H}), 6.88-6.84(\mathrm{~m}, 1 \mathrm{H}), 6.76(\mathrm{~d}, J=8.0 \mathrm{~Hz}, 1 \mathrm{H}), 4.50(\mathrm{~d}, J=8.8 \mathrm{~Hz}, 1 \mathrm{H}), 4.05(\mathrm{~d}, J$ $=8.8 \mathrm{~Hz}, 1 \mathrm{H}), 2.91-2.83(\mathrm{~m}, 2 \mathrm{H}), 1.35(\mathrm{~s}, 3 \mathrm{H})$.

${ }^{13}$ C NMR (100 MHz, $\left.\mathbf{C D C l}_{3}\right): \delta 159.5,137.5,134.8,130.3,128.1,127.9,126.4,123.3,120.2,109.7$, $81.8,46.6,46.2,24.5$.

IR (neat, $\mathbf{c m}^{-1}$ ): 3432, 2086, 1637, 1479, 1418, 1261, 1122, 1042, 750, 702.

HRMS (ESI): $[\mathrm{M}+\mathrm{H}]^{+}$calcd for $\mathrm{C}_{16} \mathrm{H}_{17} \mathrm{O} 225.1274$, found 225.1272 .
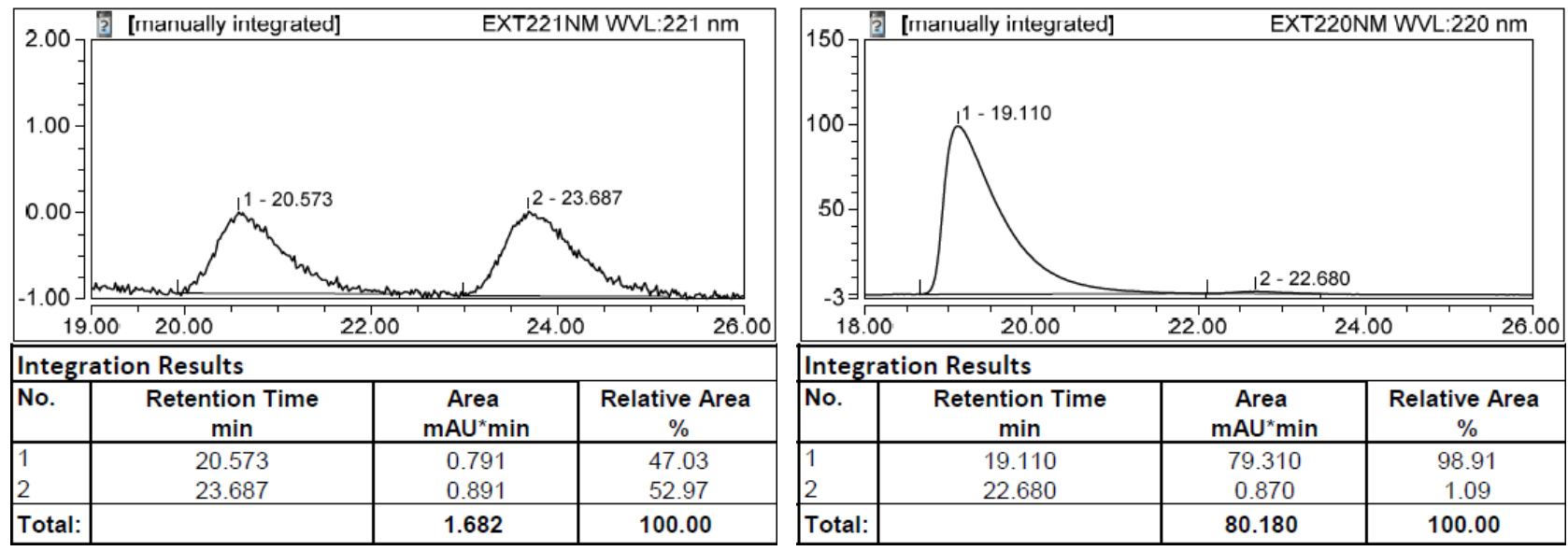

(R)-4-((5-(tert-butyl)-3-methyl-2,3-dihydrobenzofuran-3-yl)methyl)-1-tosyl-1,2,3,6-tetrahydrop yridine (3v)<smiles>C[NH+]1CC=C(C[C@@]2(C)COc3ccc(Br)cc32)CC1</smiles>

The compound was prepared according to the General Procedure from the reaction of $\mathbf{1 b}(66.0 \mathrm{mg}, 0.2 \mathrm{mmol})$ and $\mathbf{2 k}(77.0 \mathrm{mg}, 0.2 \mathrm{mmol})$. $65.9 \mathrm{mg}, 75 \%$ yield, $97 \%$ ee, colorless oil.

Chiral HPLC: CHIRALPAK IA, $25{ }^{\circ} \mathrm{C}$, ${ }^{i} \mathrm{PrOH}-$ hexanes 3/97, $1 \mathrm{~mL} / \mathrm{min}, 279 \mathrm{~nm}, t_{\mathrm{R}}($ minor $)=32.7$ $\min , t_{\mathrm{R}}($ major $)=37.8 \min$.

$[\alpha]_{\mathbf{D}}^{24}=+14\left(\mathrm{c}=1.0, \mathrm{CH}_{2} \mathrm{Cl}_{2}\right)$.

${ }^{1}$ H NMR (400 MHz, CDCl $): \delta 7.64(\mathrm{~d}, J=8.0 \mathrm{~Hz}, 2 \mathrm{H}), 7.31(\mathrm{~d}, J=8.0 \mathrm{~Hz}, 2 \mathrm{H}), 7.12(\mathrm{dd}, J=2.0$, $8.0 \mathrm{~Hz}, 1 \mathrm{H}), 7.02(\mathrm{~d}, J=2.0 \mathrm{~Hz}, 1 \mathrm{H}), 6.66(\mathrm{~d}, J=8.4 \mathrm{~Hz}, 1 \mathrm{H}), 5.29$ (s, $1 \mathrm{H}), 4.33$ (d, $J=8.4 \mathrm{~Hz}, 1$ 
H), $4.07(\mathrm{~d}, J=8.8 \mathrm{~Hz}, 1 \mathrm{H}), 3.60-3.46(\mathrm{~m}, 2 \mathrm{H}), 3.11-2.92(\mathrm{~m}, 2 \mathrm{H}), 2.42(\mathrm{~s}, 3 \mathrm{H}), 2.28(\mathrm{~s}, 2 \mathrm{H})$, 1.91-1.79 (m, $2 \mathrm{H}), 1.29$ (s, $3 \mathrm{H}), 1.27$ (s, $9 \mathrm{H})$.

${ }^{13}$ C NMR (100 MHz, CDCl 3 ): $\delta$ 157.0, 143.5, 143.4, 134.1, 133.6, 133.2, 129.6, 127.6, 124.9, 120.9, $119.7,108.7,82.2,47.9,45.5,44.8,42.9,34.3,31.7,29.9,25.7,21.5$.

IR (neat, $\mathbf{c m}^{-1}$ ): 2963, 2873, 1490, 1461, 1349, 1165, 1094, 950, 818, 736.

HRMS (ESI): $[\mathrm{M}+\mathrm{H}]{ }^{+}$calcd for $\mathrm{C}_{26} \mathrm{H}_{34} \mathrm{NO}_{3} \mathrm{~S} 440.2254$, found 440.2253.

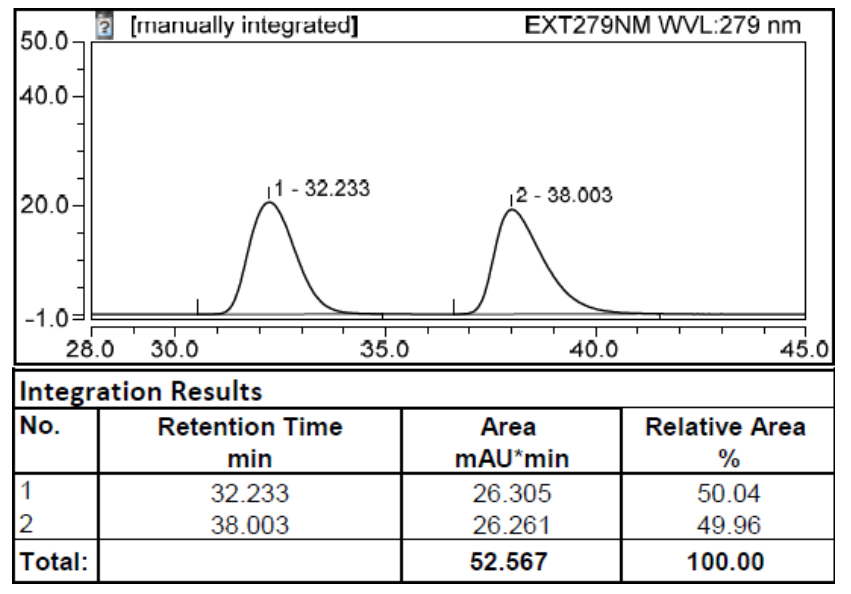

\begin{tabular}{|l|c|c|c|}
\hline 100 & [manually integrated] & \multicolumn{3}{|c|}{ EXT279NM WVL:279 $\mathrm{nm}$} \\
\\
\end{tabular}

(R)-4-((5-isopropyl-3,6-dimethyl-2,3-dihydrobenzofuran-3-yl)methyl)-1-tosyl-1,2,3,6-tetrahydr opyridine (3w)

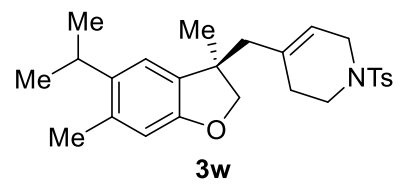

The compound (a colorless oil, $72.0 \mathrm{mg}, 82 \%$ yield, 97\% ee) was prepared according to the General Procedure from the reaction of $1 \mathrm{c}(66.0 \mathrm{mg}, 0.2$ $\mathrm{mmol})$ and $\mathbf{2 k}(77.0 \mathrm{mg}, 0.2 \mathrm{mmol})$. The gram scale reaction was conducted with 1c $(1.32 \mathrm{~g}, 4.0 \mathrm{mmol})$ and $\mathbf{2 k}(1.54 \mathrm{~g}, 4.0 \mathrm{mmol})$ to afford $\mathbf{3 w}$ with $72 \%$ yield $(1.27 \mathrm{~g})$ and $97 \%$ ee.

Chiral HPLC: CHIRALPAK IA, $25{ }^{\circ} \mathrm{C}$, ${ }^{i}$ PrOH-hexanes 3/97, $1 \mathrm{~mL} / \mathrm{min}, 287 \mathrm{~nm}, t_{\mathrm{R}}($ minor $)=30.2$ $\min , t_{\mathrm{R}}($ major $)=33.2 \mathrm{~min}$.

$[\boldsymbol{\alpha}]_{\mathbf{D}}{ }^{24}=+9\left(\mathrm{c}=1.0, \mathrm{CH}_{2} \mathrm{Cl}_{2}\right)$.

${ }^{1}$ H NMR (400 MHz, $\left.\mathbf{C D C l}_{3}\right): \delta 7.64(\mathrm{~d}, J=8.4 \mathrm{~Hz}, 2 \mathrm{H}), 7.31(\mathrm{~d}, J=8.0 \mathrm{~Hz}, 2 \mathrm{H}), 6.87(\mathrm{~s}, 1 \mathrm{H})$, $6.53(\mathrm{~s}, 1 \mathrm{H}), 5.29(\mathrm{~s}, 1 \mathrm{H}), 4.30(\mathrm{~d}, J=8.8 \mathrm{~Hz}, 1 \mathrm{H}), 4.05(\mathrm{~d}, J=8.8 \mathrm{~Hz}, 1 \mathrm{H}), 3.61-3.46(\mathrm{~m}, 2 \mathrm{H})$, 3.13-3.03 (m, $3 \mathrm{H}), 2.42$ (s, $3 \mathrm{H}), 2.27$ (s, $5 \mathrm{H}), 1.93-1.82$ (m, $2 \mathrm{H}), 1.27$ (s, $3 \mathrm{H}), 1.18-1.14$ (m, $6 \mathrm{H})$.

${ }^{13}$ C NMR (100 MHz, $\left.\mathbf{C D C l}_{3}\right): \delta 157.0,143.4,138.9,135.0,133.7,133.2,132.2,129.6,127.6,120.8$, 118.9, 111.0, 82.1, 47.9, 45.4, 44.8, 42.9, 29.9, 28.8, 25.9, 23.7, 23.4, 21.4, 19.6.

IR (neat, $\mathbf{c m}^{-1}$ ): 2960, 2926, 1489, 1459, 1349, 1165, 1094, 948, 818, 738.

HRMS (ESI): $[\mathrm{M}+\mathrm{H}]{ }^{+}$calcd for $\mathrm{C}_{26} \mathrm{H}_{34} \mathrm{NO}_{3} \mathrm{~S} 440.2254$, found 440.2252. 

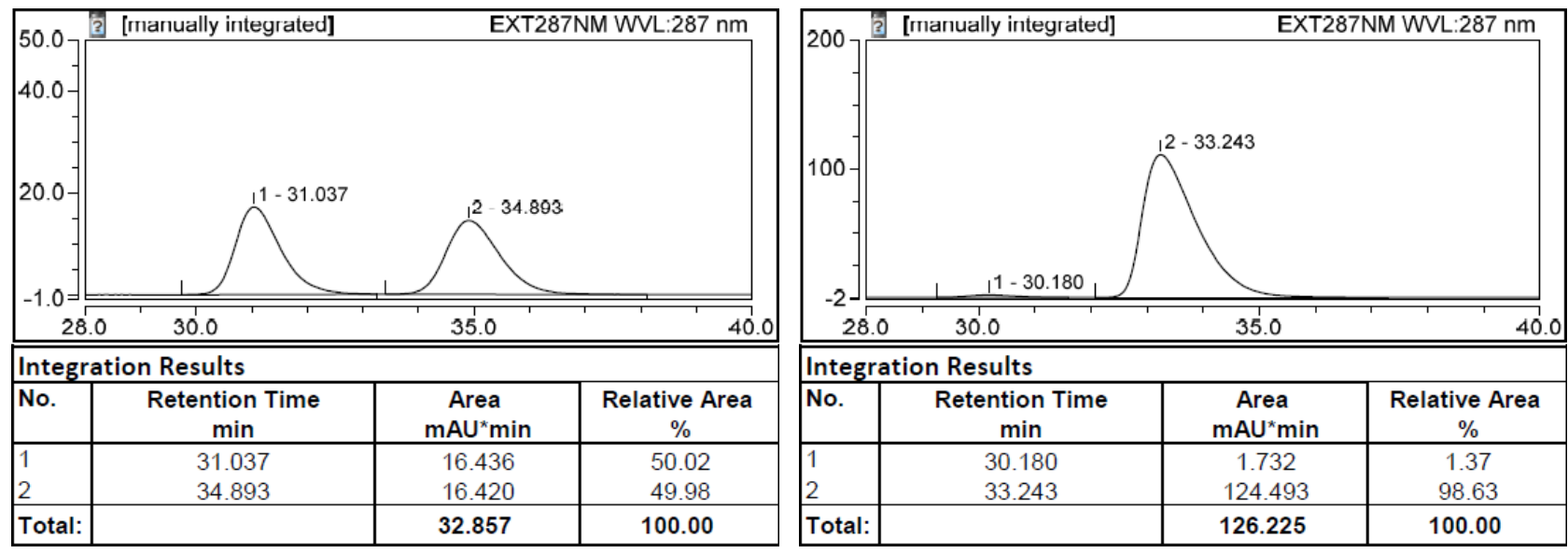

(R)-4-((3,5-dimethyl-2,3-dihydrobenzofuran-3-yl)methyl)-1-tosyl-1,2,3,6-tetrahydropyridine $(3 \mathbf{x})$

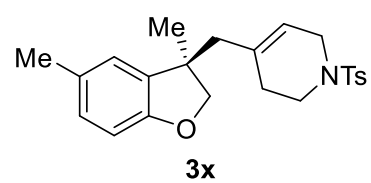
The compound was prepared according to the General Procedure from the reaction of $1 \mathbf{d}(57.6 \mathrm{mg}, 0.2 \mathrm{mmol})$ and $2 \mathbf{k}(77.0 \mathrm{mg}, 0.2 \mathrm{mmol})$. $47.6 \mathrm{mg}, 63 \%$ yield, $97 \%$ ee, colorless oil.

Chiral HPLC: CHIRALPAK IA, $25{ }^{\circ} \mathrm{C},{ }^{i} \mathrm{PrOH}-h$ exanes 10/90, $1 \mathrm{~mL} / \mathrm{min}, 281 \mathrm{~nm}, t_{\mathrm{R}}(\operatorname{minor})=17.3$ $\min , t_{\mathrm{R}}($ major $)=27.3 \mathrm{~min}$.

$[\alpha]_{\mathbf{D}}{ }^{24}=+12\left(\mathrm{c}=0.68, \mathrm{CH}_{2} \mathrm{Cl}_{2}\right)$.

${ }^{1}$ H NMR (400 MHz, CDCl 3 ): $\delta 7.64(\mathrm{~d}, J=8.4 \mathrm{~Hz}, 2 \mathrm{H}), 7.31(\mathrm{~d}, J=8.0 \mathrm{~Hz}, 2 \mathrm{H}), 6.89(\mathrm{~d}, J=8.0$ $\mathrm{Hz}, 1 \mathrm{H}), 6.83(\mathrm{~s}, 1 \mathrm{H}), 6.62(\mathrm{~d}, J=8.0 \mathrm{~Hz}, 1 \mathrm{H}), 5.30(\mathrm{~s}, 1 \mathrm{H}), 4.32(\mathrm{~d}, J=8.4 \mathrm{~Hz}, 1 \mathrm{H}), 4.05(\mathrm{~d}, J=$ $8.8 \mathrm{~Hz}, 1 \mathrm{H}), 3.61-3.49$ (m, $2 \mathrm{H}), 3.11-2.95$ (m, $2 \mathrm{H}), 2.42$ (s, $3 \mathrm{H}), 2.31-2.23$ (m, $2 \mathrm{H}), 2.26$ (s, $3 \mathrm{H})$, 1.94-1.80 (m, $2 \mathrm{H}), 1.26$ (s, $3 \mathrm{H})$.

${ }^{13}$ C NMR (100 MHz, $\left.\mathbf{C D C l}_{3}\right): \delta 157.1,143.5,134.9,133.6,133.4,129.7,129.6,128.6,127.6,123.3$, 120.9, 109.2, 81.8, 47.8, 45.4, 44.8, 42.8, 30.0, 26.0, 21.5, 20.8.

IR (neat, $\mathbf{c m}^{-1}$ ): 2967, 2924, 1490, 1459, 1344, 1165, 1094, 982, 814, 688.

HRMS (ESI): $[\mathrm{M}+\mathrm{H}]{ }^{+}$calcd for $\mathrm{C}_{23} \mathrm{H}_{28} \mathrm{NO}_{3} \mathrm{~S} 398.1784$, found 229.1785.
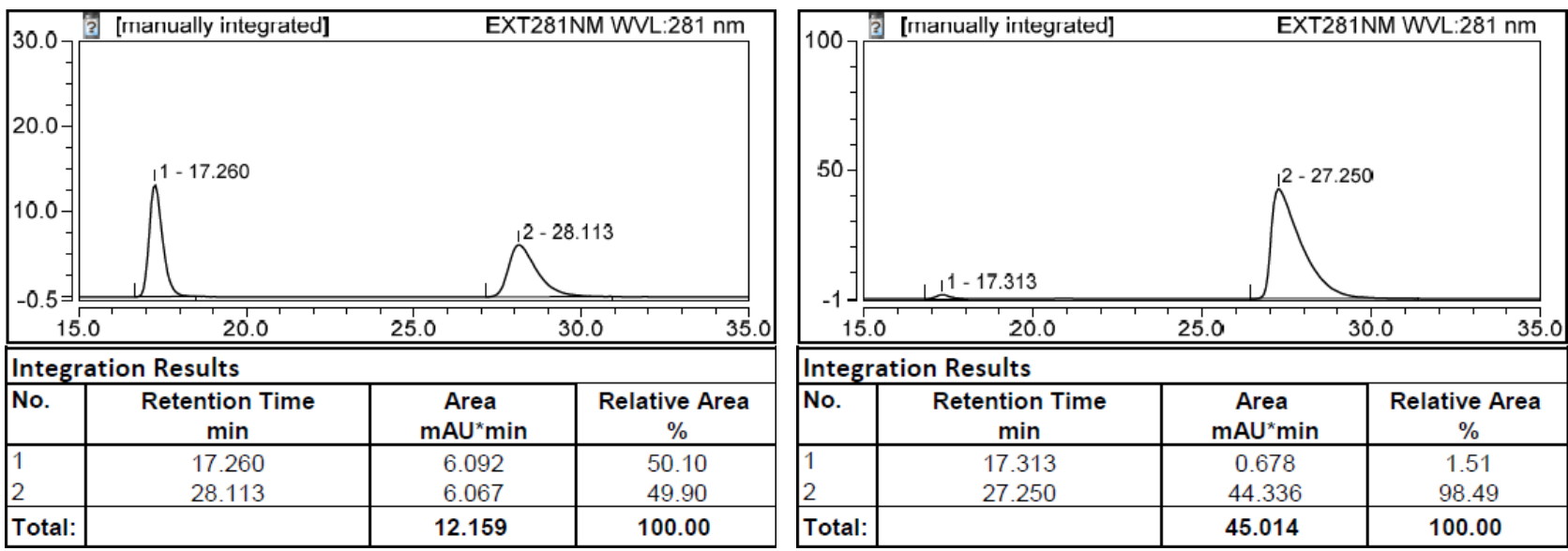

\begin{tabular}{|l|c|c|c|}
\hline \multicolumn{4}{|l|}{ Integration Results } \\
\hline No. & $\begin{array}{c}\text { Retention Time } \\
\text { min }\end{array}$ & $\begin{array}{c}\text { Area } \\
\text { AAU }^{\star} \text { min }\end{array}$ & $\begin{array}{c}\text { Relative Area } \\
\%\end{array}$ \\
\hline 1 & 17.260 & 6.092 & 50.10 \\
2 & 28.113 & 6.067 & 49.90 \\
\hline Total: & & $\mathbf{1 2 . 1 5 9}$ & $\mathbf{1 0 0 . 0 0}$ \\
\hline
\end{tabular}


<smiles>CC1(CC2=CCCCC2)COc2ccc(F)cc21</smiles>

The compound was prepared according to the General Procedure from the reaction of $\mathbf{1 e}(105.1 \mathrm{mg}, 0.36 \mathrm{mmol})$ and $\mathbf{2 a}(46.0 \mathrm{mg}, 0.2 \mathrm{mmol}) . \mathrm{NiI}_{2}(9.5 \mathrm{mg}$, $0.030 \mathrm{mmol}$ ) and L1 (11.4 $\mathrm{mg}, 0.042 \mathrm{mmol}$ ) were used.

$16.7 \mathrm{mg}, 38 \%$ yield, $99 \%$ ee, colorless oil.

Chiral HPLC: CHIRALPAK IA, $25{ }^{\circ} \mathrm{C},{ }^{i} \mathrm{PrOH}$-hexanes $0.2 / 99.8,0.75 \mathrm{~mL} / \mathrm{min}, 289 \mathrm{~nm}, t_{\mathrm{R}}($ major $)=$ $13.2 \min , t_{\mathrm{R}}($ minor $)=21.6 \mathrm{~min}$.

$[\alpha]_{\mathbf{D}}{ }^{21}=-5\left(\mathrm{c}=1.0, \mathrm{CH}_{2} \mathrm{Cl}_{2}\right)$.

${ }^{1}$ H NMR (400 MHz, CDCl $)$ ): $\delta$ 6.81-6.76 (m, $\left.2 \mathrm{H}\right), 6.68-6.65(\mathrm{~m}, 1 \mathrm{H}), 5.40(\mathrm{~s}, 1 \mathrm{H}), 4.48(\mathrm{~d}, J=$ $8.8 \mathrm{~Hz}, 1 \mathrm{H}), 4.16(\mathrm{~d}, J=8.8 \mathrm{~Hz}, 1 \mathrm{H}), 2.31-2.22(\mathrm{~m}, 2 \mathrm{H}), 1.99(\mathrm{~s}, 2 \mathrm{H}), 1.78-1.62(\mathrm{~m}, 2 \mathrm{H})$, 1.56-1.45 (m, $4 \mathrm{H}), 1.30$ (s, $3 \mathrm{H})$.

${ }^{13}$ C NMR (100 MHz, $\left.\mathbf{C D C l}_{3}\right): \delta 157.5\left(\mathrm{~d}, J_{C-F}=236.0 \mathrm{~Hz}\right), 155.1,137.3\left(\mathrm{~d}, J_{C-F}=8.0 \mathrm{~Hz}\right), 134.2$, $126.3,114.0\left(\mathrm{~d}, J_{C-F}=24.0 \mathrm{~Hz}\right), 110.2\left(\mathrm{~d}, J_{C-F}=24.0 \mathrm{~Hz}\right), 109.6\left(\mathrm{~d}, J_{C-F}=8.0 \mathrm{~Hz}\right), 82.9,48.8,46.0$, $30.2,26.1,25.4,23.0,22.1$.

${ }^{19}$ F NMR (376 MHz, $\left.\mathbf{C D C l}_{3}\right): \delta-124.40$.

IR (neat, $\mathbf{c m}^{-\mathbf{1}}$ ): 2961, 2850, 1591, 1486, 1261, 1175, 1092, 1039, 807, 751.

HRMS (ESI): $[\mathrm{M}+\mathrm{H}]^{+}$calcd for $\mathrm{C}_{16} \mathrm{H}_{20} \mathrm{FO} 247.1493$, found 247.1509.

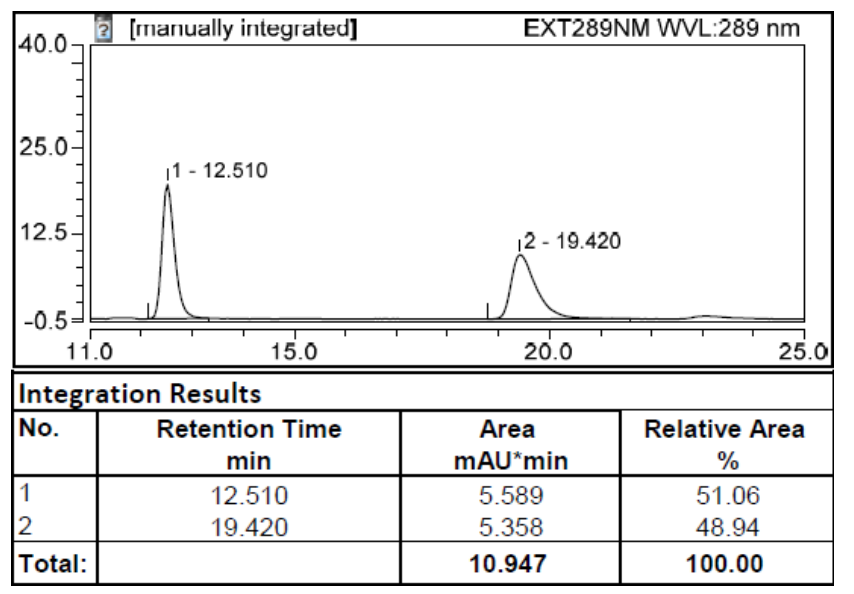

\begin{tabular}{|l|c|c|c|}
\hline 100 & [manually integrated] & \multicolumn{1}{c|}{ EXT289NM WVL:289 nm } \\
\hline \\
\end{tabular}

(R)-4-((3-methyl-5-phenyl-2,3-dihydrobenzofuran-3-yl)methyl)-1-tosyl-1,2,3,6-tetrahydropyrid ine $(3 z)$

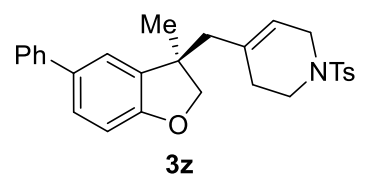

The compound was prepared according to the General Procedure from the reaction of $\mathbf{1 f}(70.0 \mathrm{mg}, 0.2 \mathrm{mmol})$ and $\mathbf{2 k}(77.0 \mathrm{mg}, 0.2 \mathrm{mmol})$. $48.7 \mathrm{mg}, 55 \%$ yield, $95 \%$ ee, white solid, $\mathrm{mp}: 60-62{ }^{\circ} \mathrm{C}$.

Chiral HPLC: CHIRALCEL OD-H, $25{ }^{\circ} \mathrm{C},{ }^{i}$ PrOH-hexanes 5/95, $1 \mathrm{~mL} / \mathrm{min}, 280 \mathrm{~nm}, t_{\mathrm{R}}($ major $)=$ 
$45.9 \min , t_{\mathrm{R}}($ minor $)=54.3 \mathrm{~min}$.

$[\alpha]_{\mathrm{D}}{ }^{24}=+75\left(\mathrm{c}=1.0, \mathrm{CH}_{2} \mathrm{Cl}_{2}\right)$.

${ }^{1}$ H NMR (400 MHz, CDCl 3 ): $\delta 7.64(\mathrm{~d}, J=8.0 \mathrm{~Hz}, 2 \mathrm{H}), 7.52-7.50$ (m, $\left.2 \mathrm{H}\right), 7.40$ (t, $J=8.0 \mathrm{~Hz}, 2$ H), $7.35(\mathrm{dd}, J=2.0,8.0 \mathrm{~Hz}, 1 \mathrm{H}), 7.31-7.28(\mathrm{~m}, 3 \mathrm{H}), 7.25(\mathrm{~d}, J=2.0 \mathrm{~Hz}, 1 \mathrm{H}), 6.80(\mathrm{~d}, J=8.0 \mathrm{~Hz}$, $1 \mathrm{H}), 5.33(\mathrm{~s}, 1 \mathrm{H}), 4.41(\mathrm{~d}, J=8.8 \mathrm{~Hz}, 1 \mathrm{H}), 4.14(\mathrm{~d}, J=8.8 \mathrm{~Hz}, 1 \mathrm{H}), 3.62-3.47(\mathrm{~m}, 2 \mathrm{H}), 3.14-2.93$ (m, 2 H), 2.42 (s, 3 H), 2.37-2.29 (m, 2 H), 1.97-1.85 (m, 2 H), 1.33 (s, 3 H).

${ }^{13}$ C NMR (100 MHz, $\left.\mathbf{C D C l}_{3}\right): \delta 158.9,143.5,141.2,135.5,134.0,133.5,133.2,129.6,128.7,127.6$, $127.4,126.7,126.6,121.6,121.1,109.8,82.2,47.9,45.4,44.8,42.8,30.0,26.1,21.5$.

IR (neat, $\mathbf{c m}^{-1}$ ): 2963, 2853, 1601, 1482, 1344, 1165, 1096, 958, 818, 738.

HRMS (ESI): $[\mathrm{M}+\mathrm{H}]^{+}$calcd for $\mathrm{C}_{28} \mathrm{H}_{30} \mathrm{NO}_{3} \mathrm{~S} 460.1941$, found 460.1941 .

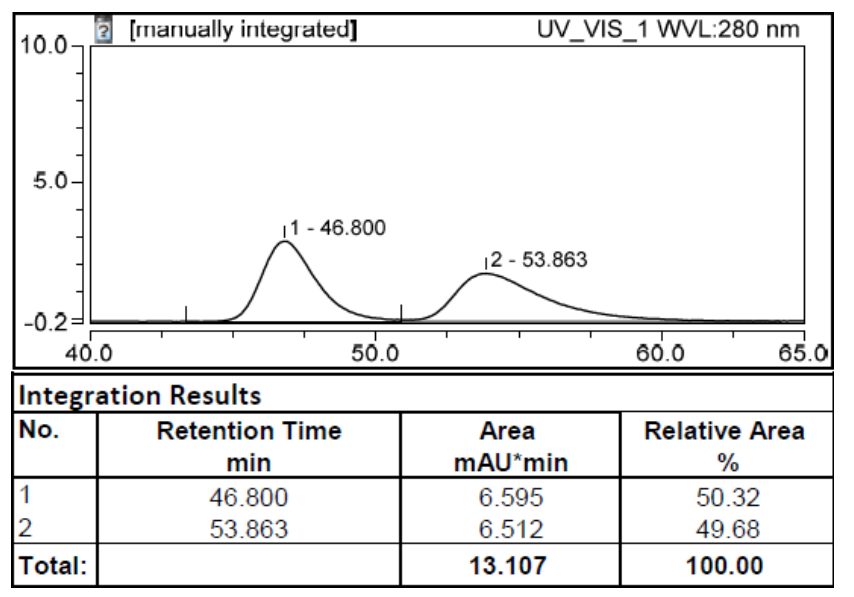

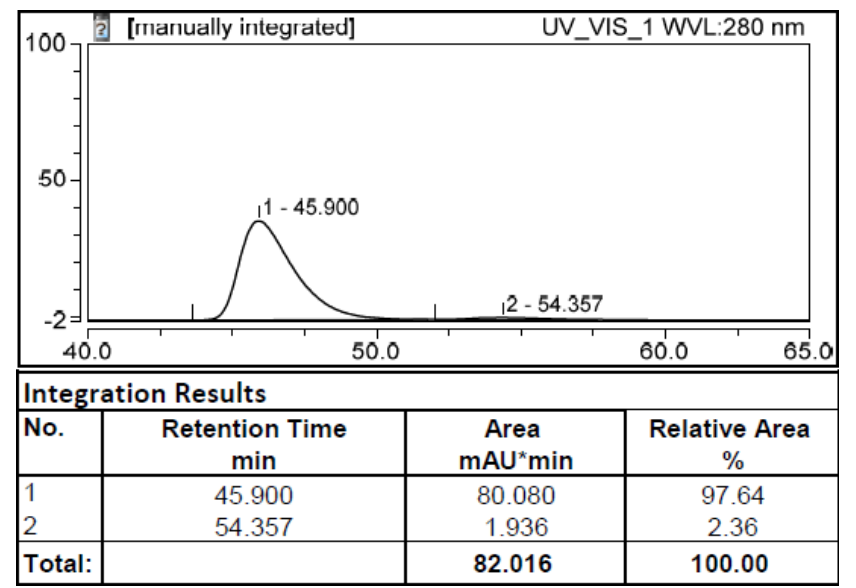

(R)-3-(cyclohex-1-en-1-ylmethyl)-3,6-dimethyl-2,3-dihydrobenzofuran (3ab)

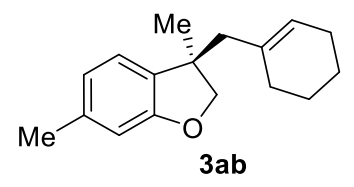

The compound was prepared according to the General Procedure from the reaction of $\mathbf{1 h}(57.6 \mathrm{mg}, 0.2 \mathrm{mmol})$ and $\mathbf{2 a}(46.0 \mathrm{mg}, 0.2 \mathrm{mmol})$.

$29.0 \mathrm{mg}, 60 \%$ yield, $98 \%$ ee, colorless oil.

Chiral HPLC: CHIRALPAK IA, $25{ }^{\circ} \mathrm{C},{ }^{i} \mathrm{PrOH}$-hexanes 0.2/99.8, $0.75 \mathrm{~mL} / \mathrm{min}, 284 \mathrm{~nm}, t_{\mathrm{R}}($ major $)=$ $11.4 \min , t_{\mathrm{R}}($ minor $)=13.5 \min$.

$[\alpha]_{\mathbf{D}}{ }^{21}=-8\left(\mathrm{c}=1.0, \mathrm{CH}_{2} \mathrm{Cl}_{2}\right)$

${ }^{1}$ H NMR (400 MHz, CDCl 3 ): $\delta 6.95(\mathrm{~d}, J=7.6 \mathrm{~Hz}, 1 \mathrm{H}), 6.83(\mathrm{dd}, J=0.4 \mathrm{~Hz}, J=7.2 \mathrm{~Hz}, 1 \mathrm{H})$, $6.59(\mathrm{~s}, 1 \mathrm{H}), 5.41(\mathrm{~s}, 1 \mathrm{H}), 4.45(\mathrm{~d}, J=8.4 \mathrm{~Hz}, 1 \mathrm{H}), 4.13(\mathrm{~d}, J=8.4 \mathrm{~Hz}, 1 \mathrm{H}), 2.30(\mathrm{~s}, 3 \mathrm{H}), 2.27(\mathrm{~d}$, $J=6.0 \mathrm{~Hz}, 2 \mathrm{H}), 1.99$ (s, $2 \mathrm{H}), 1.77-1.64(\mathrm{~m}, 2 \mathrm{H}), 1.58-1.45$ (m, $4 \mathrm{H}), 1.28$ (s, $3 \mathrm{H})$.

${ }^{13} \mathbf{C}$ NMR (100 MHz, $\left.\mathbf{C D C l}_{3}\right): \delta 159.5,138.0,134.7,133.0,125.8,122.5,121.0,110.2,82.6,49.0$, $45.2,30.2,26.3,25.4,23.0,22.2,21.5$.

IR (neat, $\mathbf{c m}^{-\mathbf{1}}$ ): 2923, 2836, 1592, 1495, 1425, 1251, 1122, 1007, 980, 751. 
HRMS (ESI): $[\mathrm{M}+\mathrm{H}]{ }^{+}$calcd for $\mathrm{C}_{17} \mathrm{H}_{23} \mathrm{O} 243.1743$, found 243.1749 .
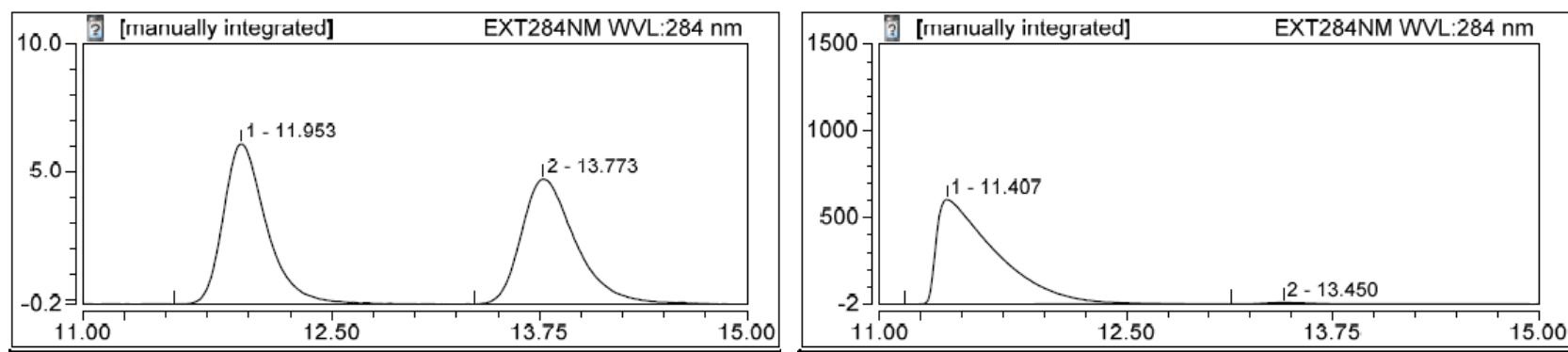

\begin{tabular}{|l|c|c|c|}
\hline \multicolumn{4}{|l|}{ Integration Results } \\
\hline No. & $\begin{array}{c}\text { Retention Time } \\
\text { min }\end{array}$ & $\begin{array}{c}\text { Area } \\
\text { mAU*min }\end{array}$ & $\begin{array}{c}\text { Relative Area } \\
\%\end{array}$ \\
\hline 1 & 11.953 & 1.905 & 50.16 \\
2 & 13.773 & 1.893 & 49.84 \\
\hline Total: & & 3.798 & 100.00 \\
\hline
\end{tabular}

\begin{tabular}{|l|c|c|c|}
\hline \hline \multicolumn{3}{|l|}{ Integration Results } \\
\hline No. & $\begin{array}{c}\text { Retention Time } \\
\text { min }\end{array}$ & $\begin{array}{c}\text { Area } \\
\text { mAU*min }^{*}\end{array}$ & $\begin{array}{c}\text { Relative Area } \\
\%\end{array}$ \\
\hline 1 & 11.407 & 251.311 & 98.93 \\
2 & 13.450 & 2.719 & 1.07 \\
\hline Total: & & $\mathbf{2 5 4 . 0 3 0}$ & $\mathbf{1 0 0 . 0 0}$ \\
\hline
\end{tabular}

(R)-3-(cyclohex-1-en-1-ylmethyl)-3,5,7-trimethyl-2,3-dihydrobenzofuran (3ac)<smiles>Cc1cc2c(c([N+](=O)[O-])c1)C[C@@](C)(CC1=CCCCC1)C2</smiles>

The compound was prepared according to the General Procedure from the reaction of $1 \mathbf{i}(60.4 \mathrm{mg}, 0.2 \mathrm{mmol})$ and $2 \mathbf{a}(46.0 \mathrm{mg}, 0.2 \mathrm{mmol})$.

$33.3 \mathrm{mg}, 65 \%$ yield, $90 \%$ ee, colorless oil.

Chiral HPLC: CHIRALCEL OD-H, $25{ }^{\circ} \mathrm{C},{ }^{i} \mathrm{PrOH}-h$ exanes $0.2 / 99.8,0.75 \mathrm{~mL} / \mathrm{min}, 290 \mathrm{~nm}$, $t_{\mathrm{R}}($ major $)=10.9 \min , t_{\mathrm{R}}($ minor $)=12.6 \mathrm{~min}$.

$[\boldsymbol{\alpha}]_{\mathbf{D}}{ }^{21}=-2\left(\mathrm{c}=1.0, \mathrm{CH}_{2} \mathrm{Cl}_{2}\right)$.

${ }^{1}$ H NMR (400 MHz, CDCl $): \delta 6.75(\mathrm{~d}, J=0.8 \mathrm{~Hz}, 1 \mathrm{H}), 6.72(\mathrm{~d}, J=0.4 \mathrm{~Hz}, 1 \mathrm{H}), 5.42(\mathrm{~s}, 1 \mathrm{H})$, $4.44(\mathrm{~d}, J=8.4 \mathrm{~Hz}, 1 \mathrm{H}), 4.12(\mathrm{~d}, J=8.4 \mathrm{~Hz}, 1 \mathrm{H}), 2.30-2.22(\mathrm{~m}, 5 \mathrm{H}), 2.17(\mathrm{~s}, 3 \mathrm{H}), 2.00(\mathrm{~s}, 2 \mathrm{H})$, 1.78-1.65 (m, $2 \mathrm{H}), 1.57-1.45$ (m, $4 \mathrm{H}), 1.27$ (s, $3 \mathrm{H})$.

${ }^{13}$ C NMR (100 MHz, $\left.\mathbf{C D C l}_{3}\right): \delta$ 155.4, 135.3, 134.8, 129.7, 129.4, 125.7, 120.8, 119.0, 82.3, 48.9, $45.8,30.2,25.9,25.5,23.1,22.2,20.8,15.0$.

IR (neat, $\mathbf{c m}^{-1}$ ): 3429, 2923, 2836, 1638, 1482, 1200, 1123,1003, 854, 749.

HRMS (ESI): $[\mathrm{M}+\mathrm{H}]^{+}$calcd for $\mathrm{C}_{18} \mathrm{H}_{25} \mathrm{O} 257.1900$, found 257.1903 .

\begin{tabular}{|l|c|c|c|}
\hline 30.0 & [manually integrated] & \multicolumn{3}{c|}{ EXT290NM WVL:290 nm } \\
\hline
\end{tabular}

\begin{tabular}{|l|c|c|c|}
\hline 1500 & \multicolumn{3}{|c|}{ EXT290NM WVL:290 nm } \\
\hline
\end{tabular}


(R)-8-((6-chloro-3-methyl-2,3-dihydrobenzofuran-3-yl)methyl)-1,4-dioxaspiro[4.5]dec-7-ene

(3ae)<smiles>C[C@]1(CC2=CCC3(CC2)OCCO3)COc2cc(Cl)ccc21</smiles>

The compound was prepared according to the General Procedure from the reaction of $1 \mathbf{k}(61.6 \mathrm{mg}, 0.2 \mathrm{mmol})$ and $\mathbf{2 h}(57.6 \mathrm{mg}, 0.2 \mathrm{mmol})$ in DMF. $41.0 \mathrm{mg}, 64 \%$ yield, $98 \%$ ee, colorless oil.

Chiral HPLC: CHIRALCEL OJ-H, $25{ }^{\circ} \mathrm{C},{ }^{i} \mathrm{PrOH}$-hexanes $0.2 / 99.8,0.75 \mathrm{~mL} / \mathrm{min}, 260 \mathrm{~nm}, t_{\mathrm{R}}(\operatorname{minor})$ $=10.4 \min , t_{\mathrm{R}}($ major $)=12.5 \min$.

$[\alpha]_{\mathbf{D}}{ }^{21}=-8\left(\mathrm{c}=0.5, \mathrm{CH}_{2} \mathrm{Cl}_{2}\right)$.

${ }^{1}$ H NMR (400 MHz, CDCl $): \delta 6.98(\mathrm{~d}, J=8.0 \mathrm{~Hz}, 1 \mathrm{H}), 6.83(\mathrm{dd}, J=1.6 \mathrm{~Hz}, J=8.0 \mathrm{~Hz}, 1 \mathrm{H})$, $6.76(\mathrm{~d}, J=1.6 \mathrm{~Hz}, 1 \mathrm{H}), 5.30(\mathrm{~s}, 1 \mathrm{H}), 4.47(\mathrm{~d}, J=8.4 \mathrm{~Hz}, 1 \mathrm{H}), 4.18(\mathrm{~d}, J=8.4 \mathrm{~Hz}, 1 \mathrm{H}), 3.96(\mathrm{~d}, J$ $=2.4 \mathrm{~Hz}, 4 \mathrm{H}), 2.31-2.26(\mathrm{~m}, 4 \mathrm{H}), 1.96(\mathrm{~d}, J=5.6 \mathrm{~Hz}, 2 \mathrm{H}), 1.66(\mathrm{t}, J=6.4 \mathrm{~Hz}, 2 \mathrm{H}), 1.32(\mathrm{~s}, 3 \mathrm{H})$.

${ }^{13}$ C NMR (100 MHz, CDCl 3$): \delta 160.2,134.3,134.0,133.3,123.6,123.4,120.4,110.4,107.6,83.2$, $64.4,47.6,45.3,35.8,31.2,29.2,26.0$.

IR (neat, $\mathbf{c m}^{-1}$ ): 2917, 1593, 1480, 1417, 1316, 1260, 1118, 1042, 875, 804.

HRMS (ESI): $[\mathrm{M}+\mathrm{H}]^{+}$calcd for $\mathrm{C}_{18} \mathrm{H}_{22} \mathrm{ClO}_{3} 321.1252$, found 321.1259 .

\begin{tabular}{|l|c|c|c|}
\hline & \multicolumn{3}{c|}{ UV_VIS_1 WVL:260 nm } \\
\hline
\end{tabular}

\begin{tabular}{|l|c|c|c|}
\hline 40.0 & \multicolumn{3}{|c|}{ EXT260NM WVL:260 nm } \\
\hline & & \\
\hline
\end{tabular}

(S)-3-(tert-butyl)-3-(cyclohex-1-en-1-ylmethyl)-2,3-dihydrobenzofuran (3af)

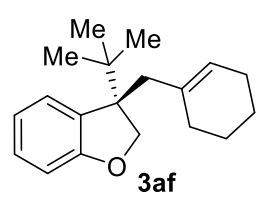

The compound was prepared according to the General Procedure from the reaction of 11 (63.2 $\mathrm{mg}, 0.2 \mathrm{mmol})$ and $\mathbf{2 a}(46.0 \mathrm{mg}, 0.2 \mathrm{mmol})$.

$33.0 \mathrm{mg}, 65 \%$ yield, $98 \%$ ee, colorless oil.

Chiral HPLC: CHIRALCEL OD-H, $25{ }^{\circ} \mathrm{C}$, ${ }^{i} \mathrm{PrOH}-$ hexanes 0.2/99.8, $0.75 \mathrm{~mL} / \mathrm{min}, 295 \mathrm{~nm}$, $t_{\mathrm{R}}($ major $)=7.7 \mathrm{~min}, t_{\mathrm{R}}($ minor $)=10.9 \mathrm{~min}$.

$[\alpha]_{\mathbf{D}}^{21}=+68\left(\mathrm{c}=0.5, \mathrm{CH}_{2} \mathrm{Cl}_{2}\right)$.

${ }^{1}$ H NMR (400 MHz, CDCl 3 ): $\delta$ 7.19-7.17 (m, $\left.1 \mathrm{H}\right), 7.10(\mathrm{dt}, J=1.2,7.6 \mathrm{~Hz}, 1 \mathrm{H}), 6.80(\mathrm{~m}, 1 \mathrm{H})$, 
$6.70(\mathrm{~d}, J=8.0 \mathrm{~Hz}, 1 \mathrm{H}), 5.31(\mathrm{~s}, 1 \mathrm{H}), 4.49-4.41(\mathrm{~m}, 2 \mathrm{H}), 2.56-2.53(\mathrm{~m}, 1 \mathrm{H}), 2.36-2.33(\mathrm{~m}, 1 \mathrm{H})$, 1.92 (s, $2 \mathrm{H}), 1.44-1.34(\mathrm{~m}, 6 \mathrm{H}), 0.93(\mathrm{~s}, 9 \mathrm{H})$.

${ }^{13}$ C NMR (100 MHz, $\left.\mathbf{C D C l}_{3}\right): \delta$ 160.6, 135.9, 131.9, 127.9, 126.4, 125.6, 119.2, 109.0, 76.1, 54.4, $42.3,37.2,30.0,25.6,25.5,23.0,22.1$.

IR (neat, $\mathbf{c m}^{-1}$ ): 2961, 2935, 1687, 1655, 1459, 1217, 1112, 1084, 835, 751.

HRMS (ESI): $[\mathrm{M}+\mathrm{H}]^{+}$calcd for $\mathrm{C}_{19} \mathrm{H}_{27} \mathrm{O}$ 271.2056, found 271.2058.
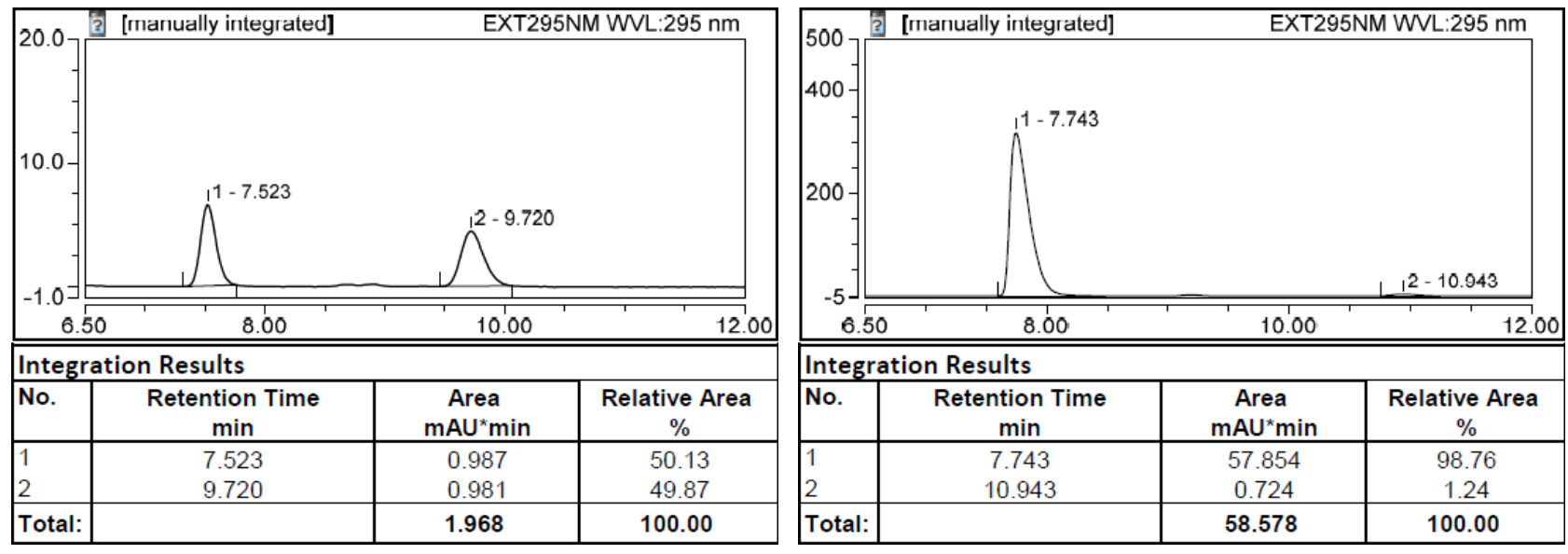

(R)-3-(cyclohex-1-en-1-ylmethyl)-3-octyl-2,3-dihydrobenzofuran (3ag)

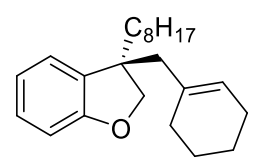

3 ag The compound was prepared according to the General Procedure from the reaction of $1 \mathbf{m}(74.4 \mathrm{mg}, 0.2 \mathrm{mmol})$ and $\mathbf{2 a}(46.0 \mathrm{mg}, 0.2 \mathrm{mmol})$. $42.4 \mathrm{mg}, 68 \%$ yield, $95 \%$ ee, colorless.

Chiral HPLC: CHIRALPAK IB, $25{ }^{\circ} \mathrm{C},{ }^{i} \mathrm{PrOH}$-hexanes 0.2/99.8, $0.5 \mathrm{~mL} / \mathrm{min}, 280 \mathrm{~nm}, t_{\mathrm{R}}$ (major) $=$ $9.0 \min , t_{\mathrm{R}}($ minor $)=10.1 \min$.

$[\boldsymbol{\alpha}]_{\mathbf{D}}{ }^{22}=-2\left(\mathrm{c}=0.5, \mathrm{CH}_{2} \mathrm{Cl}_{2}\right)$.

${ }^{1}$ H NMR (400 MHz, $\left.\mathbf{C D C l}_{3}\right): \delta 7.10(\mathrm{dt}, J=1.2,7.6 \mathrm{~Hz}, 1 \mathrm{H}), 7.04(\mathrm{dd}, J=1.2,7.6 \mathrm{~Hz}, 1 \mathrm{H}), 6.84$ $(\mathrm{dt}, J=0.8,7.6 \mathrm{~Hz}, 1 \mathrm{H}), 6.74(\mathrm{~d}, J=7.8 \mathrm{~Hz}, 1 \mathrm{H}), 5.38(\mathrm{~s}, 1 \mathrm{H}), 4.41(\mathrm{~d}, J=8.8 \mathrm{~Hz}, 1 \mathrm{H}), 4.26(\mathrm{~d}, J$ $=8.4 \mathrm{~Hz}, 1 \mathrm{H}), 2.33(\mathrm{~s}, 2 \mathrm{H}), 1.98(\mathrm{~s}, 2 \mathrm{H}), 1.70-1.42(\mathrm{~m}, 8 \mathrm{H}), 1.32-1.00(\mathrm{~m}, 12 \mathrm{H}), 0.86(\mathrm{t}, J=6.8$ $\mathrm{Hz}, 3 \mathrm{H})$.

${ }^{13}$ C NMR (100 MHz, $\left.\mathbf{C D C l}_{3}\right): \delta$ 159.8, 134.6, 134.0, 127.9, 125.9, 123.7, 120.0, 109.3, 80.5, 48.9, $47.6,39.5,31.8,30.2,30.1,29.4,29.3,25.5,24.2,23.0,22.6,22.1,14.1$.

IR (neat, $\mathbf{c m}^{-1}$ ): 2924, 2857, 1597, 1482, 1459, 1230, 1019, 975, 831, 745.

HRMS (ESI): $[\mathrm{M}+\mathrm{H}]{ }^{+}$calcd for $\mathrm{C}_{23} \mathrm{H}_{35} \mathrm{O} 327.2682$, found 327.2682. 

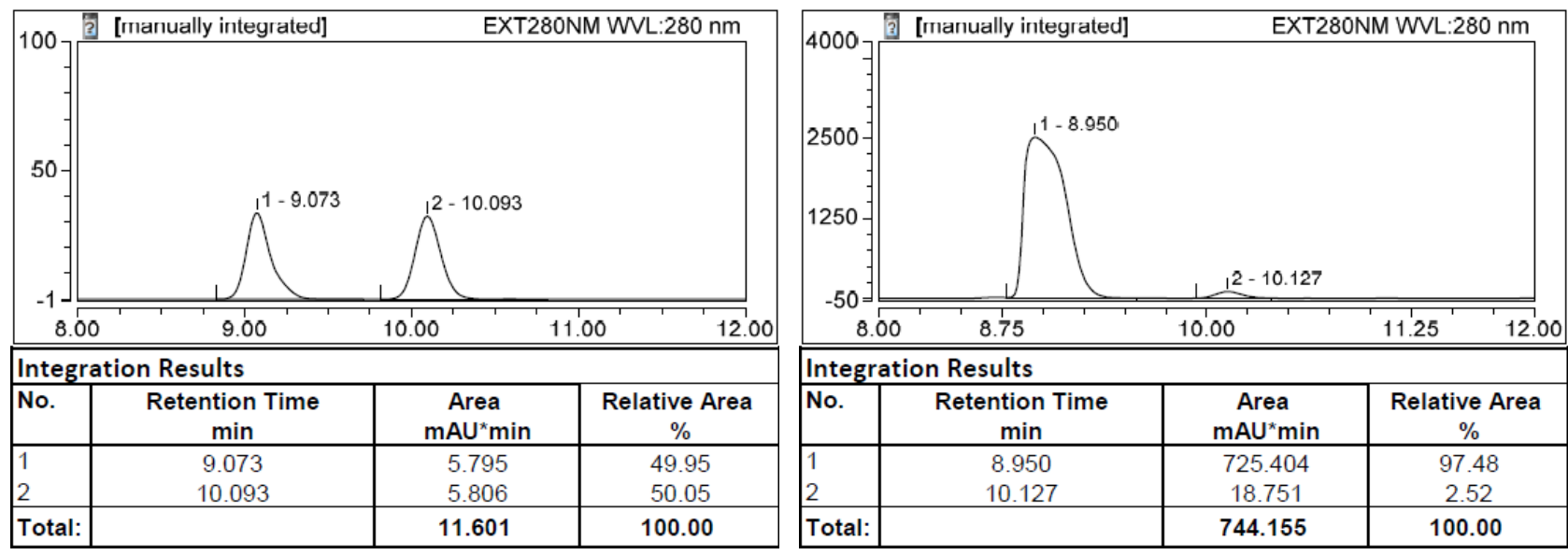

\section{(R,E)-3-(cyclooct-1-en-1-ylmethyl)-3-pentyl-2,3-dihydrobenzofuran (3ah)}

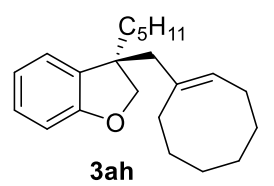

The compound was prepared according to the General Procedure from the reaction of $1 \mathbf{n}(66.0 \mathrm{mg}, 0.2 \mathrm{mmol})$ and $2 \mathrm{e}(51.6 \mathrm{mg}, 0.2 \mathrm{mmol})$.

$26.8 \mathrm{mg}, 46 \%$ yield, $93 \%$ ee, colorless oil.

Chiral HPLC: CHIRALPAK IB, $25{ }^{\circ} \mathrm{C},{ }^{i} \mathrm{PrOH}$-hexanes $0.2 / 99.8,0.75 \mathrm{~mL} / \mathrm{min}, 280 \mathrm{~nm}, t_{\mathrm{R}}($ major$)=$ $7.1 \min , t_{\mathrm{R}}(\operatorname{minor})=7.5 \min$.

$[\boldsymbol{\alpha}]_{\mathbf{D}}{ }^{22}=+10\left(\mathrm{c}=0.5, \mathrm{CH}_{2} \mathrm{Cl}_{2}\right)$.

${ }^{1}$ H NMR (400 MHz, CDCl 3 ): $\delta$ 7.12-7.08 (m, $\left.1 \mathrm{H}\right), 7.04(\mathrm{~d}, J=7.6 \mathrm{~Hz}, 1 \mathrm{H}), 6.84(\mathrm{t}, J=7.2 \mathrm{~Hz}, 1$ H), $6.75(\mathrm{~d}, J=8.0 \mathrm{~Hz}, 1 \mathrm{H}), 5.33(\mathrm{t}, J=8.0 \mathrm{~Hz}, 1 \mathrm{H}), 4.45(\mathrm{~d}, J=8.8 \mathrm{~Hz}, 1 \mathrm{H}), 4.28(\mathrm{~d}, J=8.8 \mathrm{~Hz}$, $1 \mathrm{H}), 2.42-2.31(\mathrm{~m}, 2 \mathrm{H}), 2.07-1.81(\mathrm{~m}, 4 \mathrm{H}), 1.69-1.57(\mathrm{~m}, 2 \mathrm{H}), 1.45-0.88(\mathrm{~m}, 14 \mathrm{H}), 0.83(\mathrm{t}, J=$ $6.8 \mathrm{~Hz}, 3 \mathrm{H})$.

${ }^{13}$ C NMR (100 MHz, CDCl $): \delta$ 159.8, 137.0, 134.0, 129.0, 127.8, 123.6, 120.0, 109.3, 80.3, 49.1, $45.4,39.3,32.4,30.0,29.4,28.2,26.70,26.68,26.0,23.9,22.5,14.0$.

IR (neat, $\mathbf{c m}^{-\mathbf{1}}$ ): 2963, 2851, 1595, 1459, 1260, 1122, 1093, 978, 803, 748.

HRMS (ESI): $[\mathrm{M}+\mathrm{H}]^{+}$calcd for $\mathrm{C}_{22} \mathrm{H}_{33} \mathrm{O} 313.2526$, found 313.2527.

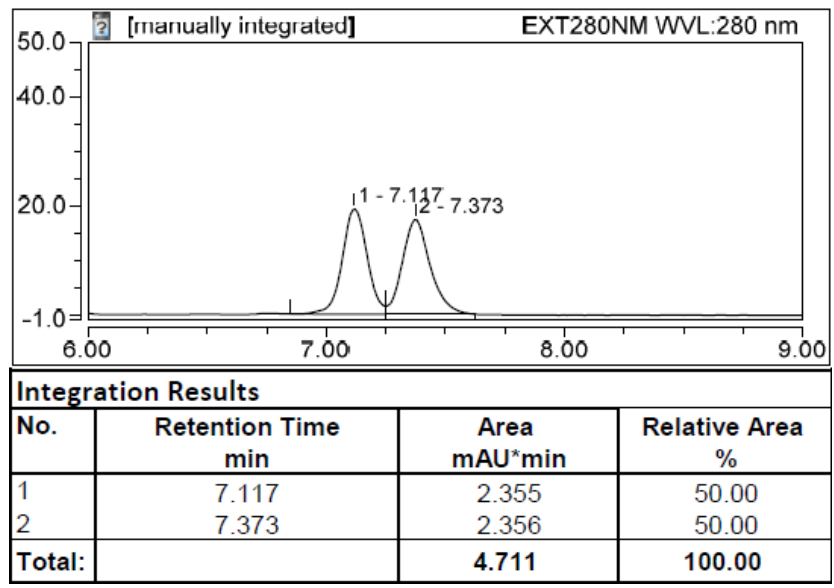

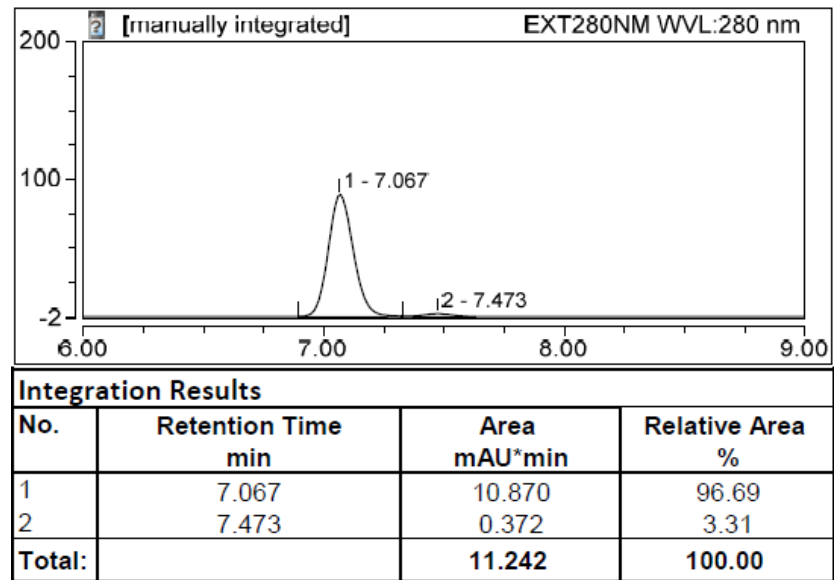




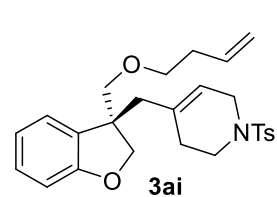

The compound was prepared according to the General Procedure from the reaction of $10(68.8 \mathrm{mg}, 0.2 \mathrm{mmol})$ and $\mathbf{2 k}(77.0 \mathrm{mg}, 0.2 \mathrm{mmol})$ in DMF at $60{ }^{\circ} \mathrm{C}$.

$37.2 \mathrm{mg}, 43 \%$ yield, $95 \%$ ee, colorless oil.

Chiral HPLC: CHIRALPAK IA, $25{ }^{\circ} \mathrm{C},{ }^{i} \mathrm{PrOH}$-hexanes 10/90, $1 \mathrm{~mL} / \mathrm{min}, 285 \mathrm{~nm}, t_{\mathrm{R}}(\operatorname{minor})=20.7$ $\min , t_{\mathrm{R}}($ major $)=24.2 \min$.

$[\alpha]_{\mathbf{D}}{ }^{22}=-1\left(\mathrm{c}=1.0, \mathrm{CH}_{2} \mathrm{Cl}_{2}\right)$.

${ }^{1}$ H NMR (400 MHz, CDCl 3 ): $\delta 7.63(\mathrm{~d}, J=8.0 \mathrm{~Hz}, 2 \mathrm{H}), 7.31(\mathrm{~d}, J=8.0 \mathrm{~Hz}, 2 \mathrm{H})$, 7.15-7.07 (m, 2 H), $6.82(\mathrm{dt}, J=1.2,7.6 \mathrm{~Hz}, 1 \mathrm{H}), 6.74(\mathrm{~d}, J=8.0 \mathrm{~Hz}, 1 \mathrm{H}), 5.82-5.75(\mathrm{~m}, 1 \mathrm{H}), 5.31(\mathrm{~s}, 1 \mathrm{H})$, 5.09-5.01 (m, 2 H), 4.28 (s, 2 H), 3.60-3.36 (m, 6 H), 3.10-2.89 (m, 2 H), 2.52-2.27 (m, 4 H), 2.42, (s, $3 \mathrm{H}), 1.87-1.81(\mathrm{~m}, 2 \mathrm{H})$.

${ }^{13}$ C NMR (100 MHz, $\mathbf{C D C l}_{3}$ ): $\delta$ 159.8, 143.5, 135.1, 133.3, 132.9, 131.1, 129.6, 128.8, 127.6, 124.2, $121.1,120.1,116.4,109.7,78.3,75.7,70.6,49.9,44.8,42.8,42.6,34.0,30.0,21.5$.

IR (neat, $\mathbf{c m}^{-1}$ ): 2924, 2857, 1597, 1482, 1347, 1165, 1098, 954, 755, 690.

HRMS (ESI): $[\mathrm{M}+\mathrm{H}]^{+}$calcd for $\mathrm{C}_{26} \mathrm{H}_{33} \mathrm{NO}_{4} \mathrm{~S}$ 454.2047, found 454.2061.
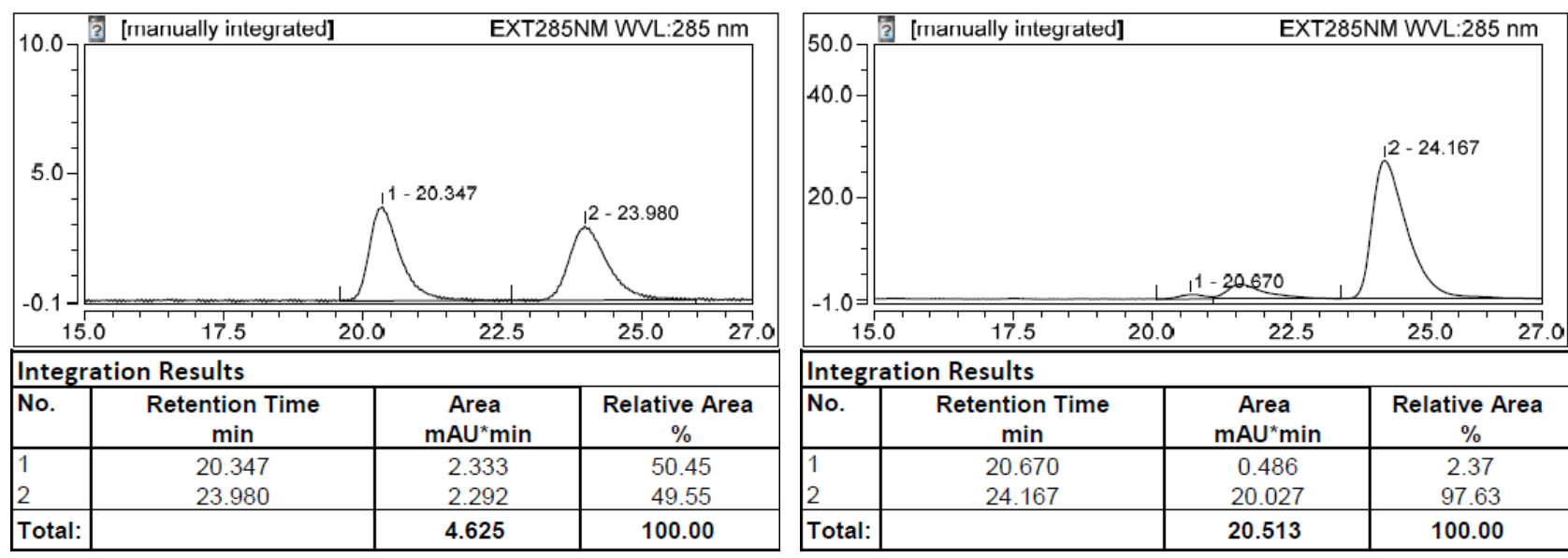

(S)-4-(cyclohex-1-en-1-ylmethyl)-4-methylchromane (3aj)<smiles>CC1(CC2CCCCC2)CCCCC1</smiles>

The compound was prepared according to the General Procedure from the reaction of $1 \mathbf{p}(57.6 \mathrm{mg}, 0.2 \mathrm{mmol})$ and $\mathbf{2 a}(46.0 \mathrm{mg}, 0.2 \mathrm{mmol})$.

$28.1 \mathrm{mg}, 58 \%$ yield, $94 \%$ ee, colorless oil.

Chiral HPLC: CHIRALCEL OD-H, $25{ }^{\circ} \mathrm{C},{ }^{i} \mathrm{PrOH}$-hexanes $0 / 100,0.3 \mathrm{~mL} / \mathrm{min}, 226 \mathrm{~nm}, t_{\mathrm{R}}($ major $)=$ $18.5 \min , t_{\mathrm{R}}($ minor $)=19.0 \min$.

$[\alpha]_{\mathbf{D}}^{21}=+1\left(\mathrm{c}=1.0, \mathrm{CH}_{2} \mathrm{Cl}_{2}\right)$. 
${ }^{1}$ H NMR (400 MHz, CDCl 3 ): $\delta$ 7.26-7.24 (m, $\left.1 \mathrm{H}\right), 7.06(\mathrm{t}, J=8.0 \mathrm{~Hz}, 1 \mathrm{H}), 6.88-6.84(\mathrm{~m}, 1 \mathrm{H})$, $6.77(\mathrm{~d}, J=8.0 \mathrm{~Hz}, 1 \mathrm{H}), 5.39(\mathrm{~s}, 1 \mathrm{H}), 4.21-4.14(\mathrm{~m}, 2 \mathrm{H}), 2.44-2.22(\mathrm{~m}, 2 \mathrm{H}), 2.05-1.66(\mathrm{~m}, 6 \mathrm{H})$, 1.55-1.46 (m, $4 \mathrm{H}), 1.31$ (s, $3 \mathrm{H})$.

${ }^{13} \mathbf{C}$ NMR (100 MHz, $\left.\mathbf{C D C l}_{3}\right): \delta$ 153.9, 135.0, 131.1, 127.5, 126.9, 126.2, 120.1, 116.9, 63.0, 51.2, $34.5,33.8,30.7,30.4,25.5,23.1,22.2$.

IR (neat, $\mathbf{c m}^{-1}$ ): 3410, 2918, 1594, 1447, 1420, 1261, 1117, 1042, 892, 750.

HRMS (ESI): $[\mathrm{M}+\mathrm{H}]^{+}$calcd for $\mathrm{C}_{17} \mathrm{H}_{23} \mathrm{O} 243.1743$, found 243.1748 .
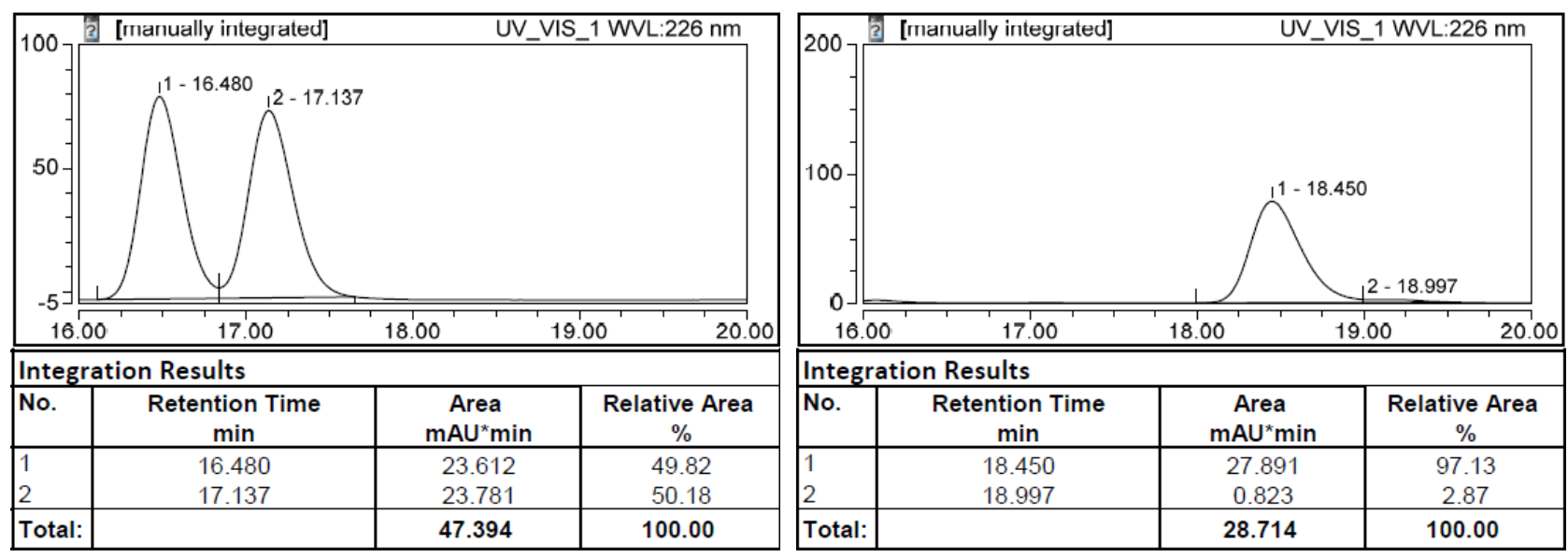

(R)-(3-(cyclohex-1-en-1-ylmethyl)-3-methyl-2,3-dihydrobenzofuran-5-yl)(piperidin-1-yl)metha none (3al)<smiles>CC1(CC2=CCCCC2)COc2ccc(C(=O)N3CCCCC3)cc21</smiles>

3al

The compound was prepared according to the General Procedure from the reaction of $1 \mathbf{r}(138.6 \mathrm{mg}, 0.36 \mathrm{mmol})$ and $\mathbf{2 a}(46.0 \mathrm{mg}, 0.2 \mathrm{mmol})$. $30.6 \mathrm{mg}, 45 \%$ yield, $92 \%$ ee, white solid, mp: $77-79{ }^{\circ} \mathrm{C}$.

Chiral HPLC: CHIRALPAK IA, $25{ }^{\circ} \mathrm{C}$, ${ }^{i} \mathrm{PrOH}$-hexanes 5/95, $1 \mathrm{~mL} / \mathrm{min}, 280 \mathrm{~nm}, t_{\mathrm{R}}$ (major) $=13.8$ $\min , t_{\mathrm{R}}(\operatorname{minor})=15.6 \min$.

$[\alpha]_{\mathbf{D}}^{22}=+22\left(\mathrm{c}=1.0, \mathrm{CH}_{2} \mathrm{Cl}_{2}\right)$.

${ }^{1}$ H NMR (400 MHz, CDCl 3 ): $\delta 7.18(\mathrm{dd}, J=2.0,4.0 \mathrm{~Hz}, 2 \mathrm{H}), 6.74(\mathrm{~d}, J=8.8 \mathrm{~Hz}, 1 \mathrm{H}), 5.40$ (s, 1 H), 4.51 (d, $J=8.4 \mathrm{~Hz}, 1 \mathrm{H}), 4.19$ (d, $J=8.4 \mathrm{~Hz}, 1 \mathrm{H}), 3.53$ (brs, $4 \mathrm{H}), 2.33-2.25$ (m, $2 \mathrm{H}), 1.98$ (s, 2 $\mathrm{H}), 1.77-1.43$ (m, $12 \mathrm{H}), 1.32$ (s, $3 \mathrm{H})$.

${ }^{13}$ C NMR (100 MHz, $\left.\mathbf{C D C l}_{3}\right): \delta$ 170.7, 160.4, 136.0, 134.2, 128.4, 127.5, 126.2, 122.6, 108.9, 82.8, 49.0, 48.7, 45.4, 43.6, 30.1, 26.4, 26.2, 25.8, 25.3, 24.6, 22.9, 22.0.

IR (neat, $\mathbf{c m}^{-1}$ ): 3569, 3450, 1655, 1638, 1473, 1277, 1262, 1074, 766, 751.

HRMS (ESI): $[\mathrm{M}+\mathrm{H}]{ }^{+}$calcd for $\mathrm{C}_{22} \mathrm{H}_{30} \mathrm{NO}_{2}$ 340.2271, found 340.2272. 

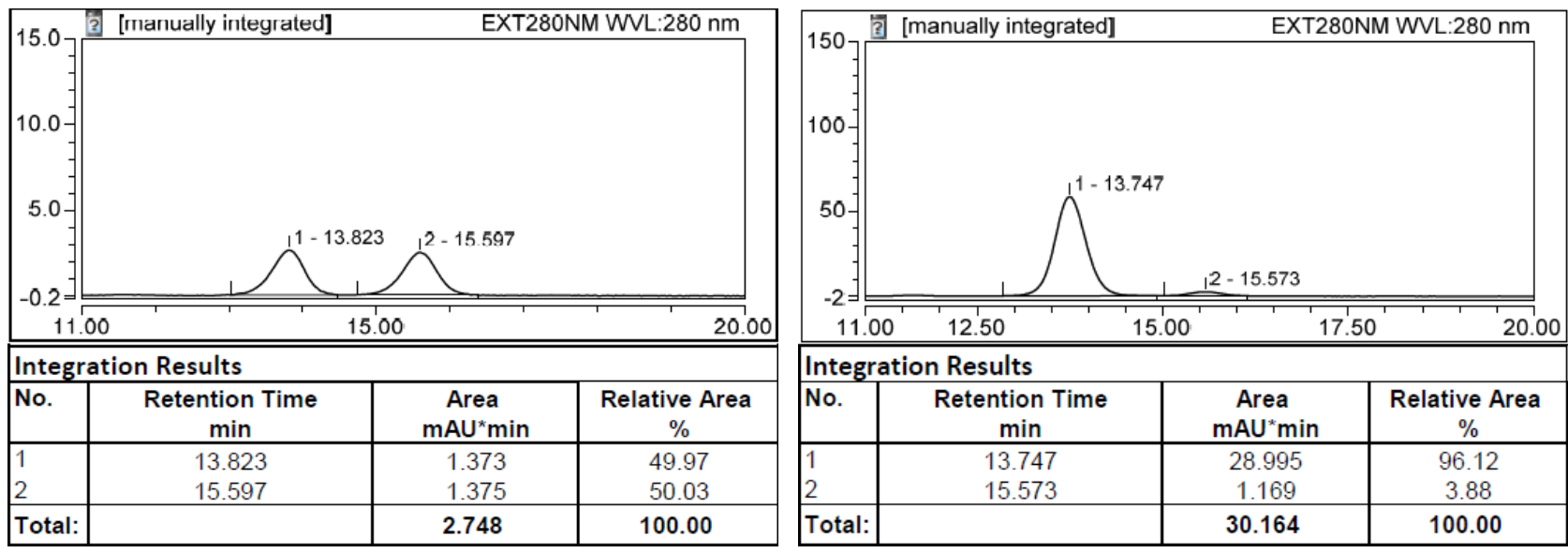

\section{Tert-butyl (R)-3-(cyclohex-1-en-1-ylmethyl)-3-methylindoline-1-carboxylate (3am)}

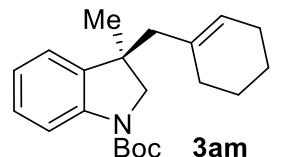

The compound was prepared according to the General Procedure from the reaction of $\mathbf{1 x}(74.6 \mathrm{mg}, 0.2 \mathrm{mmol})$ and $\mathbf{2 a}(46.0 \mathrm{mg}, 0.2 \mathrm{mmol})$ in DMF.

$30.1 \mathrm{mg}, 46 \%$ yield, $83 \%$ ee, colorless oil.

Chiral HPLC: CHIRALCEL OD-H, $25{ }^{\circ} \mathrm{C}$, ${ }^{i} \mathrm{PrOH}-$ hexanes $0.2 / 99.8,0.75 \mathrm{~mL} / \mathrm{min}, 284 \mathrm{~nm}$, $t_{\mathrm{R}}($ major $)=21.5 \min , t_{\mathrm{R}}($ minor $)=24.2 \mathrm{~min}$.

$[\alpha]_{\mathbf{D}}^{22}=-25\left(\mathrm{c}=2.0, \mathrm{CH}_{2} \mathrm{Cl}_{2}\right)$.

${ }^{1} \mathbf{H}$ NMR (400 MHz, $\mathbf{C D C l}_{3}$, mixture of rotamers): $\delta[7.81(\mathrm{~s}), 7.42(\mathrm{~s}), 1 \mathrm{H}], 7.15(\mathrm{t}, J=8.0 \mathrm{~Hz}, 1$ H), $7.04(\mathrm{~d}, J=8.0 \mathrm{~Hz}, 1 \mathrm{H}), 6.94(\mathrm{t}, J=8.0 \mathrm{~Hz}, 1 \mathrm{H}), 5.37(\mathrm{~s}, 1 \mathrm{H}), 3.97(\mathrm{~s}, 1 \mathrm{H}), 3.56(\mathrm{~s}, 1 \mathrm{H})$, 2.22(s, 2 H), 1.96 (s, 2 H), 1.64-1.40 (m, 15 H), 1.30 (s, 3 H).

${ }^{13} \mathrm{C}$ NMR (100 MHz, $\mathbf{C D C l}_{3}$, mixture of rotamers): $\delta 152.5,142.1,141.1,140.2,139.2,134.6$, $127.5,125.8,122.5,122.1,114.6,81.3,80.2,59.7,50.1,43.1,42.44,30.1,28.5,27.3,25.5,23.0$, 22.1 .

IR (neat, $\mathbf{c m}^{-\mathbf{1}}$ ): 2927, 1704, 1600, 1485, 1393, 1291, 1147, 1017, 859, 750.

HRMS (ESI): $[\mathrm{M}+\mathrm{H}]^{+}$calcd for $\mathrm{C}_{21} \mathrm{H}_{30} \mathrm{NO}_{2}$ 328.2271, found 328.2277.

NOTE: Because of the amide bond rotation equilibrium, the rotamers of 3am were observed on the NMR. This phenomenon is seen with many tertiary amides. For related references, see: ref. 19-20. 

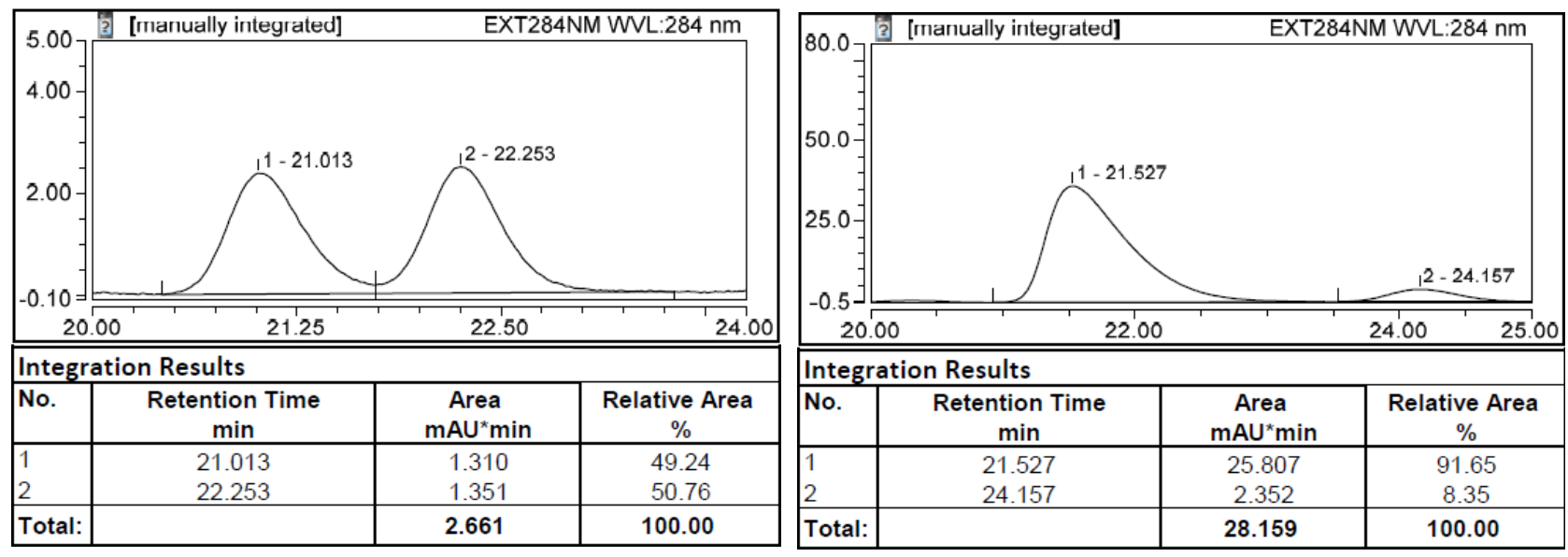

Tert-butyl(R)-3-methyl-3-((1-tosyl-1,2,3,6-tetrahydropyridin-4-yl)methyl)indoline-1-carboxylat e (3an)

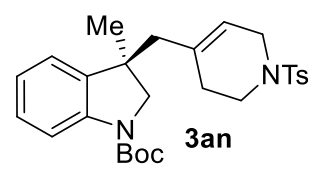

The compound was prepared according to the General Procedure from the reaction of $\mathbf{1 x}(74.6 \mathrm{mg}, 0.2 \mathrm{mmol})$ and $\mathbf{2 k}(77.0 \mathrm{mg}, 0.2 \mathrm{mmol})$ in DMF. $48.2 \mathrm{mg}, 50 \%$ yield, $91 \%$ ee, colorless oil.

Chiral HPLC: CHIRALPAK IA, $25{ }^{\circ} \mathrm{C},{ }^{i} \mathrm{PrOH}$-hexanes 10/90, $1 \mathrm{~mL} / \mathrm{min}, 280 \mathrm{~nm}, t_{\mathrm{R}}$ (major) $=15.2$ $\min , t_{\mathrm{R}}(\operatorname{minor})=16.7 \min$.

$[\boldsymbol{\alpha}]_{\mathbf{D}}{ }^{22}=-13\left(\mathrm{c}=2.0, \mathrm{CH}_{2} \mathrm{Cl}_{2}\right)$.

${ }^{1} \mathrm{H}$ NMR (400 MHz, $\mathbf{C D C l}_{3}$, mixture of rotamers): $\delta[7.78$ (s), 7.35 (s), $1 \mathrm{H}], 7.62$ (t, J = 8.0 Hz, 2 H), $7.30(\mathrm{~d}, J=8.0 \mathrm{~Hz}, 2 \mathrm{H}), 7.15(\mathrm{t}, J=8.0 \mathrm{~Hz}, 1 \mathrm{H}), 7.04(\mathrm{~d}, J=4.0 \mathrm{~Hz}, 1 \mathrm{H}), 6.94-6.91(\mathrm{~m}, 1 \mathrm{H})$, $5.29(\mathrm{~d}, J=4.0 \mathrm{~Hz}, 1 \mathrm{H}), 3.87(\mathrm{~s}, 1 \mathrm{H}), 3.50(\mathrm{~m}, 3 \mathrm{H}), 2.97(\mathrm{~d}, J=4.0 \mathrm{~Hz}, 2 \mathrm{H}), 2.42(\mathrm{~s}, 3 \mathrm{H}), 2.23$ (s, $2 \mathrm{H}), 1.89-1.67$ (m, $2 \mathrm{H}), 1.51(\mathrm{~s}, 9 \mathrm{H}), 1.27$ (d, $J=8.0 \mathrm{~Hz}, 3 \mathrm{H})$.

${ }^{13} \mathrm{C}$ NMR (100 MHz, $\mathbf{C D C l}_{3}$, mixture of rotamers): $\delta 152.2,143.4,141.9,138.9,133.5,133.1$, $129.6,127.9,127.6,122.5,122.2$, 120.1, 114.7, 81.4, 59.3, 48.6, 44.7, 42.8, 42.5, 29.9, 28.4, 27.1, 21.5.

IR (neat, $\mathbf{c m}^{-1}$ ): 2975, 2925, 1698, 1598, 1484, 1393, 1164, 1018, 951, 712.

HRMS (ESI): $[\mathrm{M}+\mathrm{H}]{ }^{+}$calcd for $\mathrm{C}_{27} \mathrm{H}_{35} \mathrm{~N}_{2} \mathrm{O}_{4} \mathrm{~S} 483.2312$, found 483.2321 .

NOTE: Because of the amide bond rotation equilibrium, the rotamers of 3an were observed on the NMR. This phenomenon is seen with many tertiary amides. For related references, see: ref. 19-20. 

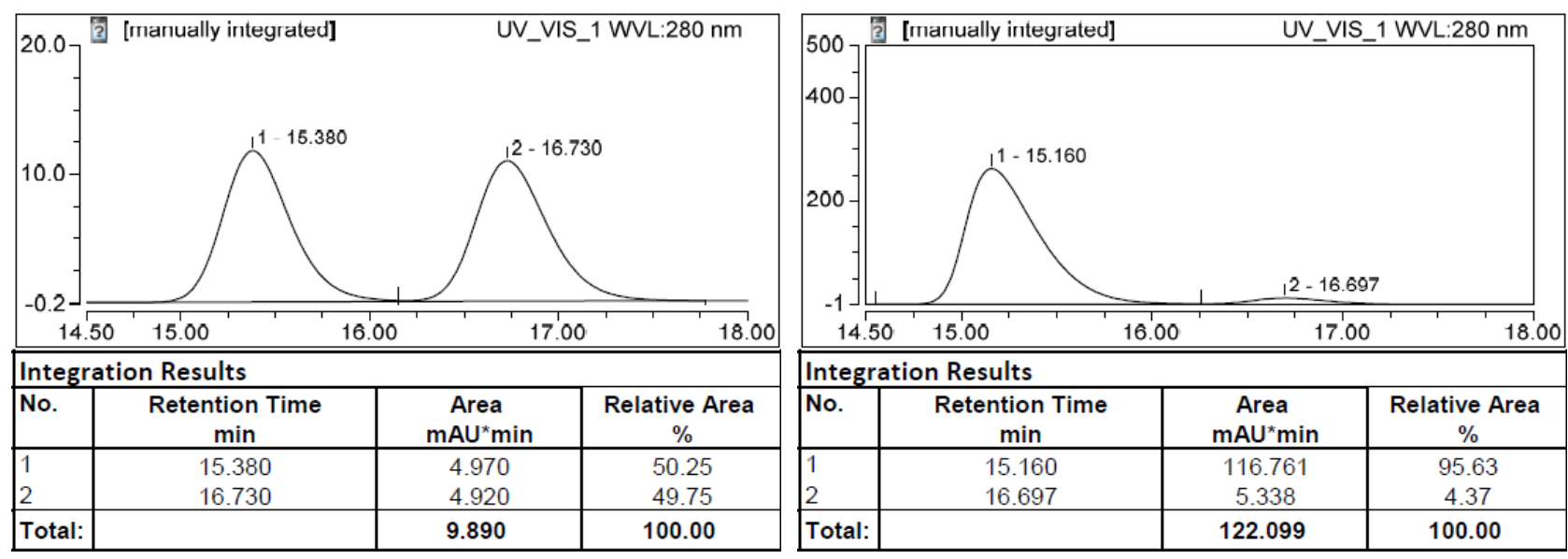

\section{Tert-butyl(R)-3-((1,4-dioxaspiro[4.5]dec-7-en-8-yl)methyl)-3-methylindoline-1-carboxylate}

(3ao)<smiles>C[C@]1(CC2=CCC3(CC2)OCCO3)CNc2ccccc21</smiles>

The compound was prepared according to the General Procedure from the reaction of $\mathbf{1 x}(74.6 \mathrm{mg}, 0.2 \mathrm{mmol})$ and $\mathbf{2 h}(57.6 \mathrm{mg}, 0.2 \mathrm{mmol})$ in DMF. $29.3 \mathrm{mg}, 38 \%$ yield, $84 \%$ ee, colorless oil.

Chiral HPLC: CHIRALPAK ID, $25{ }^{\circ} \mathrm{C},{ }^{i} \mathrm{PrOH}-h$ exanes $2 / 98,1 \mathrm{~mL} / \mathrm{min}, 280 \mathrm{~nm}, t_{\mathrm{R}}$ (major) $=9.7$ $\min , t_{\mathrm{R}}(\operatorname{minor})=12.7 \min$.

$[\boldsymbol{\alpha}]_{\mathbf{D}}{ }^{21}=-11\left(\mathrm{c}=1.0, \mathrm{CH}_{2} \mathrm{Cl}_{2}\right)$.

${ }^{1}$ H NMR (400 MHz, $\left.\mathbf{C D C l}_{3}\right): \delta[7.81(\mathrm{~s}), 7.41(\mathrm{~s}), 1 \mathrm{H}], 7.16(\mathrm{t}, J=8.0 \mathrm{~Hz}, 1 \mathrm{H}), 7.09$ (d, J = 8.0 Hz, $1 \mathrm{H}), 6.95(\mathrm{t}, J=4.0 \mathrm{~Hz}, 1 \mathrm{H}), 5.30(\mathrm{~s}, 1 \mathrm{H}), 3.97-3.89(\mathrm{~m}, 5 \mathrm{H}), 3.59(\mathrm{~s}, 1 \mathrm{H}), 2.27-2.23(\mathrm{~d}, J=4.0$ Hz), 1.89-176 (m, 2 H), 1.64-1.57 (m, $11 \mathrm{H}), 1.32$ (s, $3 \mathrm{H})$.

${ }^{13} \mathrm{C}$ NMR (150 MHz, $\mathbf{C D C l}_{3}$, mixture of rotamers): $\delta 152.5,142.1,139.9,134.3,127.7,122.7$, 122.1, 114.7, 107.6, 81.4, 80.4, 64.3, 59.9, 48.7, 43.1, 35.9, 31.3, 29.1, 28.5, 27.0.

IR (neat, $\mathbf{c m}^{-\mathbf{1}}$ ): 2925, 1703, 1599, 1485, 1393, 1256, 1147, 1080, 857, 751.

HRMS (ESI): $[\mathrm{M}+\mathrm{H}]{ }^{+}$calcd for $\mathrm{C}_{23} \mathrm{H}_{32} \mathrm{NO}_{4} 386.2326$, found 386.2337.

NOTE: Because of the amide bond rotation equilibrium, the rotamers of 3ao were observed on the NMR. This phenomenon is seen with many tertiary amides. For related references, see: ref. 19-20. 

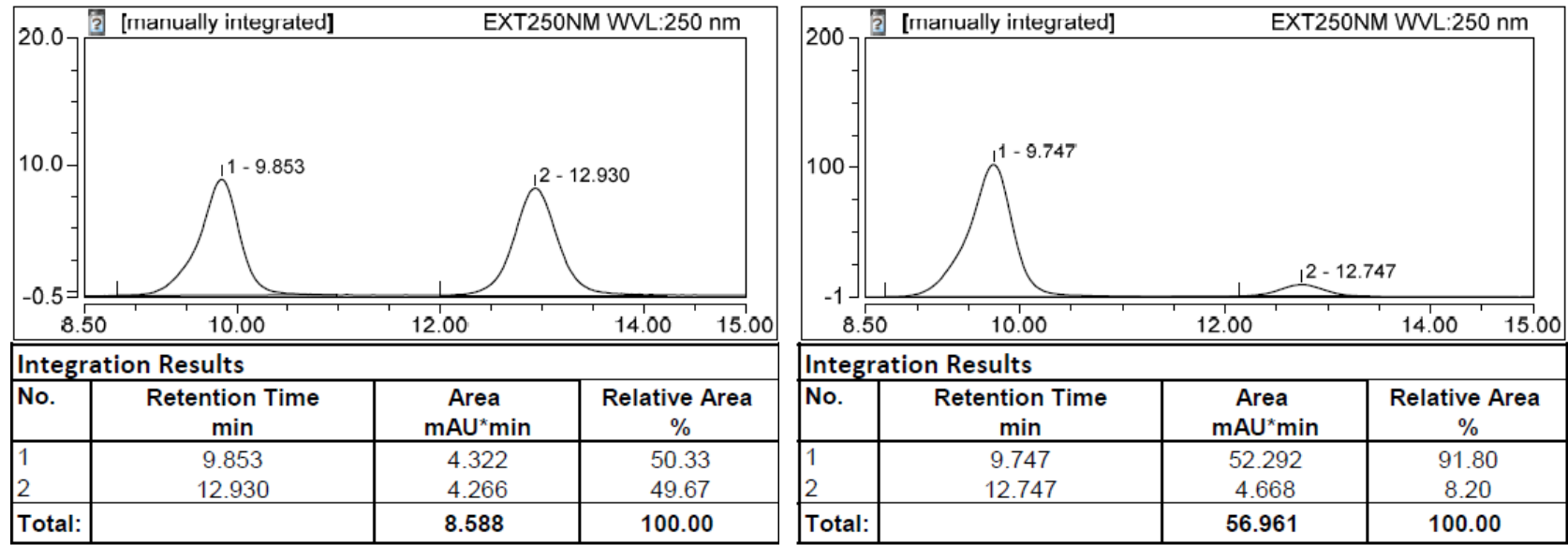

\section{Tert-butyl (R)-3-((1H-inden-2-yl)methyl)-3-methylindoline-1-carboxylate (3ap)}

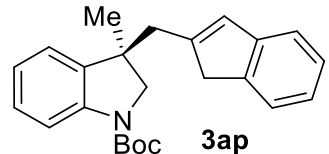

The compound was prepared according to the General Procedure from the reaction of $1 \mathbf{x}(74.6 \mathrm{mg}, 0.2 \mathrm{mmol})$ and $2 \mathrm{~m}(52.8 \mathrm{mg}, 0.2 \mathrm{mmol})$ in DMF. $38.3 \mathrm{mg}, 53 \%$ yield, $97 \%$ ee, colorless oil.

Chiral HPLC: CHIRALCEL OD-H, $25{ }^{\circ} \mathrm{C},{ }^{i} \mathrm{PrOH}-$ hexanes 4/96, $1 \mathrm{~mL} / \mathrm{min}, 284 \mathrm{~nm}, t_{\mathrm{R}}($ major$)=$ $5.4 \min , t_{\mathrm{R}}(\operatorname{minor})=6.1 \min$.

$[\alpha]_{\mathrm{D}}^{21}=-24\left(\mathrm{c}=1.0, \mathrm{CH}_{2} \mathrm{Cl}_{2}\right)$.

${ }^{1} \mathrm{H}$ NMR (400 MHz, $\mathbf{C D C l}_{3}$, mixture of isomers): $\delta$ [7.82 (s), $\left.7.39(\mathrm{~s}), 1 \mathrm{H}\right]$, 7.37-7.07 (m, $\left.6 \mathrm{H}\right)$, 7.01-6.97 (m, $1 \mathrm{H}), 6.51(\mathrm{~d}, J=4.0 \mathrm{~Hz}, 1 \mathrm{H}), 4.04-3.98(\mathrm{~m}, 1 \mathrm{H}), 3.65(\mathrm{~s}, 1 \mathrm{H}), 3.12-2.89(\mathrm{~m}, 2 \mathrm{H})$, $2.81(\mathrm{~s}, 2 \mathrm{H}), 1.50(\mathrm{~s}, 9 \mathrm{H}), 1.40(\mathrm{~s}, 3 \mathrm{H})$.

${ }^{13} \mathbf{C}$ NMR (100 MHz, $\mathbf{C D C l}_{3}$, mixture of isomers): $\delta$ 152.4, 145.8, 144.9, 143.4, 142.1, 139.1, $130.4,127.9,126.2,123.9,123.3,122.7,122.3,120.2,114.8,81.8,80.5,59.4,43.5,42.8,42.5,28.4$, 27.9.

IR (neat, $\mathbf{c m}^{-\mathbf{1}}$ ): 3424, 2975, 2928, 1700, 1599, 1460, 1392, 1147, 857, 751.

HRMS (ESI): $[\mathrm{M}+\mathrm{H}]{ }^{+}$calcd for $\mathrm{C}_{24} \mathrm{H}_{28} \mathrm{NO}_{2} 362.2125$, found 362.2123 .

NOTE: Because of the amide bond rotation equilibrium, the rotamers of 3ap were observed on the NMR. This phenomenon is seen with many tertiary amides. For related references, see: ref. 19-20. 

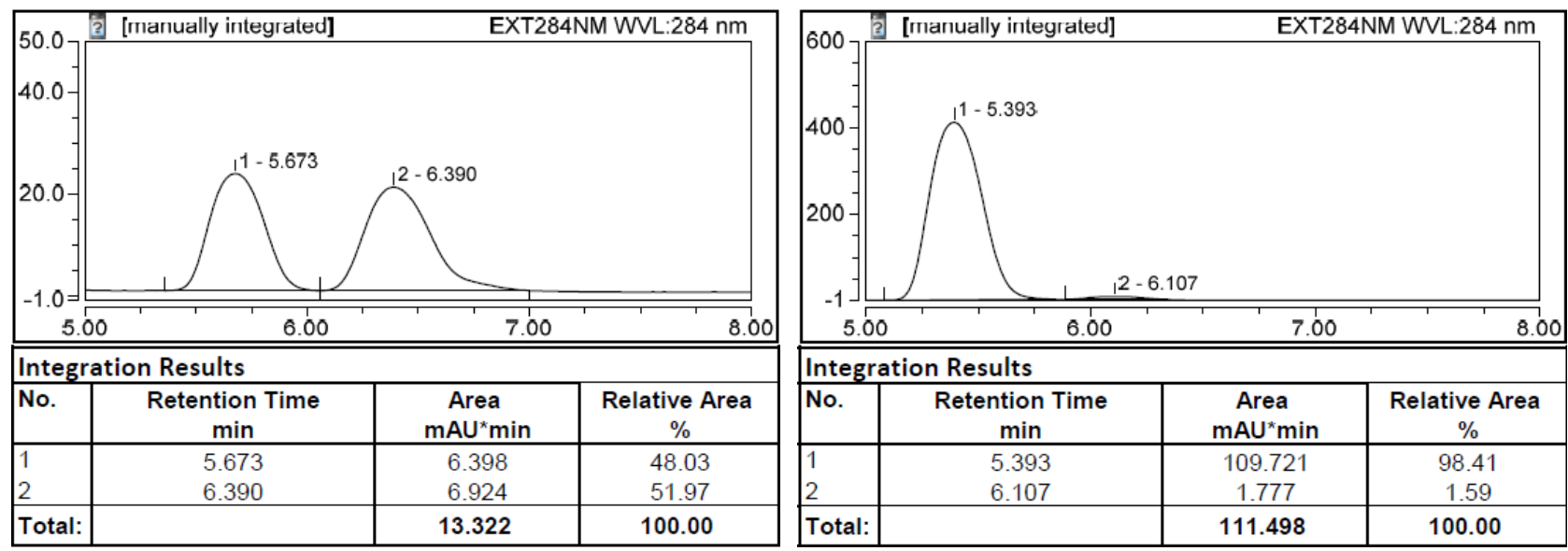

\section{(R)-1-(3-(cyclohex-1-en-1-ylmethyl)-3-methylindolin-1-yl)ethan-1-one (3aq)}

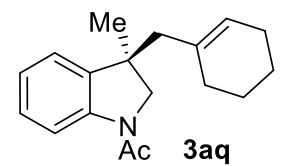

The compound was prepared according to the General Procedure from the reaction of $\mathbf{1 y}(63.0 \mathrm{mg}, 0.2 \mathrm{mmol})$ and $\mathbf{2 a}(46.0 \mathrm{mg}, 0.2 \mathrm{mmol})$ in DMF.

$24.7 \mathrm{mg}, 46 \%$ yield, $92 \%$ ee, colorless oil.

Chiral HPLC: CHIRALPAK IA, $25{ }^{\circ} \mathrm{C},{ }^{i} \mathrm{PrOH}$-hexanes $10 / 90,1 \mathrm{~mL} / \mathrm{min}, 280 \mathrm{~nm}, t_{\mathrm{R}}$ (major) $=6.7$ $\min , t_{\mathrm{R}}($ minor $)=8.3 \min$.

$[\boldsymbol{\alpha}]_{\mathbf{D}}{ }^{21}=-13\left(\mathrm{c}=1.0, \mathrm{CH}_{2} \mathrm{Cl}_{2}\right)$.

${ }^{1}$ H NMR (400 MHz, CDCl $): \delta 8.15(\mathrm{~d}, J=8.0 \mathrm{~Hz}, 1 \mathrm{H}), 7.15(\mathrm{t}, J=8.0 \mathrm{~Hz}, 1 \mathrm{H}), 7.12(\mathrm{~d}, J=8.0$

$\mathrm{Hz}, 1 \mathrm{H}), 7.04(\mathrm{~d}, J=8.0 \mathrm{~Hz}, 1 \mathrm{H}), 5.42(\mathrm{~s}, 1 \mathrm{H}), 4.03(\mathrm{~d}, J=8.0 \mathrm{~Hz}, 1 \mathrm{H}), 3.64(\mathrm{~d}, J=8.0 \mathrm{~Hz}, 1 \mathrm{H})$, 2.26-1.97 (m, $5 \mathrm{H}), 1.65$ (s, $2 \mathrm{H}), 1.57-1.36$ (m, $6 \mathrm{H}), 1.31$ (s, $3 \mathrm{H})$.

${ }^{13} \mathbf{C}$ NMR (100 MHz, $\left.\mathbf{C D C l}_{3}\right): \delta 168.4,142.1,139.6,134.9,127.8,125.9,123.6,122.4,116.9,60.8$, $50.3,43.8,30.0,27.4,25.5,24.2,22.9,22.1$.

IR (neat, cm$^{-1}$ ): 2923, 1663, 1597, 1481, 1460, 1402, 1120, 1043, 753, 618.

HRMS (ESI): $[\mathrm{M}+\mathrm{H}]^{+}$calcd for $\mathrm{C}_{18} \mathrm{H}_{23} \mathrm{NO} 270.1852$, found 270.1859 .
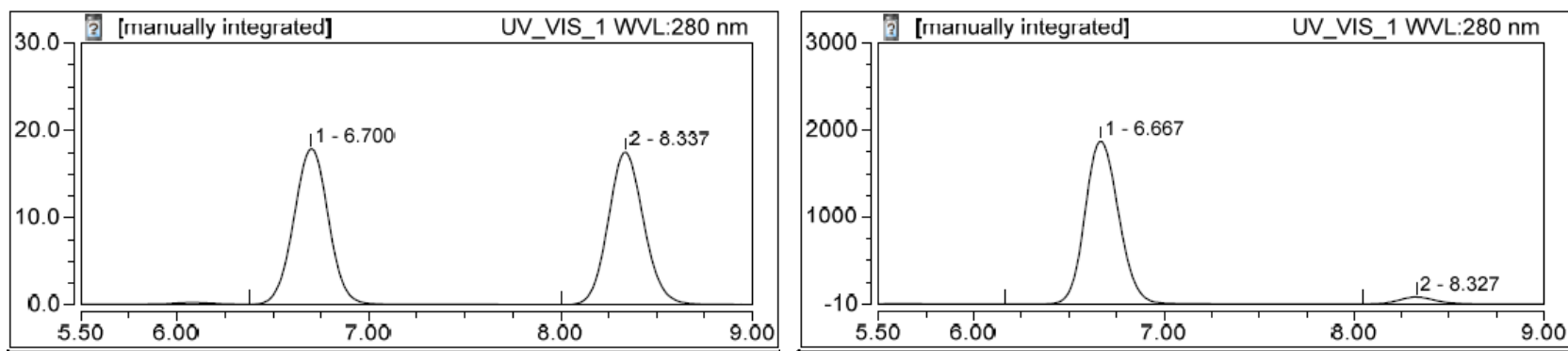

\begin{tabular}{|l|c|c|c|}
\hline \multicolumn{3}{|l|}{ Integration Results } \\
\hline No. & $\begin{array}{c}\text { Retention Time } \\
\text { min }\end{array}$ & $\begin{array}{c}\text { Area } \\
\text { AAU }^{\star} \text { min }\end{array}$ & $\begin{array}{c}\text { Relative Area } \\
\%\end{array}$ \\
\hline 1 & 6.700 & 3.726 & 49.53 \\
2 & 8.337 & 3.797 & 50.47 \\
\hline Total: & & $\mathbf{7 . 5 2 4}$ & $\mathbf{1 0 0 . 0 0}$ \\
\hline
\end{tabular}

\begin{tabular}{|l|c|c|c|}
\hline \multicolumn{3}{|l|}{ Integration Results } \\
\hline No. & $\begin{array}{c}\text { Retention Time } \\
\text { min }\end{array}$ & $\begin{array}{c}\text { Area } \\
\text { mAUmin }\end{array}$ & $\begin{array}{c}\text { Relative Area } \\
\%\end{array}$ \\
\hline 1 & 6.667 & 384.636 & 95.82 \\
2 & 8.327 & 16.788 & 4.18 \\
\hline Total: & & $\mathbf{4 0 1 . 4 2 5}$ & $\mathbf{1 0 0 . 0 0}$ \\
\hline
\end{tabular}

Tert-butyl (R)-3-(cyclohex-1-en-1-ylmethyl)-3,6-dimethylindoline-1-carboxylate (3ar) 
<smiles>[R6]N1C[C@@](C)(CC2=CCCCC2)c2cc(C)ccc21</smiles>
The compound was prepared according to the General Procedure from the reaction of $\mathbf{1 z}(77.4 \mathrm{mg}, 0.2 \mathrm{mmol})$ and $\mathbf{2 a}(46.0 \mathrm{mg}, 0.2 \mathrm{mmol})$ in DMF. $51.2 \mathrm{mg}, 75 \%$ yield, $83 \%$ ee, colorless oil.

$[\alpha]_{\mathbf{D}}^{21}=-21\left(\mathrm{c}=2.0, \mathrm{CH}_{2} \mathrm{Cl}_{2}\right)$.

Chiral HPLC: CHIRALCEL OD-H, $25{ }^{\circ} \mathrm{C}$, ${ }^{i} \mathrm{PrOH}$-hexanes $0.2 / 99.8,0.75 \mathrm{~mL} / \mathrm{min}, 280 \mathrm{~nm}$, $t_{\mathrm{R}}($ major $)=23.8 \min , t_{\mathrm{R}}(\operatorname{minor})=25.4 \mathrm{~min}$.

${ }^{1} \mathrm{H}$ NMR (600 MHz, $\mathbf{C D C l}_{3}$, mixture of rotamers): $\delta$ [7.67 (s), $\left.7.26(\mathrm{~s}), 1 \mathrm{H}\right], 6.97$ (d, J = 6.0 Hz, 1 H), $6.77(\mathrm{~d}, J=6.0 \mathrm{~Hz}, 1 \mathrm{H}), 5.37(\mathrm{~s}, 1 \mathrm{H}), 3.96(\mathrm{~s}, 1 \mathrm{H}), 3.59-3.53(\mathrm{~m}, 1 \mathrm{H}), 2.32(\mathrm{~s}, 3 \mathrm{H}), 2.21(\mathrm{t}, J$ $=12.0 \mathrm{~Hz}, 2 \mathrm{H}), 1.97(\mathrm{~s}, 2 \mathrm{H}), 1.65-1.42(\mathrm{~m}, 15 \mathrm{H}), 1.28(\mathrm{~s}, 3 \mathrm{H})$.

${ }^{13} \mathrm{C}$ NMR (150 MHz, $\mathbf{C D C l}_{3}$, mixture of rotamers): $\delta 152.5,142.3,137.5,136.7,134.8,125.7$, $122.8,122.3,115.4,80.2,60.1,50.1,42.8,30.2,28.5,27.4,25.5,23.1,22.2,21.7$.

IR (neat, $\mathbf{c m}^{-1}$ ): 2926, 2836, 1705, 1592, 1498, 1389, 1243, 1161, 1027, 764.

HRMS (ESI): $[\mathrm{M}+\mathrm{H}]{ }^{+}$calcd for $\mathrm{C}_{22} \mathrm{H}_{32} \mathrm{NO}_{2} 342.2428$, found 342.2434.

NOTE: Because of the amide bond rotation equilibrium, the rotamers of 3ar were observed on the NMR. This phenomenon is seen with many tertiary amides. For related references, see: ref. 19-20.

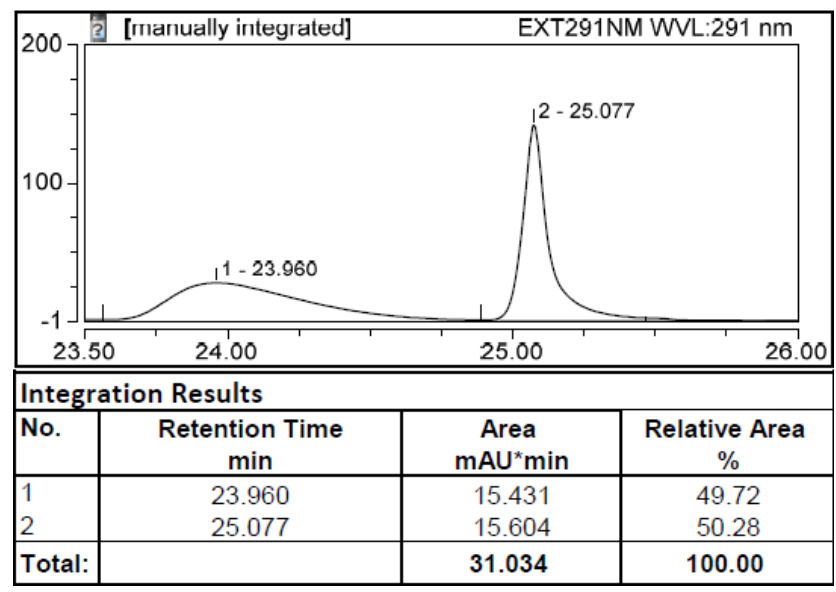

\begin{tabular}{|l|c|c|c|}
\hline 100 & \multicolumn{3}{c|}{ UV_VIS_1 WVL:280 nm } \\
\hline
\end{tabular}

Tert-butyl(R)-3,6-dimethyl-3-((1-tosyl-1,2,3,6-tetrahydropyridin-4-yl)methyl)indoline-1-carbox ylate (3as)<smiles>Cc1ccc2c(c1)[13CH2]C[C@@]2(C)CC1=CC[NH2+]CC1</smiles>
The compound was prepared according to the General Procedure from the reaction of $\mathbf{1 z}(77.4 \mathrm{mg}, 0.2 \mathrm{mmol})$ and $\mathbf{2 k}(77.0 \mathrm{mg}, 0.2 \mathrm{mmol})$ in DMF. $91.3 \mathrm{mg}, 92 \%$ yield, $96 \%$ ee, colorless oil.

Chiral HPLC: CHIRALPAK IB, $25{ }^{\circ} \mathrm{C},{ }^{i} \mathrm{PrOH}-$ hexanes 2/98, $1 \mathrm{~mL} / \mathrm{min}, 290 \mathrm{~nm}, t_{\mathrm{R}}$ (major) $=30.0$ $\min , t_{\mathrm{R}}(\operatorname{minor})=33.7 \mathrm{~min}$. $[\alpha]_{\mathbf{D}}^{21}=-25\left(\mathrm{c}=2.0, \mathrm{CH}_{2} \mathrm{Cl}_{2}\right)$. 
${ }^{1}$ H NMR (400 MHz, $\mathbf{C D C l}_{3}$, mixture of rotamers): [ $\delta 7.64$ (s), 7.27 (s), $\left.1 \mathrm{H}\right], 7.63$ (d, $J=8.0 \mathrm{~Hz}, 2$ H), $7.30(\mathrm{~d}, J=8.0 \mathrm{~Hz}, 2 \mathrm{H}), 6.91(\mathrm{~d}, J=7.6 \mathrm{~Hz}, 1 \mathrm{H}), 6.74(\mathrm{~d}, J=7.6 \mathrm{~Hz}, 1 \mathrm{H}), 5.29(\mathrm{~s}, 1 \mathrm{H}), 3.85$ (s, $1 \mathrm{H}), 3.51(\mathrm{~s}, 3 \mathrm{H}), 2.98(\mathrm{~s}, 2 \mathrm{H}), 2.41(\mathrm{~s}, 3 \mathrm{H}), 2.31$ (s, $3 \mathrm{H}), 2.22$ (s, $2 \mathrm{H}), 1.89-1.72$ (m, $2 \mathrm{H})$, $1.51(\mathrm{~s}, 9 \mathrm{H}), 1.25(\mathrm{~s}, 3 \mathrm{H})$.

${ }^{13} \mathbf{C}$ NMR (100 MHz, $\mathbf{C D C l}_{3}$, mixture of rotamers): $\delta 152.3,143.4,142.0,137.7,135.6,133.6$, 133.1, 129.5, 127.6, 122.8, 122.1, 120.7, 115.4, 81.4, 80.3, 59.5, 48.6, 44.7, 42.8, 42.5, 29.9, 28.3, 27.2, 21.6, 21.4.

IR (neat, $\mathbf{c m}^{-\mathbf{1}}$ ): 3428, 2974, 1699, 1595, 1497, 1347, 1163, 1028, 890, 737.

HRMS (ESI): $[\mathrm{M}+\mathrm{H}]{ }^{+}$calcd for $\mathrm{C}_{28} \mathrm{H}_{37} \mathrm{~N}_{2} \mathrm{O}_{4} \mathrm{~S}$ 497.2477, found 497.2469.

NOTE: Because of the amide bond rotation equilibrium, the rotamers of 3as were observed on the NMR. This phenomenon is seen with many tertiary amides. For related references, see: ref. 19-20.

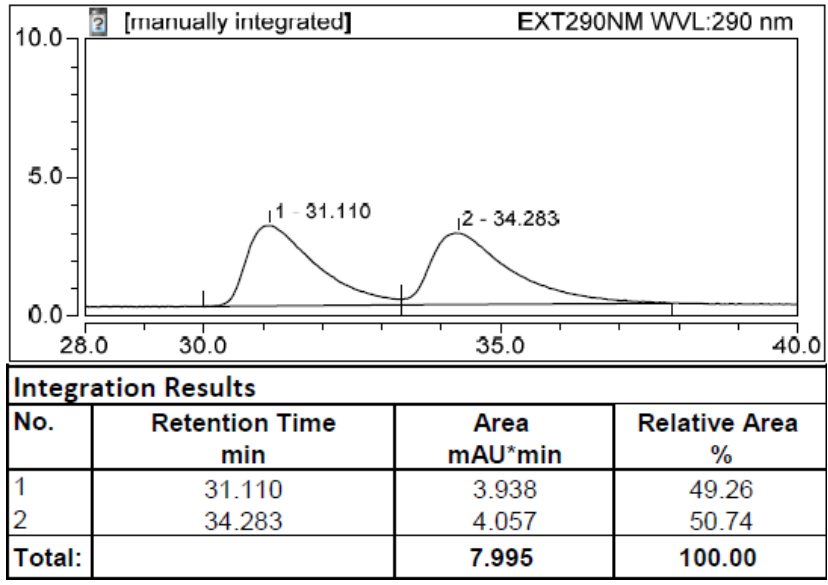

\begin{tabular}{|l|c|c|c|}
\hline 20.0 & \multicolumn{3}{|c|}{ EXT290NM WVL:290 nm } \\
\hline
\end{tabular}

\section{Tert-butyl (R)-3-(cyclohex-1-en-1-ylmethyl)-3,5-dimethylindoline-1-carboxylate (3at)}

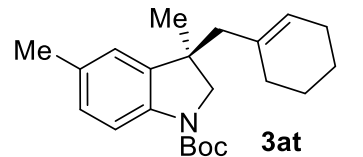

The compound wasprepared according to the General Procedure from the reaction of 1aa $(77.4 \mathrm{mg}, 0.2 \mathrm{mmol})$ and $\mathbf{2 a}(46.0 \mathrm{mg}, 0.2 \mathrm{mmol})$ in DMF. $49.1 \mathrm{mg}, 72 \%$ yield, $85 \%$ ee, colorless oil.

$[\boldsymbol{\alpha}]_{\mathbf{D}}{ }^{21}=-7\left(\mathrm{c}=1.5, \mathrm{CH}_{2} \mathrm{Cl}_{2}\right)$.

Chiral HPLC: CHIRALCEL OD-H, $25{ }^{\circ} \mathrm{C}$, ${ }^{i} \mathrm{PrOH}-h$ exanes $0.2 / 99.8,0.75 \mathrm{~mL} / \mathrm{min}, 280 \mathrm{~nm}$, $t_{\mathrm{R}}($ minor $)=17.3 \min , t_{\mathrm{R}}($ major $)=18.7 \min$.

${ }^{1}$ H NMR (400 MHz, $\mathbf{C D C l}_{3}$, mixture of rotamers): $\delta$ [7.67 (s), $\left.7.28(\mathrm{~s}), 1 \mathrm{H}\right], 6.95(\mathrm{~d}, J=8.0 \mathrm{~Hz}, 1$ H), 6.88 (s, $1 \mathrm{H}), 5.38(\mathrm{~s}, 1 \mathrm{H}), 3.96(\mathrm{~s}, 1 \mathrm{H}), 3.53(\mathrm{~m}, 1 \mathrm{H}), 2.30(\mathrm{~s}, 3 \mathrm{H}), 2.21(\mathrm{~s}, 2 \mathrm{H}), 1.96(\mathrm{~s}, 2 \mathrm{H})$, 1.55-1.44 (m, $15 \mathrm{H}), 1.28(\mathrm{~s}, 3 \mathrm{H})$.

${ }^{13} \mathrm{C}$ NMR (100 MHz, $\mathbf{C D C l}_{3}$, mixture of rotamers): $\delta 152.5,139.8,134.7,131.5,128.0,125.7$, $123.3,114.3,81.0,80.1,59.8,50.0,43.1,42.5,30.1,28.5,27.2,25.5,23.0,22.2,21.0$.

HRMS (ESI): $[\mathrm{M}+\mathrm{H}]{ }^{+}$calcd for $\mathrm{C}_{22} \mathrm{H}_{32} \mathrm{NO}_{2} 342.2428$, found 342.2434 . 
IR (neat, $\left.\mathbf{c m}^{-1}\right)$ : 3424, 2927, 1701, 1637, 1494, 1456, 1390, 1243, 1019, 858, 763.

HRMS (ESI): $[\mathrm{M}+\mathrm{H}]^{+}$calcd for $\mathrm{C}_{22} \mathrm{H}_{32} \mathrm{NO}_{2} 342.2428$, found 342.2432.

NOTE: Because of the amide bond rotation equilibrium, the rotamers of 3at were observed on the NMR. This phenomenon is seen with many tertiary amides. For related references, see: ref. 19-20.
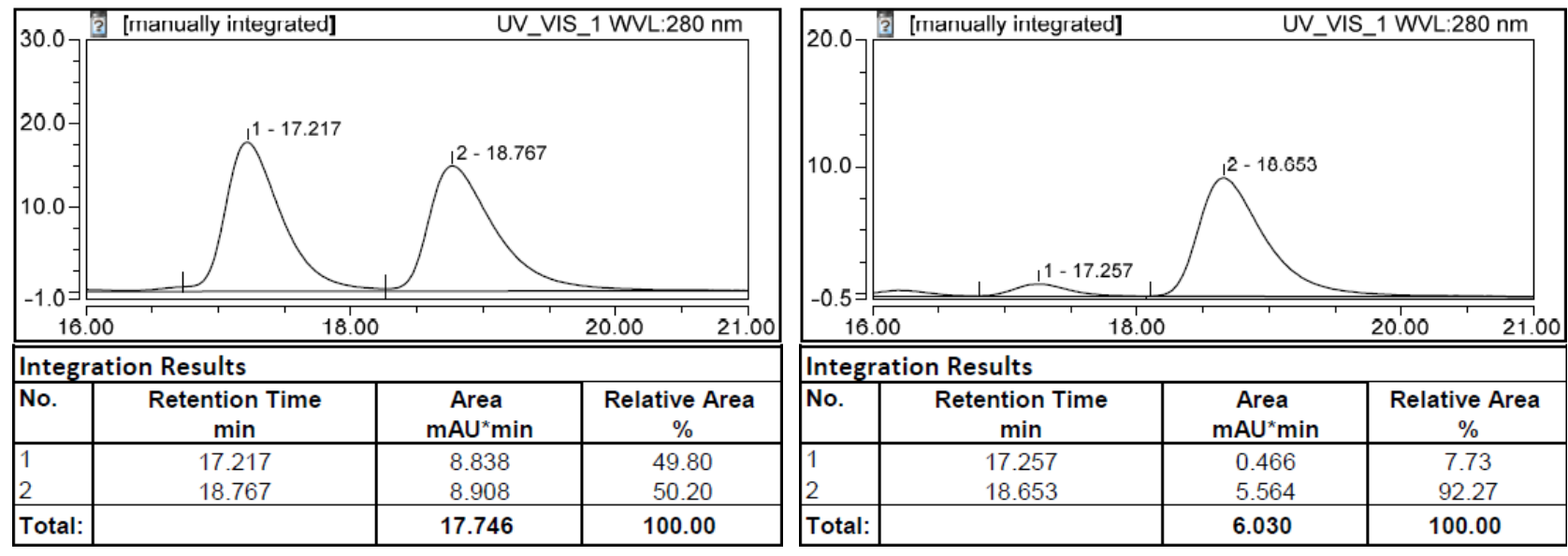

Tert-butyl(R)-6-chloro-3-(cyclohex-1-en-1-ylmethyl)-3-methylindoline-1-carboxylate (3au)

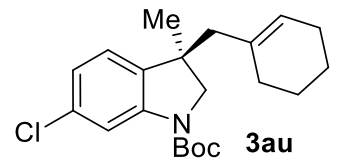

The compound was prepared according to the General Procedure from the reaction of $1 \mathbf{a b}(81.4 \mathrm{mg}, 0.2 \mathrm{mmol})$ and $\mathbf{2 a}(46.0 \mathrm{mg}, 0.2 \mathrm{mmol})$ in DMF. $41.9 \mathrm{mg}, 58 \%$ yield, $96 \%$ ee, colorless oil.

$[\boldsymbol{\alpha}]_{\mathbf{D}}{ }^{21}=-49\left(\mathrm{c}=1.0, \mathrm{CH}_{2} \mathrm{Cl}_{2}\right)$.

Chiral HPLC: CHIRALCEL OD-H, $25{ }^{\circ} \mathrm{C}$, ${ }^{i} \mathrm{PrOH}-h$ exanes $0.2 / 99.8,0.75 \mathrm{~mL} / \mathrm{min}, 280 \mathrm{~nm}$, $t_{\mathrm{R}}($ minor $)=13.8 \mathrm{~min}, t_{\mathrm{R}}($ major $)=16.3 \mathrm{~min}$.

${ }^{1} \mathrm{H}$ NMR (400 MHz, $\mathbf{C D C l}_{3}$, mixture of rotamers): $\delta[7.85(\mathrm{~s}), 7.40(\mathrm{~s}), 1 \mathrm{H}], 6.97(\mathrm{~d}, J=8.0 \mathrm{~Hz}, 1$ H), $6.91(\mathrm{dd}, J=4.0 \mathrm{~Hz}, 1 \mathrm{H}), 5.36(\mathrm{~s}, 1 \mathrm{H}), 3.98(\mathrm{~d}, J=8.0 \mathrm{~Hz}, 1 \mathrm{H}), 3.57(\mathrm{~s}, 1 \mathrm{H}), 2.20-2.19(\mathrm{~m}, 2$ H), $1.96(\mathrm{~d}, J=8.0 \mathrm{~Hz}, 2 \mathrm{H}), 1.60-1.44(\mathrm{~m}, 15 \mathrm{H}), 1.29$ (s, $3 \mathrm{H})$.

${ }^{13} \mathrm{C}$ NMR (100 MHz, $\mathbf{C D C l}_{3}$, mixture of rotamers): $\delta$ 152.2, 143.3, 137.8, 134.3, 133.1, 126.1, 123.3, 122.0, 115.0, 81.9, 80.7, 60.0, 50.0, 42.9, 42.2, 30.2, 28.4, 27.4, 25.5, 23.0, 22.1.

IR (neat, $\mathbf{c m}^{-\mathbf{1}}$ ): 2928, 2836, 1706, 1599, 1486, 1386, 1152, 1081, 921, 860.

HRMS (ESI): $[\mathrm{M}+\mathrm{H}]{ }^{+}$calcd for $\mathrm{C}_{21} \mathrm{H}_{29} \mathrm{ClNO}_{2}$ 362.1881, found 362.1889.

NOTE: Because of the amide bond rotation equilibrium, the rotamers of 3au were observed on the NMR. This phenomenon is seen with many tertiary amides. For related references, see: ref. 19-20. 

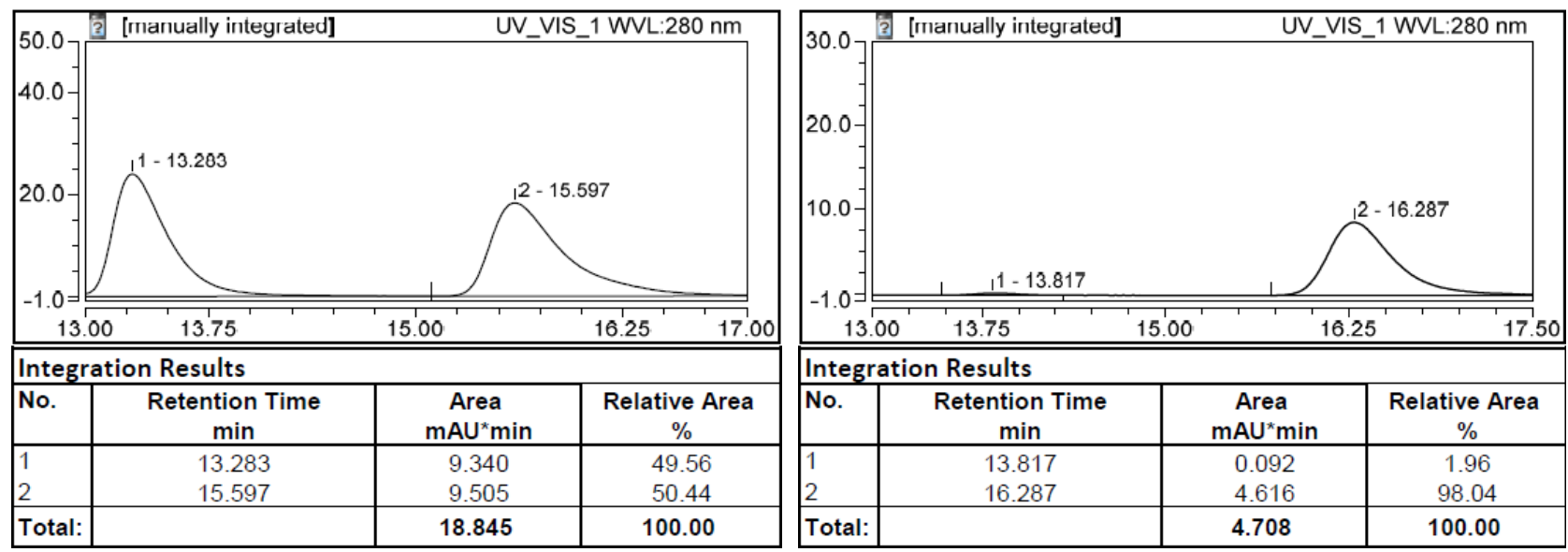

(R)-8-((1-methyl-2,3-dihydro-1H-inden-1-yl)methyl)-1,4-dioxaspiro[4.5]dec-7-ene (3av)

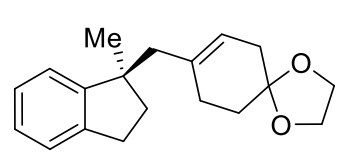

$3 a v$

The compound was prepared according to the General Procedure from the reaction of 1ac $(54.4 \mathrm{mg}, 0.2 \mathrm{mmol})$ and $2 \mathbf{h}(57.6 \mathrm{mg}, 0.2 \mathrm{mmol})$.

$43.2 \mathrm{mg}, 76 \%$ yield, $90 \%$ ee, colorless oil.

Chiral HPLC: CHIRALCEL OJ-H, $25{ }^{\circ} \mathrm{C},{ }^{i} \mathrm{PrOH}-$ hexanes 5/95, $1 \mathrm{~mL} / \mathrm{min}, 273 \mathrm{~nm}, t_{\mathrm{R}}$ (major) $=7.1$ $\min , t_{\mathrm{R}}($ minor $)=8.1 \mathrm{~min}$.

$[\alpha]_{\mathbf{D}}^{25}=+18\left(\mathrm{c}=0.78, \mathrm{CH}_{2} \mathrm{Cl}_{2}\right)$.

${ }^{1}$ H NMR (400 MHz, CDCl $\left.\mathbf{~}_{3}\right): \delta$ 7.18-7.10 (m, $\left.4 \mathrm{H}\right), 5.28(\mathrm{~d}, J=3.2 \mathrm{~Hz}, 1 \mathrm{H}), 3.97-3.90(\mathrm{~m}, 4 \mathrm{H})$, 2.93-2.78 (m, 2 H), 2.28-2.20 (m, 4 H), 2.14-2.08 (m, 1 H), 1.89-1.81 (m, 3 H), 1.66-1.59 (m, 2 H), $1.26(\mathrm{~s}, 3 \mathrm{H})$.

${ }^{13}$ C NMR (100 MHz, $\left.\mathbf{C D C l}_{3}\right): \delta$ 151.4, 143.1, 135.5, 126.2, 126.0, 124.4, 122.7, 122.0, 107.8, 64.2, $48.5,47.7,38.9,35.8,31.3,30.3,29.3,27.5$.

IR (neat, $\mathbf{c m}^{-1}$ ): 2956, 2928, 1479, 1450, 11377, 1256, 1112, 1060, 863, 759.

HRMS (ESI): $[\mathrm{M}+\mathrm{H}]^{+}$calcd for $\mathrm{C}_{19} \mathrm{H}_{25} \mathrm{O}_{2} 285.1849$, found 285.1851 .
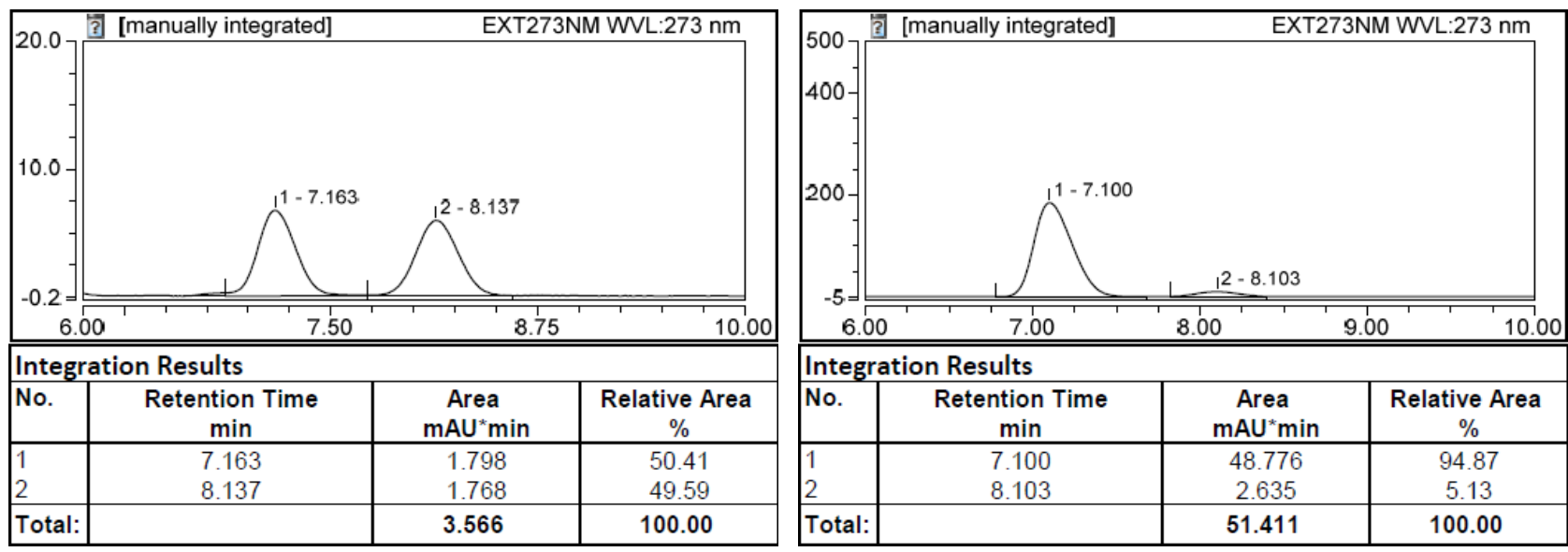

(R)-4-((1-methyl-2,3-dihydro-1H-inden-1-yl)methyl)-1-tosyl-1,2,3,6-tetrahydropyridine (3aw) 


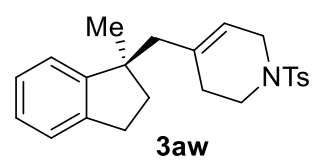

The compound was prepared according to the General Procedure from the reaction of 1ac $(54.4 \mathrm{mg}, 0.2 \mathrm{mmol})$ and $2 \mathbf{k}(77.0 \mathrm{mg}, 0.2 \mathrm{mmol})$ in DMF.

$43.4 \mathrm{mg}, 57 \%$ yield, $84 \%$ ee, colorless oil.

Chiral HPLC: CHIRALCEL AS-H, $25{ }^{\circ} \mathrm{C},{ }^{i} \mathrm{PrOH}-h$ exanes 5/95, $1 \mathrm{~mL} / \mathrm{min}, 260 \mathrm{~nm}, t_{\mathrm{R}}(\operatorname{minor})=$ $29.1 \mathrm{~min}, t_{\mathrm{R}}($ major $)=30.6 \mathrm{~min}$.

$[\alpha]_{\mathbf{D}}^{22}=-4\left(\mathrm{c}=1.0, \mathrm{CH}_{2} \mathrm{Cl}_{2}\right)$.

${ }^{1}$ H NMR (400 MHz, CDCl 3 ): $\delta 7.64(\mathrm{~d}, J=8.0 \mathrm{~Hz}, 2 \mathrm{H}), 7.30(\mathrm{~d}, J=8.4 \mathrm{~Hz}, 2 \mathrm{H})$, 7.26-7.04 (m, 4 H), 5.26 (s, $1 \mathrm{H}), 3.63-3.45(\mathrm{~m}, 2 \mathrm{H}), 3.15-2.88(\mathrm{~m}, 2 \mathrm{H}), 2.83-2.80$ (m, $2 \mathrm{H}), 2.42(\mathrm{~s}, 3 \mathrm{H}), 2.26-2.18$ (m, 2 H), 2.05-2.00 (m, $1 \mathrm{H}), 1.98-1.74(\mathrm{~m}, 3 \mathrm{H}), 1.20$ (s, $3 \mathrm{H})$.

${ }^{13}$ C NMR (100 MHz, $\left.\mathbf{C D C l}_{3}\right): \delta 150.8,143.4,142.9,134.7,133.3,129.5,127.6,126.4,126.1,124.5$, $122.5,120.0,48.8,47.6,44.8,42.9,38.4,30.3,30.2,27.6,21.4$.

IR (neat, $\mathbf{c m}^{-\mathbf{1}}$ ): 3386, 2924, 1600, 1458, 1346, 1162, 1096, 1039, 816, 761.

HRMS (ESI): $[\mathrm{M}+\mathrm{H}]^{+}$calcd for $\mathrm{C}_{23} \mathrm{H}_{28} \mathrm{NO}_{2} \mathrm{~S} 382.1835$, found 382.1842 .
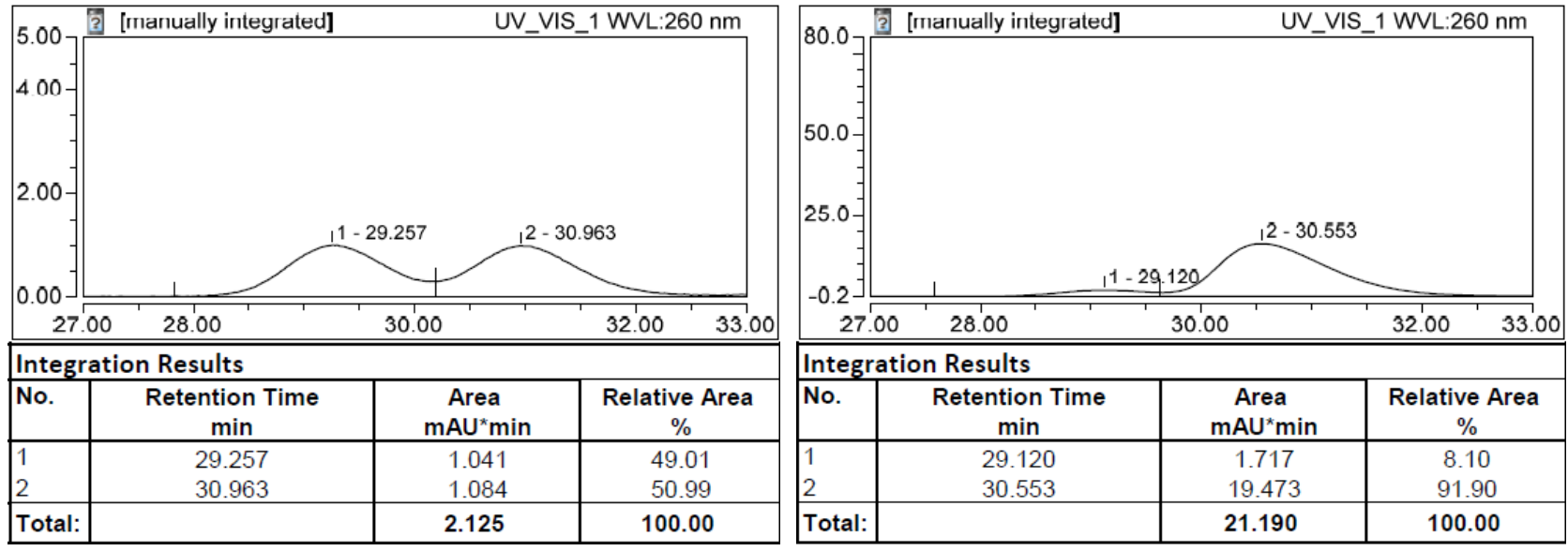

(R)-2-((1-methyl-2,3-dihydro-1H-inden-1-yl)methyl)-1H-indene (3ax)

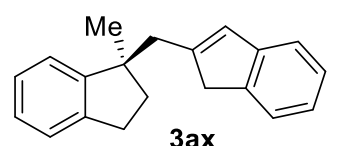

The compound was prepared according to the General Procedure from the reaction of 1ac (54.4 mg, $0.2 \mathrm{mmol})$ and $2 \mathrm{~m}(52.8 \mathrm{mg}, 0.2 \mathrm{mmol})$ in DMF. $23.4 \mathrm{mg}, 45 \%$ yield, $92 \%$ ee, colorless oil.

Chiral HPLC: CHIRALCEL OJ-H, $25{ }^{\circ} \mathrm{C},{ }^{i}$ PrOH-hexanes 5/95, $1 \mathrm{~mL} / \mathrm{min}, 260 \mathrm{~nm}, t_{\mathrm{R}}$ (minor) $=8.0$ $\min , t_{\mathrm{R}}($ major $)=10.6 \mathrm{~min}$.

$[\alpha]_{\mathbf{D}}{ }^{21}=-1\left(\mathrm{c}=1.0, \mathrm{CH}_{2} \mathrm{Cl}_{2}\right)$.

${ }^{1}$ H NMR (400 MHz, CDCl $\left.\mathbf{~}_{3}\right): \delta$ 7.23-7.18 (m, $\left.2 \mathrm{H}\right), 7.15-7.00(\mathrm{~m}, 5 \mathrm{H}), 6.99(\mathrm{~d}, J=1.2 \mathrm{~Hz}, 1 \mathrm{H})$, 6.43 (s, $1 \mathrm{H}), 3.05-2.85$ (m, $2 \mathrm{H}), 2.79-2.65$ (m, $4 \mathrm{H}), 2.15-1.76(\mathrm{~m}, 2 \mathrm{H}), 1.26$ (s, $3 \mathrm{H})$.

${ }^{13}$ C NMR (100 MHz, CDCl $)$ : $\delta$ 151.0, 147.7, 145.3, 143.6, 143.2, 129.7, 126.5, 126.3, 126.1, 124.6, $123.6,123.3,122.6,119.9,48.1,43.0,42.8,38.3,30.2,27.9$. 
IR (neat, $\mathbf{c m}^{-\mathbf{1}}$ ): 3440, 2962, 1636, 1459, 1416, 1316, 1260, 1094, 798, 755

HRMS (ESI): $[\mathrm{M}+\mathrm{H}]^{+}$calcd for $\mathrm{C}_{20} \mathrm{H}_{21}$ 261.1644, found 261.1638 .
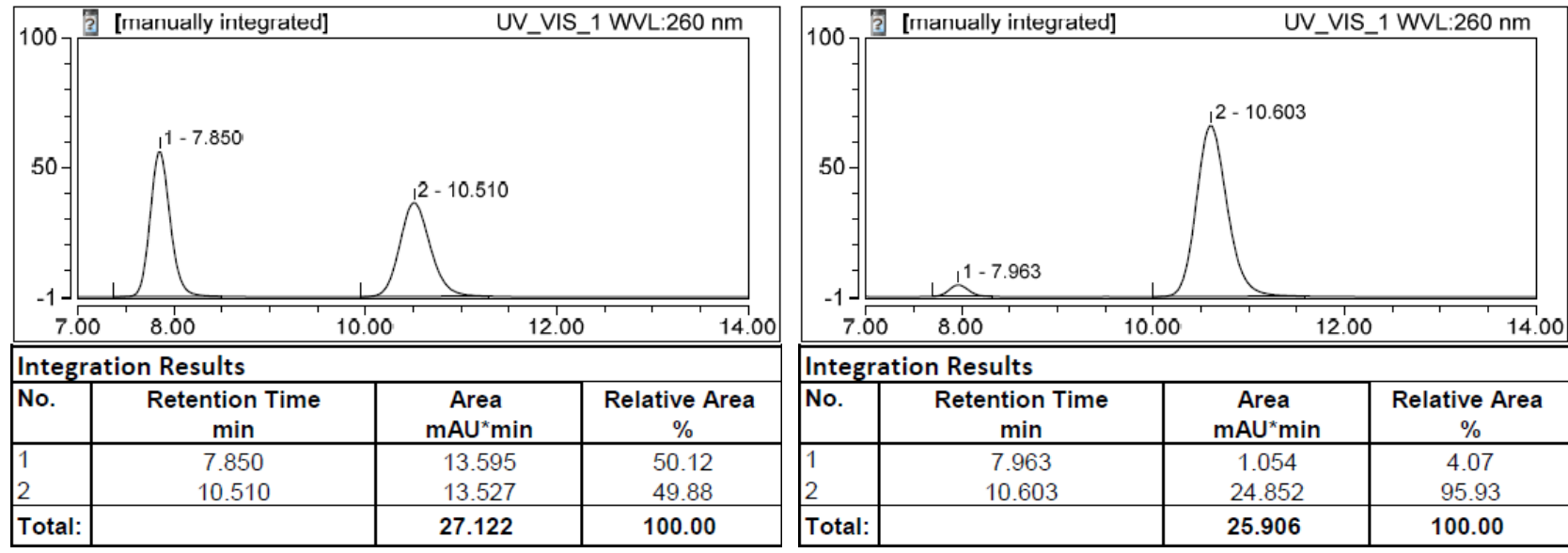

(R)-4-((1-methyl-2,3-dihydro-1H-inden-1-yl)methyl)-1,2-dihydronaphthalene (3ay)<smiles>CC1(CC2=CCCc3ccccc32)CCCC1</smiles>

The compound was prepared according to the General Procedure from the reaction of 1ac (54.4 mg, $0.2 \mathrm{mmol})$ and $2 \mathrm{n}(55.6 \mathrm{mg}, 0.2 \mathrm{mmol})$ in DMF. $25.2 \mathrm{mg}, 46 \%$ yield, $89 \%$ ee, colorless oil.

Chiral HPLC: CHIRALCEL OJ-H, $25{ }^{\circ} \mathrm{C},{ }^{i} \mathrm{PrOH}-$ hexanes 4/96, $1 \mathrm{~mL} / \mathrm{min}, 260 \mathrm{~nm}, t_{\mathrm{R}}(\operatorname{minor})=5.1$ $\min , t_{\mathrm{R}}($ major $)=5.8 \min$.

$[\boldsymbol{\alpha}]_{\mathbf{D}}{ }^{23}=-1\left(\mathrm{c}=1.0, \mathrm{CH}_{2} \mathrm{Cl}_{2}\right)$.

${ }^{1}$ H NMR (400 MHz, $\left.\mathbf{C D C l}_{3}\right): \delta 7.20-7.06(\mathrm{~m}, 8 \mathrm{H}), 5.66(\mathrm{t}, J=4.8 \mathrm{~Hz}, 1 \mathrm{H}), 2.88-2.60(\mathrm{~m}, 6 \mathrm{H})$, 2.18-2.08 (m, $3 \mathrm{H}), 1.76-1.69$ (m, $1 \mathrm{H}), 1.25$ (s, $3 \mathrm{H})$.

${ }^{13}$ C NMR (100 MHz, $\left.\mathbf{C D C l}_{3}\right): \delta 151.4,143.2,136.4,136.1,134.1,128.8,127.4,126.3,126.2,125.9$, $125.9,124.4,123.1,123.0,48.3,42.3,39.1,30.2,28.8,27.1,23.3$.

IR (neat, $\mathbf{c m}^{-1}$ ): 3023, 2930, 1945, 1600, 1478, 1311, 1109, 1023, 756, 670.

HRMS (ESI): $[\mathrm{M}+\mathrm{H}]{ }^{+}$calcd for $\mathrm{C}_{21} \mathrm{H}_{23}$ 275.1797, found 275.1794 .

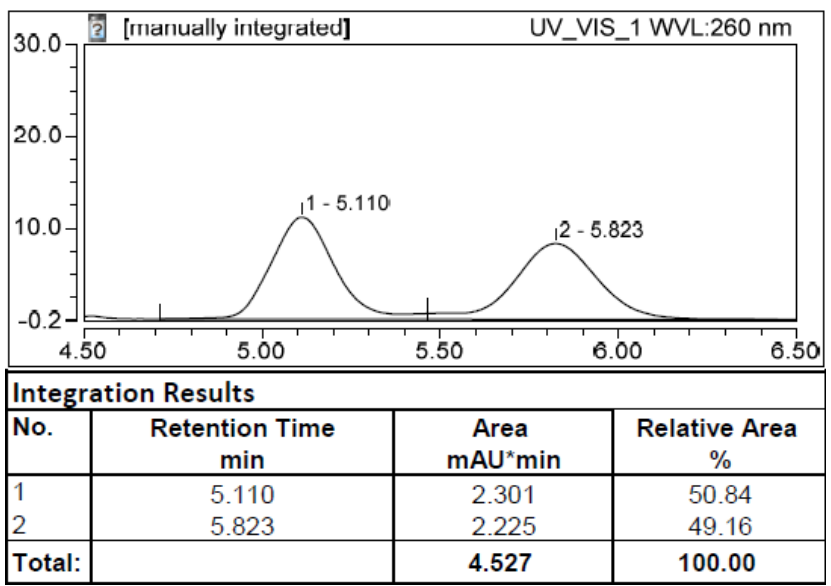

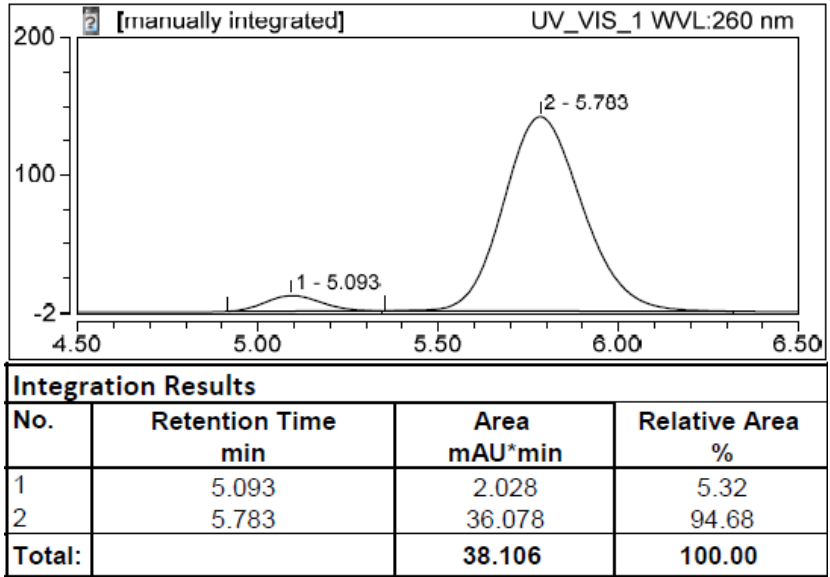


(R)-2-((1,5-dimethyl-2,3-dihydro-1H-inden-1-yl)methyl)-1H-indene (3az)

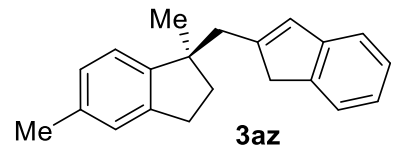

The compound was prepared according to the General Procedure from the reaction of $1 \mathrm{ad}(57.2 \mathrm{mg}, 0.2 \mathrm{mmol})$ and $\mathbf{2 m}(57.6 \mathrm{mg}, 0.2 \mathrm{mmol})$ in DMF. $18.6 \mathrm{mg}, 34 \%$ yield, $95 \%$ ee, colorless oil.

Chiral HPLC: CHIRALCEL OJ-H, $25{ }^{\circ} \mathrm{C},{ }^{i} \mathrm{PrOH}-$ hexanes 5/95, $1 \mathrm{~mL} / \mathrm{min}, 260 \mathrm{~nm}, t_{\mathrm{R}}(\operatorname{minor})=8.5$ $\min , t_{\mathrm{R}}($ major $)=9.2 \min$.

$[\alpha]_{\mathbf{D}}^{21}=+12\left(\mathrm{c}=0.5, \mathrm{CH}_{2} \mathrm{Cl}_{2}\right)$.

${ }^{1}$ H NMR (400 MHz, CDCl 3 ): $\delta$ 7.30-7.20 (m, 3 H), 7.09-7.00 (m, 4 H), 6.50 (s, 1 H), 3.14-2.96 (m, $2 \mathrm{H}), 2.82-2.70$ (m, $4 \mathrm{H}), 2.33$ (s, $3 \mathrm{H}), 2.18-1.87$ (m, $2 \mathrm{H}), 1.31$ (s, 3H).

${ }^{13}$ C NMR (100 MHz, $\left.\mathbf{C D C l}_{3}\right): \delta 147.9,147.5,145.3,143.6,143.4,136.1,129.6,127.1,126.1,125.3$, $123.6,123.3,122.4,119.9,47.7,43.1,42.8,38.6,30.1,27.9,21.3$.

IR (neat, $\mathbf{c m}^{-\mathbf{1}}$ ): 2922, 1959, 1593, 1460, 1421, 1260, 1119, 1037, 831, 799.

HRMS (ESI): $[\mathrm{M}+\mathrm{H}]^{+}$calcd for $\mathrm{C}_{21} \mathrm{H}_{23}$ 275.1794, found 275.1783 .
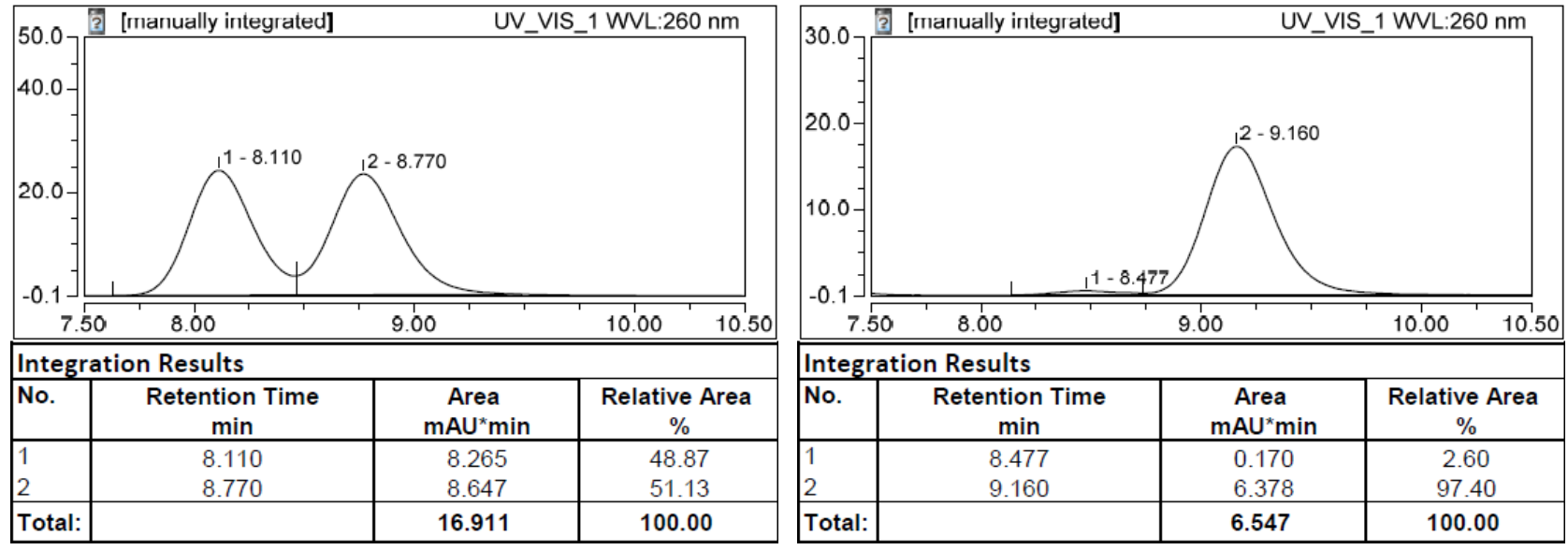

(R)-8-((1,5-dimethyl-2,3-dihydro-1H-inden-1-yl)methyl)-1,4-dioxaspiro[4.5]dec-7-ene (3ba)

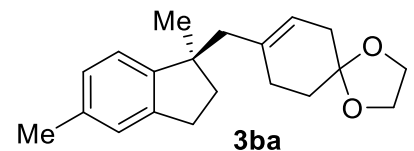

The compound was prepared according to the General Procedure from the reaction of $\mathbf{1 a d}(57.2 \mathrm{mg}, 0.2 \mathrm{mmol})$ and $\mathbf{2 h}(57.6 \mathrm{mg}, 0.2 \mathrm{mmol})$ in DMF. $22.1 \mathrm{mg}, 37 \%$ yield, $90 \%$ ee, colorless oil.

Chiral HPLC: CHIRALCEL OJ-H, $25{ }^{\circ} \mathrm{C},{ }^{i} \mathrm{PrOH}-$ hexanes 5/95, $1 \mathrm{~mL} / \mathrm{min}, 271 \mathrm{~nm}, t_{\mathrm{R}}$ (major) $=6.0$ $\min , t_{\mathrm{R}}($ minor $)=7.5 \mathrm{~min}$.

$[\boldsymbol{\alpha}]_{\mathrm{D}}{ }^{21}=+7\left(\mathrm{c}=1.0, \mathrm{CH}_{2} \mathrm{Cl}_{2}\right)$.

${ }^{1}$ H NMR (400 MHz, $\mathbf{C D C l}_{3}$ ): $\delta$ 7.02-6.96 (m, 3 H), 5.28 (s, $\left.1 \mathrm{H}\right)$, 3.97-3.93 (m, 4 H), 2.87-2.79 (m, $2 \mathrm{H}), 2.31(\mathrm{~s}, 3 \mathrm{H}), 2.25-2.18(\mathrm{~m}, 4 \mathrm{H}), 2.13-2.07(\mathrm{~m}, 1 \mathrm{H}), 1.92(\mathrm{~s}, 2 \mathrm{H}), 1.90-1.79(\mathrm{~m}, 1 \mathrm{H})$, 1.65-1.56 (m, $2 \mathrm{H}), 1.24$ (s, $3 \mathrm{H})$. 
${ }^{13}$ C NMR (100 MHz, $\left.\mathbf{C D C l}_{3}\right): \delta$ 148.7, 143.3, 135.9, 135.7, 126.8, 125.1, 122.5, 121.9, 107.9, 64.3, $48.5,47.4,39.2,35.9,31.4,30.2,29.4,27.5,21.2$.

IR (neat, $\mathbf{c m}^{-\mathbf{1}}$ ): 3370, 2921, 1590, 1453, 1424, 1378, 1259, 1115, 862, 816.

HRMS (ESI): $[\mathrm{M}+\mathrm{H}]{ }^{+}$calcd for $\mathrm{C}_{20} \mathrm{H}_{27} \mathrm{O} 298.1933$, found 298.1939 .
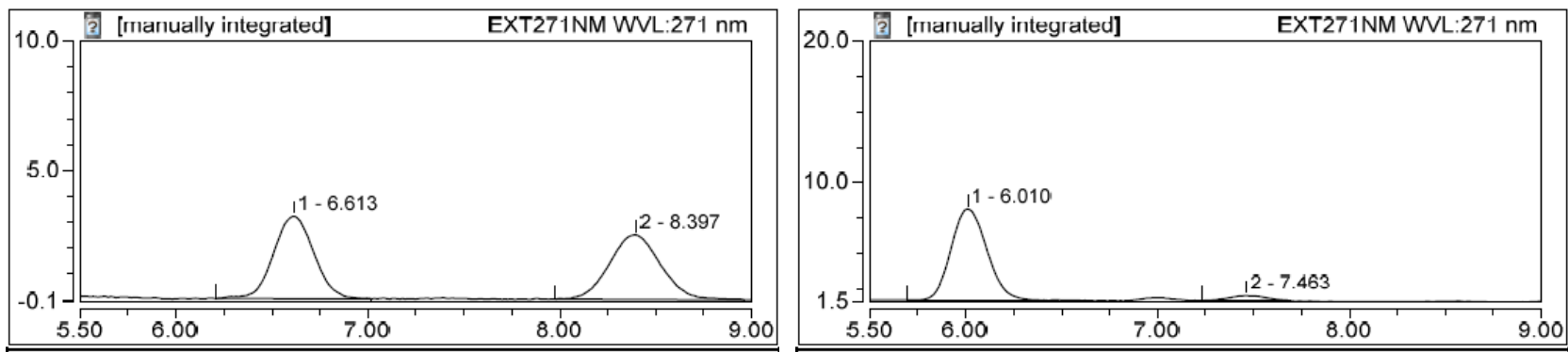

\begin{tabular}{|l|c|c|c|}
\hline \multicolumn{3}{|l|}{ Integration Results } \\
\hline No. & $\begin{array}{c}\text { Retention Time } \\
\text { min }\end{array}$ & $\begin{array}{c}\text { Area } \\
\text { mAU*min }\end{array}$ & $\begin{array}{c}\text { Relative Area } \\
\%\end{array}$ \\
\hline 1 & 6.613 & 0.777 & 50.02 \\
2 & 8.397 & 0.777 & 49.98 \\
\hline Total: & & 1.554 & 100.00 \\
\hline
\end{tabular}

\begin{tabular}{|l|c|c|c|}
\hline \multicolumn{4}{|l|}{ Integration Results } \\
\hline No. & $\begin{array}{c}\text { Retention Time } \\
\text { min }\end{array}$ & $\begin{array}{c}\text { Area } \\
\mathrm{mAU}^{\star} \text { min }\end{array}$ & $\begin{array}{c}\text { Relative Area } \\
\%\end{array}$ \\
\hline 1 & 6.010 & 1.394 & 94.93 \\
2 & 7.463 & 0.074 & 5.07 \\
\hline Total: & & $\mathbf{1 . 4 6 9}$ & $\mathbf{1 0 0 . 0 0}$ \\
\hline
\end{tabular}

(R)-4-((5-methyl-6,7-dihydro-5H-indeno[5,6-d][1,3]dioxol-5-yl)methyl)-1-tosyl-1,2,3,6-tetrahyd ropyridine (3bb)<smiles>C[NH+]1CC=C(CC2(C)CCc3cc4c(cc32)OCO4)CC1</smiles>

The compound was prepared according to the General Procedure from the reaction of 1ae $(63.2 \mathrm{mg}, 0.2 \mathrm{mmol})$ and $2 \mathbf{k}(77.0 \mathrm{mg}, 0.2 \mathrm{mmol})$ in DMF. $42.5 \mathrm{mg}, 50 \%$ yield, $94 \%$ ee, colorless oil.

Chiral HPLC: CHIRALCEL OD-H, $25{ }^{\circ} \mathrm{C},{ }^{i} \mathrm{PrOH}$-hexanes 5/95, $1 \mathrm{~mL} / \mathrm{min}, 293 \mathrm{~nm}, t_{\mathrm{R}}(\operatorname{minor})=$ $29.0 \mathrm{~min}, t_{\mathrm{R}}($ major $)=31.0 \mathrm{~min}$.

$[\alpha]_{\mathbf{D}}^{21}=+14\left(\mathrm{c}=1.0, \mathrm{CH}_{2} \mathrm{Cl}_{2}\right)$.

${ }^{1}$ H NMR (400 MHz, CDCl 3 ): $\delta 7.64(\mathrm{~d}, J=8.4 \mathrm{~Hz}, 2 \mathrm{H}), 7.31(\mathrm{~d}, J=8.0 \mathrm{~Hz}, 2 \mathrm{H}), 6.61(\mathrm{~s}, 1 \mathrm{H})$, $6.53(\mathrm{~s}, 1 \mathrm{H}), 5.90(\mathrm{dd}, J=1.2 \mathrm{~Hz}, J=7.2 \mathrm{~Hz}, 2 \mathrm{H}), 5.27(\mathrm{~s}, 1 \mathrm{H}), 3.63-3.44(\mathrm{~m}, 2 \mathrm{H}), 3.17-2.88$ (m, $2 \mathrm{H}), 2.72-2.68(\mathrm{~m}, 2 \mathrm{H}), 2.42(\mathrm{~s}, 3 \mathrm{H}), 2.20-2.13(\mathrm{~m}, 2 \mathrm{H}), 2.06-1.99$ (m, $1 \mathrm{H}), 1.86-1.73(\mathrm{~m}, 3 \mathrm{H})$, $1.15(\mathrm{~s}, 3 \mathrm{H})$.

${ }^{13}$ C NMR (100 MHz, $\left.\mathbf{C D C l}_{3}\right): \delta$ 146.5, 146.4, 143.9, 143.4, 135.5, 134.7, 133.3, 129.6, 127.6, 120.0, 105.0, 103.2, 100.9, 49.0, 47.4, 44.8, 43.0, 38.7, 30.3, 30.2, 30.0, 21.5 .

IR (neat, $\mathbf{c m}^{-1}$ ): 3435, 2086, 1638, 1474, 1417, 1349, 1162, 1097, 943, 711.

HRMS (ESI): $[\mathrm{M}+\mathrm{H}]^{+}$calcd for $\mathrm{C}_{24} \mathrm{H}_{28} \mathrm{NO}_{4} \mathrm{~S} 426.1734$, found 426.1743. 

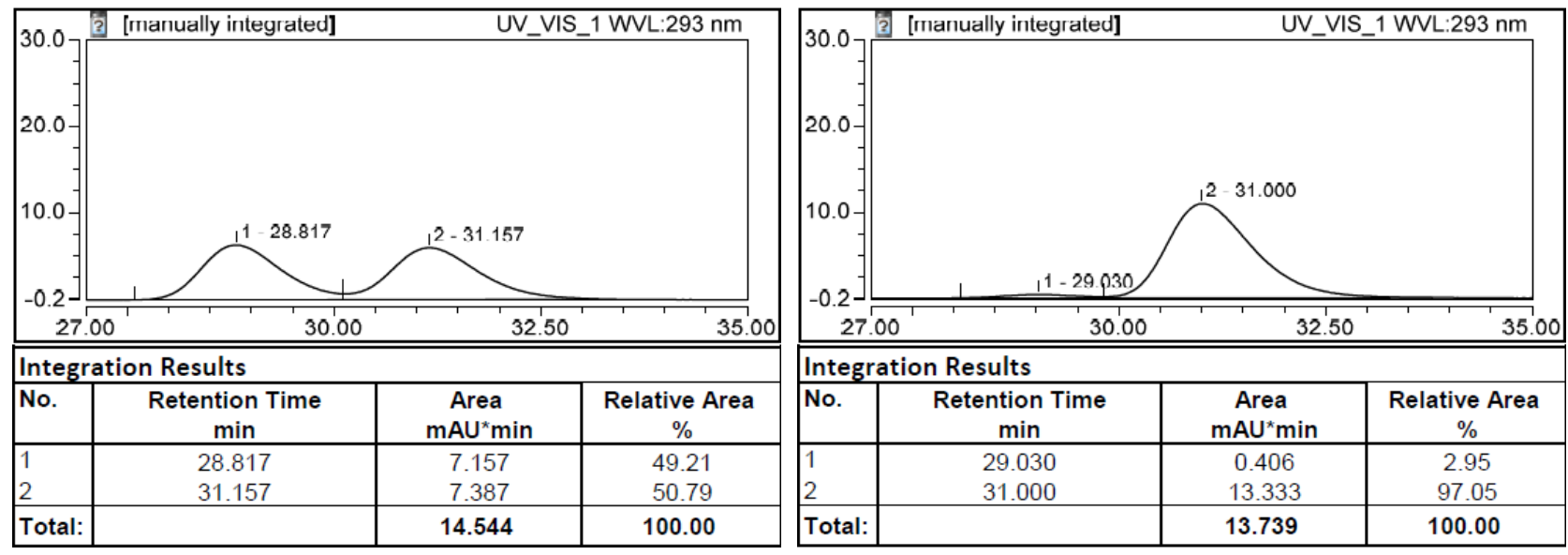

(R)-5-((1H-inden-2-yl)methyl)-5-methyl-6,7-dihydro-5H-indeno[5,6-d][1,3]dioxole (3bc)

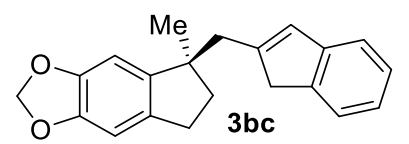

The compound was prepared according to the General Procedure from the reaction of 1ae $(63.2 \mathrm{mg}, 0.2 \mathrm{mmol})$ and $2 \mathrm{~m}(77.0 \mathrm{mg}, 0.2 \mathrm{mmol})$ in DMF.

$31.0 \mathrm{mg}, 51 \%$ yield, $94 \%$ ee, colorless oil.

Chiral HPLC: CHIRALCEL OJ-H, $25{ }^{\circ} \mathrm{C},{ }^{i}$ PrOH-hexanes 10/90, $1 \mathrm{~mL} / \mathrm{min}, 260 \mathrm{~nm}, t_{\mathrm{R}}(\operatorname{minor})=$ $13.0 \mathrm{~min}, t_{\mathrm{R}}($ major $)=24.9 \mathrm{~min}$.

$[\alpha]_{\mathbf{D}}^{21}=+55\left(\mathrm{c}=1.0, \mathrm{CH}_{2} \mathrm{Cl}_{2}\right)$.

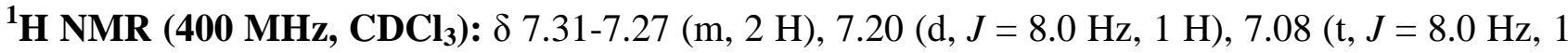
H), $6.65(\mathrm{~s}, 1 \mathrm{H}), 6.61(\mathrm{~s}, 1 \mathrm{H}), 6.49(\mathrm{~s}, 1 \mathrm{H}), 5.93(\mathrm{~d}, J=16.0 \mathrm{~Hz}, 2 \mathrm{H}), 3.15-2.96(\mathrm{~m}, 2 \mathrm{H})$, 2.77-2.65 (m, $4 \mathrm{H}), 2.20-1.87(\mathrm{~m}, 2 \mathrm{H}), 1.28$ (s, $3 \mathrm{H})$.

${ }^{13}$ C NMR (100 MHz, $\left.\mathbf{C D C l}_{3}\right): \delta 147.6,146.6,146.5,145.3,144.0,143.6,135.8,129.7,126.1,123.7$, $123.3,120.0,105.0,103.3,100.9,47.9,43.2,42.7,38.7,30.2,28.2$.

IR (neat, cm$^{-1}$ ): 2922, 1594, 1474, 1422, 1304, 1248, 1121, 1039, 941, 857.

HRMS (ESI): $[\mathrm{M}+\mathrm{H}]^{+}$calcd for $\mathrm{C}_{21} \mathrm{H}_{21} \mathrm{O}_{2}$ 305.1536, found 305.1552.
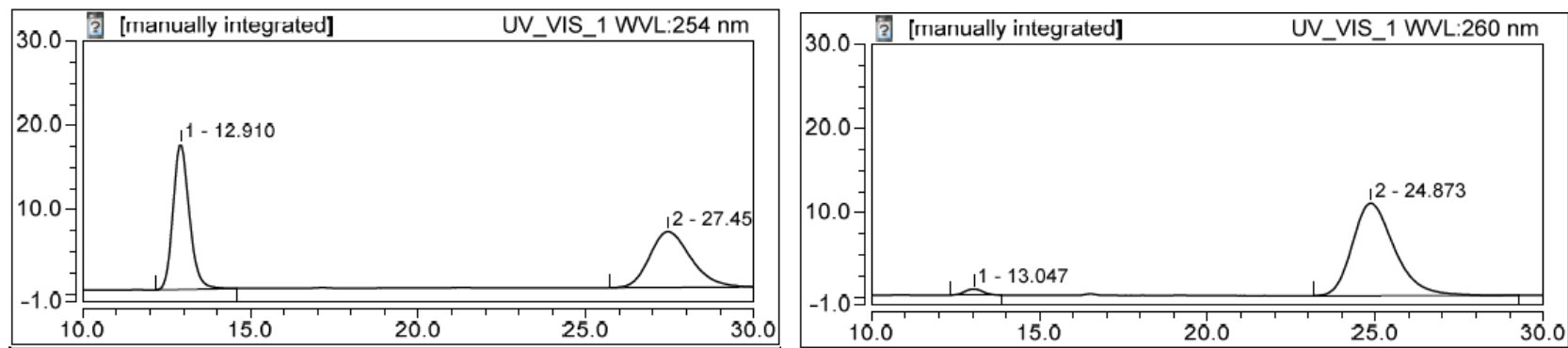

\begin{tabular}{|l|c|c|c|}
\hline \multicolumn{3}{|l|}{ Integration Results } \\
\hline No. & $\begin{array}{c}\text { Retention Time } \\
\text { min }\end{array}$ & $\begin{array}{c}\text { Area } \\
\text { mAU*min }\end{array}$ & $\begin{array}{c}\text { Relative Area } \\
\%\end{array}$ \\
\hline 1 & 12.910 & 9.726 & 50.76 \\
2 & 27.457 & 9.433 & 49.24 \\
\hline Total: & & $\mathbf{1 9 . 1 5 8}$ & $\mathbf{1 0 0 . 0 0}$ \\
\hline
\end{tabular}

\begin{tabular}{|l|c|c|c|}
\hline \hline \multicolumn{3}{|l|}{ Integration Results } \\
\hline No. & $\begin{array}{c}\text { Retention Time } \\
\text { min }\end{array}$ & $\begin{array}{c}\text { Area } \\
\text { mAU*min }\end{array}$ & $\begin{array}{c}\text { Relative Area } \\
\%\end{array}$ \\
\hline 1 & 13.047 & 0.450 & 2.73 \\
2 & 24.873 & 16.018 & 97.27 \\
\hline Total: & & 16.468 & 100.00 \\
\hline
\end{tabular}

Tert-butyl ((S)-1-(((S)-3,3-dimethyl-1-(4-(((R)-3-methyl-2,3-dihydrobenzofuran-3-yl)methyl)- 
(4)<smiles>CC(C)(C)C(NC(=O)[C@H](N)Cc1ccccc1)C(=O)N1CC=C(C[C@]2(C)COc3ccccc32)CC1</smiles>

The compound was prepared according to the General Procedure from the reaction of $\mathbf{1 a}(98.3 \mathrm{mg}, 0.36 \mathrm{mmol})$ and 2w (118.2 mg, $0.2 \mathrm{mmol})$.

$91.9 \mathrm{mg}, 78 \%$ yield, $97 \%$ de, white solid, mp: $66-68{ }^{\circ} \mathrm{C}$.

Chiral HPLC: CHIRALPAK IA, $25^{\circ} \mathrm{C},{ }^{i} \mathrm{PrOH}-$ hexanes 18/82, $0.2 \mathrm{~mL} / \mathrm{min}, 280 \mathrm{~nm}, t_{\mathrm{R}}(\operatorname{minor})=$ $131.5 \mathrm{~min}, t_{\mathrm{R}}($ major $)=138.2 \mathrm{~min}$.

$[\alpha]_{\mathbf{D}}{ }^{23}=-18\left(\mathrm{c}=0.5, \mathrm{CH}_{2} \mathrm{Cl}_{2}\right)$.

${ }^{1} \mathrm{H}$ NMR (400 $\mathbf{M H z}, \mathbf{C D C l}_{3}$, mixture of rotamers): $\delta$ 7.25-7.06 (m, $\left.7 \mathrm{H}\right), 6.89-6.71(\mathrm{~m}, 3 \mathrm{H})$, 5.40-5.34 (m, $1 \mathrm{H}), 5.15(\mathrm{~s}, 1 \mathrm{H}), 4.82-4.76(\mathrm{~m}, 1 \mathrm{H}), 4.46-4.33(\mathrm{~m}, 2 \mathrm{H}), 4.16-3.02(\mathrm{~m}, 7 \mathrm{H})$, 2.39-2.31 (m, $2 \mathrm{H}), 1.91-1.56$ (m, $2 \mathrm{H}), 1.40-1.33$ (m, $12 \mathrm{H}), 0.92-0.83(\mathrm{~m}, 9 \mathrm{H})$.

${ }^{13} \mathrm{C}$ NMR (100 MHz, $\mathbf{C D C l}_{3}$, mixture of rotamers): $\delta$ 170.8, 170.6, 169.5, 169.4, 169.0, 159.28, $159.25,159.22,159.15,155.1,155.0,136.5,135.1,134.8,134.5,134.4,133.4,133.2,129.14$, $129.10,129.0,128.5,128.34,128.25,128.20,128.15,126.8,126.6,122.8,122.7,122.1,121.2$, $121.1,120.33,120.29,109.6,109.5,81.8,81.7,81.6,81.4,79.8,55.7,54.6,54.2,54.0,48.4,48.0$, $45.6,45.43,45.41,45.3,43.4,43.3,42.04,42.00,38.8,38.7,38.3,38.0,37.9,35.80,35.75,35.7$, $35.5,30.2,30.1,29.7,29.6,28.13,28.08,26.4,26.3,26.1,26.0,25.9$.

IR (neat, $\left.\mathbf{c m}^{-1}\right)$ : 2993, 2963, 1675, 1477, 1362, 1321, 1226, 1182, 1088, 754, 688.

HRMS (ESI): $[\mathrm{M}+\mathrm{H}]^{+}$calcd for $\mathrm{C}_{35} \mathrm{H}_{48} \mathrm{~N}_{3} \mathrm{O}_{5} 590.3588$, found 590.3602 .
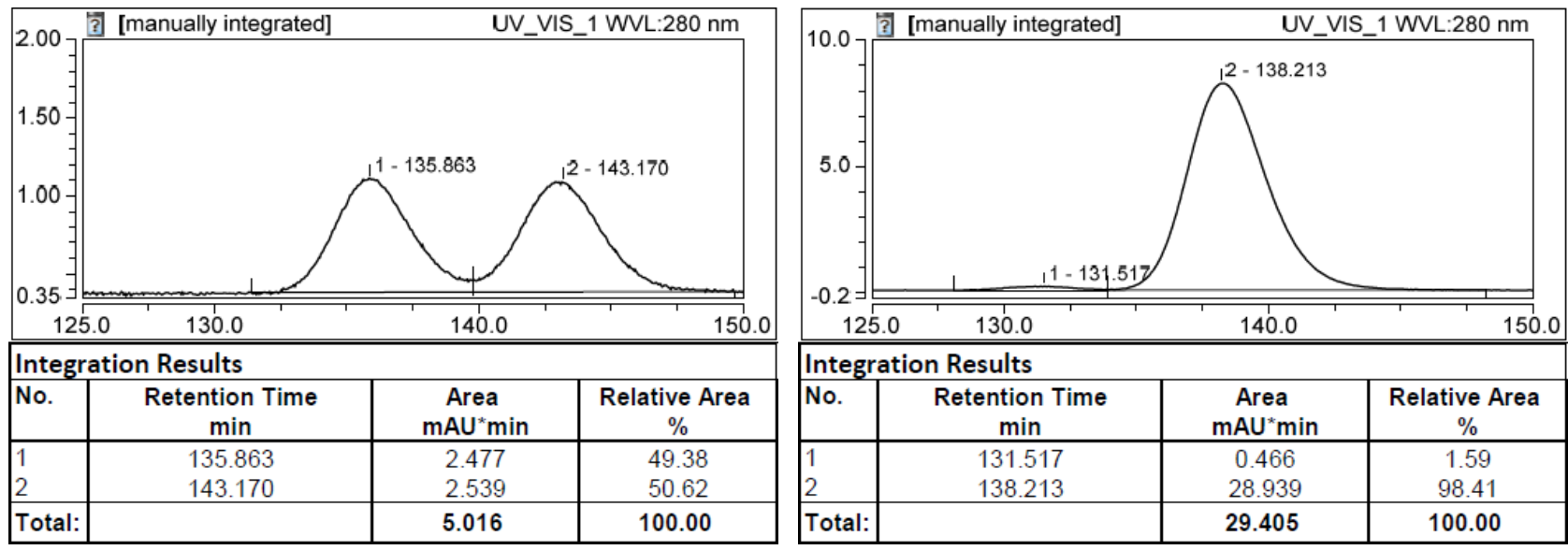

NOTE: Because of the amide bond rotation equilibrium, the rotamers of $\mathbf{4}$ were observed on the

NMR. This phenomenon is seen with many tertiary amides. For related references, see: ref. 19-20.

(R)-5-((3-methyl-2,3-dihydrobenzofuran-3-yl)methyl)hex-5-en-1-yl 2-(1-(4-chlorobenzoyl)-5- 
methoxy-2-methyl-1H-indol-3-yl)acetate (5)

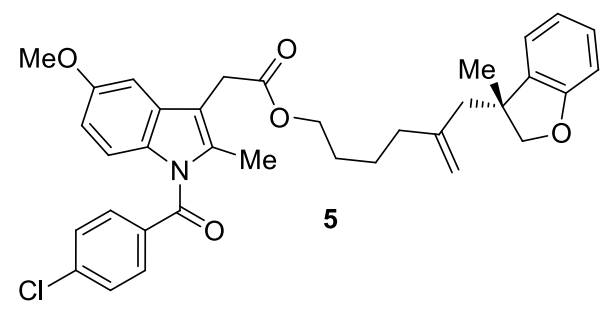

The compound was prepared according to the General Procedure from the reaction of $\mathbf{1 a}(164.4 \mathrm{mg}, 0.6 \mathrm{mmol})$ and $\mathbf{2 x}(115.6 \mathrm{mg}, 0.2 \mathrm{mmol})$.

$49.1 \mathrm{mg}, 42 \%$ yield, $94 \%$ ee, colorless oil.

Chiral HPLC: CHIRALPAK IB, $25{ }^{\circ} \mathrm{C},{ }^{i} \mathrm{PrOH}$-hexanes 3/97, $1 \mathrm{~mL} / \min , 203 \mathrm{~nm}, t_{\mathrm{R}}($ minor $)=31.8 \min , t_{\mathrm{R}}($ major $)=33.8 \mathrm{~min}$.

$[\alpha]_{\mathbf{D}}{ }^{21}=+1\left(\mathrm{c}=1.0, \mathrm{CH}_{2} \mathrm{Cl}_{2}\right)$.

${ }^{1}$ H NMR (400 MHz, CDCl 3 ): $\delta 7.65(\mathrm{~d}, J=8.4 \mathrm{~Hz}, 2 \mathrm{H}), 7.46(\mathrm{~d}, J=8.4 \mathrm{~Hz}, 2 \mathrm{H}), 7.13-7.05$ (m, 2 H), $6.96(\mathrm{~d}, J=2.4 \mathrm{~Hz}, 1 \mathrm{H}), 6.87-6.82(\mathrm{~m}, 2 \mathrm{H}), 6.76(\mathrm{~d}, J=8.0 \mathrm{~Hz}, 1 \mathrm{H}), 6.67-6.65(\mathrm{~m}, 1 \mathrm{H}), 4.80$ $(\mathrm{s}, 1 \mathrm{H}), 4.71(\mathrm{~s}, 1 \mathrm{H}), 4.47(\mathrm{~d}, J=8.8 \mathrm{~Hz}, 1 \mathrm{H}), 4.14(\mathrm{~d}, J=8.4 \mathrm{~Hz}, 1 \mathrm{H}), 4.03(\mathrm{~m}, 2 \mathrm{H}), 3.82(\mathrm{~s}, 3$ H), 3.65 (s, 2 H), 2.38 (s, 3 H), 2.34-2.27 (m, 2 H), 1.79-1.65 (m, 2 H), 1.54-1.44 (m, 2 H), 1.37-1.30 (m, $2 \mathrm{H}), 1.34(\mathrm{~s}, 3 \mathrm{H})$.

${ }^{13}$ C NMR (100 MHz, $\left.\mathbf{C D C l}_{3}\right): \delta$ 170.9, 168.2, 159.3, 156.0 145.7, 139.2, 135.8, 135.2, 133.9, 131.1, $130.8,130.6,129.1,128.1,122.8,120.3,114.9,113.9,112.7,111.6,109.6,101.3,81.8,64.8,55.6$, $46.3,45.3,36.3,30.4,28.1,26.3,24.1,13.3$.

IR (neat, $\mathbf{c m}^{-\mathbf{1}}$ ): 2943, 1735, 1687, 1597, 1480, 1321, 1224, 1167, 833, 755.

HRMS (ESI): $[\mathrm{M}+\mathrm{H}]{ }^{+}$calcd for $\mathrm{C}_{35} \mathrm{H}_{37} \mathrm{ClNO}_{5}$ 586.2355, found 586.2361.

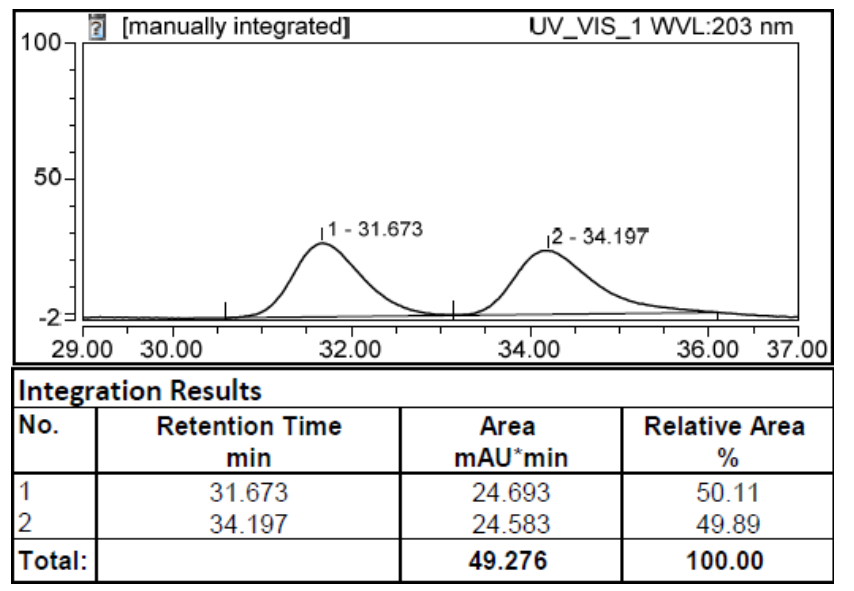

\begin{tabular}{|l|c|c|c|}
\hline 500 & \multicolumn{3}{|c|}{ UV_VIS_1 WVL:203 nm } \\
\hline
\end{tabular}

(R)-2-(1-(4-chlorobenzoyl)-5-methoxy-2-methyl-1H-indol-3-yl)-1-(4-((3-methyl-2,3-dihydroben zofuran-3-yl)methyl)-3,6-dihydropyridin-1(2H)-yl)ethan-1-one (6)

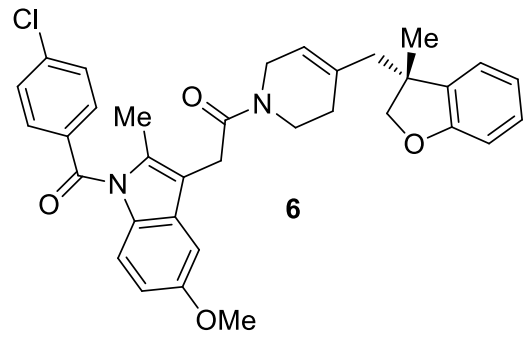

The compound was prepared according to the General Procedure from the reaction of $\mathbf{1 a}(98.6 \mathrm{mg}, 0.36 \mathrm{mmol})$ and $\mathbf{2 y}(114.0 \mathrm{mg}$, $0.2 \mathrm{mmol})$. 
$73.8 \mathrm{mg}, 65 \%$ yield, $>99 \%$ ee, white solid, mp: $66-68{ }^{\circ} \mathrm{C}$.

Chiral HPLC: CHIRALCEL OD-H, $25{ }^{\circ} \mathrm{C},{ }^{i} \mathrm{PrOH}-$ hexanes $30 / 70,1 \mathrm{~mL} / \mathrm{min}, 260 \mathrm{~nm}, t_{\mathrm{R}}(\operatorname{minor})=$ $19.7 \mathrm{~min}, t_{\mathrm{R}}($ major $)=33.2 \mathrm{~min}$. Approximate $1.25: 1$ ratio of rotamers.

$[\alpha]_{\mathbf{D}}{ }^{23}=-6\left(\mathrm{c}=0.5, \mathrm{CH}_{2} \mathrm{Cl}_{2}\right)$.

${ }^{1}$ H NMR (400 MHz, CDCl 3$): \delta 7.65(\mathrm{~d}, J=8.4 \mathrm{~Hz}, 2 \mathrm{H}), 7.45(\mathrm{~d}, J=7.6 \mathrm{~Hz}, 2 \mathrm{H})$, 7.12-7.08 (m, 1 H), $7.02(\mathrm{t}, J=6.4 \mathrm{~Hz}, 1 \mathrm{H}), 6.98(\mathrm{~d}, J=2.4 \mathrm{~Hz}, 1 \mathrm{H}), 6.86-6.81(\mathrm{~m}, 2 \mathrm{H}), 6.73(\mathrm{dd}, J=2.0,8.0 \mathrm{~Hz}$, $1 \mathrm{H}), 6.64(\mathrm{dd}, J=2.4,9.2 \mathrm{~Hz}, 1 \mathrm{H}),[5.38(\mathrm{~s}), 5.27(\mathrm{~s}), 1 \mathrm{H}], 4.39-4.34(\mathrm{~m}, 1 \mathrm{H}), 4.16-3.90(\mathrm{~m}, 3 \mathrm{H})$, $3.80(\mathrm{~d}, J=2.8 \mathrm{~Hz}, 3 \mathrm{H}), 3.68-3.32(\mathrm{~m}, 4 \mathrm{H}), 2.36-2.29(\mathrm{~m}, 5 \mathrm{H}), 1.89-1.54(\mathrm{~m}, 2 \mathrm{H}), 1.30-1.25(\mathrm{~m}$, $3 \mathrm{H})$.

${ }^{13}$ C NMR (100 MHz, $\left.\mathbf{C D C l}_{3}\right): \delta 168.71,168.67,168.1,159.2,155.8,139.1,139.0,135.1,135.0$, $134.9,134.7,134.6,133.81,133.75,132.9,131.1,130.70,130.68,130.62,130.60,128.98,128.96$, $128.20,128.15,122.7,120.9,120.31,120.28,114.7,113.2,111.4,111.3,109.6,109.5,101.4,101.3$, $81.70,81.65,55.54,55.52,48.2,45.4,45.3,44.9,42.8,42.2,38.7,30.5,30.4,30.1,29.6,25.9,25.8$, 13.4, 13.3.

IR (neat, $\mathbf{c m}^{-1}$ ): 3302, 2970, 1709, 1627, 1482, 1455, 1262, 1172, 1017, 751.

HRMS (ESI): $[\mathrm{M}+\mathrm{H}]^{+}$calcd for $\mathrm{C}_{34} \mathrm{H}_{34} \mathrm{ClN}_{2} \mathrm{O}_{4}$ 569.2202, found 569.2209.
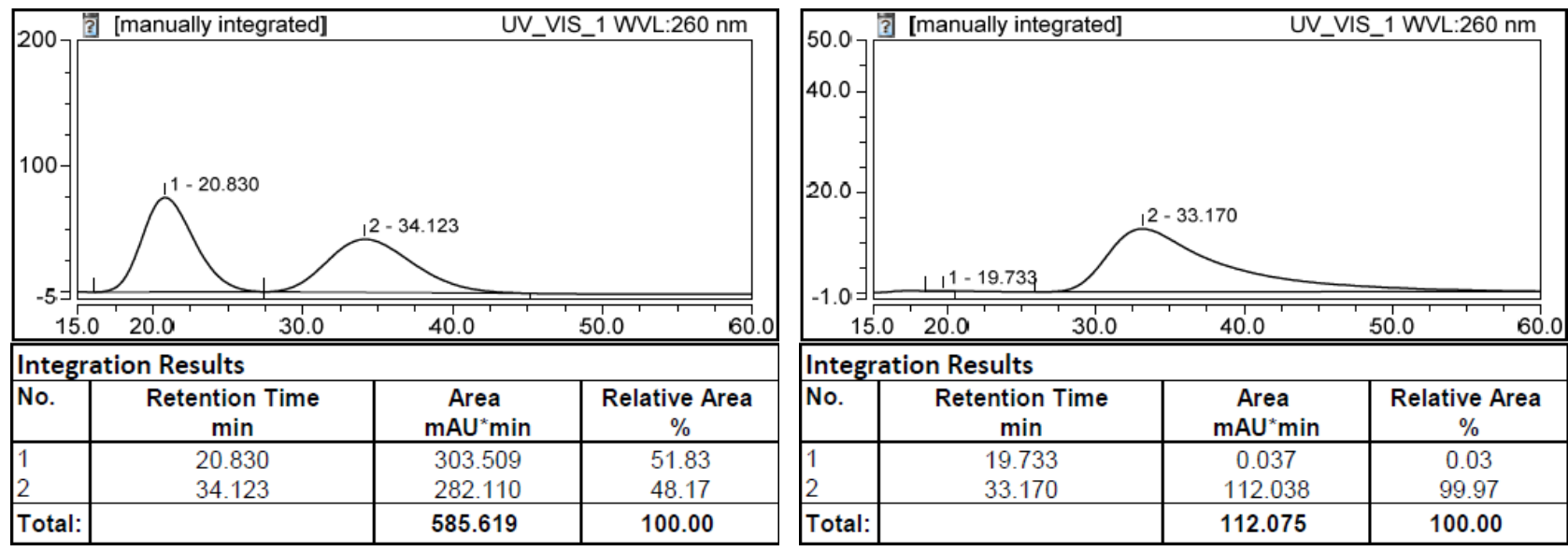

NOTE: Because of the amide bond rotation equilibrium, the rotamers of 4 were observed on the NMR. This phenomenon is seen with many tertiary amides. For related references, see: ref. 19-20.

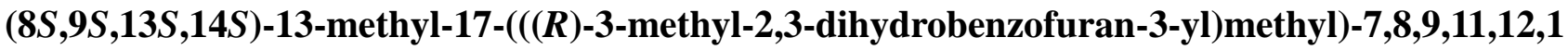
3,14,15-octahydro-6H-cyclopenta $[a]$ phenanthren-3-yl acetate (7)

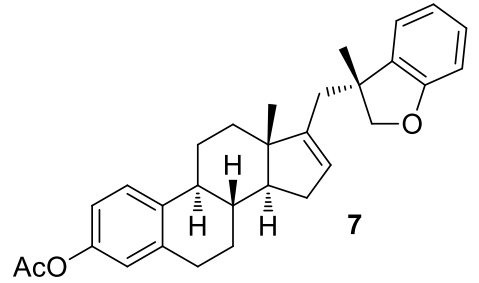

The compound was prepared according to the General Procedure from the reaction of $1 \mathbf{a}(98.6 \mathrm{mg}, 0.36 \mathrm{mmol})$ and $\mathbf{2 z}(88.8 \mathrm{mg}, 0.2 \mathrm{mmol})$. $75.1 \mathrm{mg}, 85 \%$ yield, $99 \%$ de, white solid, mp: $123-125^{\circ} \mathrm{C}$. 
Chiral HPLC: CHIRALPAK IA, $25{ }^{\circ} \mathrm{C},{ }^{i} \mathrm{PrOH}-$ hexanes 5/95, $1 \mathrm{~mL} / \mathrm{min}, 288 \mathrm{~nm}, t_{\mathrm{R}}$ (major) $=6.4$ $\min , t_{\mathrm{R}}($ minor $)=7.8 \min$.

$[\boldsymbol{\alpha}]_{\mathbf{D}}{ }^{20}=-61\left(\mathrm{c}=1.36, \mathrm{CH}_{2} \mathrm{Cl}_{2}\right)$.

${ }^{1}$ H NMR (400 MHz, CDCl 3 ): $\delta$ 7.26-7.23 (m, 1 H), 7.15-7.09 (m, 2 H), 6.90-6.78 (m, 4 H), 5.16 (d, $J=1.2 \mathrm{~Hz}, 1 \mathrm{H}), 4.53(\mathrm{~d}, J=8.4 \mathrm{~Hz}, 1 \mathrm{H}), 4.27(\mathrm{~d}, J=8.4 \mathrm{~Hz}, 1 \mathrm{H}), 2.90-2.86(\mathrm{~m}, 2 \mathrm{H}), 2.46-2.11$ (m, 8 H), 1.96-1.88 (m, 2 H), 1.76-11.73 (m, $1 \mathrm{H}), 1.62-1.33$ (m, $8 \mathrm{H}), 1.33$ (s, $3 \mathrm{H})$.

${ }^{13}$ C NMR (100 MHz, $\left.\mathbf{C D C l}_{3}\right): \delta 169.8,159.0,150.8,148.3,138.2,138.2,136.2,128.0,126.0,123.4$, $122.6,121.4,120.4,118.4,109.5,81.9,55.3,47.5,44.5,44.4,37.2$, 37.0, 34.4, 31.3, 29.4, 27.5, 26.9, $26.2,21.1,15.4$.

IR (neat, $\mathbf{c m}^{-\mathbf{1}}$ ): 2930, 2851, 1765, 1597, 1482, 1370, 1207, 1016, 974, 751.

HRMS (ESI): $[\mathrm{M}+\mathrm{H}]^{+}$calcd for $\mathrm{C}_{30} \mathrm{H}_{35} \mathrm{O}_{3} 443.2581$, found 443.2587.

\begin{tabular}{|l|c|c|c|}
\hline & \\
\end{tabular}

\begin{tabular}{|l|c|c|c|}
\hline & \multicolumn{3}{c|}{ EXT288NM WVL:288 nm } \\
\\
\end{tabular}

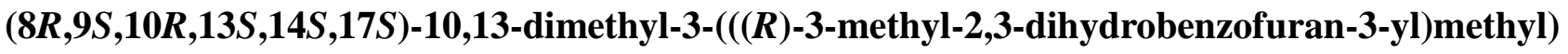
$-2,7,8,9,10,11,12,13,14,15,16,17-d o d e c a h y d r o-1 H$-cyclopenta[a]phenanthren-17-yl acetate $(8)$

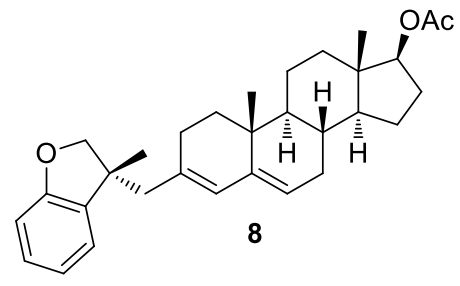

The compound was prepared according to the General Procedure from the reaction of $\mathbf{1 a}(98.6 \mathrm{mg}, 0.36 \mathrm{mmol})$ and $\mathbf{2 a a}(92.4 \mathrm{mg}, 0.2 \mathrm{mmol})$. $49.7 \mathrm{mg}, 54 \%$ yield, $94 \%$ de, white solid, mp: $47-49^{\circ} \mathrm{C}$.

Chiral HPLC: CHIRALPAK IA, $25{ }^{\circ} \mathrm{C}$, ${ }^{i} \mathrm{PrOH}-h$ exanes 5/95, 1 $\mathrm{mL} / \min , 280 \mathrm{~nm}, t_{\mathrm{R}}($ major $)=4.8 \mathrm{~min}, t_{\mathrm{R}}($ minor $)=8.2 \mathrm{~min}$.

$[\boldsymbol{\alpha}]_{\mathbf{D}}{ }^{21}=-73\left(\mathrm{c}=1.0, \mathrm{CH}_{2} \mathrm{Cl}_{2}\right)$.

${ }^{1}$ H NMR (400 MHz, CDCl 3 ): $\delta$ 7.14-7.09 (m, $\left.2 \mathrm{H}\right), 6.86(\mathrm{t}, J=7.2 \mathrm{~Hz}, 1 \mathrm{H}), 6.75(\mathrm{~d}, J=8.0 \mathrm{~Hz}, 1$ H), $5.75(\mathrm{~s}, 1 \mathrm{H}), 5.34(\mathrm{~d}, J=2.8 \mathrm{~Hz}, 1 \mathrm{H}), 4.62-4.58(\mathrm{~m}, 1 \mathrm{H}), 4.49(\mathrm{~d}, J=8.8 \mathrm{~Hz}, 1 \mathrm{H}), 4.16(\mathrm{~d}, J=$ $8.4 \mathrm{~Hz}, 1 \mathrm{H}), 2.38-2.33$ (m, $2 \mathrm{H}), 2.20-2.14$ (m, $2 \mathrm{H}), 2.04(\mathrm{~s}, 3 \mathrm{H}), 1.77-0.94(\mathrm{~m}, 18 \mathrm{H}), 0.85$ (s, 3 $\mathrm{H}), 0.82(\mathrm{~s}, 3 \mathrm{H})$. 
${ }^{13}$ C NMR (100 MHz, CDCl $)$ ): $\delta$ 171.2, 159.4, 141.7, 135.4, 133.5, 128.5, 128.0, 123.0, 121.9, 120.3, $109.5,82.7,82.1,51.2,49.2,48.2,45.8,42.5,36.7,34.7,34.2,31.6,31.3,27.9,27.5,26.4,23.5$, $21.2,20.5,18.7,12.0$.

IR (neat, $\mathbf{c m}^{-1}$ ): 2963, 1735, 1481, 1459, 1373, 1247, 1034, 978, 751, 689.

HRMS (ESI): $[\mathrm{M}+\mathrm{H}]{ }^{+}$calcd for $\mathrm{C}_{31} \mathrm{H}_{41} \mathrm{O}_{3} 461.3050$, found 461.3068 .
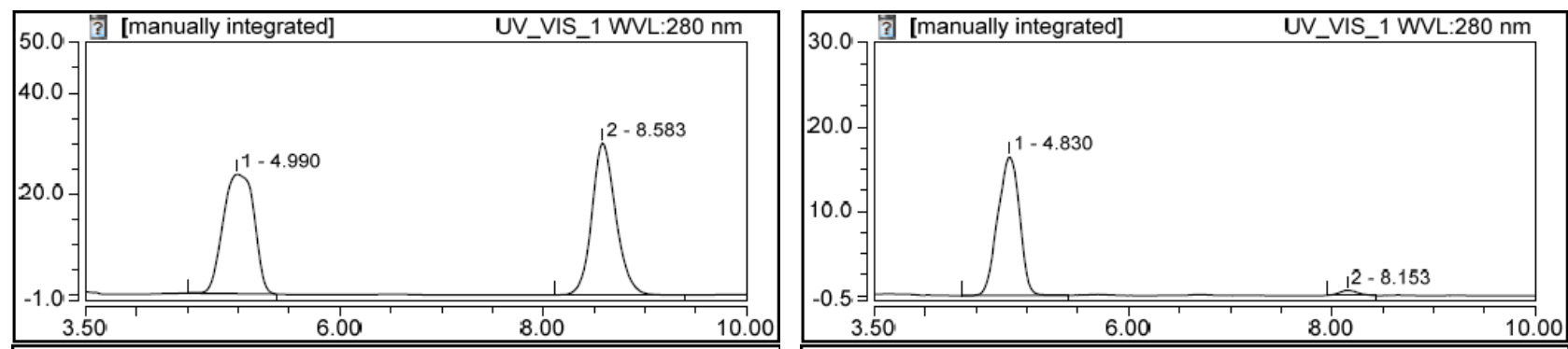

\begin{tabular}{|l|c|c|c|}
\hline \hline \multicolumn{3}{|l|}{ Integration Results } \\
\hline No. & $\begin{array}{c}\text { Retention Time } \\
\text { min }\end{array}$ & $\begin{array}{c}\text { Area } \\
\text { mAU*min }^{\star}\end{array}$ & $\begin{array}{c}\text { Relative Area } \\
\%\end{array}$ \\
\hline 1 & 4.990 & 8.353 & 50.48 \\
2 & 8.583 & 8.195 & 49.52 \\
\hline Total: & & 16.548 & 100.00 \\
\hline
\end{tabular}

\begin{tabular}{|l|c|c|c|}
\hline \hline \multicolumn{3}{|l|}{ Integration Results } \\
\hline No. & $\begin{array}{c}\text { Retention Time } \\
\text { min }\end{array}$ & $\begin{array}{c}\text { Area } \\
\text { mAU*min }\end{array}$ & $\begin{array}{c}\text { Relative Area } \\
\%\end{array}$ \\
\hline 1 & 4.830 & 4.074 & 97.15 \\
2 & 8.153 & 0.120 & 2.85 \\
\hline Total: & & 4.193 & 100.00 \\
\hline
\end{tabular}




\section{Mechanistic Investigation}

\subsection{Study of the Reaction of Alkenyl Triflate with Alkene}

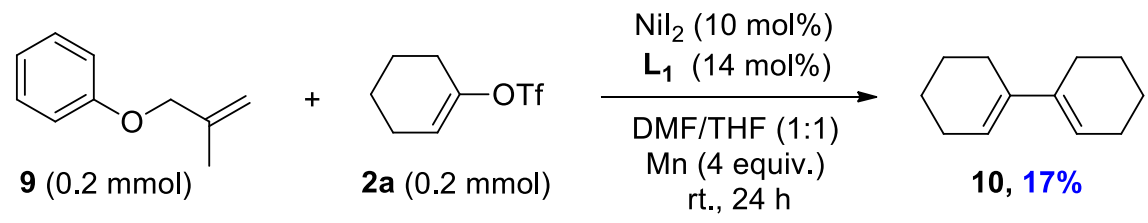

The procedure was conducted in an argon-filled glove box. To a reaction tube equipped with a magnetic stir bar was charged with $\mathrm{NiI}_{2}(6.3 \mathrm{mg}, 0.020 \mathrm{mmol}), \mathbf{L 1}(7.6 \mathrm{mg}, 0.028 \mathrm{mmol}), \mathrm{Mn}(44.0$ $\mathrm{mg}, 0.8 \mathrm{mmol})$, and $\mathrm{DMF} / \mathrm{THF}(0.5 \mathrm{~mL} / 0.5 \mathrm{~mL})$. The reaction mixture was stirred for $5 \mathrm{~min}$. Substrates 9 (29.6 mg, $0.2 \mathrm{mmol})$ and $2 \mathbf{a}(46 \mathrm{mg}, 0.2 \mathrm{mmol})$ were then added. The reaction tube was sealed with a rubber septum, and removed from the glove box. The reaction mixture was stirred at $25{ }^{\circ} \mathrm{C}$ for $24 \mathrm{~h}$. The reaction mixture was diluted with ethyl acetate $(10 \mathrm{~mL})$, washed with water, brine, and dried over anhydrous $\mathrm{Na}_{2} \mathrm{SO}_{4}$. A $0.2 \mathrm{~mL}$ of solution was collected, diluted with ethyl acetate $(2 \mathrm{~mL})$, and analyzed by GC.

The reaction afforded alkenyl dimer $\mathbf{1 0}$ with $17 \%$ yield and trace of protonated product alkenyl-H. No cross product was observed, and substrate 9 remained intact.

\subsection{The reactivity of alkene tethered Ar-I and alkenyl-OTf towards $\mathrm{Ni}(0)$}

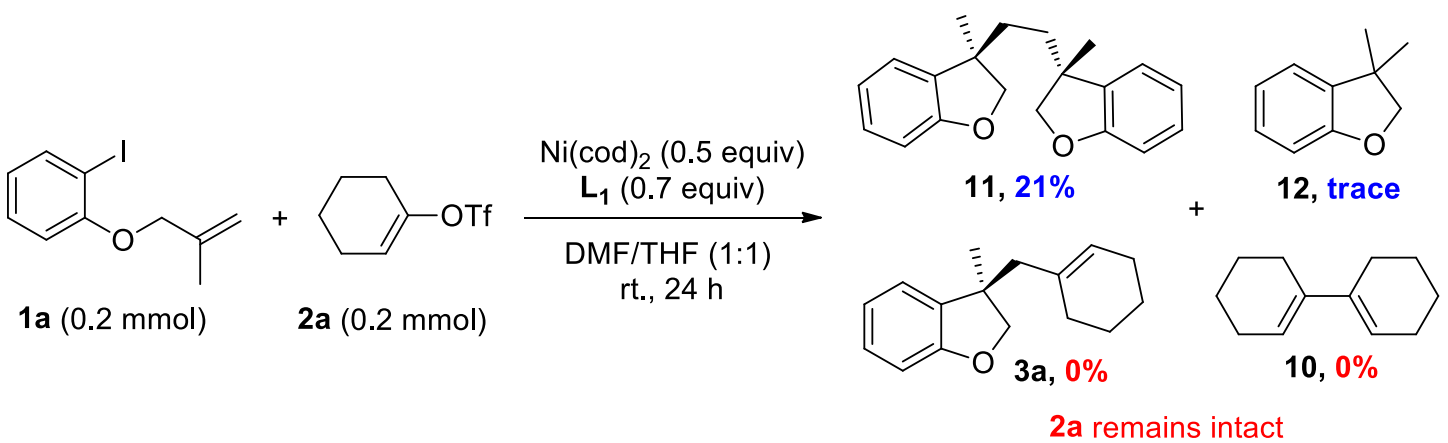

The procedure was conducted in an argon-filled glove box. To a reaction tube equipped with a magnetic stir bar was charged with $\mathrm{Ni}(\mathrm{cod})_{2}(27.4 \mathrm{mg}, 0.10 \mathrm{mmol}), \mathbf{L 1}(38.2 \mathrm{mg}, 0.14 \mathrm{mmol})$, and $\mathrm{DMF} / \mathrm{THF}(0.5 \mathrm{~mL} / 0.5 \mathrm{~mL})$. The reaction mixture was stirred for $5 \mathrm{~min}$. Substrates $1 \mathrm{a}(54.8 \mathrm{mg}, 0.2$ mmol) and $2 \mathbf{a}(46 \mathrm{mg}, 0.2 \mathrm{mmol})$ were then added. The reaction tube was sealed with a rubber septum, and removed from the glove box. The reaction mixture was stirred at $25{ }^{\circ} \mathrm{C}$ for $24 \mathrm{~h}$. The reaction mixture was diluted with ethyl acetate $(10 \mathrm{~mL})$, washed with water, brine, and dried over anhydrous $\mathrm{Na}_{2} \mathrm{SO}_{4}$. A $0.2 \mathrm{~mL}$ of solution was collected, diluted with ethyl acetate $(2 \mathrm{~mL})$, and 
analyzed by GC.

The reaction afforded dimer 11 with $21 \%$ yield, and trace of protonated product $\mathbf{1 2}$. Alkenyl triflate 2a remained intact.

\section{1,2-Bis((R)-3-methyl-2,3-dihydrobenzofuran-3-yl)ethane (11, $\left.\operatorname{known}^{39}\right)$}<smiles>C[C@]1(CC[C@@]2(C)COc3ccccc32)COc2ccccc21</smiles>

11

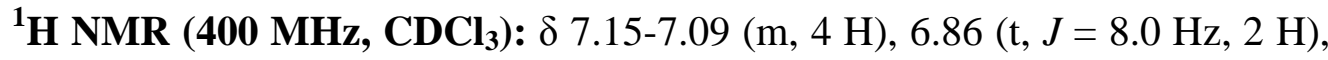
$6.75(\mathrm{~d}, J=8.0 \mathrm{~Hz}, 2 \mathrm{H}), 4.59(\mathrm{~d}, J=8.0 \mathrm{~Hz}, 2 \mathrm{H}), 4.05(\mathrm{~d}, J=8.0 \mathrm{~Hz}, 2 \mathrm{H})$, $1.84(\mathrm{~s}, 4 \mathrm{H}), 1.35(\mathrm{~s}, 6 \mathrm{H})$.

${ }^{13}$ C NMR (100 MHz, $\left.\mathbf{C D C l}_{3}\right): \delta$ 159.5, 135.4, 128.0, 126.5, 120.1, 109.6, 82.2, 46.8, 39.2, 25.0.

\subsection{Enantioselectivity of the Formation of Cross-product 3x and Protonated Byproduct 13}

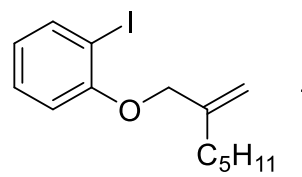

1n $(0.2 \mathrm{mmol})$<smiles>[Te]OC1=CCCCCCC1</smiles>

$2 \mathbf{e}(0.2 \mathrm{mmol})$

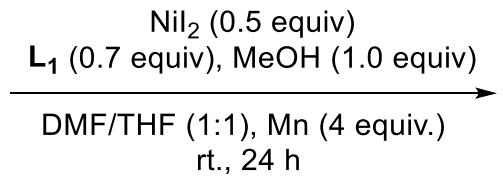

rt., $24 \mathrm{~h}$

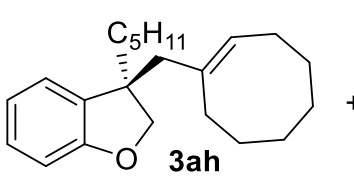

$36 \%$ yield $93 \%$ ee

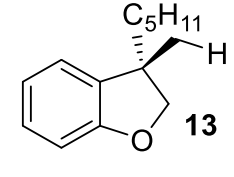

$32 \%$ yield $93 \%$ ee

The procedure was conducted in an argon-filled glove box. To a reaction tube equipped with a magnetic stir bar was charged with $\mathrm{NiI}_{2}(31.5 \mathrm{mg}, 0.10 \mathrm{mmol}), \mathbf{L 1}(38.2 \mathrm{mg}, 0.14 \mathrm{mmol}), \mathrm{Mn}(44.0$ $\mathrm{mg}, 4.0$ equiv.), and DMF/THF $(0.5 \mathrm{~mL} / 0.5 \mathrm{~mL})$. The reaction mixture was stirred for $5 \mathrm{~min}$. Substrates 1n (66.0 mg, $0.2 \mathrm{mmol}), \mathbf{2 e}(51.6 \mathrm{mg}, 0.2 \mathrm{mmol})$ and $\mathrm{MeOH}(6.4 \mathrm{mg}, 0.2 \mathrm{mmol})$ were then added. The reaction tube was sealed with a rubber septum, and removed from the glove box. The reaction mixture was stirred at $25{ }^{\circ} \mathrm{C}$ for $24 \mathrm{~h}$. The reaction mixture was diluted with ethyl acetate $(10 \mathrm{~mL})$, washed with water, brine, dried over anhydrous $\mathrm{Na}_{2} \mathrm{SO}_{4}$, and concentrated under reduced pressure. The residue was purified by flash chromatography on silica gel to afford 3ah (22.4 mg, 36\% yield, 93\% ee) and $\mathbf{1 3}$ (13.9 mg, 32\% yield, 93\% ee).

\section{(S)-3-methyl-3-pentyl-2,3-dihydrobenzofuran (13, known)}

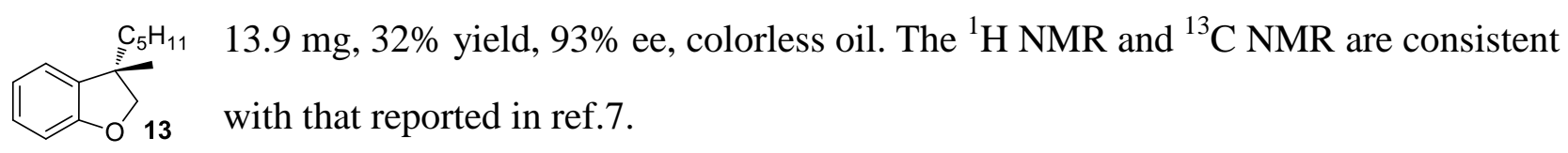

Chiral HPLC: CHIRALPAK IB, $25{ }^{\circ} \mathrm{C},{ }^{i} \mathrm{PrOH}$-hexanes $0.2 / 99.8,0.75 \mathrm{~mL} / \mathrm{min}, 280 \mathrm{~nm}, t_{\mathrm{R}}$ (major)= $7.6 \mathrm{~min}, t_{\mathrm{R}}($ minor $)=8.3 \mathrm{~min}$.

$[\boldsymbol{\alpha}]_{\mathbf{D}}{ }^{22}=+2\left(\mathrm{c}=0.5, \mathrm{CH}_{2} \mathrm{Cl}_{2}\right)$.

${ }^{1}$ H NMR (400 MHz, CDCl $\left._{3}\right): \delta$ 7.14-7.10 (m, $\left.1 \mathrm{H}\right)$, 7.08-7.06 (m, $\left.1 \mathrm{H}\right), 6.87(\mathrm{dt}, J=0.8,7.2 \mathrm{~Hz}, 1$ 
H), $6.78(\mathrm{~d}, J=8.0 \mathrm{~Hz}, 1 \mathrm{H}), 4.35(\mathrm{~d}, J=8.4 \mathrm{~Hz}, 1 \mathrm{H}), 4.15(\mathrm{~d}, J=8.4 \mathrm{~Hz}, 1 \mathrm{H}), 1.62-1.20(\mathrm{~m}, 8 \mathrm{H})$, $1.33(\mathrm{~s}, 3 \mathrm{H}), 0.85(\mathrm{t}, J=7.2 \mathrm{~Hz}, 3 \mathrm{H})$.

${ }^{13}$ C NMR (100 MHz, $\left.\mathbf{C D C l}_{3}\right): \delta 159.5,135.5,127.9,122.8,120.4,109.5,82.5,45.2,40.9,32.3$, $25.6,24.3,22.5,14.0$.
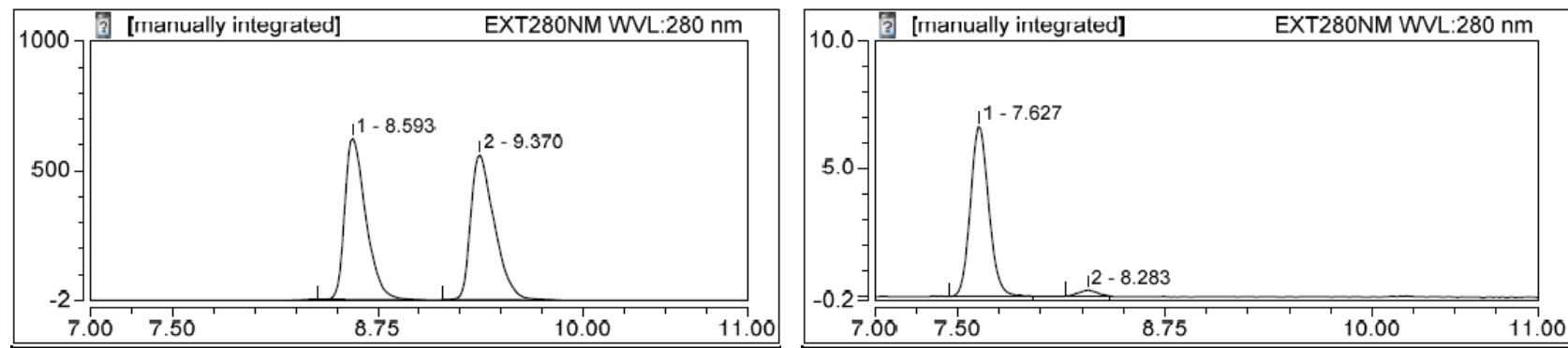

\begin{tabular}{|l|c|c|c|}
\hline \hline \multicolumn{3}{|l|}{ Integration Results } \\
\hline No. & $\begin{array}{c}\text { Retention Time } \\
\text { min }\end{array}$ & $\begin{array}{c}\text { Area } \\
\text { mAU*min }\end{array}$ & $\begin{array}{c}\text { Relative Area } \\
\%\end{array}$ \\
\hline 1 & 8.593 & 92.645 & 49.82 \\
2 & 9.370 & 93.310 & 50.18 \\
\hline Total: & & $\mathbf{1 8 5 . 9 5 5}$ & $\mathbf{1 0 0 . 0 0}$ \\
\hline
\end{tabular}

\begin{tabular}{|l|c|c|c|}
\hline \hline \multicolumn{3}{|l|}{ Integration Results } \\
\hline No. & $\begin{array}{c}\text { Retention Time } \\
\text { min }\end{array}$ & $\begin{array}{c}\text { Area } \\
\text { mAU*min }\end{array}$ & $\begin{array}{c}\text { Relative Area } \\
\%\end{array}$ \\
\hline 1 & 7.627 & 0.865 & 96.64 \\
2 & 8.283 & 0.030 & 3.36 \\
\hline Total: & & $\mathbf{0 . 8 9 5}$ & $\mathbf{1 0 0 . 0 0}$ \\
\hline
\end{tabular}




\section{Crystallographic Data for Compound 3z (CCDC 1890459)}

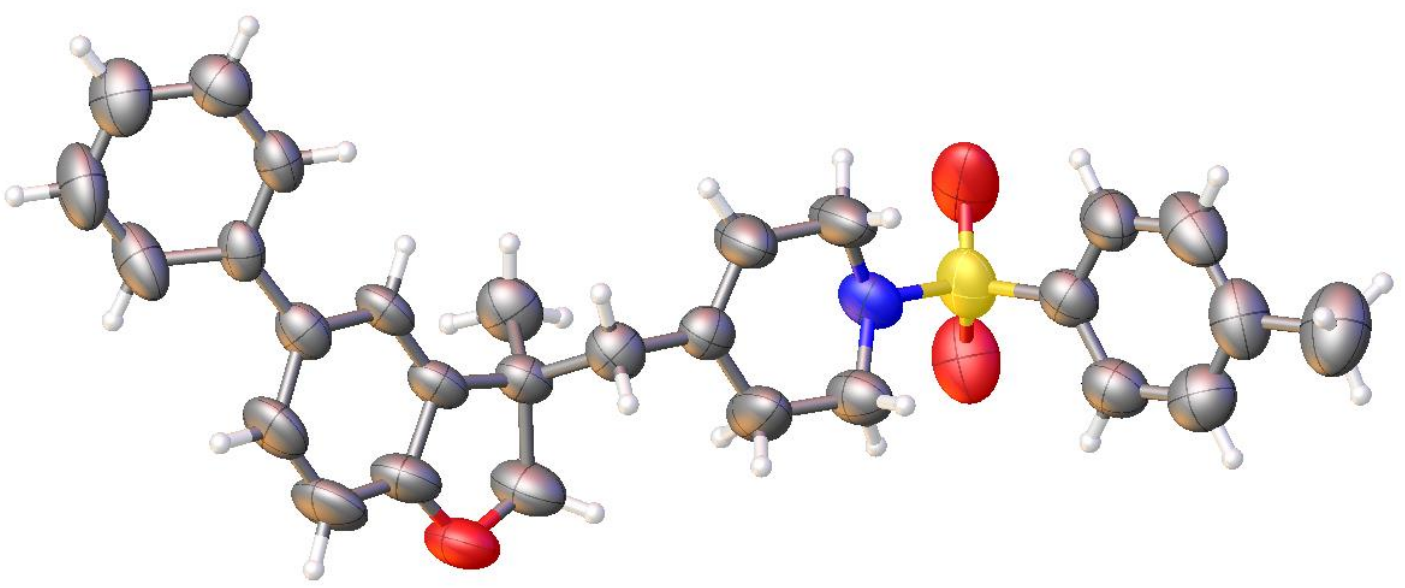

tianzhx_1015

Table 1 Crystal data and structure refinement for tianzhx_1015.

Identification code

Empirical formula

Formula weight

Temperature/K

Crystal system

Space group

$\mathrm{a} / \AA$

$\mathrm{b} / \AA$

$\mathrm{c} / \AA$

$\alpha /^{\circ}$

$\beta /^{\circ}$

$\gamma /{ }^{\circ}$

Volume $/ \AA^{3}$

Z

$\rho_{\text {calc }} \mathrm{g} / \mathrm{cm}^{3}$

$\mu / \mathrm{mm}^{-1}$

$\mathrm{F}(000)$

Crystal size $/ \mathrm{mm}^{3}$

Radiation tianzhx_1015

$\mathrm{C}_{28} \mathrm{H}_{29} \mathrm{NO}_{3} \mathrm{~S}$

460.59

295.6(2)

orthorhombic

$\mathrm{P} 2{ }_{1} 2_{1} 2_{1}$

$8.5255(6)$

10.9187(9)

26.122(3)

90.00

90.00

90.00

2431.7(4)

4

1.258

1.413

980.0

$0.21 \times 0.15 \times 0.14$

$\mathrm{CuK} \alpha(\lambda=1.54184)$ 
$2 \Theta$ range for data collection $/{ }^{\circ} 8.78$ to 133.18

Index ranges

$-6 \leq \mathrm{h} \leq 10,-12 \leq \mathrm{k} \leq 12,-31 \leq 1 \leq 29$

Reflections collected

7508

Independent reflections

$4101\left[\mathrm{R}_{\text {int }}=0.0426, \mathrm{R}_{\text {sigma }}=0.0706\right]$

Data/restraints/parameters

$4101 / 0 / 300$

Goodness-of-fit on $\mathrm{F}^{2}$

1.121

Final $R$ indexes $[\mathrm{I}>=2 \sigma(\mathrm{I})] \quad \mathrm{R}_{1}=0.0730, \mathrm{wR}_{2}=0.1894$

Final $\mathrm{R}$ indexes [all data] $\quad \mathrm{R}_{1}=0.1101, \mathrm{w}_{2}=0.2606$

Largest diff. peak/hole / e $\AA^{-3} 0.22 /-0.57$

Flack parameter

$-0.07(5)$

Table 2 Fractional Atomic Coordinates $\left(\times 10^{4}\right)$ and Equivalent Isotropic Displacement Parameters $\left(\AA^{2} \times 10^{3}\right)$ for tianzhx_1015. $U_{\text {eq }}$ is defined as $1 / 3$ of of the trace of the orthogonalised $\mathrm{U}_{\mathrm{IJ}}$ tensor.

\begin{tabular}{lrrrr} 
Atom & \multicolumn{2}{c}{$\boldsymbol{y}$} & \multicolumn{2}{c}{ U(eq) } \\
S1 & $-7953.1(18)$ & $-5233.5(19)$ & $-1431.7(7)$ & $83.3(5)$ \\
O1 & $-16535(6)$ & $-5867(5)$ & $-2890(2)$ & $93.8(16)$ \\
O2 & $-6624(5)$ & $-4766(8)$ & $-1707(2)$ & $124(2)$ \\
O3 & $-8104(7)$ & $-6521(5)$ & $-1341(2)$ & $104.8(18)$ \\
N1 & $-9511(5)$ & $-4815(5)$ & $-1751(2)$ & $74.0(14)$ \\
C1 & $-1118(5)$ & $-4366(2)$ & $64.1(14)$ \\
C2 & $-15865(6)$ & $-320(6)$ & $-4773(3)$ & $76.9(17)$ \\
C3 & $-15793(7)$ & $-510(7)$ & $-5196(3)$ & $92(2)$ \\
C4 & $-16732(9)$ & $-1480(7)$ & $-5193(3)$ & $100(3)$ \\
C5 & $-17749(10)$ & $-2271(6)$ & $-4788(3)$ & $90(2)$ \\
C6 & $-17808(8)$ & $-2107(5)$ & $-4358(2)$ & $57.5(12)$ \\
C7 & $-16866(6)$ & $-3006(5)$ & $-3934(2)$ & $61.7(13)$ \\
C8 & $-16885(6)$ & $-3694(6)$ & $-3825(3)$ & $83(2)$ \\
C9 & $-18210(6)$ & $-4643(7)$ & $-3454(3)$ & $96(2)$ \\
C10 & $-18188(7)$ & 571 & $-3234(3)$ & $78.0(18)$ \\
& $-16782(7)$ & &
\end{tabular}




\begin{tabular}{|c|c|c|c|c|}
\hline $\mathrm{C} 11$ & $-15439(6)$ & $-4256(5)$ & $-3319(2)$ & $60.5(14)$ \\
\hline $\mathrm{C} 12$ & $-15497(5)$ & $-3300(5)$ & $-3677(2)$ & $62.1(14)$ \\
\hline $\mathrm{C} 13$ & $-14100(6)$ & $-4756(5)$ & $-3016(2)$ & $60.2(13)$ \\
\hline $\mathrm{C} 14$ & $-14910(8)$ & $-5917(6)$ & $-2782(3)$ & $90(2)$ \\
\hline C15 & $-12688(7)$ & $-5088(6)$ & $-3341(3)$ & $80.5(18)$ \\
\hline C16 & $-13666(7)$ & $-3817(5)$ & $-2590(2)$ & $67.4(15)$ \\
\hline C17 & $-12237(7)$ & $-4108(5)$ & $-2283(2)$ & $60.4(13)$ \\
\hline C18 & $-10875(8)$ & $-3444(6)$ & $-2329(3)$ & $85(2)$ \\
\hline C19 & $-9535(8)$ & $-3563(7)$ & $-1979(3)$ & $97(3)$ \\
\hline $\mathrm{C} 20$ & $-11012(7)$ & $-5163(6)$ & $-1526(3)$ & $78.9(18)$ \\
\hline $\mathrm{C} 21$ & $-12284(8)$ & $-5084(9)$ & $-1905(3)$ & $100(3)$ \\
\hline $\mathrm{C} 22$ & $-7990(7)$ & $-4483(5)$ & $-846(2)$ & $67.2(15)$ \\
\hline $\mathrm{C} 23$ & $-7293(8)$ & $-3357(6)$ & $-784(3)$ & 83.2(19) \\
\hline $\mathrm{C} 24$ & $-7248(10)$ & $-2772(6)$ & $-318(4)$ & $99(3)$ \\
\hline $\mathrm{C} 25$ & $-7870(10)$ & $-3356(7)$ & $108(3)$ & $93(2)$ \\
\hline $\mathrm{C} 26$ & $-8583(9)$ & $-4458(7)$ & $65(3)$ & $90(2)$ \\
\hline $\mathrm{C} 27$ & $-8636(7)$ & $-5048(6)$ & $-417(3)$ & $79.9(18)$ \\
\hline $\mathrm{C} 28$ & $-7765(14)$ & $-2741(8)$ & $635(4)$ & $131(4)$ \\
\hline
\end{tabular}

Table 3 Anisotropic Displacement Parameters $\left(\AA^{2} \times 10^{3}\right)$ for tianzhx_1015. The Anisotropic displacement factor exponent takes the form: $-2 \pi^{2}\left[h^{2} a *{ }^{2} U_{11}+2 h k a * b * U_{12}+\ldots\right]$.

\begin{tabular}{lrrrrrr} 
Atom & \multicolumn{1}{c}{$\mathbf{U}_{\mathbf{1 1}}$} & \multicolumn{1}{c}{$\mathbf{U}_{\mathbf{2 2}}$} & \multicolumn{1}{c}{$\mathbf{U}_{\mathbf{3 3}}$} & \multicolumn{1}{c}{$\mathbf{U}_{\mathbf{2 3}}$} & $\mathbf{U}_{\mathbf{1 3}}$ & \multicolumn{1}{c}{$\mathbf{U}_{\mathbf{1 2}}$} \\
$\mathrm{S} 1$ & $51.2(7)$ & $111.1(13)$ & $87.7(11)$ & $-5.9(10)$ & $-7.5(7)$ & $15.6(8)$ \\
$\mathrm{O} 1$ & $68(3)$ & $88(3)$ & $125(4)$ & $18(3)$ & $5(3)$ & $-25(2)$ \\
$\mathrm{O} 2$ & $47(2)$ & $229(7)$ & $97(4)$ & $-7(5)$ & $6(2)$ & $12(4)$ \\
$\mathrm{O} 3$ & $101(4)$ & $91(3)$ & $122(4)$ & $-17(3)$ & $-26(3)$ & $40(3)$ \\
$\mathrm{N} 1$ & $45(2)$ & $82(3)$ & $95(4)$ & $0(3)$ & $2(2)$ & $0(2)$ \\
$\mathrm{C} 1$ & $50(3)$ & $74(3)$ & $68(3)$ & $-12(3)$ & $-13(2)$ & $4(2)$
\end{tabular}




\begin{tabular}{|c|c|c|c|c|c|c|}
\hline $\mathrm{C} 2$ & $63(3)$ & $72(4)$ & $96(5)$ & $1(4)$ & $-6(3)$ & $6(3)$ \\
\hline C3 & $89(5)$ & $85(4)$ & $103(5)$ & $-2(4)$ & $-23(4)$ & $27(4)$ \\
\hline $\mathrm{C} 4$ & $106(6)$ & $95(5)$ & $101(6)$ & $-12(4)$ & $-55(5)$ & $19(4)$ \\
\hline C5 & $78(4)$ & $74(4)$ & $118(6)$ & $-16(4)$ & $-45(4)$ & $6(3)$ \\
\hline C6 & $41(2)$ & $71(3)$ & $61(3)$ & $-8(3)$ & $-10(2)$ & $13(2)$ \\
\hline C7 & $41(2)$ & $66(3)$ & 79(4) & $-9(3)$ & $-3(2)$ & $4(2)$ \\
\hline $\mathrm{C} 8$ & $37(3)$ & $94(4)$ & $118(6)$ & $-4(4)$ & $-13(3)$ & $-5(3)$ \\
\hline C9 & $42(3)$ & $105(5)$ & $141(7)$ & $18(5)$ & 1(3) & $-22(3)$ \\
\hline $\mathrm{C} 10$ & $51(3)$ & $72(4)$ & $111(5)$ & $7(4)$ & $9(3)$ & $-14(3)$ \\
\hline C11 & $36(2)$ & $62(3)$ & $83(4)$ & $-5(3)$ & $6(2)$ & $-7(2)$ \\
\hline C12 & $32(2)$ & $65(3)$ & $89(4)$ & $-7(3)$ & $-2(2)$ & $-8(2)$ \\
\hline C13 & $47(2)$ & $62(3)$ & $72(3)$ & $-1(3)$ & $4(2)$ & $-7(2)$ \\
\hline C14 & $68(4)$ & $66(4)$ & $134(7)$ & $13(4)$ & $-2(4)$ & $-11(3)$ \\
\hline C15 & $58(3)$ & $88(4)$ & $95(5)$ & $-10(4)$ & $5(3)$ & $12(3)$ \\
\hline C16 & $58(3)$ & $68(3)$ & $77(4)$ & $-5(3)$ & $2(3)$ & $8(2)$ \\
\hline C17 & $58(3)$ & $59(3)$ & 64(3) & $-3(3)$ & $4(3)$ & $1(2)$ \\
\hline C18 & $72(4)$ & $81(4)$ & $102(5)$ & $24(4)$ & $-17(4)$ & $-21(3)$ \\
\hline C19 & $67(4)$ & $105(5)$ & $120(6)$ & $42(5)$ & $-20(4)$ & $-30(4)$ \\
\hline $\mathrm{C} 20$ & $53(3)$ & $82(4)$ & $102(5)$ & $18(4)$ & $-2(3)$ & $-2(3)$ \\
\hline $\mathrm{C} 21$ & $55(3)$ & $148(7)$ & $97(5)$ & $38(5)$ & $-5(3)$ & $-20(4)$ \\
\hline $\mathrm{C} 22$ & $50(3)$ & $70(3)$ & $81(4)$ & $-1(3)$ & $-1(3)$ & $4(3)$ \\
\hline $\mathrm{C} 23$ & $83(4)$ & $79(4)$ & $88(5)$ & $12(4)$ & $-21(4)$ & $-10(3)$ \\
\hline $\mathrm{C} 24$ & $97(5)$ & $64(4)$ & $138(7)$ & 2(4) & $-38(5)$ & $2(4)$ \\
\hline $\mathrm{C} 25$ & $95(5)$ & $81(5)$ & $101(5)$ & $-21(4)$ & $-23(5)$ & $23(4)$ \\
\hline $\mathrm{C} 26$ & $84(4)$ & $87(5)$ & $99(5)$ & $10(4)$ & $-1(4)$ & $13(4)$ \\
\hline $\mathrm{C} 27$ & $63(3)$ & $75(4)$ & $102(5)$ & $3(4)$ & $0(3)$ & $-1(3)$ \\
\hline C28 & $162(10)$ & $113(7)$ & $118(7)$ & $4(6)$ & $-35(7)$ & $32(7)$ \\
\hline
\end{tabular}


Table 4 Bond Lengths for tianzhx_1015.

\begin{tabular}{|c|c|c|c|c|c|}
\hline \multicolumn{2}{|c|}{ Atom Atom } & \multirow{2}{*}{$\begin{array}{r}\text { Length/A } \\
1.436(6)\end{array}$} & \multicolumn{2}{|c|}{ Atom Atom } & \multirow{2}{*}{$\begin{array}{r}\text { Length/Å } \\
1.371(7)\end{array}$} \\
\hline $\mathrm{S} 1$ & $\mathrm{O} 2$ & & $\mathrm{C} 10$ & C11 & \\
\hline $\mathrm{S} 1$ & $\mathrm{O} 3$ & $1.431(6)$ & $\mathrm{C} 11$ & $\mathrm{C} 12$ & $1.401(8)$ \\
\hline $\mathrm{S} 1$ & N1 & $1.633(5)$ & $\mathrm{C} 11$ & $\mathrm{C} 13$ & $1.494(8)$ \\
\hline S1 & $\mathrm{C} 22$ & $1.735(6)$ & $\mathrm{C} 13$ & $\mathrm{C} 14$ & $1.566(8)$ \\
\hline $\mathrm{O} 1$ & $\mathrm{C} 10$ & $1.390(8)$ & C13 & $\mathrm{C} 15$ & $1.516(7)$ \\
\hline $\mathrm{O} 1$ & C14 & $1.415(8)$ & C13 & C16 & $1.557(8)$ \\
\hline N1 & C19 & $1.492(8)$ & $\mathrm{C} 16$ & $\mathrm{C} 17$ & $1.493(8)$ \\
\hline N1 & $\mathrm{C} 20$ & $1.458(7)$ & $\mathrm{C} 17$ & $\mathrm{C} 18$ & $1.374(8)$ \\
\hline $\mathrm{C} 1$ & $\mathrm{C} 2$ & $1.376(9)$ & $\mathrm{C} 17$ & $\mathrm{C} 21$ & $1.452(9)$ \\
\hline $\mathrm{C} 1$ & C6 & $1.377(8)$ & $\mathrm{C} 18$ & C19 & $1.468(9)$ \\
\hline $\mathrm{C} 2$ & $\mathrm{C} 3$ & $1.381(10)$ & $\mathrm{C} 20$ & $\mathrm{C} 21$ & $1.471(9)$ \\
\hline $\mathrm{C} 3$ & $\mathrm{C} 4$ & $1.369(11)$ & $\mathrm{C} 22$ & $\mathrm{C} 23$ & $1.376(9)$ \\
\hline $\mathrm{C} 4$ & C5 & $1.367(11)$ & $\mathrm{C} 22$ & $\mathrm{C} 27$ & $1.394(9)$ \\
\hline C5 & C6 & $1.392(8)$ & $\mathrm{C} 23$ & $\mathrm{C} 24$ & $1.376(10)$ \\
\hline C6 & $\mathrm{C} 7$ & $1.480(8)$ & $\mathrm{C} 24$ & $\mathrm{C} 25$ & $1.389(12)$ \\
\hline $\mathrm{C} 7$ & $\mathrm{C} 8$ & $1.386(8)$ & $\mathrm{C} 25$ & $\mathrm{C} 26$ & $1.353(10)$ \\
\hline $\mathrm{C} 7$ & $\mathrm{C} 12$ & $1.398(7)$ & $\mathrm{C} 25$ & $\mathrm{C} 28$ & $1.533(11)$ \\
\hline $\mathrm{C} 8$ & C9 & $1.421(10)$ & $\mathrm{C} 26$ & $\mathrm{C} 27$ & $1.415(10)$ \\
\hline C9 & $\mathrm{C} 10$ & $1.362(9)$ & & & \\
\hline
\end{tabular}

Table 5 Bond Angles for tianzhx_1015.

\begin{tabular}{llllllll}
\multicolumn{2}{c}{ Atom Atom Atom } & \multicolumn{1}{c}{ Angle $^{\circ}$} & \multicolumn{1}{c}{ Atom Atom Atom } & Angle $^{\circ}$ \\
O2 & S1 & N1 & $106.7(3)$ & C12 & C11 & C13 & $130.8(4)$ \\
O2 & S1 & C22 & $106.7(4)$ & C7 & C12 & C11 & $121.4(5)$ \\
O3 & S1 & O2 & $120.2(4)$ & $\mathrm{C} 11$ & $\mathrm{C} 13$ & $\mathrm{C} 14$ & $99.5(5)$ \\
O3 & S1 & N1 & $106.6(3)$ & $\mathrm{C} 11$ & $\mathrm{C} 13$ & $\mathrm{C} 15$ & $113.4(5)$
\end{tabular}




\begin{tabular}{|c|c|c|c|c|c|c|c|}
\hline $\mathrm{O} 3$ & $\mathrm{~S} 1$ & $\mathrm{C} 22$ & $108.5(3)$ & $\mathrm{C} 11$ & $\mathrm{C} 13$ & C16 & $108.7(5)$ \\
\hline N1 & $\mathrm{S} 1$ & $\mathrm{C} 22$ & 107.6(3) & $\mathrm{C} 15$ & $\mathrm{C} 13$ & $\mathrm{C} 14$ & $112.0(5)$ \\
\hline $\mathrm{C} 10$ & O1 & C14 & $107.8(5)$ & $\mathrm{C} 15$ & $\mathrm{C} 13$ & $\mathrm{C} 16$ & $111.7(5)$ \\
\hline C19 & N1 & S1 & $118.2(4)$ & C16 & $\mathrm{C} 13$ & $\mathrm{C} 14$ & $111.0(5)$ \\
\hline $\mathrm{C} 20$ & N1 & S1 & $115.8(5)$ & O1 & $\mathrm{C} 14$ & $\mathrm{C} 13$ & $108.9(6)$ \\
\hline $\mathrm{C} 20$ & N1 & C19 & $112.8(5)$ & $\mathrm{C} 17$ & $\mathrm{C} 16$ & C13 & $115.9(5)$ \\
\hline $\mathrm{C} 2$ & $\mathrm{C} 1$ & C6 & $122.5(5)$ & C18 & $\mathrm{C} 17$ & $\mathrm{C} 16$ & $122.0(6)$ \\
\hline $\mathrm{C} 1$ & $\mathrm{C} 2$ & $\mathrm{C} 3$ & $119.8(7)$ & C18 & $\mathrm{C} 17$ & $\mathrm{C} 21$ & 118.1(6) \\
\hline $\mathrm{C} 4$ & C3 & $\mathrm{C} 2$ & $118.6(8)$ & $\mathrm{C} 21$ & $\mathrm{C} 17$ & $\mathrm{C} 16$ & $119.9(5)$ \\
\hline $\mathrm{C} 5$ & $\mathrm{C} 4$ & $\mathrm{C} 3$ & 121.1(7) & $\mathrm{C} 17$ & $\mathrm{C} 18$ & C19 & $123.8(6)$ \\
\hline $\mathrm{C} 4$ & C5 & C6 & $121.4(6)$ & C18 & C19 & N1 & $109.9(5)$ \\
\hline $\mathrm{C} 1$ & C6 & C5 & $116.5(6)$ & N1 & $\mathrm{C} 20$ & $\mathrm{C} 21$ & 111.2(6) \\
\hline $\mathrm{C} 1$ & C6 & $\mathrm{C} 7$ & $122.6(5)$ & $\mathrm{C} 17$ & $\mathrm{C} 21$ & $\mathrm{C} 20$ & $118.6(6)$ \\
\hline C5 & C6 & $\mathrm{C} 7$ & $120.8(6)$ & $\mathrm{C} 23$ & $\mathrm{C} 22$ & $\mathrm{~S} 1$ & $121.2(5)$ \\
\hline $\mathrm{C} 8$ & $\mathrm{C} 7$ & C6 & $121.4(5)$ & $\mathrm{C} 23$ & $\mathrm{C} 22$ & $\mathrm{C} 27$ & $118.2(6)$ \\
\hline $\mathrm{C} 8$ & $\mathrm{C} 7$ & $\mathrm{C} 12$ & $117.8(6)$ & $\mathrm{C} 27$ & $\mathrm{C} 22$ & $\mathrm{~S} 1$ & $120.5(5)$ \\
\hline $\mathrm{C} 12$ & $\mathrm{C} 7$ & C6 & $120.2(5)$ & $\mathrm{C} 24$ & $\mathrm{C} 23$ & $\mathrm{C} 22$ & $122.1(7)$ \\
\hline $\mathrm{C} 7$ & $\mathrm{C} 8$ & C9 & $121.7(6)$ & $\mathrm{C} 23$ & $\mathrm{C} 24$ & $\mathrm{C} 25$ & $119.1(7)$ \\
\hline $\mathrm{C} 10$ & C9 & $\mathrm{C} 8$ & $117.3(5)$ & $\mathrm{C} 24$ & $\mathrm{C} 25$ & $\mathrm{C} 28$ & 119.7(8) \\
\hline C9 & $\mathrm{C} 10$ & O1 & $124.7(5)$ & $\mathrm{C} 26$ & $\mathrm{C} 25$ & $\mathrm{C} 24$ & $120.9(8)$ \\
\hline C9 & $\mathrm{C} 10$ & $\mathrm{C} 11$ & $123.5(7)$ & $\mathrm{C} 26$ & $\mathrm{C} 25$ & $\mathrm{C} 28$ & $119.4(9)$ \\
\hline $\mathrm{C} 11$ & $\mathrm{C} 10$ & O1 & $111.8(6)$ & $\mathrm{C} 25$ & $\mathrm{C} 26$ & $\mathrm{C} 27$ & $119.6(8)$ \\
\hline $\mathrm{C} 10$ & C11 & $\mathrm{C} 12$ & 118.1(5) & $\mathrm{C} 22$ & $\mathrm{C} 27$ & $\mathrm{C} 26$ & $120.1(6)$ \\
\hline $\mathrm{C} 10$ & C11 & $\mathrm{C} 13$ & 111.1(5) & & & & \\
\hline
\end{tabular}

Table 6 Torsion Angles for tianzhx_1015.
A B C C D $\quad$ Angle $/^{\circ}$
A B C D Angle $/^{\circ}$
S1 N1 C19C18
169.1(6) C10O1 C14C13
9.7(8) 


\begin{tabular}{|c|c|c|c|}
\hline S1 N1 C20C21 & $-162.9(6)$ & $\mathrm{C} 10 \mathrm{C} 11 \mathrm{C} 12 \mathrm{C} 7$ & $1.2(9)$ \\
\hline S1 C22C23 C24 & $-177.1(6)$ & C10C11 C13 C14 & $5.4(7)$ \\
\hline S1 C22C27C26 & $176.5(5)$ & C10C11 C13 C15 & $124.5(6)$ \\
\hline O1 C10C11 C12 & $177.2(5)$ & C10C11C13C16 & $-110.8(6)$ \\
\hline O1 C10C11 C13 & $0.1(8)$ & $\mathrm{C} 11 \mathrm{C} 13 \mathrm{C} 14 \mathrm{O} 1$ & $-9.1(8)$ \\
\hline O2S1 N1 C19 & $-39.6(7)$ & C11 C13C16C17 & $-173.8(5)$ \\
\hline $\mathrm{O} 2 \mathrm{~S} 1 \mathrm{~N} 1 \mathrm{C} 20$ & $-178.0(6)$ & $\mathrm{C} 12 \mathrm{C} 7 \quad \mathrm{C} 8 \mathrm{C} 9$ & $2.0(10)$ \\
\hline $\mathrm{O} 2 \mathrm{~S} 1 \mathrm{C} 22 \mathrm{C} 23$ & $23.9(6)$ & C12C11 C13 C14 & $-171.3(6)$ \\
\hline $\mathrm{O} 2 \mathrm{~S} 1 \mathrm{C} 22 \mathrm{C} 27$ & $-151.6(5)$ & C12C11 C13 C15 & $-52.2(9)$ \\
\hline O3S1 N1 C19 & $-169.2(6)$ & C12C11 C13 C16 & $72.6(8)$ \\
\hline O3S1 N1 C20 & $52.4(6)$ & $\mathrm{C} 13 \mathrm{C} 11 \mathrm{C} 12 \mathrm{C} 7$ & 177.7(6) \\
\hline O3S1 C22C23 & $154.7(5)$ & C13C16C17 C18 & $109.4(7)$ \\
\hline O3S1 C22C27 & $-20.9(6)$ & C13C16C17 C21 & $-73.5(8)$ \\
\hline N1S1 C22C23 & $-90.3(6)$ & C14O1 C10C9 & $175.3(8)$ \\
\hline N1S1 C22C27 & $94.2(5)$ & C14O1 C10C11 & $-6.3(9)$ \\
\hline N1 C20C21 C17 & $-36.0(10)$ & C14C13C16C17 & $77.8(6)$ \\
\hline $\mathrm{C} 1 \mathrm{C} 2 \mathrm{C} 3 \mathrm{C} 4$ & $1.7(11)$ & $\mathrm{C} 15 \mathrm{C} 13 \mathrm{C} 14 \mathrm{O} 1$ & $-129.2(6)$ \\
\hline C1 C6 C7 C8 & $155.2(6)$ & C15C13C16C17 & $-48.0(7)$ \\
\hline C1 C6 C7 C12 & $-33.5(8)$ & $\mathrm{C} 16 \mathrm{C} 13 \mathrm{C} 14 \mathrm{O} 1$ & $105.3(7)$ \\
\hline C2 C1 C6 C5 & $0.5(9)$ & C16C17C18C19 & $169.6(7)$ \\
\hline C2 C1 C6 C7 & $176.6(5)$ & C16C17C21 C20 & $-165.6(6)$ \\
\hline $\mathrm{C} 2 \mathrm{C} 3 \mathrm{C} 4 \mathrm{C} 5$ & $-2.0(12)$ & C17C18C19N1 & $27.0(11)$ \\
\hline $\mathrm{C} 3 \mathrm{C} 4 \mathrm{C} 5 \mathrm{C} 6$ & $1.5(12)$ & C18C17C21 C20 & $11.7(11)$ \\
\hline C4 C5 C6 C1 & $-0.7(10)$ & C19N1 C20C21 & $56.6(9)$ \\
\hline $\mathrm{C} 4 \mathrm{C} 5 \mathrm{C} 6 \mathrm{C} 7$ & $-176.9(6)$ & C20N1 C19C18 & $-51.3(9)$ \\
\hline $\mathrm{C} 5 \mathrm{C} 6 \mathrm{C} 7 \mathrm{C} 8$ & $-28.8(9)$ & C21 C17 C18 C19 & $-7.6(12)$ \\
\hline C5 C6 C7 C12 & $142.5(6)$ & C22S1 N1 C19 & $74.6(6)$ \\
\hline $\mathrm{C} 6 \mathrm{C} 1 \mathrm{C} 2 \mathrm{C} 3$ & $-1.0(9)$ & C22S1 N1 C20 & $-63.8(6)$ \\
\hline $\mathrm{C} 6 \mathrm{C} 7 \mathrm{C} 8 \mathrm{C} 9$ & $173.5(6)$ & $\mathrm{C} 22 \mathrm{C} 23 \mathrm{C} 24 \mathrm{C} 25$ & $2.7(12)$ \\
\hline
\end{tabular}




$\begin{array}{llrr}\text { C6 C7 } & \mathrm{C} 12 \mathrm{C} 11 & -171.8(5) \mathrm{C} 23 \mathrm{C} 22 \mathrm{C} 27 \mathrm{C} 26 & 0.8(10) \\ \mathrm{C} 7 \mathrm{C} 8 \mathrm{C} 9 \mathrm{C} 10 & -4.9(12) \mathrm{C} 23 \mathrm{C} 24 \mathrm{C} 25 \mathrm{C} 26 & -3.3(12) \\ \mathrm{C} 8 \mathrm{C} 7 \mathrm{C} 12 \mathrm{C} 11 & -0.2(9) \mathrm{C} 23 \mathrm{C} 24 \mathrm{C} 25 \mathrm{C} 28 & 177.3(7) \\ \mathrm{C} 8 \mathrm{C} 9 \mathrm{C} 10 \mathrm{O} 1 & -175.7(7) \mathrm{C} 24 \mathrm{C} 25 \mathrm{C} 26 \mathrm{C} 27 & 2.7(11) \\ \mathrm{C} 8 \mathrm{C} 9 \mathrm{C} 10 \mathrm{C} 11 & 6.1(12) \mathrm{C} 25 \mathrm{C} 26 \mathrm{C} 27 \mathrm{C} 22 & -1.5(10) \\ \mathrm{C} 9 \mathrm{C} 10 \mathrm{C} 11 \mathrm{C} 12 & -4.4(11) \mathrm{C} 27 \mathrm{C} 22 \mathrm{C} 23 \mathrm{C} 24 & -1.4(10) \\ \mathrm{C} 9 \mathrm{C} 10 \mathrm{C} 11 \mathrm{C} 13 & 178.5(7) \mathrm{C} 28 \mathrm{C} 25 \mathrm{C} 26 \mathrm{C} 27 & -177.9(7)\end{array}$

Table 7 Hydrogen Atom Coordinates $\left(\AA \times 10^{4}\right)$ and Isotropic Displacement Parameters $\left(\AA^{2} \times 10^{3}\right)$ for tianzhx_1015.

\begin{tabular}{|c|c|c|c|c|}
\hline Atom & $x$ & $y$ & $z$ & $\mathbf{U}(\mathbf{e q})$ \\
\hline $\mathrm{H} 1$ & -15214 & -985 & -4086 & 77 \\
\hline $\mathrm{H} 2$ & -15113 & 346 & -4763 & 92 \\
\hline H3 & -16676 & 11 & -5477 & 111 \\
\hline H4 & -18411 & -1603 & -5471 & 121 \\
\hline H5 & -18493 & -2934 & -4800 & 108 \\
\hline H8 & -19136 & -3530 & -4000 & 99 \\
\hline H9 & -19098 & -5061 & -3364 & 115 \\
\hline H12 & -14593 & -2852 & -3744 & 74 \\
\hline $\mathrm{H} 14 \mathrm{~A}$ & -14459 & -6652 & -2930 & 107 \\
\hline H14B & -14743 & -5939 & -2415 & 107 \\
\hline H15A & -13018 & -5592 & -3622 & 121 \\
\hline H15B & -11943 & -5529 & -3136 & 121 \\
\hline $\mathrm{H} 15 \mathrm{C}$ & -12211 & -4354 & -3469 & 121 \\
\hline H16A & -13519 & -3023 & -2748 & 81 \\
\hline H16B & -14549 & -3749 & -2357 & 81 \\
\hline H18 & -10797 & -2887 & -2597 & 102 \\
\hline H19A & -9615 & -2956 & -1709 & 117 \\
\hline
\end{tabular}




$\begin{array}{lllll}\text { H19B } & -8567 & -3418 & -2165 & 117 \\ \text { H20A } & -11245 & -4627 & -1240 & 95 \\ \text { H20B } & -10945 & -5994 & -1397 & 95 \\ \text { H21A } & -12318 & -5856 & -2089 & 120 \\ \text { H21B } & -13265 & -5010 & -1719 & 120 \\ \text { H23 } & -6836 & -2979 & -1067 & 100 \\ \text { H24 } & -6808 & -1996 & -289 & 119 \\ \text { H26 } & -9035 & -4825 & 351 & 108 \\ \text { H27 } & -9104 & -5815 & -447 & 96 \\ \text { H28A } & -8182 & -3282 & 891 & 197 \\ \text { H28B } & -8359 & -1994 & 632 & 197 \\ \text { H28C } & -6689 & -2562 & 712 & 197\end{array}$




\section{References}

(1) Armarego, W. L. F.; Chai, C. C. L. Purification of laboratory chemicals, 5th ed., Butterworth- Heinemann, 2003.

(2) Gao, Y.; Xiong, W.-F.; Chen, H.-J.; Wu, W.-Q.; Peng, J.-W.; Gao, Y.-L.; Jiang, H.-F. Pd-Catalyzed Highly Regio- and Stereoselective Formation of $\mathrm{C}-\mathrm{C}$ Double Bonds: An Efficient Method for the Synthesis of Benzofuran-, Dihydrobenzofuran-, and Indoline-Containing Alkenes. J. Org. Chem. 2015, 80, 7456.

(3) Ramesh, K.; Basuli, S.; Satyanarayana, G. Microwave-Assisted Domino Palladium Catalysis in Water: A Diverse Synthesis of 3, 3'-Disubstituted Heterocyclic Compounds. Eur. J. Org. Chem. 2018, 2171.

(4) Yao, T.-L.; He, D. Palladium-Catalyzed Domino Heck/Aryne Carbopalladation/C-H Functionalization: Synthesis of Heterocycle-Fused 9, 10-Dihydrophenanthrenes. Org. Lett. 2017, 19, 842.

(5) Wu, X.-X.; Chen, W.-L.; Shen, Y.; Chen, S.; Xu, P.-F.; Liang, Y.-M. M. Palladium-Catalyzed Domino Heck/Intermolecular C-H Bond Functionalization: Efficient Synthesis of Alkylated Polyfluoroarene Derivatives. Org. Lett. 2016, 18, 1784 .

(6) Hu, M.; Gao, Y.; Wu, W. Q.; Li, J. X.; Li, C. S.; Zhang, H.; Jiang, H. F. Efficient assembly of ynones via palladium-catalyzed sequential carbonylation/alkynylation. Org. Biomol. Chem., 2018, 16, 7383.

(7) Zhang, Z.-M.; Xu, B.; Qian, Y.-Y.; Wu, L.-Z.; Wu, Y.-Q.; Zhou, L.-J.; Liu Y.; Zhang, J.-L. Palladium-Catalyzed Enantioselective Reductive Heck Reactions: Convenient Access to 3,3-Disubstituted 2,3-Dihydrobenzofuran. Angew. Chem. Int. Ed. 2018, 57, 10373.

(8) Thapa, S.; Basnet, P.; Giri, R. Copper-Catalyzed Dicarbofunctionalization of Unactivated Olefins by Tandem Cyclization/Cross-Coupling. J. Am. Chem. Soc. 2017, 139, 5700.

(9) Jaimes, M. C. B.; Weingand, V.; Rominger, F.; Hashmi, A. S. K. From Ynamides to Highly Substituted Benzo[b]furans: Gold(I)-Catalyzed 5-endo-dig-Cyclization/Rearrangement of Alkylic Oxonium Intermediates. Chem. Eur. J. 2013, 19, 12504.

(10) Rueping, M.; Leiendecker, M.; Das, A.; Poissona, T.; Bui, L. Potassium tert-butoxide mediated Heck-type cyclization/isomerization-benzofurans from organocatalytic radical cross-coupling reactions. Chem. Commun. 2011, 47, 10629.

(11) Revol, G.; McCallum, T.; Morin, M.; Gagosz, F.; Barriault, L. Photoredox Transformations with Dimeric Gold Complexes. Angew. Chem. Int. Ed. 2013, 52, 13342.

(12) Lockner, J. W.; Dixon, D. D.; Risgaard, R.; Baran, P. S. Practical Radical Cyclizations with Arylboronic Acids and Trifluoroborates. Org. Lett. 2011, 13, 5628.

(13) Zheng, H.-J.; Zhu, Y.-G.; Shi, Y. Palladium(0)-Catalyzed Heck Reaction/C-H Activation/Amination Sequence with Diaziridinone: A Facile Approach to Indoline. Angew. Chem. Int. Ed. 2014, 53, 11280.

(14) (a) Solé, D.; Mariani, F.; Fernández, I.; Sierra, M. A. Intramolecular Pd(0)-Catalyzed Reactions of (2-Iodoanilino)-aldehydes: A Joint Experimental-Computational Study. J. Org. Chem. 2012, 77, 10272. (b) Hédouin, J.; Schneider, C.; Gillaizeau, I.; Hoarau, C. Palladium-Catalyzed Domino Allenamide Carbopalladation/Direct C-H Allylation of Heteroarenes: Synthesis of Primprinine and Papaverine Analogues. 
Org. Lett. 2018, 20, 6027.

(15) Iain, D. G. W.; Stefanie. R.; Toste, F. D. Asymmetric Synthesis of Medium-Sized Rings by Intramolecular Au(I)-Catalyzed Cyclopropanation. J. Am. Chem. Soc. 2009, 131, 2056.

(16) Mariusz, J. B. A Convenient Synthesis of 2-Arylbenzo[b]furans from Aryl Halides and 2-Halophenols by Catalytic One-Pot Cascade Method. Acs. Catal. 2016, 6, 2429.

(17) Stephen, G. N.; Jennifer, K. H.; Norman, N.; Mark, L. Palladium-Catalyzed Carbohalogenation: Bromide to Iodide Exchange and Domino Processes. J. Am. Chem. Soc. 2011, 133, 14916.

$$
\text { Luo, Z.-S.; Mohamed, }
$$

$\mathrm{N}$.

A

synthetic

approach

for

(S)-(3-benzyl-3-methyl-2,3-dihydro-benzofuran-6-yl)-piperidin-1-yl-methanone, a Selective CB2 Receptor Agonist. Tetrahedron Letters. 2012, 53, 3316.

(19) Croft, A. K.; Foley, M. K. Proline-rich Proteins-deriving a Basis for Residue-based Selectivity in Polyphenolic Binding. Org. Biomol. Chem., 2008, 6, 1594.

(20) Alt, I. T.; Guttroff, C.; Plietker, B. Fe-catalyzed Intramolecular Aminations of C(sp3 )-H-bonds of alkylarylazides. Angew. Chem. Int. Ed. 2015, 54, 10545.

(21) Shan, X. H.; Yang, B.; Zheng, H. X.; Qu, J. P.; Kang, Y. B. Phenanthroline-tBuOK Promoted Intramolecular C-H Arylation of Indoles with ArI under Transition-Metal-Free Conditions. Org. Lett. 2018, 20, 7898.

(22) Su, X.-L.; Huang, H.-G.; Yuan, Y.-F.; Li, Y. Radical Desulfur-Fragmentation and Reconstruction of Enol Triflates: Facile Access to $\alpha$-Trifluoromethyl Ketones. Angew. Chem. Int. Ed. 2017, 56, 1338.

(23) (a) Overman, L. E.; Tanis, P. S. Origin of Stereocontrol in the Construction of the 12-Oxatricyclo[6.3.1.02,7]dodecane Ring System by Prins Pinacol Reactions. J. Org. Chem., 2010, 75, 455. (b) Crisp, G. T.; Scott, W. J. A Convenient One-Pot Procedure for the Conversion of Terminal Acetylenic Alcohols (and O-Derivatives) into (E)-Olefinic Alcohols (or Derivatives). Synthesis. 1985, 335.

(24) Dürr, A. B.; Yin, G.; Kalvet, I.; Napolya, F.; Schoenebeck, F. Nickel-catalyzed trifluoromethylthiolation of Csp2-O bonds. Chem. Sci. 2016, 7, 1076.

(25) Lippincott, D. J.; Linstadt, R. T. H.; Maser, M. R.; Lipshutz, B. H. Synthesis of Functionalized [3], [4], [5] and [6]Dendralenes through Palladium-Catalyzed Cross-Couplings of Substituted Allenoates. Angew. Chem. Int. Ed. 2017, 56, 847.

(26) Navendu, J.; Quyen, N.; Tom, G. D. Development of a Suzuki Cross-Coupling Reaction between 2-Azidoarylboronic Pinacolate Esters and Vinyl Triflates To Enable the Synthesis of [2,3]-Fused Indole Heterocycles. J. Org. Chem. 2014, 79, 2781.

(27) Si, T.-D.; Li, B.-W.; Xiong, W.-R.; Xu, B.; Tang, W.-J. Efficient cross-coupling of aryl/alkenyl triflates with acyclic secondary alkylboronic acids. Org. Biomol. Chem. 2017, 15, 9903.

(28) Tsuyoshi, U.; Hideyuki, K.; Kei, M. Trichlorophenyl Formate: Highly Reactive and Easily Accessible Crystalline CO Surrogate for Palladium-Catalyzed Carbonylation of Aryl/Alkenyl Halides and Triflates. Org. Lett. 2012, 14, 5370 .

(29) Clark, J. R.; Feng, K.-B.; Sookezian, A.; White, M. C. Manganese-Catalysed Benzylic C(sp3)-H Amination for Late-stage Functionalization. Nat. Chem. 2018, 10, 583. 
(30) Jigajinni, V. B.; Wightman, R. H. Hydrogenolysis of Enol Triflates; A New Method for the Reduction of Ketones to Methylene Compounds. Tetrahedron Letters, 1982, 23, 117.

(31) Patel, H. H.; Sigman, M. S. Enantioselective Palladium-Catalyzed Alkenylation of Trisubstituted Alkenols To Form Allylic Quaternary Centers. J. Am. Chem. Soc. 2016, 138, 14226.

(32) Peter, J. S.; Thomas, E. D. Preparation of Vinyl Trifluoromethanesulfonates: 3-Methyl-2-Buten-2-yl Triflate. Org. Synth. 1974, 54, 79.

(33) Hu, J. T.; Zheng, B.; Chen, Y. C.; Xiao, Q. Expedient synthesis of 9,10-phenanthrenes via LiOPiv-promoted and palladium-catalysed aryne annulation by vinyl triflates. Org. Chem. Front., 2018, 5, 2045.

(34) Huang, H.; Ash, J.; Kang, J. Y. $\mathrm{Tf}_{2} \mathrm{O}$-Promoted Activating Strategy of Phosphate Analogues: Synthesis of Mixed Phosphates and Phosphinate. Org. Lett. 2018, 20, 4938.

(35) Zhang, X.-J.; Xie, X.; Liu, Y.-H. Nickel-Catalyzed Highly Regioselective Hydrocyanation of Terminal Alkynes with $\mathrm{Zn}(\mathrm{CN})_{2}$ Using Water as the Hydrogen Source. J. Am. Chem. Soc. 2018, 140, 7385.

(36) Goh, S. S.; Baars, H.; Gockel, B.; Anderson, E. A. Metal-Catalyzed Syntheses of Abridged CDE Rings of Rubriflordilactones A and B. Org. Lett., 2012, 14, 6278.

(37) Odriozola, A.; Oiarbide, M.; Palomo, C. Enantioselective Synthesis of Quaternary $\Delta 4$-and $\Delta 5$-Dehydroprolines Based on a Two-Step Formal [3+2] Cycloaddition of $\alpha$-Aryl and $\alpha$-Alkyl Isocyano(thio)acetates with Vinyl Ketones. Chem. Eur. J. 2017, 23, 12758.

(38) Bischo, A. J.; Nelson, B. M.; Niemeyer, Z. L.; Sigman, M. S.; Movassaghi, M. Quantitative Modeling of Bis(pyridine)silver(I) Permanganate Oxidation of Hydantoin Derivatives: Guidelines for Predicting the Site of Oxidation in Complex Substrates. J. Am. Chem. Soc. 2017, 139, 15539.

(39) Wang, W.; Zhou, R.; Jiang, Z.-J.; Wang, X.; Fu, H.-Y.; Zheng, X.-L.; Chen, H.; Li, R.-X. Palladium-Catalyzed Domino Mizoroki-Heck/Intermolecular $\mathrm{C}\left(\mathrm{sp}^{3}\right)-\mathrm{H}$ Activation Sequence: An Approach to the Formation of C( $\left(\mathrm{sp}^{3}\right)-\mathrm{C}\left(\mathrm{sp}^{3}\right)$ Bonds. Eur. J. Org. Chem. 2015, 2015, 2579. 
8. Copies of NMR Spectra

1c-1; ${ }^{1} \mathrm{H}$ NMR (400MHz, $\left.\left.\mathrm{CDCl}_{3}\right) ;{ }^{13} \mathrm{C} \mathrm{NMR} \mathrm{(100MHz,} \mathrm{CDCl}_{3}\right)$

$\stackrel{q}{\stackrel{q}{i}} \stackrel{\substack{0 \\ i}}{i}$

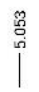

隹

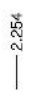

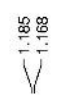

$\stackrel{8}{i}$
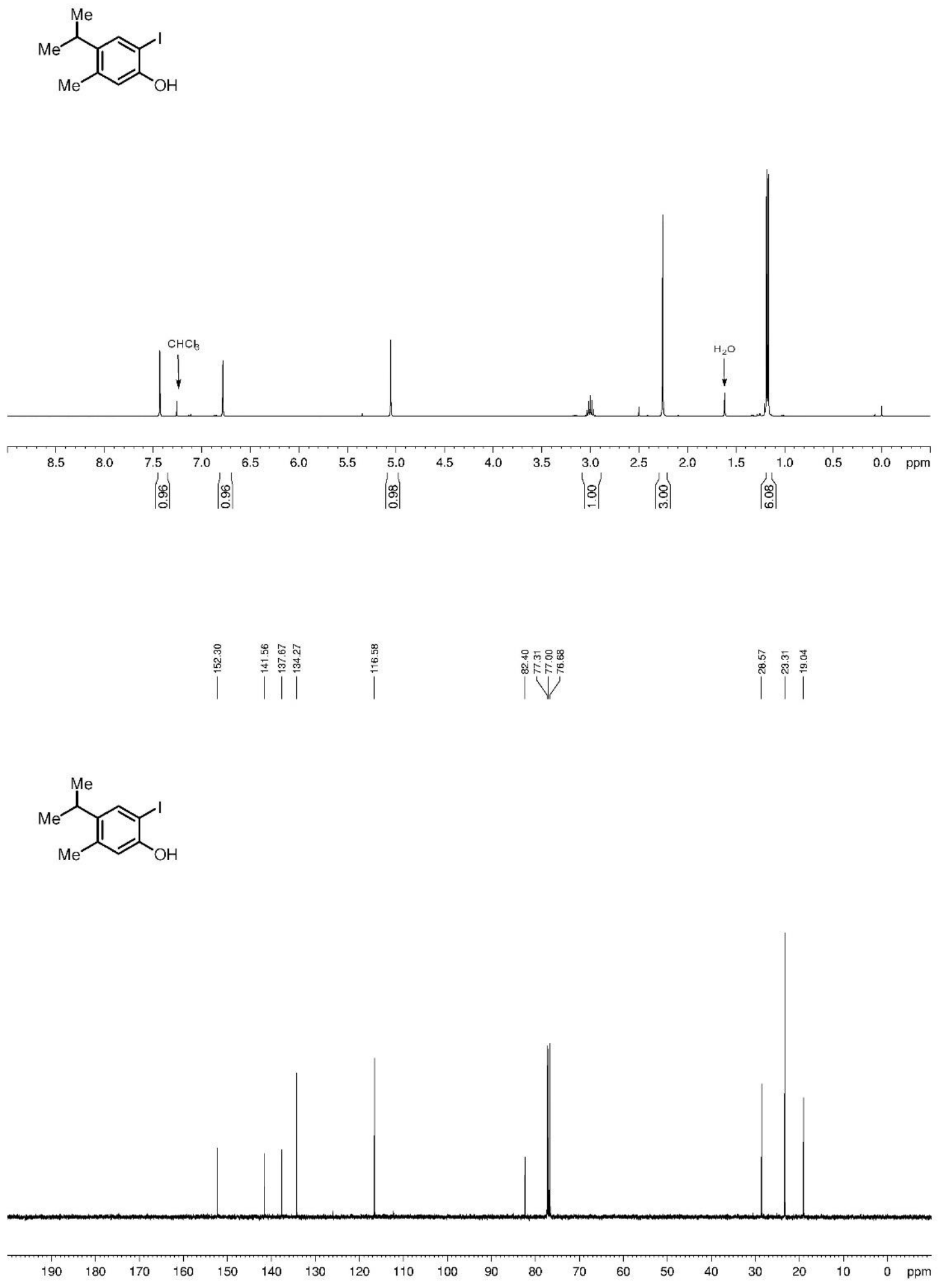

S82 
1c; ${ }^{1} \mathrm{H}$ NMR (400MHz, $\left.\mathrm{CDCl}_{3}\right) ;{ }^{13} \mathrm{C}$ NMR (100MHz, $\left.\mathrm{CDCl}_{3}\right)$

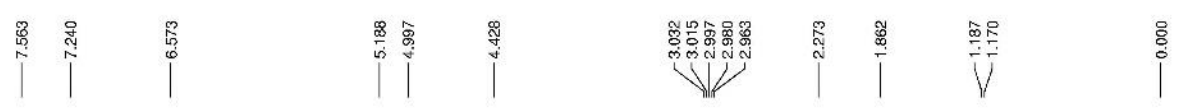<smiles>C=C(C)COc1cc(C)c(C(C)C)cc1I</smiles>
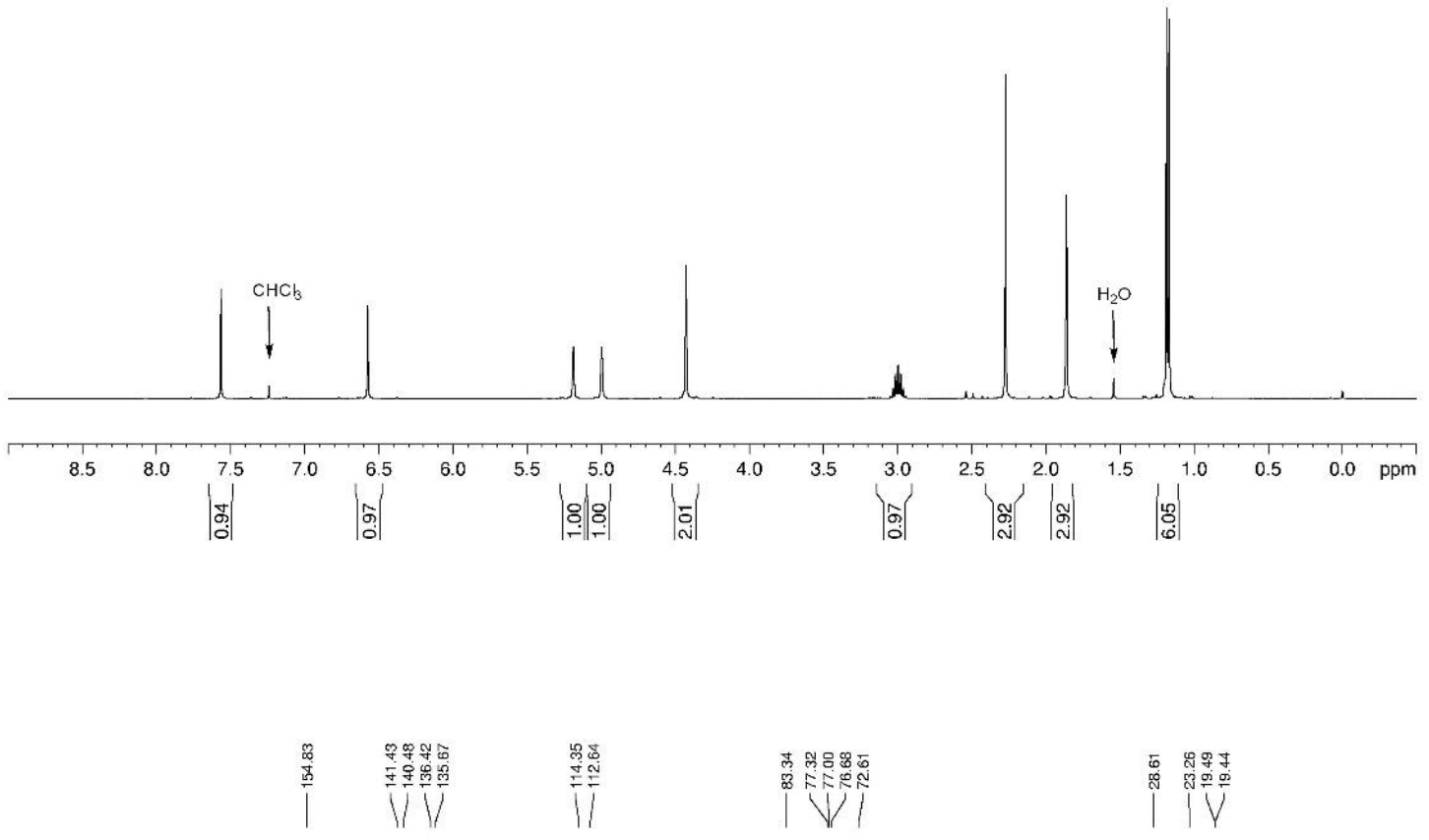<smiles>C=C(C)COc1cc(C)c(C(C)C)cc1I</smiles>

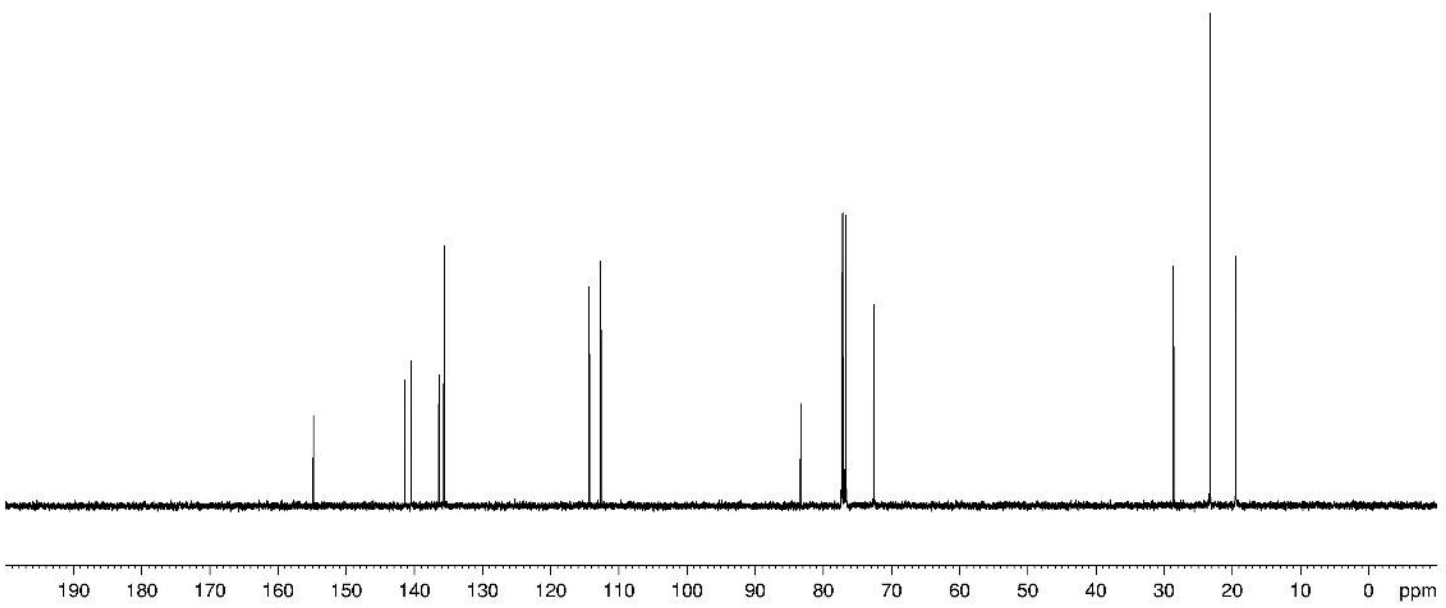


1g; ${ }^{1} \mathrm{H}$ NMR $\left(400 \mathrm{MHz}, \mathrm{CDCl}_{3}\right) ;{ }^{13} \mathrm{C}$ NMR (100MHz, $\left.\mathrm{CDCl}_{3}\right)$

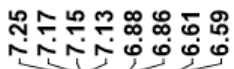
กู่
$\stackrel{\text { స }}{\stackrel{\infty}{\leftarrow}}$
$\stackrel{0}{i}$<smiles>C=C(C)COc1cccc(C)c1I</smiles>

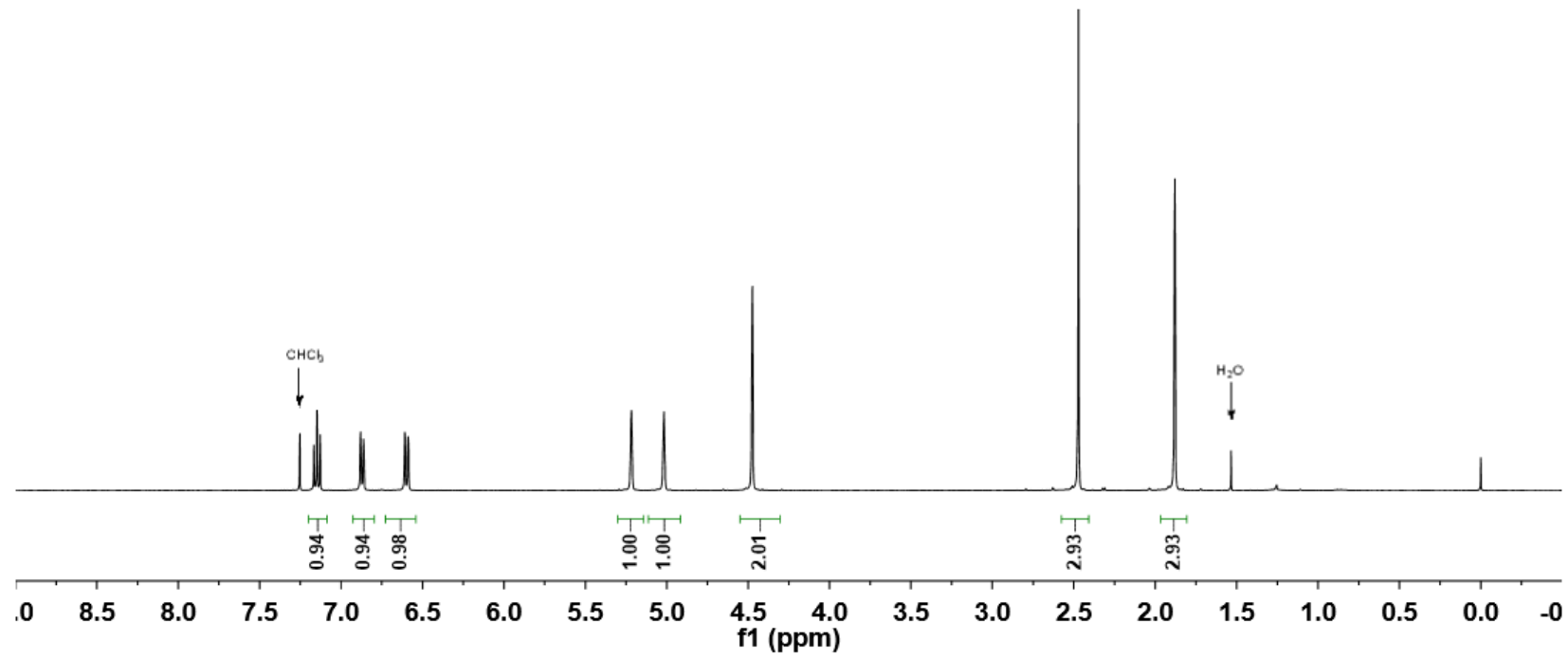

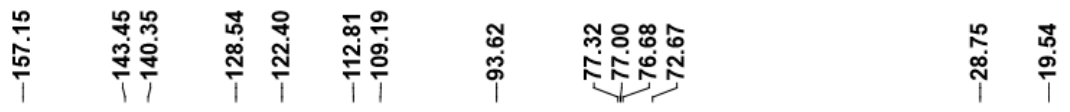<smiles>C=C(C)COc1cccc(C)c1I</smiles>

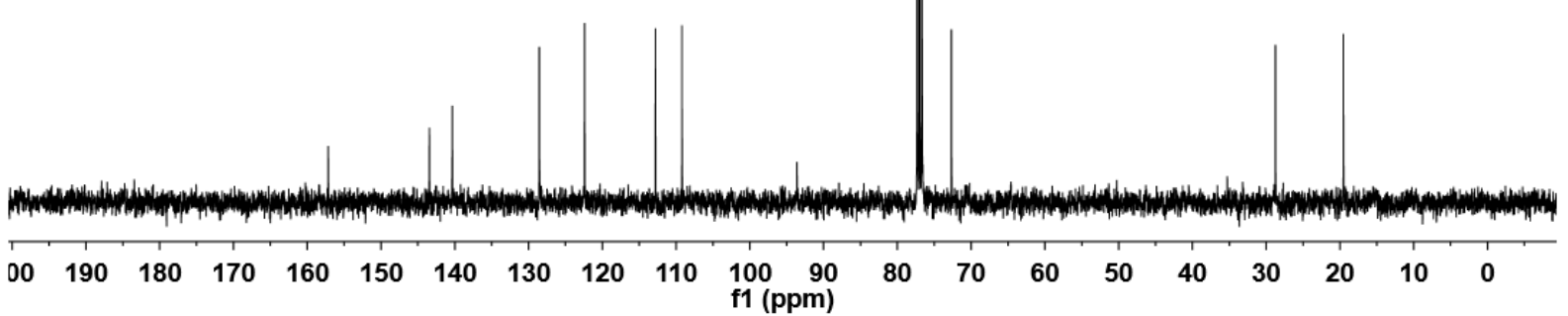


1i; ${ }^{1} \mathrm{H}$ NMR $\left(400 \mathrm{MHz}, \mathrm{CDCl}_{3}\right) ;{ }^{13} \mathrm{C}$ NMR (100MHz, $\left.\mathrm{CDCl}_{3}\right)$
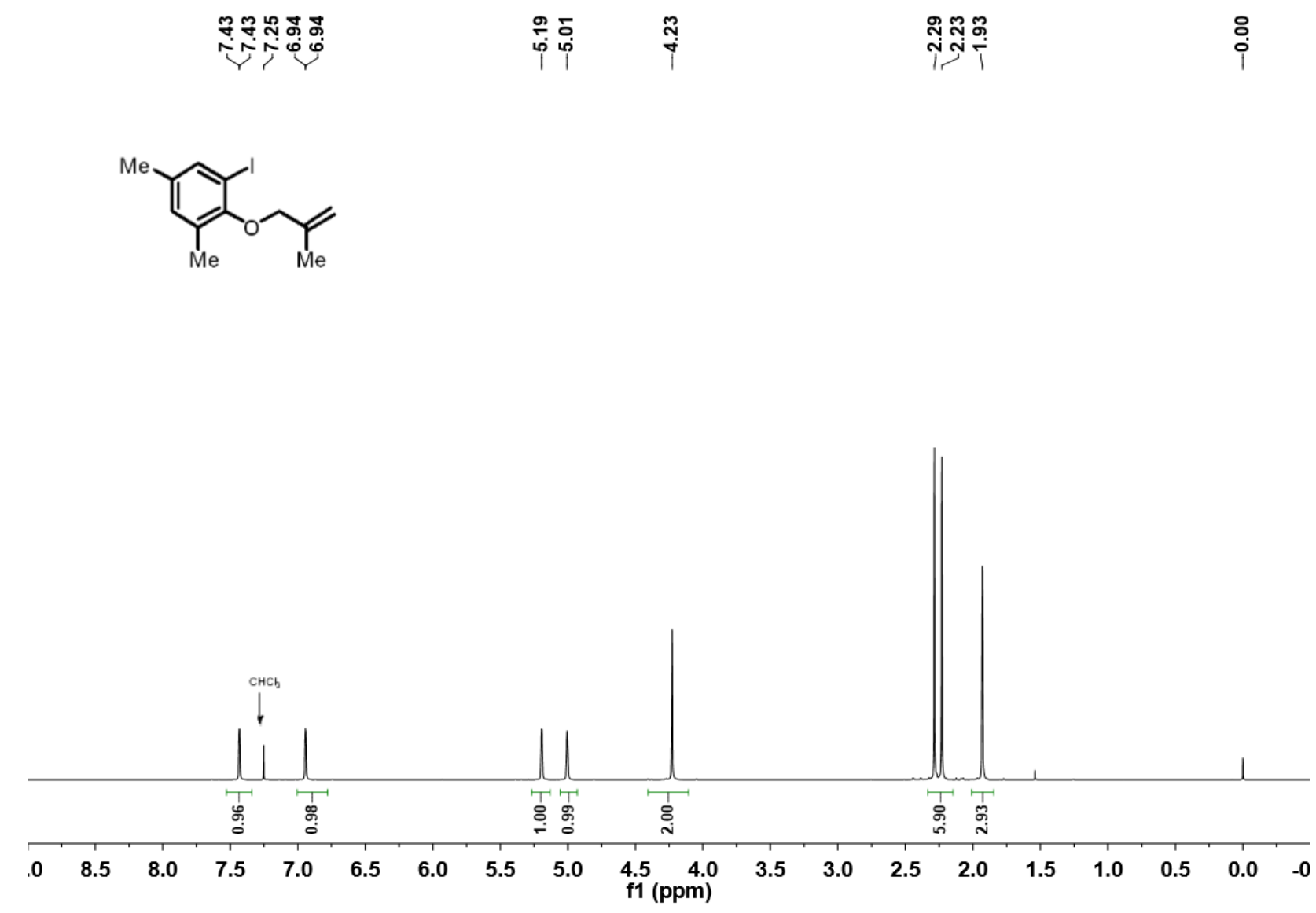

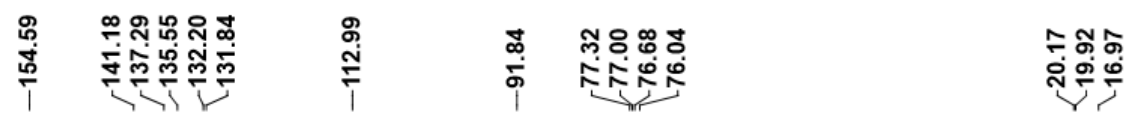
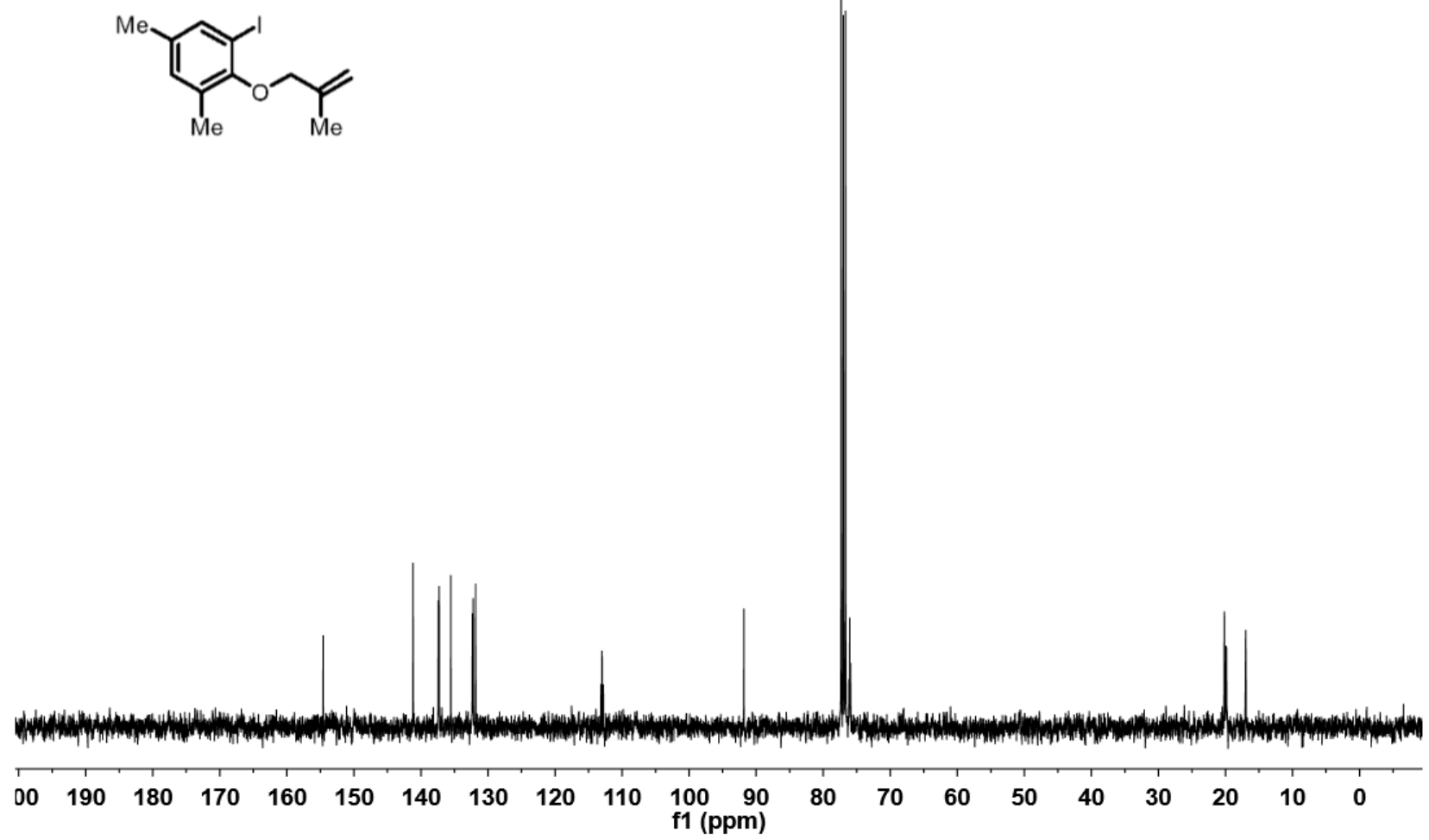
1j; ${ }^{1} \mathrm{H}$ NMR (400MHz, $\left.\mathrm{CDCl}_{3}\right) ;{ }^{13} \mathrm{C}$ NMR (100MHz, $\left.\mathrm{CDCl}_{3}\right)$
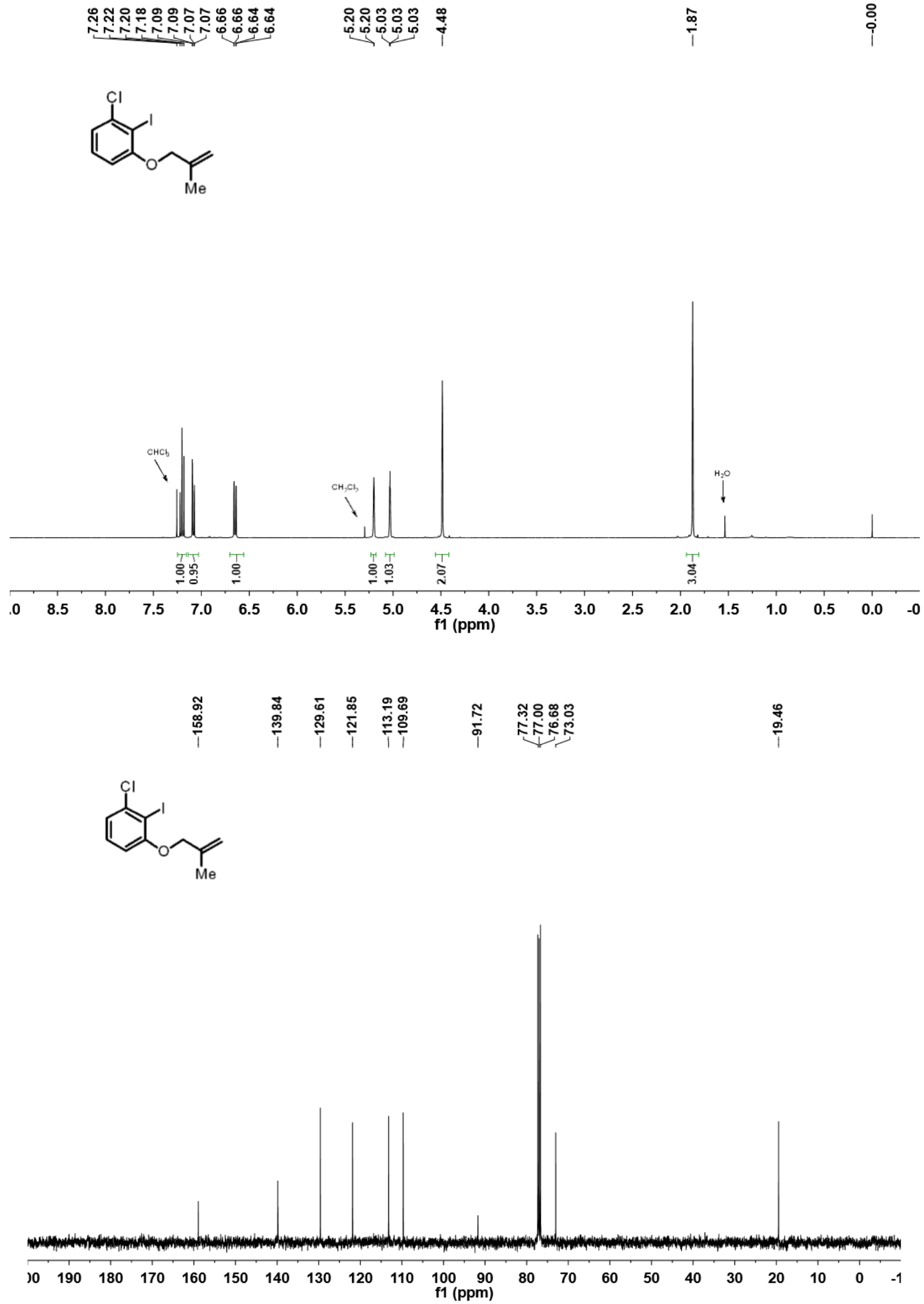
11; ${ }^{1} \mathrm{H}$ NMR (400MHz, $\left.\mathrm{CDCl}_{3}\right) ;{ }^{13} \mathrm{C}$ NMR (100MHz, $\left.\mathrm{CDCl}_{3}\right)$

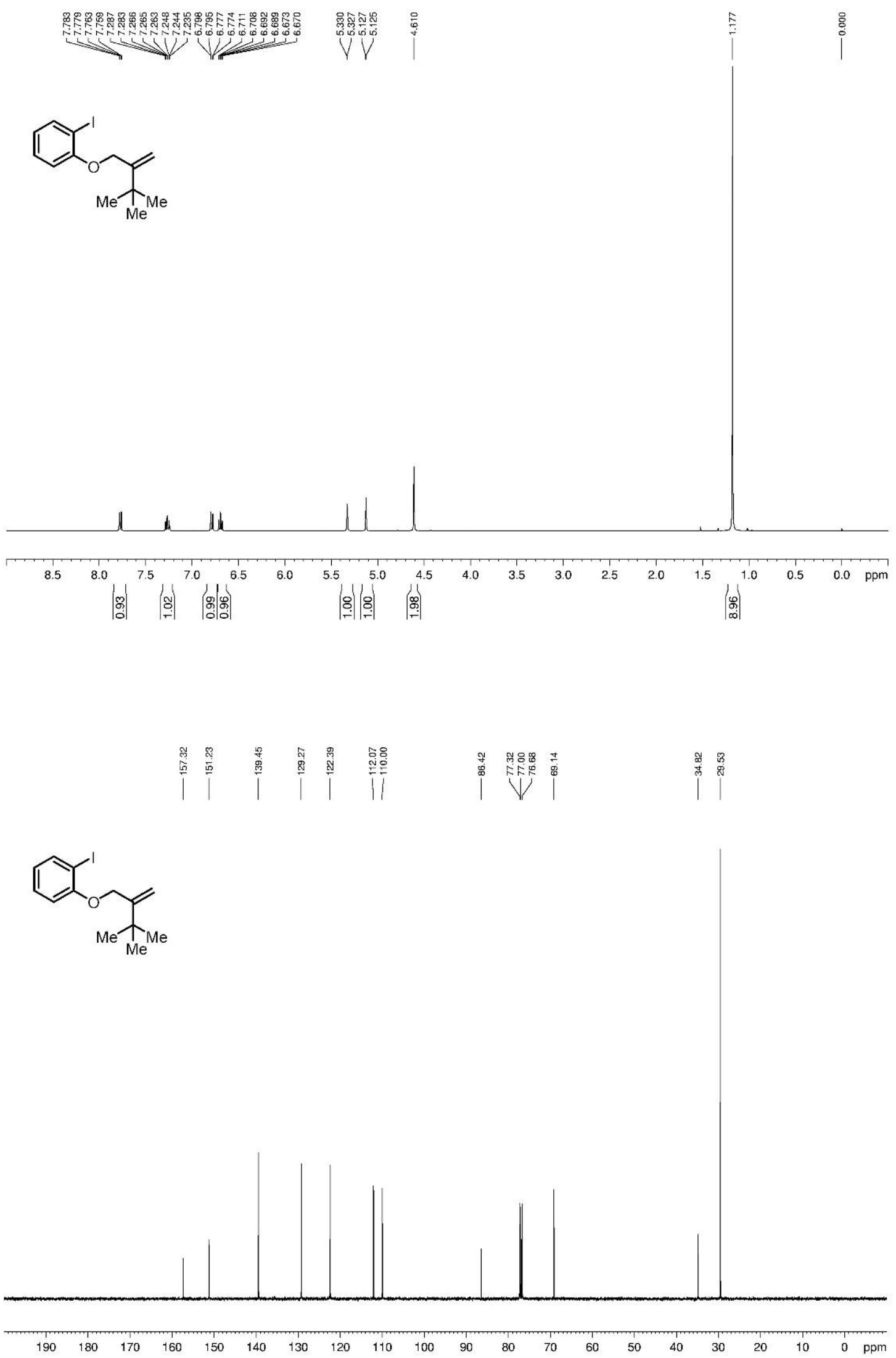


1m; ${ }^{1} \mathrm{H}$ NMR (400MHz, $\left.\mathrm{CDCl}_{3}\right) ;{ }^{13} \mathrm{C}$ NMR (100MHz, $\left.\mathrm{CDCl}_{3}\right)$

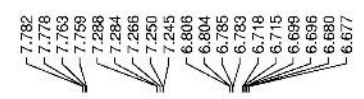

为器器

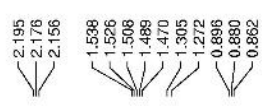

i
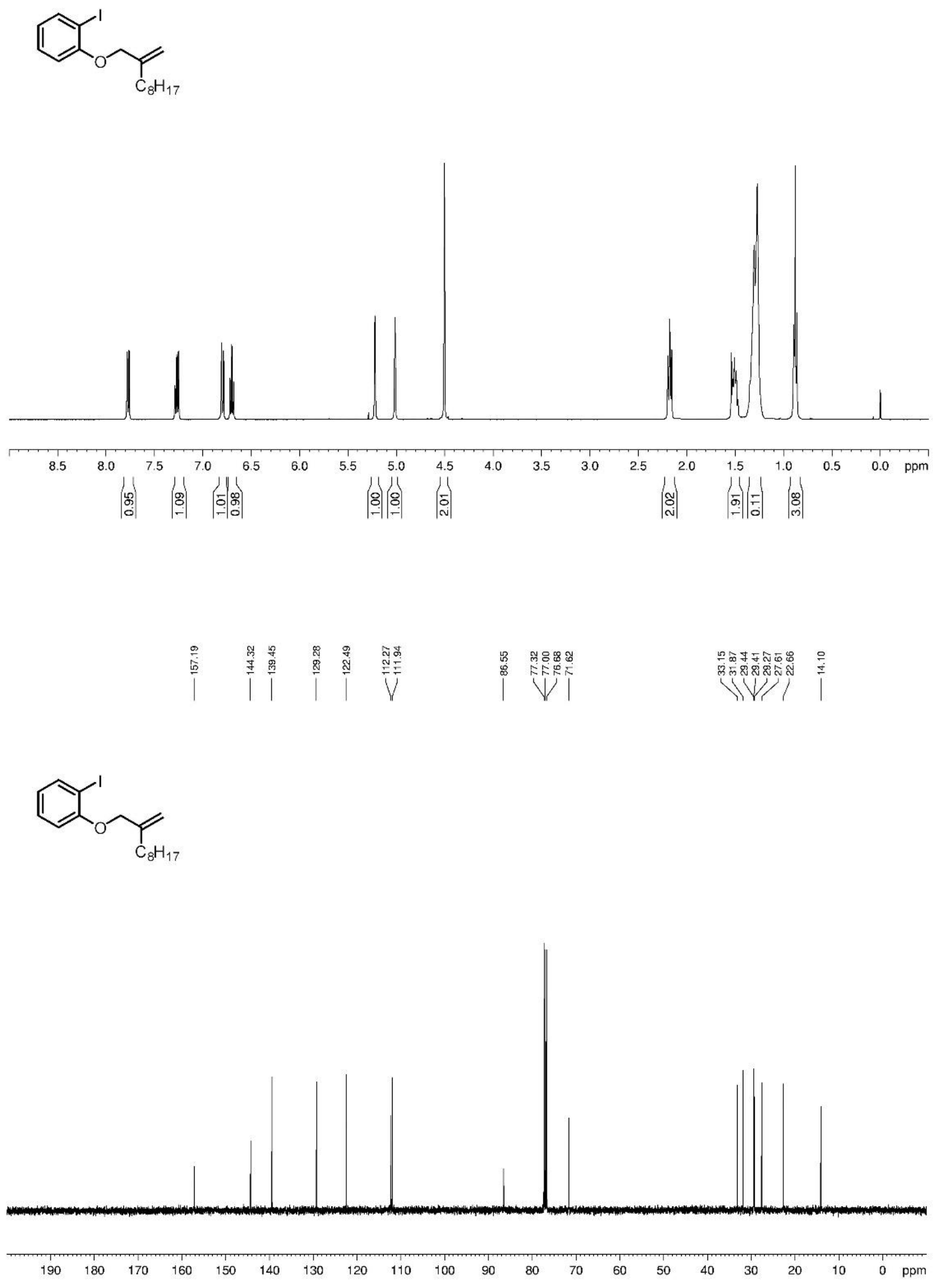

S88 
1o; ${ }^{1} \mathrm{H}$ NMR (400MHz, $\left.\mathrm{CDCl}_{3}\right) ;{ }^{13} \mathrm{C}$ NMR (100MHz, $\left.\mathrm{CDCl}_{3}\right)$

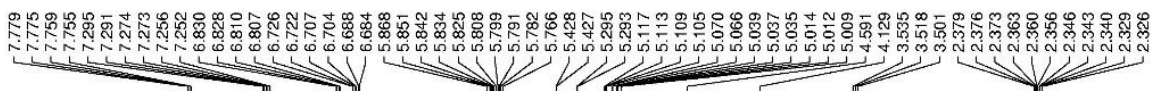

i
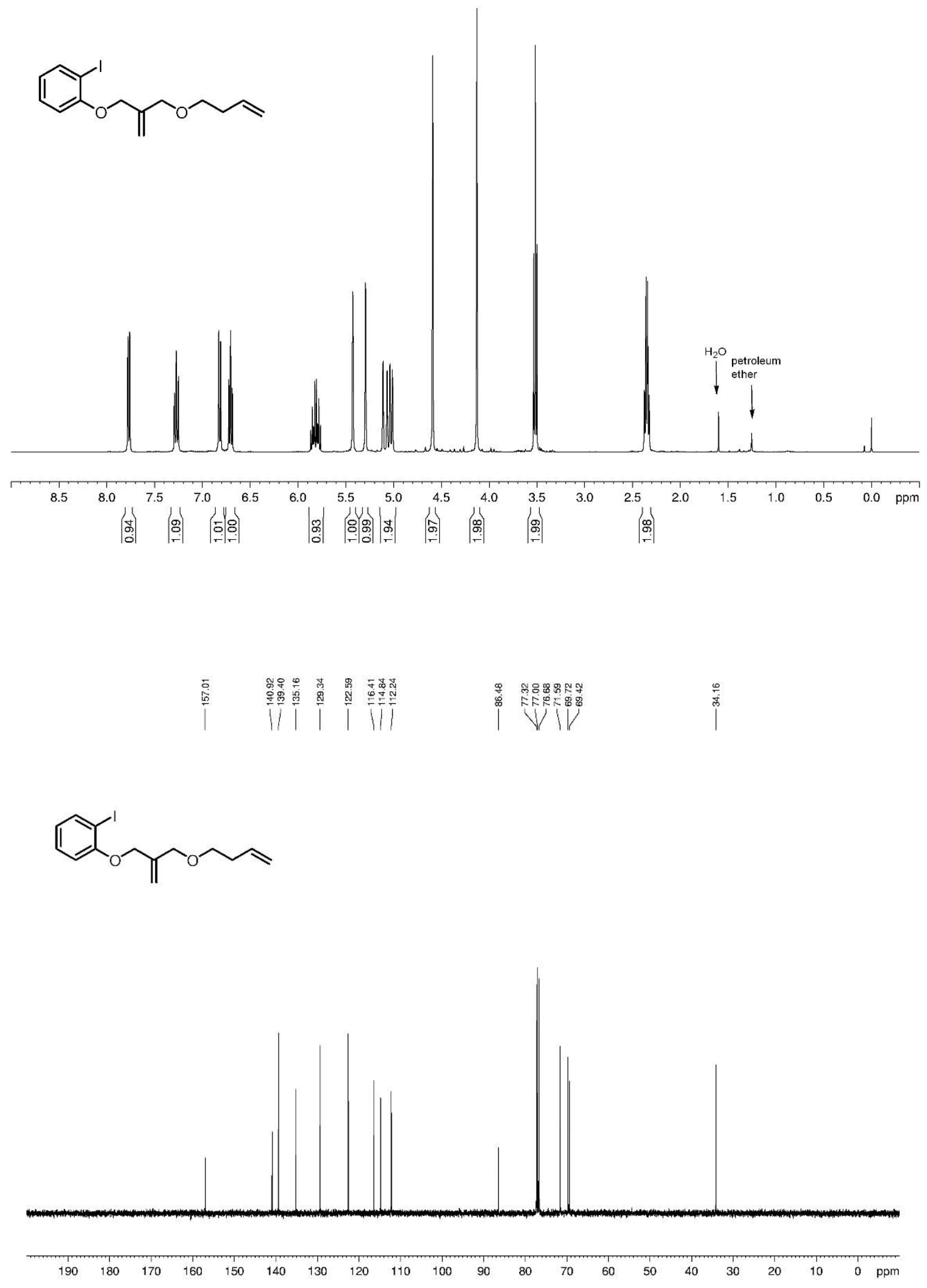

S89 
1q; ${ }^{1} \mathrm{H}$ NMR $\left(400 \mathrm{MHz}, \mathrm{CDCl}_{3}\right) ;{ }^{13} \mathrm{C}$ NMR (100MHz, $\left.\mathrm{CDCl}_{3}\right)$

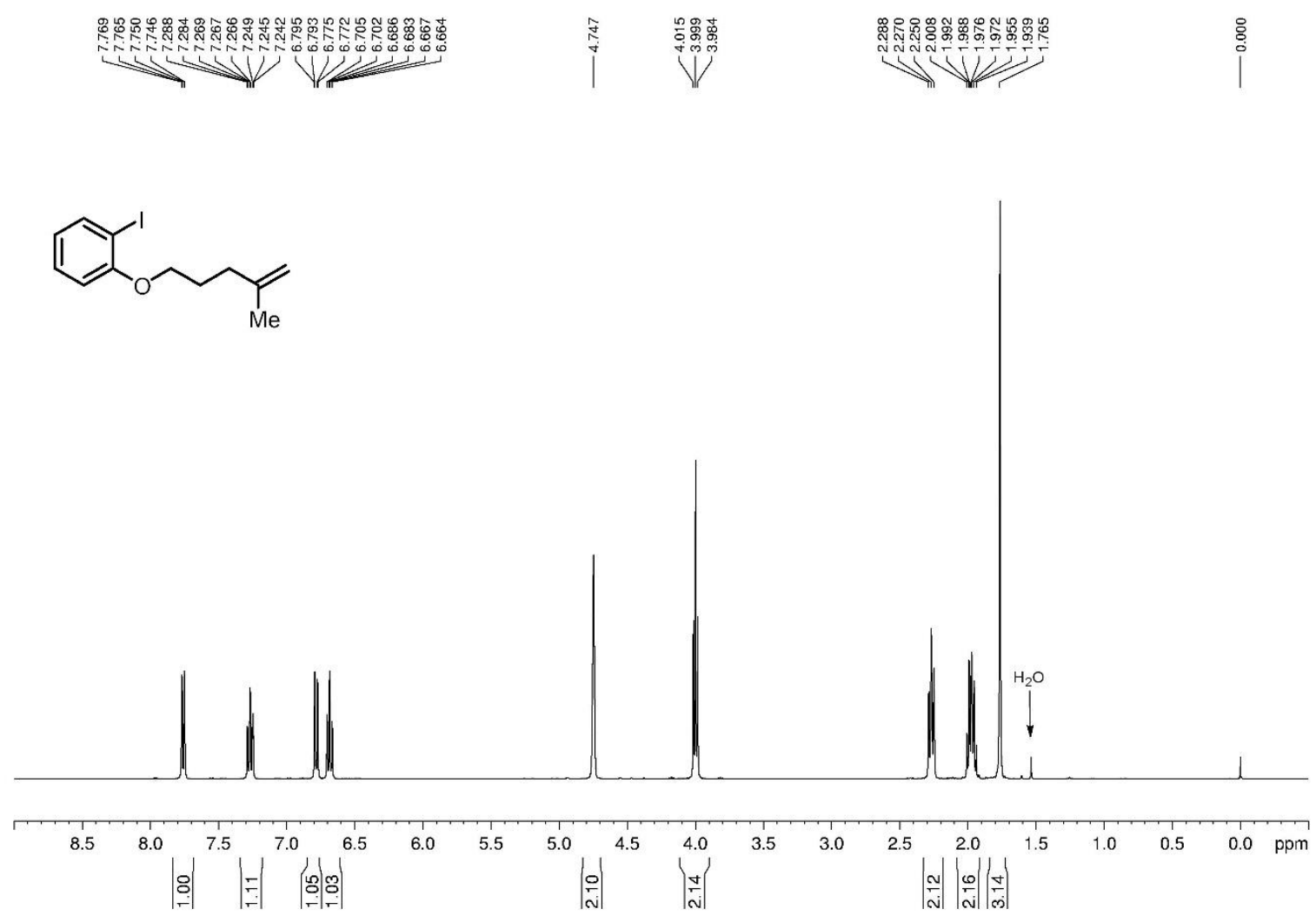

|
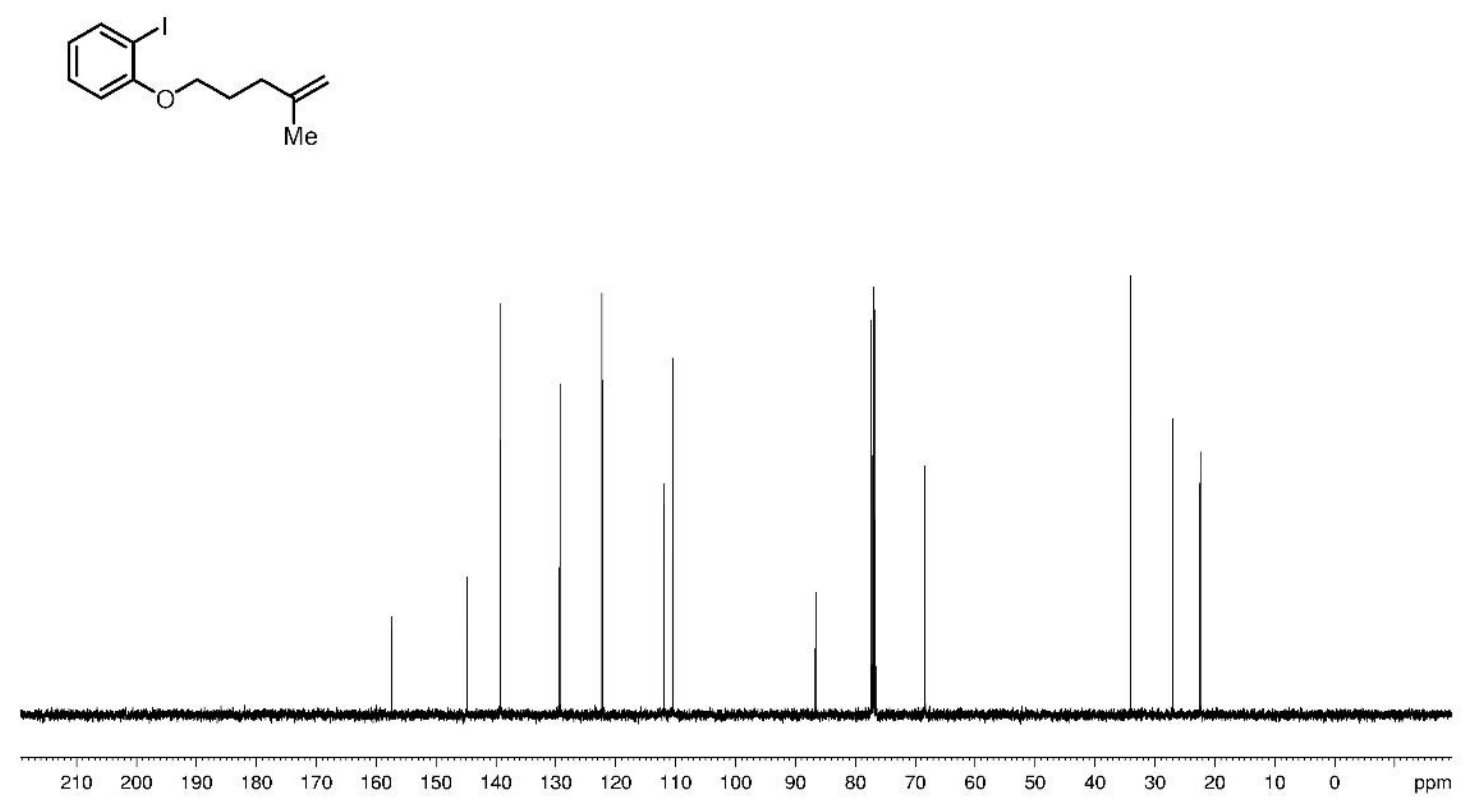
1r-1; ${ }^{1} \mathrm{H}$ NMR (400MHz, $\left.\mathrm{CDCl}_{3}\right) ;{ }^{13} \mathrm{C}$ NMR $\left(100 \mathrm{MHz}, \mathrm{CDCl}_{3}\right)$

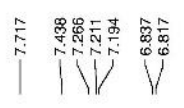

P

$\prod^{\text {糔 }}$

¿
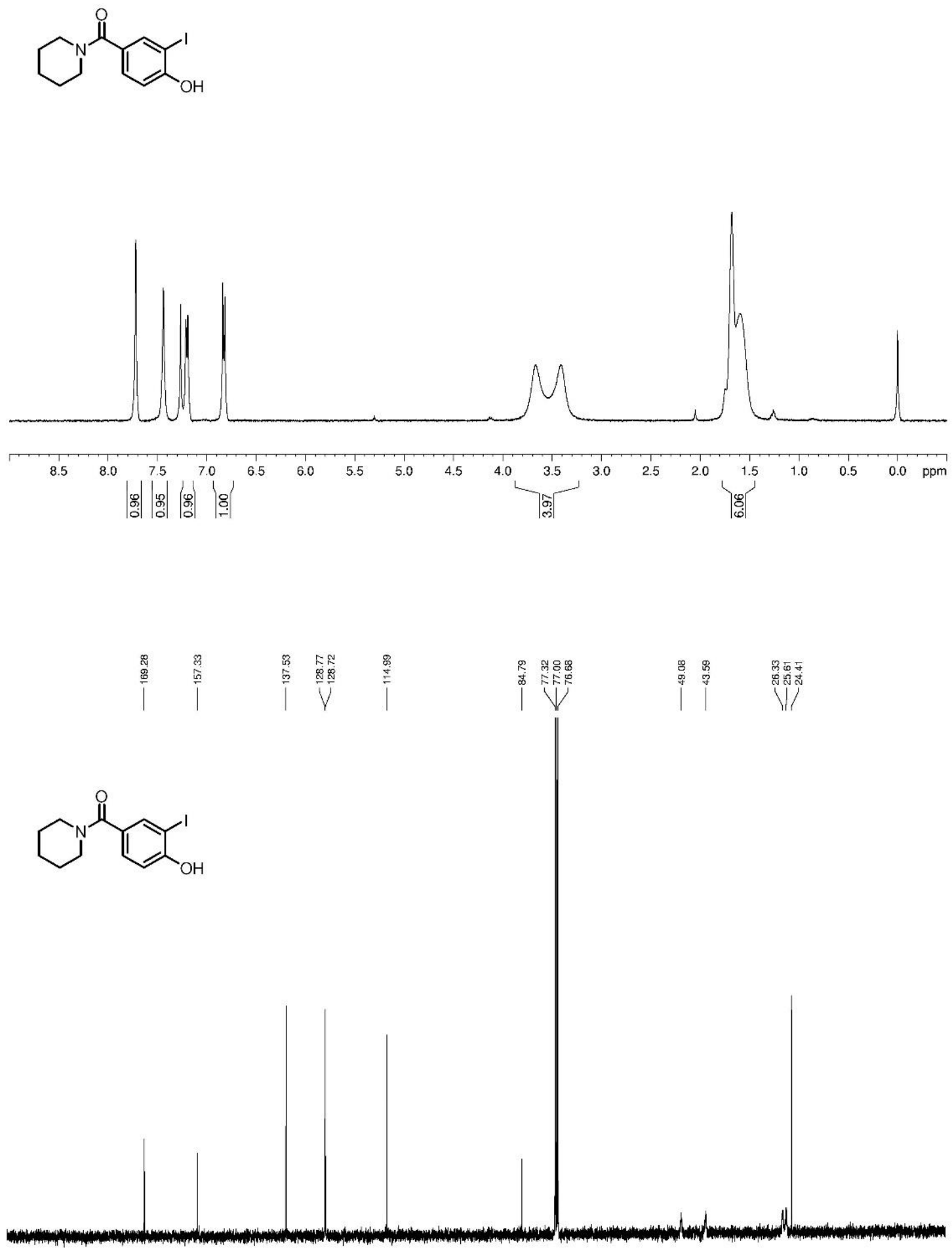

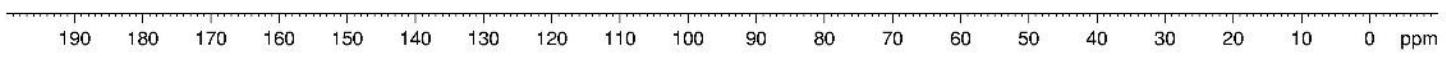

S91 
1r; ${ }^{1} \mathrm{H}$ NMR (400MHz, $\left.\mathrm{CDCl}_{3}\right) ;{ }^{13} \mathrm{C}$ NMR (100MHz, $\left.\mathrm{CDCl}_{3}\right)$

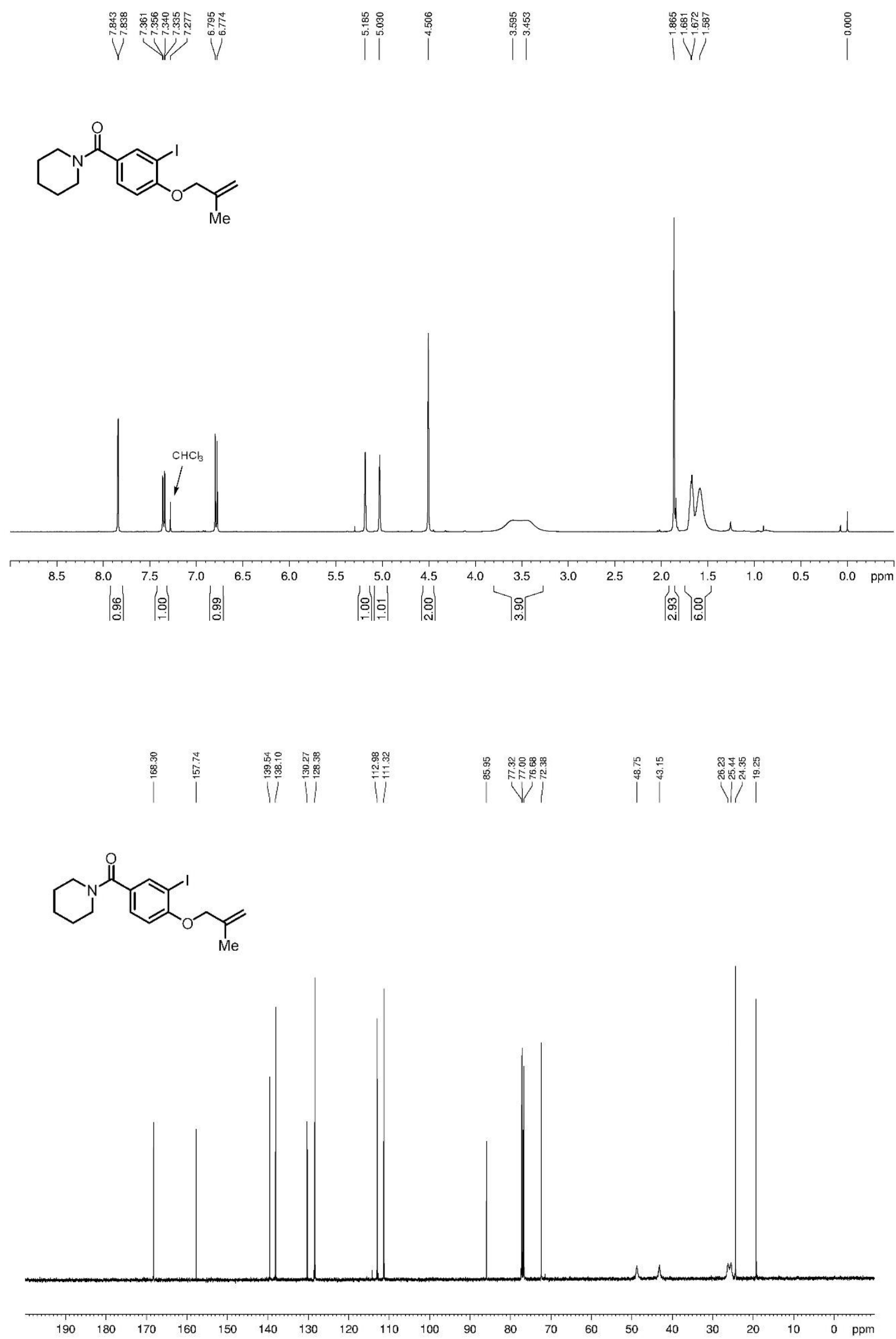


1z; ${ }^{1} \mathrm{H}$ NMR (400MHz, $\left.\mathrm{CDCl}_{3}\right) ;{ }^{13} \mathrm{C}$ NMR (100MHz, $\left.\mathrm{CDCl}_{3}\right)$
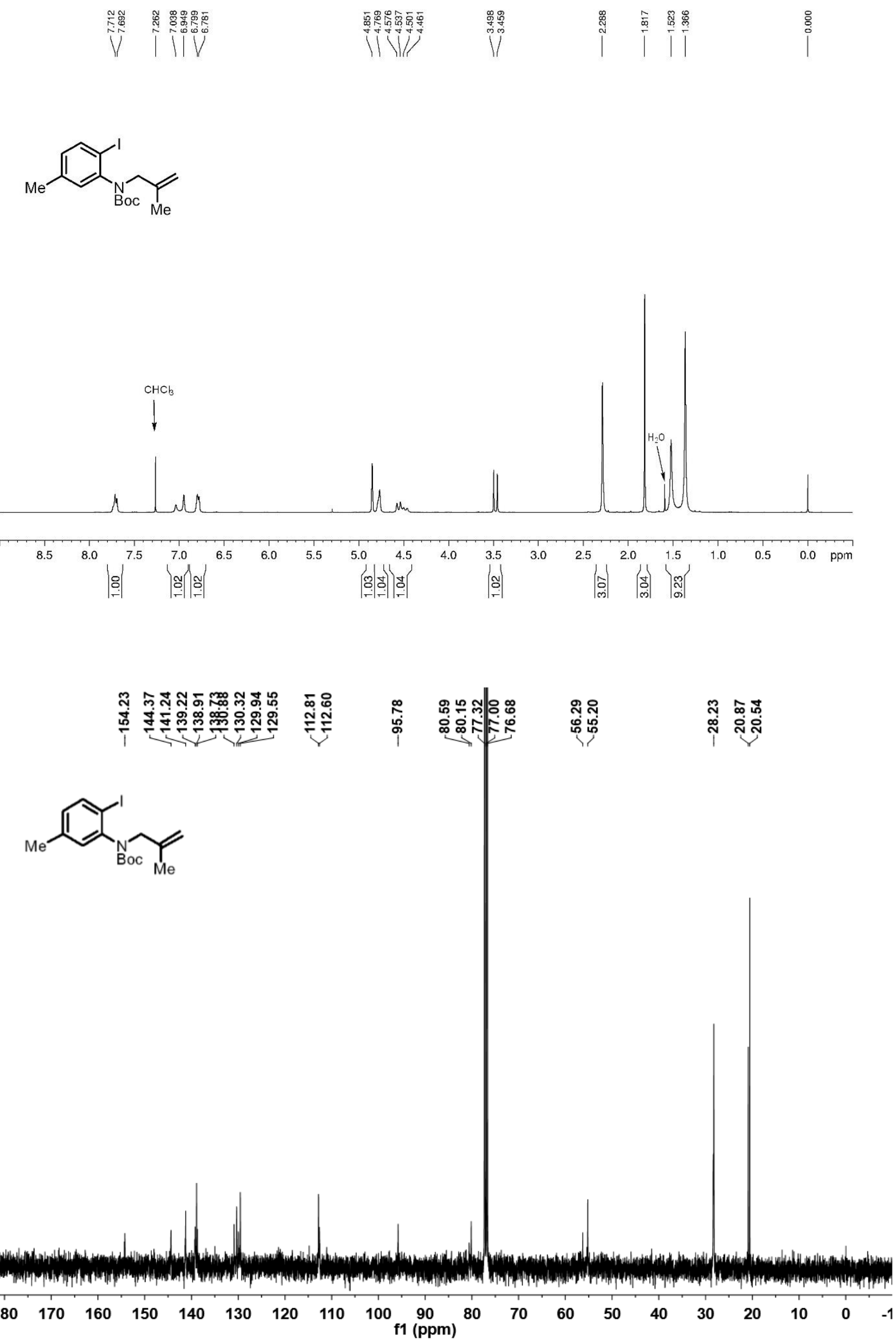
1aa; ${ }^{1} \mathrm{H}$ NMR $\left(400 \mathrm{MHz}, \mathrm{CDCl}_{3}\right) ;{ }^{13} \mathrm{C}$ NMR $\left(100 \mathrm{MHz}, \mathrm{CDCl}_{3}\right)$

\section{I waw}

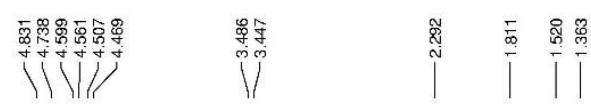<smiles>C=C(C)CNc1ccc(C)cc1I</smiles>

oc
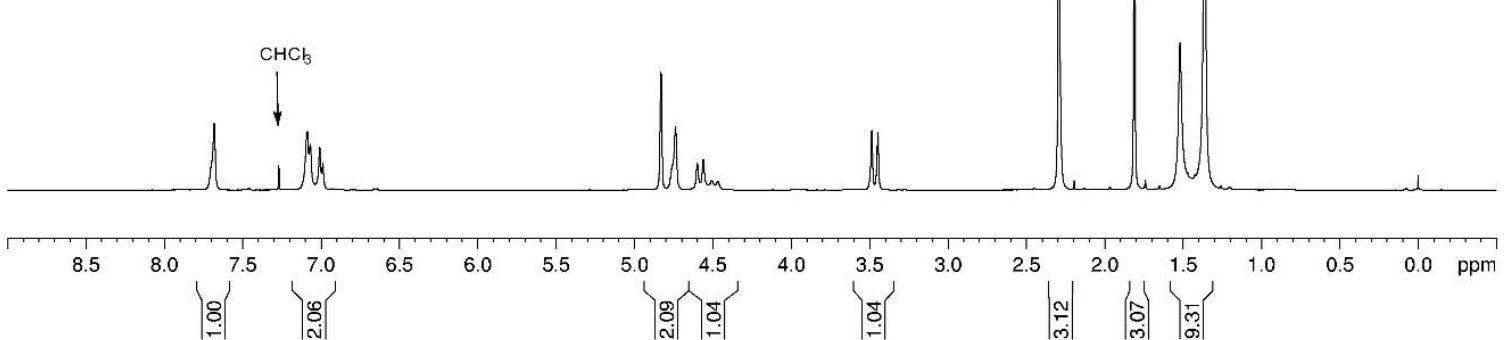

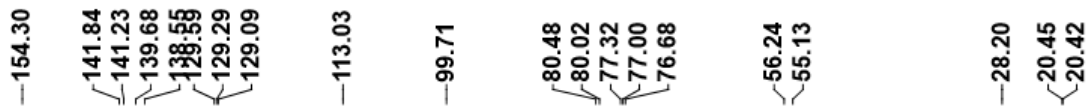
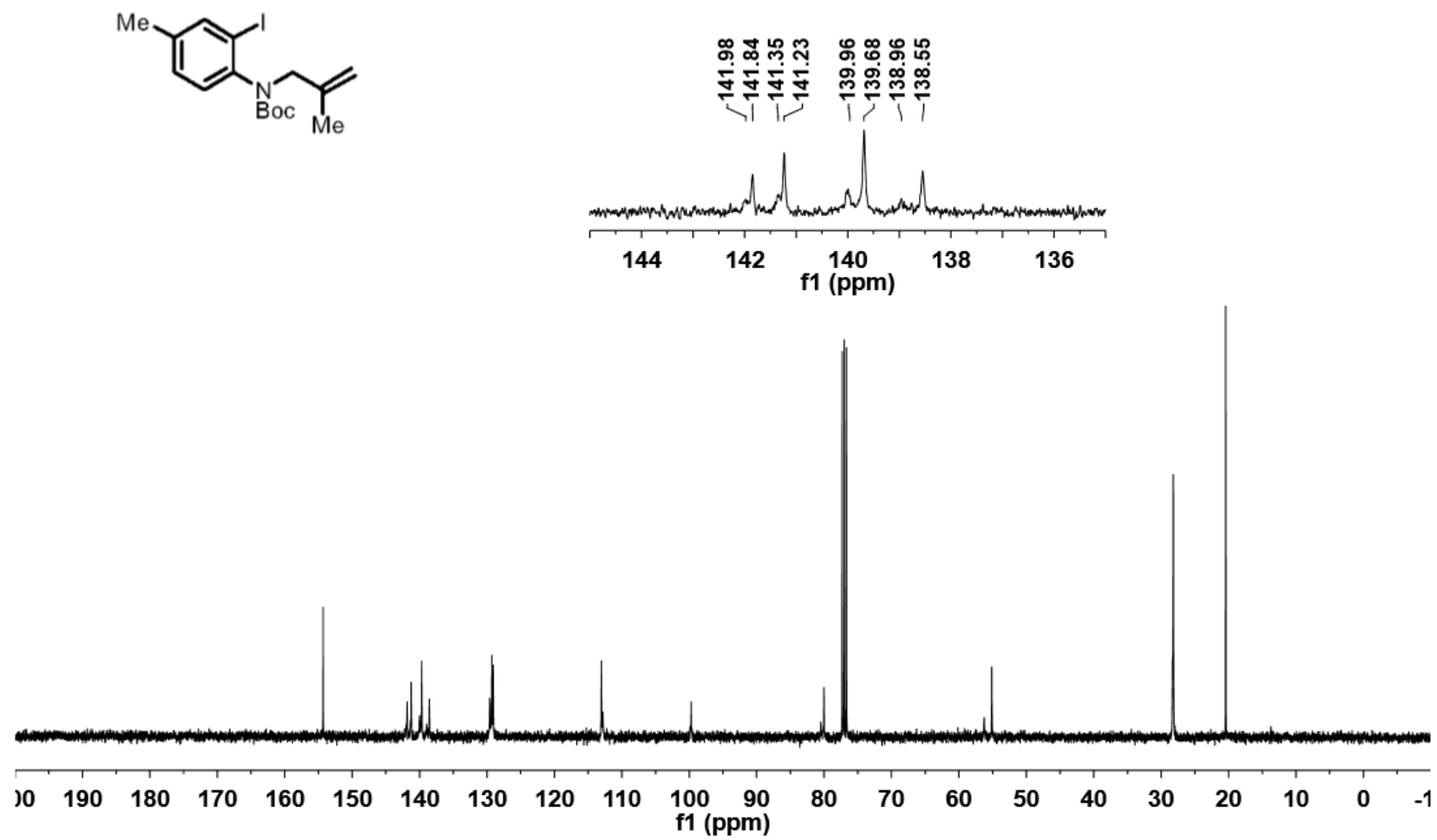
1ab; ${ }^{1} \mathrm{H}$ NMR $\left(400 \mathrm{MHz}, \mathrm{CDCl}_{3}\right) ;{ }^{13} \mathrm{C}$ NMR $\left(100 \mathrm{MHz}, \mathrm{CDCl}_{3}\right)$
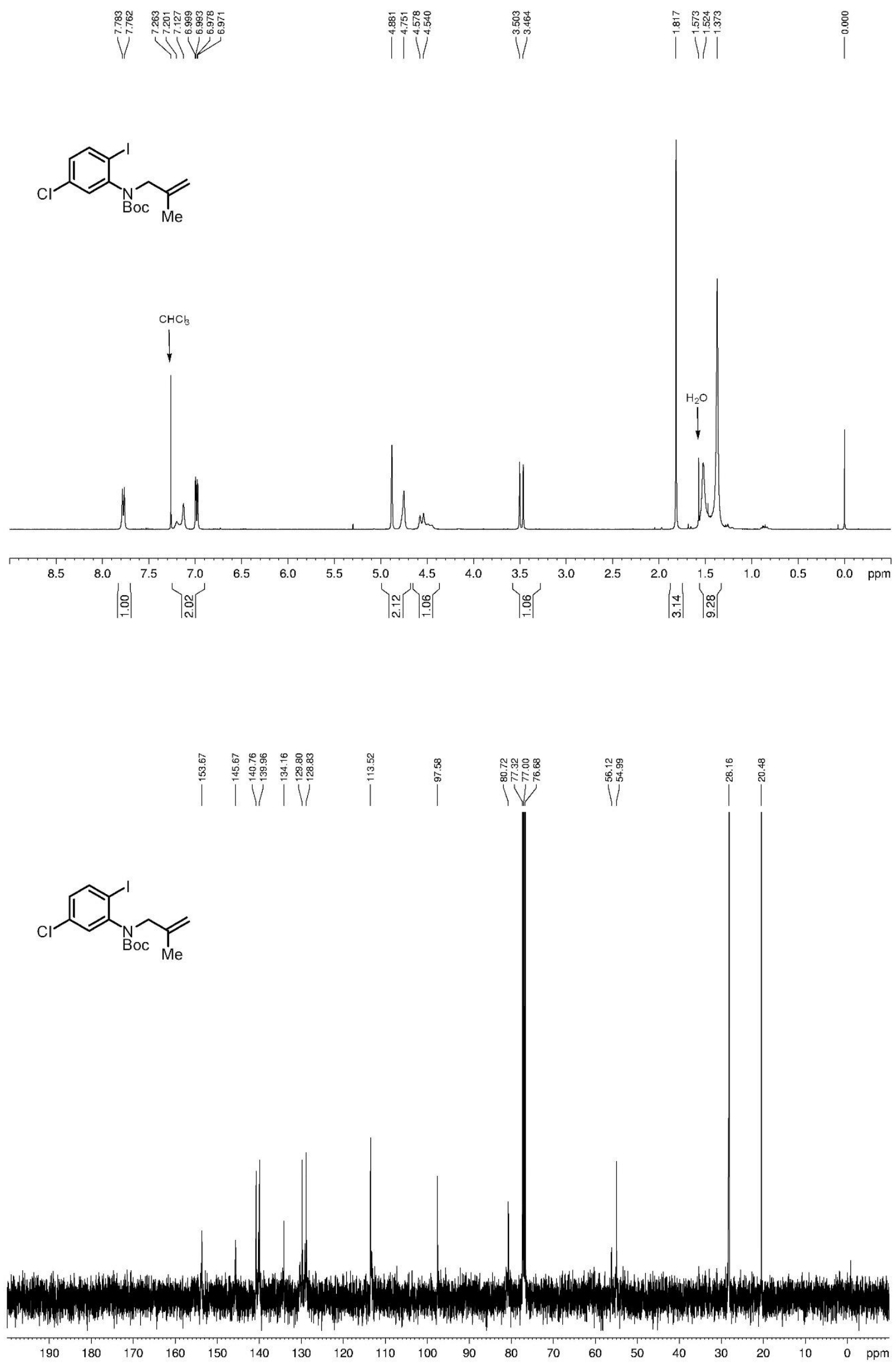
Iad; ${ }^{1} \mathrm{H}$ NMR $\left(400 \mathrm{MHz}, \mathrm{CDCl}_{3}\right) ;{ }^{13} \mathrm{C}$ NMR $\left(100 \mathrm{MHz}, \mathrm{CDCl}_{3}\right)$
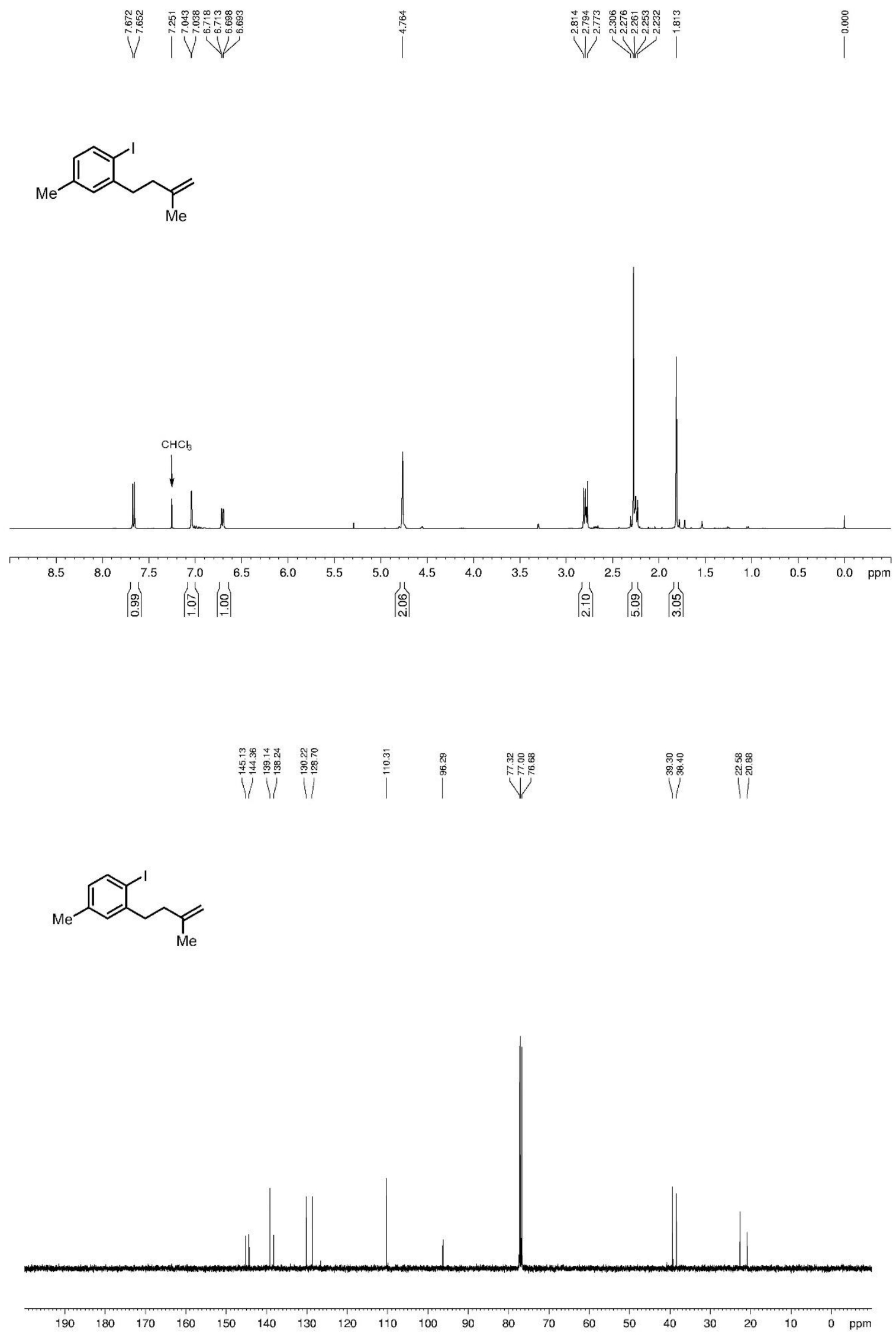
1ae; ${ }^{1} \mathrm{H}$ NMR (400MHz, $\left.\mathrm{CDCl}_{3}\right) ;{ }^{13} \mathrm{C}$ NMR (100MHz, $\left.\mathrm{CDCl}_{3}\right)$

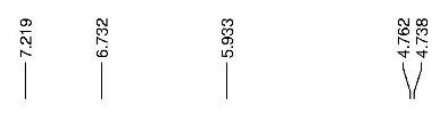

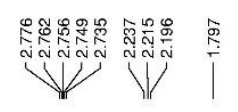

i

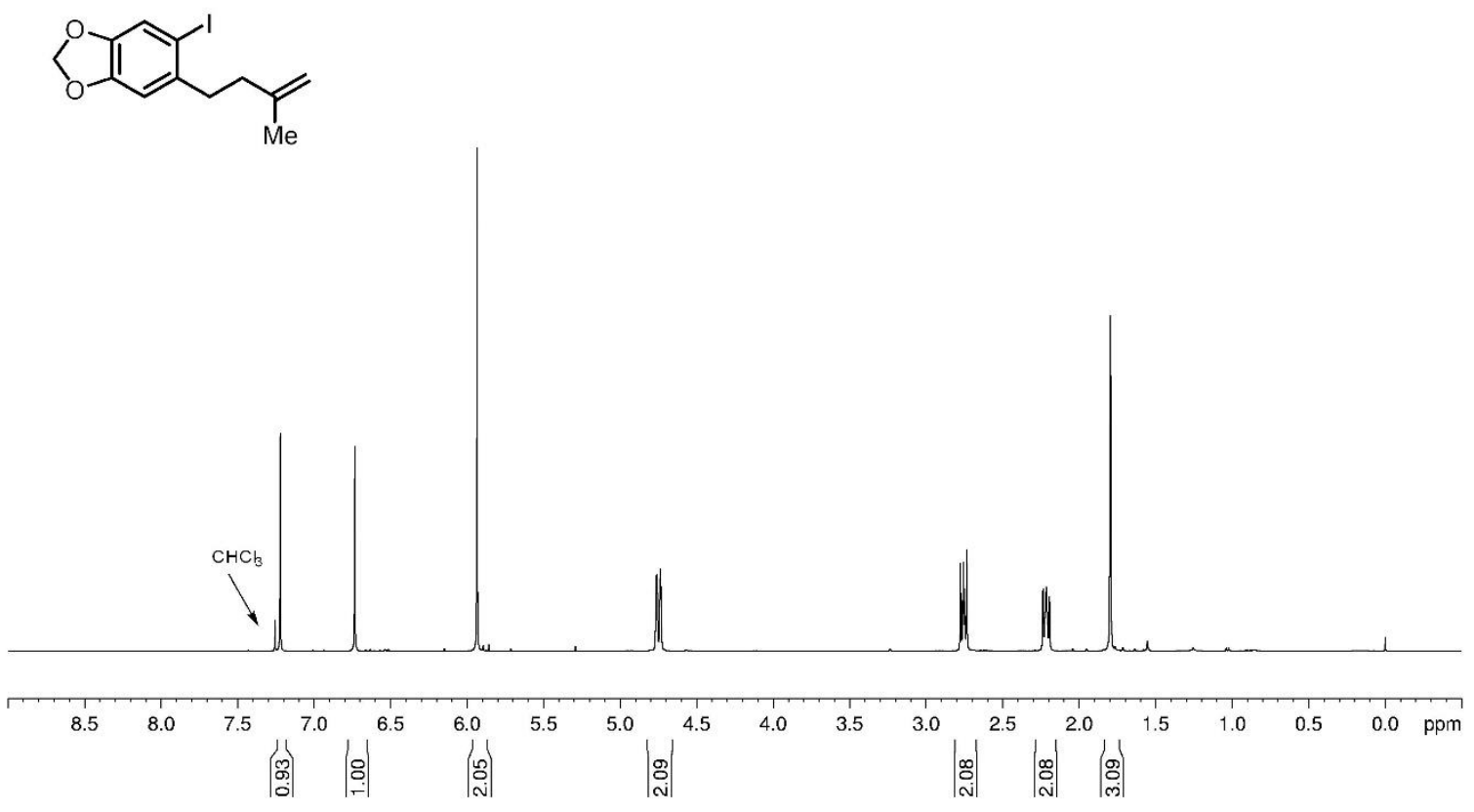

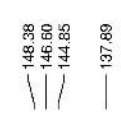
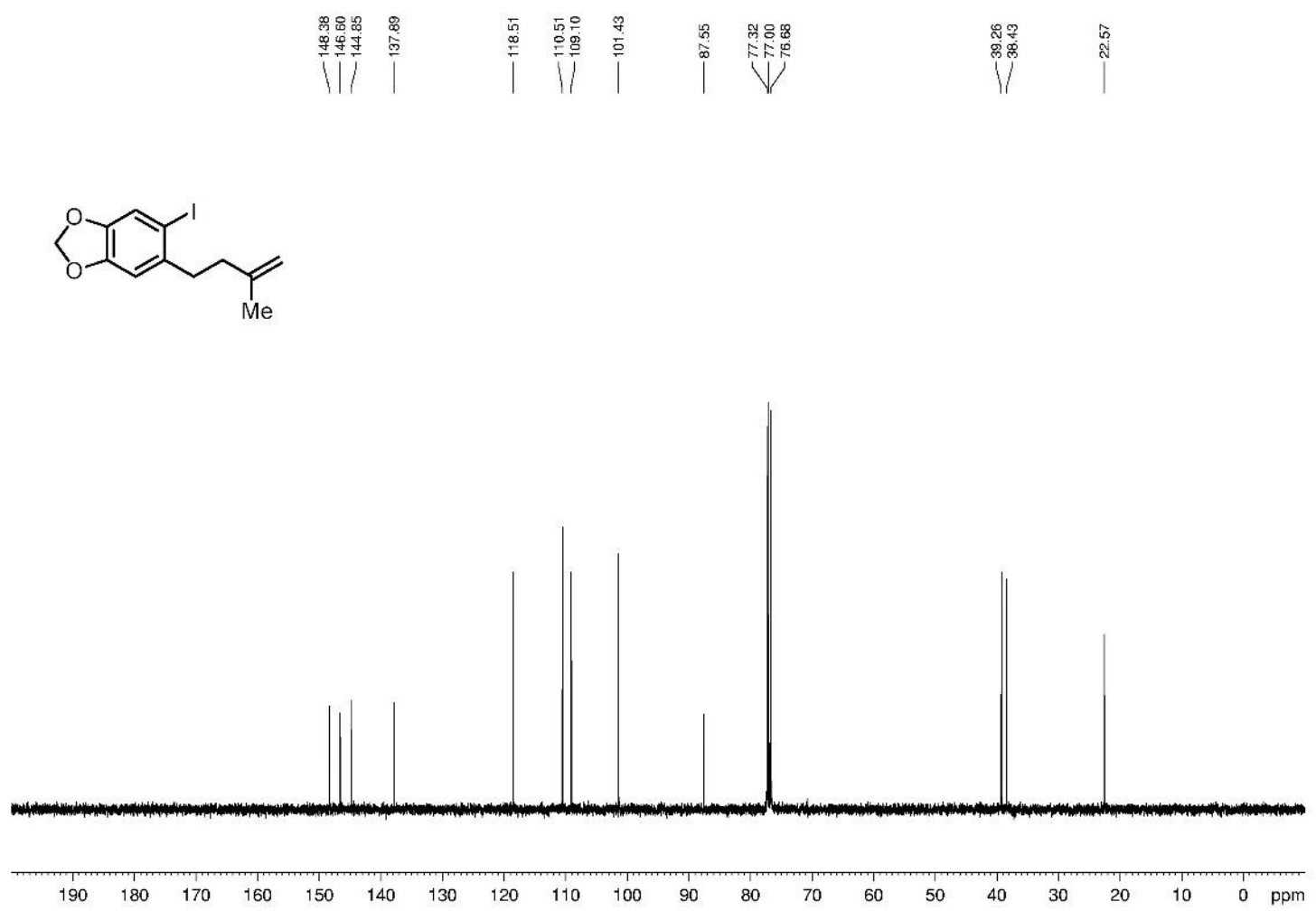

S97 
1af; ${ }^{1} \mathrm{H}$ NMR (400MHz, $\left.\left.\mathrm{CDCl}_{3}\right) ;{ }^{13} \mathrm{C} \mathrm{NMR} \mathrm{(100MHz,} \mathrm{CDCl}_{3}\right)$

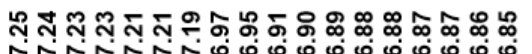

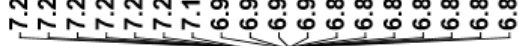

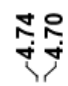

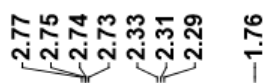

$\stackrel{0}{i}$<smiles>C=C(C)CCc1cc(F)ccc1I</smiles>

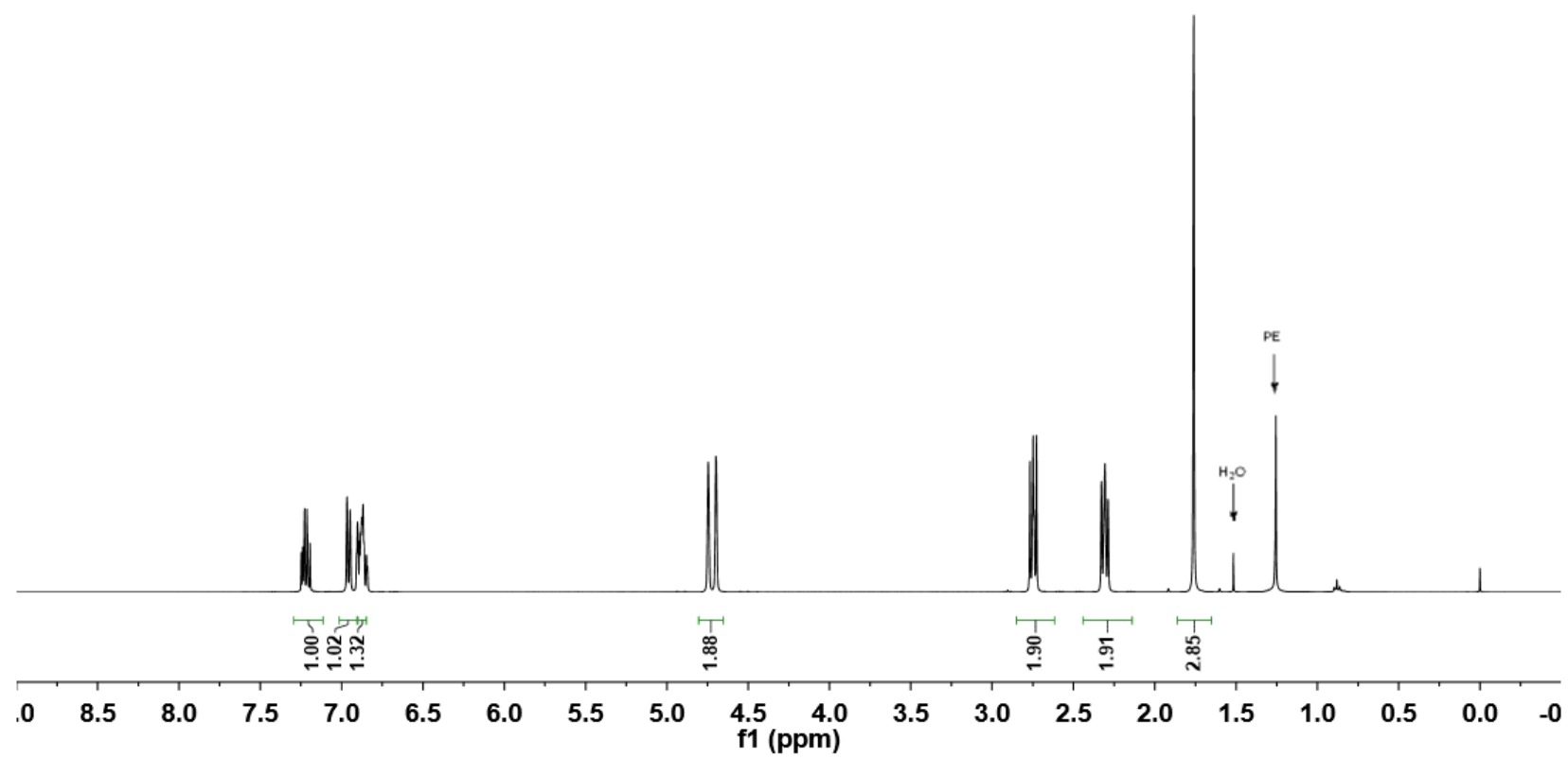

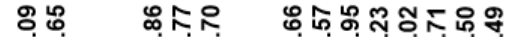

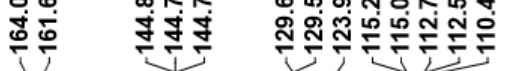

న్లి:

กิ์i

융<smiles>C=C(C)CCc1cc(F)ccc1I</smiles>

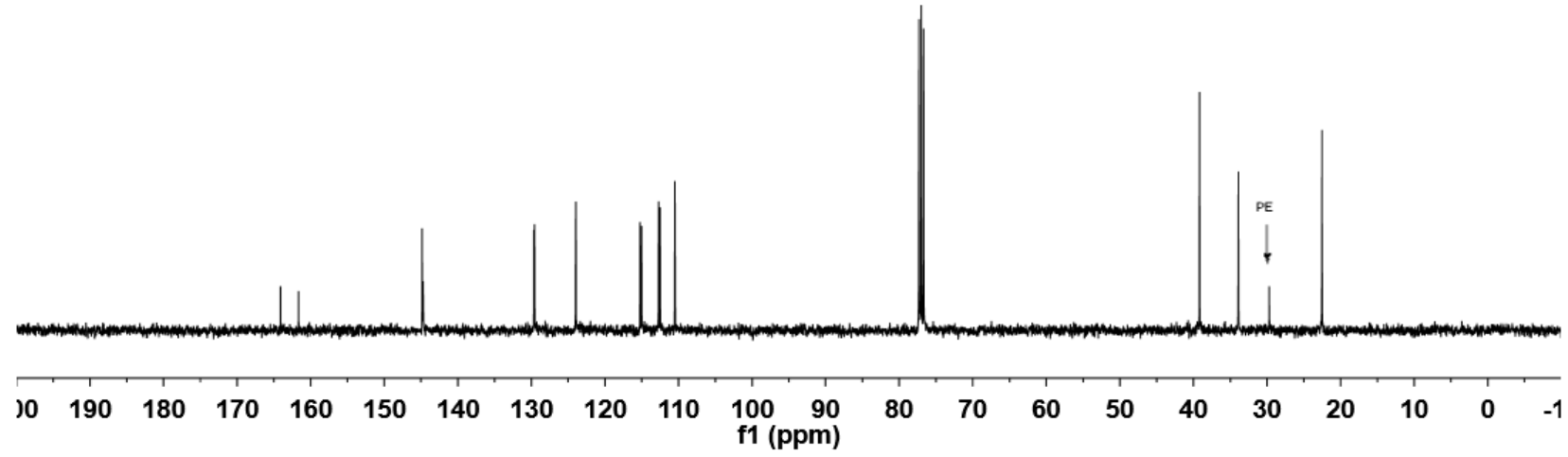


1af; ${ }^{19}$ F NMR (376MHz, $\left.\mathrm{CDCl}_{3}\right)$

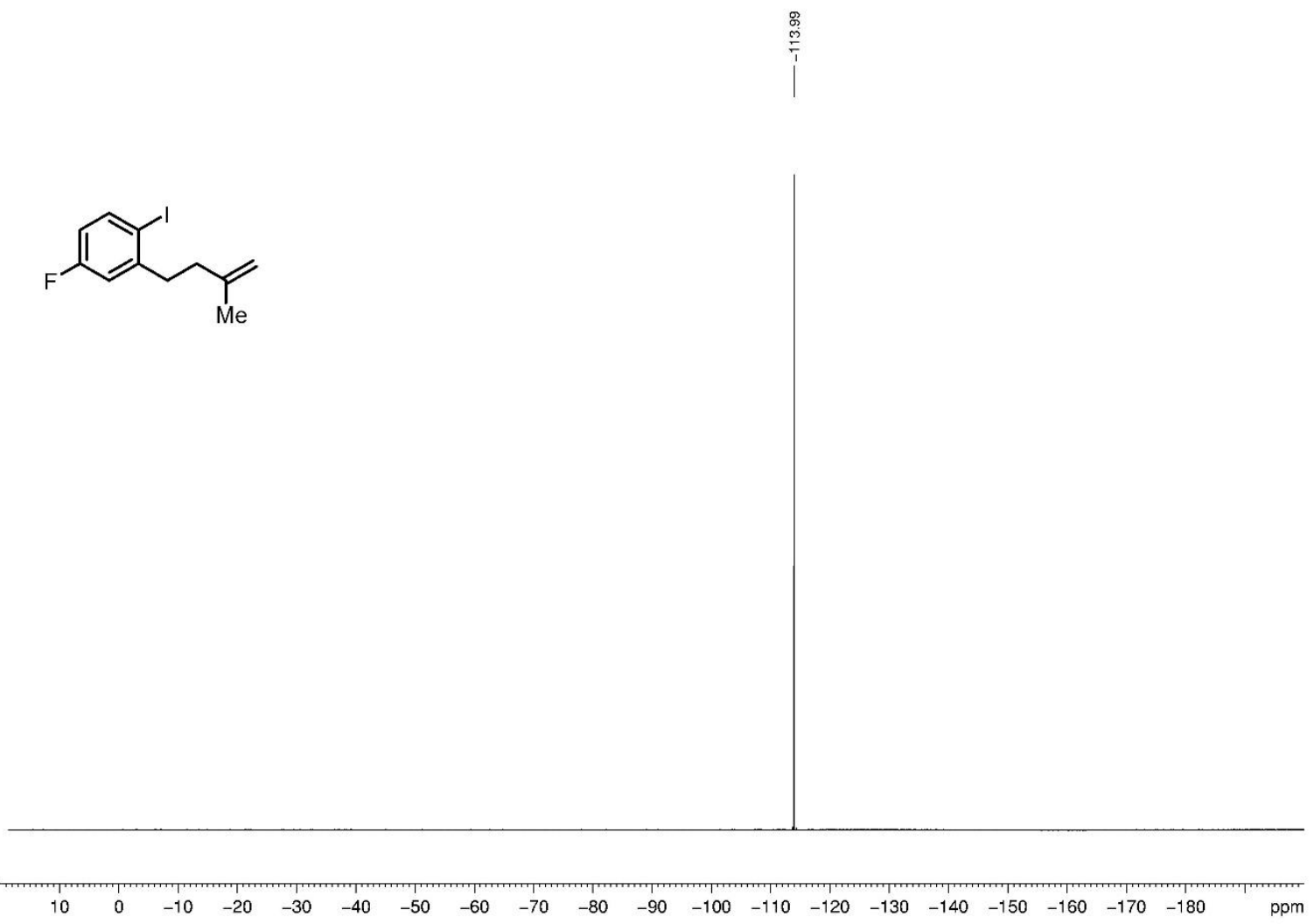


2o; ${ }^{1} \mathrm{H}$ NMR (600MHz, $\left.\mathrm{CDCl}_{3}\right) ;{ }^{13} \mathrm{C}$ NMR $\left(150 \mathrm{MHz}, \mathrm{CDCl}_{3}\right)$

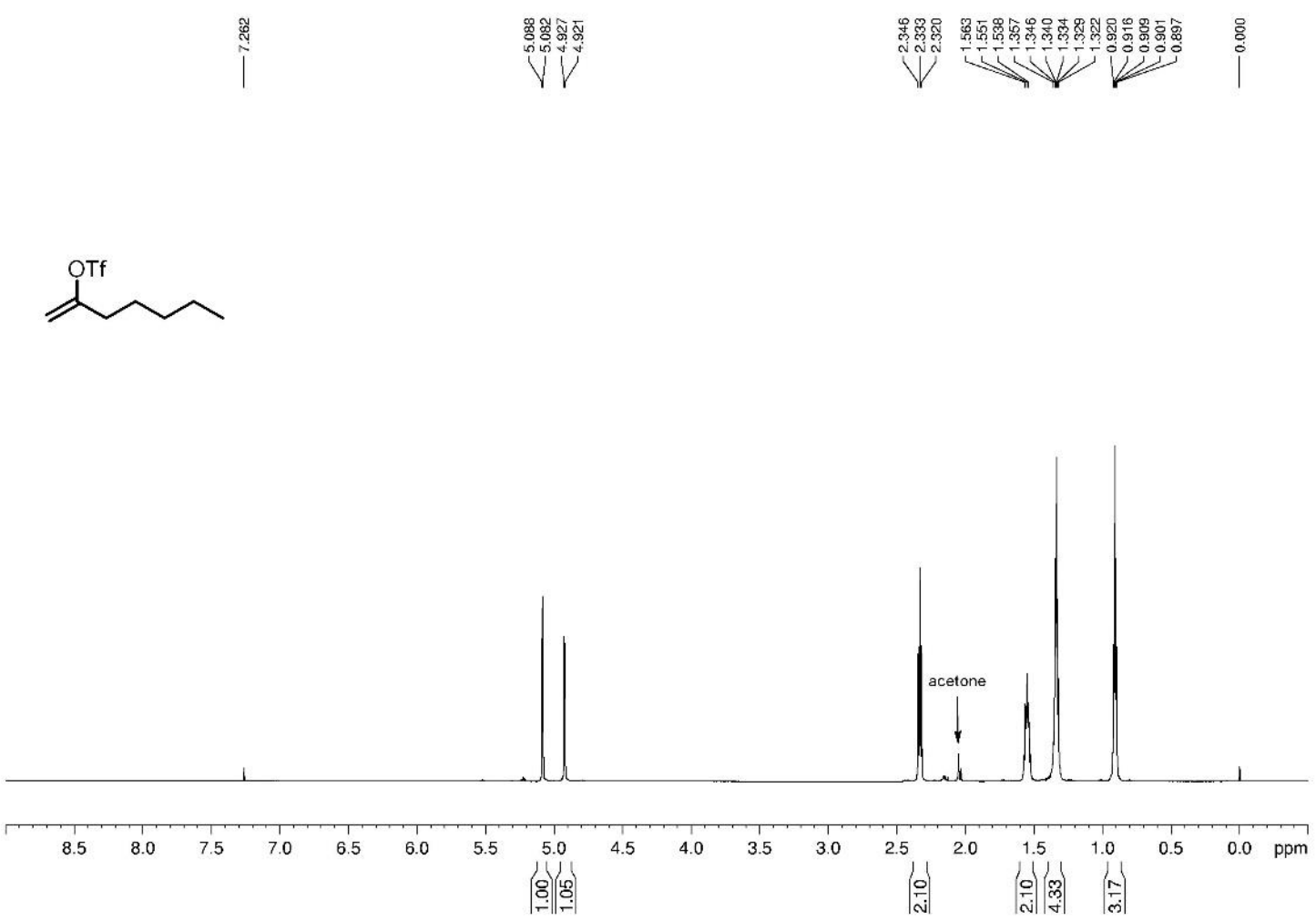

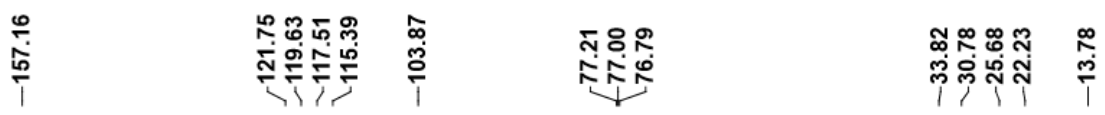

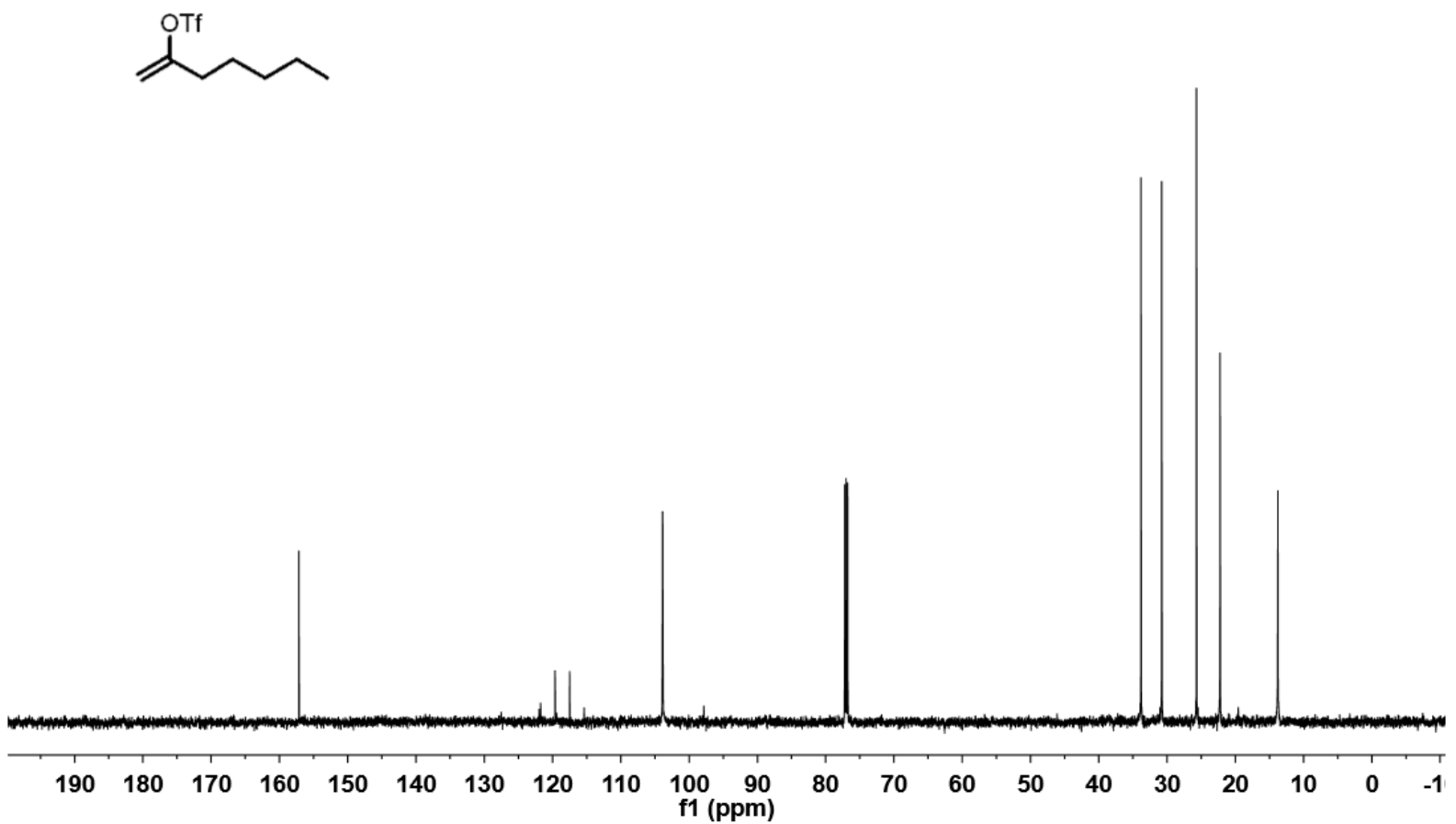


2o; ${ }^{19}$ F NMR (564MHz, $\left.\mathrm{CDCl}_{3}\right)$

$\overbrace{}^{\text {OTf }}$

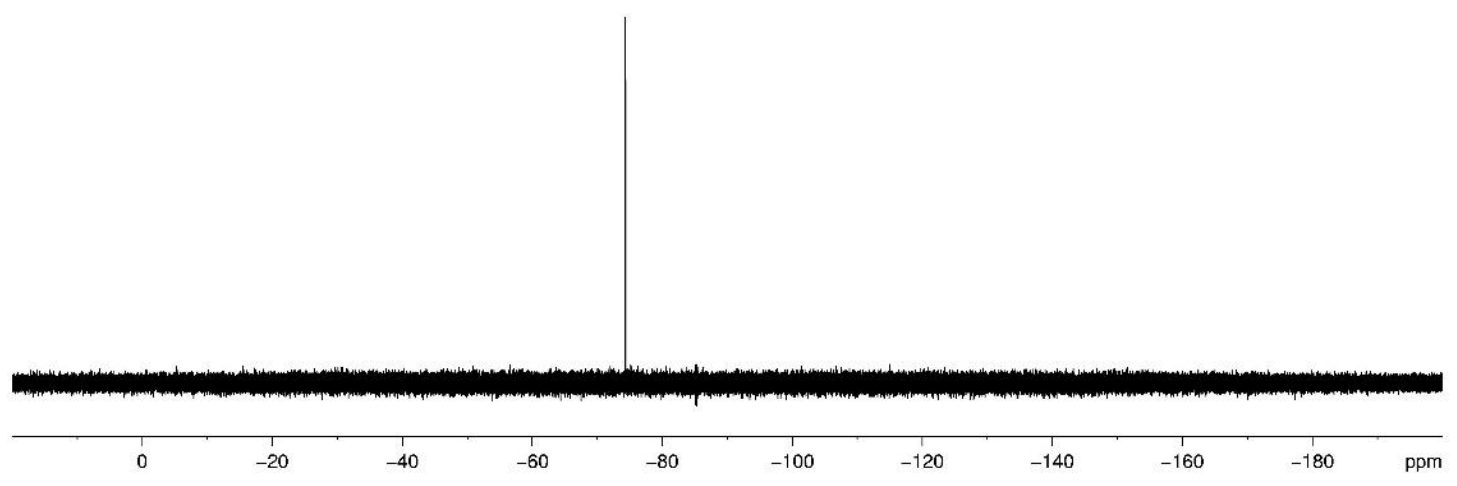


2p; ${ }^{1} \mathrm{H}$ NMR (400MHz, $\left.\mathrm{CDCl}_{3}\right) ;{ }^{13} \mathrm{C}$ NMR (100MHz, $\left.\mathrm{CDCl}_{3}\right)$

i

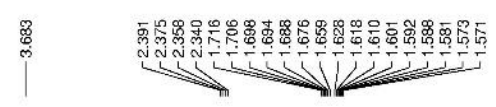

i
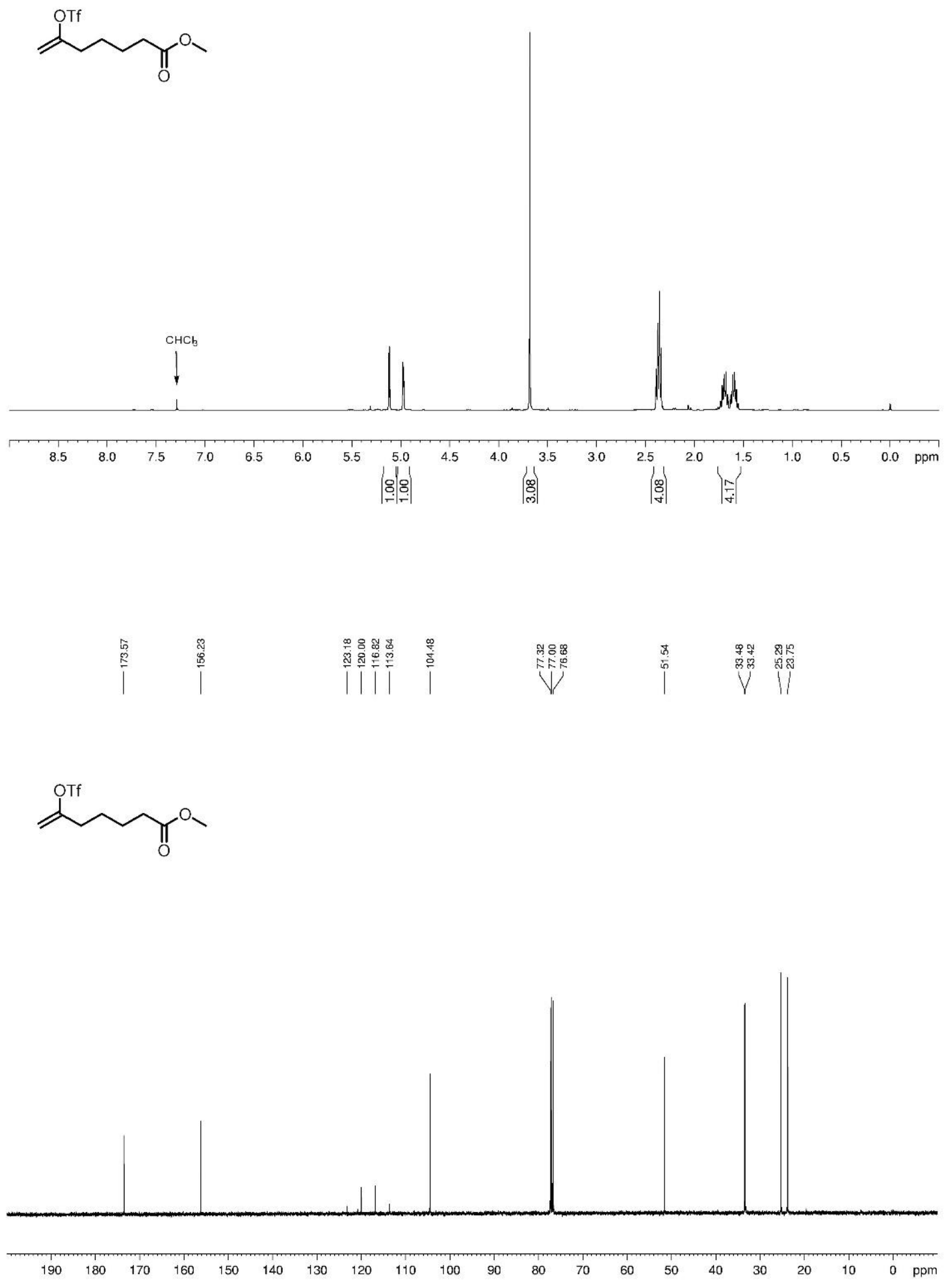

S102 
2p; ${ }^{19}$ F NMR (376MHz, $\left.\mathrm{CDCl}_{3}\right)$

$\overbrace{}^{\text {Tff }}$

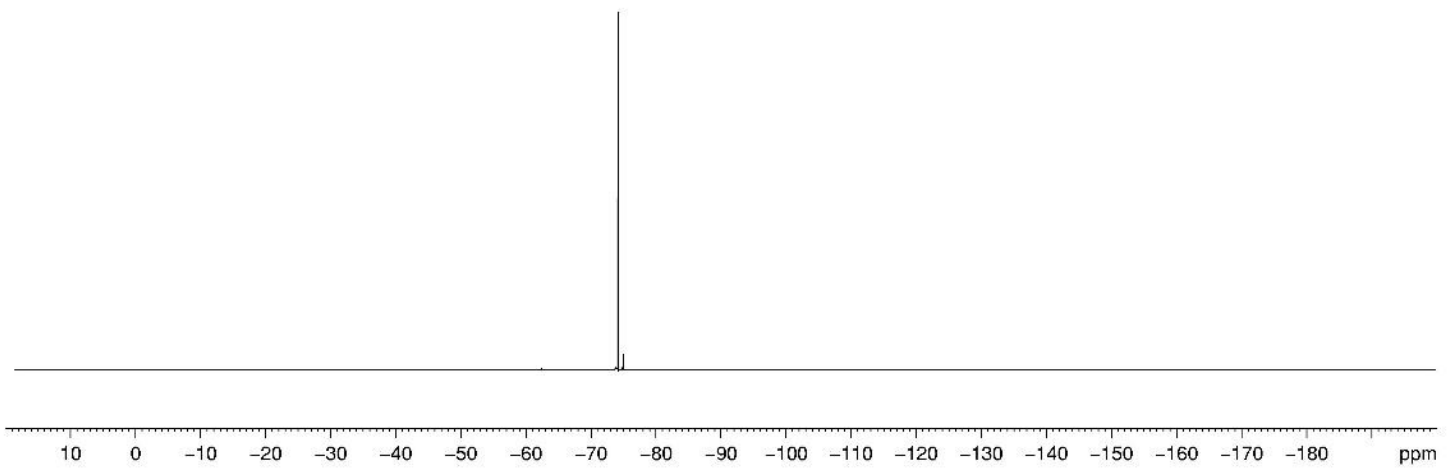


2q; ${ }^{1} \mathrm{H}$ NMR (400MHz, $\left.\mathrm{CDCl}_{3}\right) ;{ }^{13} \mathrm{C}$ NMR (100MHz, $\left.\mathrm{CDCl}_{3}\right)$
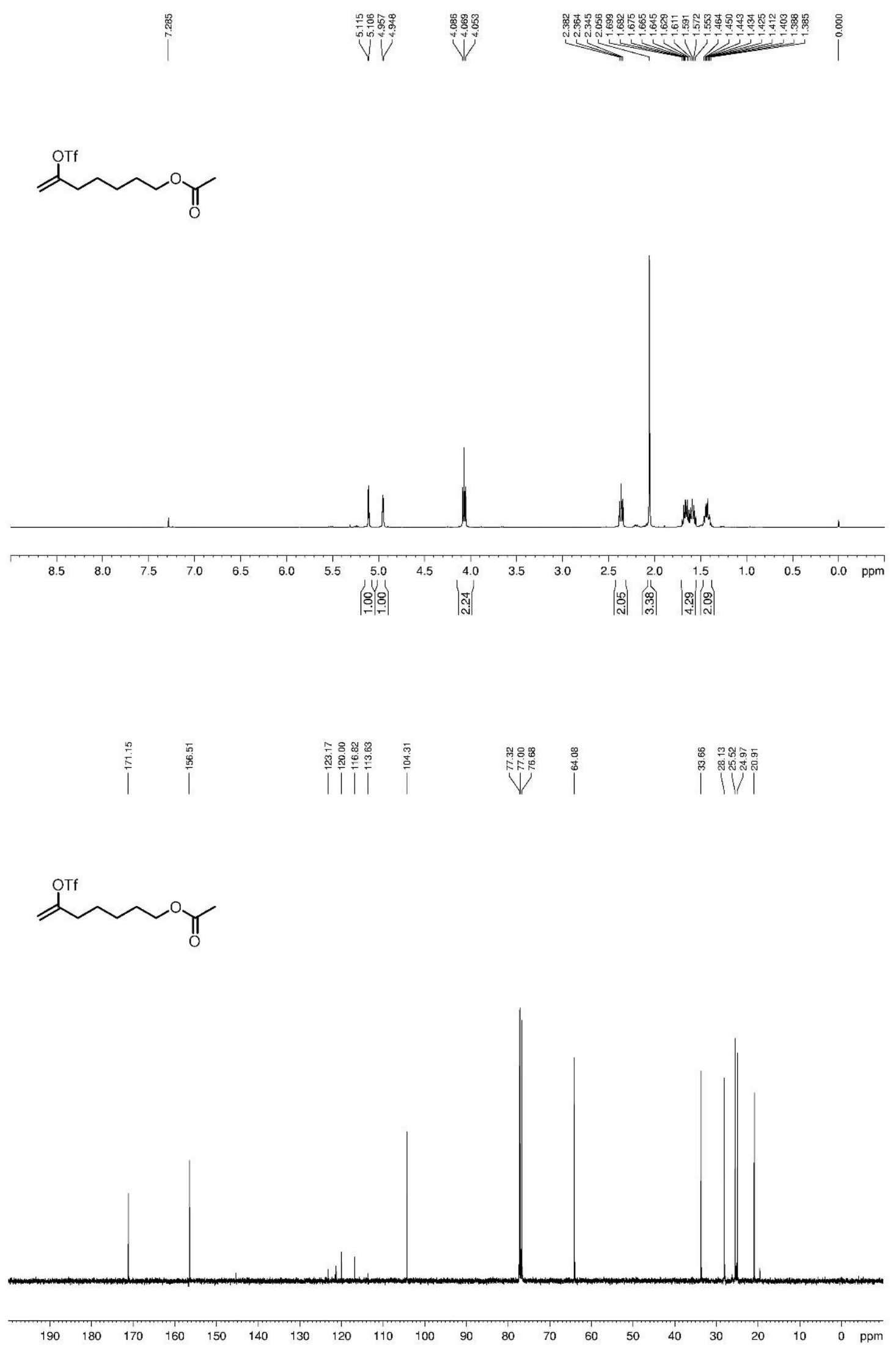

S104 
2q; ${ }^{19}$ F NMR (376MHz, $\left.\mathrm{CDCl}_{3}\right)$

放

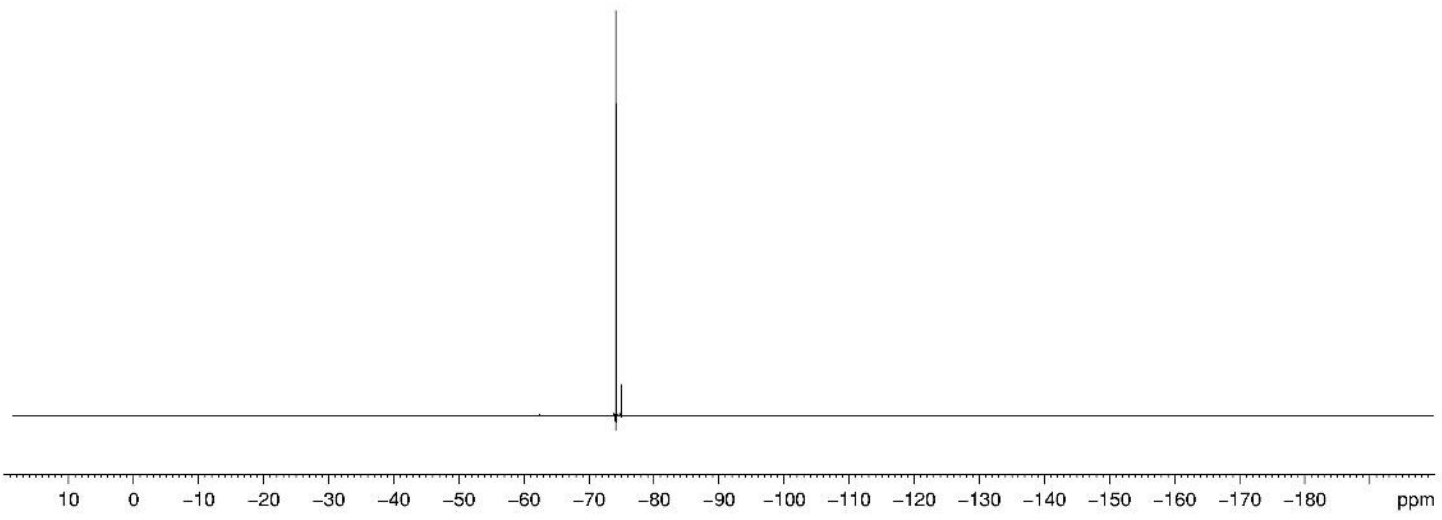


2w-1; ${ }^{1} \mathrm{H}$ NMR (400MHz, $\left.\mathrm{CDCl}_{3}\right) ;{ }^{13} \mathrm{C}$ NMR (100MHz, $\left.\mathrm{CDCl}_{3}\right)$

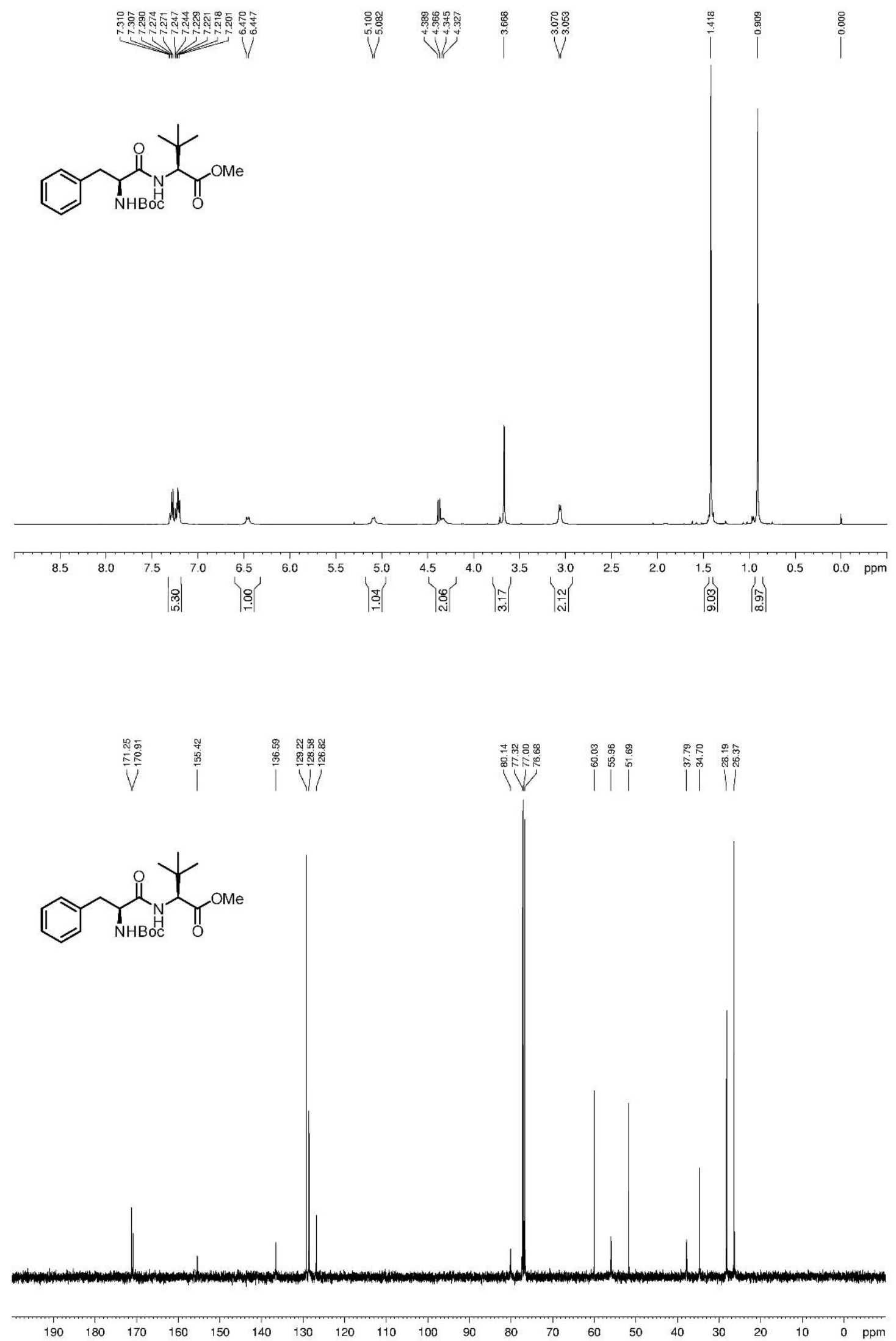


2w; ${ }^{1} \mathrm{H}$ NMR (400MHz, $\left.\mathrm{CDCl}_{3}\right) ;{ }^{13} \mathrm{C}$ NMR (100MHz, $\left.\mathrm{CDCl}_{3}\right)$
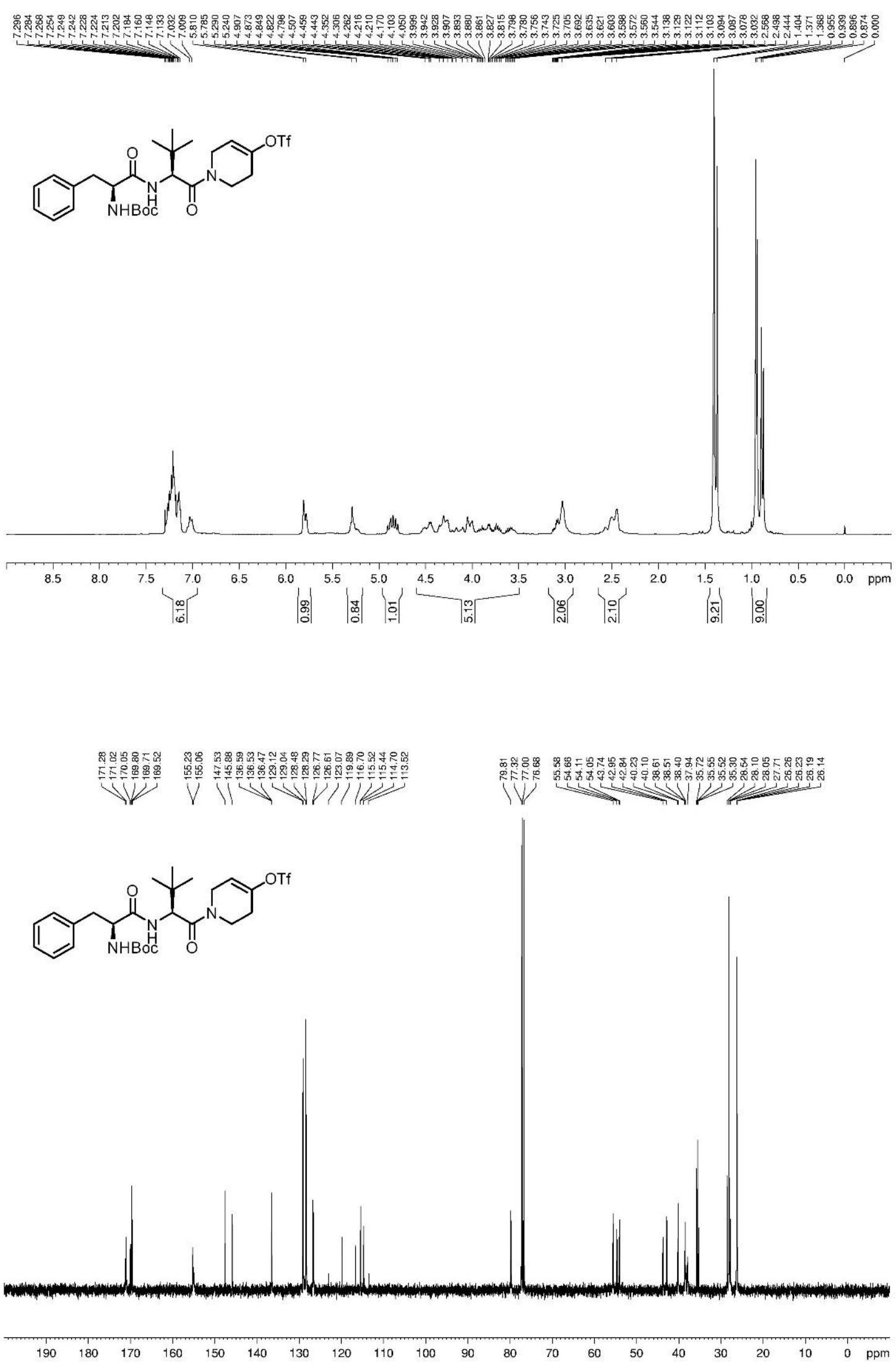
2w; ${ }^{19}$ F NMR (376MHz, $\left.\mathrm{CDCl}_{3}\right)$

Vִ<smiles>CCCC1=CCN(C(=O)C(NC(=O)C(N)Cc2ccccc2)C(C)(C)C)CC1</smiles><smiles>C1=C[As][As]1</smiles>

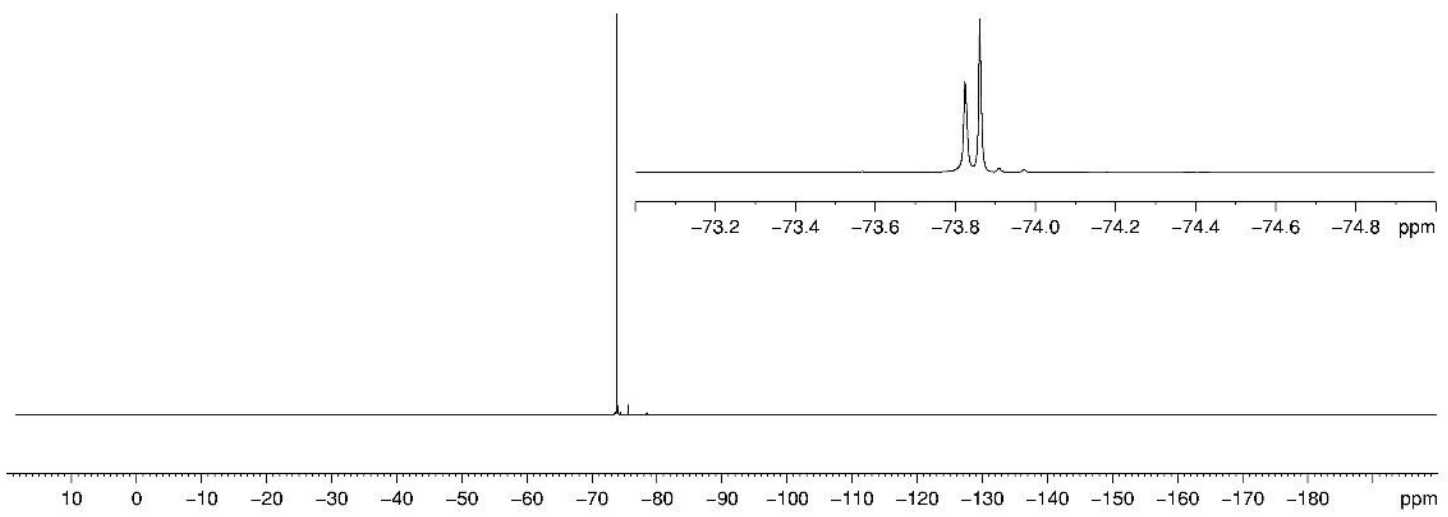


2x-1; ${ }^{1} \mathrm{H}$ NMR (400MHz, $\left.\mathrm{CDCl}_{3}\right) ;{ }^{13} \mathrm{C}$ NMR (100MHz, $\left.\mathrm{CDCl}_{3}\right)$

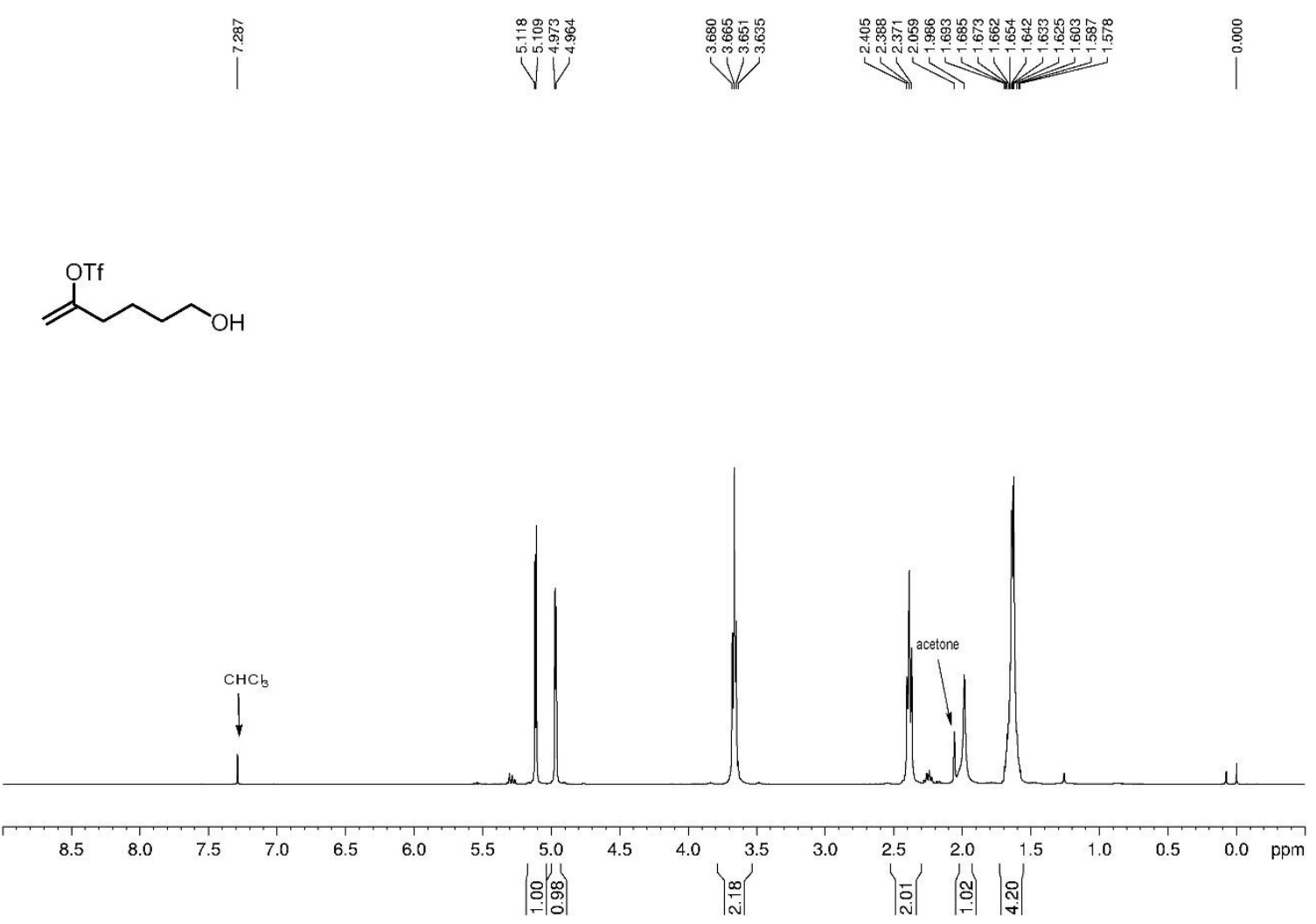

|

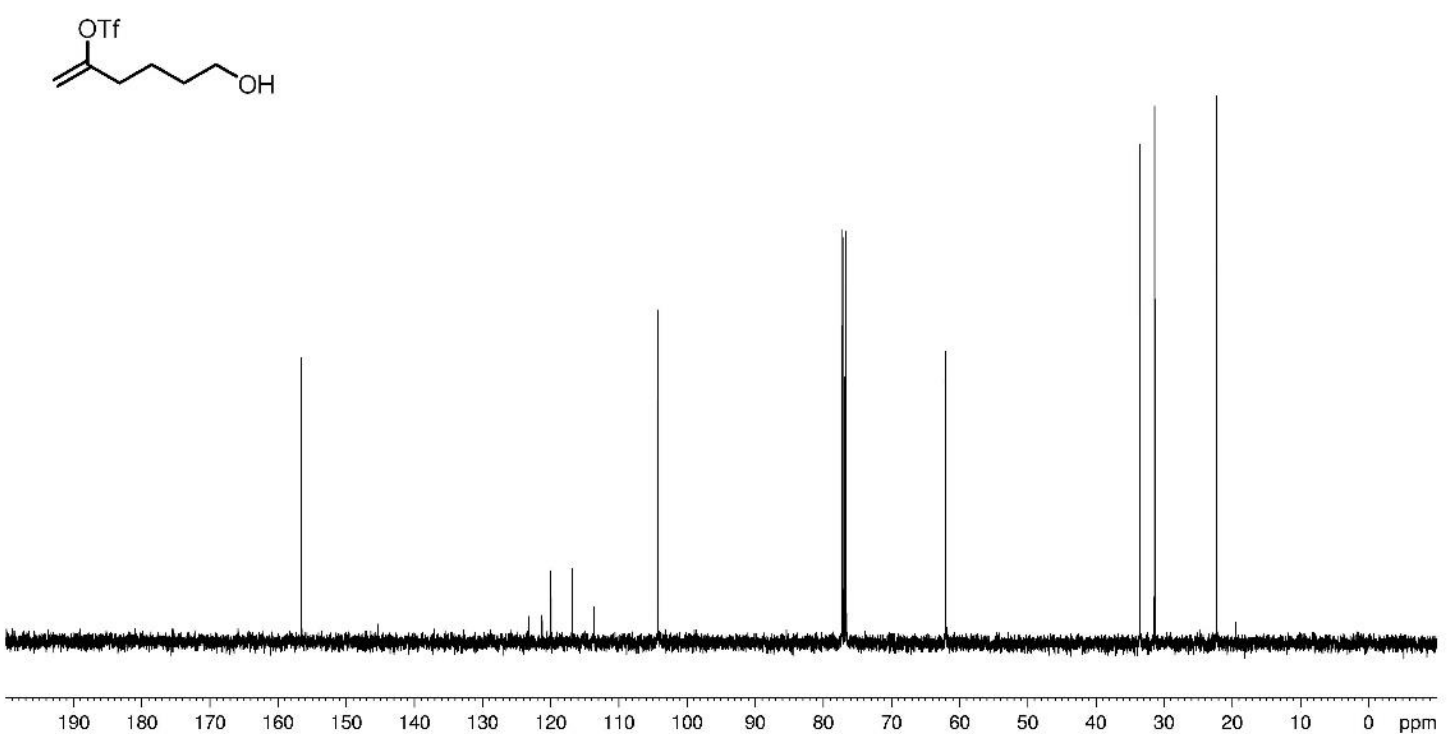


2x-1; ${ }^{19}$ F NMR (376MHz, $\left.\mathrm{CDCl}_{3}\right)$

$\overbrace{}^{\mathrm{OTf}}$

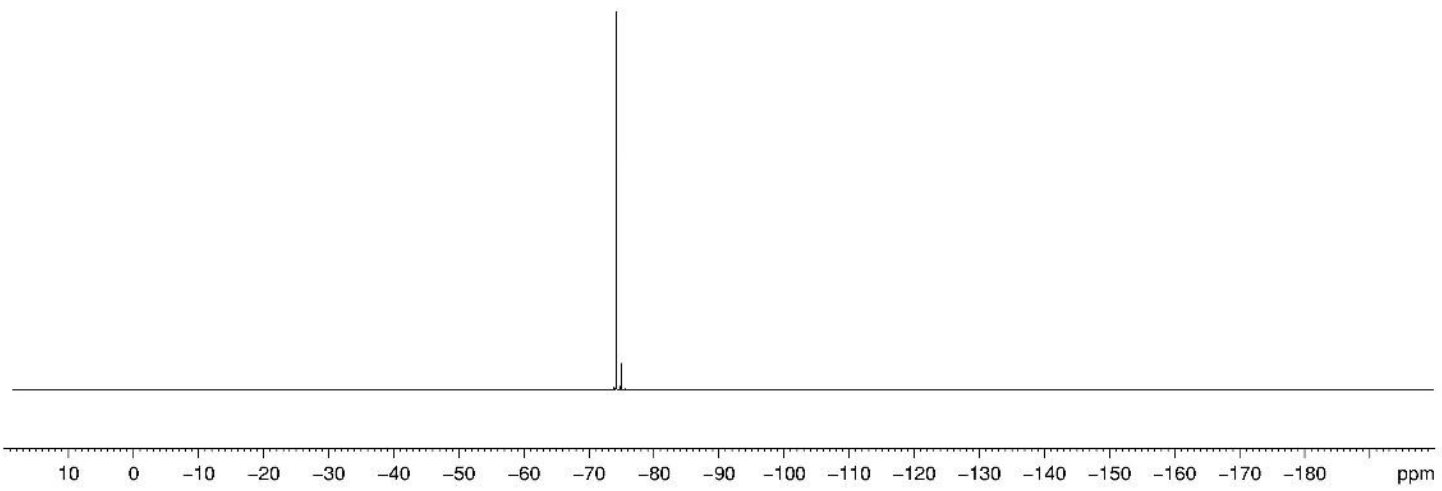


$2 \mathrm{x} ;{ }^{1} \mathrm{H}$ NMR (400MHz, $\left.\mathrm{CDCl}_{3}\right) ;{ }^{13} \mathrm{C}$ NMR (100MHz, $\left.\mathrm{CDCl}_{3}\right)$

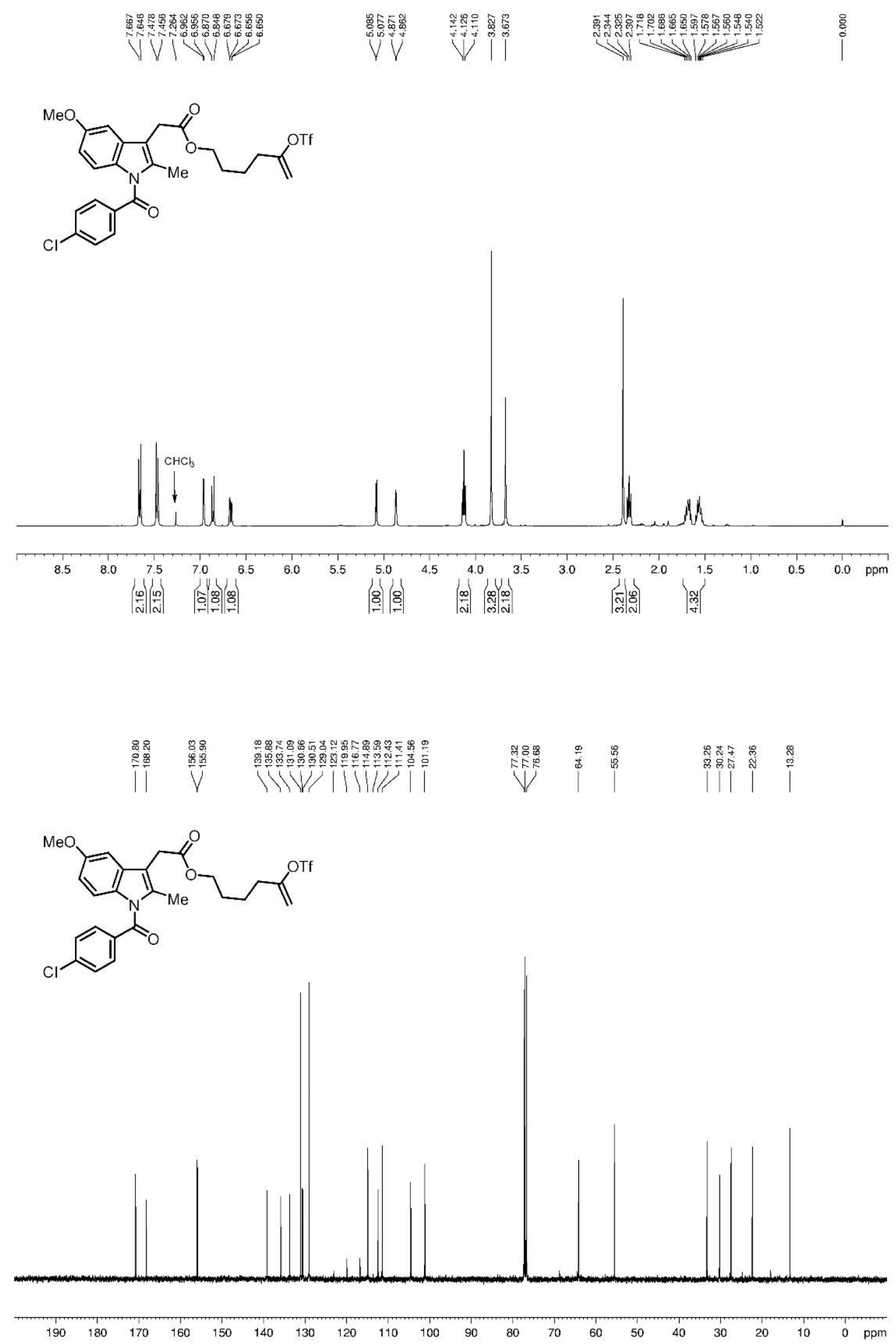


$2 \mathrm{x} ;{ }^{19}$ F NMR (376MHz, $\left.\mathrm{CDCl}_{3}\right)$<smiles>C=C(CCCCOC(=O)Cc1c(C=O)n(C(=O)c2ccc(Cl)cc2)c2ccc(OC)cc12)OC</smiles>

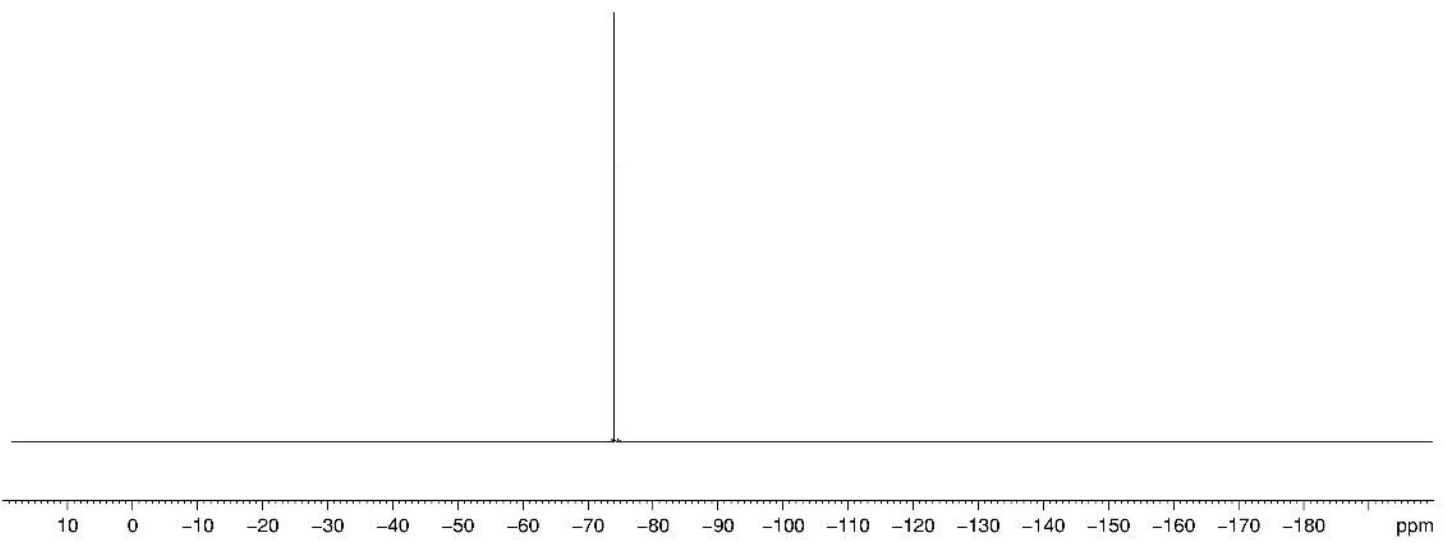


$2 \mathrm{y} ;{ }^{1} \mathrm{H}$ NMR $\left(600 \mathrm{MHz}, \mathrm{CDCl}_{3}\right) ;{ }^{13} \mathrm{C} \mathrm{NMR}\left(150 \mathrm{MHz}, \mathrm{CDCl}_{3}\right)$

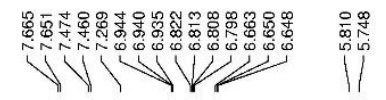<smiles>COc1ccc2c(c1)c1c(n2C(=O)c2ccc(Cl)cc2)C2CC(O)=CCN2C(=O)C1</smiles>
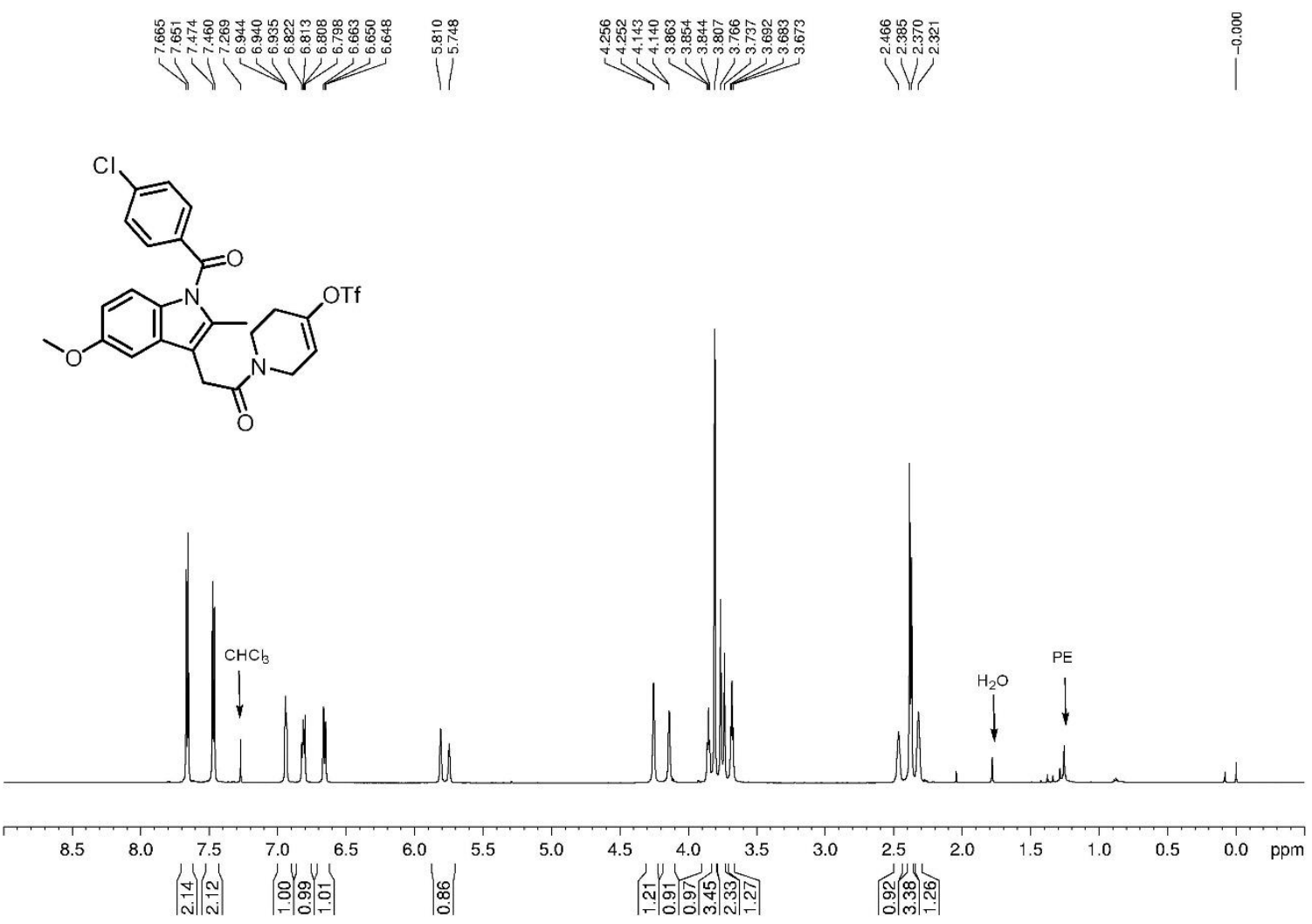

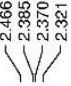

\section{$\stackrel{8}{\circ}$} . 
$2 \mathrm{y} ;{ }^{19} \mathrm{~F}$ NMR (376MHz, $\left.\mathrm{CDCl}_{3}\right)$

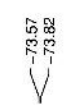<smiles>COc1ccc2c(c1)CC(=O)N1CC=C(O)CC21</smiles><smiles>PCCP</smiles>

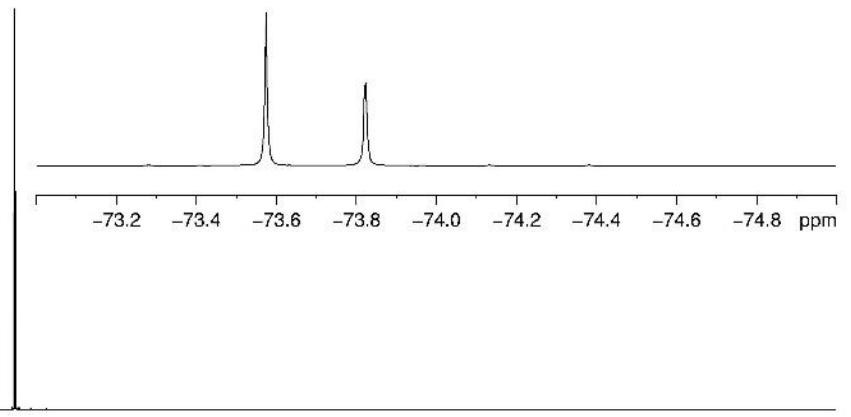

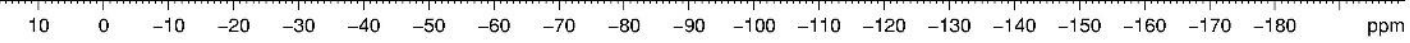


3a; ${ }^{1} \mathrm{H}$ NMR (400MHz, $\left.\mathrm{CDCl}_{3}\right) ;{ }^{13} \mathrm{C}$ NMR (100MHz, $\left.\mathrm{CDCl}_{3}\right)$

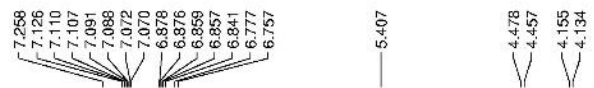

幽<smiles>C[C@]1(CC2=CCCCC2)COc2ccccc21</smiles>

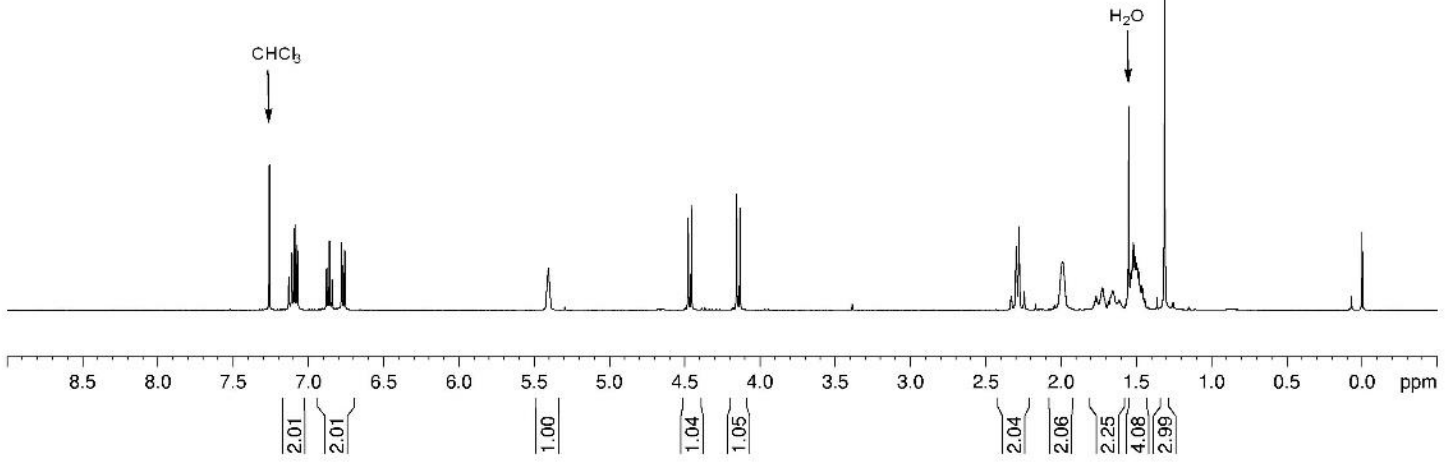

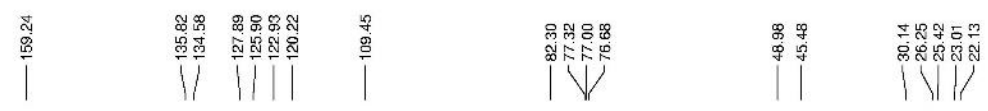<smiles>C[C@@](COc1ccccc1)(CC1=CCCCC1)C1CCCCC1</smiles>

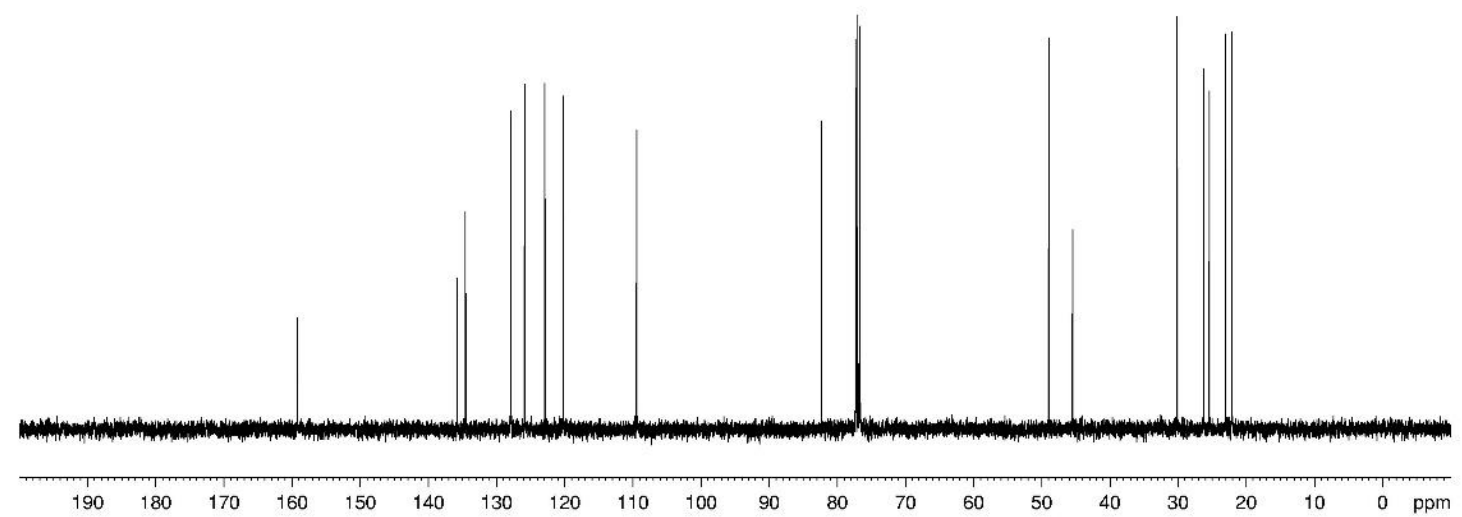


3b; ${ }^{1} \mathrm{H}$ NMR (400MHz, $\left.\mathrm{CDCl}_{3}\right) ;{ }^{13} \mathrm{C}$ NMR (100MHz, $\left.\mathrm{CDCl}_{3}\right)$

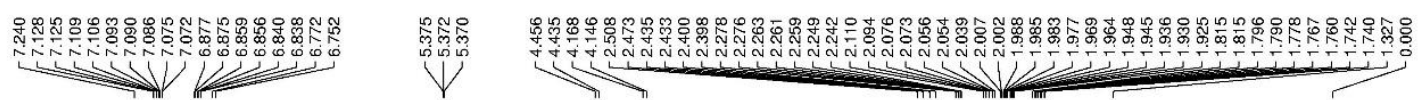<smiles>N[C@@]1(CC2=CCCC2)COc2ccccc21</smiles>

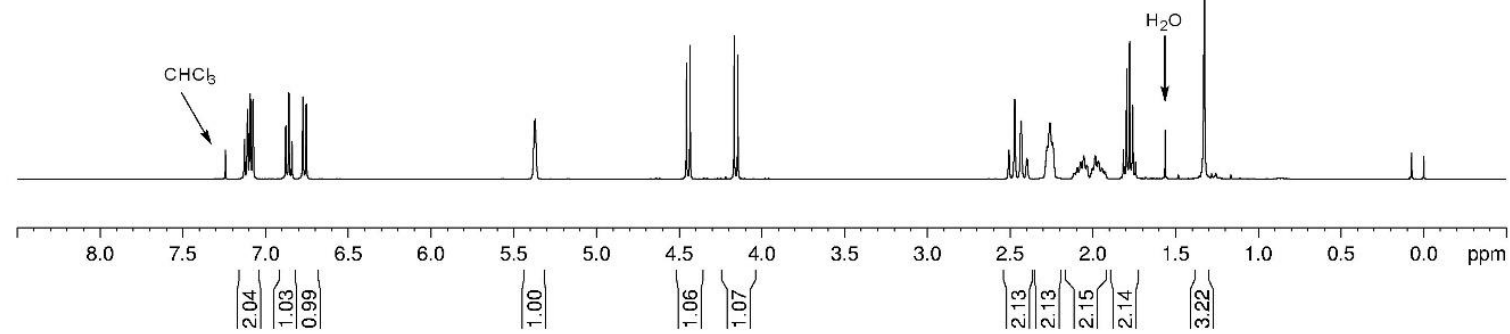

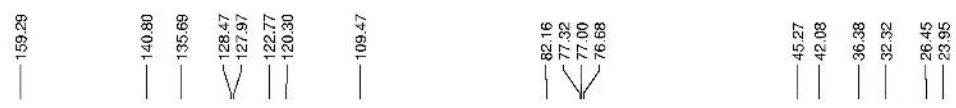<smiles>C[C@]1(CC2=CCCC2)COc2ccccc21</smiles>

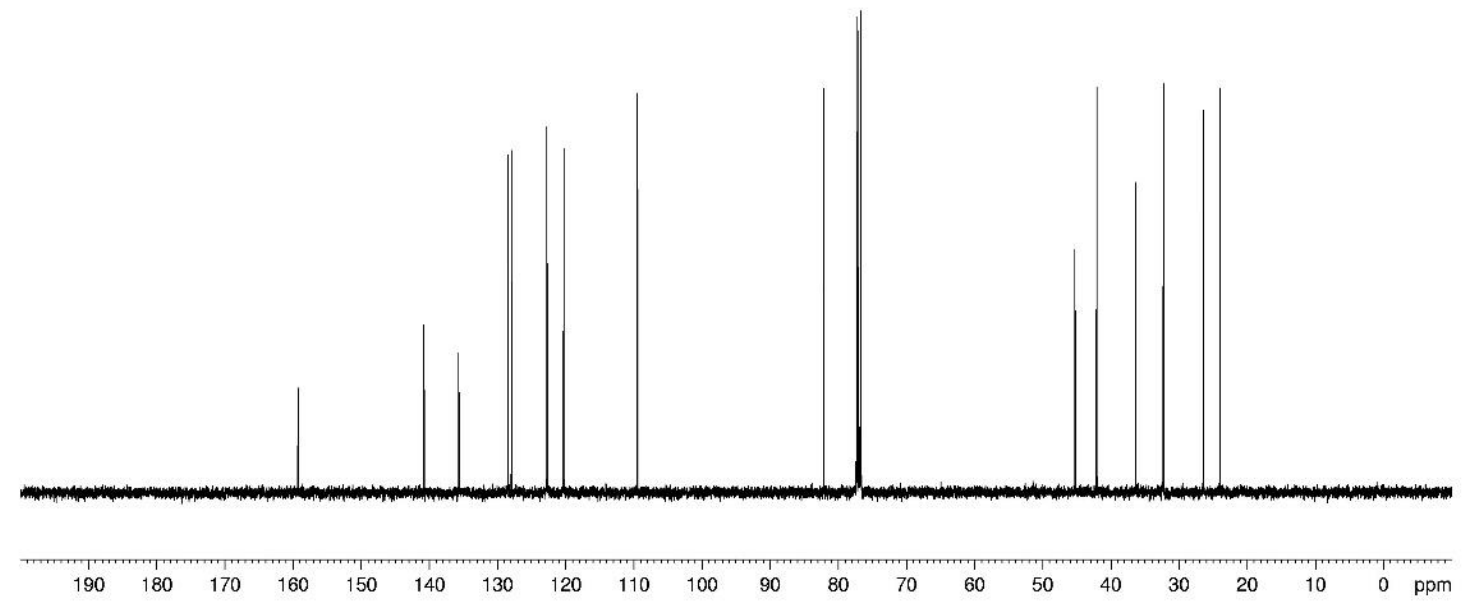


3c; ${ }^{1} \mathrm{H}$ NMR (400MHz, $\left.\mathrm{CDCl}_{3}\right) ;{ }^{13} \mathrm{C}$ NMR (100MHz, $\left.\mathrm{CDCl}_{3}\right)$

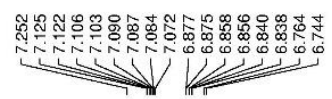

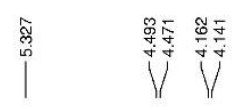
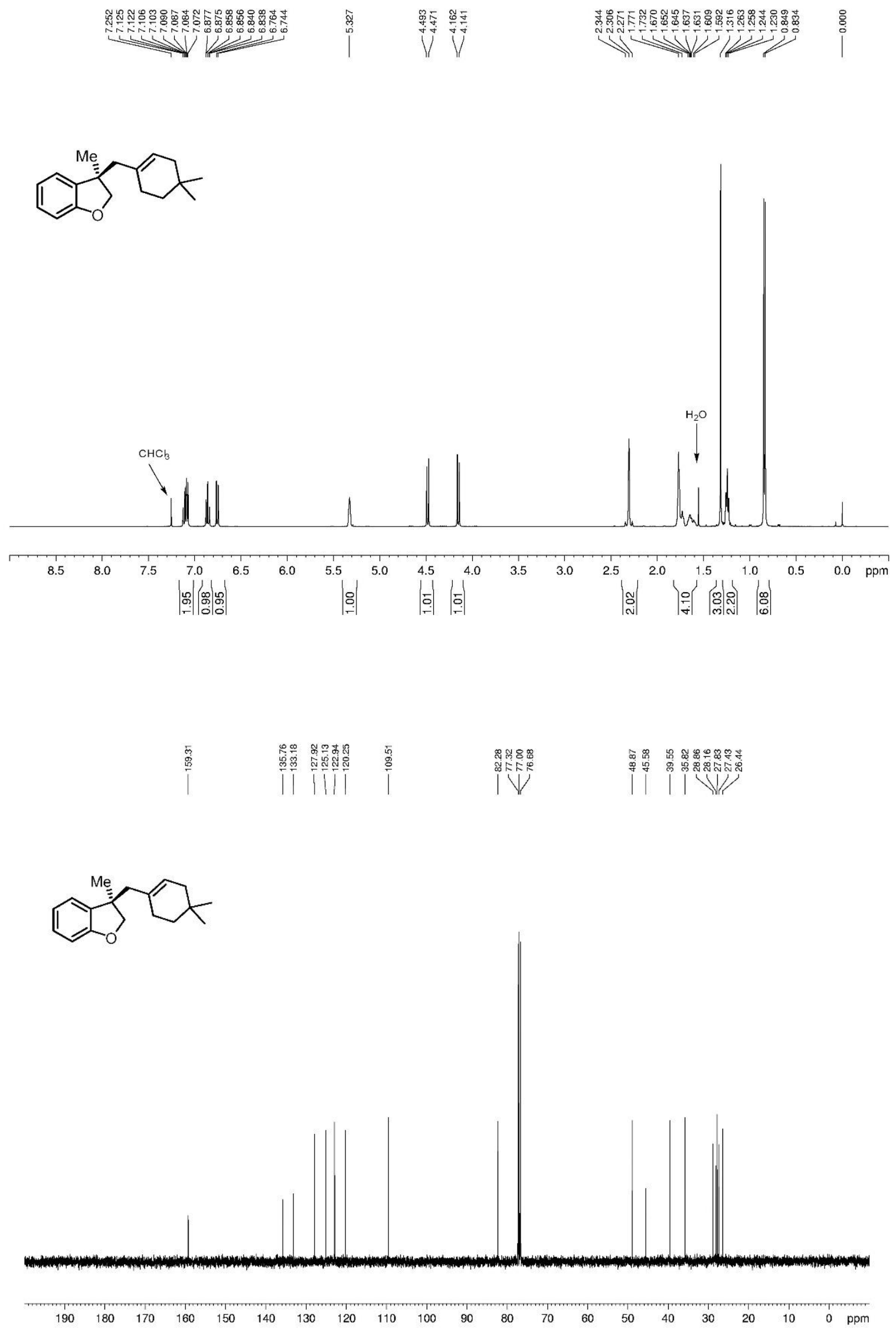

S117 
3d; ${ }^{1} \mathrm{H}$ NMR (400MHz, $\left.\mathrm{CDCl}_{3}\right) ;{ }^{13} \mathrm{C}$ NMR $\left(100 \mathrm{MHz}, \mathrm{CDCl}_{3}\right)$

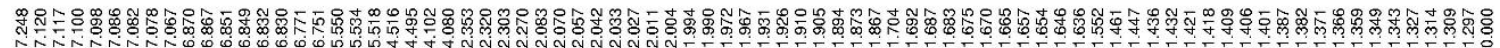<smiles>C[C@]1(CC2=CCCCCC2)COc2ccccc21</smiles>
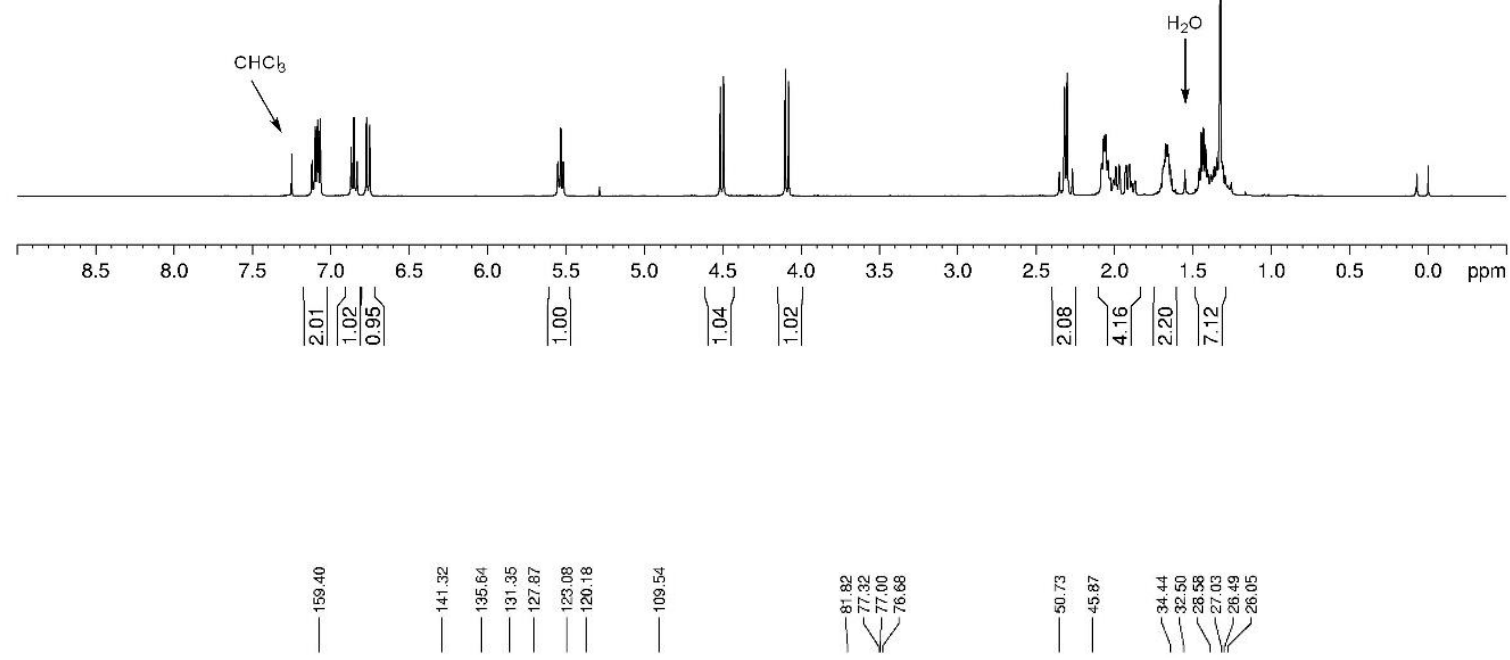<smiles>C[C@]1(CC2=CCCCCC2)COc2ccccc21</smiles>

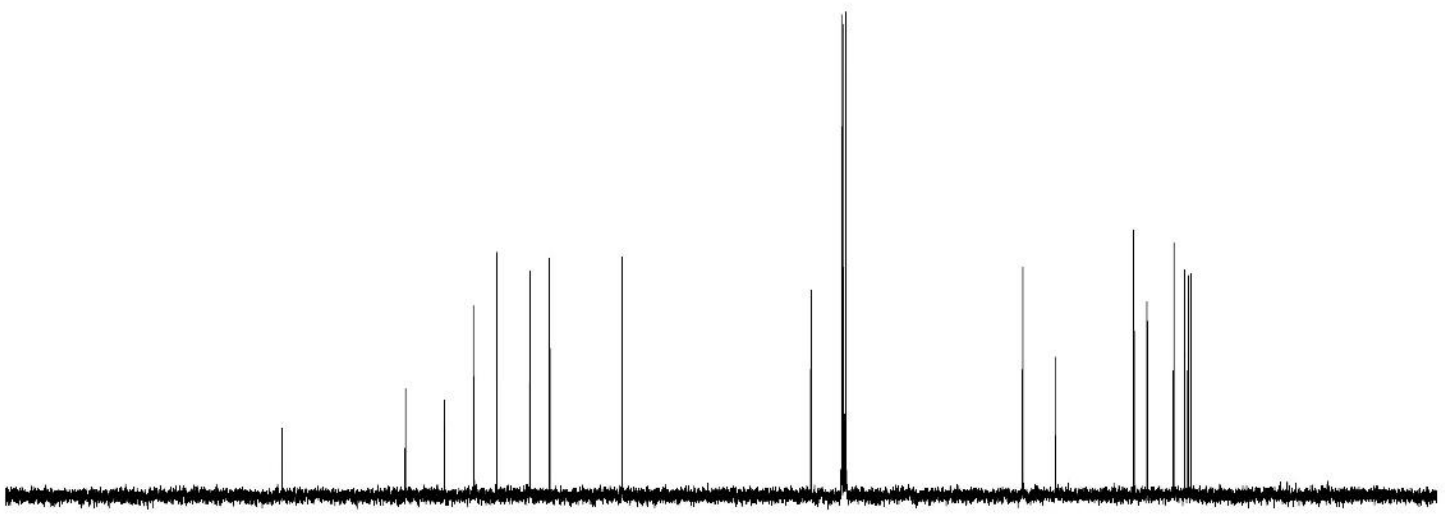

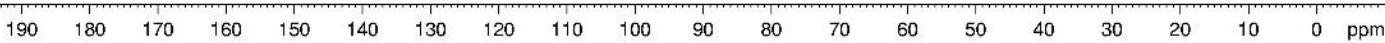


3e; ${ }^{1} \mathrm{H}$ NMR (400MHz, $\left.\mathrm{CDCl}_{3}\right) ;{ }^{13} \mathrm{C}$ NMR (100MHz, $\left.\mathrm{CDCl}_{3}\right)$

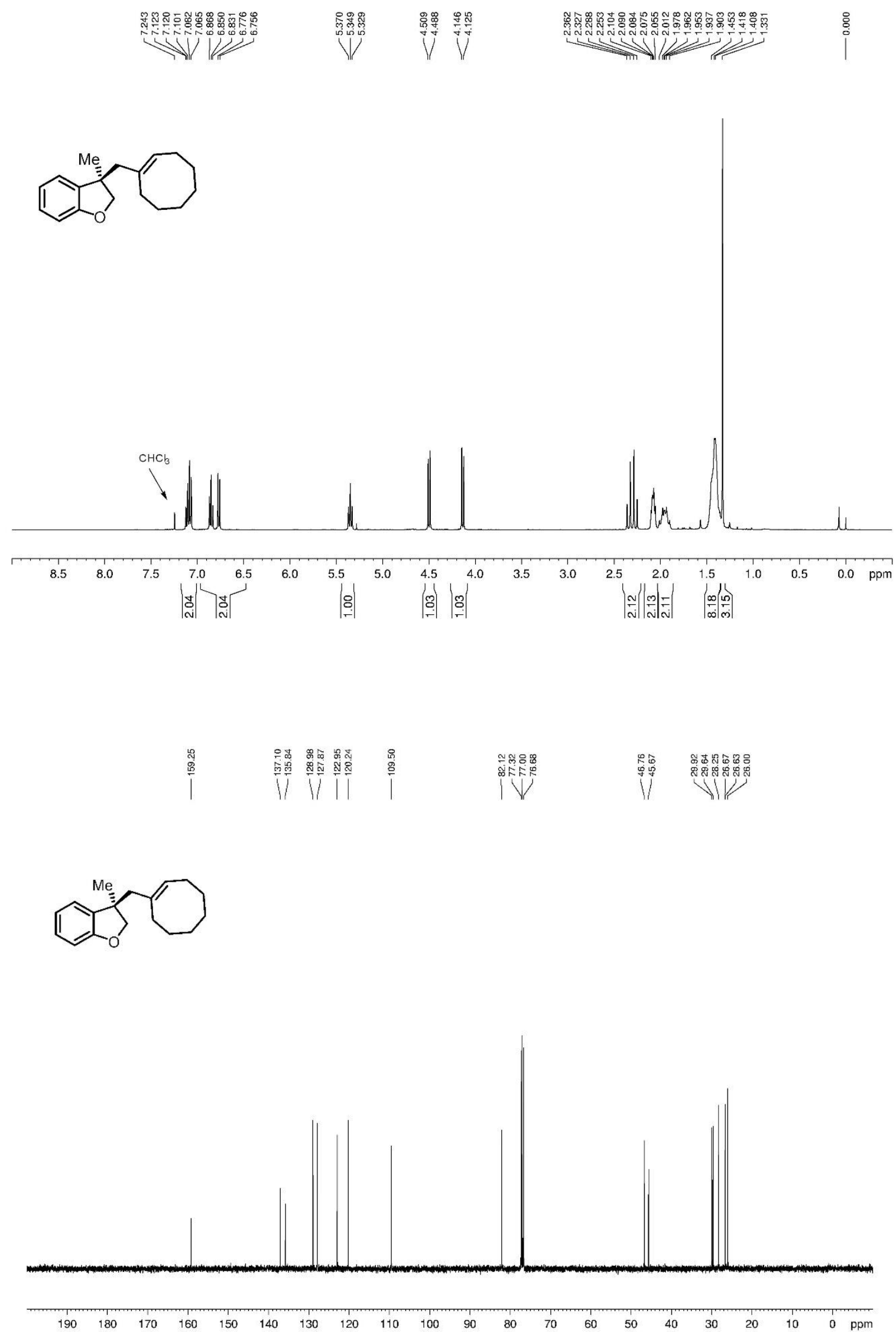


3f; ${ }^{1} \mathrm{H}$ NMR (400MHz, $\left.\mathrm{CDCl}_{3}\right) ;{ }^{13} \mathrm{C}$ NMR $\left(100 \mathrm{MHz}, \mathrm{CDCl}_{3}\right)$
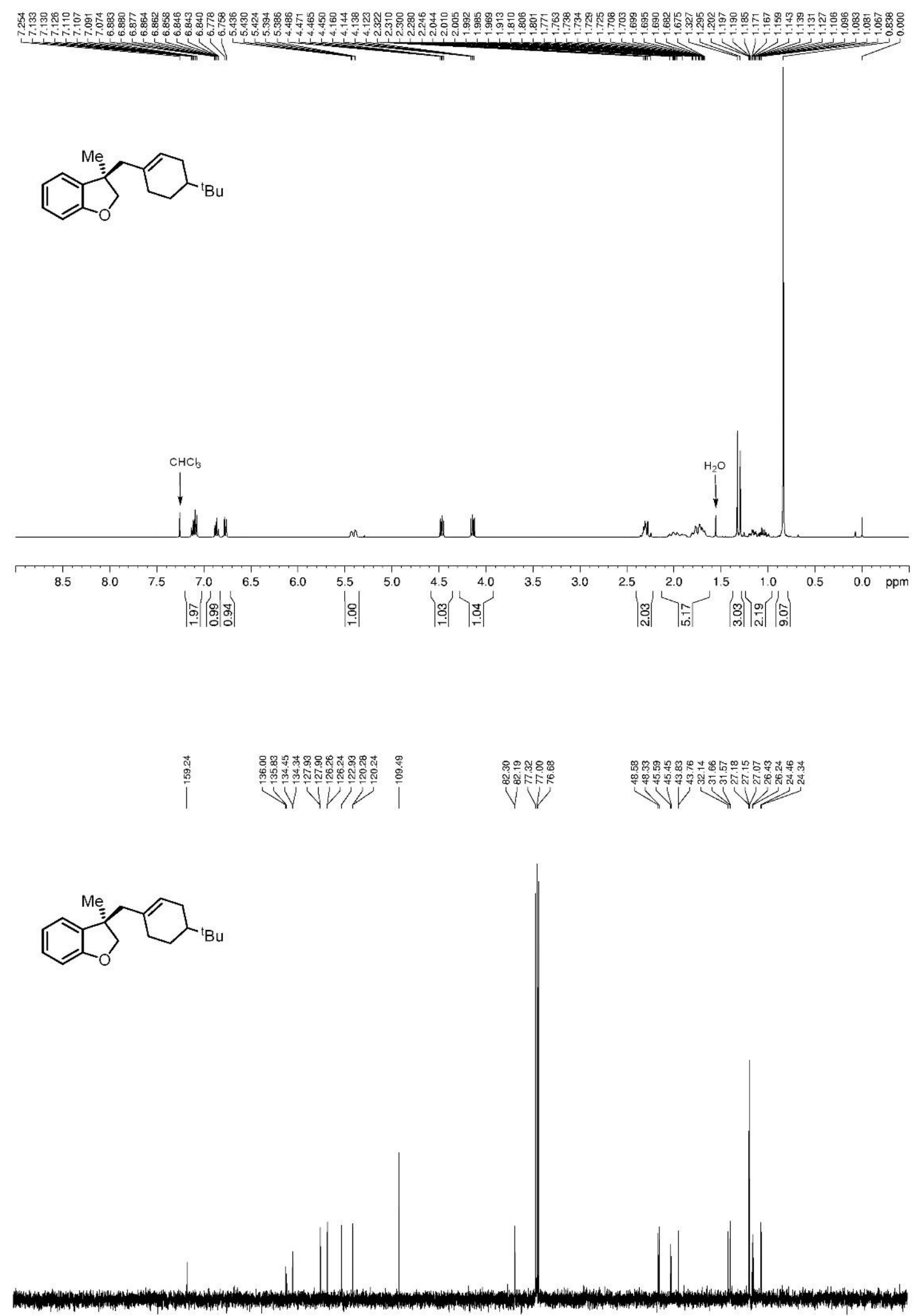

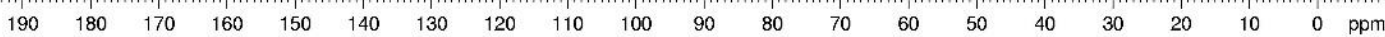


3g; ${ }^{1} \mathrm{H}$ NMR (400MHz, $\left.\mathrm{CDCl}_{3}\right) ;{ }^{13} \mathrm{C}$ NMR $\left(100 \mathrm{MHz}, \mathrm{CDCl}_{3}\right)$

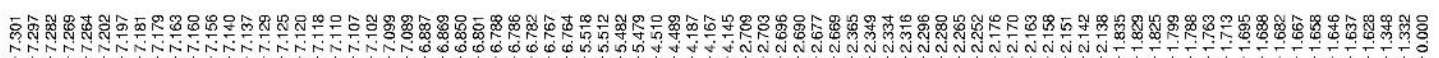<smiles>C[C@]1(CC2=CCC(c3ccccc3)CC2)COc2ccccc21</smiles>

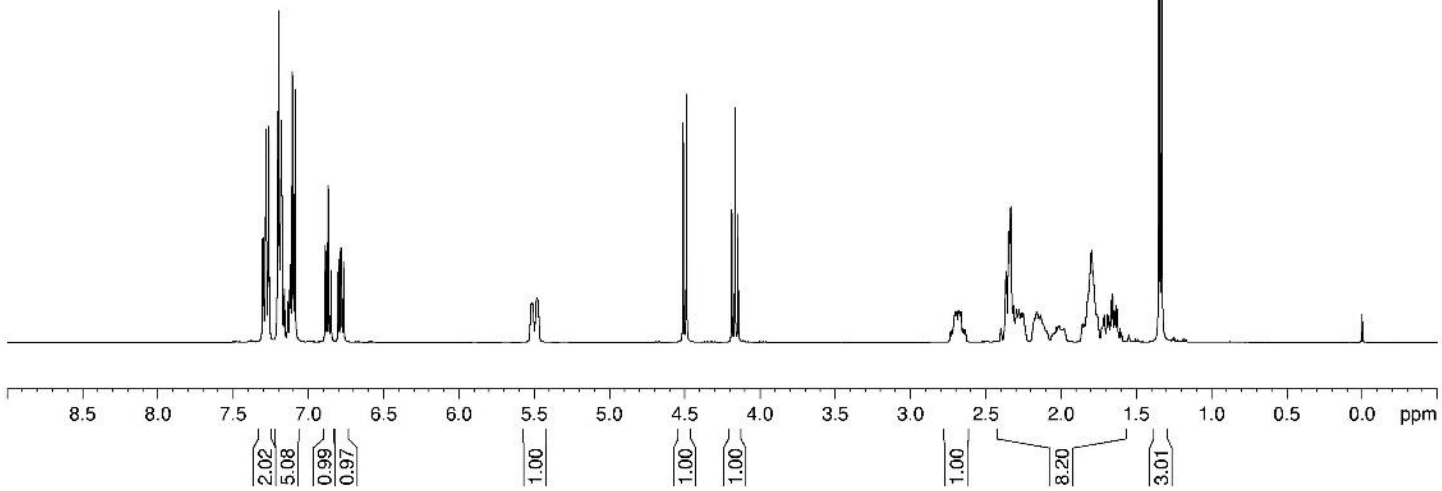

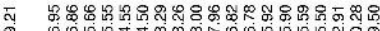

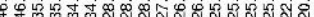

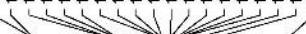<smiles>C[C@]1(CC2=CCC(c3ccccc3)CC2)COc2ccccc21</smiles>

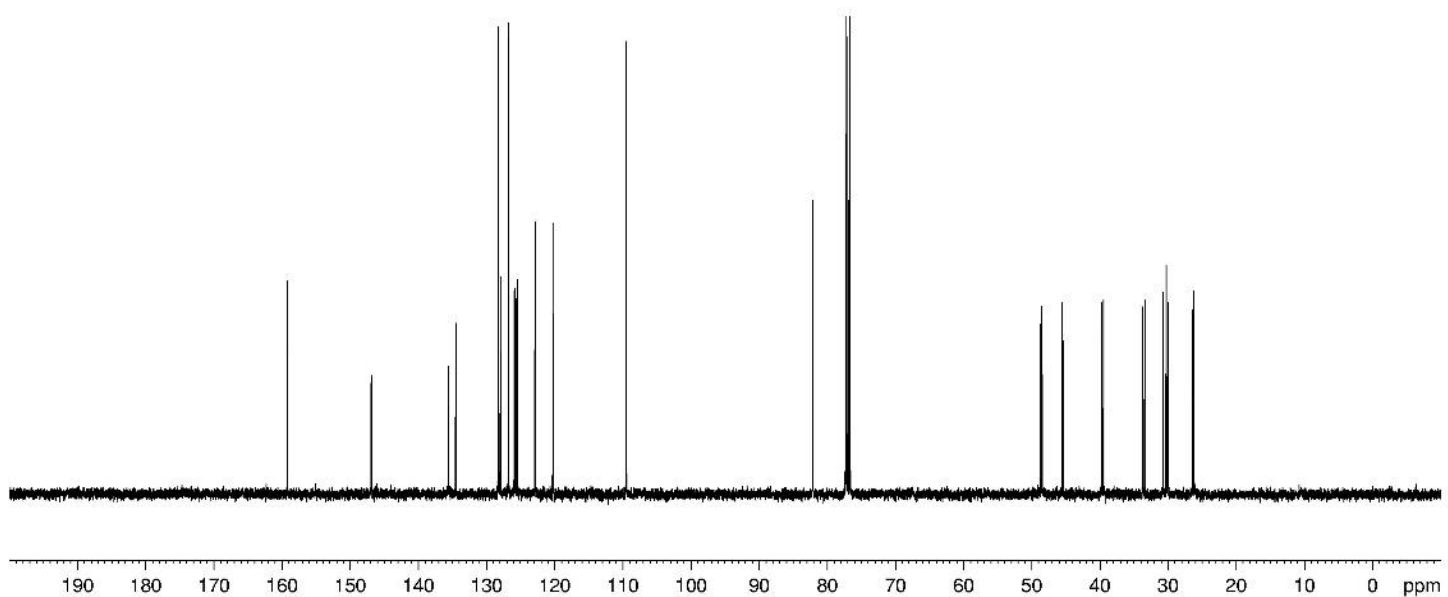


3h; ${ }^{1} \mathrm{H}$ NMR (400MHz, $\left.\mathrm{CDCl}_{3}\right) ;{ }^{13} \mathrm{C}$ NMR $\left(100 \mathrm{MHz}, \mathrm{CDCl}_{3}\right)$

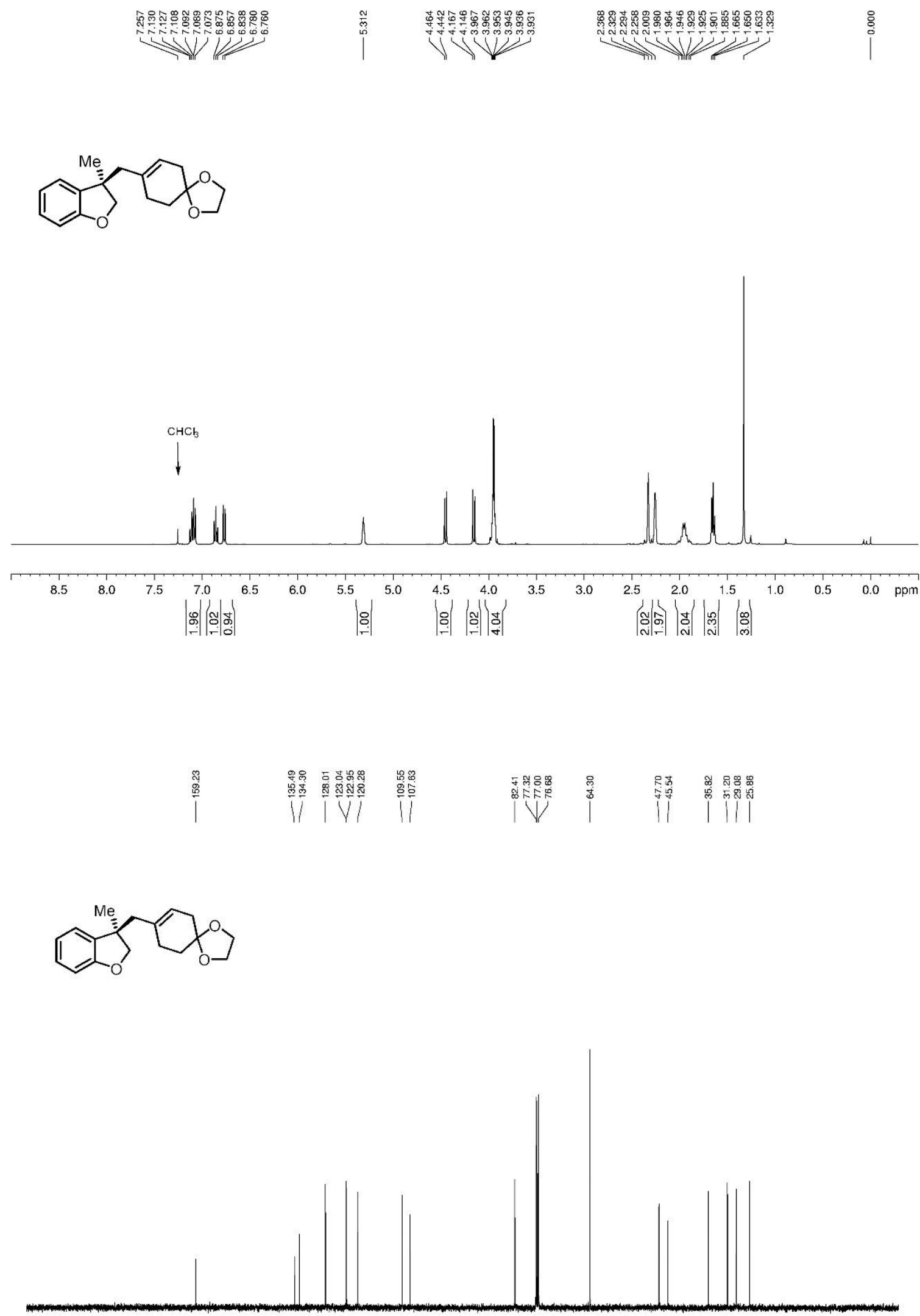

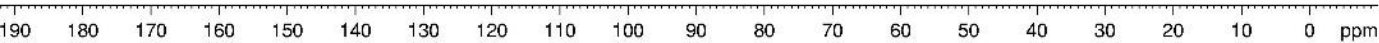


3i; ${ }^{1} \mathrm{H}$ NMR (400MHz, $\left.\mathrm{CDCl}_{3}\right) ;{ }^{13} \mathrm{C}$ NMR $\left(100 \mathrm{MHz}, \mathrm{CDCl}_{3}\right)$

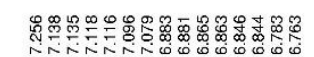 \\ N-Nin}

$\rightarrow$ ill

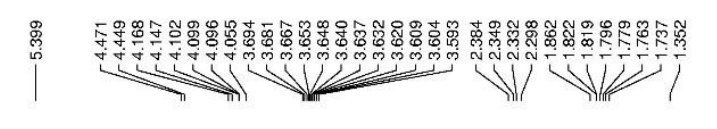

$\stackrel{8}{i}$
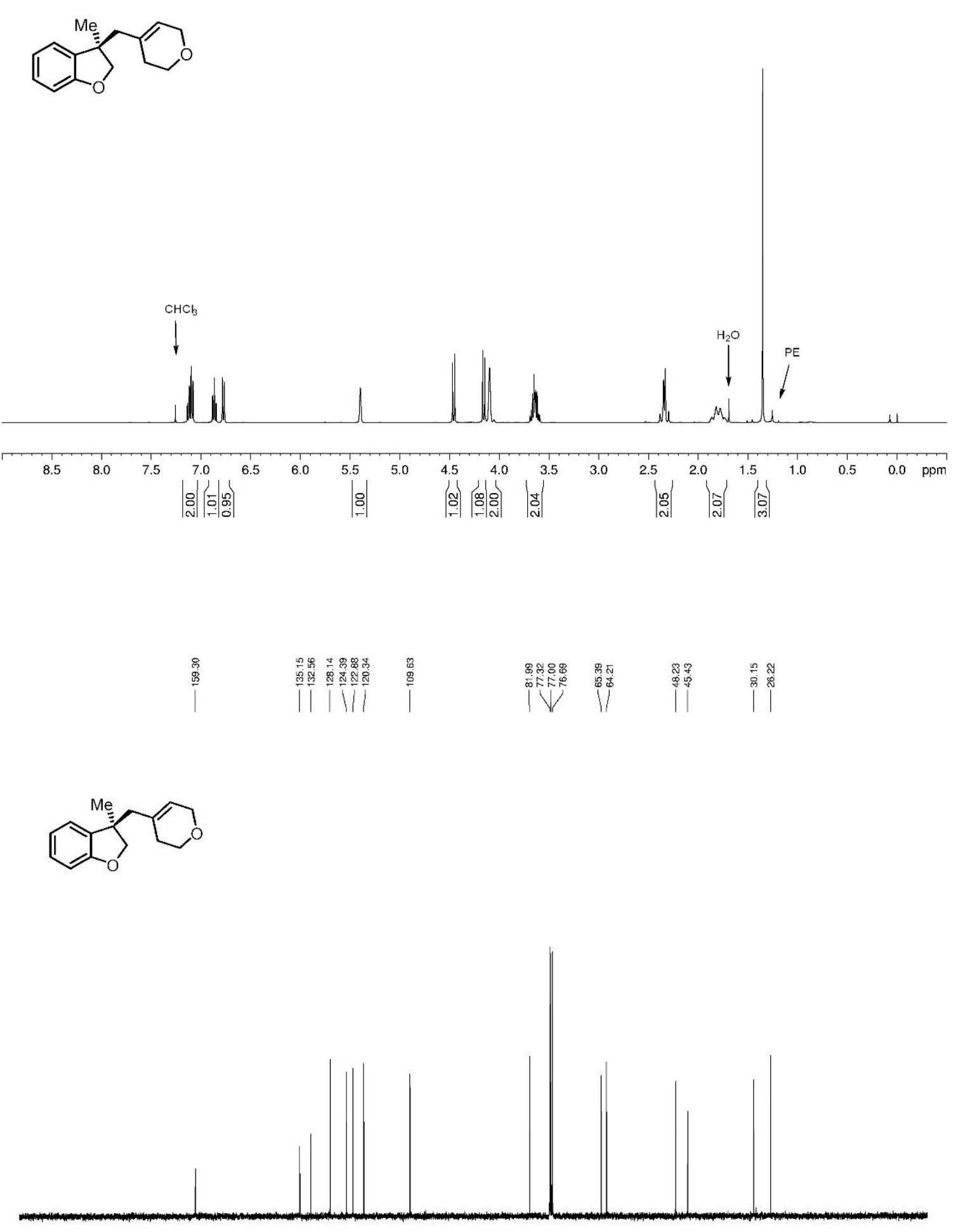

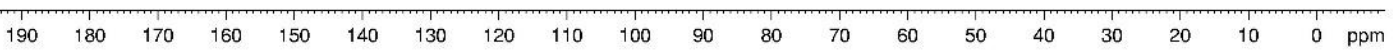


3j; ${ }^{1} \mathrm{H}$ NMR (400MHz, $\left.\mathrm{CDCl}_{3}\right) ;{ }^{13} \mathrm{C}$ NMR $\left(100 \mathrm{MHz}, \mathrm{CDCl}_{3}\right)$

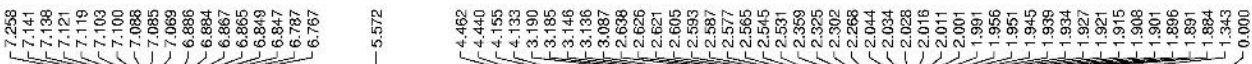<smiles>C[C@]1(CC2=CCSCC2)COc2ccccc21</smiles>

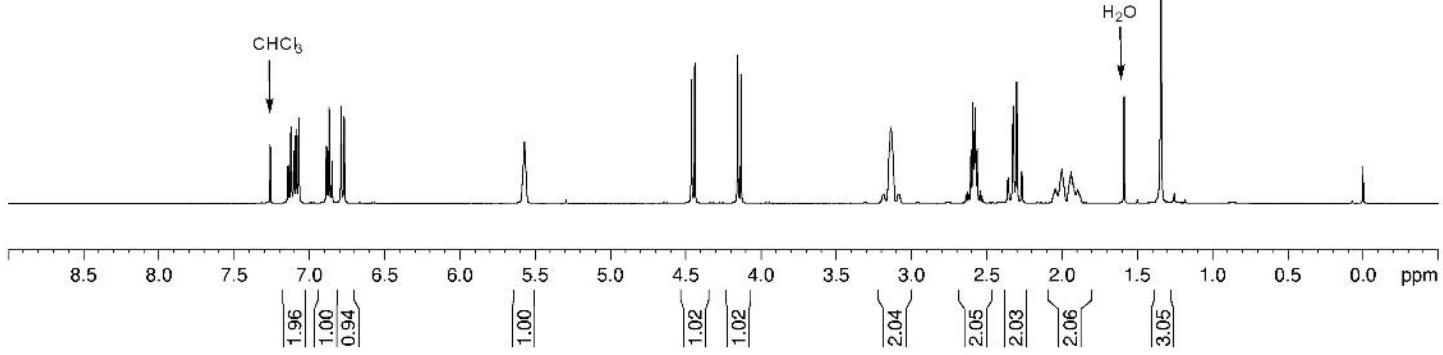

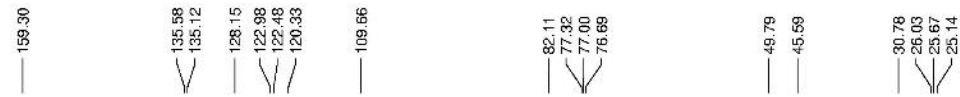<smiles>C[C@]1(C2=CCSCC2)COc2ccccc21</smiles>

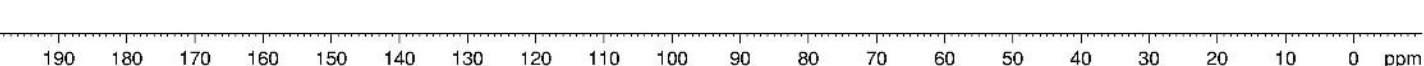


3k; ${ }^{1} \mathrm{H}$ NMR (400MHz, $\left.\mathrm{CDCl}_{3}\right) ;{ }^{13} \mathrm{C}$ NMR $\left(100 \mathrm{MHz}, \mathrm{CDCl}_{3}\right)$

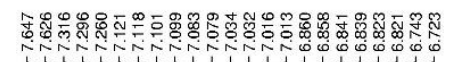

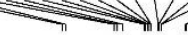

|

$\overbrace{0}^{N T S}$

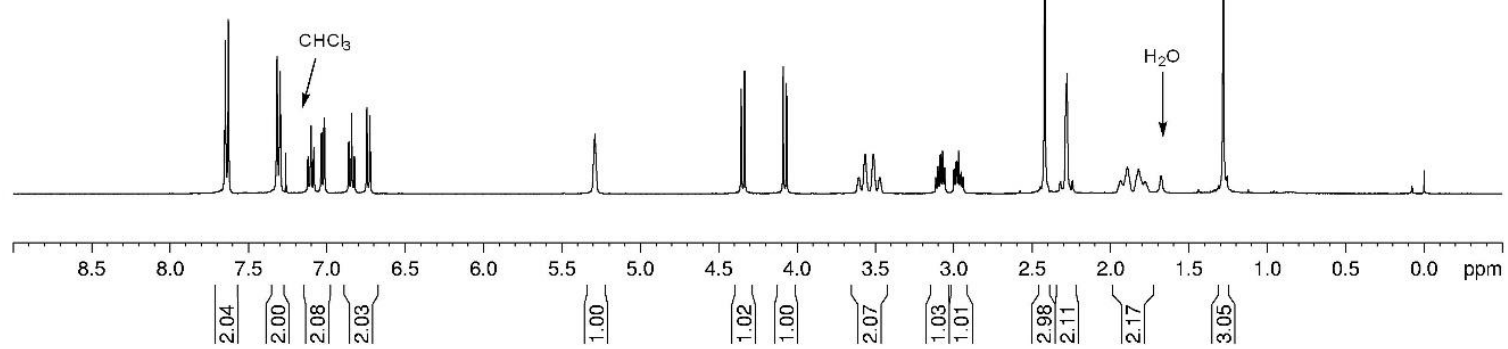

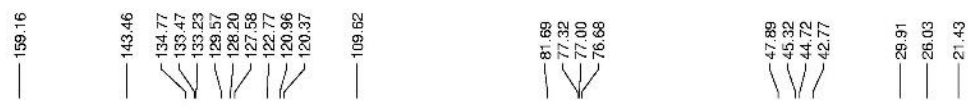<smiles>C[C@]1(CC2=CC[NH+](S)CC2)COc2ccccc21</smiles>

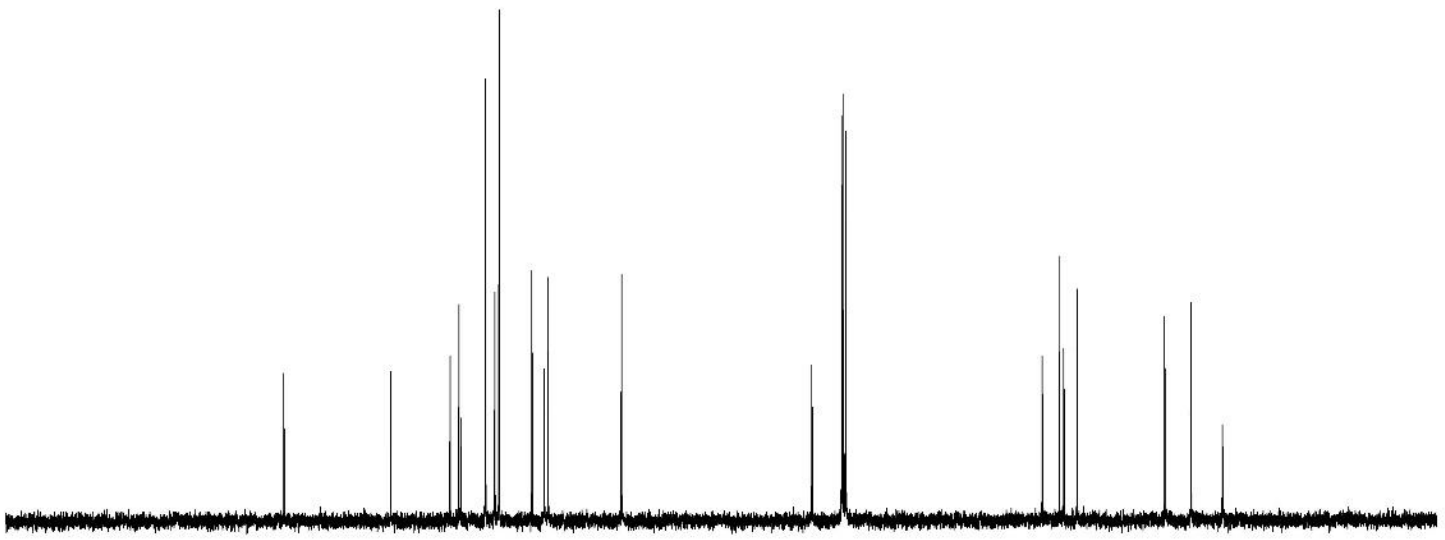

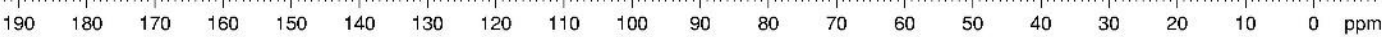


31; ${ }^{1} \mathrm{H}$ NMR (400MHz, $\left.\mathrm{CDCl}_{3}\right) ;{ }^{13} \mathrm{C}$ NMR (100MHz, $\left.\mathrm{CDCl}_{3}\right)$
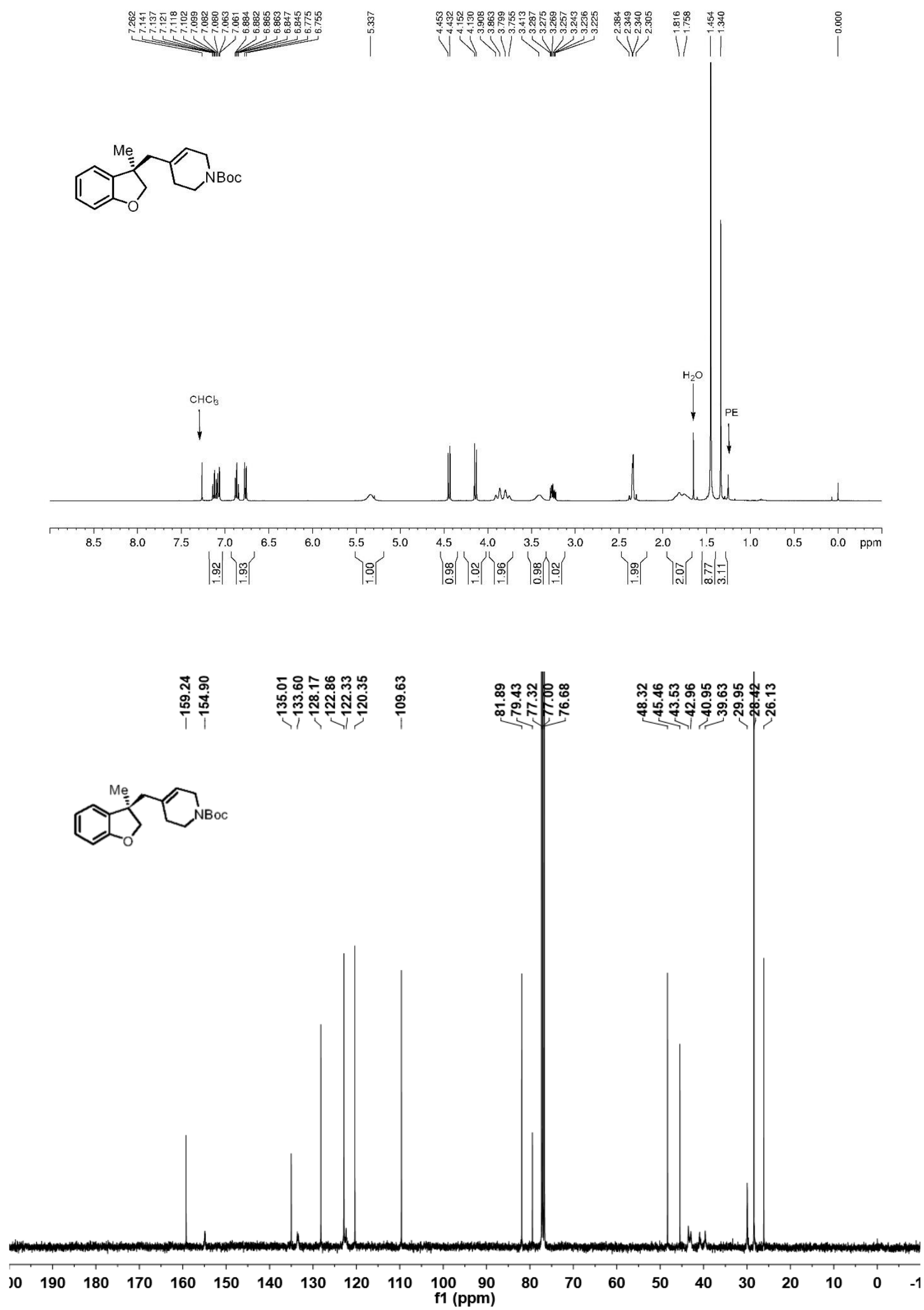
3m; ${ }^{1} \mathrm{H}$ NMR (400MHz, $\left.\left.\mathrm{CDCl}_{3}\right) ;{ }^{13} \mathrm{C} \mathrm{NMR} \mathrm{(100MHz,} \mathrm{CDCl}_{3}\right)$

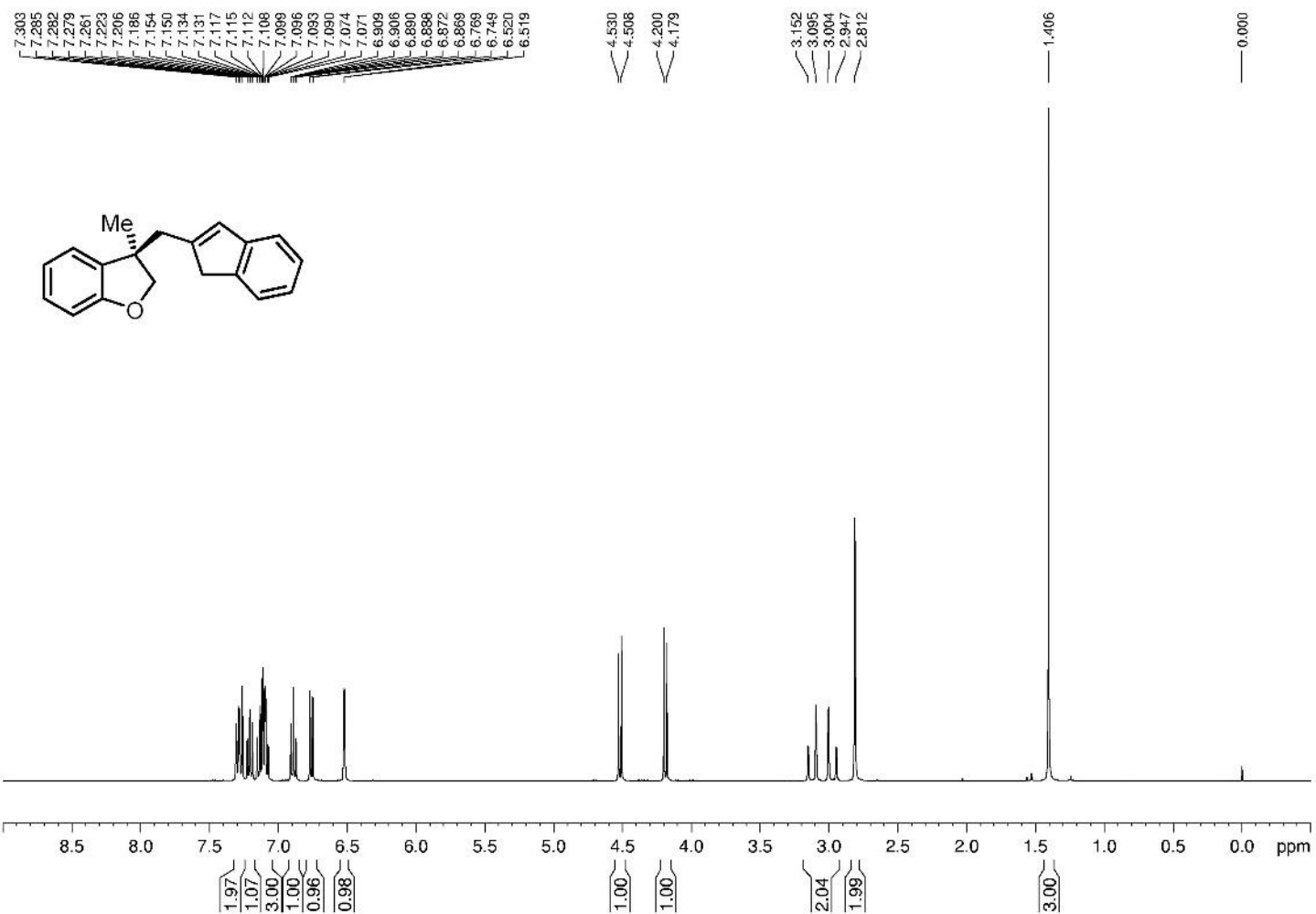

|<smiles>C[C@]1(CC2=Cc3ccccc3C2)COc2ccccc21</smiles>

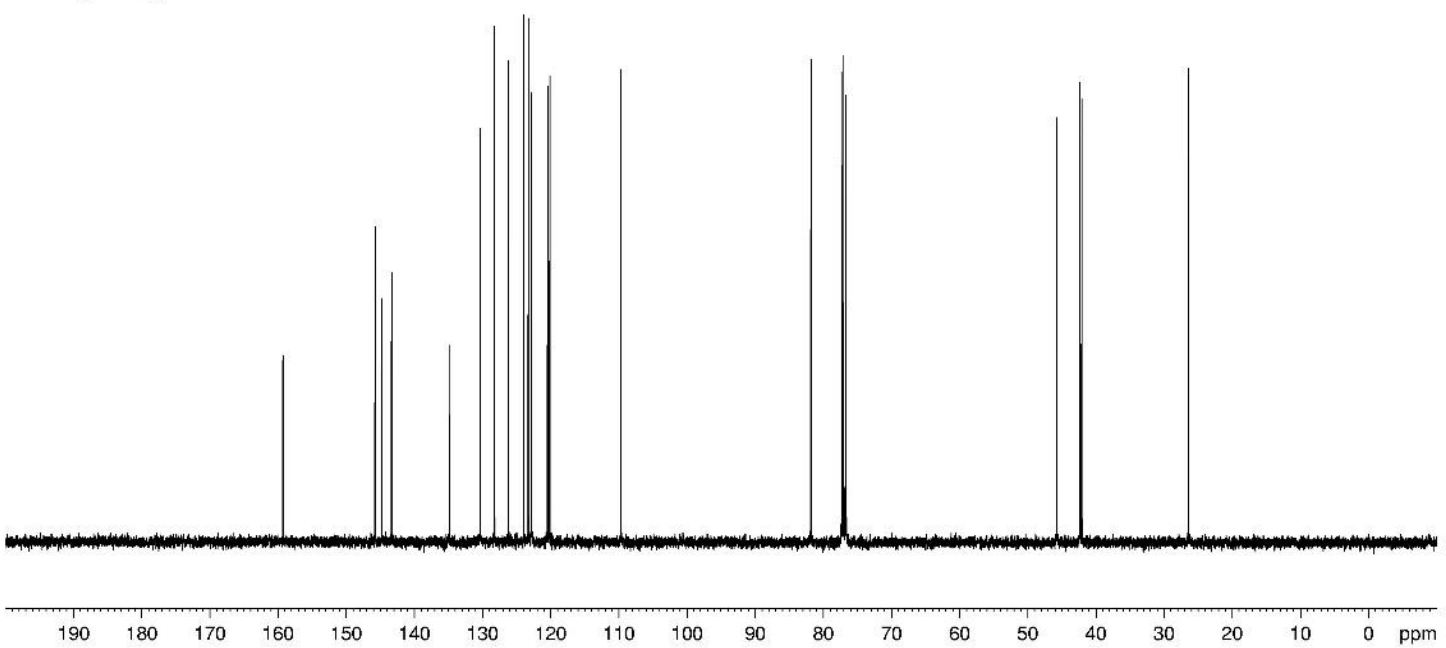


3n; ${ }^{1} \mathrm{H}$ NMR (400MHz, $\left.\mathrm{CDCl}_{3}\right) ;{ }^{13} \mathrm{C}$ NMR (100MHz, $\left.\mathrm{CDCl}_{3}\right)$

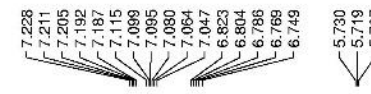

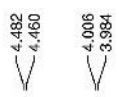

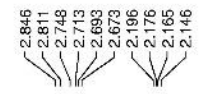<smiles>C[C@]1(CC2=CCCc3ccccc32)COc2ccccc21</smiles>

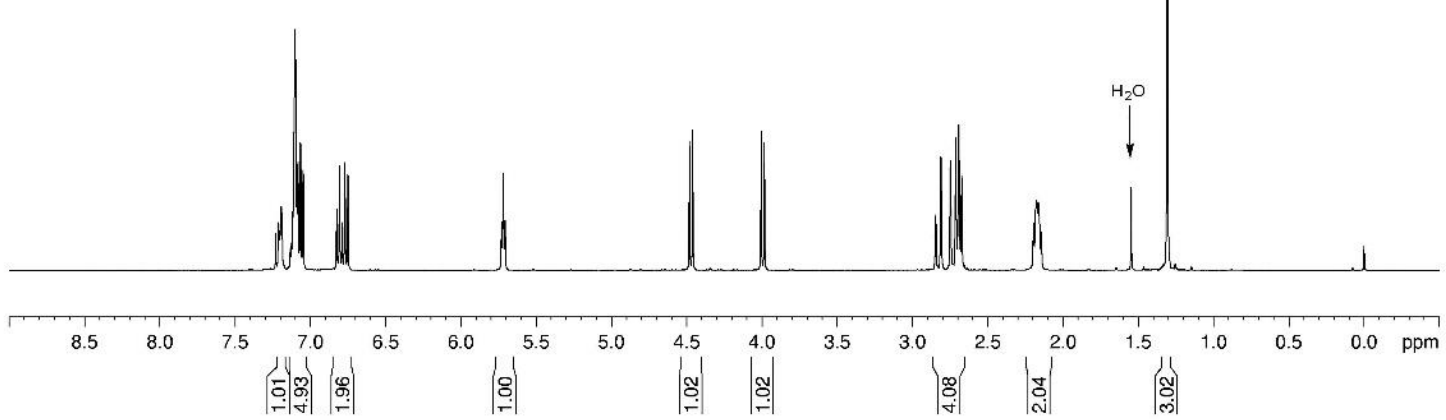

lo

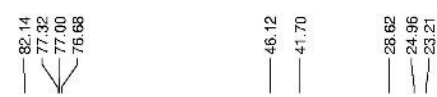<smiles>C[C@]1(CC2=CCCc3ccccc32)COc2ccccc21</smiles>

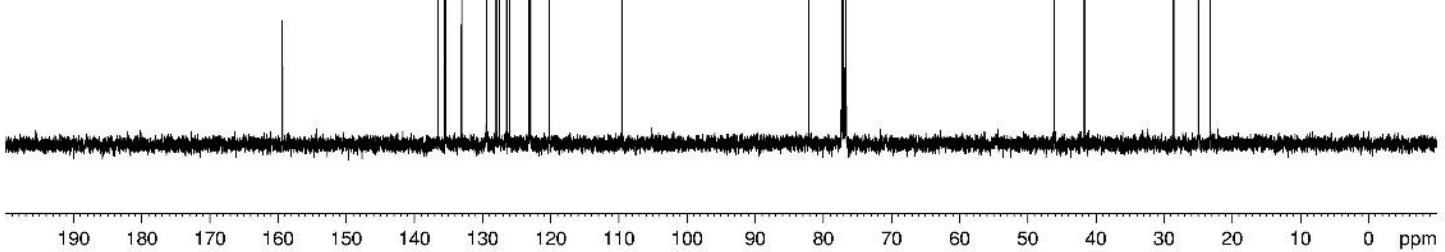


3o; ${ }^{1} \mathrm{H}$ NMR (400MHz, $\left.\mathrm{CDCl}_{3}\right) ;{ }^{13} \mathrm{C}$ NMR $\left(100 \mathrm{MHz}, \mathrm{CDCl}_{3}\right)$

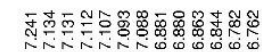

我

(1) VII

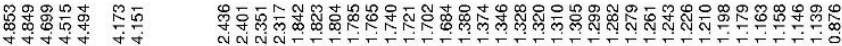

$\overbrace{0}^{M e} \pi_{=}^{C_{5} H_{11}}$

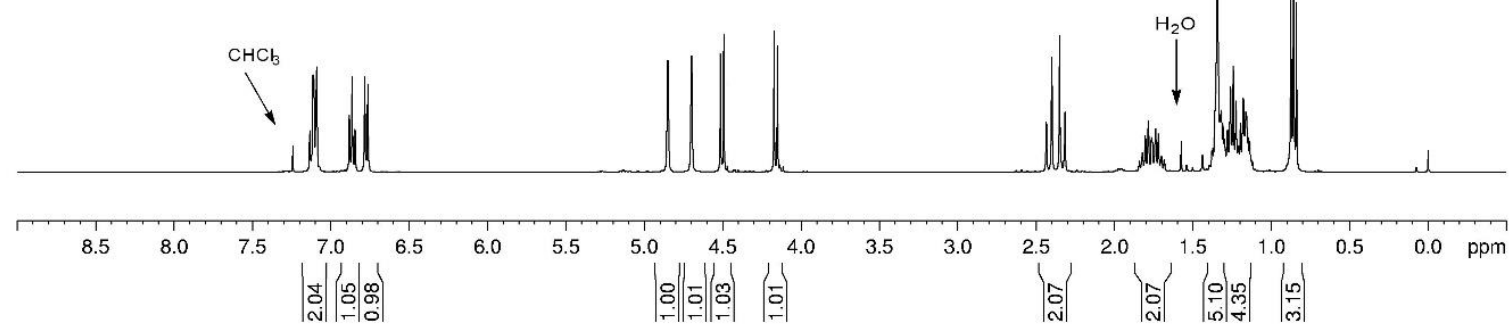

|<smiles>C=C(CCCCCC)C[C@@]1(C)COc2ccccc21</smiles>

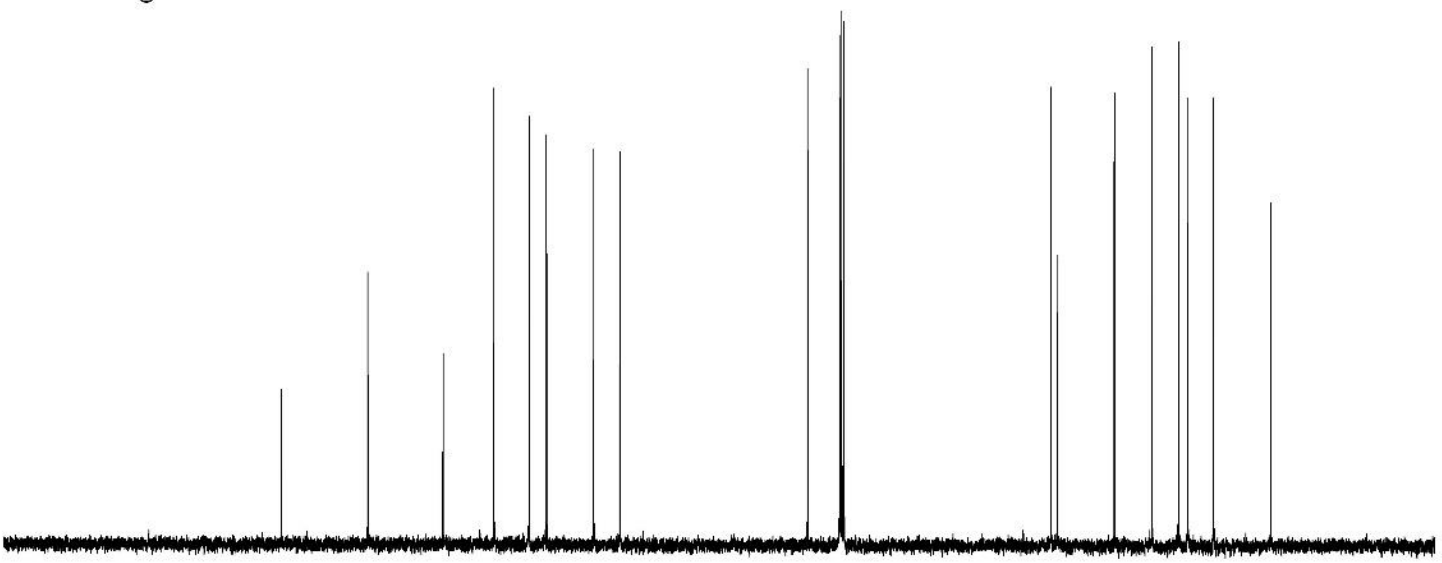

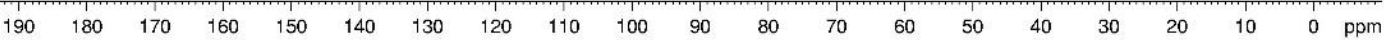


3p; ${ }^{1} \mathrm{H}$ NMR (400MHz, $\left.\mathrm{CDCl}_{3}\right) ;{ }^{13} \mathrm{C}$ NMR (100MHz, $\left.\mathrm{CDCl}_{3}\right)$
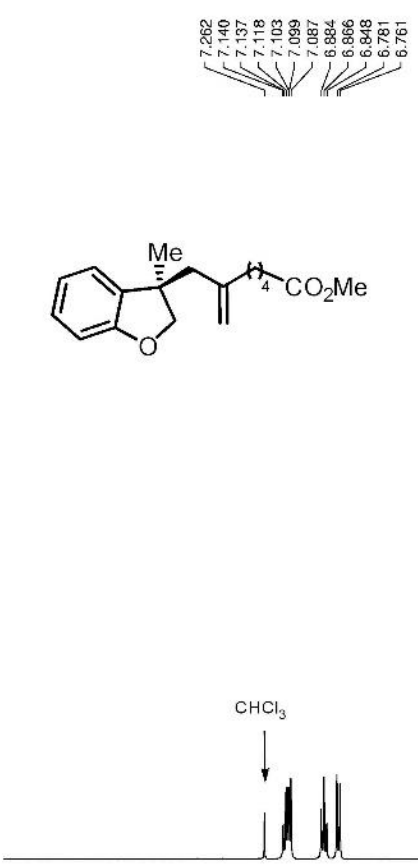
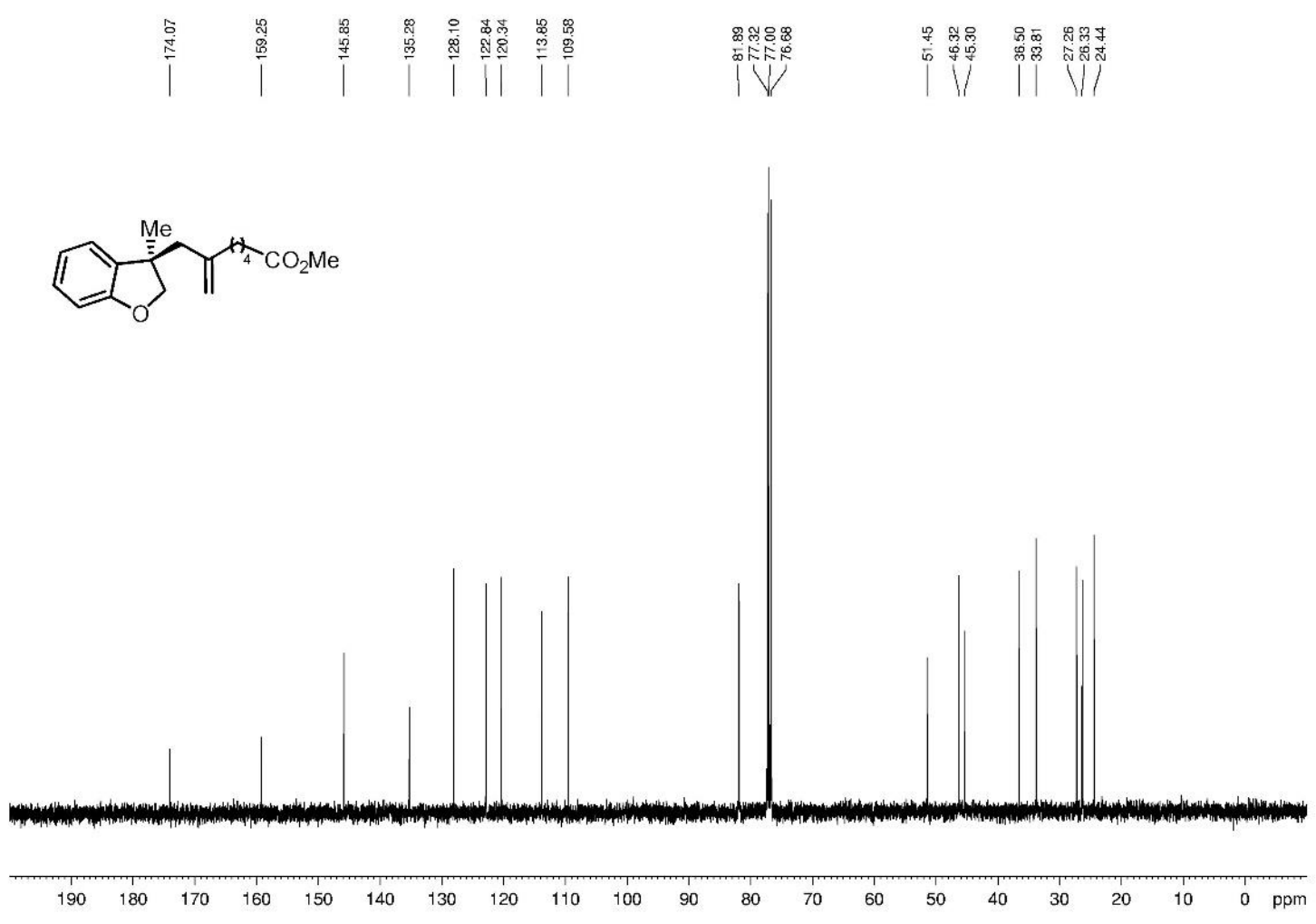

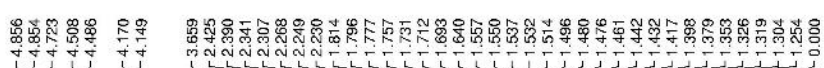

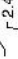

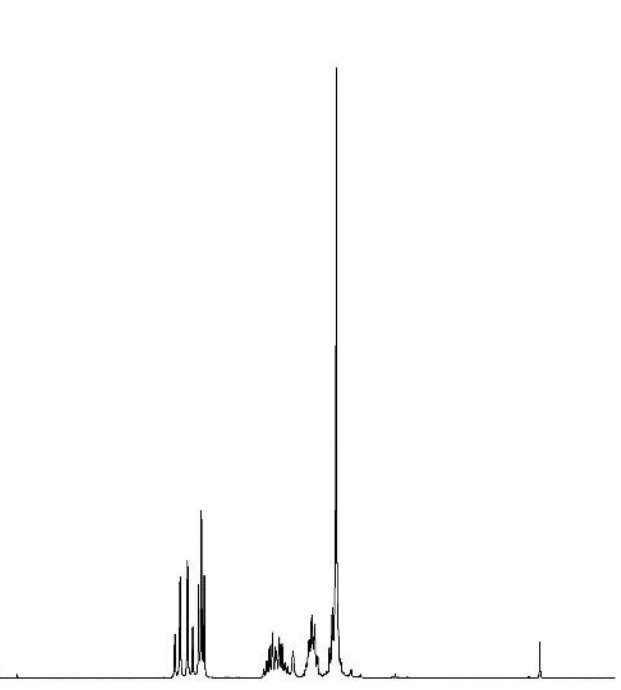

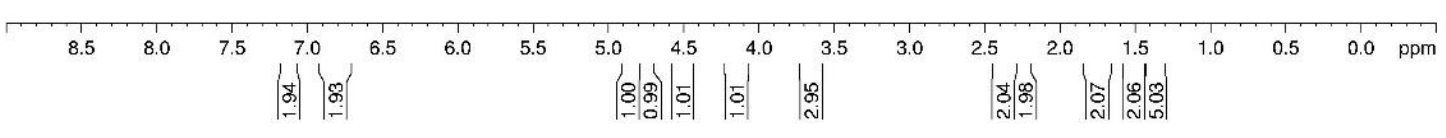

S130 
3q; ${ }^{1} \mathrm{H}$ NMR (400MHz, $\left.\mathrm{CDCl}_{3}\right) ;{ }^{13} \mathrm{C}$ NMR (100MHz, $\left.\mathrm{CDCl}_{3}\right)$

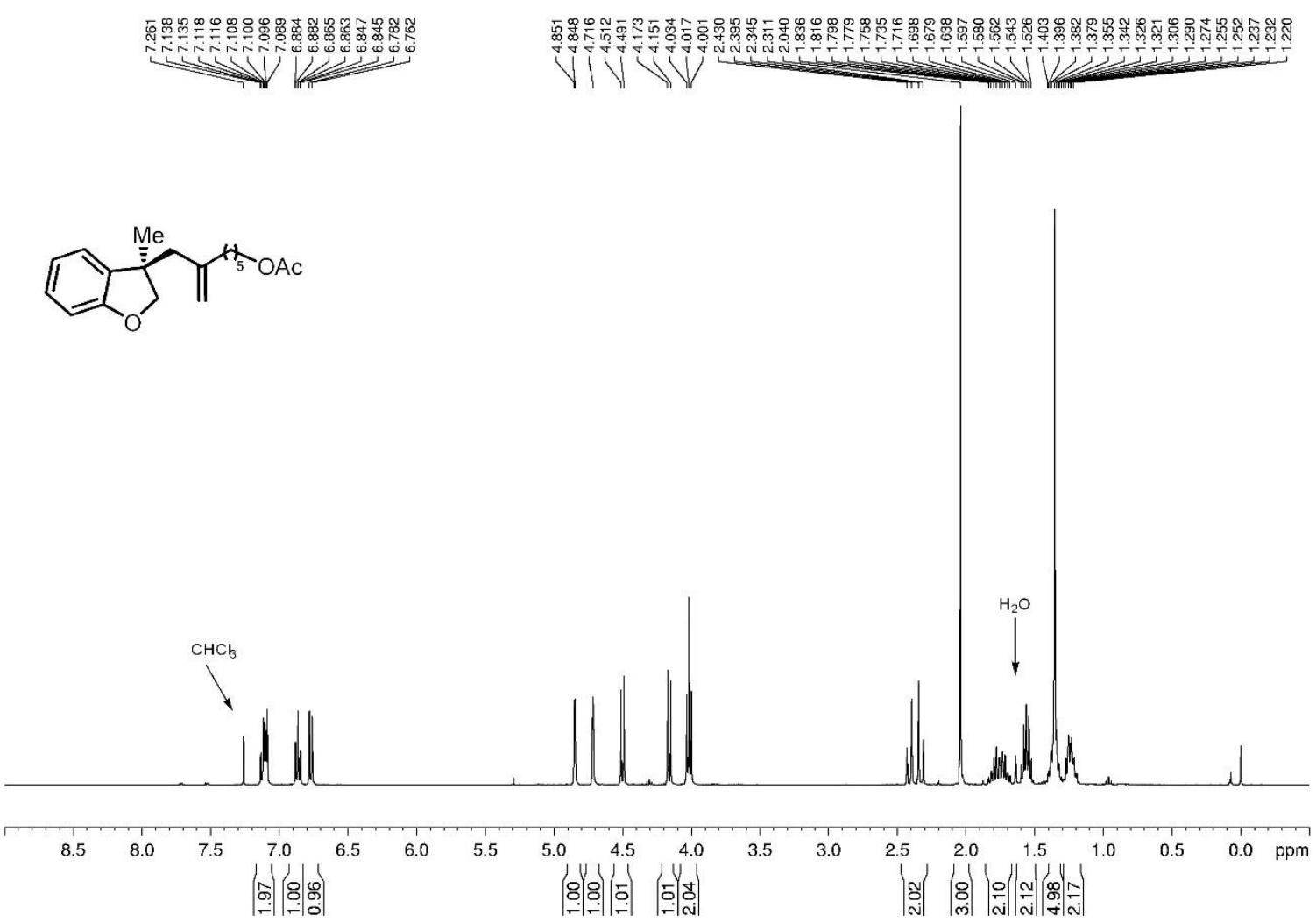

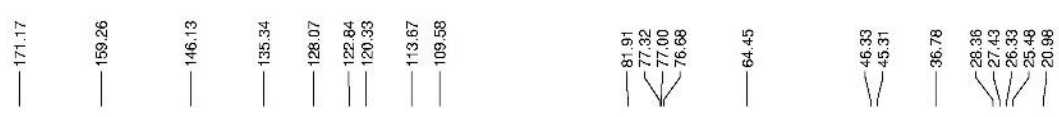<smiles>C=C(CC1(C)COc2ccccc21)NOC(C)=O</smiles> 
3s; ${ }^{1} \mathrm{H}$ NMR (400MHz, $\left.\mathrm{CDCl}_{3}\right) ;{ }^{13} \mathrm{C}$ NMR (100MHz, $\left.\mathrm{CDCl}_{3}\right)$
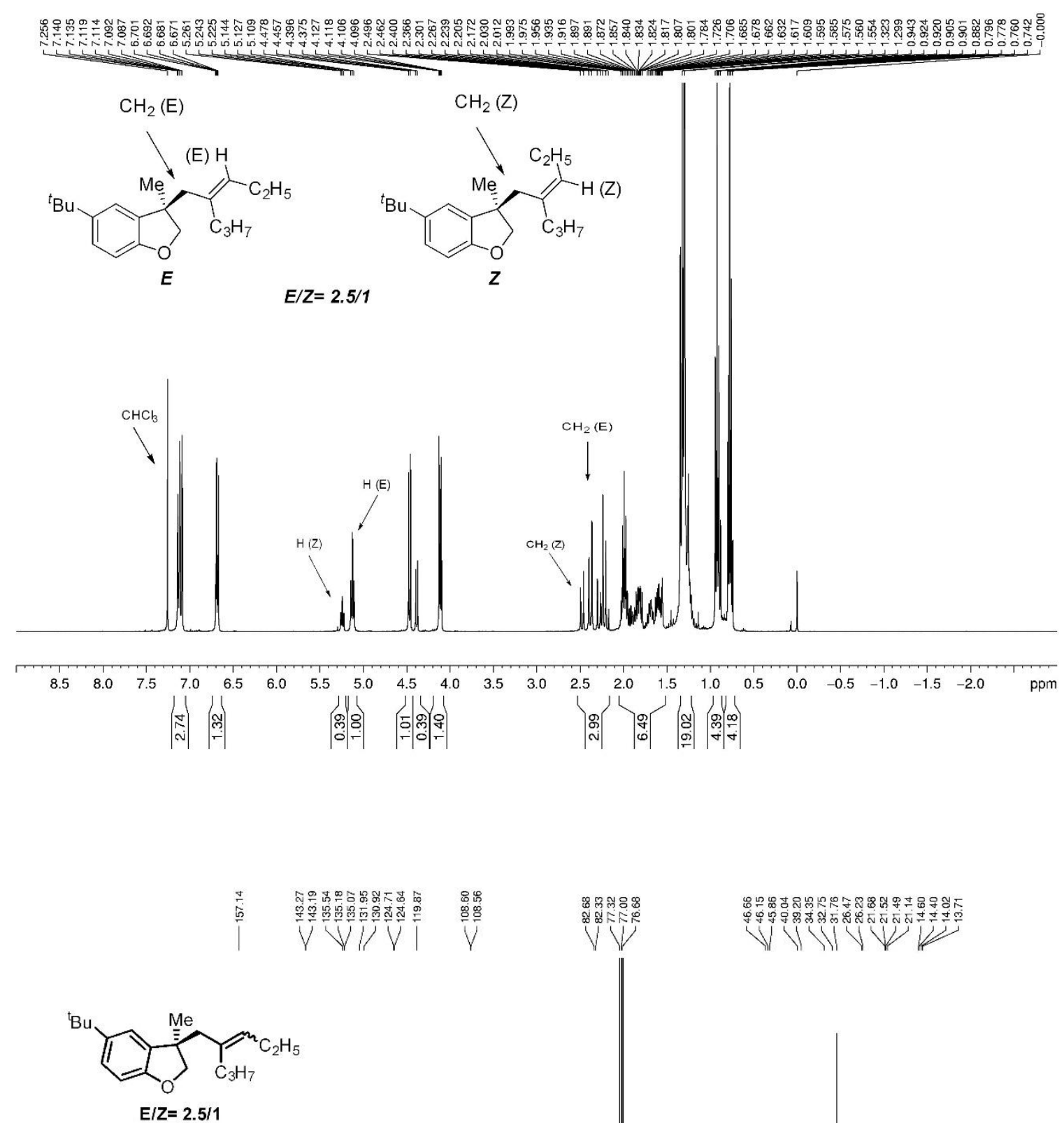
1D-NOE spectra of $E-3 \mathrm{~s}$

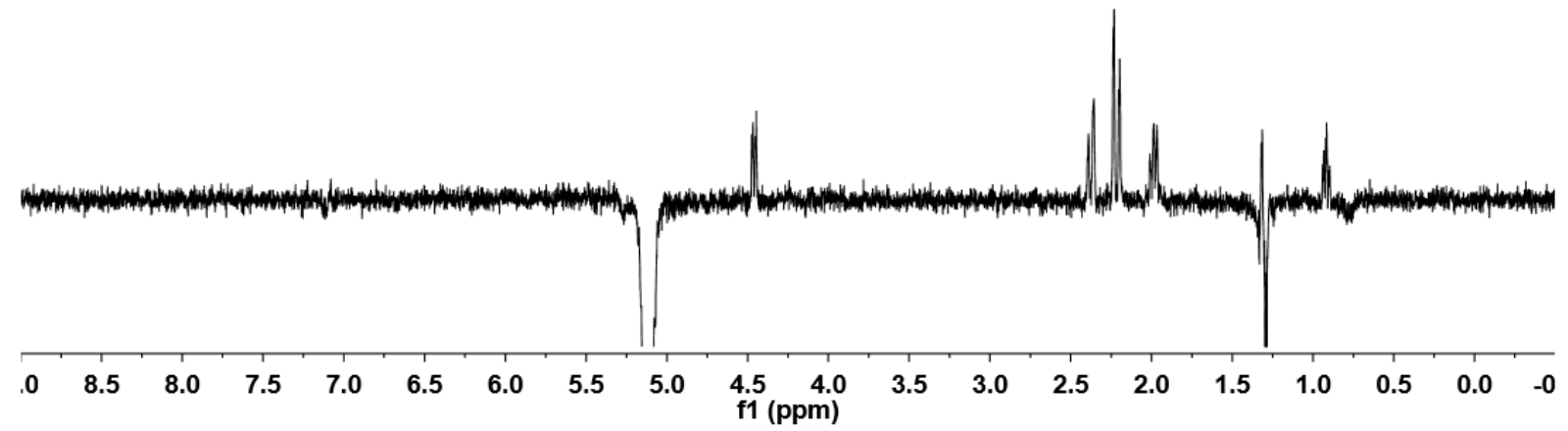

1D-NOE spectra of $Z-3 \mathrm{~s}$

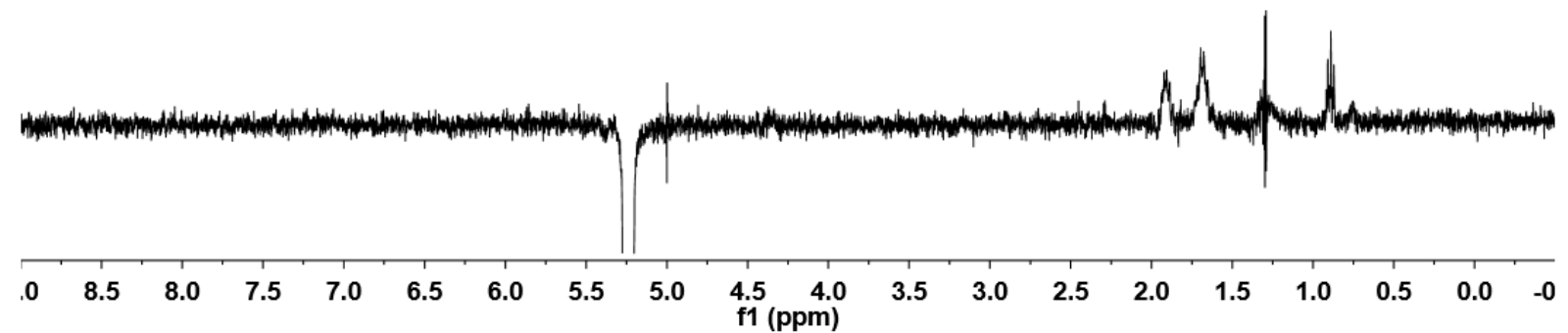


3t; ${ }^{1} \mathrm{H}$ NMR (400MHz, $\left.\mathrm{CDCl}_{3}\right) ;{ }^{13} \mathrm{C}$ NMR (100MHz, $\left.\mathrm{CDCl}_{3}\right)$
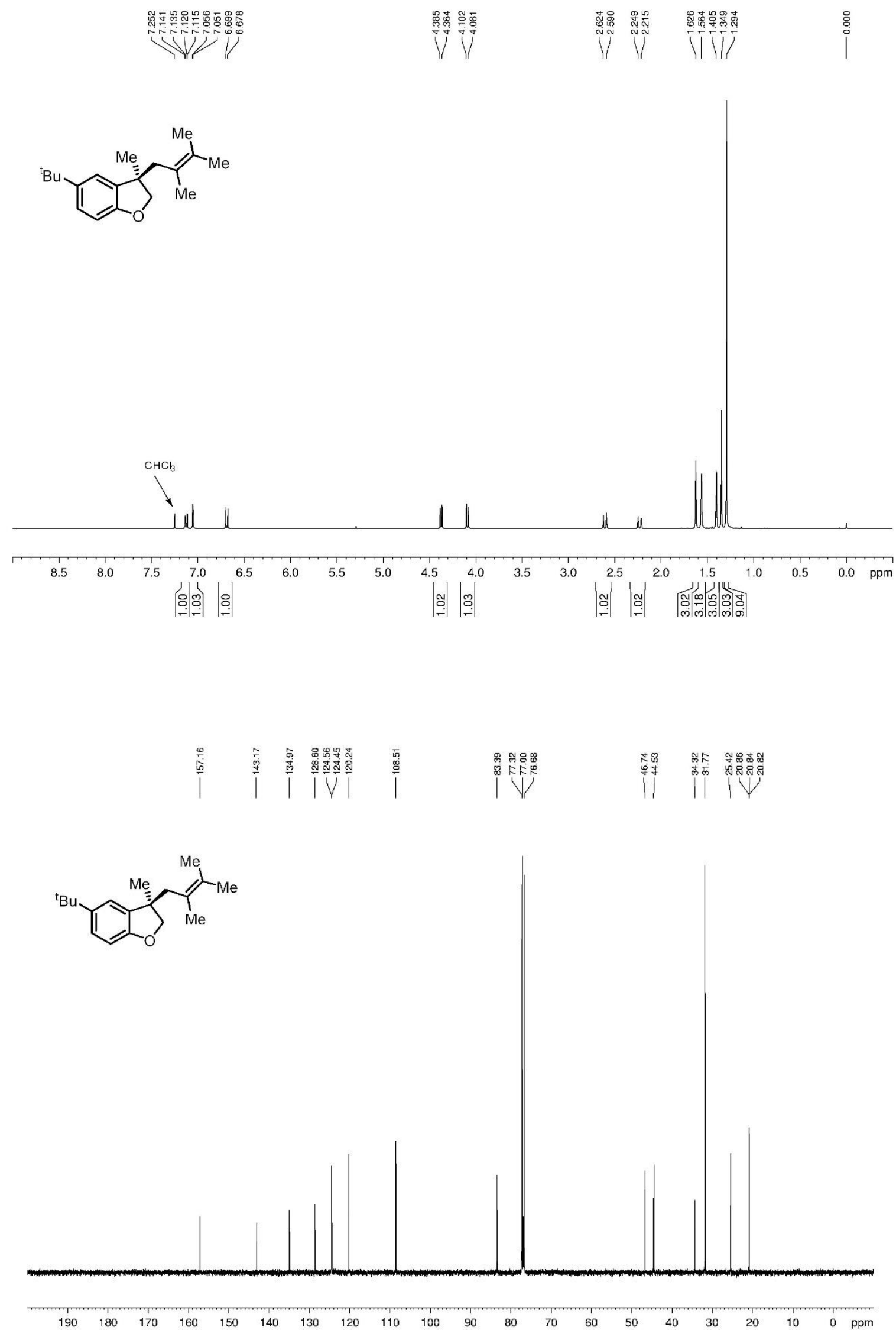
3u; ${ }^{1} \mathrm{H}$ NMR (400MHz, $\left.\mathrm{CDCl}_{3}\right) ;{ }^{13} \mathrm{C}$ NMR (100MHz, $\left.\mathrm{CDCl}_{3}\right)$

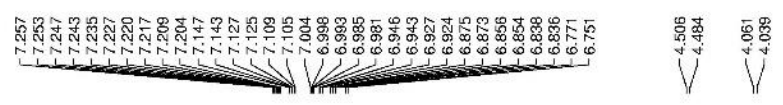
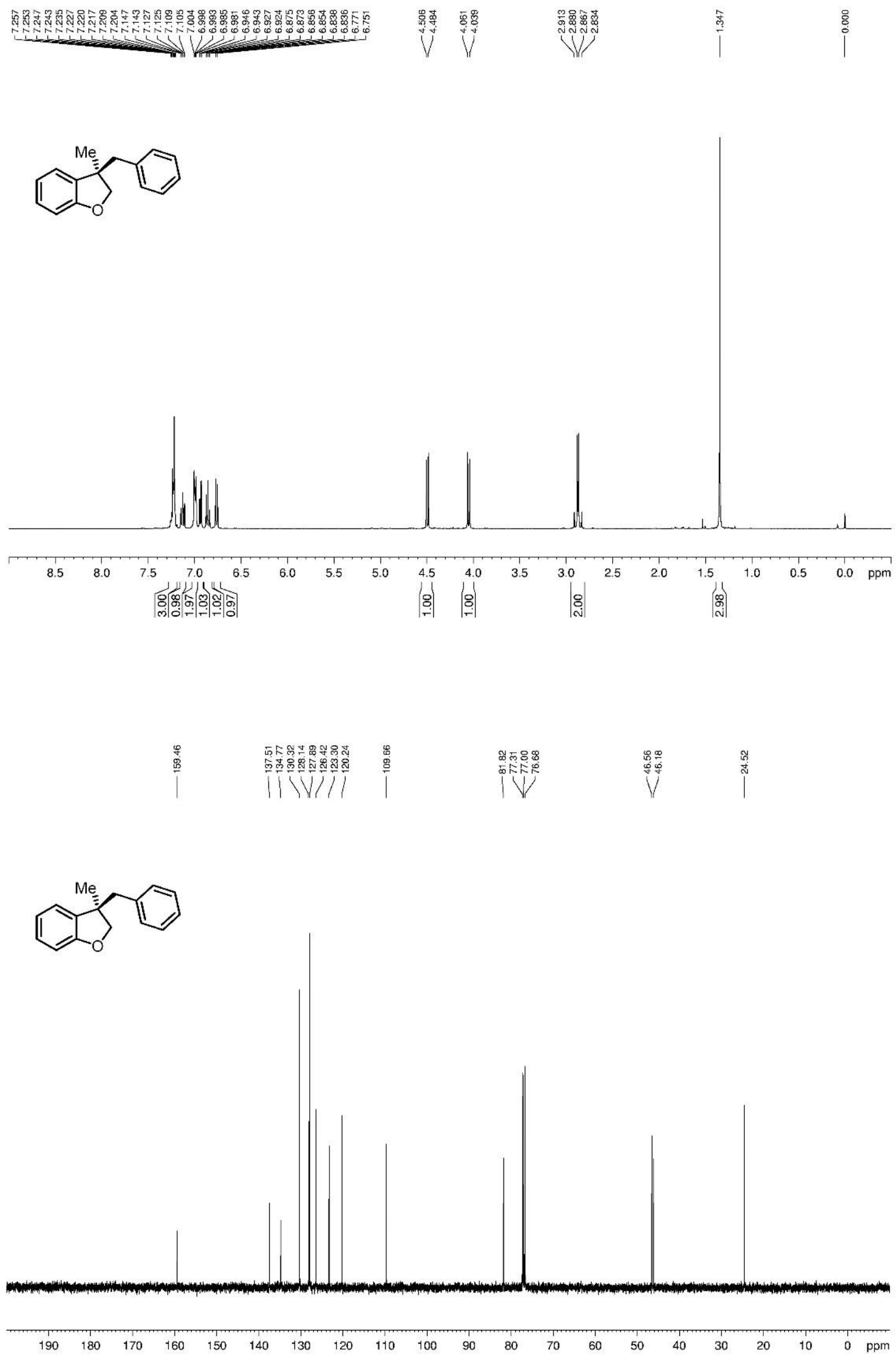

S135 
; ${ }^{1} \mathrm{H}$ NMR $\left(400 \mathrm{MHz}, \mathrm{CDCl}_{3}\right) ;{ }^{13} \mathrm{C}$ NMR $\left(100 \mathrm{MHz}, \mathrm{CDCl}_{3}\right)$

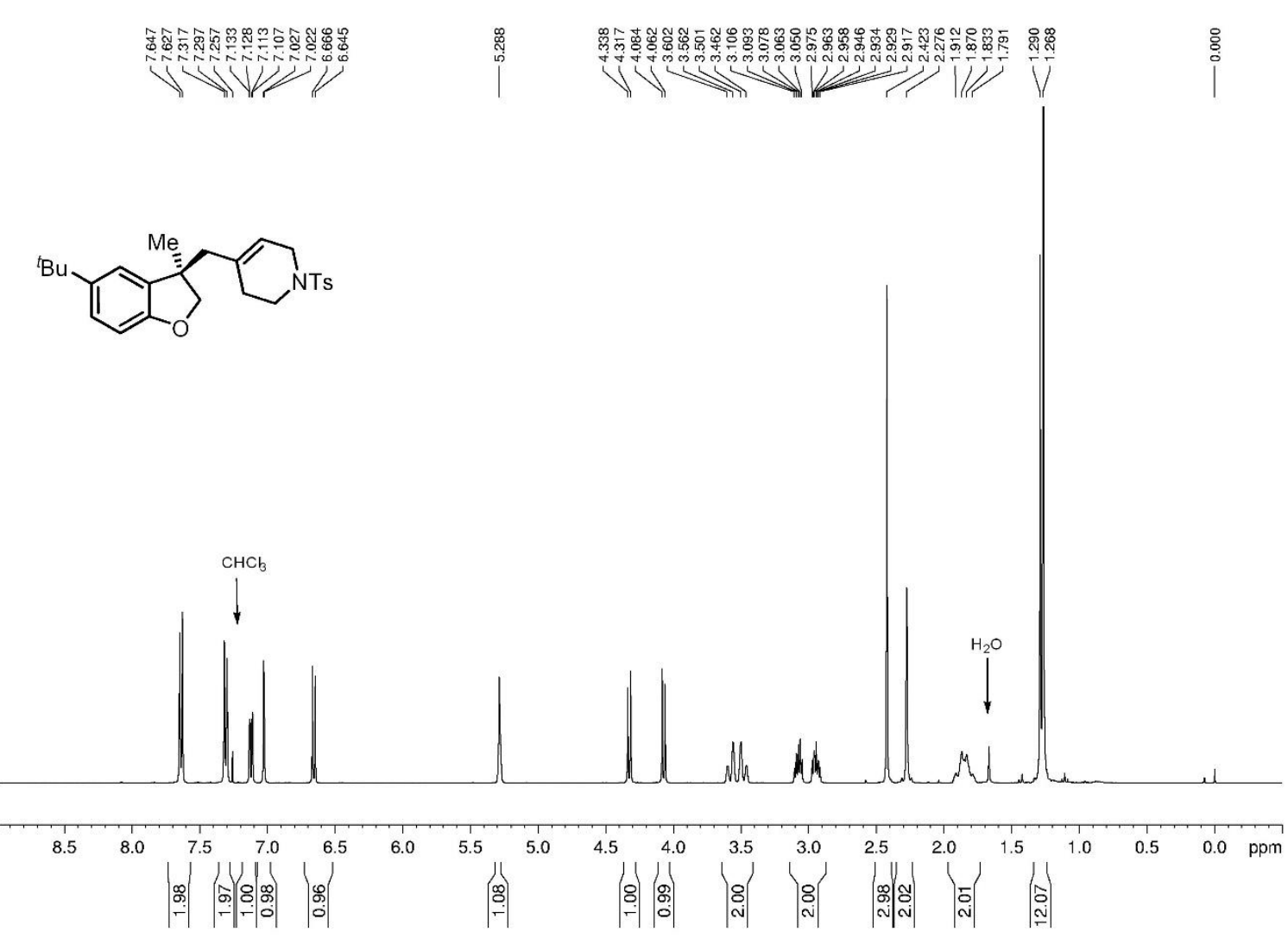

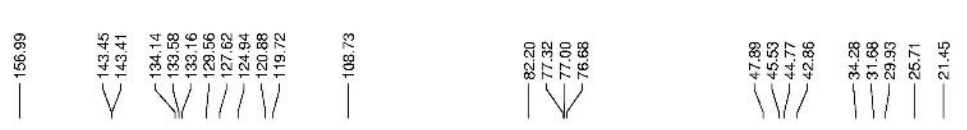

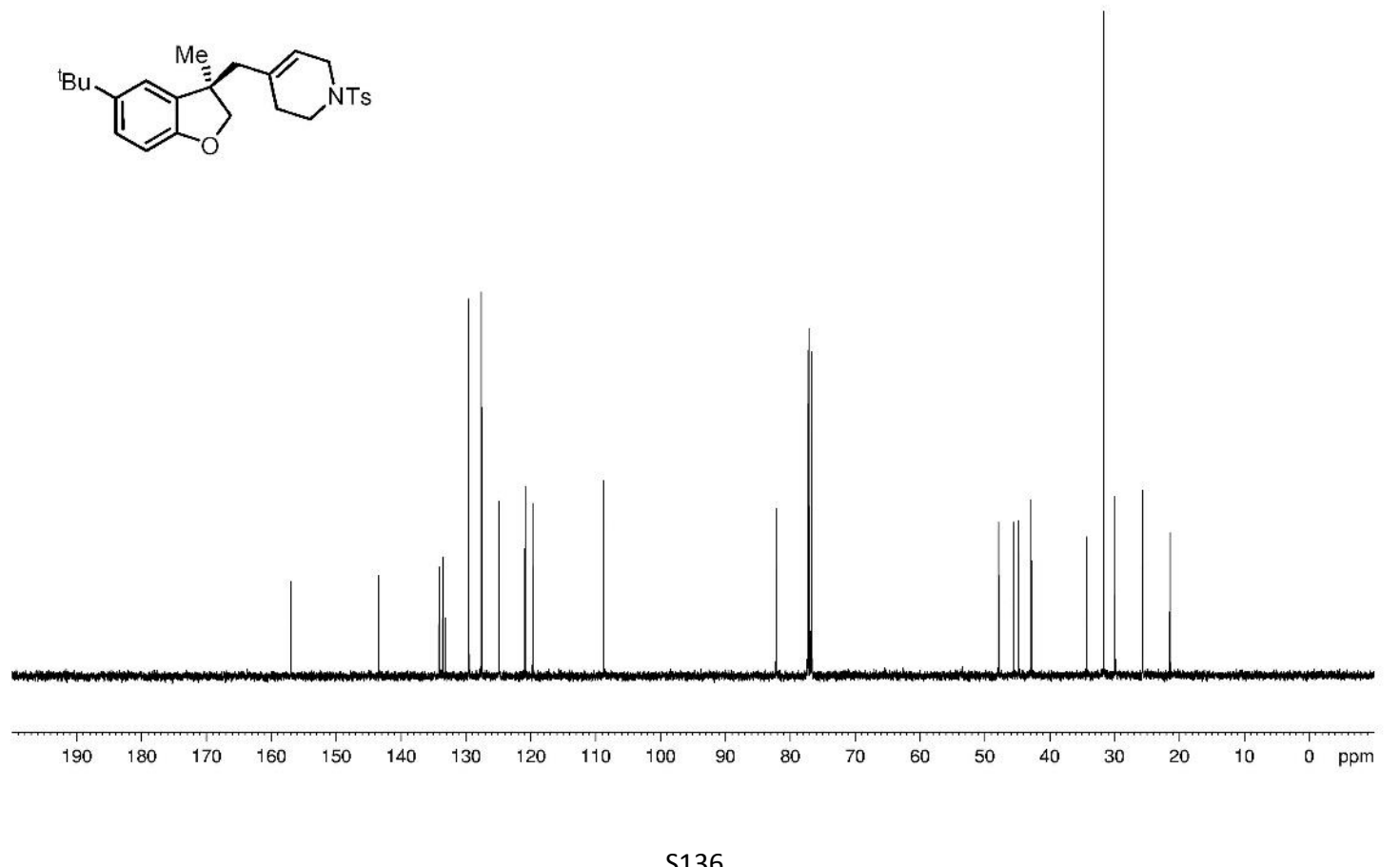


3w; ${ }^{1} \mathrm{H}$ NMR (400MHz, $\left.\mathrm{CDCl}_{3}\right) ;{ }^{13} \mathrm{C}$ NMR (100MHz, $\left.\mathrm{CDCl}_{3}\right)$

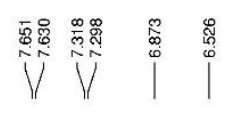
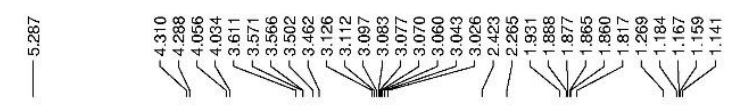

i
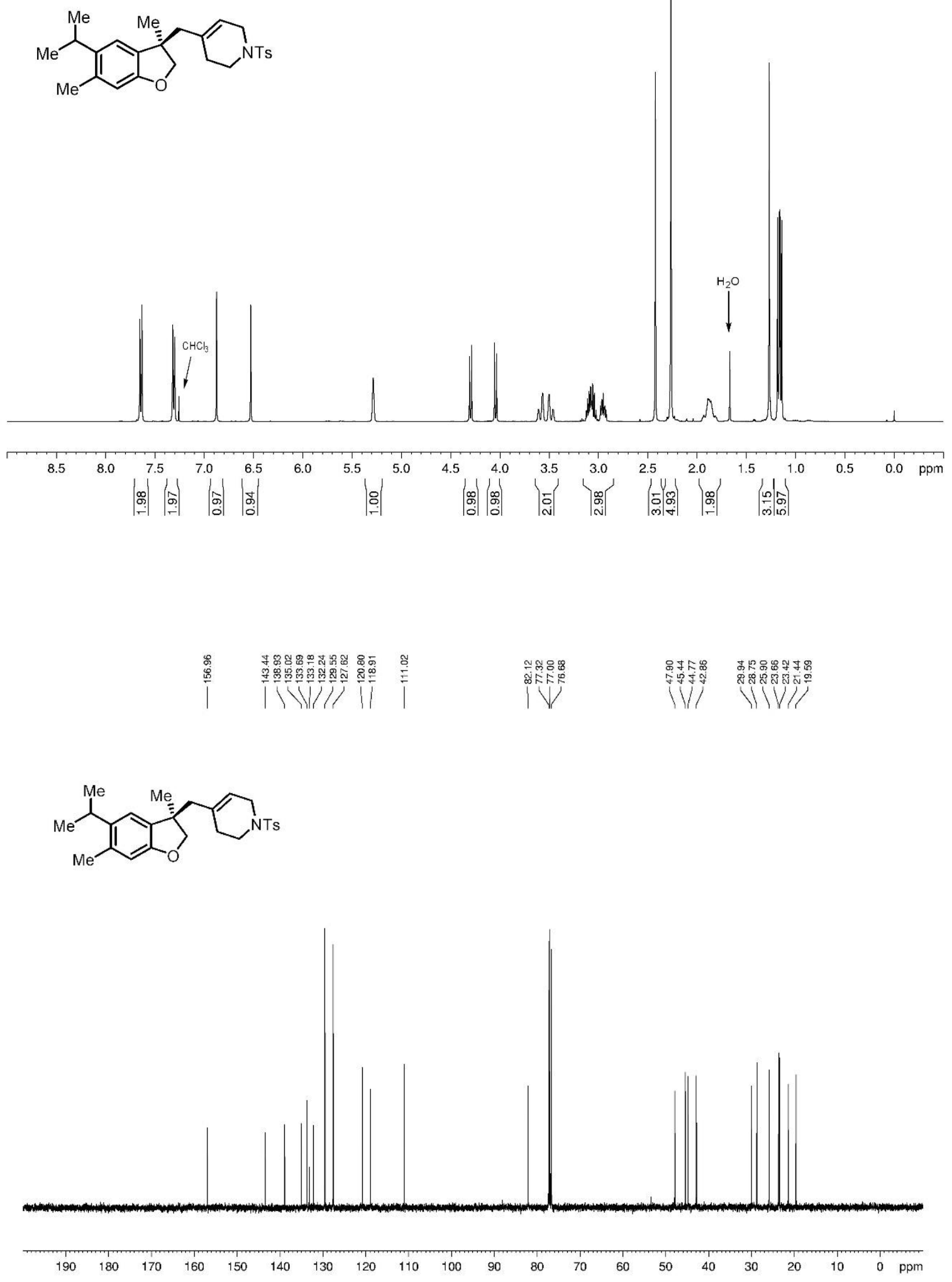

S137 
$3 \mathrm{x} ;{ }^{1} \mathrm{H}$ NMR (400MHz, $\left.\mathrm{CDCl}_{3}\right) ;{ }^{13} \mathrm{C}$ NMR (100MHz, $\left.\mathrm{CDCl}_{3}\right)$

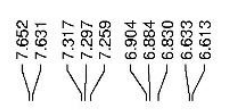

i.

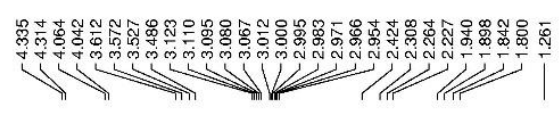

i
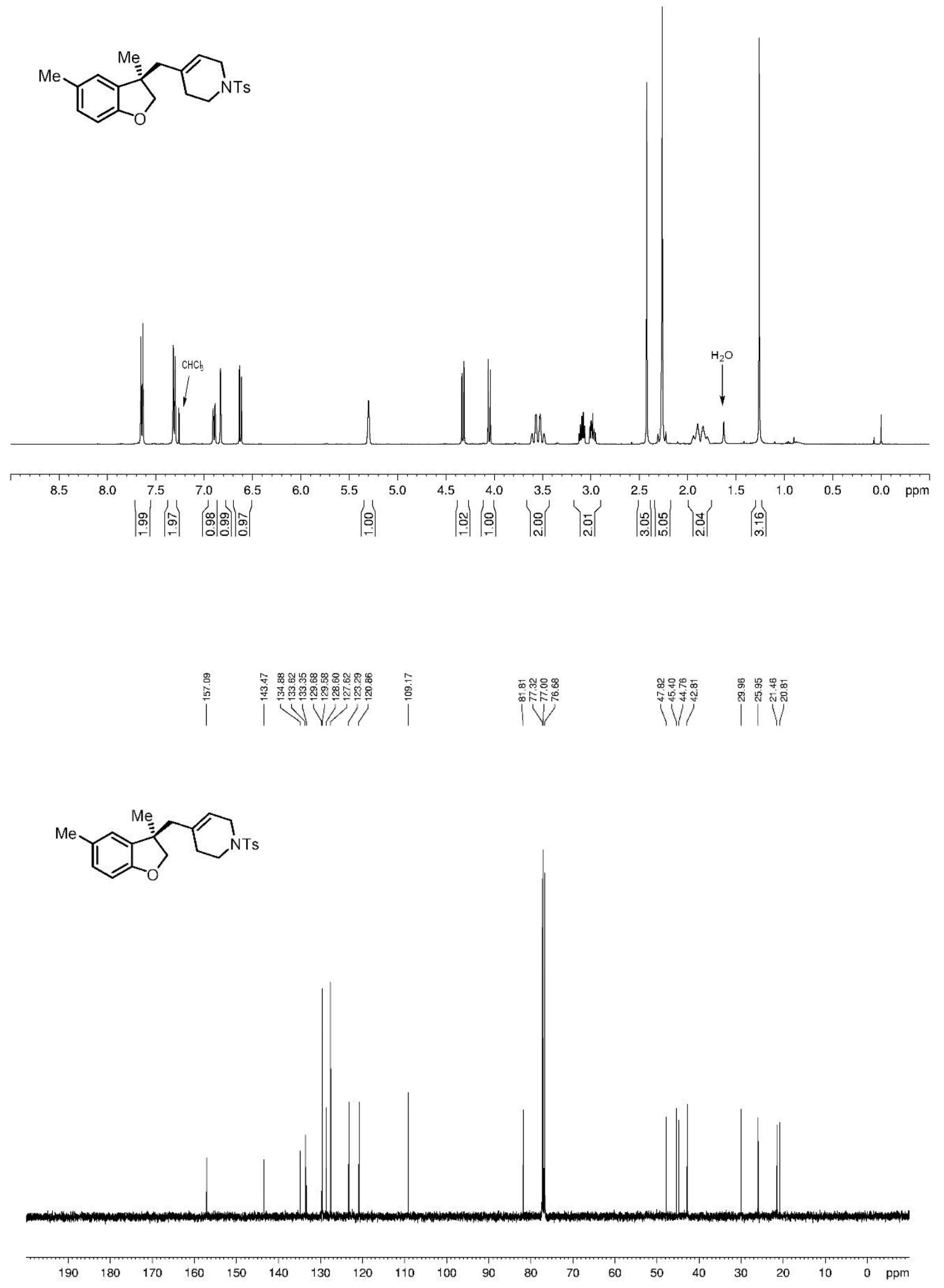

S138 
$3 y ;{ }^{1} \mathrm{H}$ NMR $\left(400 \mathrm{MHz}, \mathrm{CDCl}_{3}\right) ;{ }^{13} \mathrm{C}$ NMR $\left(100 \mathrm{MHz}, \mathrm{CDCl}_{3}\right)$

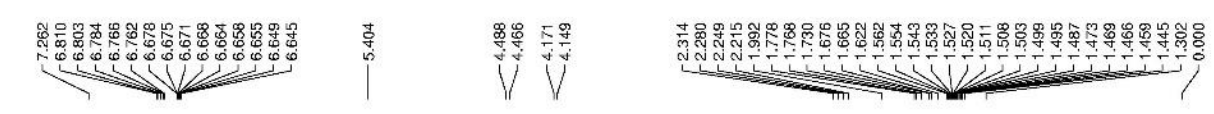<smiles>C[C@]1(CC2=CCCCC2)COc2ccc(F)cc21</smiles>
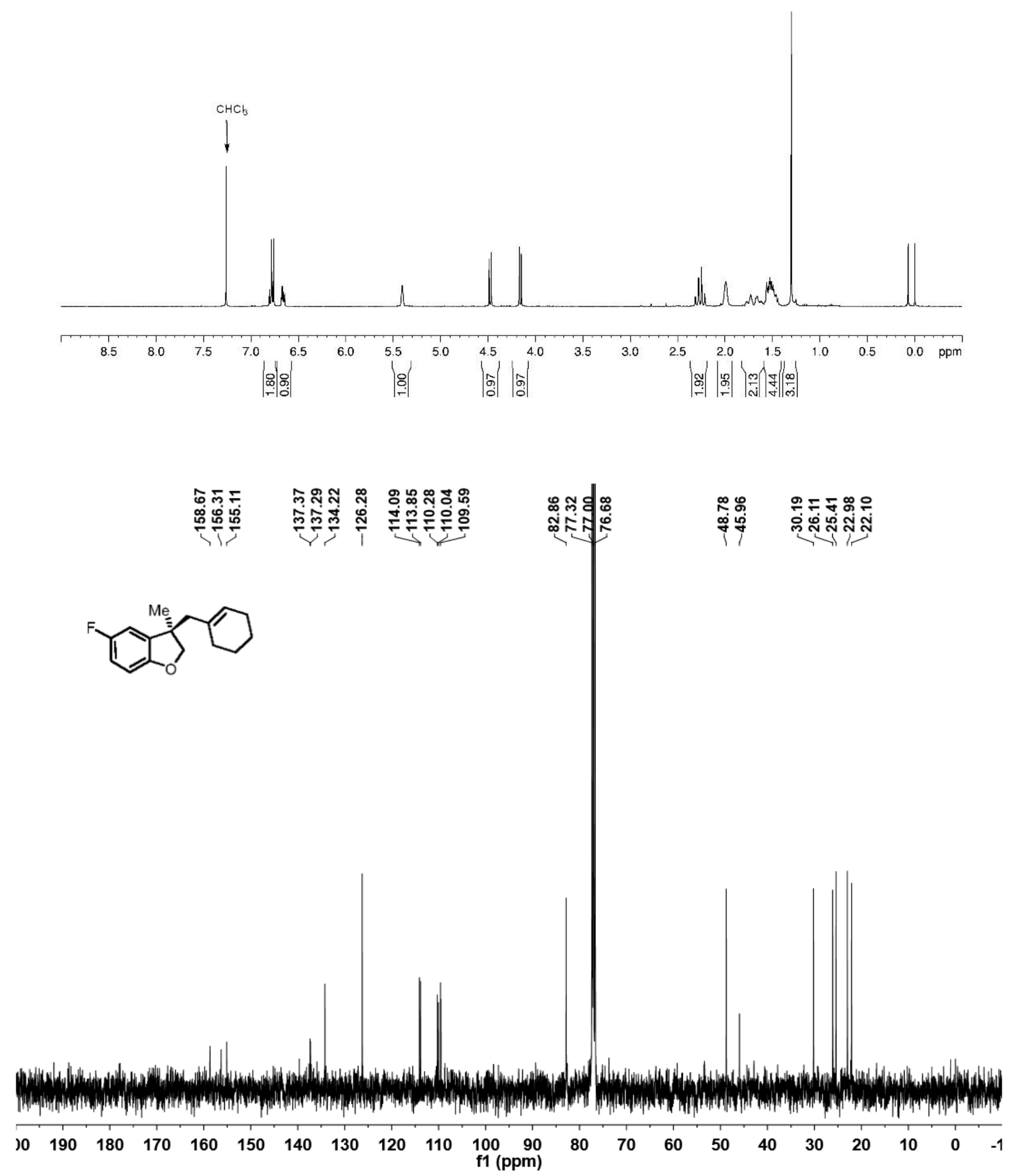
$3 \mathrm{y} ;{ }^{19} \mathrm{~F}$ NMR $\left(376 \mathrm{MHz}, \mathrm{CDCl}_{3}\right)$

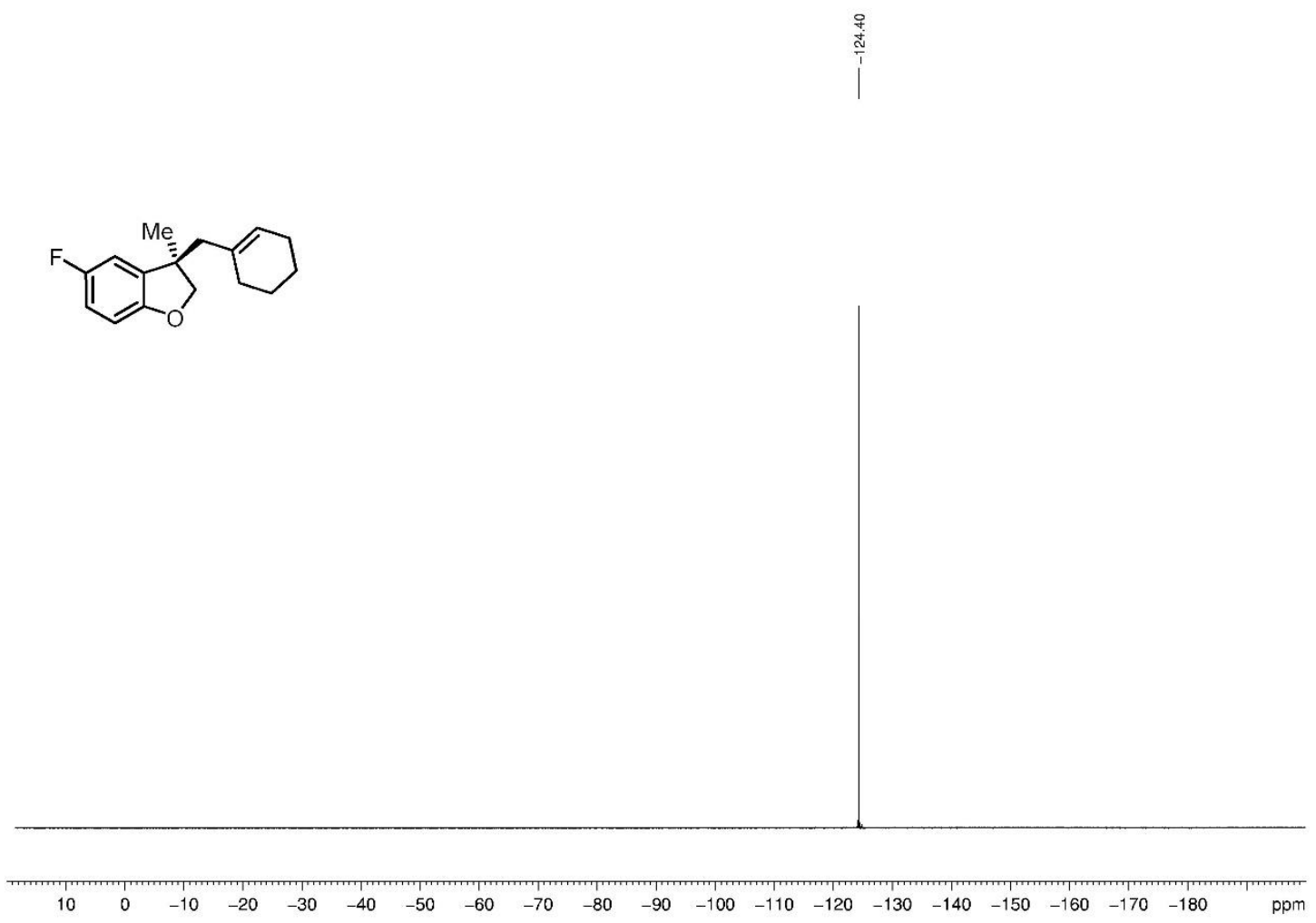


3z; ${ }^{1} \mathrm{H}$ NMR (400MHz, $\left.\mathrm{CDCl}_{3}\right) ;{ }^{13} \mathrm{C}$ NMR (100MHz, $\left.\mathrm{CDCl}_{3}\right)$
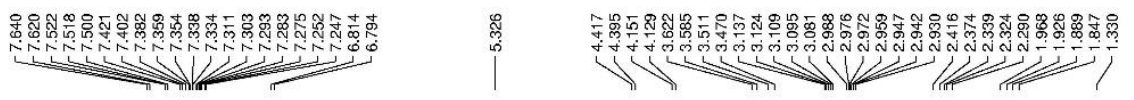

i
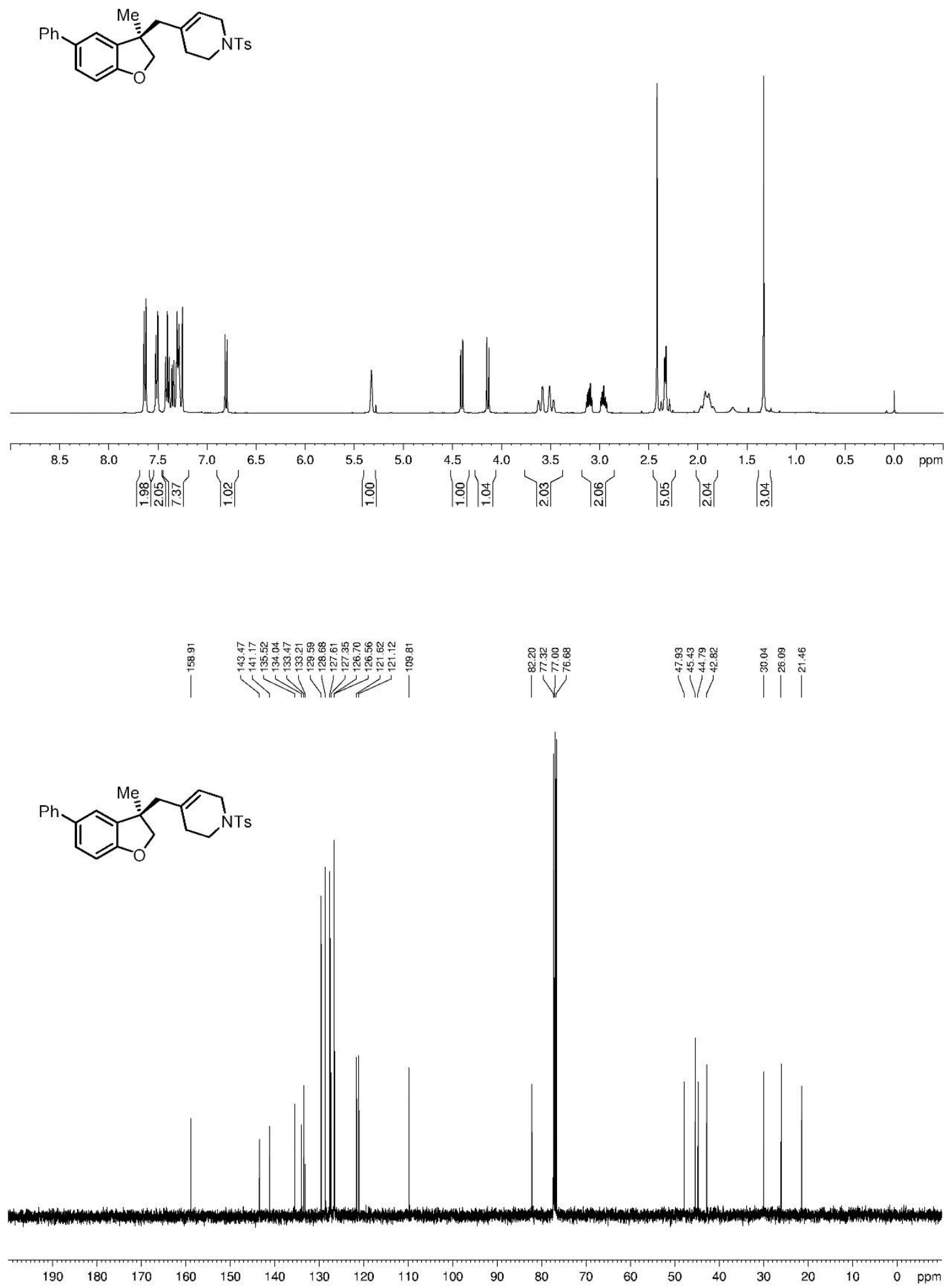

S141 
3ab; ${ }^{1} \mathrm{H}$ NMR $\left(400 \mathrm{MHz}, \mathrm{CDCl}_{3}\right) ;{ }^{13} \mathrm{C}$ NMR $\left(100 \mathrm{MHz}, \mathrm{CDCl}_{3}\right)$

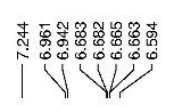

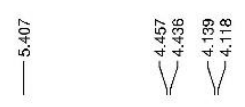
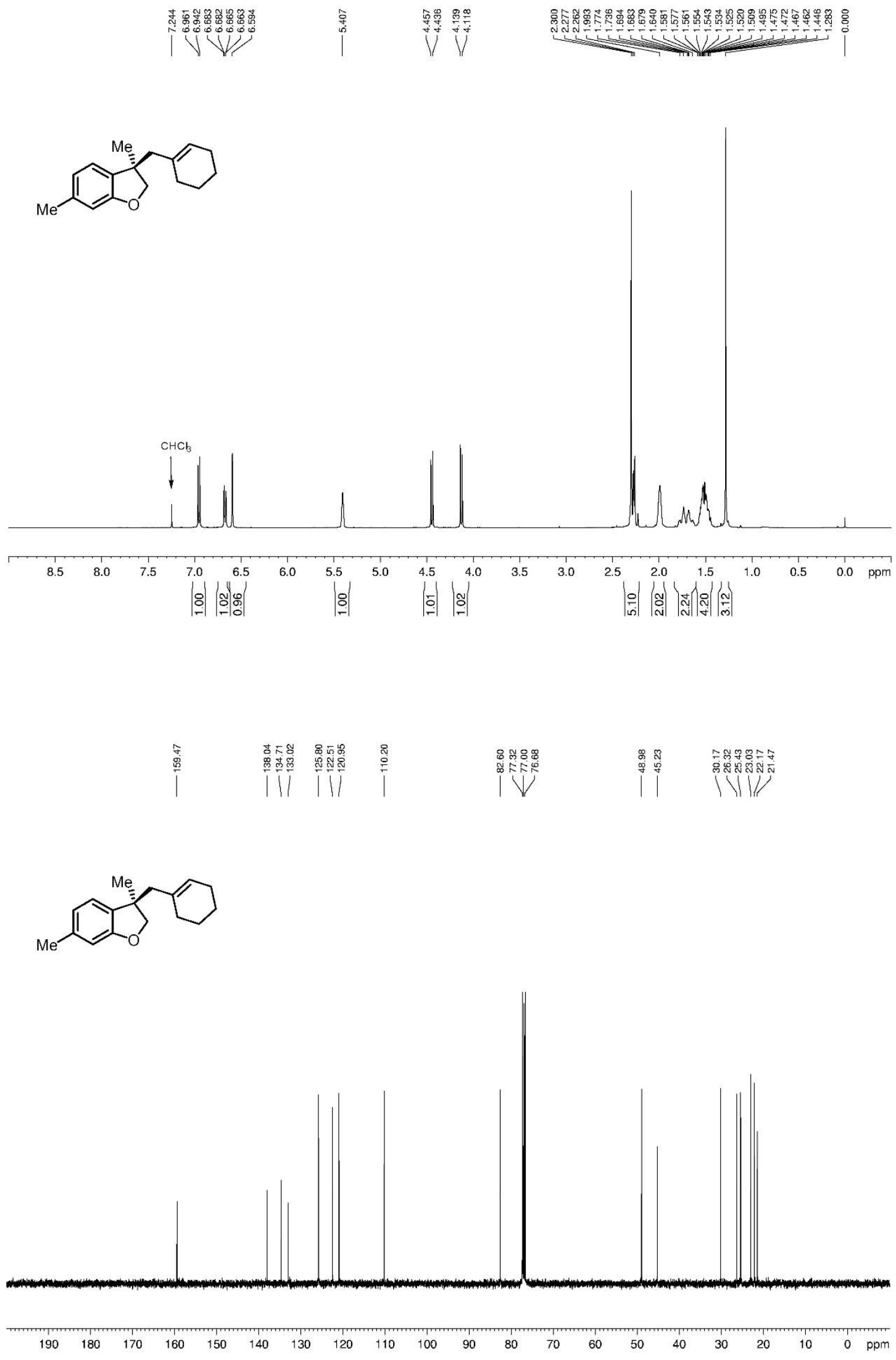

S142 
3ac; ${ }^{1} \mathrm{H}$ NMR $\left(400 \mathrm{MHz}, \mathrm{CDCl}_{3}\right) ;{ }^{13} \mathrm{C}$ NMR $\left(100 \mathrm{MHz}, \mathrm{CDCl}_{3}\right)$
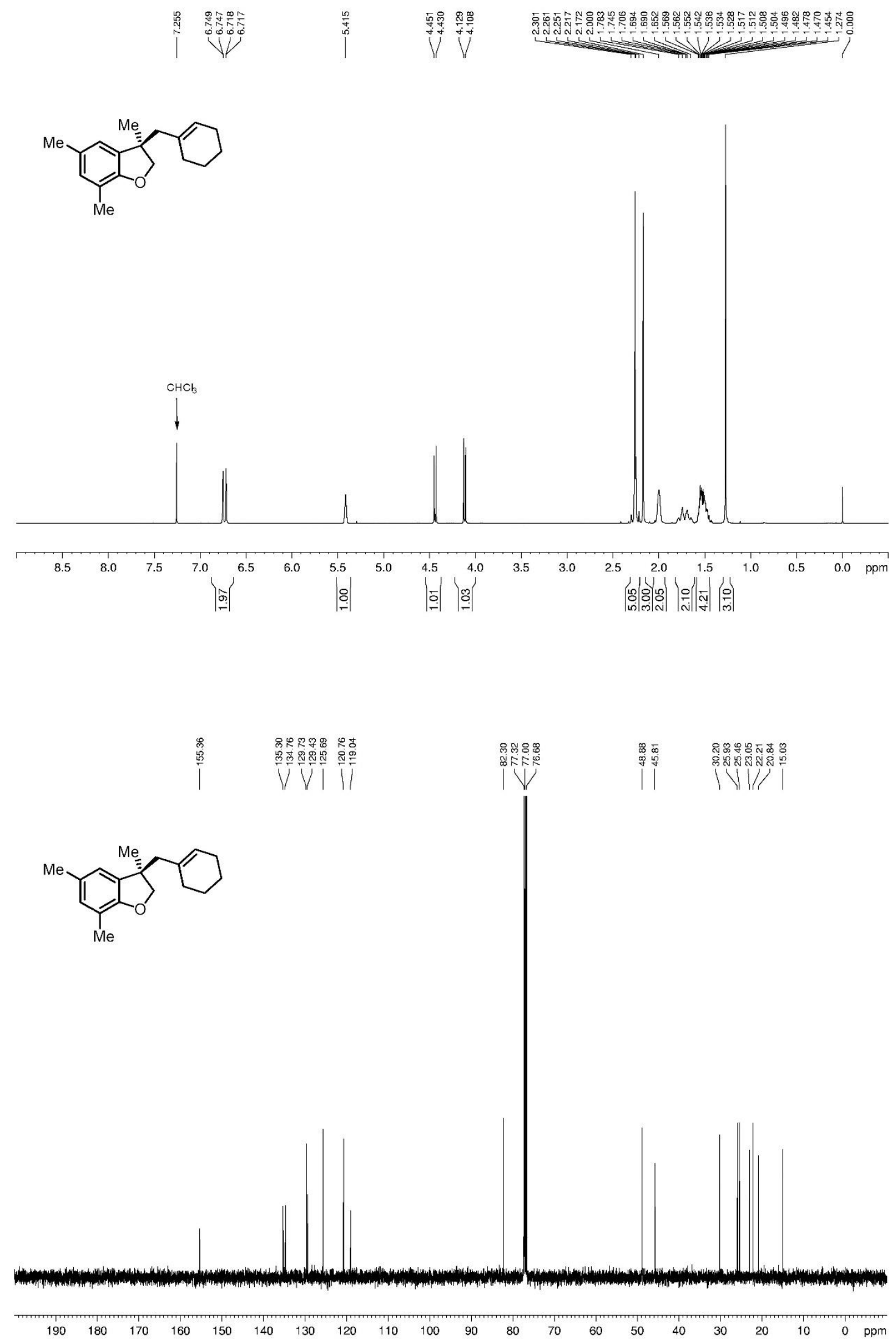
3ae; ${ }^{1} \mathrm{H}$ NMR $\left(400 \mathrm{MHz}, \mathrm{CDCl}_{3}\right) ;{ }^{13} \mathrm{C}$ NMR $\left(100 \mathrm{MHz}, \mathrm{CDCl}_{3}\right)$
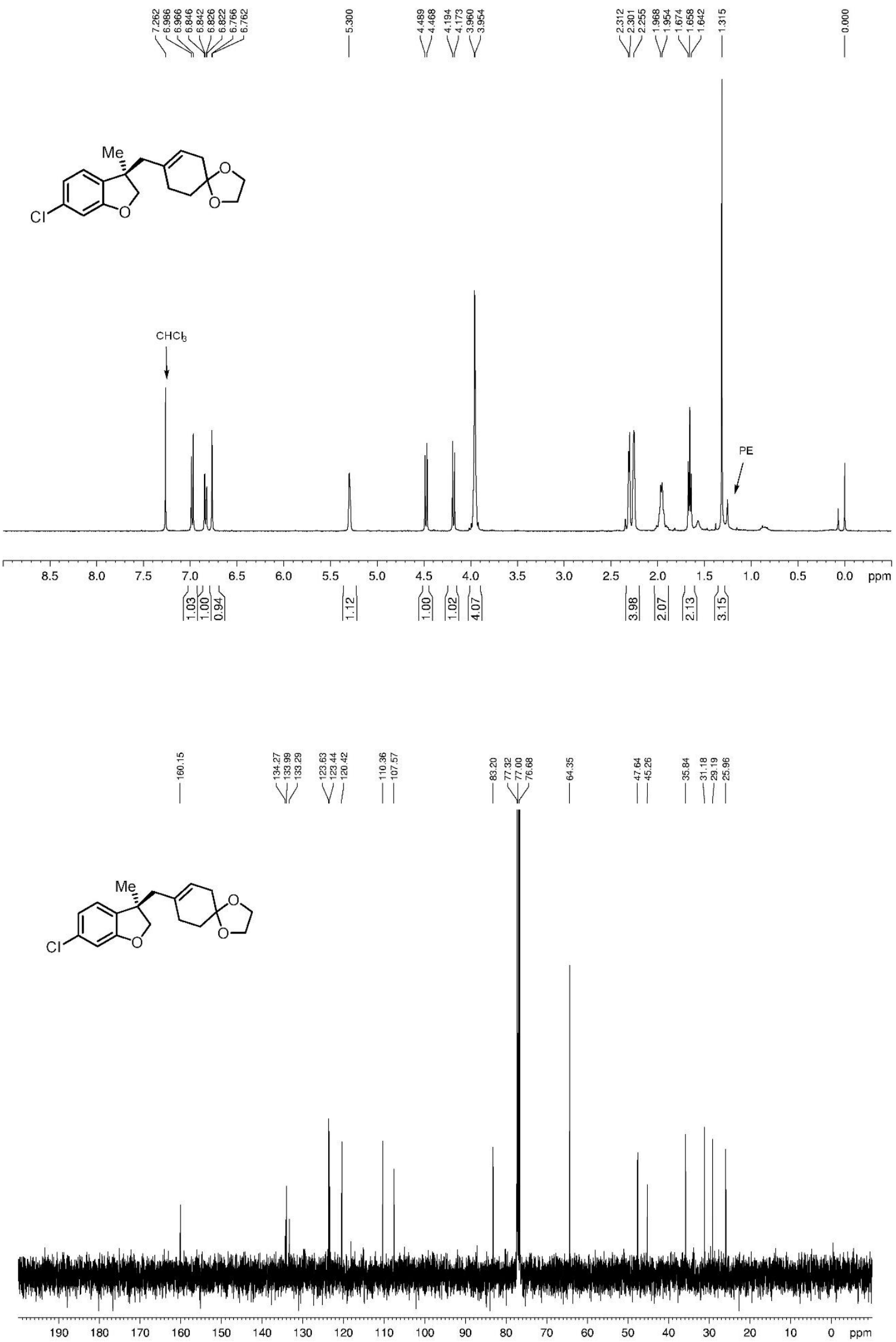
3af; ${ }^{1} \mathrm{H}$ NMR (400MHz, $\left.\mathrm{CDCl}_{3}\right) ;{ }^{13} \mathrm{C}$ NMR $\left(100 \mathrm{MHz}, \mathrm{CDCl}_{3}\right)$
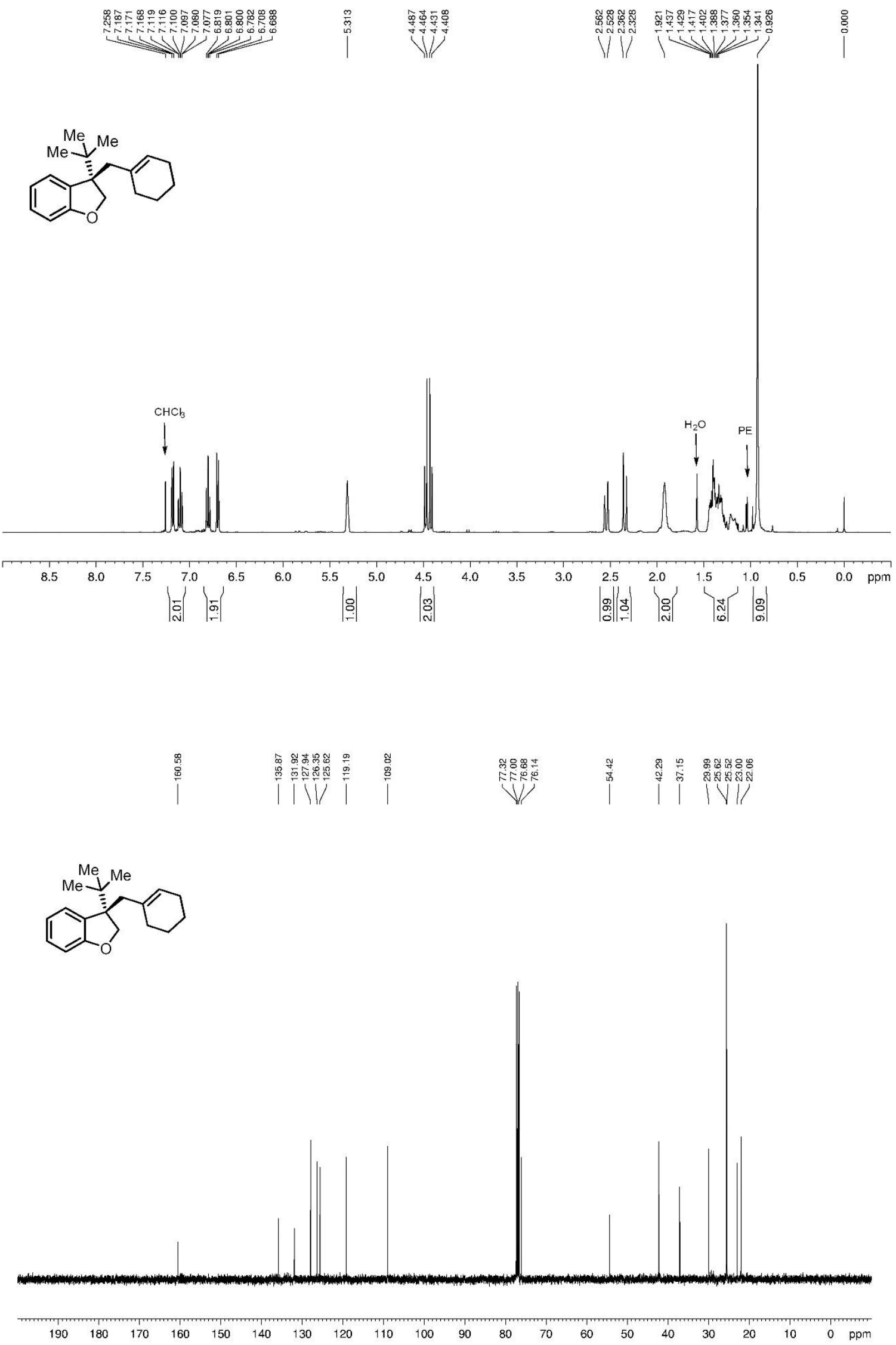
3ag; ${ }^{1} \mathrm{H}$ NMR (400MHz, $\left.\mathrm{CDCl}_{3}\right) ;{ }^{13} \mathrm{C}$ NMR $\left(100 \mathrm{MHz}, \mathrm{CDCl}_{3}\right)$

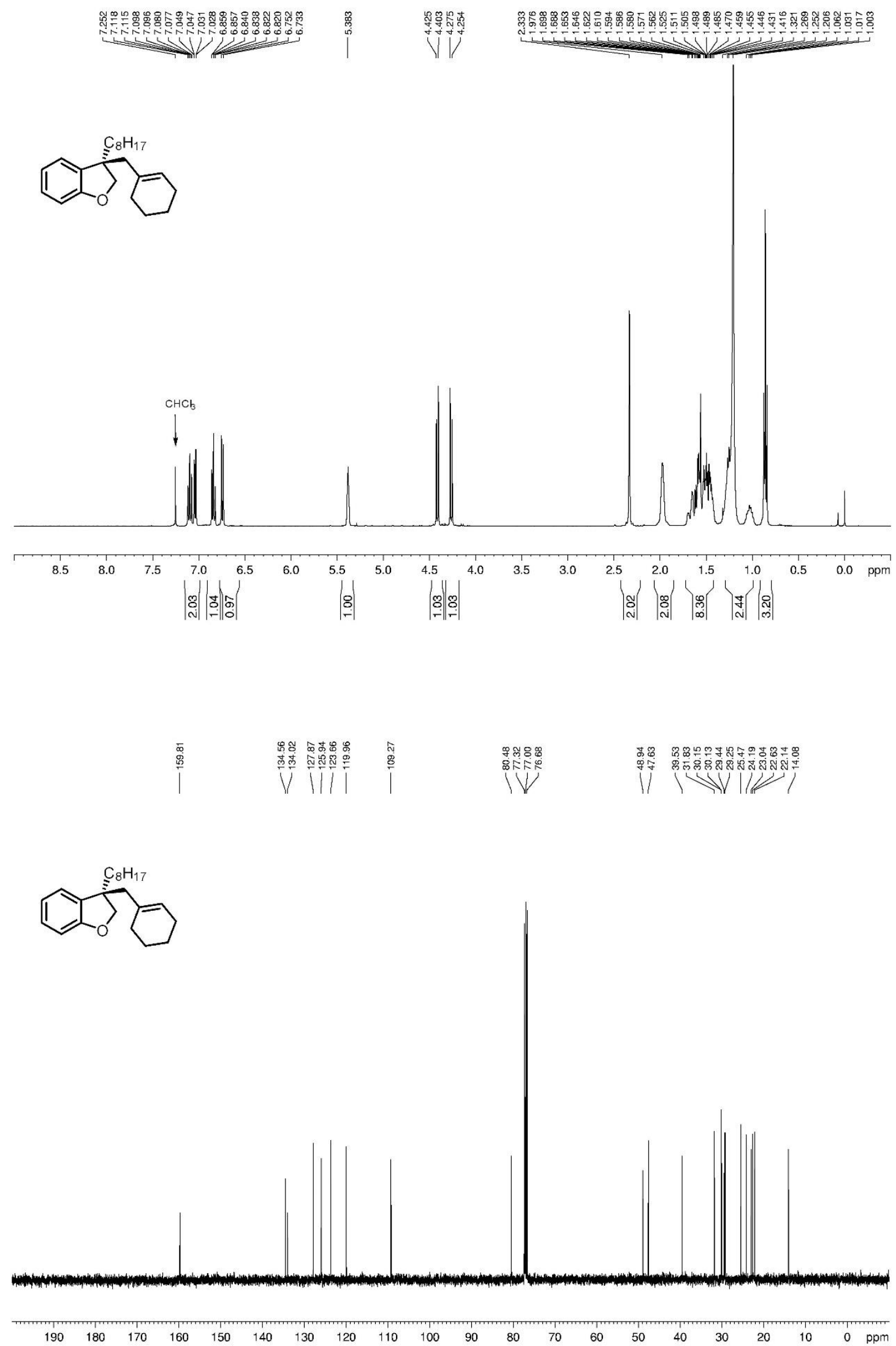


3ah; ${ }^{1} \mathrm{H}$ NMR $\left(400 \mathrm{MHz}, \mathrm{CDCl}_{3}\right) ;{ }^{13} \mathrm{C}$ NMR $\left(100 \mathrm{MHz}, \mathrm{CDCl}_{3}\right)$

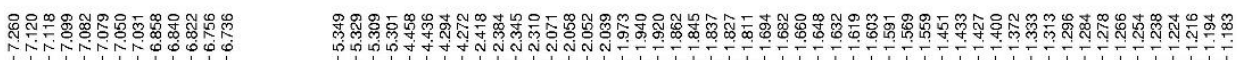
$\sqrt{1}$

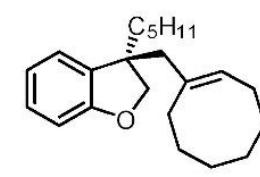

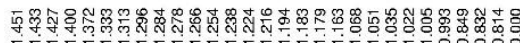
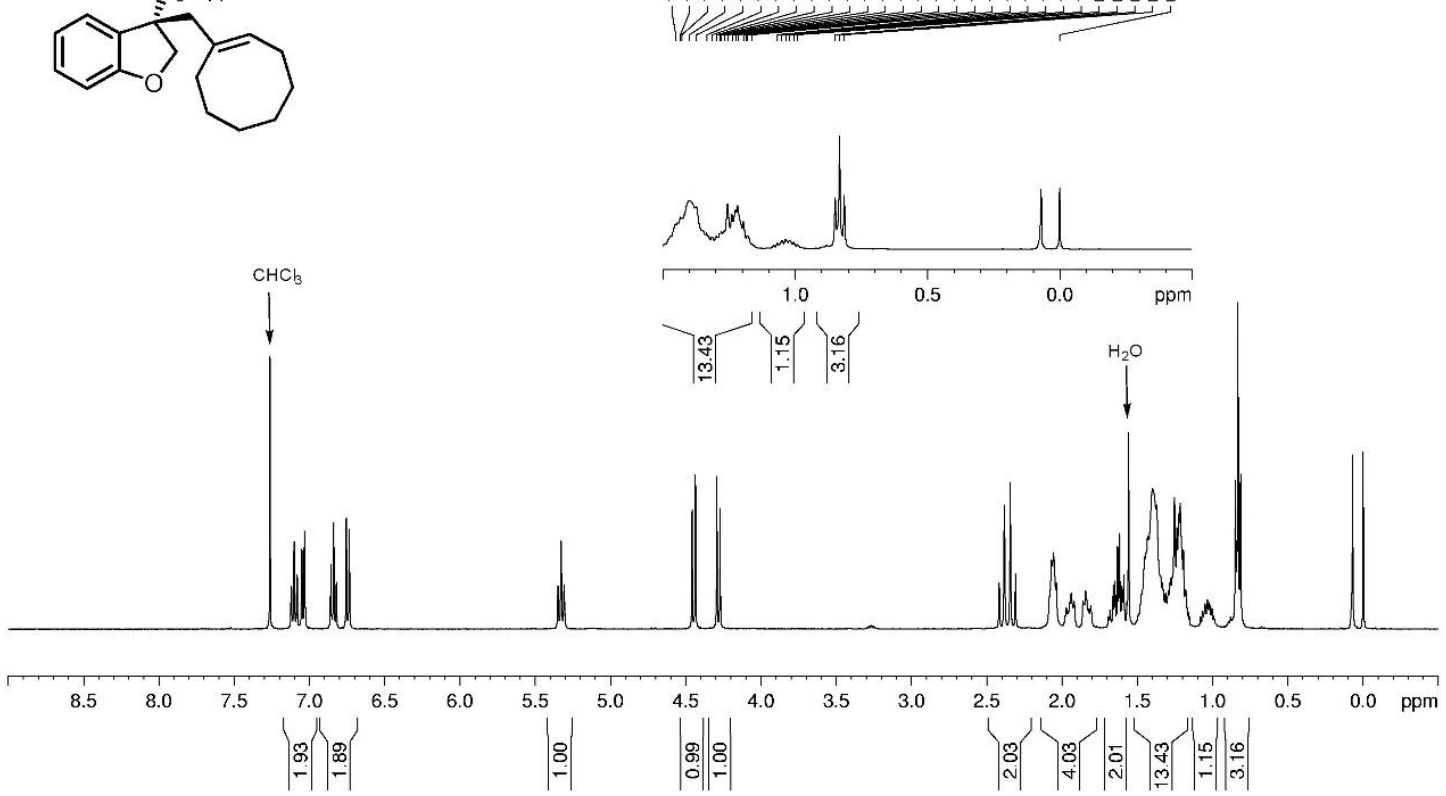

क्ष

.

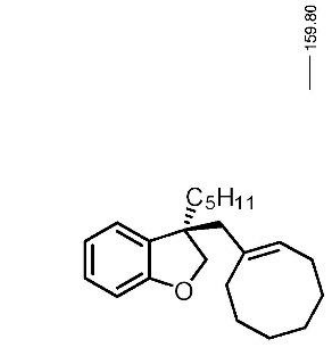

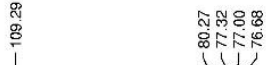

1
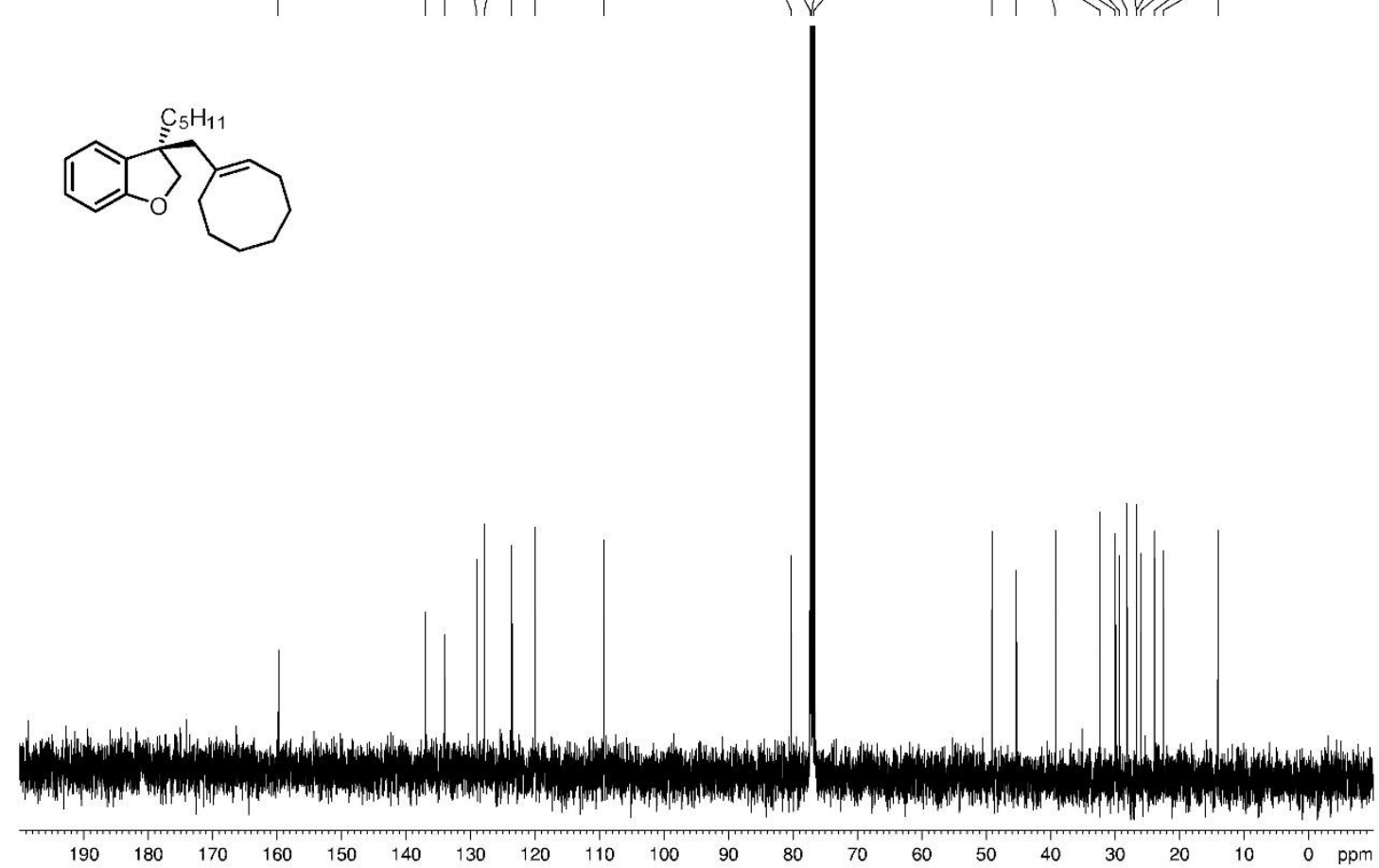
3ai; ${ }^{1} \mathrm{H}$ NMR (400MHz, $\left.\mathrm{CDCl}_{3}\right) ;{ }^{13} \mathrm{C}$ NMR $\left(100 \mathrm{MHz}, \mathrm{CDCl}_{3}\right)$

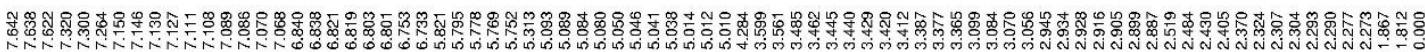

$\overbrace{0}^{N T S}$
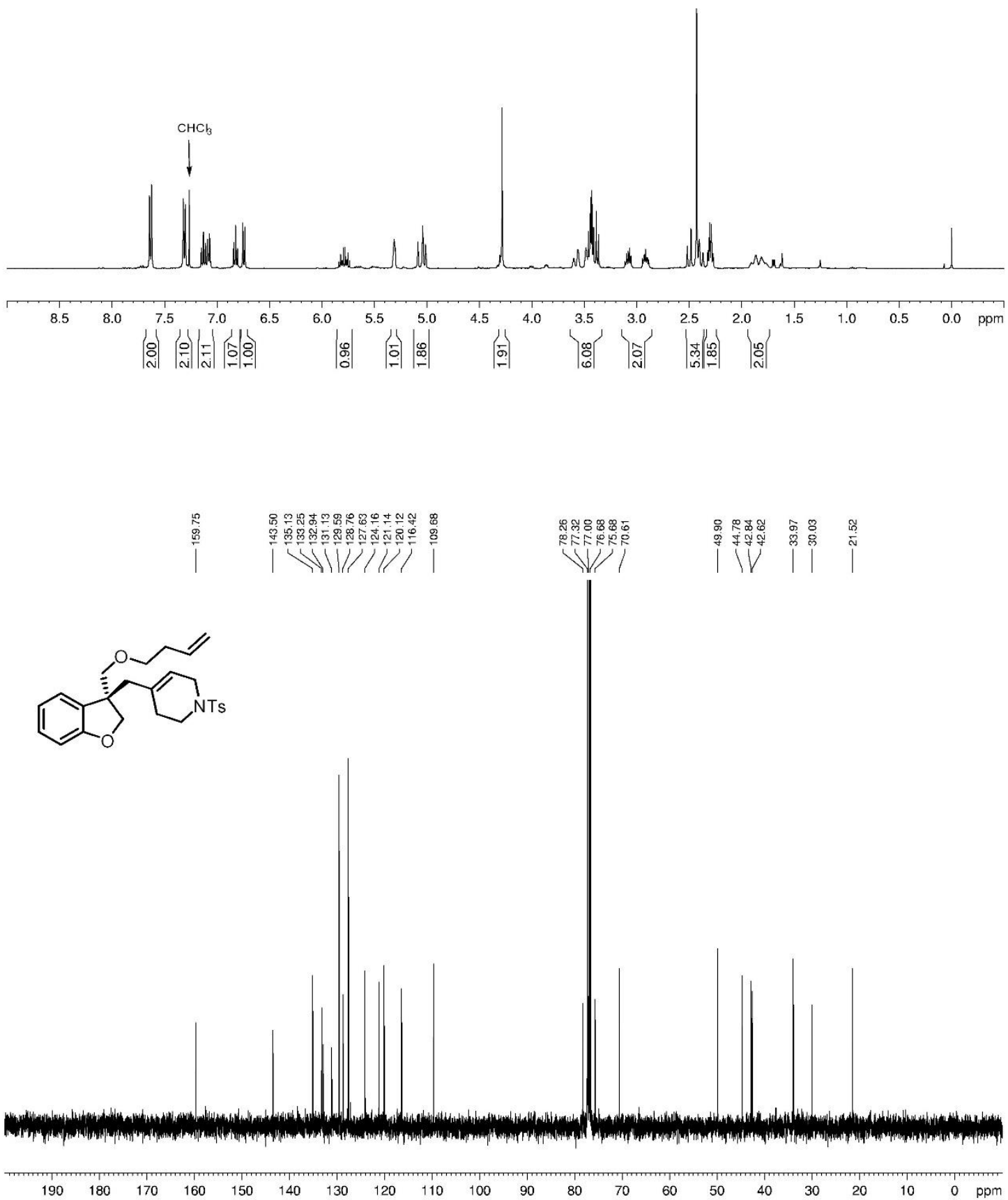
3aj; ${ }^{1} \mathrm{H}$ NMR (400MHz, $\left.\mathrm{CDCl}_{3}\right) ;{ }^{13} \mathrm{C}$ NMR $\left(100 \mathrm{MHz}, \mathrm{CDCl}_{3}\right)$

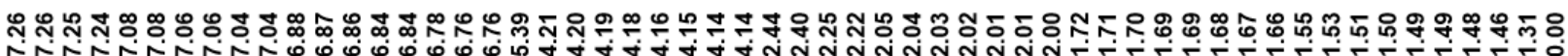<smiles>C[C@]1(CC2=CCCCC2)CCOc2ccccc21</smiles>

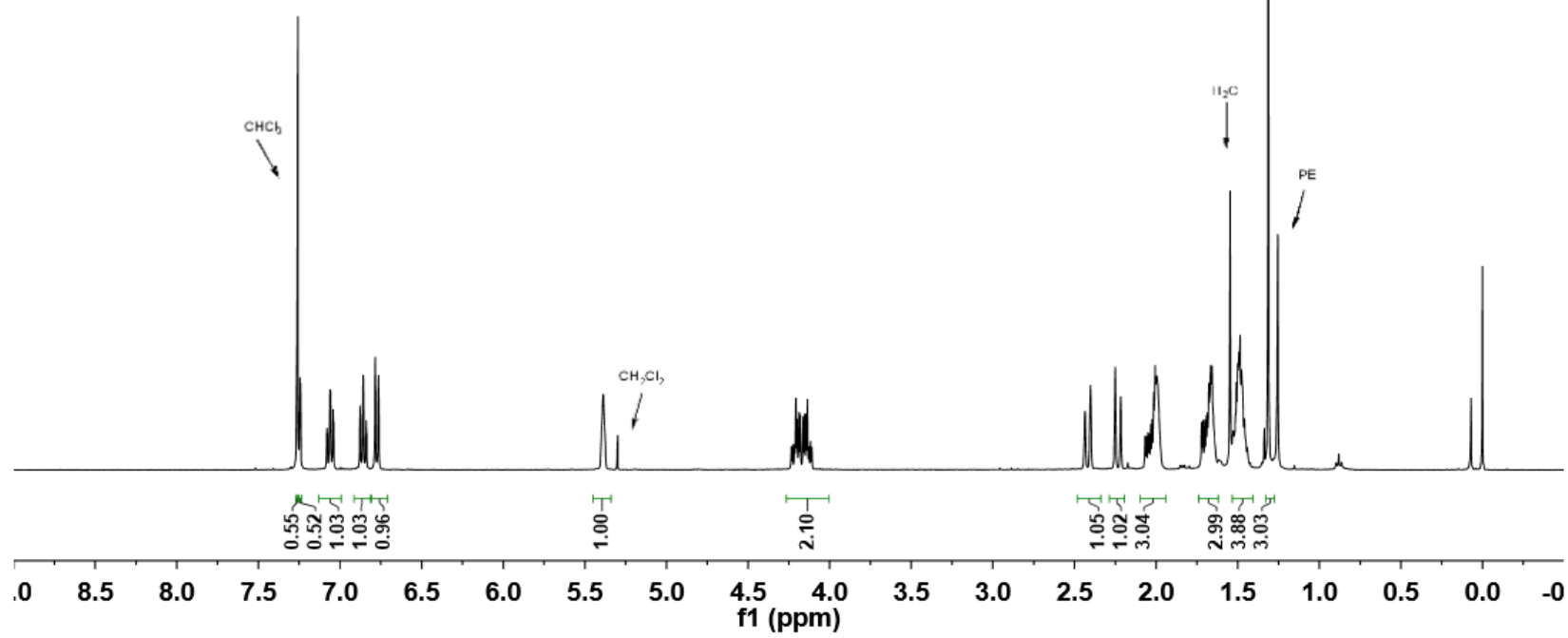

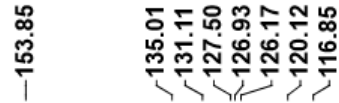

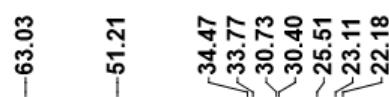<smiles>CC1(CC2=CCCCC2)CCOc2ccccc21</smiles>

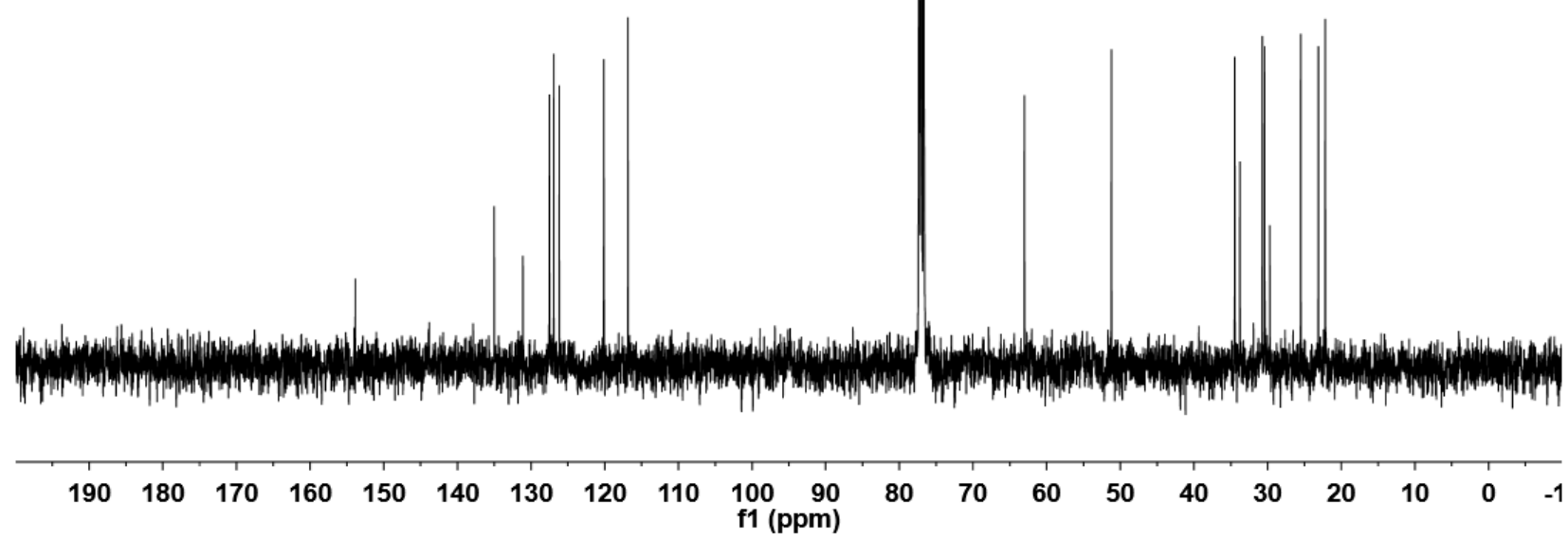


3al; ${ }^{1} \mathrm{H}$ NMR (400MHz, $\left.\mathrm{CDCl}_{3}\right) ;{ }^{13} \mathrm{C}$ NMR (100MHz, $\left.\mathrm{CDCl}_{3}\right)$

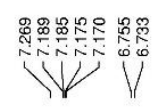

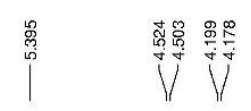

帝
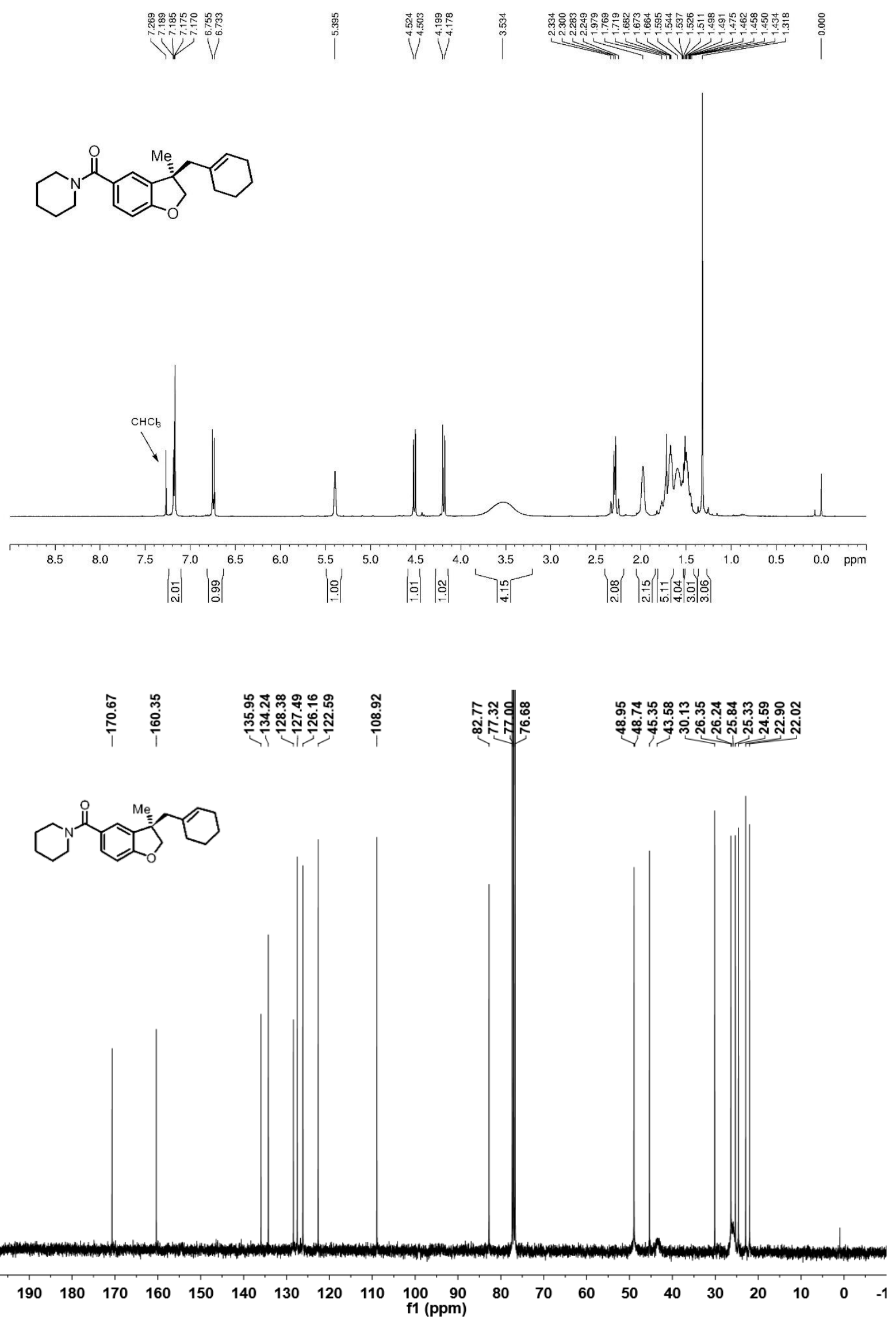

S150 
3am; ${ }^{1} \mathrm{H}$ NMR (400MHz, $\left.\mathrm{CDCl}_{3}\right) ;{ }^{13} \mathrm{C}$ NMR $\left(100 \mathrm{MHz}, \mathrm{CDCl}_{3}\right)$

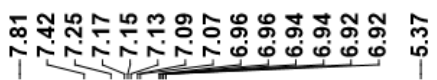

๙ิ<smiles>CC(C)(C)OC(=O)N1C[C@@](C)(CC2=CCCCC2)c2ccccc21</smiles>

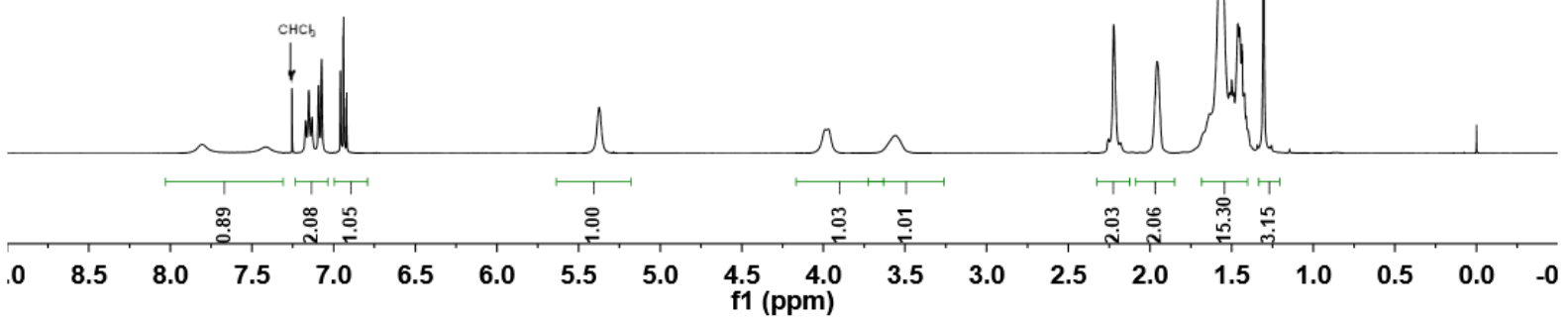

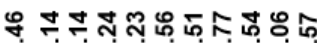

กิ่<smiles>CC1(CC2=CCCCC2)CNc2ccccc21</smiles> 
3an; ${ }^{1} \mathrm{H}$ NMR (400MHz, $\left.\mathrm{CDCl}_{3}\right) ;{ }^{13} \mathrm{C}$ NMR $\left(100 \mathrm{MHz}, \mathrm{CDCl}_{3}\right)$

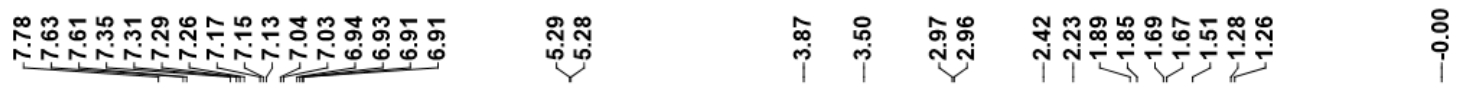<smiles>C[NH+]1CC=C(C[C@@]2(C)CNc3ccccc32)CC1</smiles>

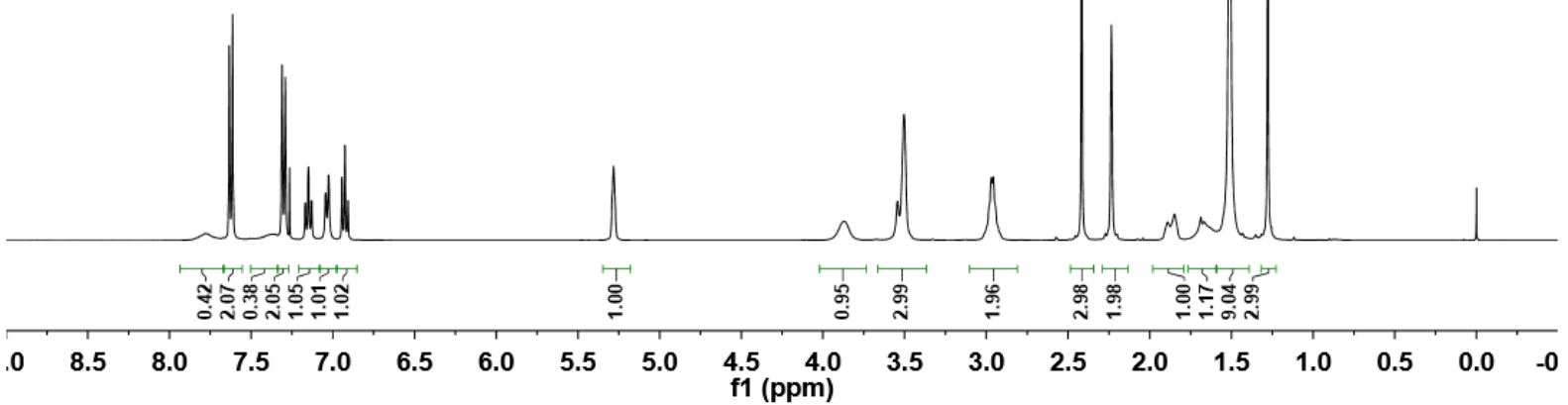

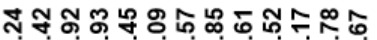
สุ่าำ

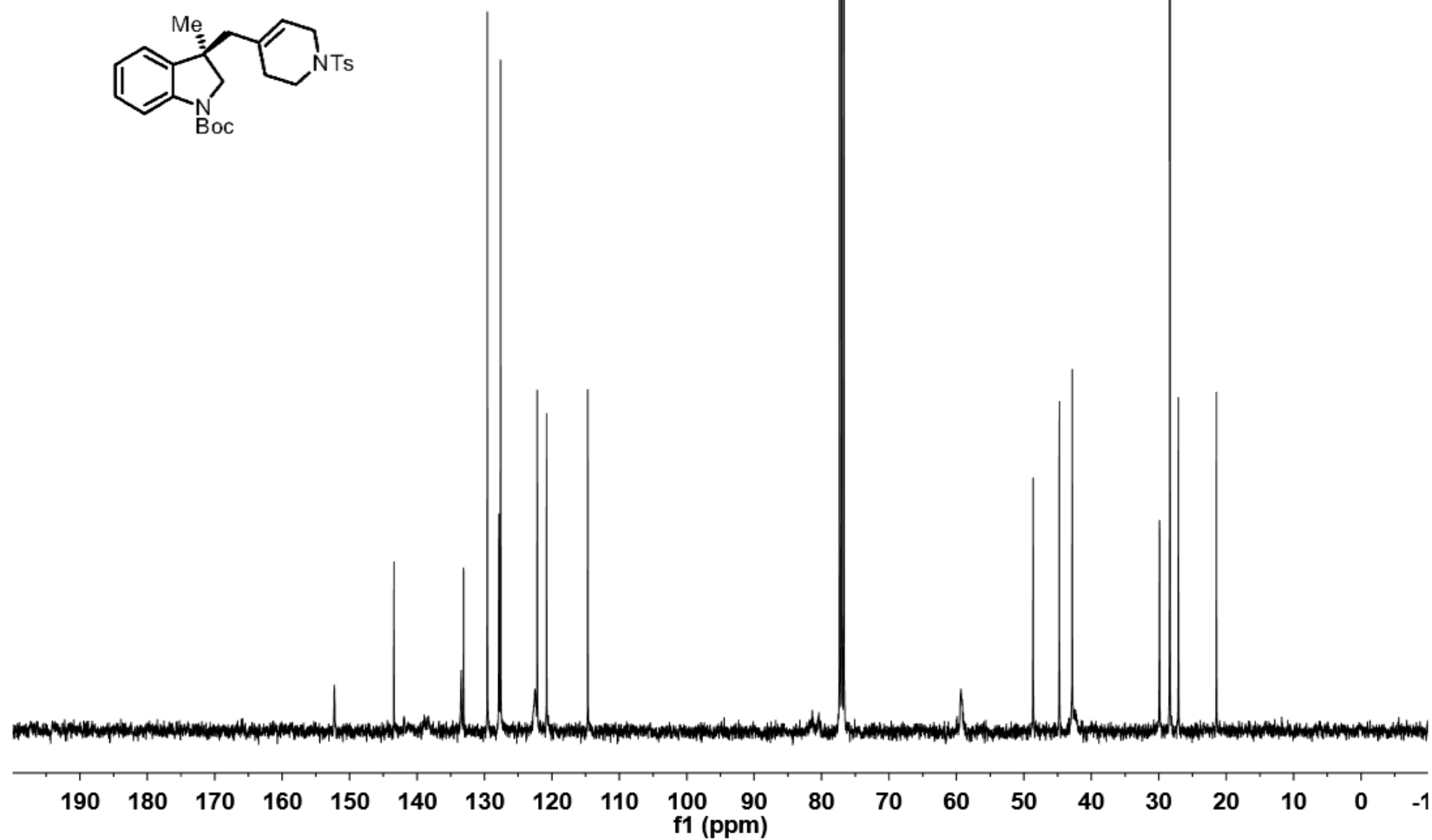

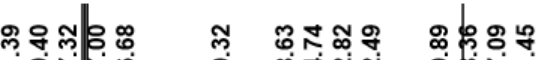

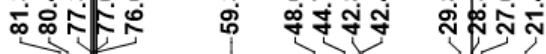


3ao; ${ }^{1} \mathrm{H}$ NMR $\left(400 \mathrm{MHz}, \mathrm{CDCl}_{3}\right) ;{ }^{13} \mathrm{C}$ NMR $\left(150 \mathrm{MHz}, \mathrm{CDCl}_{3}\right)$

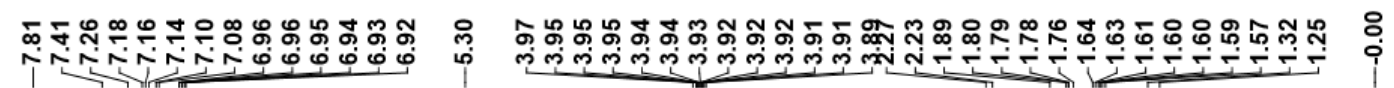<smiles>C[C@]1(CC2=CCC3(CC2)OCCO3)C[NH2+]c2ccccc21</smiles>
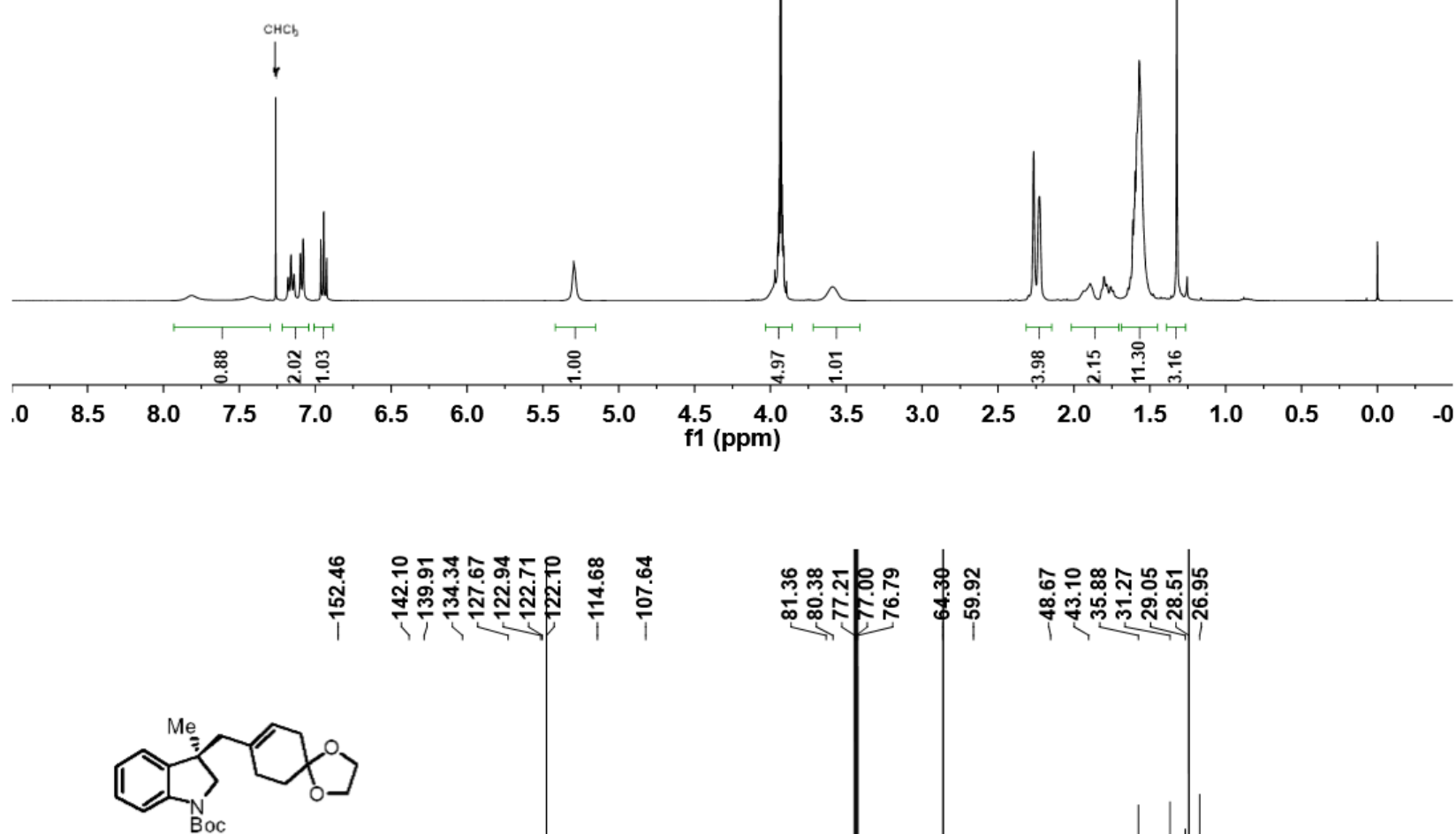

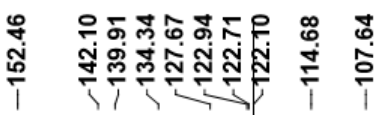

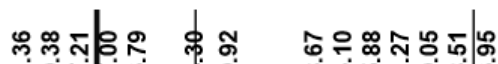

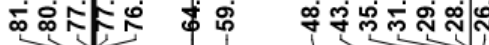

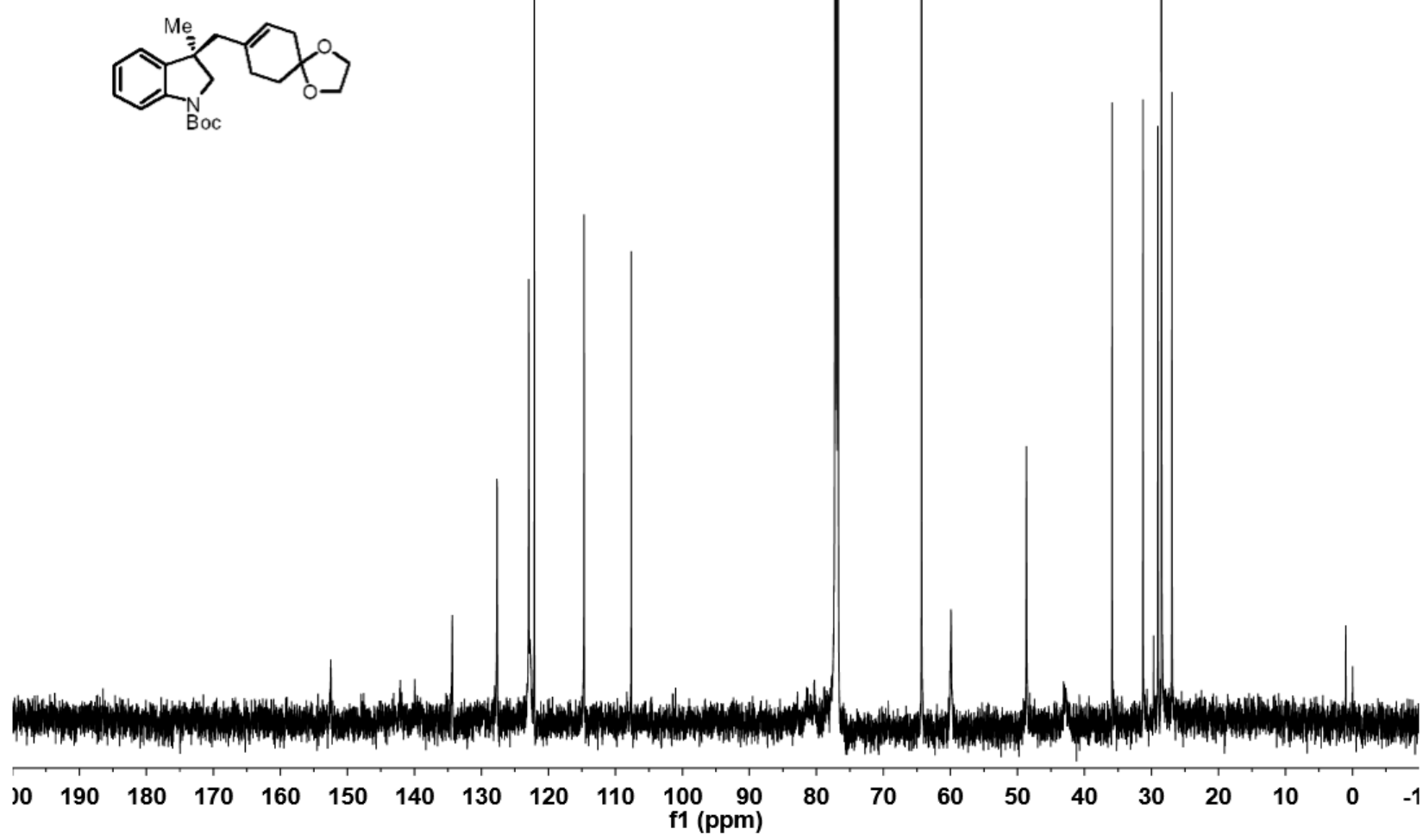


3ap; ${ }^{1} \mathrm{H}$ NMR $\left(400 \mathrm{MHz}, \mathrm{CDCl}_{3}\right) ;{ }^{13} \mathrm{C}$ NMR $\left(100 \mathrm{MHz}, \mathrm{CDCl}_{3}\right)$
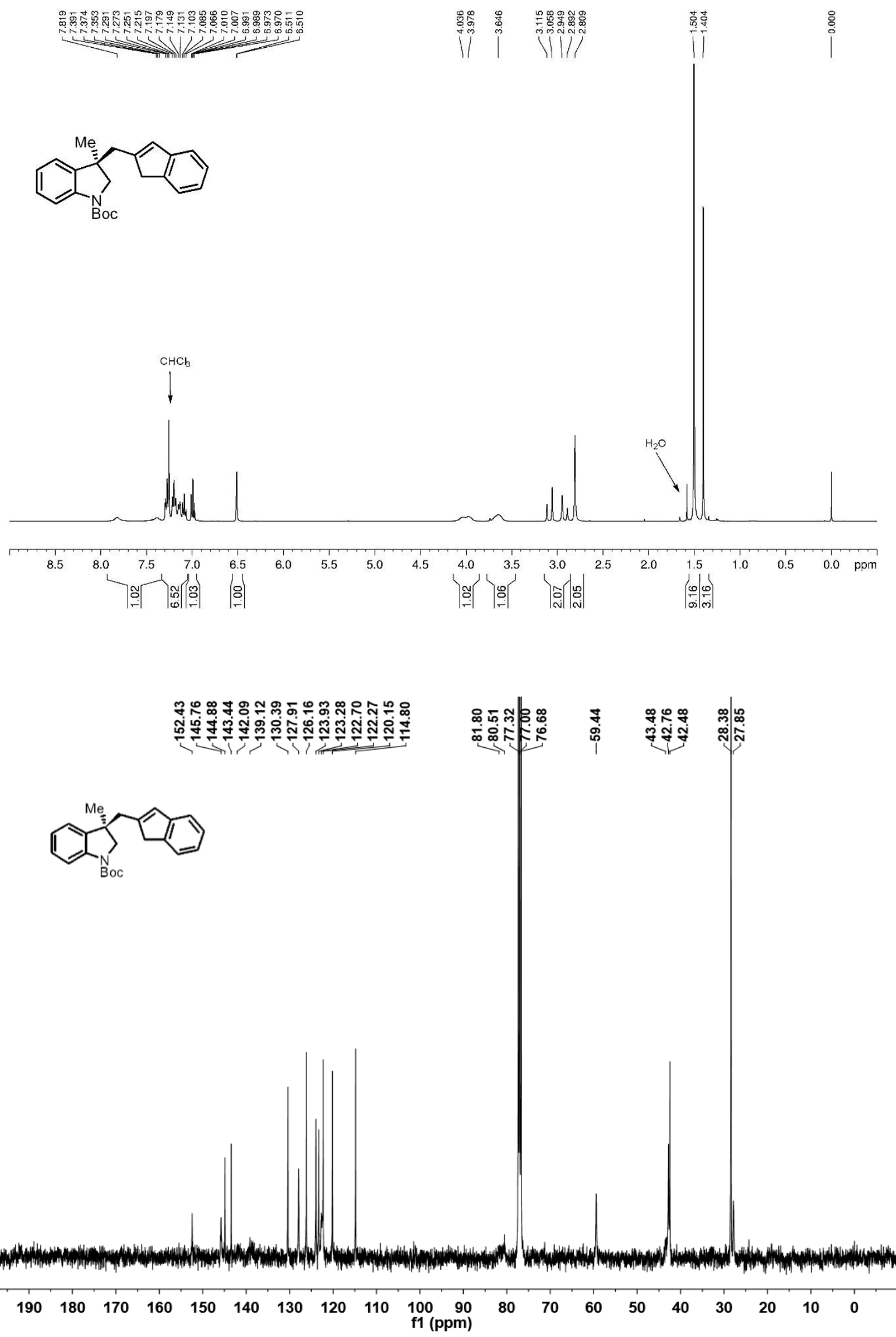
3aq; ${ }^{1} \mathrm{H}$ NMR $\left(400 \mathrm{MHz}, \mathrm{CDCl}_{3}\right) ;{ }^{13} \mathrm{C}$ NMR $\left(100 \mathrm{MHz}, \mathrm{CDCl}_{3}\right)$

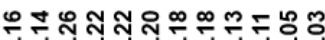
o on

พิ่

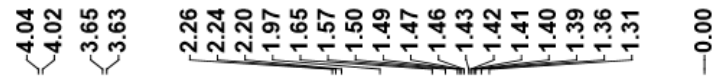<smiles>C[C@]1(CC2=CCCCC2)CN([14CH3])c2ccccc21</smiles>

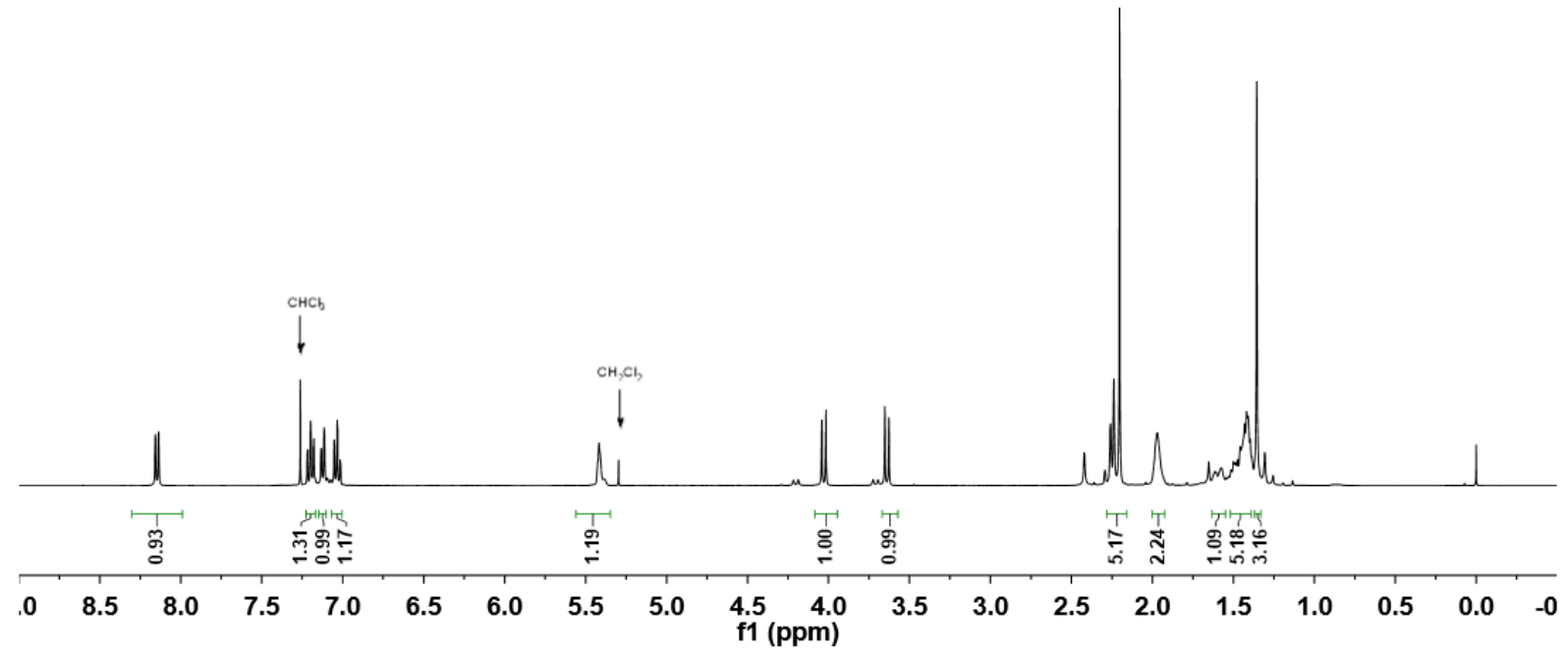

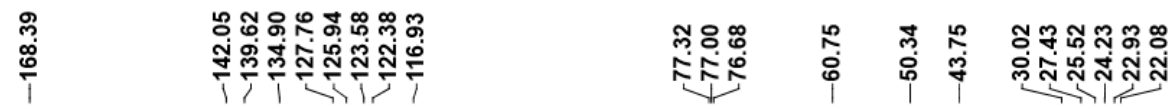<smiles>CN1CC(C)(CC2=CCCCC2)c2ccccc21</smiles>

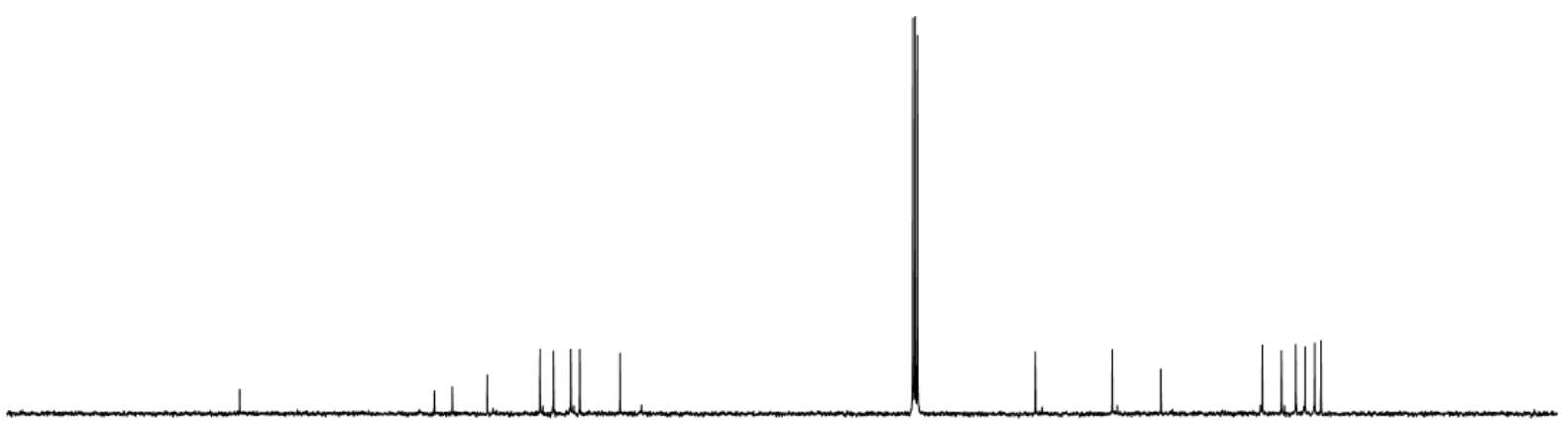

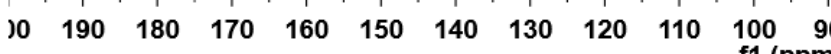

$\begin{array}{llllllllll}80 & 70 & 60 & 50 & 40 & 30 & 20 & 10 & 0 & -1\end{array}$ 1 (ppm) 
3ar; ${ }^{1} \mathrm{H}$ NMR (600MHz, $\left.\mathrm{CDCl}_{3}\right) ;{ }^{13} \mathrm{C}$ NMR $\left(150 \mathrm{MHz}, \mathrm{CDCl}_{3}\right)$

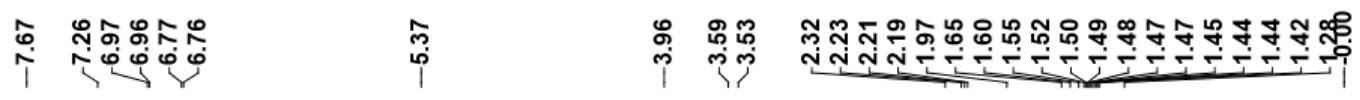
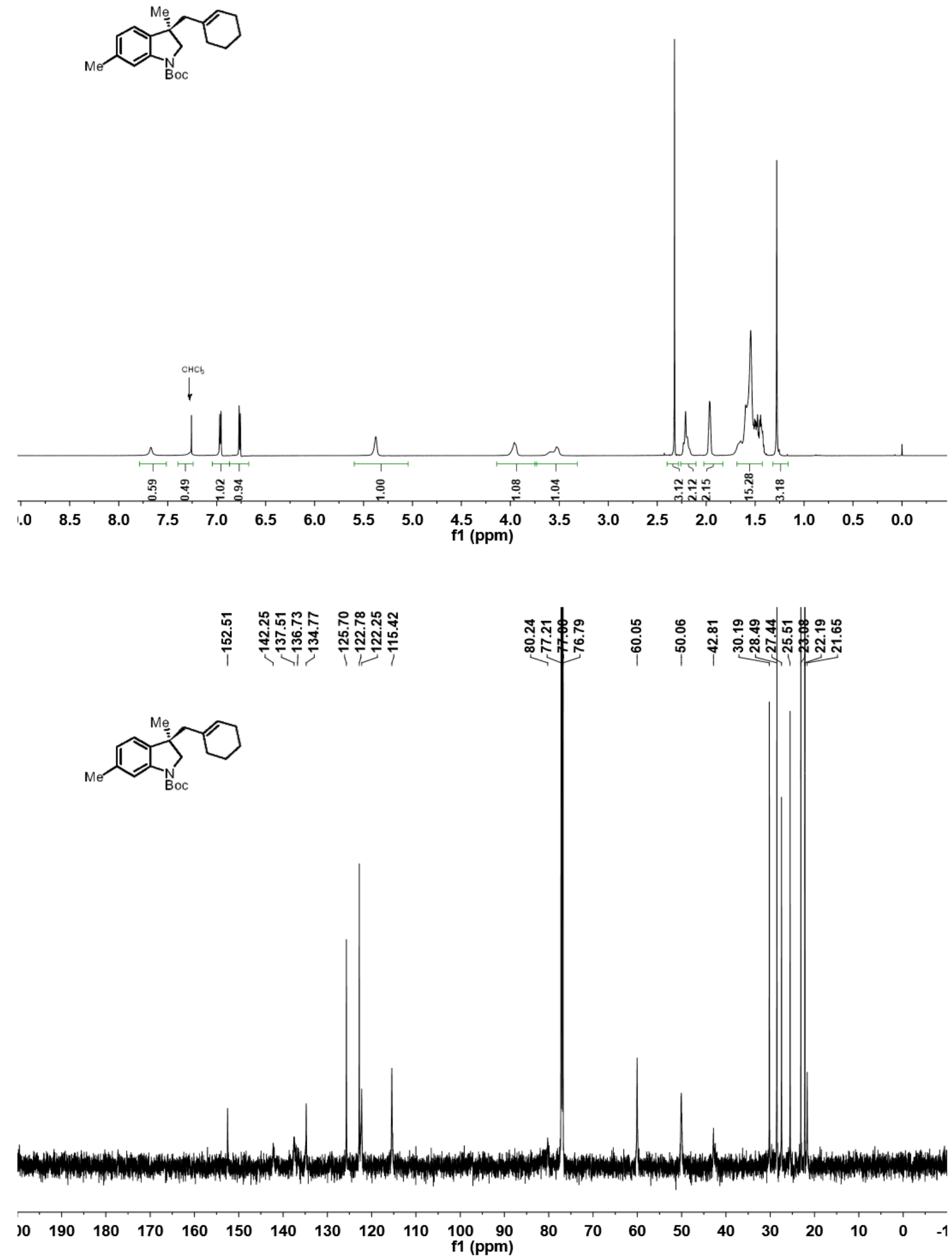

i

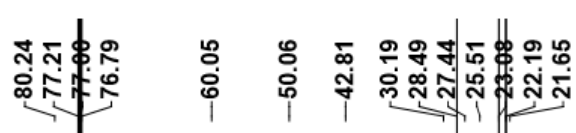


3as; ${ }^{1} \mathrm{H}$ NMR $\left(400 \mathrm{MHz}, \mathrm{CDCl}_{3}\right) ;{ }^{13} \mathrm{C}$ NMR $\left(100 \mathrm{MHz}, \mathrm{CDCl}_{3}\right)$

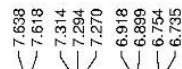

VVV VU<smiles>Cc1ccc2c(c1)[NH+]([O-])CC2(C)CC1=CC[NH+](C)CC1</smiles>

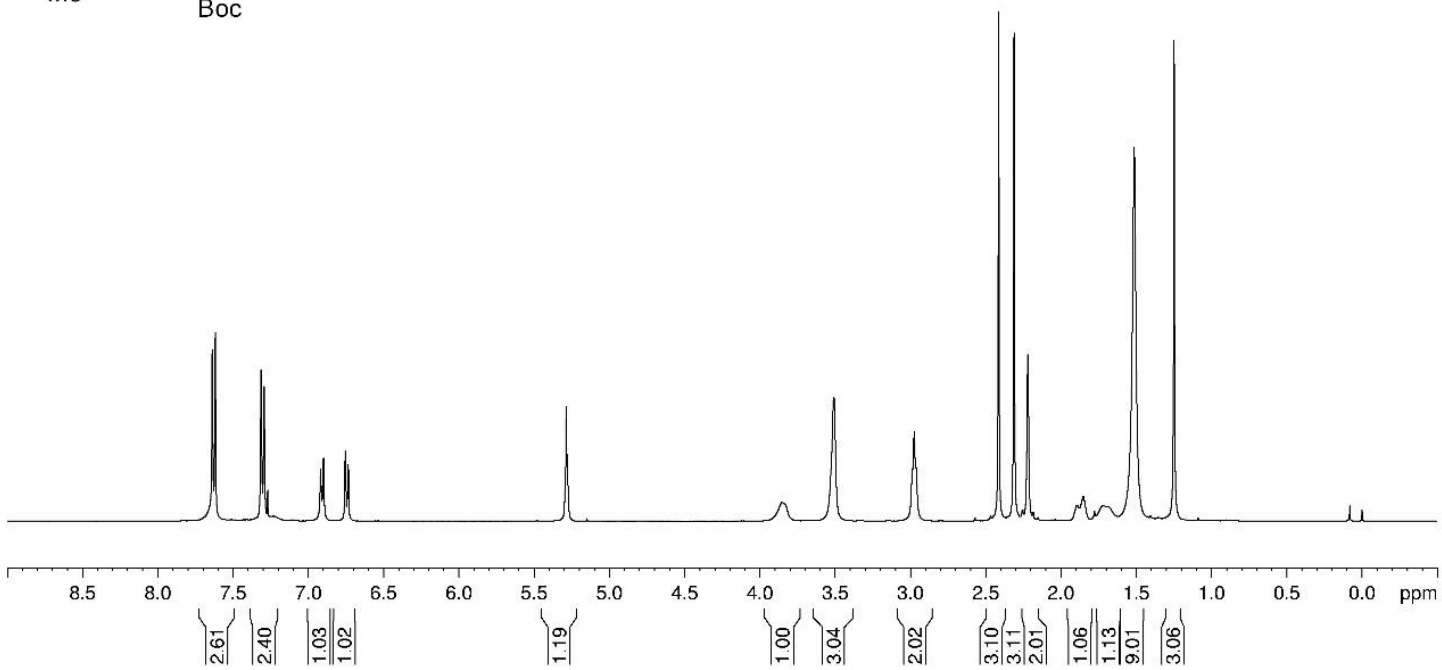

$\mid$<smiles></smiles>

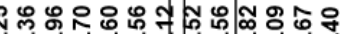
ำㄱำ

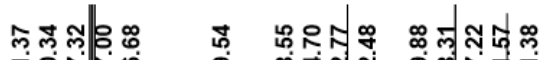

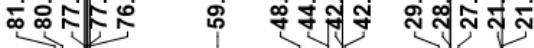
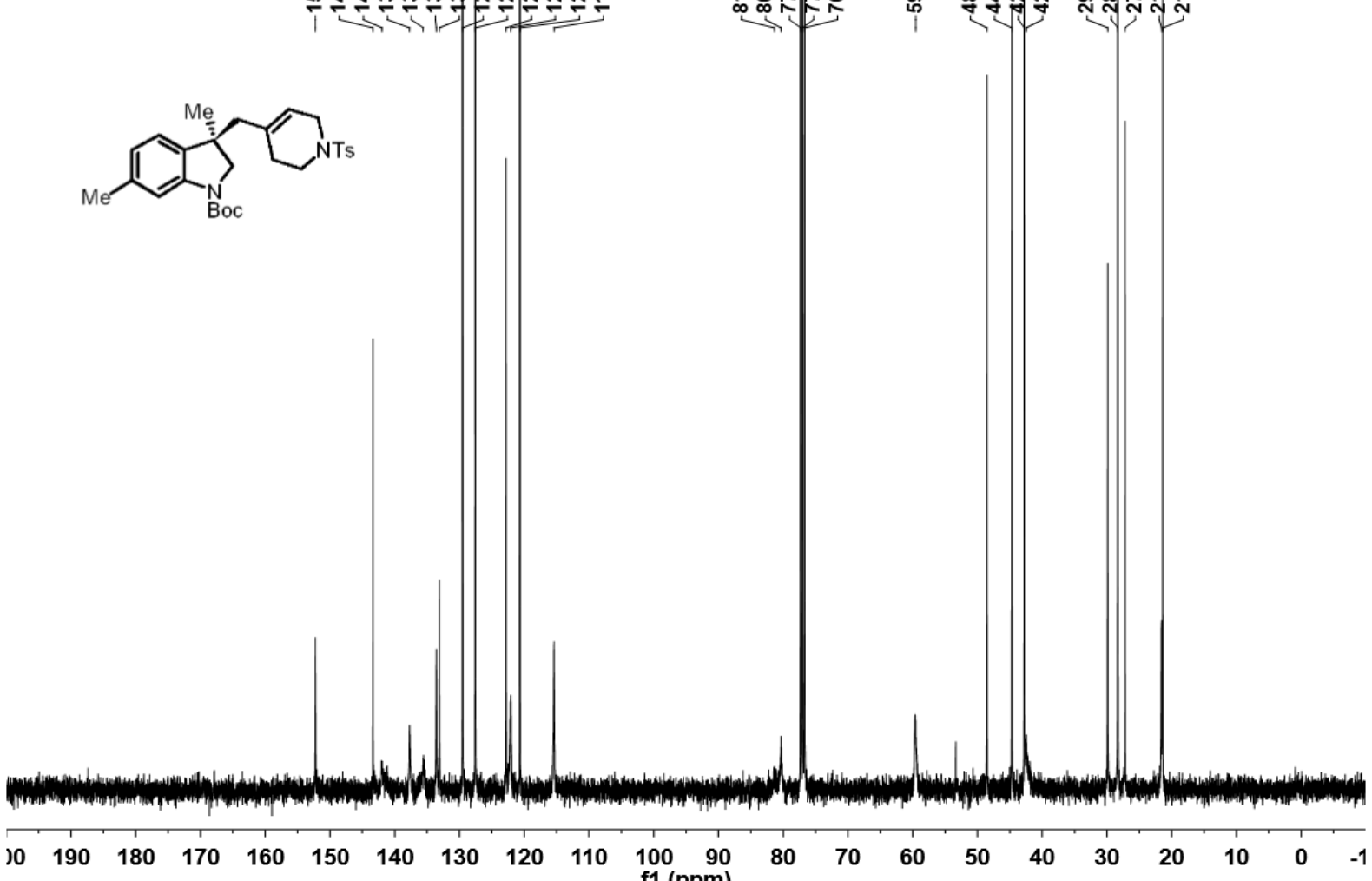
3at; ${ }^{1} \mathrm{H}$ NMR (400MHz, $\left.\left.\mathrm{CDCl}_{3}\right) ;{ }^{13} \mathrm{C} \mathrm{NMR} \mathrm{(100MHz,} \mathrm{CDCl}_{3}\right)$

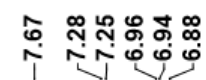

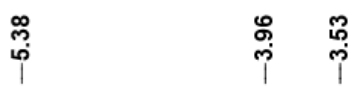

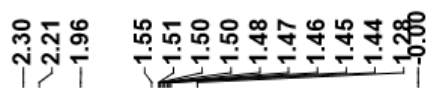<smiles>Cc1ccc2c(c1)[C@](C)(CC1=CCCCC1)C2</smiles>

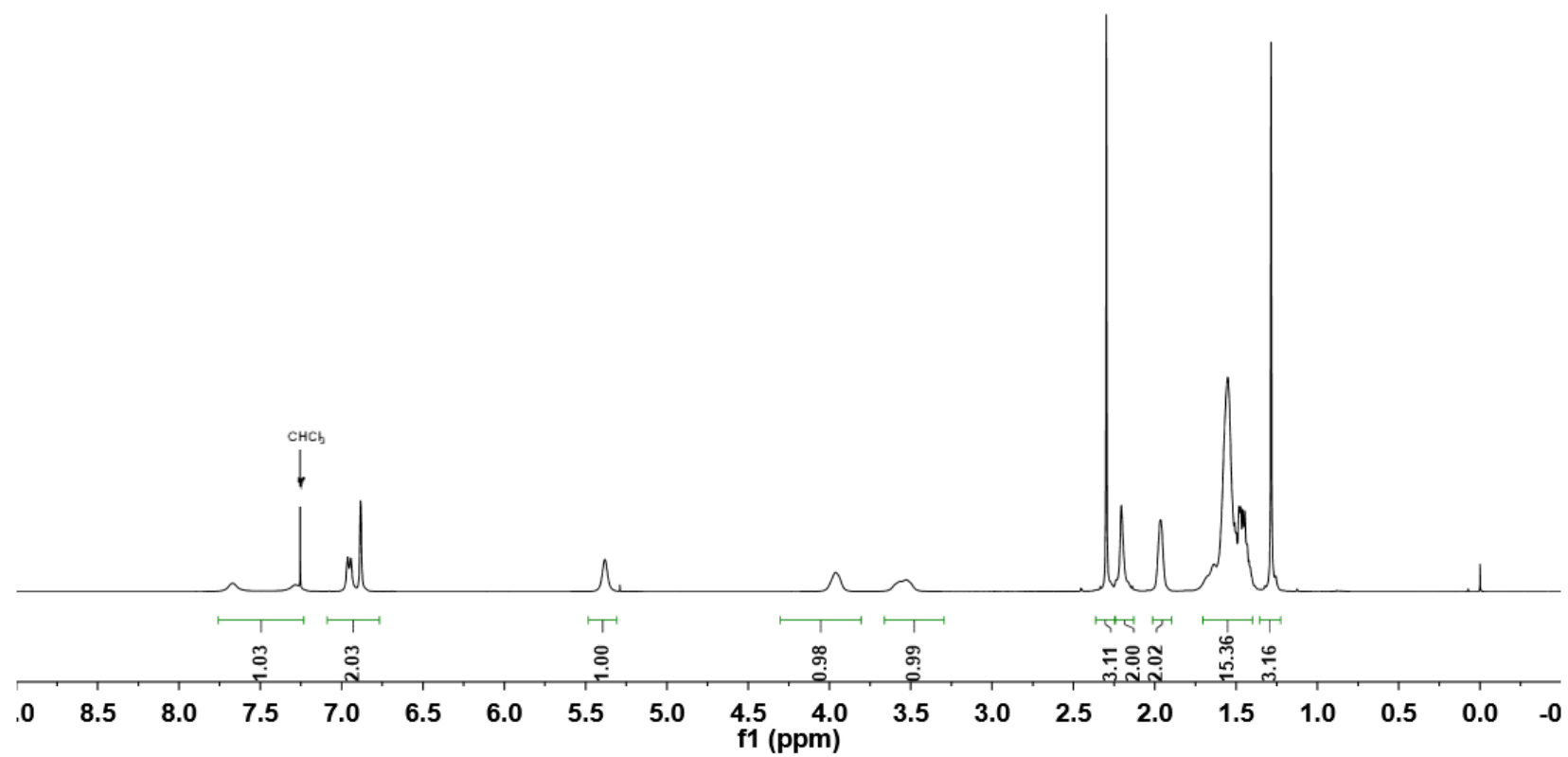

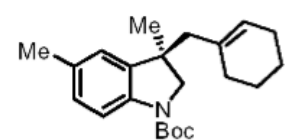

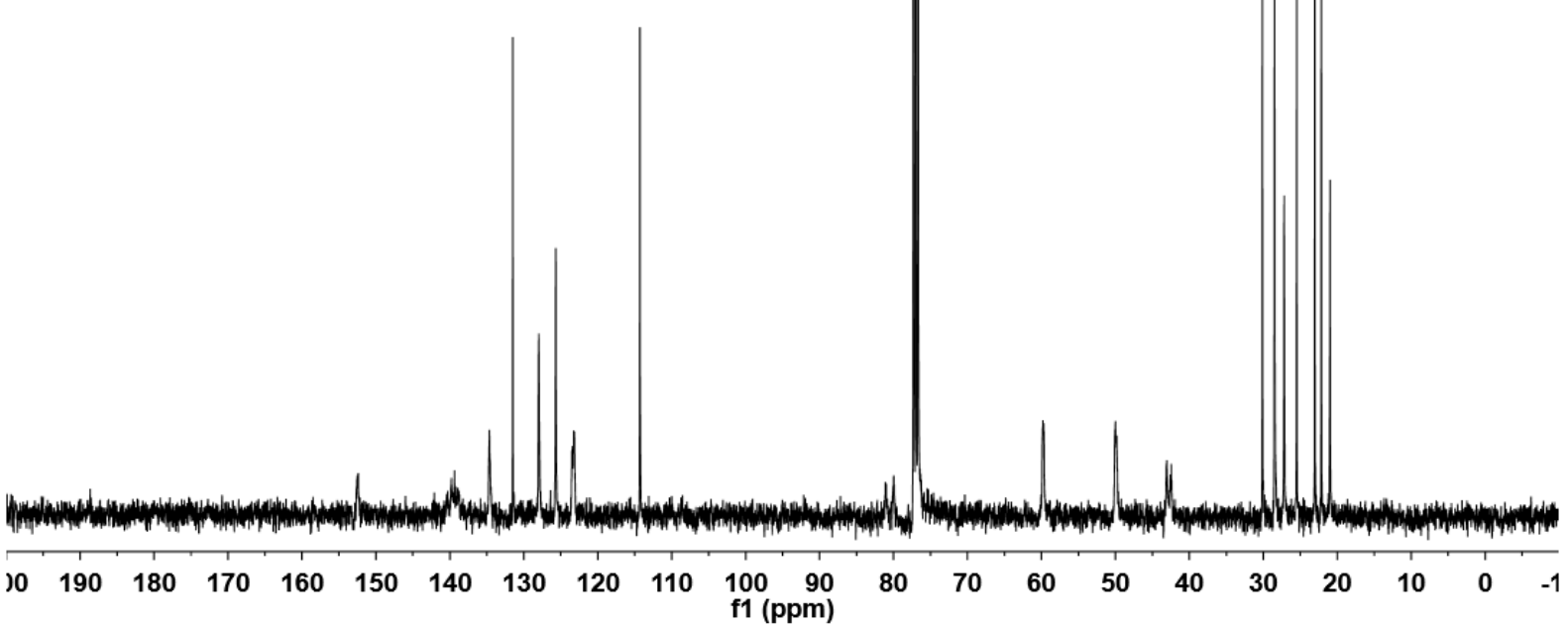


3au; ${ }^{1} \mathrm{H}$ NMR (400MHz, $\left.\mathrm{CDCl}_{3}\right) ;{ }^{13} \mathrm{C}$ NMR (100MHz, $\left.\mathrm{CDCl}_{3}\right)$

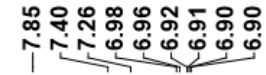
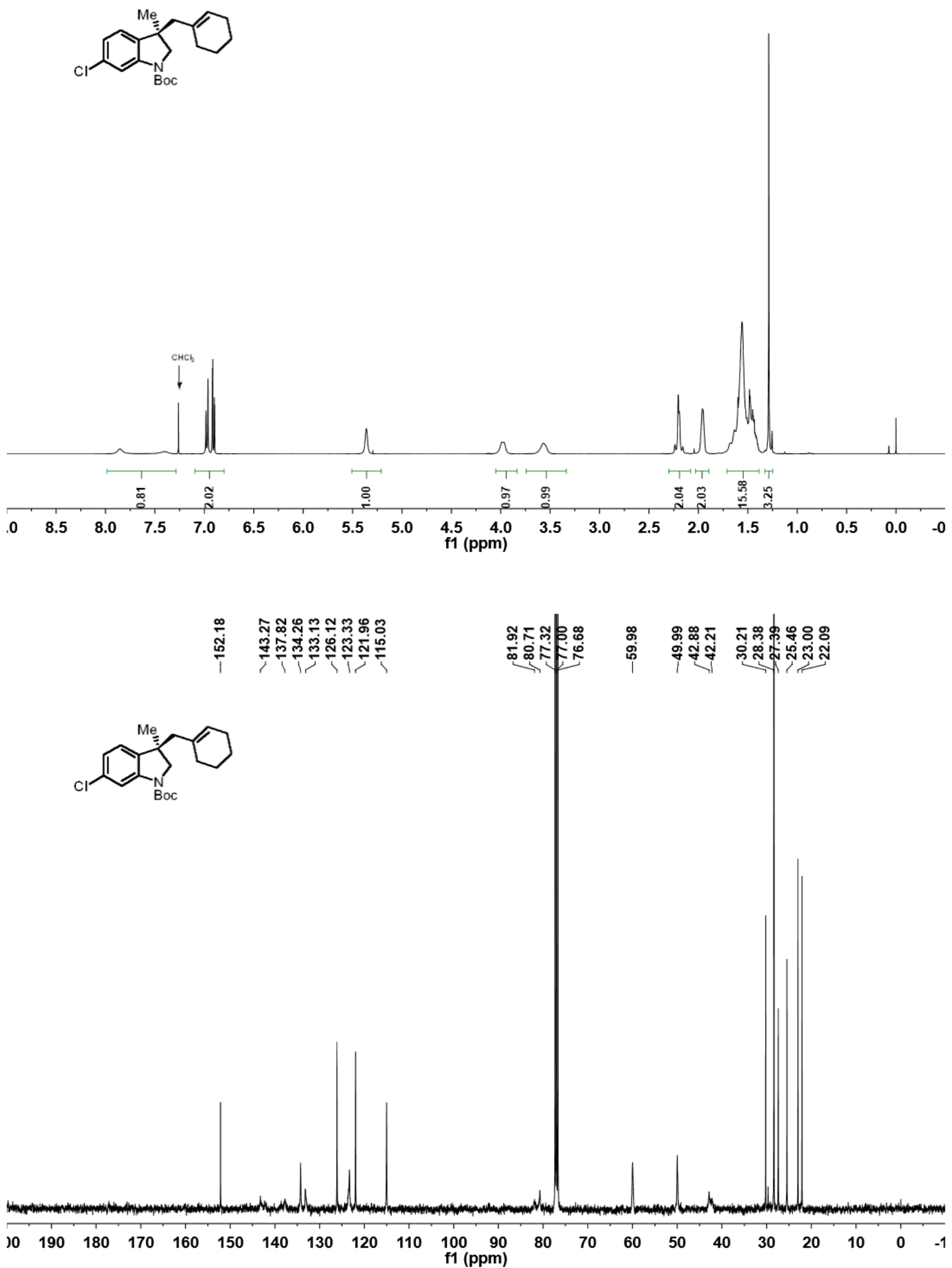

S159 
3av; ${ }^{1} \mathrm{H}$ NMR (400MHz, $\left.\mathrm{CDCl}_{3}\right) ;{ }^{13} \mathrm{C}$ NMR $\left(100 \mathrm{MHz}, \mathrm{CDCl}_{3}\right)$

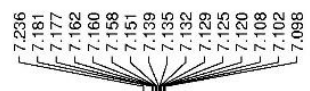

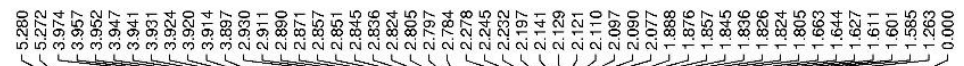
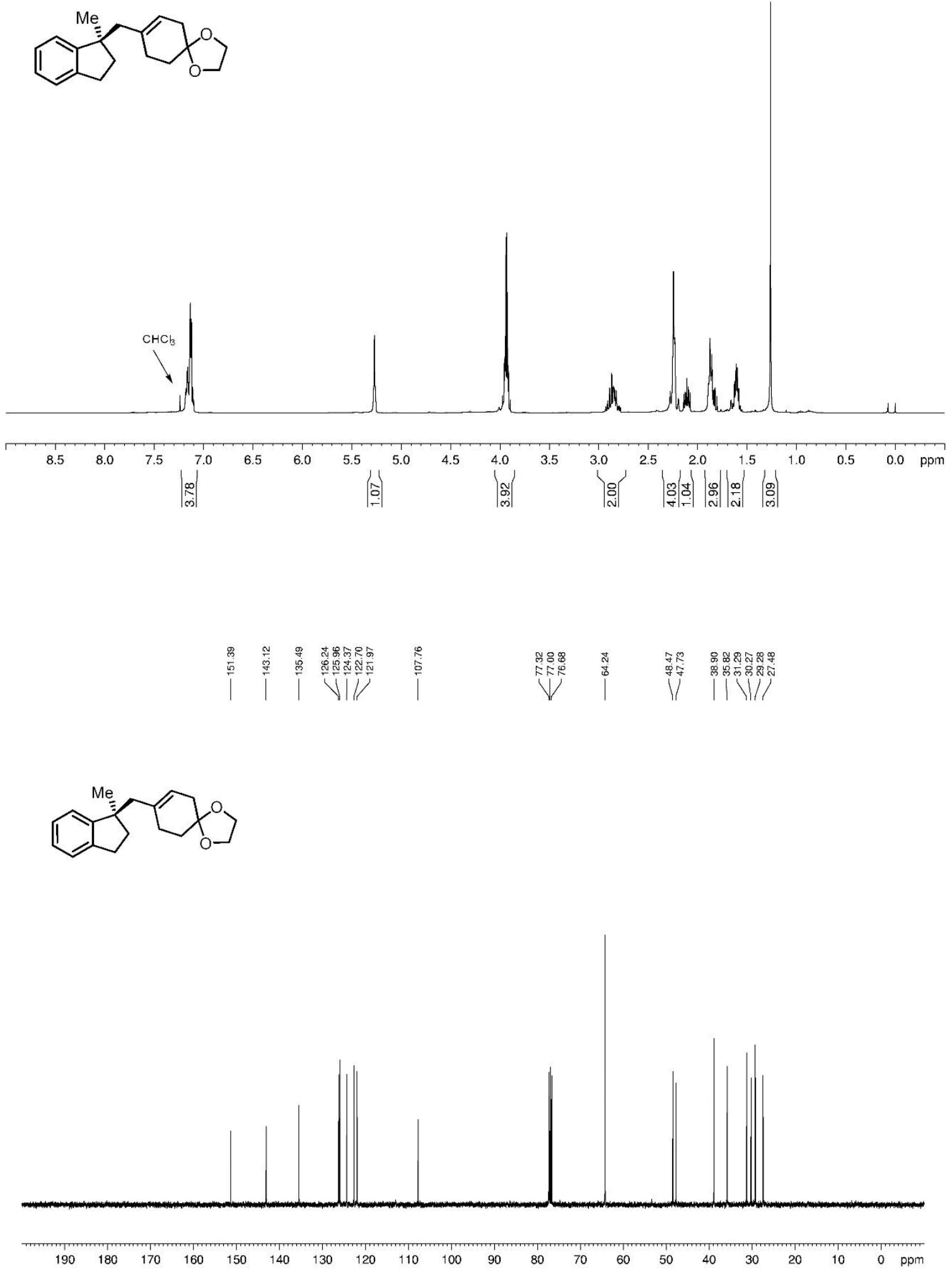

S160 
3aw; ${ }^{1} \mathrm{H}$ NMR (400MHz, $\left.\mathrm{CDCl}_{3}\right) ;{ }^{13} \mathrm{C}$ NMR $\left(100 \mathrm{MHz}, \mathrm{CDCl}_{3}\right)$

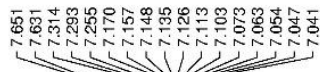

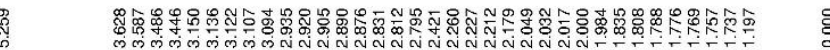

(N)

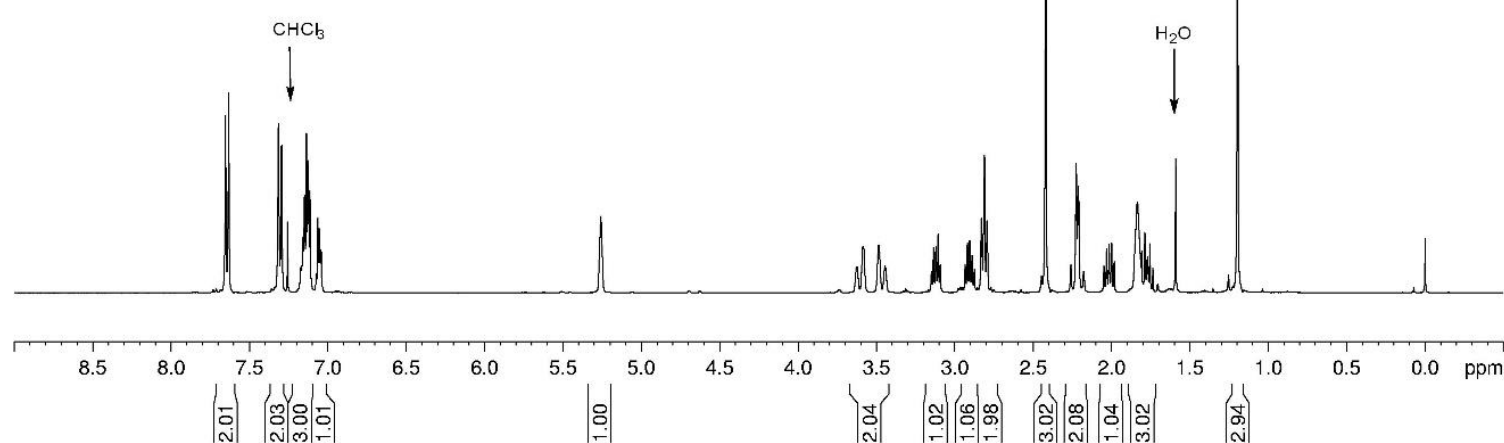

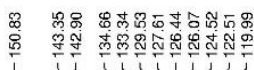

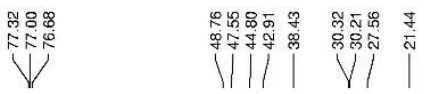

NTS

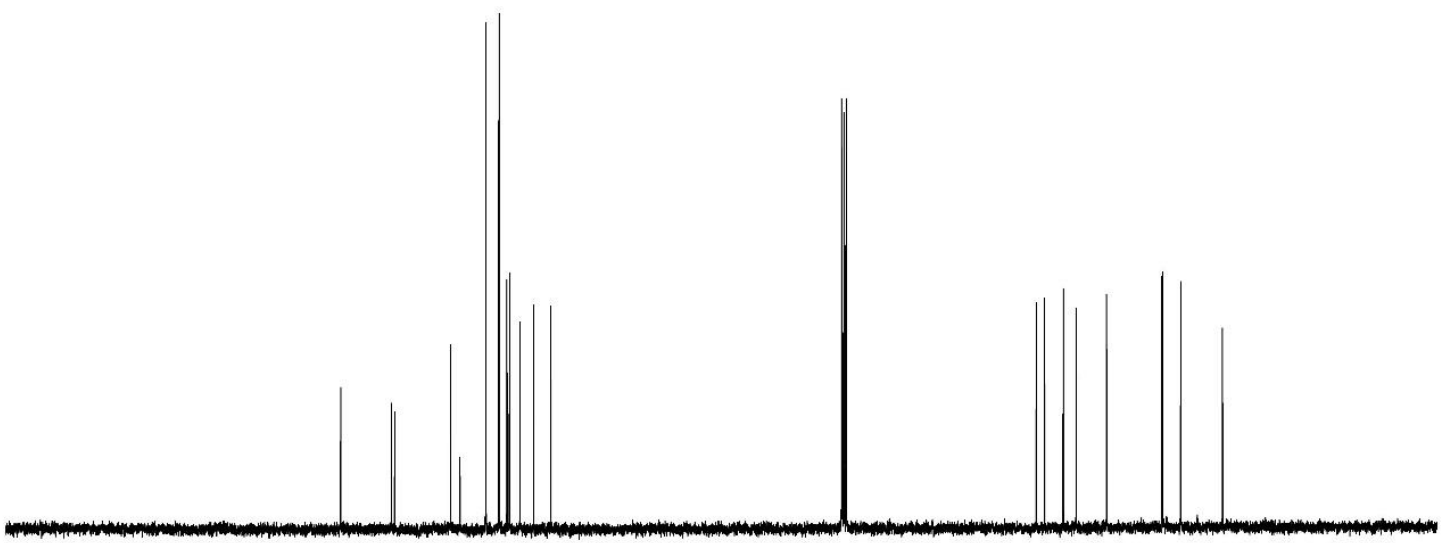

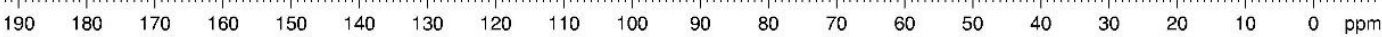


3ax; ${ }^{1} \mathrm{H}$ NMR $\left(400 \mathrm{MHz}, \mathrm{CDCl}_{3}\right) ;{ }^{13} \mathrm{C}$ NMR $\left(100 \mathrm{MHz}, \mathrm{CDCl}_{3}\right)$

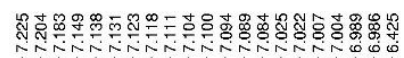

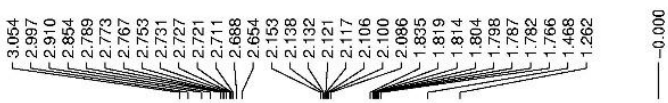

(1)

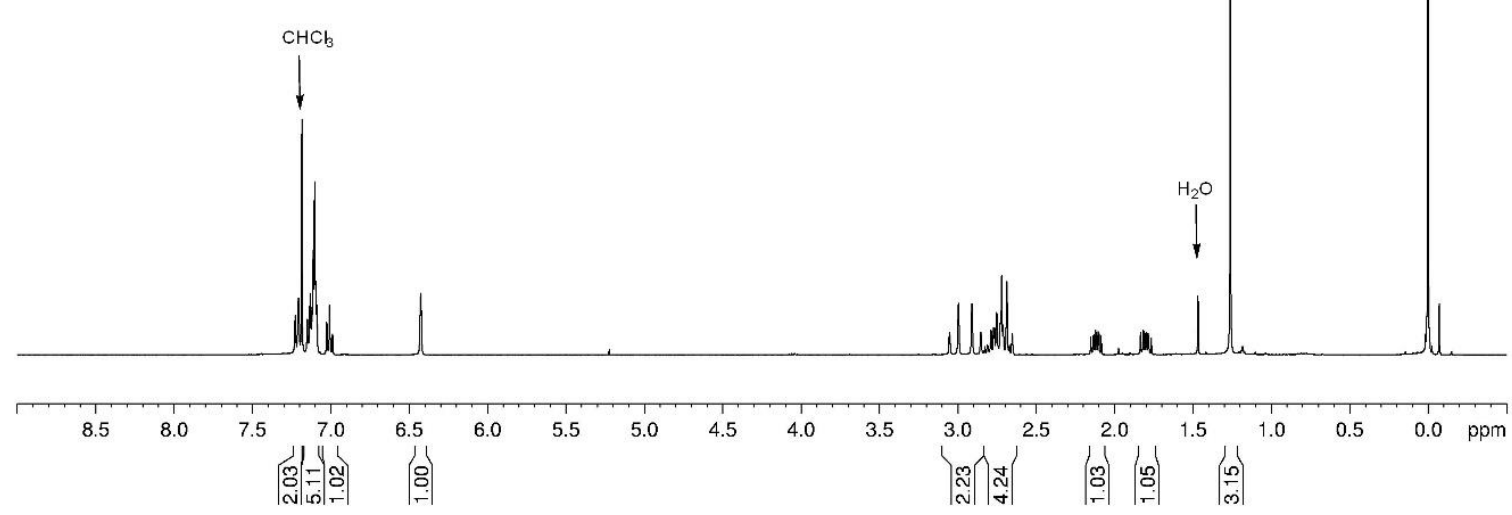

(1)

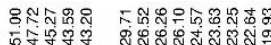

ITi wiVI

요용

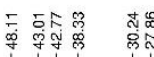

| V| |

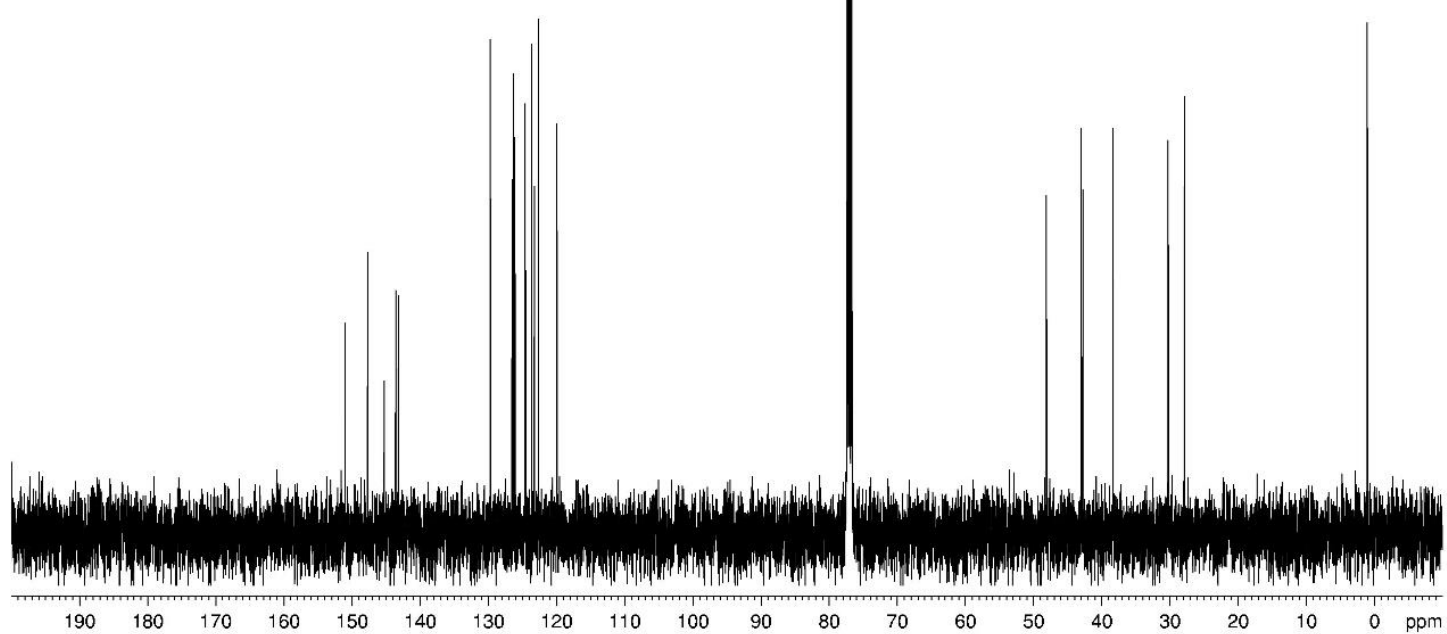


3ay; ${ }^{1} \mathrm{H}$ NMR (400MHz, $\left.\mathrm{CDCl}_{3}\right) ;{ }^{13} \mathrm{C}$ NMR $\left(100 \mathrm{MHz}, \mathrm{CDCl}_{3}\right)$

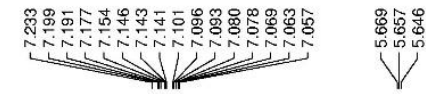

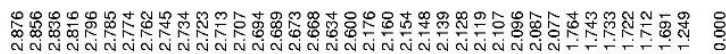

(1)

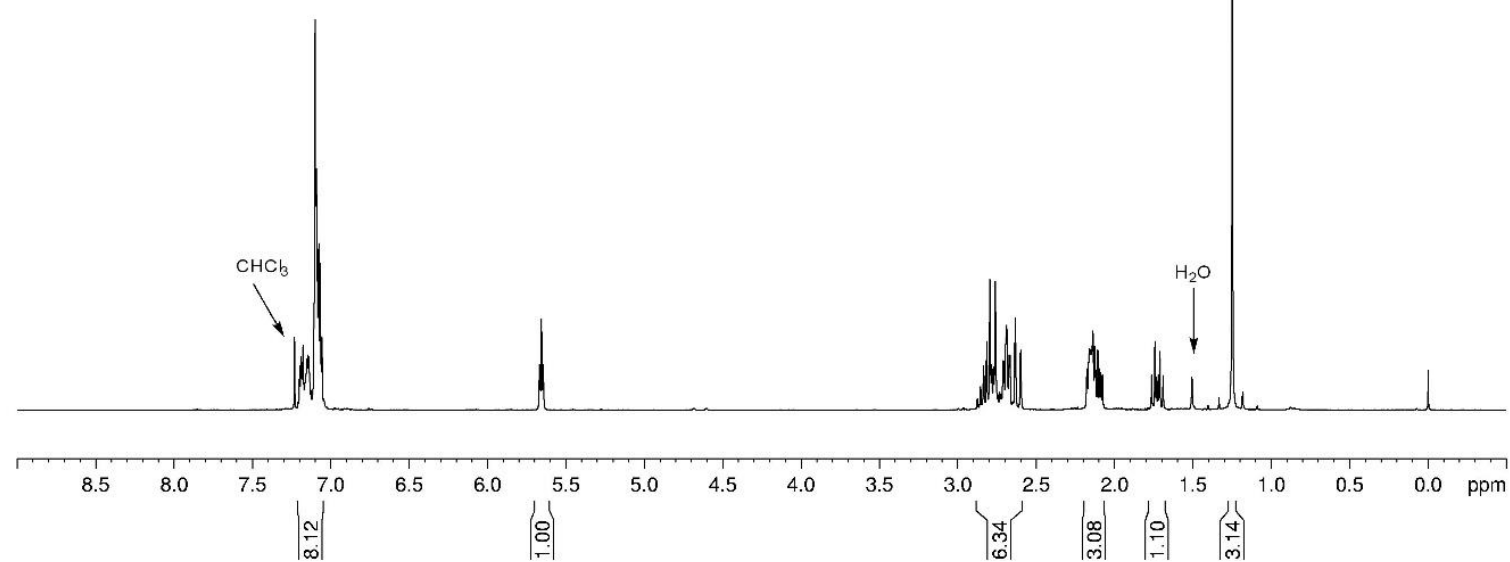

ले लिष

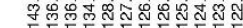

ivivit

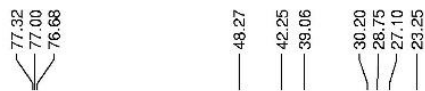

(1)

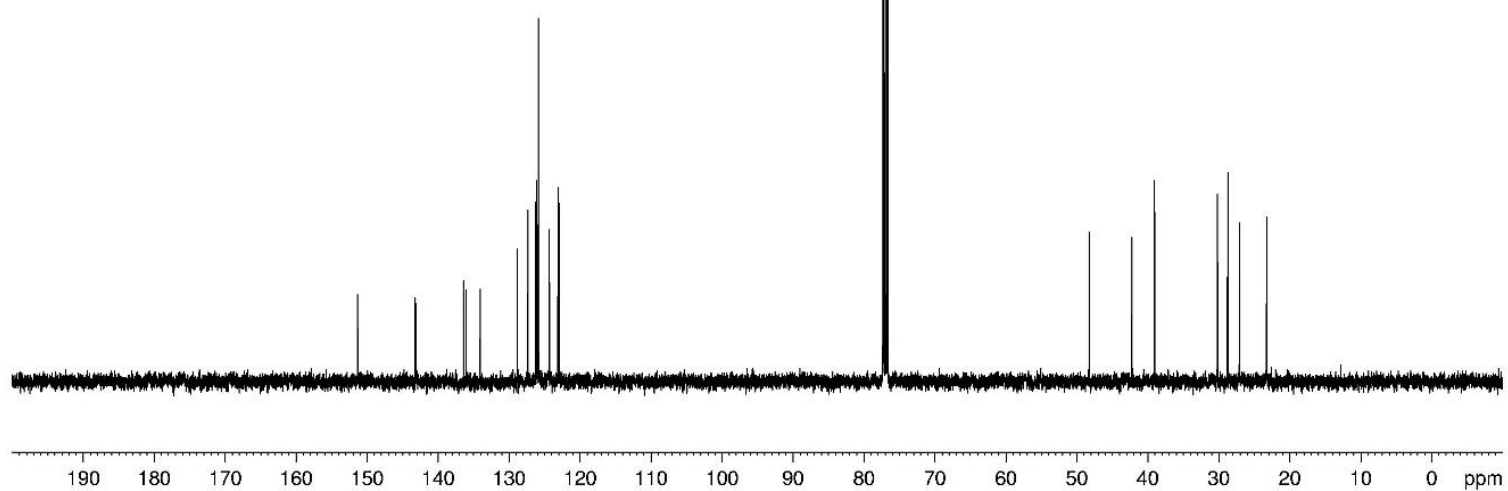


3az; ${ }^{1} \mathrm{H}$ NMR (400MHz, $\left.\mathrm{CDCl}_{3}\right) ;{ }^{13} \mathrm{C}$ NMR $\left(100 \mathrm{MHz}, \mathrm{CDCl}_{3}\right)$

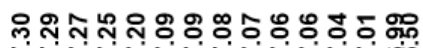

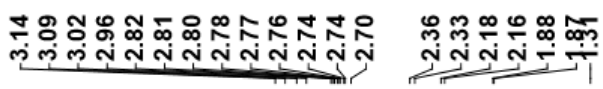

$\stackrel{\circ}{i}$
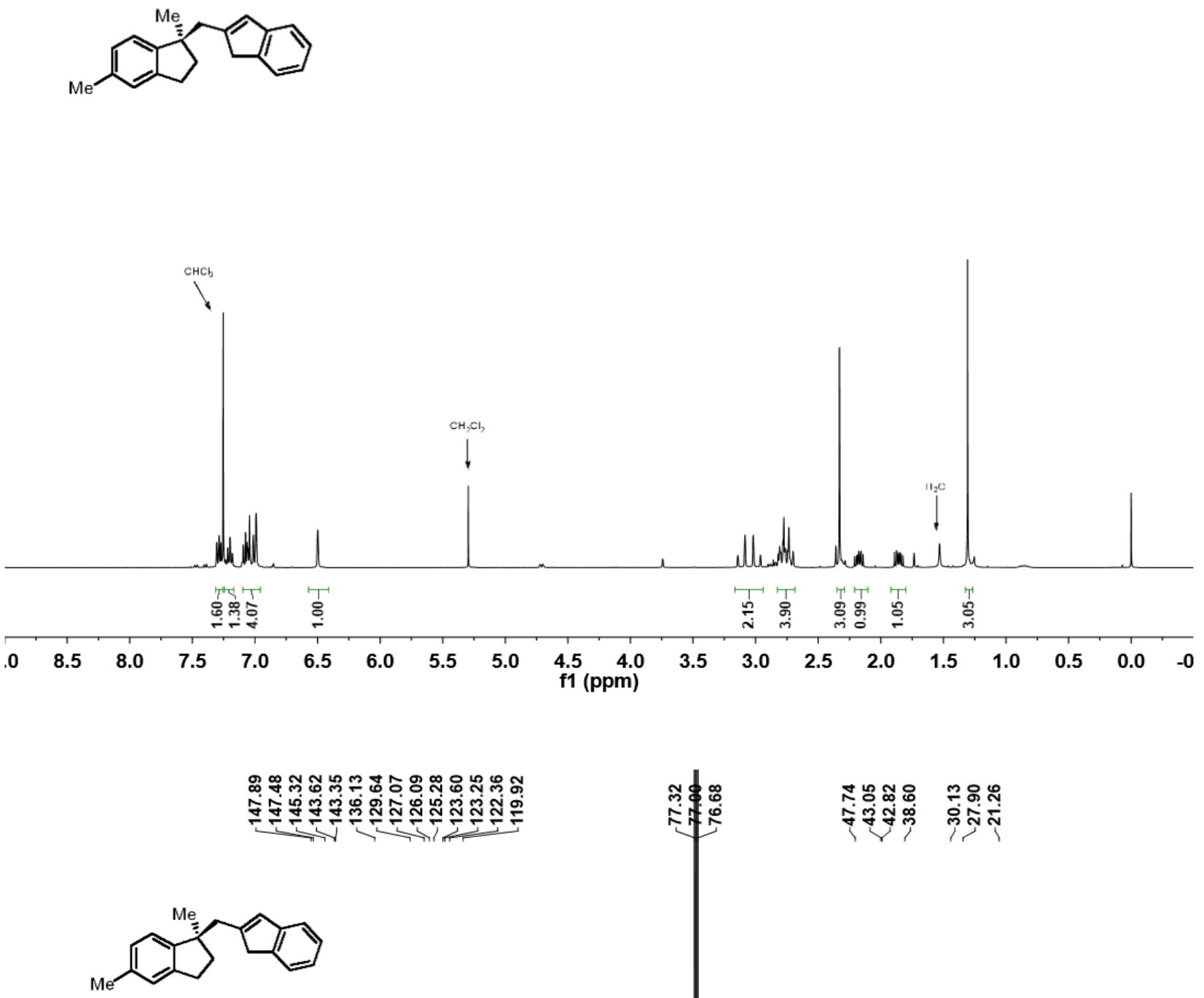

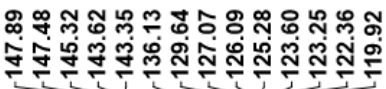

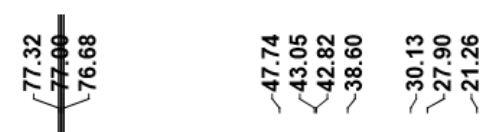

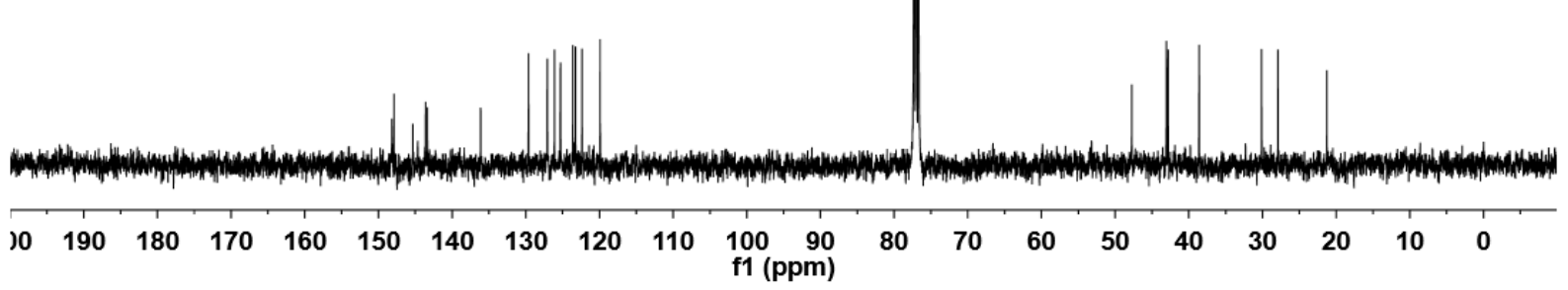

S164 
3ba; ${ }^{1} \mathrm{H}$ NMR $\left(400 \mathrm{MHz}, \mathrm{CDCl}_{3}\right) ;{ }^{13} \mathrm{C}$ NMR $\left(100 \mathrm{MHz}, \mathrm{CDCl}_{3}\right)$
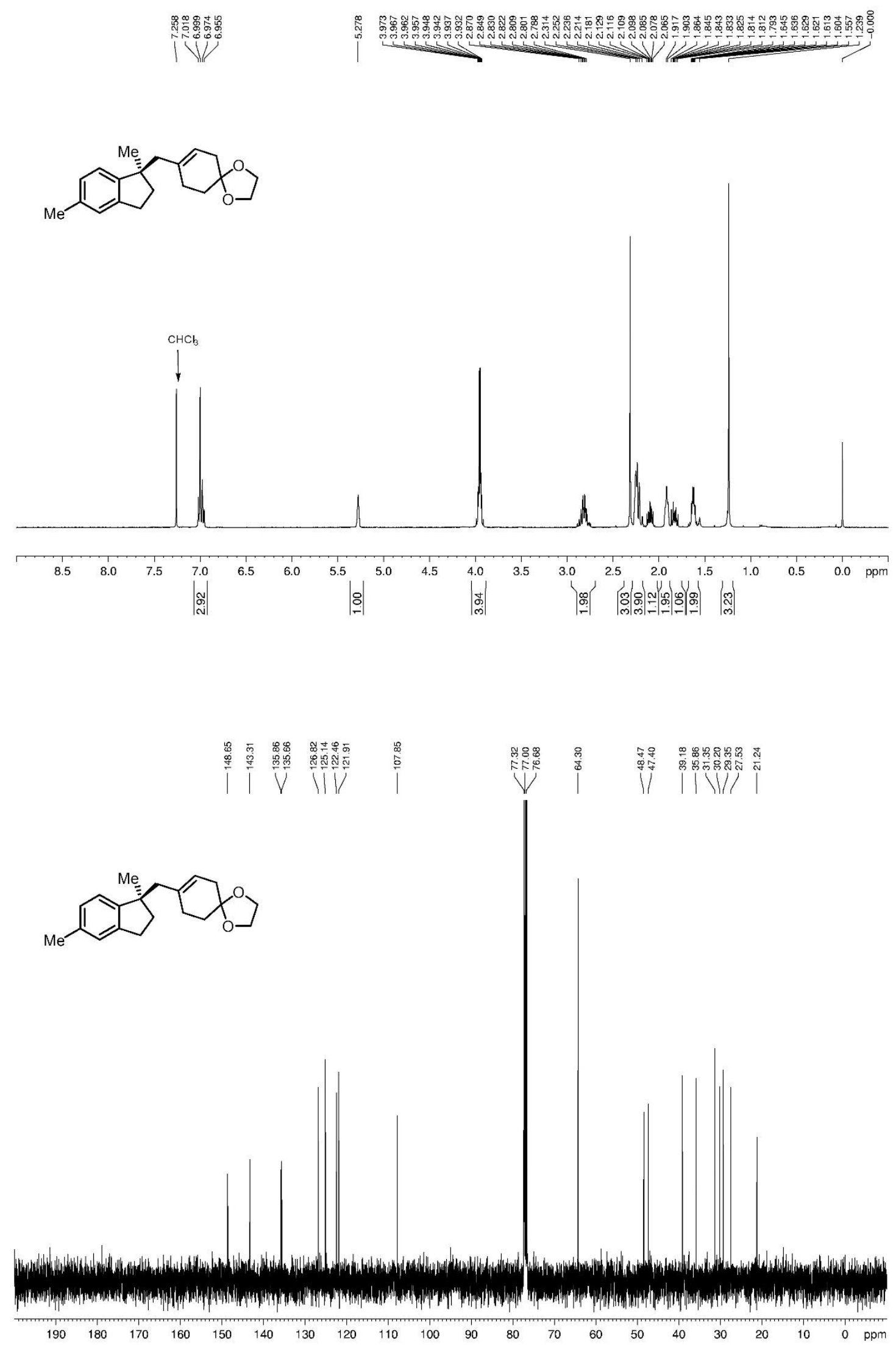
3bb; ${ }^{1} \mathrm{H}$ NMR (400MHz, $\left.\mathrm{CDCl}_{3}\right) ;{ }^{13} \mathrm{C}$ NMR $\left(100 \mathrm{MHz}, \mathrm{CDCl}_{3}\right)$
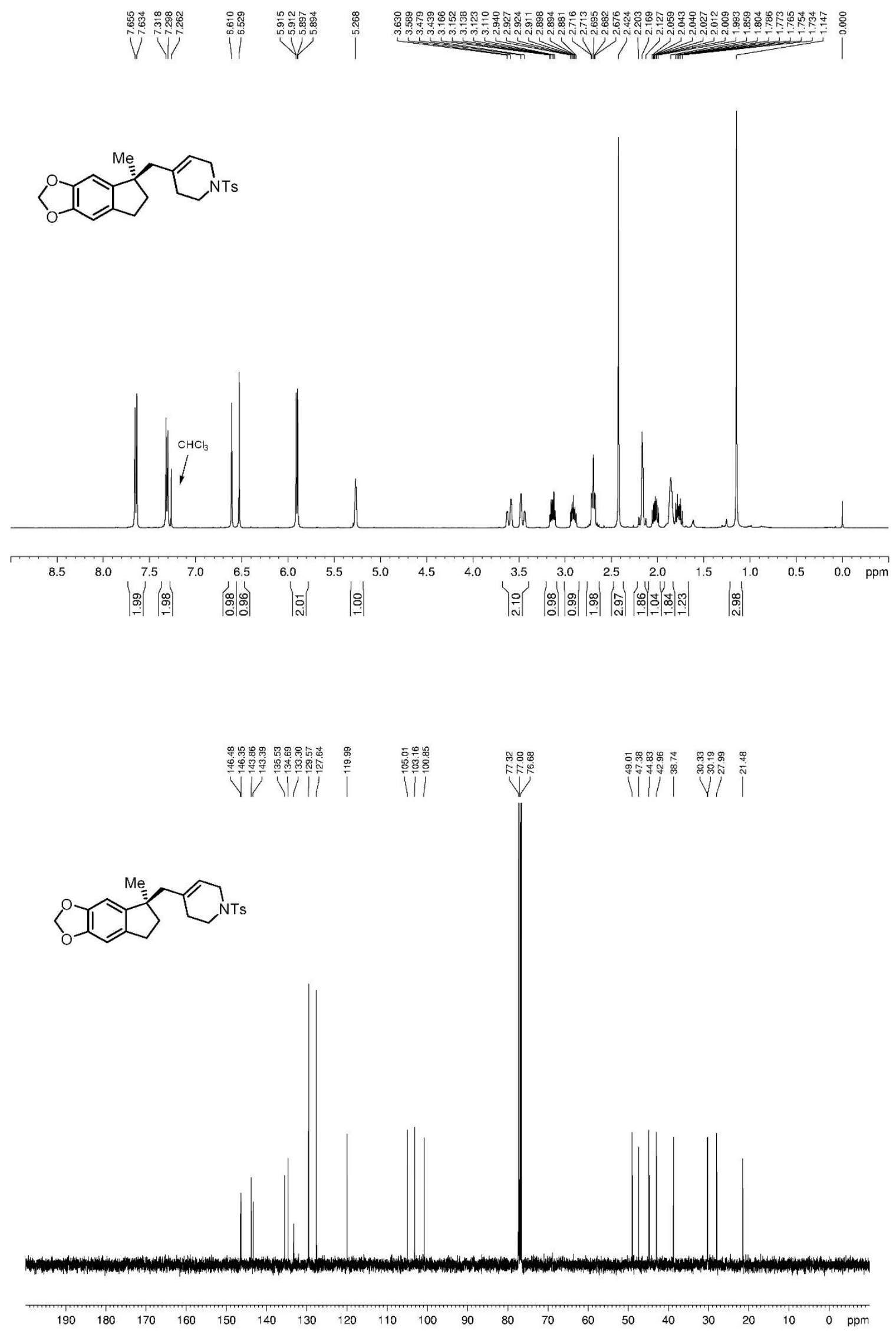

S166 
3bc; ${ }^{1} \mathrm{H}$ NMR (400MHz, $\left.\mathrm{CDCl}_{3}\right) ;{ }^{13} \mathrm{C}$ NMR $\left(100 \mathrm{MHz}, \mathrm{CDCl}_{3}\right)$

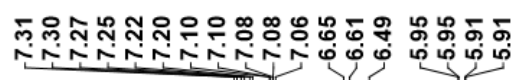

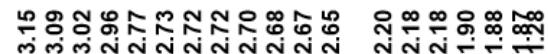

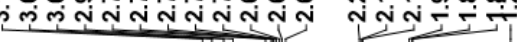

$\stackrel{\circ}{i}$<smiles>C[C@]1(CC2=Cc3ccccc3C2)CCc2cc3c(cc2C1)OCO3</smiles>

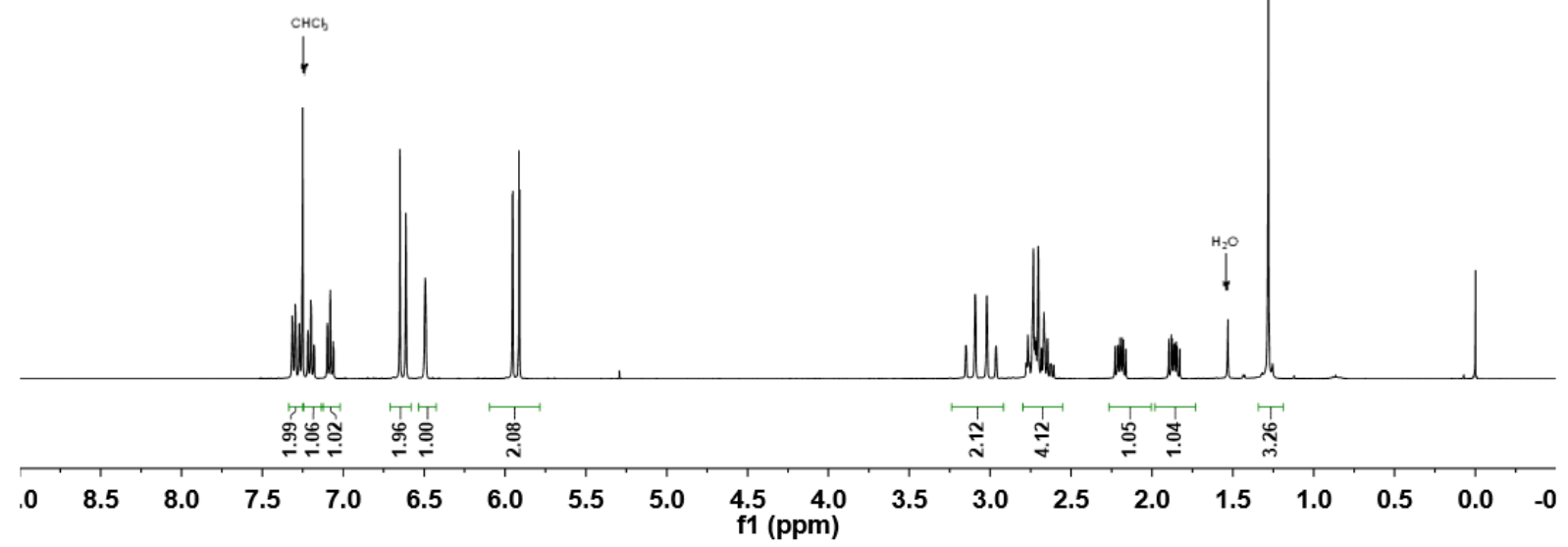

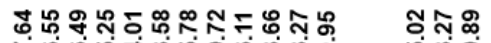

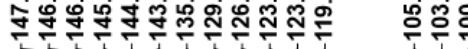

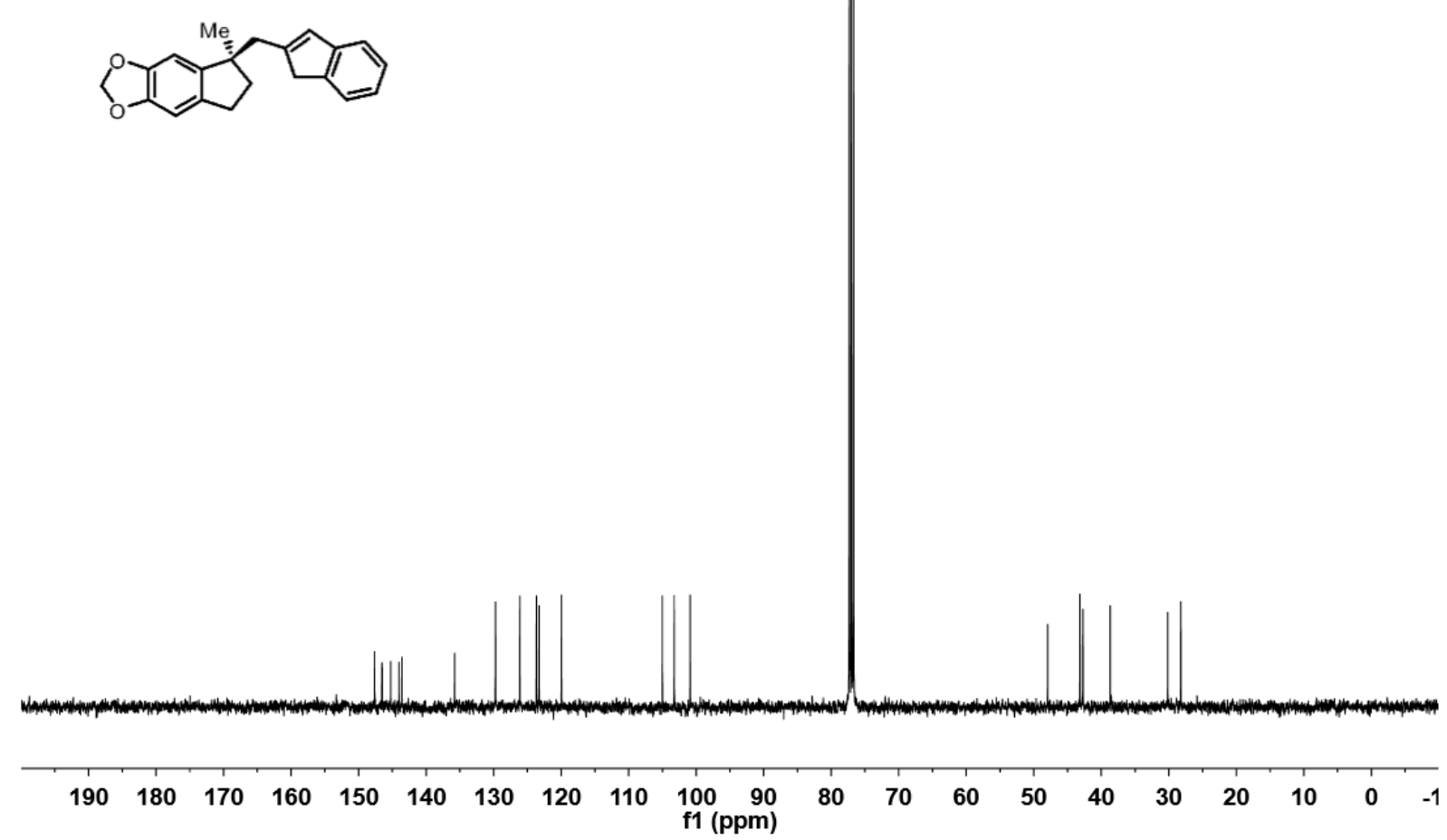




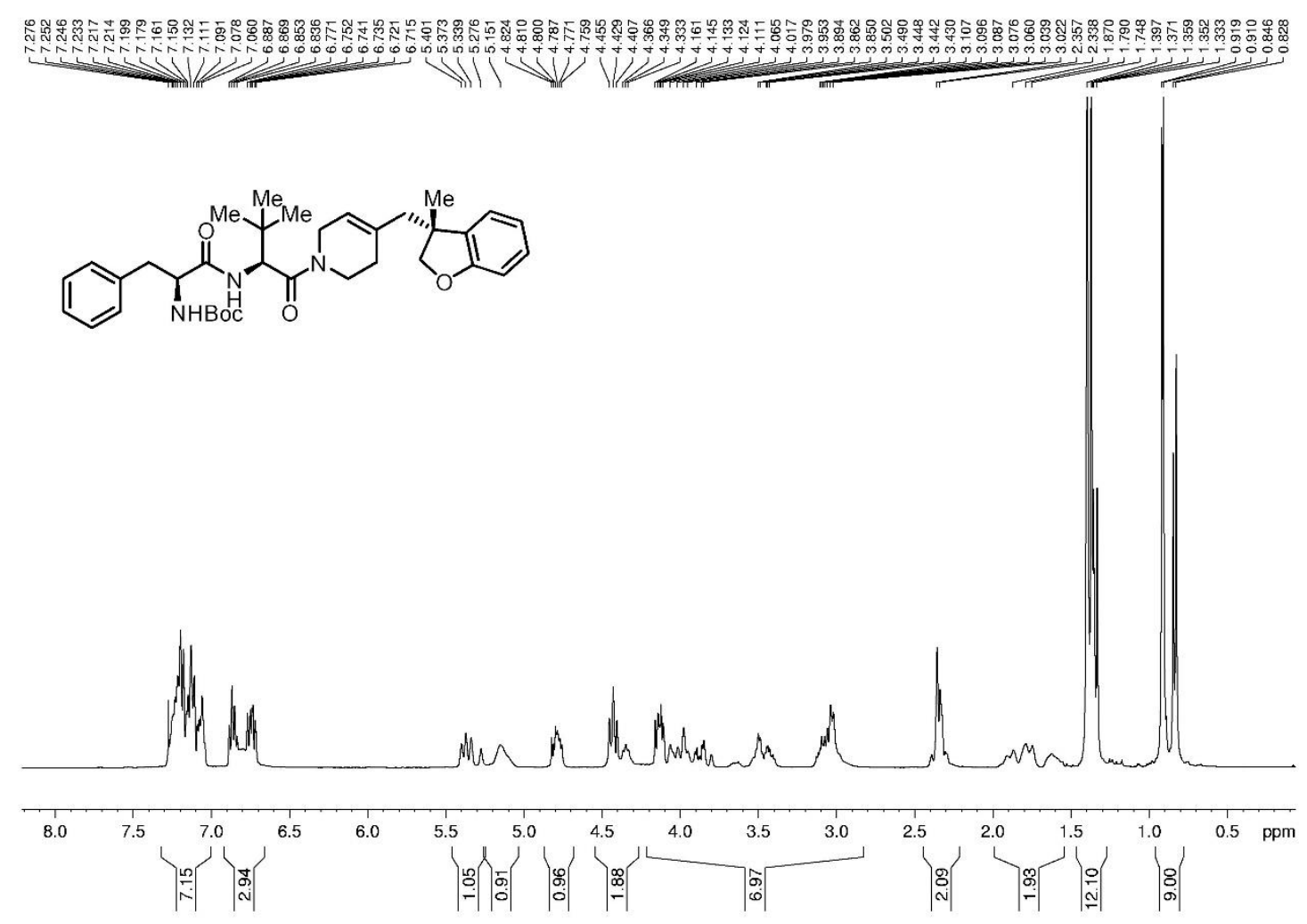

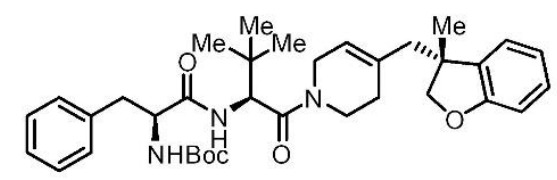

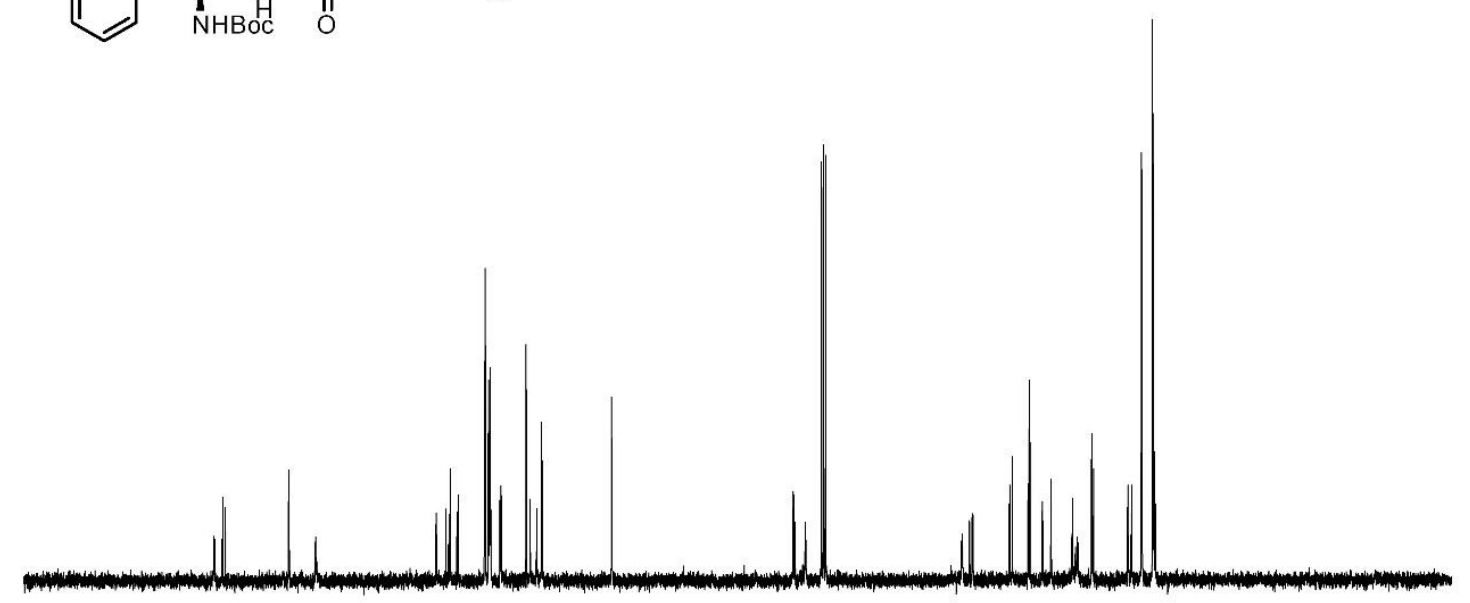

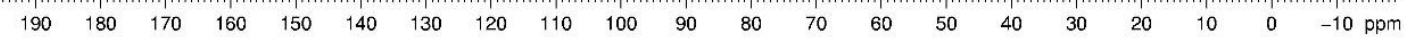


5; ${ }^{1} \mathrm{H}$ NMR (400MHz, $\left.\mathrm{CDCl}_{3}\right) ;{ }^{13} \mathrm{C}$ NMR (100MHz, $\left.\mathrm{CDCl}_{3}\right)$
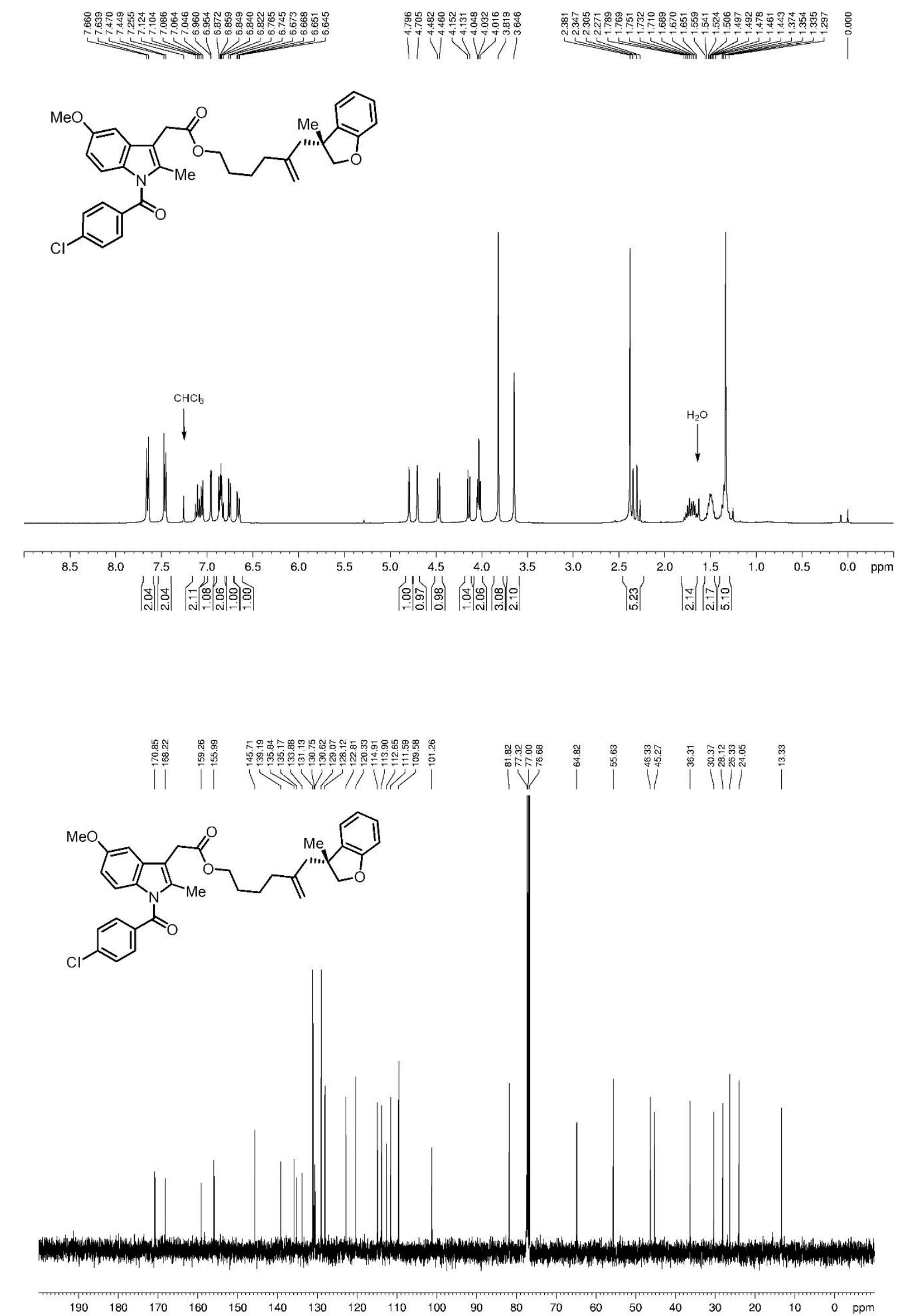
6; ${ }^{1} \mathrm{H}$ NMR (400MHz, $\left.\mathrm{CDCl}_{3}\right) ;{ }^{13} \mathrm{C}$ NMR (100MHz, $\left.\mathrm{CDCl}_{3}\right)$

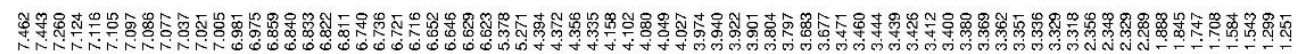<smiles>COc1ccc2c(c1)c(CC(=O)N1CC=C(C[C@]3(C)COc4ccccc43)CC1)c(C)n2C(=O)c1ccc(Cl)cc1</smiles>
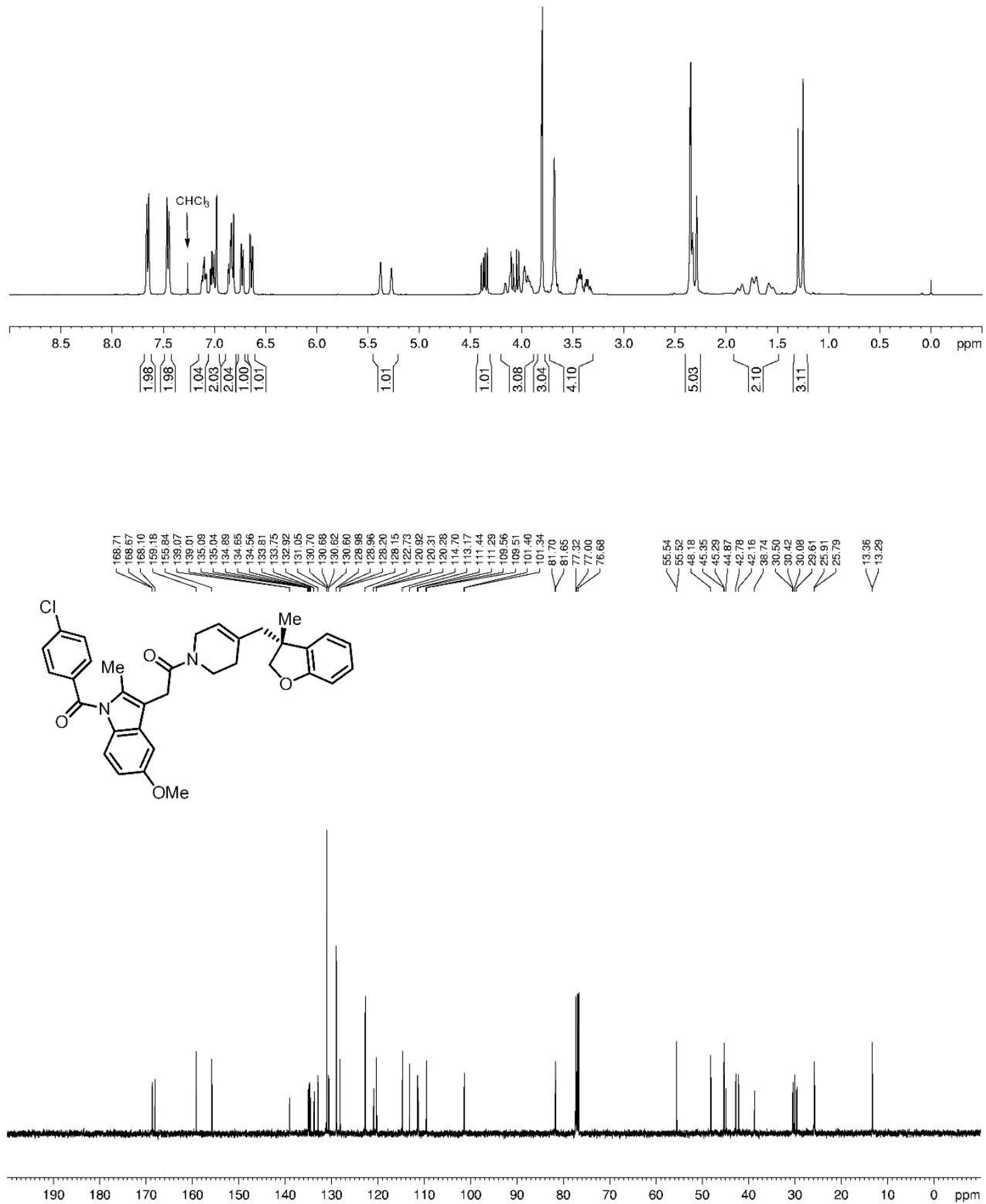
7; ${ }^{1} \mathrm{H}$ NMR (400MHz, $\left.\mathrm{CDCl}_{3}\right) ;{ }^{13} \mathrm{C}$ NMR (100MHz, $\left.\mathrm{CDCl}_{3}\right)$

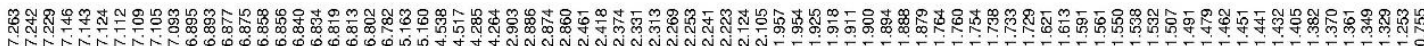<smiles>COc1ccc2c(c1)CCC1C3CCC(CC4(C)COc5ccccc54)C3CCC21C</smiles><smiles>[Te]C1CCC1</smiles>

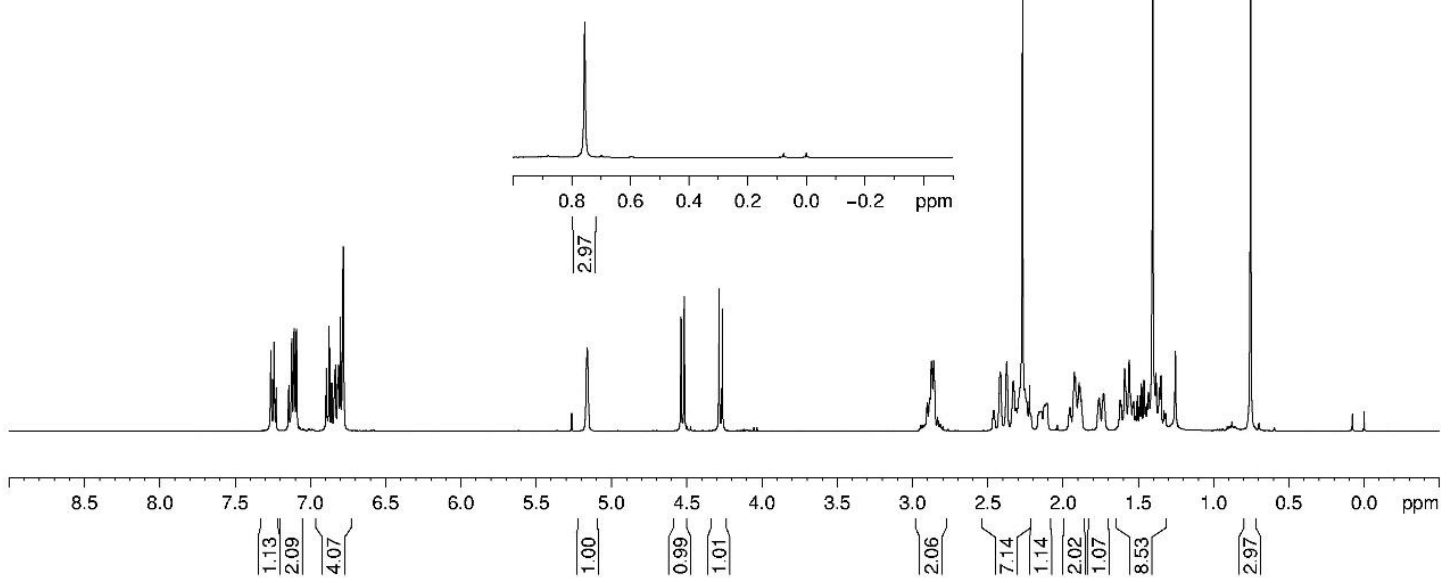

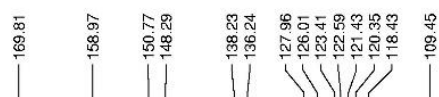

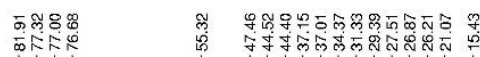

V

$|V| i|| \mid$<smiles>CC(=O)Oc1ccc2c(c1)CCC1C3CC=C(C[C@@]4(C)COc5ccccc54)C3(C)CCC21C</smiles>

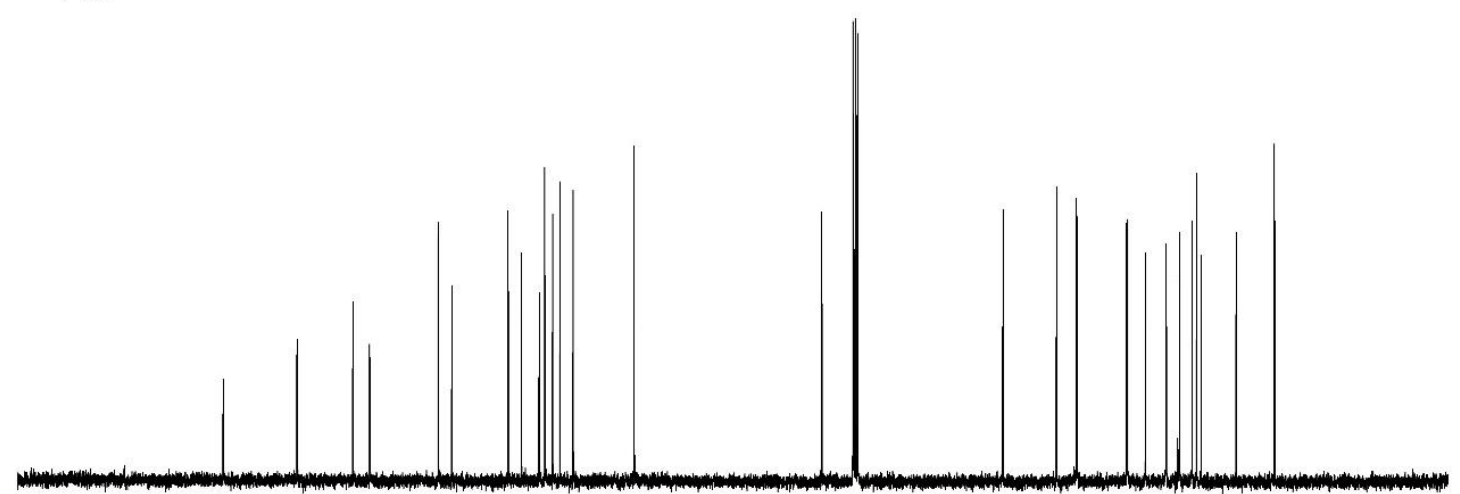

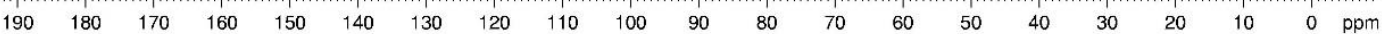


<smiles>CC(=O)OC1CCC2C3CC=C4C=C(C[C@]5(C)COc6ccccc65)CCC4(C)C3CCC12C</smiles>
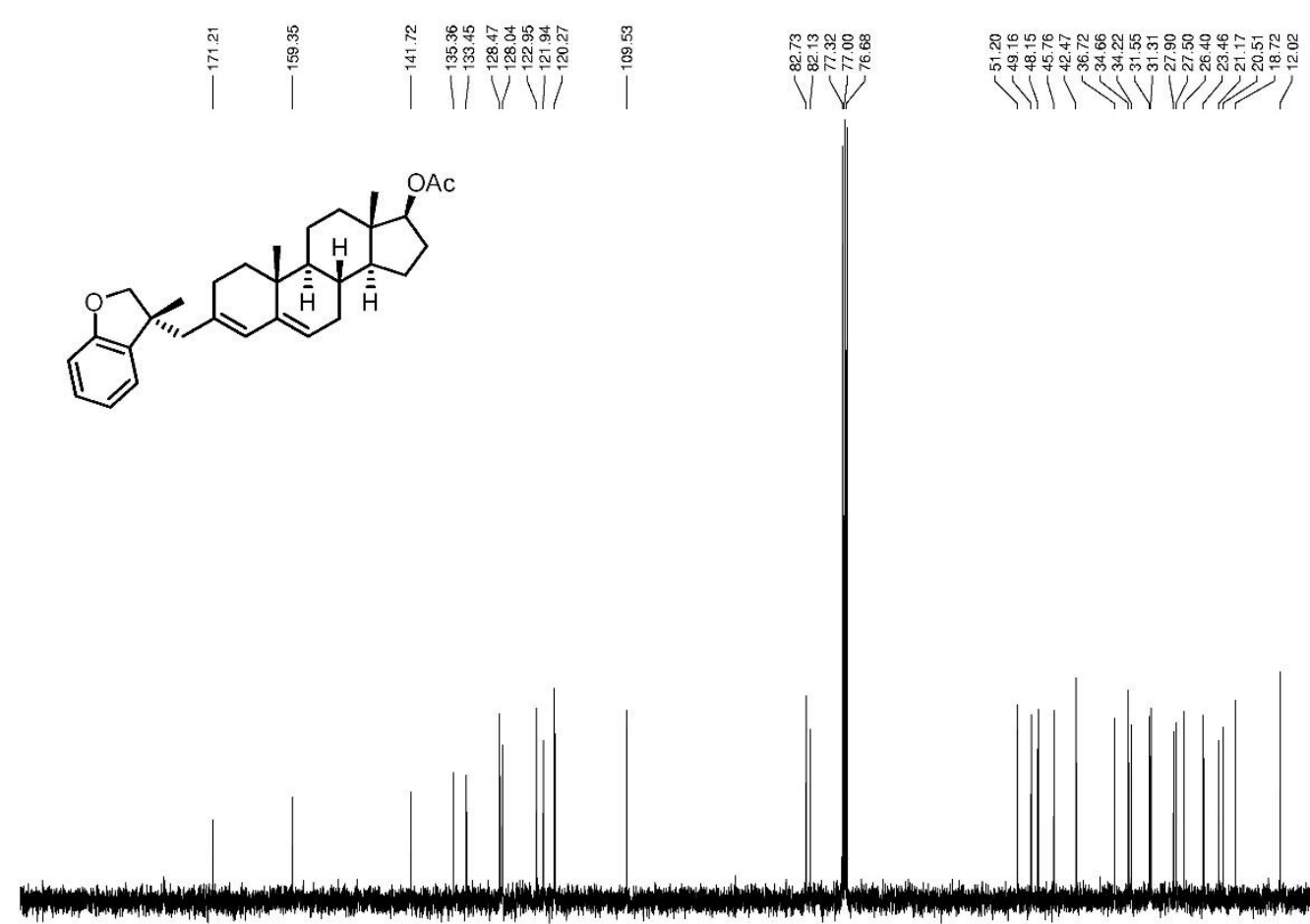

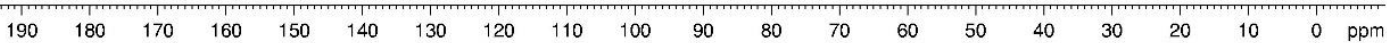

